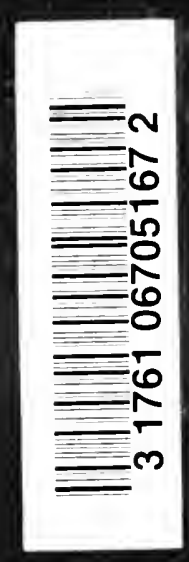

UNiv. of Taromero LIERAKY 
$j+\quad \because \cdots+\cdots$ 
BLWDING LIST DEC 151920 
Digitized by the Internet Archive in 2007 with funding from Microsoft Corporation 


\title{
THE \\ OXFORD MEDICINE ADVANCE PAGES
}

\author{
EDITED BY \\ HENRY A. CHRISTIAN SIR JAMES MACKENZIE
}

CONTRIBUTORS VOLUME I

LEWELLYS F. BARKER

FRANK BILLINGS

RICHARD C. CABOT

HENRY A. CHRISTIAN

CHAS. BENEDICT DAVENPORT

EUGENE F. DU BOIS

FREDERICK P. GAY

LAWRENCE J. HENDERSON

A. WALTER HEWLETT

GUY HINSDALE

Volume I
WALTER B. JAMES

WILLIAM B. JOHNSTON

SIR JAMES MACKENZIE

JOHN J. MACKENZIE

ELMER V. McCOLLUM

FRANCIS W. PEABODY

LEONARD G. ROWNTREE

HENRY SEWALL

DONALD D. VAN SLYKE

WILLIAM H. WELCH

PART I

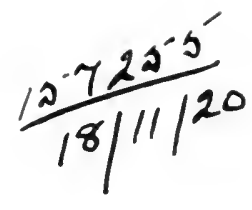

NEW YORK

OXFORD UNIVERSITY PRESS

AMERICAN BRANCH: 35 WEST 32 ND STREET 


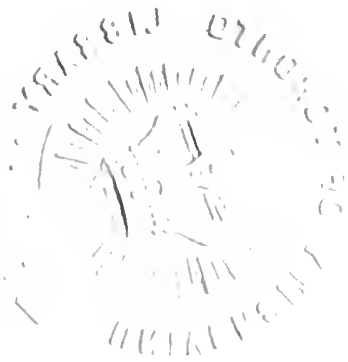

\author{
COPYRIGHT I9I8 \\ EY THE \\ OXFORD UNIYERSITY PRESS \\ American Branch
}


TO THE

\section{MEDICAL MEN OF THE ALLIED NATIONS}

Who Whether in Active Service or at Home

Doing the Work of Absent Colleagues in Addition to Their Own Have Given of Their Best to the Cause of Democracy

These Volumes are Dedicated 

CONTENTS OF PART I, VOLUME I-OXFORD MEDICINE

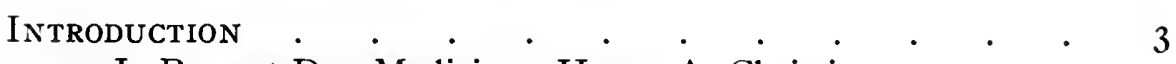

I. Present-Day Medicine-Henry A. Christian.

II. The Future of Medicine-James Mackenzie.

III. The Heritage of Modern Medicine-William B. Johnston.

Chapter

I. The Part Played in Diet by Foods of Unknown Chemical Nature-Elmer V. McCollum. . . . . . 43

\section{Chapter}

II. Climate in Relation to Health and Disease-Henry Sewall 6r 


\section{.}




\section{PREFACE}

IN the midst of war a new system of medicine has been undertaken. The reasons lie in part in conditions created by the war, in part they are found in the present situation of medicine. War in itself has changed the current of thought in regard to all topics and it has created new conditions. Following the war necessarily there will be a period of reconstruction and readjustment. Medicine will play an important part in these changes, and in turn all sorts of new conditions will react to affect medicine. A summary of the present state of medicine should be ready to play its part in this new era.

In the period just prior to this war, medicine was undergoing rapid changes. A functional concept of medicine, to a considerable extent, had supplemented the older structural concept. This brought into use many new methods of studying patients, and from these much new data had been accumulated, correlated, and interpreted in relation to disease. Anglo-Saxon physicians were active in this work, and Anglo-Saxon medicine as a result has contributed much to these newer developments. A number of years have elapsed since a system of medicine in English has been published, and now is an opportune time to issue a medicine by American and English physicians and to present their viewpoints for the reconstructive period of the war and immediately thereafter.

Newer publishing methods make it possible to issue in parts, to supplement at will, and to replace from time to time as views change. Under these conditions a medicine written for the present can be maintained in tune with succeeding periods, and need not lose its value, since it will not get out of date. Such a method will be used in this work.

Representative American and English men of medicine will contribute. Emphasis throughout will be placed on function. Structure, however, will not be neglected, but when possible, it will be interpreted in terms of function. Treatment will receive adequate discussion. A simplified statement of methods of treatment in relation to function is 


\section{PREFACE}

needed. Treatment is perhaps the most difficult part of medicine to deal with, because so much of it remains empirical and inexact. Still we do treat our patients with much benefit to them, and it should be possible to describe the methods advised in such a way that the reader may carry them out for himself. Such an attempt will be made in these volumes.

The first volume will be composed of discussions of general topics. This will be issued in advance in order that it may be read first. Here will be found the general principles underlying much of the newer medicine. These need to be known for an adequate understanding of the discussions of special diseases which will occupy subsequent volumes. 


\title{
INTRODUCTION
}

\section{PRESENT-DAY MEDICINE}

\author{
By Henry A. Christian
}

Medicine, like other sciences, advances by a periodic progression; for a certain time a methor or a viewpoint dominates and the investigations and consequent progress are apt to be along the same general lines. Then comes a new method of study to change the aspect of medicine and a new period is inaugurated. The length of the period varies in relation to the dominance of a method and its productivity. With different periods of development we associate certain names of men, but methods and viewpoint, rather than men, determine periods in the history of medicine; names persist because the strong minds of a period dominate as they develop and apply the methods or interpret their results to their colleagues. Names loom large in retrospection rather than at the time. Perspective often is needed to rate properly the men of a period; names currently prominent too frequently pass into oblivion when it is realized that their part, after all, has been but interpretation and application, while others have been the contributors of the new to the period, and a rare man has been the creative genius whose work largely has instigated the investigations and the methods characteristic of the period.

Virchow's name stands out from the middle of the nineteenth century as the prominent one of a period in medicine which may be termed a period of the dominance of a structural pathology. In the last quarter of the same century a new period in medicine was inangurated by the invaluable investigations of Pasteur, Lister, and Koch, a bacteriological or etiological period. During both these periods, in clinical medicine, as is pointed out in the next part by Sir James Mackenzie, physical signs have been the center of interest as the expression during life of a structural pathology and as the result of causative agents. The physician has busied himself chiefly in the greater part of the nineteenth century with the discovery and the elucidation of physical signs of disease, with 
methods of investigating and recording them in ways bringing them into the ken of our special senses, and with the effort by therapeutic measures to influence physical signs as an index of a change in the diseased condition.

In a similar sense to that which has led us to speak of the third quarter of the nineteenth century as a period of dominance in medicine of a structural pathology the present may be designated as a period of physiological (including biochemical) or functional dominance which had its beginning in the early days of the twentieth century and is now in full swing. Today our interest is more in what an organ or a group of organs can do as an effect of the changed conditions of disease, and in how the body activity as a whole may be influenced by the general or local effects of abnormal conditions, than it is in structure. Structure still is important, for in last analysis no adequate understanding of disease can come until a correlation between structure and function can be made. Today, however, advances seem to be made more along the lines of study of function than of structure.

Unfortunately in this very fact lies a danger. It is but natural to turn toward those methods of study which seem most productive of advance and to concentrate on them to the neglect of other knowledge whose significance is none the less great in the final understanding of the whole. Already we see the evil result of this in the new generations of medical men quite ignorant of many of the facts of pathological anatomy which are fundamental to an understanding of disease, its recognition, and its treatment. We find pathological physiologists and bacteriologists, as well as clinicians, whose interpretation of observations falls into error by reason of a lack of proper fundamental training and knowledge of the methods of pathological anatomy. Both teachers and practitioners of medicine need to keep this in mind and to guard against the fault of neglect of pathological anatomy and its structural concept.

One effect of present-day interest in the utilization of physiological and chemical methods in the study of disease seems to have been a tendency to minimize the value of pathological observation, with a consequent decrease in the number of post-mortem examinations. The percentage of autopsies made on our fatal cases, at least in America, is decreasing. This is most unfortunate for medicine. Even if we grant, as some would claim, that little further advance in medicine is to be expected from post-mortem study - a claim which in my judgment is not true-still thorough examination after death remains the most valuable check which we possess as to the correctness of the results of our ante-mortem studies of patients, and for this reason alone, if nothing 
else was to be contributed, every effort should be made to obtain permission for a post-mortem examination of the body of every patient that dies. That physician who continues to practice medicine and never submits his theories of disease and his diagnosis to the test of a careful inspection of the end-result of disease fails to secure for himself the most valuable check against fanciful theory and absolute misconception. Without it he acquires a dangerous facility in misdiagnosis and a selfsatisfaction in his methods that early become fatal to his progress. The autopsy which reveals to the physician or surgeon his error in diagnosis and treatment stimulates to renewed effort to improve the quality of his work. His next case is examined more thoroughly, is thought over more carefully, and receives a better treatment by reason of his having had demonstrated to him his previous mistake in deduction and conclusion. It is a poor physician, indeed, that does not learn something of value from each autopsy, and this is just as true today as it was in the period of dominance of pathological anatomy in medicine.

Many explanations have been sought for the distressingly small number of autopsies obtained at the present time. Undoubtedly many factors contribute, but I feel sure a large part of the blame lies at the door of the physician himself. In a hospital the chief-of-service fails to appear at the time of the autopsy. His neglect to come tends to create in the juniors the feeling that the autopsy, after all, is relatively unimportant and they grow less keen to obtain the necessary permission for autopsy. Failure to attend is not due solely to carelessness or to having no time available. Too often the chief-of-service of today knows too little about what he sees at autopsy to appreciate fully its value or learn its lesson, for his training has been physiological or chenical to the neglect of pathology, and some do not care to expose their ignorance of pathological anatomy. In hospitals where the visiting staff attends and understands the autopsies, the percentage of autopsies is much larger. Chiefs of staffs and hospital attendants would do well to ponder their own figures for autopsies and search themselves for a cause of the poor showing, if such there be.

In private practice the autopsy has become wellnigh obsolete. The difficulties here are greater, we grant. However, if we turn to the great names of the past we find that our predecessors found means for postmortem examination in private work. Many made their own examinations and became quite excellent pathologists. This custom should be revived. Particularly does its revival become important if Sir James Mackenzie's ideas as to the future advances of medicine come true, advances to be made by the careful observation of the general practitioner to whom come the beginnings of disease, when structural changes 
cannot be detected, and whose opportunity it is to study disease from . inception to ending. How important it is for that type of clinician to follow his problem to its finish, and after death to observe the final effect of what for years he has been watching, as little by little it grew from the stage of symptoms alone to that of physical signs, from the stage of little interference with activity to the stage of bedridden disability and on to the culmination in death!

In this question of post-mortem examination and a knowledge by the clinician of pathological anatomy the medicine of today has taken a backward step. Pathology plays as large a part as ever in medicine, only it is not the sole or chief part. Function rather than structure is our chief interest today, and yet function is in large measure dependent on structure, and to be understood thoroughly anatomical changes need to be known. For this, pathological anatomy is needed as before; only now it is not the final stage of the knowledge that we seek, it is rather one of several means to a larger understanding of medicine.

That our interest has turned more and more toward function is not unnatural. After all, it is what the body can do rather than how the organs look which concerns both patient and physician. Even with the utmost refinements of the technique of the present, the highest powers of the microscope sometimes fail to reveal evidences of structural change in patients, where during life there was every evidence of disorder. These negative findings at autopsy have stimulated investigators to utilize other methods of studying disease. A search for causes has developed other methods of observation than the microscope. The discovery of ultra-microscopic or non-filterable organisms, for example, has necessitated other means of examination than those depending on any form of visibility. These organisms are recognized and classified by their effects, really a functional method.

In the progress of physiology much apparatus was devised to record the function of animal tissues; to seek to apply these in the clinic became the almost obvious thing for the clinician. The clinician's attempts stimulated the effort to create better means of recording and apparatus more suitable to use on man. Soon, as the result of the use of this apparatus, a large body of data became available for correlation and interpretation. Some of this was of a nature that reasonably could be harmonized with what might be expected as the effect of changes in structure, and as anticipated these changes in structure subsequently were found. In many instances, however, by applying these physiological methods changes in function were observed to take place, and yet careful search failed to show any corresponding change in structure. These things stimulated interest in function, and in methods for recog- 
nizing and recording variations in function whether they could be correlated with structural changes or not.

During these same years remarkable developments were taking place in chemistry. Both analytic and synthetic chemical methods rapidly were being applied to the study of organic substances. With increasing knowledge of organic chemistry the chemists turned to the problem of the chemical constitution of living matter. While as yet relatively little is known of the actual chemical structure of living matter, great progress has been made in our knowledge of biochemistry. From the chemists have come many methods applicable to the study in man of the products of body or organ activity. These have yielded new clata expressive of the function of tissues and, as with physiological methods, have often recorded evidences of change where no structural change could be made out.

Very lately important extensions of knowledge have been made with the methods of physical chemistry, and these applied to the living organism have furnished us with more information in regard to the mechanism of body activity. The laws of physical chemistry are very important in an understanding of the equilibrium of body fluids which seems so necessary for life itself. Slight changes in tissue fluids, such as are to be detected by the methods of physical chemistry, frequently are the index of variations in body function where other means fail to show a change and where no structural variation can be detected.

From the bacteriologist, the physiologist, and the chemist (both biochemist and physical chemist) the clinician obtained methods; these methods he has improved in their applicability to man and with them has observed and recorded changes, until he has accumulated a vast store of data in regard to function. Methods of study of function have come to have a place of dominance and from them has been built up a functional concept of disease which, at the present time, plays a very large part in medicine. Functional methods give many new means of approach to diagnosis, and they are now included of course among the ways taught to the student of recognizing diseased processes.

The functional concept of today has had a very large effect on the treatment of disease. The structural viewpoint of the pathological anatomists of the last century tended toward skepticism as to the possibility of improving conditions by treatment. It was natural for the plysician, who appreciated the anatomical changes in such processes as the cirrhotic liver or tuberculous lung, to feel that the damage done was irreparable, and that the destroyed cells could not be regenerated. For many of these not unnaturally there developed a veritable nihilism toward therapeutics. 
In contrast to them the physician of today with means of determining by test the functional efficiency of an organ has learned that often the organ which shows extensive anatomical change does its required work efficiently. Moreover, besides a large factor of safety in function of single organs, they have learned that, under the stimulus of need, other tissues may take on the work of the damaged one. The correlative activities of organs have become of great importance in both an understanding of body activities and an appreciation of ways and means of influencing body function.

Animal experimentation has shown the very great extent of repair and regeneration that is possible after injury of cellular organs. In fact, the extreme power of repair in their tissues makes it very difficult to produce in animals chronic changes such as we see in man in the later stages of disease. Presumably human tissues, too, have great powers to regenerate; only in man we have little opportunity to study them anatomically in these stages. Functional study, however, indicates that extensive reparative processes are possible.

The effects of fatigue and the improvement from rest which we know to occur do not show themselves in structure. Tests of function give us a measure of these fatigue changes, whether they result from overuse or are caused by ordinary use of an organ impaired by disease. In recent years our knowledge has been increased greatly in respect to many kinds of depressed and deranged function where we find no parallel structural change.

The factor of safety in tissues, the power of repair and regeneration, and the effects of fatigue and rest, are portions of the functional concept of today which change our views as to treatment, and give us a different opinion as to the possibility of improvement when adequate measures are applied to the body. Instead of therapeutic nihilism there is the expectancy of good results from properly conceived and adequately carried out forms of therapeutics, in the sense that function is improved even though the anatomical change produced by the disease is permanent.

Another cause has been operative, too, in influencing our methods of treating disease. Investigations in parasitology and bacteriology have yielded much information as to the ways in which organisms act on the body tissues and how these tissues react to the organisms. From such studies have come the protective inoculations against disease on the principle of increasing body resistance in a specific way, and the curative sera, whether antitoxic or antibacterial. A better understanding of nonspecific tissue resistance also has been obtained, and these principles are used in managing infectious diseases.

Of the very greatest importance are the advances which have been 
made in specific chemotherapy. Workers skilled in chemistry have made synthetically long series of substances closely allied in structural formula, and the action of these has been tested on pathogenic organisms in animals, until one has been found which combines the properties of maximal injurious action on the pathogenic organism with minimal deleterious effect on the tissues of the host. In this way we have been supplied with drugs capable of the most marked effect on diseases produced by parasites.

For these various reasons much change has been brought about in the present-day therapeutics, whether the agent used is drug, serum, diet, or physical method. Treatment has become more rational. Of course much of our knowledge of treatment remains empirical, but even purely empirical methods are capable of being tested by means of studying function, and though we may not know the how, still we can feel sure that a given means will influence function in a given direction and so be indicated under certain conditions.

At present we are in a position to make pretty definite statements in regard to the treatment of many conditions. Our knowledge justifies us in eliminating some of the methods of the past and replacing them by better. Some drugs now discarded may find new uses in the future when more is learned in regard to them. It is to be expected that treatment will remain a changing subject about which the final word for any disease rarely can be given. Still, it is possible to outline a simplified method of management for many diseased conditions, a management which in the broad sense is treatment or therapeutics. This should be an important part of a new medicine based on our present knowledge.

In the training of the medical man to provide him with a proper knowledge of the relationship between function and structure, there is needed a balance between the fundamental or basal parts of medicine (anatomy, physiology, biochemistry, pathology, bacteriology, and pharmacology) and their practical applications to man which constitute the practice of medicine. There is an optimum balance to be sought in such applications between general medicine and the specialties, and between the laboratory and the clinic. First the fundamental parts need to be studied by themselves; then the student must be instructed in the methods of examining patients. Next should come drill and practice in methods on the sick man in varying stages of disease with observation as to the results of methods of treatment. It is most important in acquiring a conception of disease to get a proper integration between these two more or less separable parts of the beginnings of a medical education. Ordinarily this requires some return to the fundamental branches, after their application has been practiced for a time in the clinic or in general 
practice. Obviously specialization should come relatively late in development, but the specialties are no immediate concern of this discussion of present-day medicine.

Finally, the man ready to practice modern medicine needs to possess a proper balance between so-called laboratory methods and those of history taking and simple observation. In the immediate past there has been a distinct tendency to overvalue the laboratory and to undervalue the means that made of our predecessors very sound practitioners of the medicine of their day. In respect to these two phases of medicine a changing balance is needed and the medical man must remain versed in both. This balance would seem to be maintained best by an organization of the clinic so that each worker spends part of his time in the clinic and part in the laboratory, finding his problems in the former and aiding their solution by means and methods in the latter. Too continued isolation in either the laboratory or the clinic is bad for the man who is to be a leader in medicine. Neglect of the methods of either would be equally harmful.

In private practice just the same balances need to be maintained. In fact, the medicine of today for the practitioner is dependent upon the power to utilize information about his patients obtained from both laboratory and direct observation. In both some of the work almost necessarily must be done for the practitioner, but the final interpretation of the data has to be made by the single individual, the clinician. Complex methods, especially those yielding numerical values, often have an apparent accuracy which may be very misleading. It is highly important to keep this in mind so as not to be tempted to neglect the old-fashioned and simple methods of observation of the patient, in connection with his story of his symptoms and their development.

Diagnosis, in final analysis, depends on proper evaluation of symptoms and signs, recorded by all available means and interpreted with the critical judgment of a large common-sense. It needs to be recognized that symptoms antedate signs, and that the beginnings of disease may be present and productive of symptoms, when no available method can detect abnormality in structure, and even tests of function record no change. Treatment to be successful must depend on a correct diagnosis. For a diagnosis often many methods of study must be applied to the patient. Methods of treatment always must be properly adjusted to the individual idiosyncrasy of the patient. Continued observation of the most careful sort is needed to estimate the effects of treatment. All of this is required by the man who is to become a good practitioner of medicine. Common-sense and judgment cannot be replaced, safely, by graphic methods of recording, and yet many mechanical methods are 
of the greatest value when properly used and interpreted. The medicine of today furnishes us with many ways of studying our patients; some new, many old, some to detect structural changes, others to measure disturbances in function. For some patients few methods need to be used; for others, many ways of examining must be employed. Experience and critical judgment serve as guides to the necessary procedure in the individual patient.

To the man who works either in the hospital or in the family, the opportunity offered by the medicine of today is great. Much is known; much remains to be discovered. Both the beginnings and endings of diseased processes contain much as yet unexplored. Present-day methorls of studying function and structure furnish to all the means of being investigators. In both private practice and in institutions observations may be made which, as they accumulate, necessarily must throw light in dark places. He who is in medicine both to advance our knowledge and to alleviate suffering in the individual remains, as always, the ideal physician. 


\title{
THE FUTURE OF MEDICINE
}

\author{
By James Mackenzie
}

A NEw work on medicine which has been compiled by authorities on the different diseases may be taken as representative of the state of medical knowledge at the time of issue. The occasion may be taken to consider the stage which medical progress has reached in order to find out if the advance is being made along those lines likely to achieve the best results. This will be found necessary if we reflect upon the history of medicine. The aim of all interested in the investigation of disease is to make of medicine a science, and to be a science it is necessary that there should be a certain degree of exactitude in our knowledge. It is not easy to define exactly what means should be employed to make such a subject as medicine a science. Each science has to be pursued by methods peculiar to itself, and those familiar with the methods in one science are not capable of determining the methods best fitted for conducting investigations in other sciences. Medicine is so distinct from all other sciences that no one, unless engaged for a long time in its practice, can fully understand its peculiar features, and the need for methods specially adapted to its pursuits. It has scarcely been realized, even by those who are engaged in some branch of medicine, how complicated a matter it is, that there are fields absolutely necessary to its purpose which have so far been hardly touched, and that there are members of the profession who have opportunities for the prosecution of research, essential for the advancement of medical knowledge, whose services have not been employed.

Many sciences pass through stages during which they are but a mass of facts without system and arrangement, clouded by beliefs and superstition. Medicine has not yet thrown off much of the credulity that has hampered its progress, and as yet the facts upon which it is based have not been differentiated and classified with that accuracy which is necessary before it can become a science. It is necessary, therefore, from time to time that those who are engaged in the practice of medicine should pause and ask themselves if the object they aim at is being pursued on the right lines. 


\section{The Progress of Medicine during the Past Fifty Ycars}

The methods of investigation, and the type of individual who prosecutes research, have altered during the last fifty years. Before that time the investigator was a man engaged in medical practice, who. recognizing in his patients problems that needed solving, sought their solution by experiments conducted by himself or under his supervision. This was the method pursued by such men as Harvey, Hunter, Jenner. and Lister. This type of investigator has almost disappeared, and research has passed mainly into the hands of the laboratory worker. This has been due to the elaboration of laboratory methods. It is readily conceivable that any new country when it is first opened up will reveal a great many new features. The development of laboratory methods had yielded a remarkable number of new facts and greatly advanced our knowledge. The perfection of mechanical instruments for the examination of the body during life and of the tissues after death has been of the greatest service. Moreover, the germ theory of disease has thrown such a flood of light upon many diseases that the impression has been made, almost universally, that it is only by laboratory methods that progress in medicine towards a science can be made. To such an extent has this conception prevailed that distinguished laboratory workers can assert without fear of contradiction that, in the future, progress in medical knowledge can be made only through the laboratory. The outcome of this conception is seen in the manner in which research in medicine is encouraged. The various governments as well as wealthy donors, desirous of helping humanity by the relief of suffering, give liberally for this purpose. Naturally they turn to medical authorities for guidance, and these invariably direct that the money should be spent on laboratories. Hence, in all countries we see magnificent buildings erected, and able men appointed for the carrying out of research. To these laboratories are attracted all the brilliant and able among the younger members of the medical profession. As generation after generation is brought up under the guidance of the laboratory worker, the belief becomes established that on these lines alone can medical research be advanced, and other lines of investigation are neglected.

Underlying all this seemingly reasonable belief there lies a fallacy. Investigators are now specially trained for their work under men who have spent their lives in the laboratory. The pursuit of knowledge tends to assume ever more and more an academic character, with little reference to the problems confronting the doctor who practices medicine. This is in contrast with the methods pursued by the great pioneers whose 
names I have mentioned. Laboratory workers now get a limited view of disease, and we must recognize that their opportunities permit them to see but a very small part of the field of medicine.

The reaction of these views upon medical practice is seen in the breaking up of clinical medicine into a series of specialities. The patient's examination is not to be restricted to one intelligent practitioner, but he must be scrutinized by a number of specialists. Thus, when a patient consults a physician, the physician not content with his own examination sends him to a series of specialists, and each of these sends in a report dealing with the organ or method in which he specializes. The physician studies these reports and is supposed then to be in a position to treat the patient. This method seems so learned and thorough, and it has so impressed the community, medical and lay, that it threatens to become a necessary procedure in the examination of patients, for we find not only physicians in their private capacity practicing it and medical men associating together for the purpose of carrying it out, but we find distinguished physicians urging its adoption by the state.

This procedure seems so reasonable that it is difficult at first sight to see wherein the fallacy lies. That it is fallacious there can be no doubt, for otherwise one would despair of the future of medicine. It is manifest that if humanity at large is to benefit by medical progress, the bulk of the people would fail to receive adequate medical provision by this method. It is only a few that can afford the high prices that such specialists charge. If institutions, even under the government, are started, only a limited number of the people can be reached. Anyone who has practiced in a large community knows that such a scheme would have little effect, for many patients are bedridden, and the number of ailing people is so great that no institutional means could examine them. Moreover, to such an institution the cases sent would not be necessarily those that were most in need of help. The type of case sent would be those who were manifestly ill, usually with some definite sign which indicated that the disease had already caused destruction or alteration of tissue. The cases in the early stages, before these things happen, would not likely be sent, and if sent, their ailments would not likely be recognized, for the phenomena created by the early stage of disease are not revealed by mechanical means or laboratory methods. The physician trained in the laboratory or hospital ward can recognize neither the mechanism of the production nor the significance of such symptoms as arise in the early stages of disease.

Furthermore, granted that an exhaustive report comes in from specialists who deal with the examination by means of $\mathrm{x}$-rays, blood- 
pressure instruments, electrocardiographs, bismuth meals, test meals, blood counts, urine analyses, microscopic and chemical examinations of the blood, discharges, and excreta, and other methods, for it is manifest that there is no standard that can limit the number of specialists, who is qualified to assess the value of all these reports? The effect of disease is rarely limited to one organ; in many cases the change in one organ is secondary to conditions elsewhere. The specialist whose horizon is restricted to his particular subject is incapable of recognizing the remote causes and effects. Suppose a physician directs these researches, the very fact that he has had to call in the help of the others implies that he is not able to detect the phenomena produced by disease, and if he is not capable of doing this how can he be qualified to assess their value? As a matter of fact, the profession have not yet recognized the importance of this feature, the assessing of the value of symptoms, while in the practice of medicine they have not employed the appropriate methods by which the knowledge can be acquired.

\section{The Life History of Disease}

The best way to understand how medical investigation should be pursued is to appreciate the defects in medical knowledge, and to do this the life history of disease in the body and the phases that have been studied so far should be considered. The onset of disease in the body is invariably insidious and causes little disturbance to the economy and no visible sign of its presence. By and by the patient becomes conscious that all is not well with him, and there is a loss of that feeling of wellbeing which accompanies the healthy state. Disagreeable sensations arise, at first vague, but later they become more definite, and so urgent, that he seeks advice. Still, no evident sign of disease may be perceived on the most careful examination. The disease situated in some organ, as it continues to advance, modifies the tissues of the organ, so that a physical sign is produced and its presence detected. The course hereafter varies; it may end in death or in impaired health, or being of a temporary nature, in recovery. The course of the disease may be a matter of days or of many years, but the general characteristics are the same.

Doctors detect the beginning of disease by the presence of symptoms. Hitherto in clinical medicine the chief progress has been made in the minute study of disease after it has produced a physical sign or after the patient has died; that is to say, after the tissues have been clamaged. It would not be far wrong to describe the last fifty years of clinical medicine as being the era of physical signs. 


\section{Early Stages of Disease}

It is accepted universally that the earlier a disease is detected the more amenable it is to treatment. It behooves us, then, to recognize disease in its earliest stages. If careful consideration be given to the matter, the importance of recognizing the early stages of disease will be realized, and moreover, from the patient's point of, view, it is vastly more important to observe the early stages of disease than to recognize its peculiarities when it has produced physical signs, or when it is found on the post-mortem table.

What are we doing to discover these early stages? The truth of the proposition that the earlier a disease is discovered the more hopeful it is for treatment may be admitted, but it has never been realized how little is done to provide the necessary knowledge to understand disease at this stage.

In the recent past research has been restricted chiefly to laboratories and in a less degree to hospital wards. Consider the stages of disease that are studied there. It is unnecessary to dwell upon the careful study in the post-mortem room, as that is a stage which does not immediately interest the patient and does not concern the beginnings of disease. In hospital wards we find the patients with disease so far advanced as to present a physical sign, and we all know what time and care is spent on teaching the physical signs. Little interest is excited in the student unless there is something he can either see, hear, or feel, with the result that the well-trained student leaves the hospital convinced that he has grasped the essence of medicine because of his ability to detect a physical sign and to know the mechanical basis of its production. Moreover, in the more advanced schools, where there are clinical laboratories, it is usually to the wards that he is attached. This gives the opportunity of confirming the knowledge of the cause of the physical signs, and stamps the impression on the student of the essential importance of physical signs. We see, therefore, that disease, when it has reached an advanced stage or after it has killed the patient, has been thoroughly studied.

But what of the stages that preceded? What of the time, it may be years, of the gradual progress of disease before the production of a physical sign, the time when the disease was amenable, it may be, to treatment, or more amenable to mitigation than in the later stages? The patient in the early stages shows no objective sign, but he has a definite sensation which tells him that all is not well. We all know that such patients go to the out-patient department. Here we have the younger members of the hospital staff, men who have been trained in the hospital wards, where the training has made them adepts in the detection of 
physical signs. What is their attitude? I have visited many hospitals in different countries, and the procedure is wonderfully alike; wherever I have gone, a patient with no physical sign receives, as a rule, little consideration, but when a physical sign is detected he may be sent to the hospital ward. This attitude toward a patient without a physical sign is revealed by the scant knowledge we possess of the symptoms in the early stages of disease.

It will be granted that subjective sensations are the earlier signs of disease, but so far the profession have been so little trained in examining a patient when the disease presents only subjective sensations that the out-patient physician has not known how to set about the examination. The reason why I make this statement is that the sensations of a patient have never been studied with the care and precision which would enable any teacher of the present day fully to appreciate them.

There is not a single sensation which man is capable of experiencing that has been thoroughly investigated. Until that is done we shall be incapable of detecting the early stage of disease, and it is because this has not been done that I state that the out-patient department has not yet been fully utilized. Take the most universal of all sensations, that of pain, a sensation which, if understood, would reveal the early stages of disease in a large number of cases. Yet we are ignorant of the most elementary facts necessary to its understanding. We do not even know the tissues capable of producing pain, and we have but the slightest knowledge of the nature or kind of stimulation that can induce pain. We are to a great extent ignorant of the mechanism by which it is produced, and of the laws governing its radiation we have no knowledge. We know, for instance, how informative the spread of pain is in heart disease or renal colic, because the distribution of the pain is so remarkable; but the distribution is equally informative in many other diseases of which we know little or nothing. As one who has labored much at the subject, I assert that, once we have discovered all the facts concerning pain, a stride forward in our knowledge of clinical medicine will have been made which will lead to some of the greatest discoveries in merlicine. What is true of the neglect of the study of pain is true of every other sensation, such as exhaustion, giddiness, faintness, palpitation, nausea, heartburn, and breathlessness.

It is evident that the study of the early stage of disease is of the very first importance, that it is fraught with difficulties, and that the recognition of the nature of the disease is infinitely more difficult at this stage than when a physical sign is present. It will be necessary to alter the practice in hospitals. At present the easily discovered stage of disease, and the most hopeless for treatment, is by custom handed over to 
the experienced physician, while that which is most difficult to diagnose and the most hopeful for treatment is placed in the hands of the least experienced. These conditions need to be reversed.

\section{Secondary Diseases}

There is another matter that has never been clearly understood, and that is the aspect of disease as presented to the family doctor. Those whose experience of medicine has been limited to the laboratory, the hospital ward, and the consulting-room, have no idea of the difference between their practice and that of the general practitioner. I may point out that the bulk of the general practitioner's patients present none of the physical signs that were taught him in hospital. The complaints are chiefly subjective in character and the physical signs of an elusive and evanescent nature that were overlooked or ignored in his hospital experience. I have already said that these indicate the early stage of disease, but many do more than this; they indicate the presence of some affection which in itself may be of little moment, but which may, by lowering the vitality of the patient, prepare him for the onset of other more serious diseases. When engaged in general practice I was struck with this difference in the character of the complaints, and as time went on I found that the symptoms to which I had paid little attention frequently were evidences of the onset and progress of serious diseases, or diseases appeared in other organs remote from the site of the original complaint. Thus, in an analysis of one thousand consecutive cases there was a vast predominance of digestive troubles, but the full extent of this predominance could not be made out, as many patients suffering from diseases elsewhere gave a history of early digestive trouble. In later years in consultation practice in London I saw many cases of heart affection, and I found a large proportion of the cardiac disabilities were secondary in development to intestinal affections. In seeking an explanation for this I became aware of Arbuthnot Lane's views, and found in my own experience much to support them.

It must strike everyone that, notwithstanding the enormous amount of work and money that has been spent on the study of consumption, we are still far from understanding its real cause or cure. It is manifest that there is more in the matter than the presence of the tubercle bacillus. It had long seemed to me there was another factor because, when I reviewed all the cases that occurred in my family practice, I found that every one had suffered from other complaints, years before the outbreak of consumption. I was at a loss to understand whether this was due to a latent infection or to a predisposing complaint. When Lane put forth 
the view that the toxins from intestinal stasis lowered the vitality and rendered the individual susceptible to infection, I reconsidered my series of cases of consumption, and found that a considerable number had been under my care for years suffering from gastric ulcer, a condition which Lane maintains is itself secondary to the intestinal stasis. A simple illustration may help to give a clue to the mystery still surrounding consumption. A man suffered from a pustular eruption in his hands and arms and consulted a bacteriologist, who, examining the pus microscopically, detected an organism. He cultivated the organism and made a vaccine from it. This he injected into the man. The treatment was persisted in for six months, and there was no improvement. The man consulted another doctor, who found he was suffering from scabies and promptly cured him.

There are several morals to be drawn from this experience, but the one that is appropriate here is the suggestion that the failure to cure consumption by all the elaborate means at our disposal is probably due to the fact that the tubercular process, like the pustular eruption, is a superadded disease, and the proper line of treatment would be the removal of the primary cause of impaired health. Inasmuch as it is now known that a great many people have a tubercular infection, and never suffer from consumption or other serious tubercular disease, it is manifest there must be some other factor than the tubercle bacillus. The recognition of this factor can be attained only by the study of the patient before he shows any signs of consumption, and the opportunity for doing this exists neither in a laboratory nor in a consumptive hospital, but is afforded the family doctor.

\section{The Classification of Diseases}

The reason given for the need of a body of specialists to examine one patient is that medicine is becoming such a complicated concern that one man is incapable of understanding all its phases. This view should at once arouse the suspicion that the pursuit of medicine is not on right lines, for the more a subject tends to become a science the more it becomes simple and easy to understand. That this should be so, a little consideration will render clear. A great number of phenomena may be perceived and so long as they are isolated there may be a difficulty in studying them while their individual qualities form the only points for observation. But when they are viewed as the manifestations of a common cause, then they can be grouped or classified according to natural affinities and their presence and peculiarities can be accounted for easily. This is seen in the evolution of such sciences as chemistry, botany, and astronomy. 
The advance of medicine, so far, has not permitted diseases to be classified on any sound principle. Medicine is still in that stage when it consists of more or less disconnected facts. Diseases themselves are not clearly recognized, and often the symptoms they produce are mistaken for the disease itself. Such classifications as exist, as that based on the affections of separate organs, imply a limited outlook, and a failure to recognize the fundamental principles involved in the production of disease. Some glimmer of the true nature of what a scientific classification should be is to be found in taking a broad outlook on such a condition as disease of the arteries. Where there is a general affection of the arterial system, little evidence can be obtained from the examination of the arteries themselves, except in advanced degeneration, as in aneurism. The evidence of arterial disease is shown not by observing the arteries, but by recognizing the perverted functions of a great variety of organs. As the blood supply of these organs is diminished, the impaired nutrition produces effects on the structure of the organs and on their functions. In some people these effects are more pronounced in one organ, while in other people other organs are more affected. In this way we get a representation of a great variety of diseases, while in any given case the organ whose symptoms are the most in evidence dominates the field, and custom associates the patient's state with these dominant manifestations. Where organs have a variety of functions, as the kidney, heart, and brain, the effects of the impaired nutrition may affect chiefly one or more of these functions, and then we get the disease of the organs split up into different varieties and these varieties are called diseases, and named according to the disordered function whose symptoms are most in evidence. But even in such an illustration we realize only part of the true state of affairs, for there is some underlying cause producing the arterial disease. We do know that in some cases syphilis may be an agent, and there is a great variety of other conditions assumed to be the cause, but most of these assumptions are little better than guesswork, as the methods of study hitherto pursued are incapable of bringing the real causes to light. Hence we get the position we are in at present, a vast accumulation of data with no clear conception of their significance. The disadvantage of this confused accumulation of disconnected or imperfectly related facts is shown in the measures taken to combat disease, particularly in the field of therapeutics. Anyone, who seriously studies the attitude of the profession towards remedies, will find great differences of opinion among authorities, some having faith in certain remedies, others faith in other remedies, and others again with no faith in any. From this the conviction arises that there is something at fault in the conception of medicine. This misconception will be found to lie 
in the fact that we are not yet in a position to know the meaning and significance of the symptoms of disease.

\section{The Classification of the Symptoms of Discase}

The presence of disease is revealed by certain manifestations which it produces and which we call "symptoms." It is, therefore, incumbent that these evidences of disease be studied with the greatest care. Considering that the account of every disease described in textbooks consists of a record of symptoms, and that there are large volumes published specially directed to symptoms, it might be assumed that this subject at least had been thoroughly investigated. No doubt a great deal of labor has been spent in finding symptoms, and an enormous number of symptoms have been recognized, but the data thus accumulated have never been analyzed and systematized properly, so as to bring out the full significance of symptoms, and progress will be hampered so long as the significance of individual symptoms is not understood. At present symptomatology is so confused that the information which symptoms are capable of giving has not been appreciated. It may be taken for granted that until symptoms are classified on a basis having reference to the principles underlying their production, medical progress will be delayed.

I have referred to the fact that the past fifty years has been a period devoted to the recognition of physical signs. There is much more required than the detection of such signs. As a rule a physical sign merely demonstrates that there is something abnormal in the condition of the organ, and it rarely gives information of the functional efficiency of the organ. Phenomena provoked by impaired efficiency have received relatively little attention, and there has been no clear differentiation between the symptoms produced in these two ways. There is another group of symptoms, which is entirely missed by the laboratory worker and production of which has been realized only imperfectly by clinical observers, and that is the group of symptoms produced by the stimulation of the central nervous system by the diseased organ. I have referred to the lack of knowledge concerning pain. In addition to pain there are a number of other symptoms which indicate clearly the region in the central nervous system stimulated by a remote organ. It never occurs to the average clinical observer that the phenomena by which he recognizes many diseases are produced by an irritable focus in the spinal cord. The symptoms in appendicitis for instance are mainly, and in many cases entirely, the outcome of such a focus. Thus, the situation of the pain, the hyperalgesia of the skin and deeper structure of the external body wall, the increased reflexes of certain muscles, and the tonic con- 
traction of some part of the musculature and the abdominal wall, with, in some cases, great irritability of the bladder, point to an irritation of the spinal cord in a well-defined region. This irritable focus, no doubt, arises from a peculiar stimulation worked by the diseased appendix. The symptoms in angina pectoris and of certain diseases of other organs are produced in a similar manner. A study of this phase of the problem is needed badly.

\section{The Assessment of the Value of Symptoms}

The final and most important of all branches of medicine is the assessing of the value of symptoms. As soon as a doctor is engaged in general practice he finds out the importance of this aspect of symptomatology. After detecting a sign, the patient's inquiry, expressed or implied, is naturally-What is going to happen to me? Is this a sign of disease that will shorten my life or cripple me? Does it call for treatment or is it amenable to treatment? The answers imply a knowledge of symptoms which no hospital or laboratory training can ever completely supply, and yet it is the most important of all forms of knowledge, if we are ever successfully to combat disease.

This knowledge of how to assess the value of symptoms so absolutely necessary to the progress of medicine distinguishes clinical medicine from all other sciences and all other branches of medicine. For its acquirement, methods and opportunities peculiar to itself are necessary. While its importance and even its cardinal necessity are widely recognized, yet the methods by which this knowledge can be acquired have not yet been understood. It has been assumed tacitly that this knowledge has already been acquired, and that it is a field of knowledge that requires no special training for its acquisition. This, however, is a great mistake. We can easily find evidence of the failure of medicine from this lack of knowledge. At this time of national stress, when every available man is called upon for war service, the burden is thrown upon the doctor of determining the fitness of individuals. The military medical authorities thought that the knowledge was easily acquired and that any qualified man, however young and inexperienced, was capable of undertaking the work. To assist them, certain regulations, vague and often misleading, were drawn up. It is now everywhere acknowledged that the results of recruits' examinations have been so unsatisfactory that radical changes have had to be made, and this has resulted from the fact that the knowledge of how to assess the value of the most ordinary symptoms nowhere exists. Even in life assurance, where the most experienced members of the profession are engaged, the basis on which the prospect of life 
is valued when some abnormal sign is detected, is to a great extent a matter of guesswork. We can see that by reading the books of those who reckon to guide the profession in such matters. Occasionally one physician, more critical than usual, realizes this and describes his limitations. Thus, Sir William Gairdner, in discussing the matter in relation to heart murmurs, states: "Whether any data can be procured that will enable our successors to deal with cases of extra risk-we have none, and so deal with them either by loading or rejecting, or shutting our eyes." How true this is, can be verified by anyone who turns to a textbook to find out the prognostic significance of the simplest and most obvious signs. When it is borne in mind that such signs daily are confronting the general practitioner, and that the question of assessing their value is arising continually, it will be recognized that the matter is one of urgent concern. Nevertheless, it is a matter dealt with in so vague a manner that no clear information is anywhere to be had.

But the defect in the knowledge hampers medicine in every way. There are people who, desirous of keeping in good health, reckon that if they are periodically examined by a doctor they may be prevented from falling ill. But more harm than good may be the outcome; for if some aberration is detected, it may be a shadow in the lungs when examined by the $\mathrm{x}$-ray, or a murmur or irregular action of the heart, the doctor, unable to assess the value of these signs, may give such warnings as seriously to disturb the patient and put him to endless. trouble and expense to avoid an illness that was never likely to arise. When there is distinct evidence of an active disease the same uncertainty prevails. We all know that many patients with such diseases as gastric ulcer or appendicitis recover without operation procedures. There are nowhere to be found clear indications when to operate and when not to operate. Some surgeons would remove every suspicious appendix, because, though they recognize that some are without danger, yet being unable to distinguish them from the dangerous, they prefer that the patient should not run the risk. What is this but an acknowledgment that they cannot assess the value of the symptoms? When such a surgeon says that the symptoms are not sufficient to serve as a guide, he but deceives himself, for the data are there, only he is unable to interpret them.

\section{The Opportunities of the General Practitioncr}

It is manifest that if symptoms are ever to be properly valued, it can be done only by those who have the opportunity to watch the individual patients through long periods of time, who see the disease at the earliest 
stage, or even before its inception, and who can observe its progress through all the vicissitudes of life. Manifestly that cannot be done by the worker in a laboratory or in a hospital ward. On the other hand, consider the opportunities of the general practitioner. $\mathrm{He}$ is the only individual in the medical community who has a broad outlook on medicine, whose life work gives him the opportunity of seeing all parts of medical knowledge in its true perspective. He sees the conditions which predispose to disease; he sees its inception and the course it pursues, when it is amenable to medical treatment, or passing to the time when it calls for surgical interference. He sees the after-effects of the operation, when the surgeon may claim it as a success. If he cares to inquire into the symptoms of disease he is brought into contact with every special department, and has opportunities for estimating them at their true value.

Yet the general practitioner has no say in medical education or research, or even in making the laws which bind him to an unintelligent performance of his duties. There are fields of research which block the advance of medicine, which can be worked only by him. Yet what is done to encourage him? Money is poured out for research, but no one ever thinks of giving him a grant or helping him to make use of his opportunities.

\section{The Place of the Laboratory Worker in Clinical Medicine}

It has been a misfortune to clinical medicine that the laboratory worker has been allowed to dominate the field of research. In many cases he has gone after purely academic problems, leaving unrevealed a great many phenomena which baffle the physician in his work. The faith in an involved technique has resulted in the simple and easily recognized manifestations being too often ignored, while those that are revealed by some elaborate mechanical implement are extolled as being the more scientific. This view has been forced upon me by studying the attitude of the profession towards heart failure. Over fifty years' careful study of this matter has revealed to me that heart failure can be perceived only in its early stages by recognizing the sensations evoked by the patient in his response to effort. These are varied and at first difficult to distinguish from sensations due to other conditions. Nevertheless, a long-continued study enables one to get a true comprehension of the heart's efficiency. Yet we find the vast bulk of investigators unable to recognize these very simple manifestations, placing their trust in mechanical methods, never realizing that these instruments probably cannot reveal what is essential in the study of heart affections-the efficiency of the heart muscle. 
The chief use of laboratory methods is to throw light on the manifestations of disease which can be perceived by the unaided senses. Once any particular method has given help in elucidating a sign or symptom it need not be employed in practice, though it may be used for demonstration purposes in teaching and for the prosecution of further inquiries. It should, therefore, be the aim of those who have devised some technical method to hasten to get rid of its need for employment in everyday practice. The importance of this is manifest. If, for instance, a bacteriologist discovers an organism which produces disease in man, he should not limit his observation to studying its peculiar behavior in culture media, or in guinea-pigs, but should recognize the manifestations of its presence when it has gained entrance into the human body. It must be evident to everyone that if bacteriology is ever to have its full influence on medicine, other methods of investigation must be pursued besides those which are undertaken in laboratories. The vast majority of the population cannot have the benefit of laboratory investigation, nor is it necessary that they should have, for this reason, that on the entrance of the microbe into the human body certain phenomena are produced, and these phenomena are peculiar to the individual microbe. We infer, for instance, that measles, scarlet fever, and smallpox are all due to microbic invasion, and although the microbes have not been identified, the evidences of their invasion are well known. The invasion of the body by other microbes will give just as clear evidence, only as yet we do not know how to search for and recognize the evidence as pointing to that organism as the cause.

The use of special methods can be superseded in a great many cases. It is stated by those who advocate the examination of patients by specialists, that their heart's action should be recorded by means of the polygraph or electrocardiograph. These instruments undoubtedly have been of the greatest value in differentiating the different forms of heart irregularity. Beyond this they have failed to shed any light of practical value. By carefully relating the records obtained by these instruments with the signs revealed by the finger on the pulse; by auscultation, and other simple means, the different irregularities can now be recognized without the aid of these instruments, and if so recognized, they need no longer be used. These instruments will always be of use for teaching purposes and for the investigation of obscure irregularities and the action of remedies, but only in very exceptional cases will they be required in practice.

If in other conditions a systematic attempt be made to correlate the symptoms perceived by the doctor's unaided senses with the results of observation made by elaborate methods evolved in the laboratory the 
same result will be attained, whether these involve instruments like the $\mathrm{x}$-ray, or chemical or microscopic examinations. Of course, there will always be some cases where these methods will help, just as there will always be a residuum on which they will fail to shed any light.

\section{Conclusion}

In dealing with the future of medicine in this manner the object is to call attention to the defects in our knowledge. If it is once grasped clearly and definitely, where there is a lack of knowledge, the first step is taken to make good that defect. Where there is an absence of a clear perception of the deficiencies in knowledge, even if it is recognized that our knowledge requires supplementing, the efforts taken to supply the deficiencies are certain to be so ill-directed that much labor will be spent in unprofitable undertakings. From the foregoing considerations it can be inferred that there is an urgent call to the clinical investigator to view his position from a standpoint different from that to which he has been accustomed. There is a great field still unexplored for the detection, and for the assessment of the value of symptoms recognizable by the unaided senses, or by simple mechanical aids. The class of investigators must be enlarged to include those who have the opportunities of seeing disease in its earliest stages. The clinician must take a part in the laboratory investigations in order to solve the numerous problems that will arise if he sets himself to find out the significance of the signs of disease. If he does not himself actually carry out laboratory experimentation, he must at least suggest the theme or direct the method.

This does not imply the supersession of the laboratory investigator, but rather the extension of the laboratory conception to include the man who comes with a problem direct from the practice of medicine. This calls for the closer association of the clinical observer with the laboratory worker, so that on the one hand the clinician will be enabled to understand the mechanism of the symptoms of disease, while on the other hand he will be able to detect in the human body the signs which correspond or are related to the phenomena discovered by the laboratory technician. 
$96^{a}$

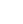




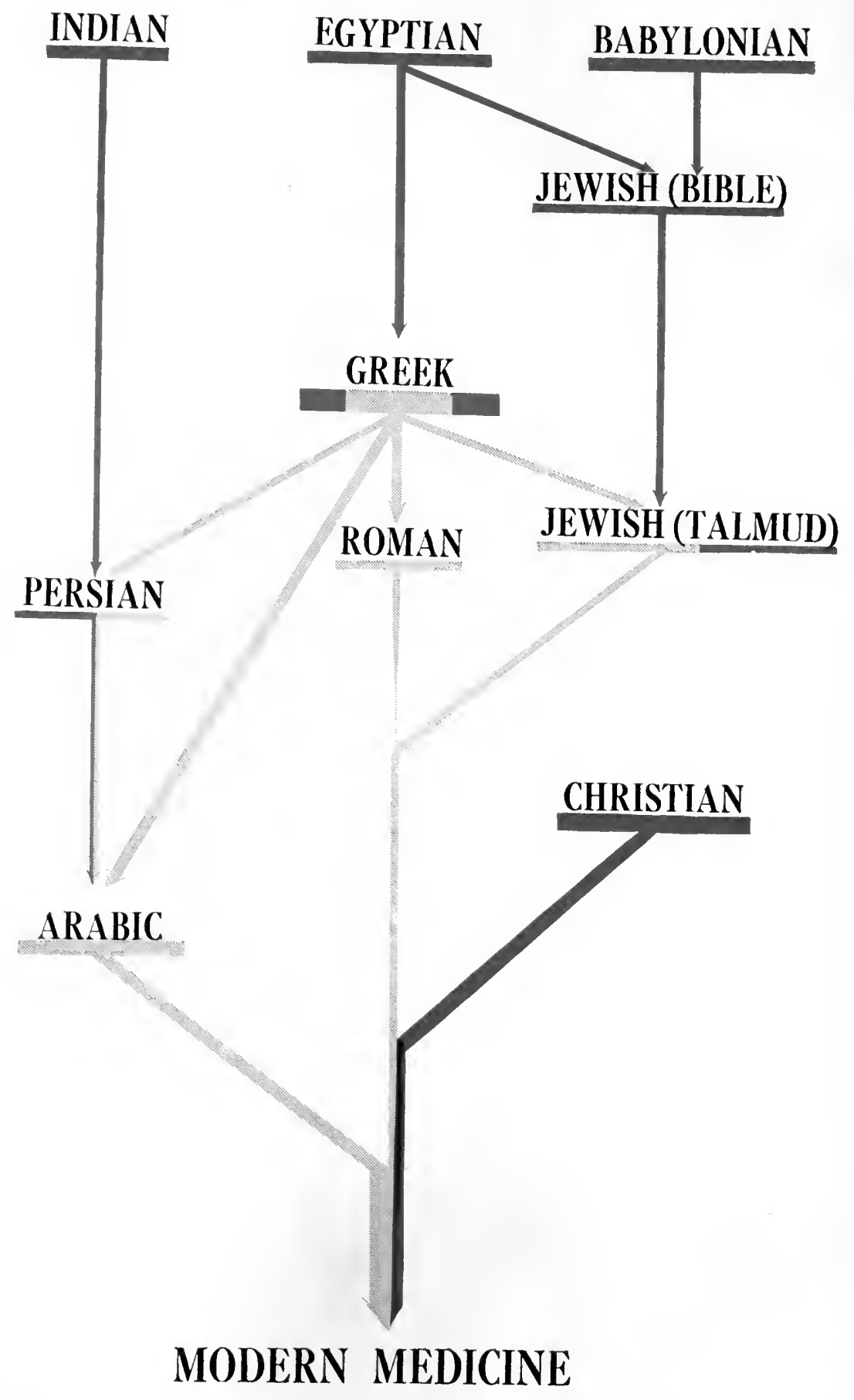

SACRED

SCIENTIFIC

ALTRUISTIC

PLATE I 


\title{
III
}

\section{THE HERITAGE OF MODERN MEDICINE}

\author{
By William B. Johnston
}

MANY of the facts upon which the practice of medicine is based are as old as the history of the human race itself; but the ever-widening stream of intellectual and social activities, scientific and altruistic, embraced within the term Modern Medicrne has its sources in the cultivated classes of Greece and among the simple folk of Palestine. Hippocrates, the friend of poets and philosophers, was the first physician to free his own mind and the minds of his contemporaries from the inherited obsession of the divine or demon origin of disease, and to lay the foundation of modern medical and surgical science. The organized care of the sick and of the incapable was the direct outcome of the teaching of Christ and his disciples.

The earliest historical records picture the practice of medicine entirely in the hands of the priests or priest-physicians, who, as interpreters of the will of the unseen gods and goddesses, claimed to possess the most potent charms and therapeutic measures. Magic was more powerful than medicine. There is abundant proof, however, that in India, Assyrio-Babylonia, and in Egypt gradually there grew up a body of lay-physicians who were in excellent standing in the community, who were protected by law, and even were maintained at the expense of the state. Medical instruction was given in the temples or in schools attached to the temples, which were the forerunners of our modern universities. The immemorial custom of committing to memory those parts of the sacred writings and traditions which dealt with medicine, and in learning the rites and incantations appropriate to the performance of operations and to the administration of drugs, was supplemented by observation of the sick who came to consult the priests or to sacrifice in the temple.

Egyptian specialists, with their multitude of salves and medicinal preparations, were celebrated throughout the Eastern Mediterranean and were in demand among their Assyrio-Babylonian neighbors, whose practice, of a crude sort, was much concerned with priestly interpretations of the stars and with the ritual of examining the viscera of sacrificial 
animals. On the Indian peninsula practical instruction in surgery, the acquiring of manual dexterity by operating upon bladders, upon gourds, and upon the stems of plants, developed a proficient class of surgeons, who, with the help of narcotics, performed successfully difficult operations. Influenced by his many years in Egypt, Moses, in creating his ideal state, placed the supervision of the public health in the hands of the priests, who even exercised the powers of sanitary inspectors. Meat inspection, so important in the life of the Jews, was probably a development of the Babylonian ritual gradually introduced into the sacred law after the Babylonian captivity.

Though warped and distorted by superstitious beliefs, these early civilizations showed, in addition to their extraordinary skill in art, architecture, and engineering, a keen sense of justice and a marked capacity for administration. Their point of view in regard to the ordinary affairs of life greatly resembled that of the present day, and, though detailed evidence is slight, we can conclude that in their hands the practice of medicine was not entirely divorced from careful observation, intelligent consideration, or from sane and skillful treatment.

It is impossible to say exactly how much of this Eastern medical lore and practice was known to the ancient Greeks. In common with all early peoples they believed in the supernatural origin of disease, evolved a class of priest-physicians, and depended for the cure of their ills upon sacrifices to their gods and upon the interpretation of dreams and omens. Unlike the more easterly races, however, the Greeks showed a very marked tendency to take a more rational and less grotesquely superstitious view of life. The Greek gods and goddesses, generalizations of human qualities, possessed the form and characteristics of human beings and were supposed to take a lively interest in the welfare of their worshipers.

The temples, built in honor of the mythical God of Medicine, the first physician. were, in many respects, large sanitoria where buildings, decorations, site, surroundings, and organization combined to create in the most health-giving situations an atmosphere of serenity and of hope. The attendant priests, though disclaiming to cure otherwise than through the divine aid of Æsculapius, whose colossal image in ivory and gold filled the inner sanctuary of each beautiful temple, placed as much importance upon a carefully regulated regimen of diet, baths, and recreations as upon the sacrifices and the expectant sleep before the statue of the god.

As the apparently dying and incurable patients were excluded from the precincts of the temples, and as the God of Healing did not always 
deign to bring about a cure, there were gradually formed in the temple neighborhoods groups of those lay-physicians, the Asclepiadæ, who, claiming descent from Æsculapius and being inheritors through many generations from father to son of the Hellenic medical traditions, had long practiced their art among the Greeks. Between them and the priests there existed no unfriendly rivalry or competition. They were free to follow cases under treatment within the temple and to consult the votive tablets, records of cures, placed in the sanctuary by generations of devout and grateful pilgrims. These groups, gradually increasing in size and in importance and admitting into their society all who wished to become physicians, formed schools or guilds where the theories and practices of the day, differing slightly in each locality, were taught to individual pupils by discourses and at the bedside. The famous socalled Hippocratic oath, to which all students had to subscribe before being admitted to membership in the society of the Asclepiadx, witnesses to the seriousness of purpose and to the high ethical standards of these early physicians.

The tendency to philosophize inherent in the Greek mind and the eminence of the philosophers, many of whom were also physicians, colored the teaching in all the schools. At the Greek colonial town of Croton in southern Italy, Pythagoras, who spent many years in Egypt. taught, in addition to the importance of understanding the structure and functions of the body, a philosophic doctrine tinctured with Eastern ideas of metempsychosis and the prevalence of demons and other mystical beings as the causes of disease. Empedocles, in his attempts to reach the four essential elements, influenced profoundly the current conceptions of physiology and pathology.

It was into this world of active speculation, where philosophy and medicine were inextricably entangled, that Hippocrates, the son and grandson of distinguished physicians tracing their ancestry to Æsculapius himself, was born. Genius can develop in a desert, but the richer the soil the more luxuriant and the more perfect will be the growth. Fortunately for the world Hippocrates was from his earliest years surrounded by influences which enabled him to become not only a great practitioner of medicine and surgery, but one of the most high-minded, modest, kindly, well-balanced, and intellectually fearless men of the world. The end of Greek education was not technical training, but the harmonious development of body and mind. The study of music and of the poets was intended to inspire lofty emotions, to soften and to humanize the soul. Rhetoric, which included training in eloquence and the study of ethics and politics, sought to arrive at clear conceptions of the basic principles of conduct, to induce clear thinking and a careful 
wording of the thoughts expressed. Training in the gymnasium aimed to produce a harmonious development of all the muscles, to achieve physical perfection.

Under the direct supervision of his father, as was the custom among the Asclepiadæ, the young Hippocrates began his medical studies on the island of Cos, his birthplace. His naturally acute powers of observation and his definite bent in a practical direction rapidly developed in the congenial atmosphere of the long-established and already famous Asclepiad school at Cos. The objective investigation of disease, the study of etiology, and an insistence upon the value of the expectant treatment combined with mild therapeutic measures, which distinguished the teachings of Cos from the more subjective methods of the nearby school of Cnidus, gave him the foundation upon which he built with such power and effect. A further period of study in the stimulating world of Athens, then at the height of its glory, followed by travel in Thrace, Thessaly, and Macedonia, increased his knowledge of men and of disease and brought him into close contact with currents of contemporary thought and the accumulated knowledge of the past. True to the Greek type, his mind was constantly concerned with the search for fundamental principles. His writings, simple, forceful, and direct, give the picture of disease rather than of diseases. They include all that was important and essential in the teachings of the schools and declare with a distinctness remarkable for that superstitious and speculative age that disease is the result of natural causes which are to be searched out and considered in the most critical spirit until all sources of error are, as far as is humanly possible, finally eliminated.

The necessary forward step had been taken. Following upon Alexander's ambitious conquests, the writings of Hippocrates and of his followers were spread over the greater part of the known world, setting a standard of professional conduct and of scientific attainment and opposing a bulwark against the still superstitious mental attitude of the East.

With the final intellectual life of the Greek creative impulse, the center of intellectual life shifted to Alexandria, where Alexander's general, Ptolemy, and his descendants concentrated the trade and culture of East and West. Here, in the handsome and spacious Museum and Serapeum, the spirit of Hippocrates and of Aristotle, the son of a physician, founder of comparative anatomy and embryology, and the second great scientist of the Hellenic Golden Age, dominated the minds of Greeks, Egyptians, and Jews. Alexandria offered all the external stimuli to intellectual activity; such as, well-stocked libraries and museums, highly paid professorships, and a large body of students drawn from 
many countries. Enriched and stimulated by the work and example of Euclid and Archimedes and by the continued efforts of the Ptolemies, themselves men of capacity and cultivation, Alexandria became the clearing-house and point of distribution for all knowledge. For the first time in the history of the world anatomy was systematically taught by dissection of the human cadaver. The supply of material was abundant. Two physicians, Herophilus and Erisistratus, pupils of Cos and Cnidus, made many accurate and valuable contributions to the newly born science of human anatomy. They rose to great prominence, advanced the practice of surgery, and founded schools of medicine which for many centuries continued to attract the students of the world.

The Jews, who, as a race, were decidedly opposed to magic of all kinds, showed a great aptitude for scientific medicine and flocked to Alexandria. They carried with them into Palestine and Asia Minor the standard of Greek medicine and helped to maintain a closer connection between Alexandria and the extensive Hellenic kingdom which Seleucus, another of Alexander's generals, had established in Babylon and in Persia. They helped also, at a later date, to carry the lore of Egypt and the classical learning of Greece to the new Empire of Rome.

Roman medicine, like Roman art and general culture, was from the first a borrowed product. The worship of Æsculapius had long preceded the arrival of the scientifically trained Greek physicians, the first of whom, Asclepiades, a man of wide education, practiced in Rome during the last century before the coming of Christ. Of the many highly trained and cultivated Greeks who, in the first centuries of the Christian Era, lived under the protection of the powerful Roman Empire, Aretrus, Dioscorides, and Galen exercised the greatest influence upon the future development of medicine. The works of Aretæus, of whose personality practically nothing is known, show an originality, breadth of view, comprehensiveness, and clearness found only in the writings of Hippocrates. He described vividly many distinct forms of discase. Dioscorides, a military physician, who had unusual opportunites for travel throughout the Empire, compiled the first Materia Medica; i.e., a complete list of the medicinal agents then in use, with a description of their therapeutic action. Galen brought from his birthplace at Pergamum in Asia Minor, where there long had been a celebrated school of medicine, from travels in Egypt, and from the still famous schools of Alexandria, a wealth of scientific information and a skill in practice which soon placed him at the head of his profession. He was much sought as a lecturer and teacher, and his voluminous writings on every branch of medicine summed up the knowledge of his day. He made many original contributions to the science of anatomy, particularly of 
the nervous system, and attempted by experiments upon animals to investigate the functions of the various organs.

Increased familiarity with anatomy enabled the surgeons to make even greater advances than were made in medicine. With a variety of efficient instruments they performed major operations, resections, and difficult feats in plastic surgery.

The Roman genius for organization did not neglect the public health. District physicians, archiatri populares, attended the poor and served as a board of consultation in the matter of controlling epidemics. The principle of hydrotherapy, introduced with the cult of Æsculapius, was elaborated until the baths (the gorgeous baths of Caracalla and Diocletian accommodated from sixteen hundred to three thousand persons) became part of the public life. At Baden-Baden and Wiesbaden, at Aixles-Bains, and at Bagnères de Bigorre in the Pyrenees, at Bath and wherever the Romans found natural springs, large establishments were built.

The general disorganization of the Empire following the successive waves of barbarian invasion and the persistent infiltration of the Christian faith, which, antagonistic to everything connected with the ancient pagan world, was finally adopted by both conquerors and conquered, practically put an end to the long uninterrupted progress in medical organization, knowledge, and practice. The baths, certain schools of medicine in Rome and the Western provinces, and the institution of the archiatri populares, were adapted to the needs of the conquerors. Under the slightly less disturbed conditions of the Eastern Empire a few men of unusual ability, Oribasius and Aëtius, physicians to the Byzantian emperors, Alexander of Tralles, Paul of Agina, and John Actuarius, collected and arranged the writings of the ancients, practiced surgery, and, throughout the fourth, fifth, sixth, and seventh centuries, made original observations upon disease. However, by the beginning of the sixth century the temples of Esculapius had long been closed, the schools of philosophy abandoned, and Greek culture was seeking asylum in Asia Minor and in the kingdom of Persia. Throughout the West and along the shores of the Mediterranean the ideals and the wisdom of the Old World had disappeared gradually, and all branches of education were passing under the control of the Christian priests.

Destructive and obstructive as were these years of constant warfare and emotional upheaval, a powerful constructive force had been at work. Except in India, where Asoka, the first great Buddhist king, had established hospitals for men and animals, no general attempt to care for the sick and unfortunate had ever been made. In Egypt and in Babylon it had been the custom to place the sick in the streets, where friends 
and passers-by exchanged experiences with and gave advice to the sufferers. In Greece the well-arranged and well-supplied iatreia were usually attached to the houses of physicians and served more for the treatment of out-patients than as hospitals. The Roman tabcrnce medicce were copies of the Greek iatreia, and the convalescent homes and sick rooms built by the great landed proprietors were open only to slaves and dependents on the Roman estates. The army had its hospitals and its stalls for sick horses. The early Christian Church had been organized not so much for the purposes of religious worship as for the care of the sick and the destitute, and throughout its early history the social service side of its activities absorbed the energies of its rapidly increasing converts. Archdeacons, deacons, and deaconesses administered a communistic charitable organization, and the parabaloni, male and female nurses, sought out the sick and needy, whom the Church cared for and protected. Beginning in the poorest and simplest class of society; the impulse gradually spread to the upper classes. Fabiola, a well-to-do Roman widow, whose example was followed by others, founded a Christian infirmary and cared for the sick in person. By the middle of the fourth century the teachings of Christ had so penetrated the Roman world and had gathered such force and importance that large hospitals began to appear. The institution founded by St. Basil at Cæsarea in Asia Minor was one of the earliest and also one of the largest. Special doctors and nurses were in attendance, and with its workshops and industrial school it soon reached the proportions of a small town. There were hospitals in Constantinople, in Antioch, in Alexandria, and by the sixth century there were hospital foundations in France and in Spain.

The inclusion of the monastic system within the Church organization broadened the social service aspect of Christianity. Each monastery had its hospice or hostel, and even small communities boasted their homes for the aged, for cripples, for orphans, and for foundlings. At Cæsarea a special department had been devoted to the care of the lepers, and in many parts of Italy and in Switzerland the establishment of lazar-houses preceded the founding of hospitals. When, in the eleventh century, after the Crusades, this long-known and already prevalent disease began to spread at an alarming rate, the lazar-houses dotted the continent of Europe. The religious fervor of the Crusades produced a great increase in the associations, such as the Brothers of St. Anthony, the Knights and Ladies of St. John, the Lazarists, and the Black Sisters, who deroted their energies to nursing, and such were the strictness, intelligence, and concentrated effort with which the lepers were cared for that by the sixteenth century the disease had been stamped out. 
It was only natural that the emotional enthusiasm, the general ignorance of past achievements, and the power and prominence of the priests should cause a revival of the sacred or priestly type of medical practice. Yet, in spite of the renewed faith in prayers, exorcisms, charms, and amulets, the active interest in the careful study and rational treatment of disease never wholly disappeared. Many of the earliest bishops and abbots were physicians. They have left careful descriptions of smallpox epidemics, and it was a bishop who first used the term variola. The Order of Benedictine Monks paid particular attention to the study of medicine. Alcuin, an English Benedictine, aided Charlemagne in establishing the Cathedral Schools, where, as in the Temple Schools of Egypt, medicine was taught. The Monastery of Monte Cassino near Naples, built by St. Benedict upon the ruins of a temple of Apollo, gradually developed into the School of Salerno, the earliest of the many famous European schools of medicine.

In the East, Nestorius, the deposed patriarch of Constantinople, removing with his followers to Edessa, a fortified Roman outpost, and carrying with him the spirit of ancient Greek learning, founded both a hospital and a school of medicine. The influence of the Nestorians soon spread to Nisibis, also a fortified town, where, under Greeks and under Jews trained at Alexandria, eight hundred students are said to have gathered. Other similar centers of learning sprang up in Eastern Asia Minor, but the persistent persecution of the Christian emperors at Constantinople eventually drove Nestorians and philosophers southeastward until, about the year 500, they sought safety at the Court of Chosroes, king of Persia.

Chosroes I and his son, Chosroes the Conqueror, the strongest kings of Persia since the days when Artaxerxes and his son had raised their new kingdom on the ruins of the extensive realm of Seleucus, were champions of Zoroastrianism, the native religion of Persia, and were violently opposed to Christianity. While waging a constant and eventually suicidal war against the Byzantine emperors, they permitted Nestorians and philosophers to settle at Ctesiphon, on the Tigris, near modern Bagdad, and at the long-established schools of Gondisapur near the ruins of Susa. Since the decay of the kingdom of Seleucus, Persia had been more in touch with India than with Greece, and at the famous schools of Gondisapur Greek and Indian culture came once more into intimate contact. The Nestorians taught and practiced in the hospital, and it was here that the conquering Arabs, irresistibly spreading eastward, found one of the largest and most important centers of Greek culture fused with the medical traditions and practices of India.

The Bedouins of Arabia were not a naturally imaginative or religious 


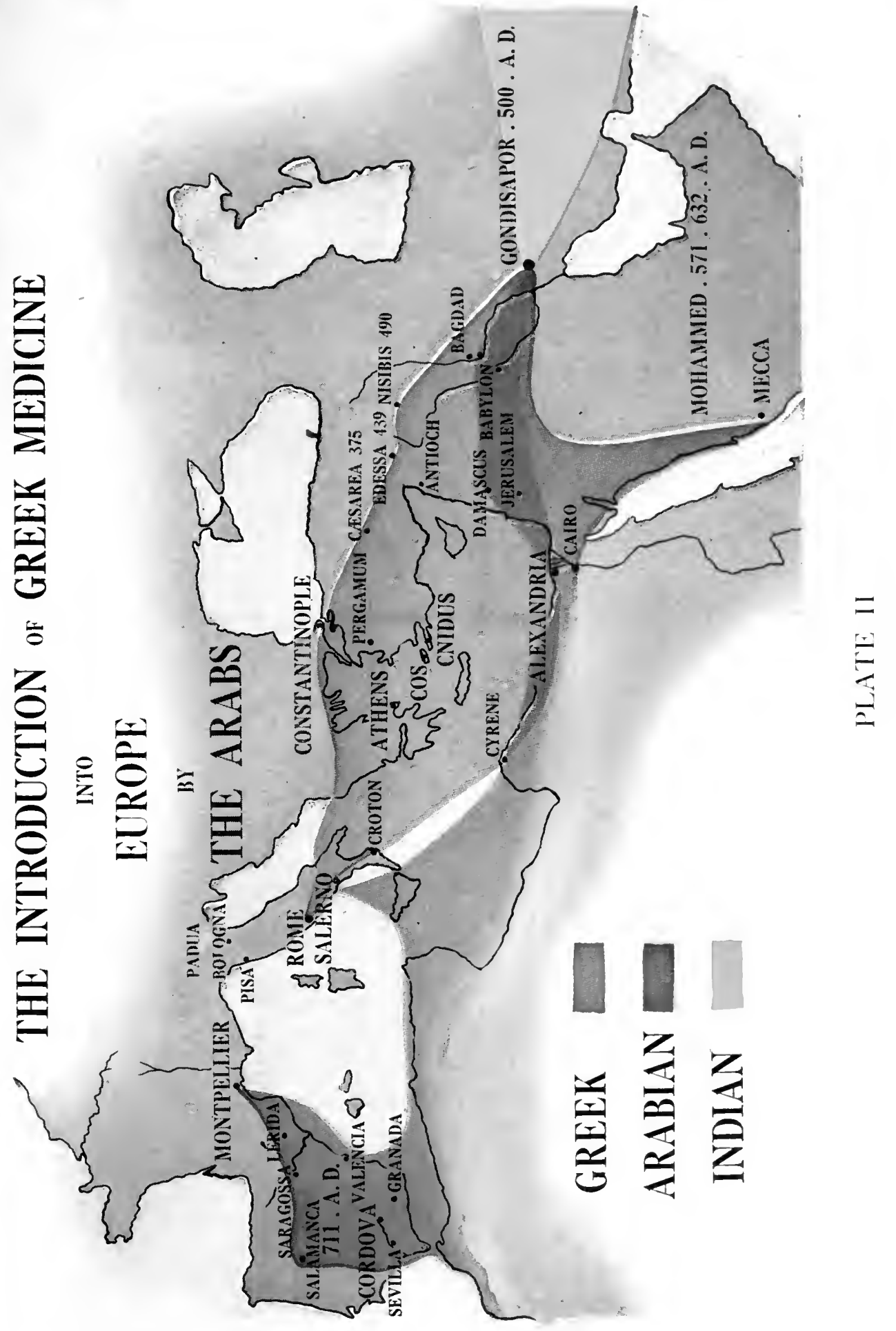



race. They had no mythology, and the religion preached by Mohammed was a simple monotheism, the least mysterious of all the positive religions. The "Arabian Nights" are of Indian or Persian origin, and only the pictures and anecdotes of actual life are truly Arabian. To this inherent interest in facts there was soon added a passion for general culture, and, as their political dominion was established, they revived the schools of Alexandria and turned their mosques into free institutions of learning. The simplicity of their religion encouraged the growth of a spirit of tolerance. Learned men and physicians of all faiths and nationalities, particularly the Jews, with whom the Arabs had much in common, were welcomed and employed by the Caliphs. The lay and medical literature of the Greeks, Jews, Persians, Indians, and Egyptians was carefully collected, translated into Arabic, and absorbed with avidity. Bagdad and Damascus, Alexandria and Cordova vied with each other in securing rare volumes and in founding libraries. Abd-ar-rahman III and his successor, Hakam II, the most capable of the Caliphs of Cordova, had agents in all countries who bought, regardless of cost, ancient and modern manuscripts, whose care added to the labors of copyists, binders, and illuminators, already attached to the enormous Cordovan library. Hakam's court was crowded with scholars from every land, and all branches of learning flourished. Thousands of students were attracted to the university. In the twenty-seven public seminaries of Cordova the children of the poor received an education, and centers of learning existed in all the larger towns of Spain. While in Christian countries only the priest could read or write, these accomplishments were common to nearly all Arabs. The exact sciences, mathematics, mechanics, and physics, were highly developed. The science of chemistry and its sister science, pharmacology, were practically reborn. A great number of the new remedies and preparations were added to the Pharmacopœia, and the Arabian works on Materia Medica ranked throughout the Middle Ages with the volume of Dioscorides. Apothecary shops were first introduced by the Arabs.

The study of medicine was considered an important branch of general culture. From the end of the ninth to the beginning of the thirteenth century Arabian physicians from Rhazes and Hali-ben-Abbas, the Persian, to Avenzor and Moses Maimonides, physician to Saladin, dominated the medical world. They were sought as body physicians by Christian kings and even became viziers to the Caliphs. Their theory and practice were based upon the works of Hippocrates, Galen, and Aristotle. The elaborate writings of Avicenna, physician-in-chief to the Bagdad hospital, were the standard work of the Middle Ages. Albucasis of Cordova published a great medico-chirurgical treatise picturing the instru- 
ments of his day, which, unfortunately, owing to the Mohammedan prejudice against dissection and cutting, were being gradually discarded for the cautery. Averrhoes, also of Cordova, was a fearless logician, philosopher, and free-thinker, and added to the reputation of those of his race for great independence in the practice of their profession.

The practical Arab was quick to grasp the essential usefulness of the Christian hospital development. They not only continued and enlarged existing institutions, but erected others, notably the great Al-Mansur Hospital at Cairo, which was more elaborate and socially efficient than any under control of the Church. Their treatment of the insane, whom they regarded as divinely inspired, was much more gentle and humane than was that employed by the Christians. The decline of the Arabian civilization was as rapid and extensive as had been its rise. By the beginning of the fourteenth century the fierce energy aroused by the teaching of Mohammed had burned itself out. No great discoveries had been made, nor had any far-reaching or fruitful idea been born; but many branches of science had been enriched, the treasures of ancient learning had been saved, and the stagnant intellectual life of Christian Europe had been refreshed and stimulated.

The revivifying spirit worked mainly through two channels: the medical schools of Montpellier and Salerno. If the Arabs took no part in the founding of the famous school of Salerno, they had much to do with its development. It is probable that the gathering of health-seekers in or near the Benedictine monastery and ecclesiastical hospitals of Monte Cassino gave an opportunity to monks and novices interested in medicine to establish themselves in practice in the equally soothing and stimulating air of nearby Salerno. The church officials, who occupied positions as directors and professors in the gradually evolved school of medicine, welcomed the Jews and Arabs from Sicily. Arabic translations of the medical classics retranslated into Latin soon formed the basis of medical education, and by the tenth century the doctors of Salerno had become so celebrated that their aid and advice were sought by distant sovereigns and by prominent travelers. So great was the unprejudiced spirit of intellectual freedom that women were admitted to the profession and even to the professorships. Many women became celebrated as medical authoresses.

Southern Italy and the island of Sicily, parts of the Mohammedan world, had been conquered by a powerful Norman prince, and this vigorous, brilliant, and tolerant Norman state soon exercised a decided influence upon the school of Salerno. In II40 Roger of Normandy, king of Sicily and Naples, promulgated a law that "Whoever from this time forth desires to practice medicine must present himself before 
our officials and judges and be subject to their decision. Anyone audacious enough to neglect this shall be punished by imprisonment and confiscation of goods. This decree has for its object the protection of our kingdom from the dangers arising from the ignorance of practitioners." A century later Frederick II, king of Sicily and Naples, and, as grandson of Frederick Barbarossa also emperor of the Holy Roman Empire, one of the most remarkable personalities of the Middle Ages, and an enthusiastic friend to all men of learning irrespective of their creed, took an intense interest in medicine. He not only renewed the law of his Norman grandfather, King Roger, in whose kingdom he had spent his early years, but drew up a set of rules and regulations for the study and practice of medicine which, in their intelligent comprehension of the needs of the student, the public, and the profession, set a standard for all future medical legislation. A three years' preliminary education in logic, the then classical course of study, had to precede five years' devotion to the study of medicine based upon the Hippocratic writings. After successfully passing the state examination, for which a Latin diploma was granted, the young graduate, before starting an independent practice, was expected to gain a year's experience under the guidance of an established practitioner. Those wishing to practice surgery were required to present certificates testifying that they had acquired a thorough knowledge of human anatomy and had spent at least one year in practical surgical work. All candidates had to promise on oath that they would give free advice to the poor and that they would inform the magistrates of mistakes in filling prescriptions made by the apothecaries. The apothecaries had to swear that they would dispense only drugs of known purity, and physicians and apothecaries were forbidden by law to form partnerships. The preparation of drugs was supervised by inspectors.

Frederick's regulations were later adopted by the rival school of Montpellier, where; on account of its nearness to Spain and the broadminded attitude of the Church, Jews and Arabs early had exercised a like influence in stimulating and developing the original Christian hospital and school of medicine. Salerno long retained its prominent position as a center for medical studies. After a slow and gradual decay it was, in I8II, finally closed by order of Napoleon. To the Medical Faculty at Montpellier soon were added faculties of Law and Theology, and the University of Montpellier, though no longer attracting enormous numbers of students and distinguished invalids, exists today.

The almost insurmountable prejudice against dissection of the human body, which had expressed itself in legal ordinances, was the only great handicap to the progress of the thousands of keen-minded medieval 
medical students and professors. Even the bold Frederick was not able to furnish, except once every five years, the material necessary to the requirements that he himself had laid down. The carcases of bears, monkeys, pigs, and dogs revealed only approximate truth, and the "Anatomy of the Pig," by Copho, the Jew, of Salerno, had to serve as the student's handbook. During the great plagues bodies were frequently opened in the hope of discovering the cause of the devastating pestilence, and the law occasionally sanctioned post-mortem examinations in suspected cases of poisoning. Whenever a body could be procured by stealth hurried dissections were carried out. In I3I6 Mondino di Luzzi, professor at the University or Studium Generale of Bologna, circulated his "Anatomia" or practical guide to dissection, and little by little the students, not satisfied with lectures and scholastic discussions of the medical classics and desiring first-hand information, demanded a relaxation of the proscriptions. Popular prejudice was not so easily overcome. In 1368 the Senate of the Republic of Venice issued a decree that a dissection should be performed once at year at Padua. Montpellier later received this legal permission, and within a few years this privilege was granted to the University of Lerida in northern Spain. The bodies were those of criminals. By the beginning of the fifteenth century dissections became more common at Bologna, and the third-, fourth-, and fifth-year students, who paid the expenses of procuring, preparing, and burying the corpse, were invited to attend, the ceremony. being so arranged that before the completion of his course of studies each student should have had the opportunity to follow the dissecting of a female and two male bodies. Where the bodies of criminals were lacking, the corpses of those who were not natives of the town were used. By the middle of the century the authorities of Bologna, Padua, Ferrara, and Pisa regularly supplied two corpses a year to the universities. At Paris, Oxford, Cambridge, Prague, and in the universities of Germany dissections were not arranged for till at a much later date. The universities of Bologna and Padua were the first to build anatomical theaters, and Italy long held the lead in the teaching of anatomy. For two hundred and fifty years, however, dissections remained, on account of their infrequency, among the most important and thrilling events in university life. John Evelyn, who in 1645 began at Padua a course of lectures in "physic and anatomy" by "the most famous professors in Europe," records in his diary that he was "present at the famous anatomy lecture, celebrated here with extraordinary apparatus, lasting almost a whole month," and that during this time he saw "a woman, a child, and a man dissected with all the manual operations of the Chirurgeon on the human body." 
These "operations of the Chirurgeon" were undoubtedly, at this date, extremely varied and interesting. Though the Church, for some time, had forbidden the practice of surgery to the clergy and had, by so doing, cast a social stigma upon those who used the knife, interest and progress in this branch of the profession had not been stifled. A few French and Italian operators had compelled recognition not only for themselves but for their art. Guglielmo Calicetti, city physician (the old Roman archiater) of Bologna and later of Verona, a universitytrained man with a wide experience in the hospitals and on the battlefield, had at the beginning of the thirteenth century boldly abandoned the Arabian cautery. His pupil, Lanfranchi of Milan, going to Paris and associating himself with the Surgical College of Saint-Come, had with the assistance of his pupils given practical demonstrations in surgery. His contemporary, Henri de Mondeville, an original and picturesque figure, had forcibly described the advantages to the surgeon of absolute cleanliness. Guy de Chauliac, a country boy from Auvergne, priest and surgeon, educated at Toulouse, Montpellier, Paris, and Bologna, and physician and chaplain to three successive Popes of Avignon, had compiled his "Chirurgia Magna," in which he described the narcotic inhalations used throughout the Middle Ages to produce anesthesia. Branca, of Sicily, and his son, Antonio, itinerant surgeons, had caused the greatest excitement by their skill in repairing injuries and deformities of the face.

In 1543 Vesalius, the vigorous and self-reliant Belgian, after five years as public prosecutor of Padua under Sylvius, professor of anatomy, had published his beautiful plates and woodcuts of the human body and was teaching and demonstrating in the crowded amphitheaters of Padua, Bologna, and Pisa that there were many facts not contained in the works of Greek and Arabian authors. The eager spirit of inquiry, the desire to study Nature at first hand, to no longer follow tradition or to depend upon authority, which in the fifteenth and early sixteenth centuries indicated the dawning of the modern scientific era, had been nowhere more marked than among the painters and sculptors of Italy. Every Renaissance artist had become a realist and a pseudo-scientist, interested in problems of perspective and of human anatomy. Leonardo da Tinci, who finally abandoned Art for Science, had been a most brilliant. careful, and discriminating dissector. Michelangelo, already an old man when Vesalius began to teach, knew more of human anatomy than did the professors of the schools. The notes and drawings of these two great men, unfortunately, had not been accessible to inquisitive students and practitioners, and to Vesalius alone is due the credit of having placed Anatomy at last upon a firm scientific footing. 
Surgery had made immediate profit of these new and accurate discoveries, which had been corroborated and added to by Vesalius' pupil, Fallopius. The revival of Greek learning, which followed the tireless researches of Petrarch and of his indefatigable helper, Boccaccio, and the arrival in Italy of Greek scholars and original manuscripts from conquered Constantinople, had led to a rediscovery of ancient surgical procedures. The surgeon of the Renaissance, essentially a practical man and not a scholar, had received a greater stimulus, however, from the introduction of firearms, which, producing wounds unlike any that had ever been encountered, forced a recasting of old conceptions and the invention of new methods of treatment. No surgeon used the discoveries of Vesalius, the new Greek learning, and the experiences on the battlefield to greater advantage than did Ambroise Paré, the barber's apprentice and surgical dresser of the Hôtel-Dieu, who, when opportunity offered, developed into one of the great men of France. He popularized the works of Vesalius, invented many instruments, demonstrated the uselessness of cauterization, and, by reintroducing the ligature, which had not been used since Roman times, blazed the pathway for future progress in major surgery.

During the thirteenth century, while the successful campaign against leprosy was at its height, the control of the ecclesiastical hospitals passed into the hands of the municipal authorities. Many new institutions, modeled after the hospital of Montpellier, were erected, and the custom of using hospitals for the purposes of teaching gradually became more common. The impulse given to scientific inquiry by the monk, Roger Bacon, bore fruit in carefully kept case records, which were compiled and circulated by university professors. The enthusiasm for Greek learning and the consequent establishment of Platonic academies made a knowledge of the Greek tongue essential to the cultivated physician. Thomas Linacre, educated in Italy, and, with Henry VIII and Cardinal Wolsey, founder of the College of Physicians, published a Latin translation of Galen's original works, and Rabelais, priest and physician, translated the Aphorisms of Hippocrates. The invention of movable type, which had permitted the publication of semi-popular medical works written in the vernacular, weakened the importance of classical learning and prepared the stage for the appearance of Paracelsus, a man of strong religious feeling and of enormous vitality, who, possessing the independence of spirit that characterized both Vesalius and Ambroise Paré, asserted loudly and combatively the superiority of the personal observation of disease over slavish dependence upon the experiences of Galen, Avicenna, and the ancients.

The breadth and variety of Paracelsus' own experiences gave added 
force to his teaching. He was the son of a learned Swiss physician, had been educated at the University of Basle, and had spent many months at Swaz in the Austrian Tyrol in the mines and laboratories of the Fuggers, the bankers and merchant princes of Augsburg. Here he had made an exhaustive examination of metals, had discovered zinc, made zinc ointment, and had formed the conclusion that the aim of chemistry was not alchemy, the manufacture of gold, but the preparation of medicines. He had visited the universities of Vienna and Cologne, and the schools of Italy, had spent some time at Montpellier, and, speaking Latin, the language of scholars throughout the world, had visited Spain and England. He had served as barber-surgeon in the army of the Netherlands, had traveled from the North Sea to the Bosphorus, and had served three years as surgeon in the Venetian army.

His first teaching in Tübingen and in Strassburg, while fascinating to students, aroused the antagonism of the settled practitioners, and his stay at Basle, where he had been called by the Protestants, was hardly more successful. His crowded lecture rooms, where he spoke in German, his bedside teaching, his kindness to the poor students, who lodged at his house and who helped him in the preparation of his mineral medicines, and his uncompromising opposition to the accepted matter and manner of instruction, aroused the enmity both of the apothecaries and of his Catholic confrères. During a students' celebration he had thrown the "Canon" of Avicenna into a huge bonfire burning in the market-place of Basle, and though he had remained true to the Church, his opponents compared him to Luther. Persecution and injustice finally led him into publishing a vitriolic reply to his enemies, and, threatened with arrest, he was forced to leave the university. He fled to the mountains, where, believing with his friend, Erasmus, that the Church should be reformed from within, he began to teach religion and to distribute the Bible. Again his activities and personality aroused the enmity of the Catholics. He had been refused permission to practice medicine in Switzerland, and being at last reduced to great poverty, he fled into Austria, where, until his early death (he was killed by a blow upon the head when he was only forty-eight years old), he continued his chemical experiments. After much opposition he finally published his "Greater Surgery," which brought him again into prominence, and he was, for many years, the most widely known physician in Europe. His treatise on the diseases of miners was a valuable contribution to clinical medicine, and his other writings, though permeated with his strong religious and mystical feeling, greatly aided the further development of medical chemistry.

Great intellectual movements call for great leadership, and great leaders require more than the possession of original ideas and intellectual 
independence. Had Vesalius, Paré, and Paracelsus not possessed the added qualities of restless force, personal magnetism, and moral and physical fearlessness, the dead weight of the past might have continued to paralyze all scientific progress. By force of personality these three pioneers broke through the encircling wall of tradition and led their eager followers into a new and living world of progressive scientific activity. 


\title{
CHAPTER I
}

\section{THE PART PLAYED IN DIET BY FOOD SUBSTANCES OF UNKNOWN CHEMICAL NATURE}

\author{
By E. V. McCollum
}

Is recent years great interest has centered in the relation betwcen diet and disease, and numerous researches have been conducted the object of which was to determine the exact nature of the faults in the diet which are concerned in the etiology of several syndromes. Beriberi, scurvy, and pellagra for a decade have been classed by sonie investigators definitely as diseases which are due to the lack of specific chemical substances in the diet, and it has been suggested likewise, although no clear evidence has been presented in support of this view, that rickets is to be assigned to a similar cause. The present article will treat briefly of the experimental studies which relate to the etiology of these diseases.

Beri-beri has been recognized for a long time as a multiple neuritis, and its cause has been assigned variously to an infective agent, to arsenical poisoning, to poisoning with moldy foods, and to lack of suitable diet. The relation of the disease to faulty diet first was supported by convincing evidence by the experience of the Japanese navy. Beri-beri was prevalent constantly among sailors of the Japanese navy until $188_{4}$, when the diet was improved. This was followed by a striking and progressive diminution of beri-beri which continued until the disease wholly disappeared. The prominent place of rice in the diet of those who suffered from beri-beri led to its incrimination as the causative agent, and an investigation by Eijkman ( $\left.{ }^{1}\right)$ in 1897 formed the starting-point of modern researches on the diseases referable to faulty diet. Eijkman restricted the diet of fowls and pigeons to polished rice, and observed that they developed the typical symptoms of polyneuritis. $\mathrm{He}$ found that the administration of rice polishings caused prompt relief of the symptoms, and drew the conclusion that the outer coat of the rice kernel contained some substance which was essential to the normal nutrition of birds. In 1907 Fraser and Stanton $\left({ }^{2}\right)$ extracted rice polishings with alcohol and found that the part soluble in alcohol was highly effective in inducing a "cure" of polyneuritic birds. In I9Io Funk $\left({ }^{3}\right)$ began an extensive study of the problem of isolation of the curative substance, 
employing reagents which precipitate organic bases (phosphotungstic acid, mercuric chloride, etc.), and extended his studies to other natural foodstuffs, especially yeast, in order to throw light on the distribution of the antineuritic substance in the more common foods. The results of administering minute doses of certain of his preparations to birds suffering from polyneuritis as the result of feeding exclusively upon polished rice were so striking as to form convincing evidence that the disease is in reality due to the lack of some chemical substance in the diet. To this substance he gave the name vitamine. Later he extended the meaning of the term to include a number of hypothetical substances which he believed to be individually specific in protecting against scurvy, pellagra, and rickets. Funk, however, presented no experimental evidence in support of the view that the last-named syndromes actually were the result of lack of specific substances in the diet in a manner analogous to beri-beri. Still the theory, supported so strongly by the studies of polyneuritis, proved very attractive, and resulted in the wide acceptance of the view that all of the diseases enumerated above belong to a class which has been designated as avitaminosis.

Previous to the year 1915 the etiology of the diseases referable to faulty diet had been investigated only by methods directed toward their relief by the administration of preparations made from natural foods, the object being to separate the "curative" constituent or constituents of these in a state of purity in the hope that their identity might be established. Within recent years a new line of attacking the problems in this field has been opened up by the studies of simplified diets. A detailed discussion of the validity of Funk's "vitamine hypothesis," which postulates the existence of a group of special chemical substances, the absence of which from the diet leads to the development of pathological states due to faulty metabolism, the type differing according to which one is lacking in sufficient amount, best can be left until the normal diet has been considered in the light of recent researches.

For many years it has been assumed by dietitians and students of nutrition that the essential constituents of an adequate diet are protein, fat, carbohydrate, and certain inorganic salts. Osborn and Mendel $\left({ }^{4}\right)$ in I9I I and Hopkins ( $\left.{ }^{5}\right)$ in I9I2 demonstrated that food mixtures made up of carefully purified food substances and including all of the recognized dietary essentials in approved proportions failed to induce any growth or to maintain long the life of experimental animals. Hopkins $\left({ }^{5}\right)$ showed that the addition of milk sufficient to furnish about four per cent of the solids of the food mixture was sufficient to convert such a mixture into complete rations, capable of inducing growth for short periods. He interpreted this to mean that milk contained certain uniden- 
tified chemical substances which were indispensable to the diet, and that the failure of animals to grow and to have a normal length of life, when restricted to rations consisting of carefully purified food substances, was due to the absence of these, which he designated as "accessory food substances."

McCollum and Davis in $1912\left({ }^{6}\right)$, employing a ration composed of purified casein (protein), dextrin, lactose, fat, and a suitable inorganic salt mixture, found that no growth could be obtained in young rats when lard or olive oil was used as the source of fat, whereas growth took place when the fat of the diet was supplied in the form of butter fat or egg yolk fat. It was, therefore, demonstrated that there was something associated with the latter fats which had not been recognized hitherto and which was essential to the animal as a constituent of its food. Further studies showed, however, that the same food mixture, when made up with one of the growth-producing fats but with the milk sugar replaced by dextrin or starch, failed to support growth. When milk sugar which had been subjected to a thorough process of purification was used in place of the ordinary laboratory article (Merck, Kahlbaum) no growth could be secured, but growth proceeded at once when the pure lactose was replaced by a less pure preparation. They, therefore, arrived at the conclusion that the lactose carried some impurity which constituted a second dietary essential and one which was not associated with fats. Many experiments by several investigators have since confirmed these conclusions; viz., that in addition to the long recognized dietary factors, protein, carbohydrate, fats, and mineral elements. the diet, in order to be adequate, must contain suitable amounts of two as yet chemically unidentified substances. Some investigators still hold that an antiscorbutic substance exists, and, since both of the two newly recognized substances have been clearly shown not to play a rôle in the etiology or relief of this disease, this view would necessitate the assumption that there are at least three chemically uncharacterized food essentials. There is, however, much evidence against the view that there are three such substances.

It is well established now that a diet consisting of carefully purified food substances and containing one of the growth-promoting fats will, if properly constituted, promote growth when supplemented with a very small amount of substance which can be extracted from almost any natural food by treatment with hot alcohol. The same result can be obtained by employing a water extract of a natural foodstuff, but water extracts so many other constituents that the preparation is less desirable in this type of experiment than is an alcoholic extract. The point of importance is, that the physiologically active substance is soluble in both 
water and alcohol, and, as stated above, is never associated with any fats or oils of either animal or vegetable origin. It is, therefore, possible to add one or the other of the two unknown substances to an experimental diet without adding the other. As a working hypothesis, McCollum and Davis ( $\left.{ }^{7}\right)$ in I9I 5 proposed the provisional assumption that there is but one unidentified chemical substance in butter fat and egg fats which is indispensable in nutrition, and that the alcoholic extracts employed for the addition of the other unidentified dietary essential likewise contain but a single indispensable substance. This idea was at variance with the views of Funk that there were several deficiency diseases in the same sense as beri-beri and accordingly a corresponding number of specific protective substances.

Hopkins had designated as accessory food substances the unknown constituents in milk which made it capable of supplementing a mixture of purified food substances so as to make it a complete food. Funk called them "Vitamines," and McCollum and Kennedy $\left({ }^{8}\right.$ ) proposed the terms fat-soluble A and water-soluble B, in order to differentiate the two types which can be clearly recognized by the effects produced when they are absent from the diet. Since the existence of two (or possibly more) individual substances of this class has come to be generally recognized by investigators, the term " food hormones" likewise has been employed frequently to designate them.

All the above terms except fat-soluble $\mathrm{A}$ and water-soluble $\mathrm{B}$ are misnomers. The word accessory carries the idea that the substances in question are dispensable. Condiments may be desirable, but they are dispensable and are properly designated as accessory food substances. An indispensable food complex cannot properly be designated by this term. The term "vitamine" is objectionable because it connotes an importance of these dietary essentials, greater than other equally indispensable constituents of the diet such as certain of the amino-acids which play a rôle in protein metabolism. The ending amine has a definite and specific meaning in organic chemistry, being used to designate a compound derived from ammonia by the substitution of one or more of its hydrogen atoms by various organic radicals. There is no evidence that either of the substances in question is an amine, and indeed fat-soluble A probably contains no nitrogen, for it is especially abundant in butter fat, and the latter is practically free from this element. "Food hormones" is an objectionable term because all the evidence available indicates that both fat-soluble $\mathrm{A}$ and water-soluble $\mathrm{B}$ are never-failing constituents of the cells of both animal and plant tissues. They have nothing in common with the hormones. The latter are chemical substances which are formed in the body by special tissues and contributed 
to the blood stream, where they cause the stimulation of certain other tissues to physiological activity. They are chemical messengers, while the substances under discussion are food complexes, apparently necessary for all the living cells of the body. The terms "growth substances" and "growth determinants," which are employed frequently for these dietary essentials, are not suitable, for the reason that they are just as essential for the maintenance of health in the adult as they are for the support of growth in the young. Furthermore, in actual experience, rations are met with in which the amounts of certain protein cleavage products or of certain mineral elements are limiting factors which "determine" the quality of the ration. When the addition of an aminoacid or of calcium, for example, will cause growth, while the same food mixture without such addition will not, these substances with just as much propriety might be called "growth determinants," as the still unidentified dietary essentials in question. These terms might be applied fittingly to any one of the indispensable components of the diet.

All the natural foods such as the seeds, leaves, tubers, edible roots, and fruits of plants, the animal tissues, such as muscles and glandular organs, and likewise milk and eggs, contain in all cases more or less of each of the long-recognized constituents of the normal diet, and of the dietary essentials, fat-soluble A and water-soluble B. There is, however, great variation in the amount of each of these in different foodstuffs. Some, as meats, are good principally as sources of protein; others, such as the potato, are of value primarily as sources of energy; butter possesses the peculiar property, not found in any vegetable fats or oils, of carrying an abundant amount of the fat-soluble A, etc.

The facts now thoroughly established concerning the requirements of an adequate diet may be summarized as follows :

I. No matter how satisfactory the chemical character of the proteins, carbohydrates, fats, and mineral elements of the diet may be, an animal cannot long remain alive on a diet containing only these substances, though these substances formerly were believed to be the only: essential factors in the diet. There must be furnished in addition both the unidentified dietary factors, fat-soluble A and water-soluble B. Fatsoluble $\mathrm{A}$ is not abundant in nature. It is found in greatest amounts in the fats of milk and of egg yolk, and is found in smaller amounts in the glandular organs such as liver, kidney, etc., and in the leaves of plants, such as, lettuce, cabbage, spinach, celery tops, etc., and is present, though less abundant, in the seeds of plants. Experiments in which the diets employed derived their sole content of fat-soluble $A$ from a single seed have shown that wheat, maize, rye, barley, oats, peas, and 
beans, or kaffir corn furnish approximately half the amount of this substance which is necessary in order to support normal growth and successful reproduction and rearing in young. Millet seed is distinctly richer in this substance than are the other seeds named. Fat-soluble A is almost entirely absent from such foods as are derived from the endosperm of seeds. The most important foods of this class are wheat flour (bolted), degerminated corn meal, polished rice, starches, glucose, and the sugars from cane and milk. It is entirely absent from the vegetable fats and oils. It is apparently present in the plant tissue in some form of chemical union, which is not soluble in fats and is not extracted in the process of isolation of the fats and oils. Unless the diet is properly planned there is some danger that it may not contain enough of the fat-soluble $\mathrm{A}$. When the diet is adequate in other respects but lacks this substance, there soon results a peculiar disease of the eyes.

2. The second unidentified dietary essential, water-soluble $B$, is everywhere abundant in natural foods. The cereal grains and other seeds, such as peas and beans, contain roughly about three to six times as much of it as is required to maintain an animal in excellent nutrition. The leafy vegetables (cabbage, lettuce, spinach, parsley, celery, etc.) contain it in great abundance. It is also found in the animal tissues used as food, and is present in liberal amounts in milk and egg yolk, but not in butter fat or any other animal fats. It has been stated already that the water-soluble B is never found in fats or oils of either animal or vegetable origin. The disease beri-beri, which is common in those parts of the world where the poorest classes live on a diet restricted to polished rice and fish, is due to a lack of a sufficient amount of this dietary essential in the food supply. The only foodstuffs which do not contain it are polished rice, starch, the sugars and syrups, and the fats and oils. Foods very poor in water-soluble B are bolted wheat flour, polished rice, and degerminated corn meal.

3. It is a matter of the greatest importance that the diet shall contain a satisfactory mineral content. The only elements required are sodium, potassium, calcium, magnesium, chlorine, iodine, sulphur, phosphorus, and iron. If any elements other than these and those characteristic of the organic compounds are necessary, they are required in extremely small amounts and it would be difficult to secure foods which would not contain enough to supply the needs of man or animals.

Leaving out of consideration the possibility that the water supply may in certain regions contain a suitable inorganic content to make good their deficiencies, it is not possible to secure a food mixture derived from the seeds of plants, no matter how complex the mixture, which contains a suitable mineral content to permit a young animal to grow. It is like- 
wise not possible to secure any appreciable growth in a young animal on combinations of seeds with muscle tissue (beefsteak, ham, chops, etc.), or on mixtures of seeds with the tubers such as the potato, though the mixture of seeds may contain any or all of the following: wheat, millet seed, oats, maize, rye, barley, peas or beans. The only elements which are not furnished in sufficient amounts by such food mixtures, as have just been described, are sodium, chlorine, and calcium. When these three elements are supplied by suitable additions to the above mixtures, growth can be secured on any of the above described food mixtures. It will not be normal in rate over a prolonged period, however, and the animals restricted to such diets will fall far below the normal performance in ability to produce and rear young, and, in all cases, will have but a short span of life, varying from one-sixth to one-third of the normal in the case of experimental rats. The reason for this lies in the low biological value of the seed proteins in general, and in their limited content of the fat-soluble A.

The only combinations of natural foodstuffs which furnish satisfactory inorganic mixtures for the nutrition of an animal during growth are such as result from the employment of seeds, tubers, roots or fruits, either singly or collectively, with liberal amounts of milk or of leafy vegetables. When the leafy vegetables, all of which contain in the dry state from two to six times as much total ash-forming elements as do the seeds, are employed as the sole supplements for seeds, they must be used very liberally, so liberally, in fact, that it is difficult for man to eat a sufficient amount of these to entirely correct the mineral deficiency of the seed moiety of his diet. As much as twenty to thirty per cent of the total solids of the ration should come from the leaf of the plant, if best results are to be attained with diets derived solely from leaf and seed products. The Japanese and Chinese peoples and a few others occasionally approximate this type of diet, and, apparently, with a fair degree of success. In general those peoples, who do not use dairy products, live principally on seed and leaf foods, but improve the quality of the protein and increase its quantity by the addition to their diets of small amounts of eggs, meats, fish, and poultry. Meats alone have very little power to improve the character of the mineral content of the seeds, and any amount of improvement in the protein of the diet in such mixtures will not enable an animal to thrive. The problem of securing a satisfactorily constituted inorganic content in the diet is one of the three great problems in human nutrition and animal production. The other two are of about equal gravity and are to secure protein mixtures of high biological value, and to secure an adequate amount of the unidentified essential fat-soluble $\mathrm{A}$. 
4. The best proteins are found in milk, meat, and eggs. There is great variation in the food value of the proteins from different sources. Those from the cereal grains are, in general, of relatively poor quality when they serve as the sole source of protein. Their value is greatly enhanced by the use with them of moderate amounts of milk, eggs or meats. It is not difficult to secure rations which supply an abundance of protein of good quality by the employment of such mixtures.

Eijkman $\left({ }^{1}\right)$, of the Dutch East Indian Service, first called attention to the relation of the disease beri-beri to the diet. He first produced experimentally a condition closely analogous to beri-beri in man by feeding pigeons and fowls a diet restricted to polished rice, and made the discovery that the disease was relieved by feeding the birds rice polishings. This result suggested that there was lacking from polished rice something which was essential to the maintenance of health in the bird, and that this something was present in the rice polishings. This was the first indication that there is necessary in the diet something other than proteins, carbohydrates, fats, and inorganic salts.

Beri-beri is a disease common in the Orient among people who limit their diet largely to polished rice and fish. Its most striking characteristic is a general paralysis and frequently it is referred to as polyneuritis. In recent years it has been observed in Labrador among people eating too largely of white flour and meat (fish). It is fairly common in Brazil.

The observations of Eijkman attracted but little attention until 19I0, when the Polish chemist, Casimir Funk $\left({ }^{3}\right)$, took up the study of the substance which is responsible for the relief of polyneuritis in birds. He made an extensive investigation directed toward the isolation and identification of this most interesting substance. It is due to Funk's researches that the subject of the so-called "deficiency" disease attained such widespread popularity in recent years. $\mathrm{He}$ observed that the amount of substance in the form of material separated from rice polishings by extraction with alcohol and precipitation methods, which was necessary to relieve polyneuritis in a pigeon, was no more than two to five thousandths of a gram. Such small amounts suffice to restore to an apparently normal condition a bird which is in a helpless condition and near death because of starvation for this still unidentified food complex.

There can no longer be any doubt that beri-beri is a deficiency disease in the sense in which Funk regarded it; viz., as due to the lack in the diet of a specific chemical substance, of which but a surprisingly small amount is necessary. To this substance he gave the name vitamine. He postulated without experimental evidence, however, the theory that certain other diseases, scurvy, pellagra, and rickets, like beri-beri, were caused by the absence from the diet of an adequate amount of similar 
protective substances. Thus, frequently he used the term " antiscorbutic vitamine" for the supposed protective substance against scurvy. In order to explain certain confusing experimental observations, Funk likewise suggested the existence of certain other "vitamines" essential for growth, which were not necessary for the maintenance of an animal. The idea of a group of "deficiency" diseases in this sense was very attractive, and the "vitamine" hypothesis has become very popular in the last few years. McCollum and his coworkers do not agree with Funk in including scurvy, pellagra, and rickets in the group of deficiency diseases, but they recognize beri-beri as such, and have described another, a type of xerophthalmia.

When an animal is fed a diet of purified protein, casein, carbohydrates, and fat, together with a satisfactory mixture of inorganic salts, it will live no longer than if it were given no food. It needs to be supplied with the two unidentified substances, fat-soluble $A$ and watersoluble B. If a food rich in the latter is freed from fats by extraction with ether, in which the water-soluble $B$ is insoluble, and the fat-free food is then extracted with hot alcohol, the substance which relieves beri-beri will be found in the alcohol. From the material soluble in alcohol it can be dissolved in hot benzene, although the latter solvent will not extract the substance directly from the natural foods. Exceedingly small doses of the material, which by this procedure becomes dissolved in benzene, will correct the deficiency of a ration of purified foodstuffs such as was just described, so far as the dietary factor water-soluble $\mathrm{B}$ is concerned. Fat-soluble $\mathrm{A}$ can be added most conveniently to such a diet by including three or four per cent of butter fat in place of such "non-growth-promoting" fat as the mixture may contain. When such a food mixture is supplemented with such a benzene extract together with a fat of the "growth-promoting" class, it becomes adequate for the support of growth and the maintenance of physiological wellbeing.

When the mixture of purified foodstuffs is fed along with the growth-promoting fat (fat-soluble A) but without the addition of the water-soluble $B$, the animals fail to grow, and in the course of a few weeks develop polyneuritis, becoming helpless with paralysis. If the same food mixture is fed with the addition of the water-soluble $B$, but without fat-soluble A, they will fail to grow but will never become paralyzed. Instead, within a few weeks, the eyes become buried because of swelling of the surrounding tissues. The eyes become inflamed and greatly irritated and permanent blindness will result within a short time, unless the missing dietary essential be supplied. There is but one way to produce this condition of the eyes; viz., to selectively fast 
an animal for the fat-soluble $A$; and there is but one way to effect its cure; that is, by supplying this missing dietary complex. The incorporation of a small per cent of butter fat in the food will cause the eyes to return to the normal appearance within a short time, usually within two weeks. The eye disease, just described, is a "deficiency" disease in the same sense as beri-beri, in that there is for it a specific protective substance. McCollum and Simmonds $\left({ }^{9}\right.$ ) have pointed out clinical evidence that the eye trouble, which is a type of xerophthalmia, has occurred repeatedly as a human disease due to faulty diet. That this xerophthalmia is the second and only other "deficiency" disease in the same category with beri-beri is made practically certain by recent researches relating to the cause of scurvy and of pellagra. The evidence concerning these diseases, both of which are referable to faulty diet, will next be discussed.

For a long time clinicians have been almost universally convinced that scurvy is a disease which is the result of a faulty diet. It was in the days of long voyages on sailing vessels of distressingly frequent occurrence among sailors. The men were forced to subsist for long periods on sea biscuits and salt meat. Scurvy is characterized by profound changes in the walls of the fine capillaries resulting in hemorrhages beneath the skin, in the mucous membranes and other tissues, and in a spongy condition with hemorrhage of the gums. There is also anemia and great weakness. The symptoms of scurvy promptly disappear when fresh vegetables, lime or lemon juice are taken in liberal amounts. Such observations led naturally to the belief that scurvy was due to the lack of something in the diet of stale bread and meats, which was furnished by the fresh vegetables and fruit juices. Since the relief of this serious condition by orange juice or other suitable additions to the diet is little less spectacular than is the "cure" of beri-beri in a pigeon, it is not surprising that Funk adopted the idea that scurvy, like beri-beri, is caused by the absence from the diet of a specific chemical substance, or "vitamine."

There is now apparently satisfactory proof that scurvy in infants and adults is due to the consumption of unwholesome food. It may result in certain species of animals as the result of taking food which is comparatively safe and wholesome for others. As an example; the guineapig, in nearly all cases, will suffer an attack of scurvy within a few weeks if fed exclusively upon a diet of rolled oats and milk, even of good quality. On such a diet the human infant, young rats or young farm pigs may grow well and remain in good condition. There can be nothing lacking from this mixture which is essential for the mammal during growth. Why does the guinea-pig usually develop scurvy, and why does 
the human infant sometimes develop the disease and sometimes thrive on such food? The answer seems to be supplied by recent investigations in which both human infants and guinea-pigs served as subjects.

McCollum and Pitz $\left({ }^{10}\right)$, after demonstrating that several diets which are entirely satisfactory for the rat during the entire growth period caused speedy decline and death in guinea-pigs, found that the latter animals could be relieved of the symptoms of scurvy, which they had developed as the result of being confined strictly to a diet of oats and fresh milk, by such remedial agents as liquid petrolatum, phenolphthalein, and also by the administration of an "artificial orange juice" prepared from pure inorganic salts, cane sugar, and crystalline citric acid, in proportions similar to those found in the edible portion of the orange. Orange juice has long been recognized as a very efficient food for the relief of scurvy in man, both infants and adults. The accepted explanation of its potency has been that orange juice contains an antiscorbutic substance which, according to Funk, is to be classed as "vitamine."

The real explanation of the susceptibility of the guinea-pig to scurvy is, according to McCollum and Pitz, to be found in the peculiar conformation of its digestive tract. The cecum, a pouch lying between the small and large intestine, varies remarkably in different species of animals. In the guinea-pig, the cecum is of extraordinary size and its walls are very thin and delicate. Their power of contraction is small, and it is not possible for the animals to pass the contents of the cecum into the large intestine unless the material possesses very favorable physical properties. The guinea-pig can thrive only on a diet which contains a liberal amount of some succulent vegetable, such as cabbage or carrots. These produce bulky, easily eliminable feces, and such a diet tends to keep the digestive tract in a hygienic condition. Both oats and milk are constipating foods. When this species is confined to a diet of oats and milk, the cecum soon becomes packed with feces, and extensive putrefaction takes place in the stagnated contents. Scurvy is in some way related to the bacterial factor which accompanies this condition, but whether bacteria actually invade the tissues, when the cecal wall is injured by prolonged contact with decomposing feces, or whether the destruction of the capillaries is the result of the absorption of toxic products of bacterial origin, is not determined. Jackson and Moore $\left({ }^{11}\right)$ have found a diplococcus in the hemorrhagic areas of guinea-pigs suffering from scurvy as the result of a milk and oat diet, and they are inclined to attribute to this organism a causal relationship to the disease.

Following the studies just described, Hess $\left({ }^{12}\right)$ demonstrated that infants fed on milk, which had been pasteurized immediately before feed- 
ing, do not develop scurvy, while others fed the same milk, which was kept twenty-four to forty-eight hours after the heat treatment, may develop the disease. Boiled milk, on the other hand, is not liable to cause scurvy in infants. The reason for these results appears to be found in the bacteriological condition of the milk treated in these different ways. Pasteurization destroys the lactic acid forming organisms but not certain spore-forming bacteria. The latter develop rapidly in milk in which there is not the normal rise in acidity, i.e., pasteurized milk. After a day or two, therefore, such milk is a well-developed culture of organisms which may in the delicate intestine of the human infant cause abnormal decomposition of the proteins with the formation of products which are irritating to the mucosa, cause injury, and possibly permit the invasion of the tissues by organisms. Boiled milk is nearly free from bacteria, so it is less dangerous than old pasteurized milk. Milk which has been pasteurized and is fed soon thereafter is without detrimental qualities, because the forms of organisms, which cause the damage, are not permitted to develop and accordingly but few are ingested.

The evidence is convincing that scurvy is liable to follow the use of stale foods. If, however, the disease develops in those who use desiccated foods, canned or preserved foods, the cause is to be sought in the bacteriological condition of the food, and not, as has been frequently asserted, in the destruction of some hypothetical "vitamine." There is no protective substance against scurvy in the sense that there is against beri-beri, or the type of xerophthalmia described as resulting from specific starvation for the dietary essential, fat-soluble A.

Scurvy in the long sailing voyages of the past was probably the result, first, of the poor quality of the food (biscuits and stale meat), which did not form an adequate diet and caused depletion of the vitality of the sailors. Constipation and perhaps also, as Hess suggested from his observations on infants, failure to urinate sufficiently formed the preliminary conditions which permitted the development of the bacterial flora which finally brought on an attack of the disease. Fresh vegetables and fruit juices relieved the condition for three reasons; first, they did much to render the diet complete from the physiological standpoint, which alone would greatly promote recovery; second, they were good correctives for the faulty elimination, because of their bulk and the water-holding capacity of their indigestible residues; and third, especially in the fruit juices, the diuretic property would cause a thorough washing out of the tissues. A diet of wheat biscuits and meat is inadequate with respect to two chemical factors; its organic content is entirely inadequate, and it is lacking in the factor, fat-soluble $\mathrm{A}$. What 
there is of indigestible residue consists mainly of protein residues, which would favor the development of a pernicious flora in the intestine.

Such a tentative explanation as that just offered harmonizes well with the experimental facts. The most convincing evidence of the general correctness of this view of the etiology of scurvy lies in its experimental production with diets which are known to be complete chemically, and which nourish well certain species of mammals that do not suffer from special anatomical disabilities, because of the peculiar form of the digestive tract. The relief of the disease by several means, none of which can possibly be interpreted as supplying a hypothetical curative substance, an "antiscorbutic substance," which would be considered as analogous to those which are concerned with the relief of beri-beri and xerophthalnia, eliminates this from the list of so-called "deficiency" diseases.

Pellagra is a very serious disease which has been common in Europe for centuries. It occurs extensively in northern Italy, where it has been called Alpine scurvy, in Spain, and in the South of France. It was not observed in America until about 1907, but has since become common in several of the Southern States. In I9I7 it was estimated that there were one hundred and sixty-five thousand pellagrins in the United States.

That the diet is of basic import either as the causative agent or as a contributing factor has been accepted generally by observers. In Europe and in America the disease has been confined, in general, to the poorer classes, especially in the country districts and villages. It is more prevalent in times of scarcity of food. In Europe it was long supposed to be the result of eating diseased corn as the chief article of diet. It is known now that the consumption of corn has nothing to do with the disease. The trouble begins usually with the digestive disturbances of indefinite character, later followed by skin eruptions and the formation of dark crusts, which suppurate. The digestive symptoms become worse, the mouth is very sore, and diarrhea develops. In severe cases there are pronounced nervous symptoms.

The disease, in its early stages, yields fairly readily to dietetic treatment. Indeed, it is generally recognized that there is no other effective treatment. The liberal use of milk, meats, and eggs, together with an abundant supply of leafy vegetables, such as, cabbage, collards, and lettuce, leads, as shown by the studies of Goldberger $\left({ }^{13}\right)$, to the disappearance of the symptoms and to the failure of recurrence of the disease.

In the United States, especially, pellagra tends to a seasonal occurrence, most new cases appearing in the spring or better at the end of winter. Goldberger has called attention to the very poor quality of the diet of the class of people who are the principal sufferers from the disease. It is not uncommon for poor families to restrict their diet during 
the winter practically to corn bread, pork, and molasses. From what has been said of the properties of the several classes of natural foodstuffs, it is to be expected that serious nutritional disturbances will follow confinement to such a diet for a hundred days or more. Pellagra is prevalent among people who live too exclusively upon molasses, sweet potatoes, and foods derived from the endosperm of seeds of plants, together with meat in small amounts. Milk, eggs, and leafy vegetables are conspicuously absent from their diet, or taken in very small amounts. Their diet is deficient in at least three respects; viz., it contains but a very low content of protein, and this of poor biological value; its inorganic content is entirely inadequate in character and amount; and it is very poor in the fat-soluble A. In addition to these faults there is in many instances a destruction of a part of the water-soluble $B$ in the process of making soda biscuits, for in alkaline reaction this substance is readily destroyed. A typical diet of many institutions and of some districts, where pellagra is prevalent, is made up of dishes prepared from bolted flour, degerminated corn meal, rice, starch, syrup, pork fat, and a relatively small amount of the total energy of the diet in the form of sweet potatoes, cabbage, and other leafy vegetables. Such a diet has been shown by Goldberger to produce in man a condition identical with incipient pellagra.

Chittenden and Underhill $\left({ }^{14}\right)$ have described the results of restricting dogs to a diet of crackers (wheat flour), cooked peas, and cottonseed oil. In from two to eight months the animals developed the typical sore mouth, severe diarrhea, and skin changes strikingly suggestive of pellagra in man. McCollum, Simmonds, and Parsons $\left({ }^{15}\right)$ have recently shown that this diet is deficient only in respect to its inorganic content, the relatively poor quality of its proteins, and a shortage of the fatsoluble A. When these are corrected by the addition of purified food substances, the diet is rendered capable of supporting growth and the production of young in the rat. This experiment demonstrates that this mixture of seed products is not deficient in any element of mystery, which might be termed " vitamine."

Rickets is a nutritional disease, the most prominent feature of which is an alteration of the growth of the bones. These become enlarged at the extremities and so soft that they bend as the result of muscular contraction or by reason of the weight of the body resting upon them. It is a disease of the first two years of life and is associated with faulty diet. It is of frequent occurrence in cases where milk in the diet is replaced too largely by cereals or other foods not suited to the digestive tract of the young child. In many cases tuberculosis and syphilis are predisposing factors. The symptoms develop gradually. There is rest- 
lessness and perspiration during the night and great sensitiveness of the limbs, even a light touch being very painful. There are gastric disturbances, especially colic and distention of the intestine with gas, so that the abdomen protrudes. The bones become thickened and nodules develop at the juncture of the ribs with the costal cartilages, forming the characteristic "beaded ribs." There is defective ossification of the skull; the teeth appear later than normal and in unusual order. Various deformations of the head, spine, chest, and limbs result as the child develops. Recovery with deformity is of frequent occurrence.

Although there has been no thorough study of this disease from the dietary standpoint, the well-known dietary deficiencies of the cereal grains, together with the extensive injury to the intestine, which is evident from the distended abdomen, its occurrence only in very early life and its association with infectious disease, make it unnecessary to invoke the aid of any hypothetical "vitamine," to a lack of which the disease may be attributed. The facts are now sufficiently established, therefore, to warrant the complete confidence in the belief that scurvy, pellagra, and rickets, while referable to faulty diet, are not to be regarded as "deficiency" diseases in the sense in which this term has been generally employed during the past seven or eight years.

In the light of what has been said, certain conclusions concerning the choice of foods become obvious. With the passing of the practice of local milling of grains into flour and meal, the length of time between milling and consumption has greatly increased, and the quality of the flour or meal which appears on the market has changed, and for the worse. Previous to the entrance of the United States into the war, the public had become educated to demand a white flour. This product represents only material which is derived from the endosperm of the wheat kernel. This is more deficient in inorganic salts than is the entire seed, and is decidedly poorer in both the fat-soluble A and water-soluble $B$ than is the entire seed. Furthermore, the germ contains proteins of better quality than are those of the endosperm, so that the bolted wheat flour is deficient in quality with respect to four factors of great importance from the standpoint of nutrition, as compared with the seed from which it is prepared. The justification for the marketing of such a product is found in the good keeping qualities of bolted wheat flour. Insects which deposit their eggs in the grain place them in the germ, the portion which is the most nearly complete from the dietary standpoint, and any product which contains the germ will in warm weather harbor weevils. Since the interval which elapsed between milling of wheat flour and its delivery to the consumer, either in the form of flour or of bread, was in normal times about six months, the keeping qualities 
of the marketed product was an important commercial matter. What has been said of bolted wheat flour was likewise true of corn meal and polished rice. The degerminated product could be handled in commerce without hazard, whereas the marketing of the products which contained the germ required much more careful handling and there was great danger of economic loss. The milling of high extraction flours (i.e., containing a high percentage of the grain) is feasible only when the flour, meal, etc., is to be consumed without great delay. The polishing of rice effects the removal of the germ at the same time with the outer coating of the kernel.

If in normal times it is desirable to employ low extraction flours and other degerminated cereal products, as in the case of corn and rice, with the utilization of the by-products of the milling industry as stock feeds, it is of the utmost importance for the preservation of the public health that certain foods should always be included in the diet. These are to be so constituted as to correct the deficiencies of those foodstuffs which are derived from the endosperm of seeds, and from tubers and edible roots. In fact, those foodstuffs from plant sources, which have the functions of storage organs, have very comparable dietary properties. They all resemble the seeds in a general way, in their content of the two chemically unidentified dietary essentials and in the amount and character of their inorganic elements. The roots and tubers all have lower protein values than the seeds.

It has been pointed out that experimentally diets have not been found satisfactory when derived solely from seeds, and the same may be said of diets derived from the storage organs of plants. McCollum and his coworkers have shown that any combination of seeds, tubers, and edible roots will require for the satisfactory nutrition of an animal more fatsoluble $\mathrm{A}$, and certain inorganic salt additions. In certain cases protein supplements will likewise be necessary. In experimental work diets have been found satisfactory only when they contain a liberal amount of either the leaf of the plant, eggs, or milk. These foods have special values in that they are so constituted as to correct the faults in that portion, usually large, which comes from seed products, tubers, and roots. Within recent times the practice of eating liberally of the leafy portion of the plant has become in America almost discontinued among city dwellers, largely because of the high cost of such articles as lettuce, spinach, and such leaves as turnip, and beet tops, dandelions, etc., which are in some districts used liberally in the form of greens. It is true that the nutritive values with respect to energy and protein are low, but dietitians have employed these too extensively as the criteria of the relative values of foodstuffs in general. In the light of modern researches the peculiar 
qualities of the inorganic content of the leaf as contrasted with the rest of the edible portion of the plant have become appreciated as a factor of great importance. The more liberal consumption of dairy products is still more important, but the more liberal use of the leafy vegetables is to be advised. We have in the United States derived for many years between fifteen and twenty per cent of our food supply from milk, butter, cream, and cheese, and this has contributed in no small measure to maintaining a high standard of health and efficiency among our people. It is in those districts where milk and its products are not taken in the diet that pellagra and tuberculosis are a menace, and the researches of the last few years seem to establish the view that faulty diet is an important predisposing factor in the etiology of both of these diseases. The well-nourished and vigorous may be exposed to infection with tuberculosis without great danger, and the same appears to be true of pellagra, which in the view of several investigators is caused by an infectious agent. The studies of Goldberger practically establish the fact that where milk is used liberally in the diet, pellagra does not occur, and it is generally relieved by the patient taking a highly satisfactory diet. The relation between the character of the diet and the incidence of pellagra is a close one, but not in the way in which Funk believed. The disease attacks those whose resistance has been reduced by long adherence to a faulty diet.

Meats are of particular value only as respects the quality of their proteins and their extraordinary palatability. They are poor in both the fat-soluble $\mathrm{A}$ and water-soluble $\mathrm{B}$, and their inorganic content is not of a character suitable for the correction of the deficiencies of diets derived from seed products, tubers, and roots. Fruits are, after meats, the only highly palatable foods. They are poor in protein, poor in the fat-soluble A, and of some value for the correction of the inorganic faults in such diets as are in common use. The greatest single factor in the maintenance of good nutrition lies in the liberal use of milk and its products either in cookery, confections, ice cream, or as a beverage.

There has been much written during the past few years about the special value of freshness in vegetable foods as contrasted with cooked, canned, and preserved articles. This has, without doubt, been overrated. In carefully conducted investigations on animals it has been shown that cooking in the ordinary way, or even subjecting foodstuffs to such temperatures as are employed in the processing of canned fruits and vegetables, does not tend to destroy rapidly the two unidentified dietary essentials, unless the reaction of the food is alkaline, under which condition they are rapidly destroyed. Such a condition is met with only occasionally, as in the preparation of soda biscuits or the cooking of 
greens or beans with soda for the purpose of hastening the rate of cooking. This practice need not necessarily be condemned, however, for if such a selection of foods is made as will insure that the protein and the inorganic content of the diet as well as the two unidentified factors are all satisfactorily represented, it will do no harm to destroy the latter substances in such a small fraction of the food as is ordinarily derived from beans, greens, and soda biscuits. Safety in nutrition is best to be safeguarded by emphasizing the importance of taking liberal amounts of dairy products and the leafy portions of the plants. The selection of the rest of the diet safely may be left to the appetite of the individual.

\section{BIBLIOGRAPHY}

I. EIJKMAN, C.: Arch. f. Hyg., I906, LVIII, I50.

2. FRASER, H. and STANTON, A. T.: Lancet, London, I9Io, I, 733.

3. FUNK, C.: Lancet, London, II, I266.

4. OSBORN, T. B. and MENDEL, L. B.: Bull. I56, Pub. Carnegie Inst., Washington, I9II. Parts I and II.

5. HOPKINS, F. G.: Jour. Physiol., I9I2, XLIV, 425.

6. McCOLLUM, E. V. and DAVIS, M.: Jour. Biol. Chem., 1913, XV, I67.

7. McCOllUM, E. V. and DAVIS, M.: Jour. Biol. Chem., I9I5, XXIII, I $8 \mathrm{I}$ and $23 \mathrm{I}$.

8. McCOLlUM, E. V. and KENNEDY, C.: Jour. Biol. Chem., I9I6, XXIV, 491 .

9. McCOLlUM, E. V. and SIMMONDS, N.: Jour. Biol. Chem., I9I7, XXXII, I 81 .

Io. McCOLLUM, E. V. and PITZ, W.: Jour. Bio. Chem., I9I7, XXXI, 229.

II. JACKSON, L. and MOORE, J. J.: Jour. Infect. Dis., I9I6, XIX, 478.

12. HESS, A. F.: Amer. Jour. Dis. Children, I917, XIV, 337 and 478 .

I3. GOLDBERGER, JOSEPH: Jour. Amer. Med. Assoc., I916, LXVI, 47 I. Public Health Reports, Nov. I7, I916, 3159: Nov. 12, I915, 3.

I4. CHITTENDEN, R. H. and UNDERHILL, F. P.: Amer. Jour. Physiology, I9I7, XLIV, I3.

15. McCOLLUM, E. V., SIMMONDS, N., and PARSONS, H. T.: Jour. Biol. Chem., I918, XXXIII, 4II. 
CHAPTER II

CLIMATE IN RELATION TO HEALTH AND DISEASE

By Henry Sewall

\section{THE GENERAL BIOLOGICAL RELATIONS OF CLIMATE}

Climate is the "summation of atmospheric conditions as recorded for a long period of time; or, in other words, it is the totality of weather, while weather is the physical condition of the atmosphere at a given time, or during a limited period." $\left.{ }^{1}\right)$ It is obvious that these atmospheric conditions are themselves determined by the integration of many diverse factors, among the most important of which are the motions of the earth, latitude, altitude, the distribution of land and water, the nature of the soil and its surface features, ocean currents, and prevailing winds.

It is the province of meteorology, through the aid of refined apparatus, to exploit the physical data of climate, but the subject owes its medical interest wholly to the effects which the elements exert upon the living body. It is obvious that meteorology thus applied demands a consideration of all physiological processes both in health and disease, and the terms "physiological climatology" and "medical climatology" are descriptive of the fields of knowledge involved in a study of the subject. It would seem self-evident that forms of life and physiological processes must be intimately related to climate; the distribution of vegetation is crucial evidence, at least, for plant life. As regards animals, and especially man, the problem is more complex. The importance of climate to human welfare must always have held something like an inverse relation to civilization according as this involved means of protection against the elements.

When culture advanced to the stage where study of disease began, climatic or at least weather influences must have entered largely into the schemes of treatment. Our recently acquired knowledge of the etiology of diseases has gone far towards eliminating false notions as to the physiologic effects of certain climates. Phases of debility which until recently were ascribed universally to climatic influence are now known to be due wholly to the pathogenic effects of certain preventable infections whose agents and media of transmission happen to find a congenial 
environment in hot climates. This is strikingly true of most, if not all, of the so-called "tropical diseases" which were formerly supposed to depend directly upon the physical conditions of the equatorial belt. It has been maintained that the distribution of races has, in certain regions, been ordered by the prevalence of malaria in the past, and today there are areas wherein the "sleeping sickness" forbids community life. In short, there can be no solid foundation of knowledge in physiological and medical climatology until the relations of endemic and transmissible diseases and of human habits of association are clearly recognized.

When numbers of men are suddenly removed from their homes and congregated in concentration camps, as in the preparation of our National Army, we are prone to attribute the outbreak of epidemic disease among them to climatic influence; but it is open to conjecture that other factors, such as lowered vital resistance from physical fatigue and nostalgia, undue exposure to infection by the too close proximity of the well to the sick, etc., more effectually explain the epidemiology. Investigation of the incidence of epidemic disease in the concentration camps throughout the United States has developed the significant fact that the morbidity rate has borne a definite relation to the previous habitat of the sick soldier. Troops drawn from rural communities suffered under the same conditions much more in the epidemics than those accustomed to city life. It is appropriate to add that practitioners in Denver are frequently impressed with unwonted development of colds and sore throat among the robust class of miners and others who visit the city at rare intervals from their mountain environment. Obviously the epidemicity in question is rather a biological than a climatological problem. When all is said, we must admit the possibility of unsuspected factors which link together biologic and meteorologic states.

A classification of the geographical distribution of diseases is a useful adjunct to a description of the vital influence of climate in suggesting causal relations between climate and disease, but especially is it useful in leading to detection of popular errors of assumption as to the incidence of such relations. The study of medical climatology really embraces, then, within its subject-matter nearly the whole range of our knowledge not only of inorganic nature but of human physiology both in health and disease.

During the last half-decade the literature has been wellnigh bare of contributions to Climatology, even in the shape of compilation. The medical mind appears to have lost interest in the subject. It is worth considering whether the deficiency is inherent in the medical mind or in the value of the subject. For example, pulmonary tuberculosis is the disease, par excellence, for which climatic change was traditionally 
recommended. When with advancing knowledge it was found that excellent therapeutic results could be obtained by home treatment in cases which it was once thought could be helped only by a far journey, the average medical mind hastened to generalize the truth and fell into the well-known error of treating the disease instead of the patient.

As will be pointed out presently, the vital relations of climate depend upon definite biological reactions caused by it, and these reactions may be either helpful or harmful to a given organism. Manifestly, further advance in knowledge must depend upon the application to the problem of the highest technical skill and trained observation. Two general methods are available for the advance of our knowledge of the influence of climate on life; namely, by observation and by experiment.

The first method finds its exercise in what may be called "field work," noting and classifying biological reactions in a given locality and comparing them with those manifested under different climatic conditions. Alexander von Humboldt's work $\left({ }^{2}\right)$ has long been a classic in this field, but it is a source of knowledge to which every adequately endowed observer may venture to add. The journal literature is already ricl in contributions on medical climatology applied to the treatment of certain diseases and a considerable number of admirable monographs present the facts and principles of Meteorology and Climatology as ascertained from observation. Among the more indispensable of these in chronological order are those of, Moore $\left({ }^{1}\right)$, Williams $\left({ }^{3}\right)$, Solly $\left({ }^{4}\right)$, Weber $\left({ }^{5}\right)$, Hann $\left({ }^{6}\right)$, Huggard $\left({ }^{7}\right)$, and Hinsdale $\left({ }^{8}\right)$. The general range covered embraces a presentation of meteorologic data, a discussion of the effect of meteorologic and climatic conditions on the body in health and disease, and a classification of health resorts as to their adaptability to various pathologic conditions.

The second method of investigation is by laboratory experiment, which, of course, entails not only the use of certain physical apparatus but special training in the technique and principles of research. Paul Bert $\left(^{\circ}\right)$ in his monumental work was the pioneer in practically all the great advances which have been made in our knowledge of the vital relations of climate during the past forty years. The essence of the experimental method consists in an analysis of a complex problem into its simplest variables and a study of these seriatim. It was by laboratory demonstration that proof was first offered as to the importance of the heat and humidity of the air in ventilation. Laboratory methods have been applied with great success to a study of the physiological infuence of altitude by a considerable number of highly trained observers, notably Mosso $\left({ }^{10}\right)$, Zuntz $\left({ }^{11}\right)$ and his colleagues in the high Alps, and especially Haldane $\left({ }^{12}\right)$ and his coworkers on Pike's Peak, Colorado. These 
investigations form a model for research in many other climatic conditions.

One basic concept underlies an understanding of the principles and facts of physiological and medical climatology; it appears to have been first clearly stated in the admirable work of Huggard ( $\left.{ }^{7}\right)$. "The most fundamental point in the action of climate is its influence on tissue change." It is, in other words, the physiological reaction of the organism that determines climatic influence. It is obvious, therefore, that the vital effect of climate is a problem of protoplasmic irritability. Physiological reactions to climate obey the fundamental law for the stimulation of nervous tissue, long ago announced by du Bois-Reymond, namely, that the vital effectiveness of a stimulus is determined, not by its absolute intensity but by its rate of change of intensity:

To this must be added Weber's law of the relation of sensation to stimulus, according to which, "when a stimulus is continually increased, the smallest increase of sensation which we can appreciate remains the same so long as the proportion which the increase of the stimulus bears to the whole stimulus remains the same; that is to say, one varies directly as the other." $\left({ }^{13}\right)$ Huggard $\left({ }^{7}\right)$ dwells on the well-known fact that "heat and cold in alternation have a very much more marked effect than has either one when continued for some time without change. A given amount of cold following heat has a much more stimulating action than has the same amount of cold long continued, and in like manner heat following cold brings about greater lassitude or stimulation according to the individual reaction than does long-continued heat. . . . Ross tells, that when he and his party had.been for a long time exposed to a temperature of $-47 \mathrm{~F}$., a temperature of -20 and $-25 \mathrm{~F}$. was found agreeable; and Captain Parry records that when his men had been for some time exposed to a temperature of - $3 \mathrm{~F}$. they complained of the heat when the temperature rose to $26 \mathrm{~F}$." The effect of an environment, as implied above, largely depends on the phase of irritability at the moment characterizing the reacting organism. This principle lies at the basis of the difference in the effects of a given climate on individuals according to race and age. Ás the grade of physiological irritability, which reacts to what may be called the centripetal influence of environment, is determined by a long chain of antecedent organic events, so no less truly does the centrifugal response of the organism to stimulation depend upon the more or less gradually acquired grade of metabolic power inherent in the tissue cells at any moment of stress. That is, biologic reactions are largely determined by training and acclimatization. A proper course of training modifies every physiological mechanism so that, after a longer or shorter time, an individual may accomplish feats 
with ease, the attempts at which before training would have proved disastrous.

Zuntz and his coworkers on Monte Rosa found excessive physiological friction and lost effort to be maintained by Alpine guides at the beginning of the climbing season, while later in the year, after continued practice, the metabolic waste gave way to more perfect machine efficiency. Haldane $\left({ }^{12}\right)$ and his colleagues dwell upon the profound differences in the physiological powers and reactions manifested at high altitudes by the acclimatized as contrasted with the unacclimatized individual. It is self-evident that these conceptions lie at the basis of the climatic reactions of the sick man as of the well. Whether the pathologic state is due to infection, as in tuberculosis, or to metabolic insufficiency, as in diabetes, the essential fact is the limitation in the range of physiological accommodation to cellular stress. The wise clinician attempts to adapt the vital burden to its bearer according to his strength, at first by a régime of absolute rest and then, with developing adjustment, with increase of burden through a course of training in which effort must never exceed the limits of elasticity.

The medical climatologist will avoid error in his therapeusis by keeping in mind a postulate recently well elaborated by Krause $\left({ }^{14}\right)$, that a disease-producing agent may have opposite vital effects according as the affected tissue is healthy or is already diseased. "Microorganisms that are brought to a tissue that is already inflamed fall on relatively resistant soil; but as regards the further development and spread of an already established focus inflammation can exert an effect that may not be so favorable." This is a special corollary of the general proposition which we have set forth.

A broad conception of the fundamental relationship between climatic stimuli and racial civilization and efficiency has been elaborated by Huntington $\left({ }^{15}\right)$. He has collected evidence that the most progressive peoples have always lived in the most variable climates; climates in which the meteorologic conditions vary extensively from day to day, but still within ranges which do not include extremes of temperature, humidity, etc. "Whenever civilization has risen to a high level, the climate appears to have possessed the qualities which today are most stimulating." Huntington studies the effects of weather upon the efficiency of groups of factóry employees, students, etc. "The results are surprising. Changes in the barometer seem to have little effect. Humidity possesses a considerable degree of importance, but the most important element clearly is temperature. The people here considered are physically most active when the average outside temperature is from 60 to 65 degrees, that is, when the room temperature rises to 70 degrees 
or even more. . . Mental activity reaches a maximum when the outside temperature averages about 38 degrees, that is, when there are mild frosts at night. Another highly important condition is the change of temperature from one day to the next. People do not work well when the temperature remains constant. Great changes are also unfavorable. The ideal conditions are moderate changes, especially a cooling of the air at frequent intervals."

That the emotional states as well as productive mental concentration are modified greatly by weather influences is the thesis of a monograph by Dexter $\left({ }^{16}\right)$. He concludes that " the quality of the emotional state is plainly influenced by weather states. . . . Those meteorological conditions which are productive of misconduct in a broad sense of the word, are also productive of health and mental alertness; as a corollary, misconduct is the result of an excess of reserve energy not directed to some useful purpose."

Philosophers, from Herbert Spencer to Jacques Loeb $\left({ }^{17}\right)$ and L. J. Henderson $\left({ }^{18}\right)$, have dilated upon the conception of life as a dynamic equilibrium in the interaction between external and internal factors. Perhaps the richest but most neglected treasury of information upon this point of view is found in Medical Climatology. Henderson says, "Fitness is a mutual or reciprocal relationship between the organism and the environment." Osborn $\left({ }^{19}\right)$ has emphasized the dominance of force as contrasted with matter and form in a recent work on "The Origin and Evolution of Life on the Theory of Action, Reaction, and Inter-action of Energy." Enough has been said, at least, to show that leaders of modern thought agree that the conception of a continually adjusted balance between environment and organic function is funclamental to an explanation of vital phenomena. The adjustment by which equilibrium is maintained depends upon the physiological reaction of the organism to external conditions and changes.

The relation between environment and the living, acclimated organism may be represented crudely as an equation in which physiological functions are grouped on one side and climatic factors on the other. As has been pointed out, the number of physiological variables to be considered is exceedingly great. The state of the vital organism is not merely the relatively simple one of health, but the living mechanism may have its metabolism, and consequently the power of adjustment, modified in all sorts of pathologic conditions. Perhaps a relatively simple mechanical-clinical illustration of the reality of such a vital-climatic balance may serve to condense the ideas which have been set forth. Such an illustration is afforded by the effect of what is essentially lowered barometric pressure on the functional capacity of the heart. 
The writer's observations have been made at Denver, which is situated on a rolling plain just one mile above sea-level. To this moderate elevation the average healthy organism adjusts itself without apparent sensory or motor disturbance. In a large proportion of cases of cardiac debility and disease life is here not only comfortable, but there is reason to believe that the circulatory powers are actually strengthened by the residence. However, the clinician constantly has forced on his attention the fact that in another, not inconsiderable, proportion of cases, compensation for cardiac defects is difficult to achieve and impossible to maintain. It is a common practice of the Denver physicians to refer their refractory cardiac patients to southern California, and the reports that return of the salutary influence of the change have well established a belief that even moderately high altitudes are distinctly contraindicated in the treatment of certain forms and stages of cardiac disability. A special form of heart failure is exhibited not infrequently by tuberculous invalids, newly arrived in Denver, who show no sign of organic heart disease, but who suffer from a mechanical obstruction to the lesser circulation because of pulmonary cirrhosis and probably attendant arterial sclerosis. Such a person, coming from sea-level and beguiled by the stimulating atmosphere to indulge in exercise beyond his strength, within a day or two may begin to suffer extraordinary dyspnea on exertion. His sense of well-being is otherwise little impaired and it is difficult to convince him of the necessity for absolute rest. A physical examination shows a somewhat dilated and turbulently acting heart and, most significant, the moist râles of edema throughout those areas of the lungs which were formerly probably in normal condition.

In the writer's experience this is a state of affairs which nearly always terminates fatally within one to several days. The author is convinced that such a history is only characteristic of broken compensation in un-acclimatized persons. He has not observed it in cases, however debilitated, who have been in Colorado a fortnight or more. The break in compensation may affect persons in a rather advanced stage of tuberculosis who have gone home for a visit and then returned and been led by overconfidence to an excess in exercise. The experienced physician in Colorado is prone to advise complete mental and physical rest for a few days for patients recently arrived from low altitudes. Eastern practitioners are liable to err in neglecting to direct patients, whom they refer to higher altitudes, to seek at once the advice of a competent doctor on their arrival.

These are facts without one word of theory. To leap to the conclusion that all cardiopathic cases should avoid moderate elevations above the sea would be contrary to reason and experience. The heart, like other 
muscles, demands for its best health an amount of exercise carefully adjusted to what Mackenzie $\left({ }^{20}\right)$ calls "its range of physiological response." In prescribing therapeutic rules it is of fundamental importance to realize, that, while a temporary overstrain thrown upon a normal organ rarely causes a permanent disorder, a similar insult offered to a diseased or degenerated tissue is prone to produce a disastrous lapse of function. Discussion of the physiological-physical basis of such clinical experiences will be deferred to a later section. The simple facts are sufficient evidence for the belief that there is some special relation inherent between barometric pressure and cardiac compensation.

With this introduction we shall outline the principal physical factors of climate and briefly discuss their individual and collective influence upon physiologic functions and, finally, we shall present a synopsis of actual climates according to their therapeutic indications.

\section{METEOROLOGICAL CLIMATOLOGY}

An instructive and elaborate classification of climates is that given by W. F. R. Phillips $\left({ }^{21}\right)$, but for our purpose a somewhat different arrangement will be useful. Physiological Climatology is essentially a study of metabolism as affected by atmospheric conditions. These fundamental conditions are Temperature, Wind, Humidity, and Barometric Pressure, to which may be added the more or less hypothetical influences of Radiant Energy and Electricity. The intensities of these meteorological variables are determined by certain terrestrial constants which readily fall into five groups, as represented in the table. In the following brief survey of meteorological data the atmospheric factors will be treated according as they obviously influence physiological reactions.

\section{TERRESTRIAL}

A Classification of Conditions Which Affect the Atmosphere

Determining Conditions

Conditions Determined

I. Distribution of Land $\left\{\begin{array}{c}\text { Temperature } \\ \text { and Water }\end{array}\right.$ Humidity $\left\{\begin{array}{c}\text { Temperature variations } \\ \text { (winds) } \\ \text { Insolation } \\ \text { ture) }\end{array}\right.$ (tempera- $\}$ Metabolism

II. Latitude

$\left\{\begin{array}{l}\text { Insolation (tempera- } \\ \text { ture) (winds) } \\ \text { Wind Direction }\end{array}\right\}$ Metabolism 
Determining Conditions

III. Soil and Topography $\left\{\begin{array}{l}\text { Temperature (winds) } \\ \text { Humidity } \\ \text { Radiant Energy and } \\ \text { Electricity }\end{array}\right\}$ Metaholism

IV. Altitude (Barometric $\left\{\begin{array}{l}\text { Temperature (winds) } \\ \text { Humidity } \\ \text { Radiant Energy and } \\ \text { Electricity }\end{array}\right\}$ Metabolism

V. Terrestrial Rotation
and Revolution $\left\{\begin{array}{l}\text { Seasons } \\ \text { Day and Night } \\ \text { Insolation (tempera- } \\ \text { ture) Metabolism } \\ \text { Wind Direction }\end{array}\right\}$ M

It will be observed that at any definite altitude there are but three interrelated variables which concern the meteorologist; namely, temperature, humidity (and precipitation), and winds. The factors of radiant energy and electric potential do not, as yet, submit to calculation. Meteorology exploits the laws of interaction of these physical phenomena and furnishes rules for the deduction of future weather conditions, which have made of it one of the most noteworthy of applied sciences. Unfortunately, it is still far from possible to deal with the data collected by the meteorological observer with mathematical exactness; judgment based on experience is an essential agent in the construction of all weather maps. $\left({ }^{22}\right)$

It is interesting to observe that the physical elements which engage the meteorologist have definite influences upon the metabolism and physiological reactions of living forms and that there is a close parallelism in the relative importance of the physical agents in inanimate and animate nature. Temperature is the dominant factor in both fields. Winds are the outcome of temperature differences, humidity modifies temperature, and the various states of water are determined by it.

L. J. Henderson $\left({ }^{18}\right)$ has elaborated a view of the vital relations of inorganic media which illuminates the underlying principles of Climatology. This author's purview reaches beyond the processes of adaptation through which organisms are brought into harmony with their environment, and studies the special fitness of the materials forming the environment for the support of life. He argues that the specific properties of water and of carbon dioxide, and the chemical properties of the elements. carbon, hydrogen, and oxygen, distinguish these substances from all 
others in fitness to sustain life; so that, when living matter was developed, it was in a world the preponderant mass of which had already been selected, as it were, with special reference to the nutrition of organisms.

Distribution of Land and Water. Effect on Temperature. About three-fourths of the surface of the earth is covered by water. Nearly three-fourths of the land is aggregated in the Northern Hemisphere. Temperature is the dominant factor in meteorological, as it is in vital, phenomena, and it is necessary to trace the conditions leading to its distribution and variation.

Since in the Southern Hemisphere the earth is nearer the sun in the summer and farther away in the winter than in the Northern Hemisphere, it might be expected that the Southern Hemisphere would present a greater range of seasonal extremes in temperature than the Northern Hemisphere. Observation shows the reverse to be true, and the explanation is to be found in the relative excess of water south of the equator. The specific heat of water is said to be four times that of land; that is, the absorption of a given amount of solar energy would raise the temperature of soil four times as high as that of an equal weight of water. Land, as a rule, reflects and conducts heat poorly, but absorbs and radiates it readily. Water is a good reflector and a fairly good conductor of heat; the proportion of solar energy absorbed penetrates rather deeply and is evenly distributed. When this mobile body is unequally warmed convection currents are set up which tend to equalize the temperature throughout.

Among the results of such unequal heating are the ocean currents which may modify profoundly the normal temperatures of the latitudes which they visit. But, as pointed out by Moore $\left({ }^{1}\right)$, there is popular misconception as to the mode of action of such currents, especially as illustrated by the Gulf Stream. Thus, that western Europe is warmer, moister, and of more equable temperature than the eastern coast of America for similar latitudes, is due to the fact that the prevailing winds of the European coast are westerly and have traversed a broad stretch of ocean to whose surface the Gulf Stream has transported an immense body of relatively warm water. Similar differences of climate probably would obtain between the two coasts were other conditions to remain the same but without the Gulf Stream. On the equator there is a nearly constant surface temperature of the ocean of $82^{\circ}$ to $84^{\circ} \mathrm{F}$. "At the depth of 2,400 feet the temperature is $44^{\circ} \mathrm{F}$. and unchangeable, below 6,000 feet it is little above the freezing-point of fresh water; namely, $34^{\circ}$ to $36^{\circ} \mathrm{F}$. In the middle latitudes the surface variation is from $50^{\circ}$ in winter to $68^{\circ}$ in summer. At latitude of $70^{\circ}$ the surface temperature 
has but a small diurnal variation and a yearly range of from $35^{\circ}$ for winter to $45^{\circ}$ for summer; at a depth of 2,400 feet it remains steady at $32^{\circ} \mathrm{F}$. From this level there is a gradual decrease to a depth of 6,000 feet, where a constant temperature of about $28^{\circ} \mathrm{F}$. exists, and below this to the bottom there is no change." $\left({ }^{1}\right)$. These figures impress one with the magnitude of the forces constantly disturbing the uniformity of density in the ocean and causing compensatory movements in its water.

It may be asssumed that the earth owes its warmth to solar radiation and it is obvious that the intensity of insolation at any point on the surface decreases with the obliquity of the sun's rays. When the sun is over the equator, the amount of solar energy received by a given area on any meridian at midday is said to vary approximately as the cosine of the latitude. As dust-free air, through its content of watery vapor and carbon dioxide, has great power of absorbing and making latent heat, the amount of solar energy reaching the surface of the earth in higher latitudes is further reduced by the increased atmospheric envelope presented to the oblique rays. Suspended impurities exert an additional variable extent of absorption. Latitude also determines the relative length of day and night, and this effects warmth. Moore writes, "The rapidly increasing length of the day toward the poles during the summer soon more than compensates for the decreasing angle at which the solar rays strike the earth." The amount of insolation received at the poles during the summer is considerably in excess of that reaching the equator. The favorable effects of increasing latitudes in magnifying the access of solar energy in summer is proportionately reversed in winter, because, where there is increase in the length of clay in one season, there is a corresponding increase in the length of the night in the other. It is said that "annually the pole receives $4 \mathrm{I}$ per cent of the amount of insolation that reaches the equator." $\left.{ }^{1}\right)$

Altitude, or elevation above sea-level, is an important constant in the determination of temperature. The increasing cold of the higher atmosphere is well known in aeronautics. The temperature of interstellar space is said to approach absolute zero. Mountain peaks in the tropics may remain snow-covered throughout the year. "Dry air cools by expansion in ascending at the rate of about $\mathrm{O}^{\circ} 55^{\prime \prime}$ of a degree Fahrenheit for every 100 feet of elevation. . . If air at a temperature of $60^{\circ} \mathrm{F}$. and a pressure of 30 inches has its pressure doubled, its volume decreases to one-half and its temperature rises to $175.5^{\circ}$, the first increase of pressure of one inch effecting an increase in temperature of $5^{\circ}$. If its pressure is diminished one-half, its volume will expand to double the original size and its temperature will fall from $60^{\circ}$ to $\left.2.4^{\circ} . "{ }^{1}\right)$ As 
will be seen later, the temperature characteristic of high altitudes is largely dependent on the diminution of aqueous vapor in the air. "The general law of the diminution of vapor with altitude depends principally on the law of the diminution of temperature." $\left({ }^{1}\right)$

The nature of the soil influences the temperature of the air above it according to its properties of reflection, absorption, and radiation. Practically the problem is always complicated by the water content of the ground, not to speak of the vegetation which may cover it. "Drainage lowers the specific heat of the soil and decreases the cooling effect of evaporation; therefore, under sunshine the dry soil becomes warmer than the wet and . . . it has a higher temperature and therefore radiates more freely to the air above. A covering of sand likewise lowers the specific heat of the surface, and thereby conduces to a higher temperature during the day and increased radiation at night." $\left({ }^{1}\right)$

A soil having a high specific heat and a moderate temperature may transmit to the cooler air above a moderate degree of heat for a long time. A soil, like dry sand, having low specific heat and a high temperature transmits considerable heat comparatively rapidly. As will be seen, the development of dew and frosts and the effect on vegetation is closely dependent on these facts.

While latitude is the most important factor determining the distribution of temperature over the earth, the intensity of insolation at a given place is greatly modified by the pressure and the composition of the air, especially as regards its content of moisture. This agent absorbs the heat rays of the sun and provides a uniformly warm blanket for the surface. Its presence tends to equalize the difference in temperature between sun and shade. As will be seen later, where atmospheric moisture is deficient, as at high altitudes, the air temperature may be low in the shade while a few feet distant under the direct rays of the sun the heat may be scorching. We must, therefore, distinguish between solar and air or telluric temperatures.

To obtain the temperature of the air a thermometer should be suspended in the shade at a considerable distance above the ground and away from the proximity of objects which radiate and reflect heat. Solar temperature is measured by a special form of thermometer having a blackened bulb inclosed in vacuo and suspended in the direct rays of the sun. It is evident that the difference between the sun and air temperatures is variable at any place and increases markedly with elevation above sea-level.

The lowest temperature of the day occurs shortly before sunrise while the highest is registered an hour or two after noon. There is a similar retardation of seasonal temperatures; the warmest period of the summer 
in the Northern Hemisphere tends to follow about a month after the summer solstice (June 2I), and the coldest weather is found at a similar interval after the winter solstice (December 22).

At meteorological stations most atmospheric data are registered graphically by instruments operating continuously, but observers are accustomed to make records, especially of the air temperatures, at certain hours, as 7 A.M., 2 P.M., and 8 P.M. The average of these temperatures is known as the mean temperature of a place. Hann $\left({ }^{6}\right)$ recommends the formula (7 A.M. +2 P.M. +9 P.M. +9 P.M. $) \div 4$, as giving a close approximation to the true mean temperature.

Lines drawn upon a map through all the localities having the same temperature are known as isotherms. Isotherms may be used to connect together places having the same daily, monthly, seasonal or annual mean temperatures. In weather forecasting it is necessary to know the distribution of temperatures at the same absolute time. On maps of the U. S. Weather Bureau isotherms represent the simultaneous distribution of temperatures at the hour of 8 A.M. on the $75^{\text {th }}$ meridian which runs slightly eastward of the city of Philadelphia. Seasonal maps of isotherms represent the intensity and geographical variation of temperature. In high latitudes of the Southern Hemisphere the isotherms run nearly parallel to the equator throughout the year, that is, the temperature variations are slight. In the Northern Hemisphere, especially over the continents, the isotherms bend far to the north in summer and to the south in winter. The prime reasons for these differences lie, of course, in the relative distribution of land and water. Hann $(6, p .17)$ writes, "Organic life is much more closely affected by the irregular changes of temperature which occur during shorter periods, as by the changes within the same month, and especially by those which take place from one day to the next. The value of such changes as these is a gauge of what is known as the variability of temperature. If these changes are slight, the climate is known as an even or a uniform one; if they are considerable, we speak of the climate as variable or changeable as regards its temperature."

Winds. It is obvious from the difference in the specific heat and other physical properties of land and water that, exposed to the same solar radiation, there would be a much greater rise of temperature in a given surface of the former than the latter; conversely, in the absence of the sun the cooling of the land would proceed more rapidly than that of the water. Through radiation and conduction the air above the respective surfaces partakes of their temperature. The volume of a given weight of air varies directly with its temperature, therefore air which is warmed over a restricted area becomes relatively lighter than 
the surrounding medium and rises through it as in a chimney, being replaced at the surface by cooler and denser air flowing in from all sides. It is believed that the ascending column overflows in the upper atmosphere and thus a continuous current is initiated. Thus, temperature differences determine the relative weights and barometric pressures of air columns at any given altitude and when the barometric pressures in adjoining areas differ there is always an air movement or wind from the region of higher to that of lower pressure. The velocity of the wind is proportional to the pressure gradient, which, at a given place, " is the difference in barometric pressure at the ends of a line of small length taken horizontally and at right angles to the direction of the isobars (lines drawn through places having the same barometric pressure) cutting the line, divided by the length of the line. The effect of gravity in causing the air to move in the direction perpendicular to the isobars is proportional to this pressure gradient." $\left({ }^{22}\right)$ That is, the breeze is stronger the closer together are the points of a given pressure difference.

"Atmospheric circulation, whether manifesting itself, for example, in a monsoon or in a gentle lake breeze, is a gravitational phenomenon induced and maintained by temperature differences. If there were no differences whatsoever in temperature throughout its different parts, the atmosphere of the earth would quickly settle into a state of rest.relative to the surface of the earth. The distribution of atmospheric pressure would then be that demanded by gravity on a rotating globe." $\left({ }^{22}\right)$

Perhaps the simplest illustration of the temperature cause of winds is to be found in the diurnal land and sea breezes-familiar phenomena of the seacoast. "As the temperature of the land rises from increasing insolation during the morning hours, the air near the surface expands. In consequence there is a flow of air in the upper levels out over the sea, increasing the surface pressure there, and causing a surface flow of air in toward the land. This flow will be maintained until the land cools off after sundown, when a reverse circulation will be established. As the air over the land cools and contracts, air flows in from the sea, increasing the surface pressure over the land and causing a surface current from the land to the sea, which is usually gentle as compared with the sea breeze." $\left({ }^{1}\right)$

Wholly similar in origin to the land and sea breezes are those seasonal air movements known as monsoons, which have opposite directions in summer and winter. The term is usually restricted to winds originating in regions where the difference in temperature between land and sea is especially great, as in India, China, Australia, and portions of Africa. "If the term 'monsoon' be extended, as it properly should, to include all 
winds whose prevailing directions and velocities undergo distinct alterations as a result of seasonal changes in temperature, it clearly follows that this class of winds is wellnigh universal." $\left({ }^{22}\right)$

Winds that travel a considerable distance become affected as to direction by the rotation of the earth. As the velocity of surface movement of the earth becomes progressively less from the equator to the poles, air proceeding from lower to higher latitudes in the Northern Hemisphere acquires an eastwaird direction and vice versa. In the Southern Hemisphere the same rule applies and gives reversed deflections. "Any objects whatsoever moving over the surface of the earth are subjected to a more or less pronounced deflective influence that tends to cause the body to swerve to the right of its onward course in the Northern, and to the left in the Southern Hemisphere." $\left({ }^{22}\right)$

The "trade winds" of the sub-tropics blow almost continuously over the ocean from the northeast in the Northern Hemisphere and from the southeast in the Southern Hemisphere; the direction of each verges to nearly east as the equator is approached. Over continental areas the inequalities of heating due to the physical features of the land complicate the condition of air movement. The weather maps issued daily by the U. S. Department of Agriculture epitomize graphically the physical conditions of pressure, temperature, etc., prevailing over the United States, and from them the meteorologist is able to make forecasts which have considerable accuracy for limited periods. On weather maps centers of highest pressure are marked as "high" and those of lowest pressure as "low." "Under the influence of gravity the air at any point presses equally in all directions, thus causing it to flow from a region of greater pressure toward one of less. The velocity with which the wind mores from the high toward the low will depend largely on the difference in air pressure." ( $\left.{ }^{1}\right)$

From the point of view of temperature and movement the atmosphere may conveniently be considered to be made up of three superimposed layers. The first is predominantly the region of terrestrial disturbance. It reaches vertically from the ground about 3,000 meters ( 9,842 feet). The temperature gradient is here very irregular and is often reverse. It is the region of strong convection and of storms. From the top of this layer up to about 10,000 meters $(32,808$ feet) above sea-level is the second layer, a region where the temperature is always much below the freezing-point and its gradient is nearly constant. There is here comparatively little condensation. "During storms it may be the seat of vertical convections of various extents and intensities, but its normal condition is one of stability, as shown by its usual freedom from clouds." Above this layer comes the third. In the explored region of this third 
layer above the I0,000-meter level, the temperature is said to be nearly uniform, though it generally increases slowly with ascent and, therefore, vertical convection is impossible. $\left({ }^{1}\right)$ (These facts, of merely theoretical interest when discovered, have become indispensable practical knowledge since the aeroplane has extended the activities of man within the second zone of the atmosphere.) An additional division of the lowermost stratum of the air, of great biological interest, should distinguish a shallow layer directly contiguous to the ground and subject to the radiations and precipitations of the surface.

Atmospheric Moisture or Humidity. The air everywhere contains a variable proportion of watery vapor whose most distinctive physical property is its power of modifying temperature through its great capacity for absorbing and retaining heat. Water vapor has a little more than half the density of dry air. Its proportion varies from less than one per cent in certain arid lands to five per cent of the weight of the air in hot humid regions.

The amount of watery vapor which can be taken up by a given space is hardly affected by the gases already present. The temperature of the space determines the amount of moisture which it can maintain. A given volume of dry air at a certain temperature can take up only a definite amount of water in the form of vapor; it is then said to be saturated. If the air is cooled ever so little a certain proportion of the vapor is condensed to water. It is important, therefore, to distinguish between two views of humidity. One regards the mass or amount of watery vapor per unit volume. This can reach a maximum (saturation) which is strictly determined by temperature. It is known as the absolute humidity. Thus, at -20 degrees F. saturated air contains 0.166 grains of vapor per cubic foot; at 60 degrees the weight of vapor is 5.745 grains, and at 100 degrees it is 19.766 grains.

The other view takes account of the practical fact that air is rarely saturated with moisture, but only contains a fractional percentage of the moisture necessary to saturation. This is relative humidity. Instead of expressing the relative humidity in terms of moisture percentage actually present in the air, it may be convenient to consider the percentage necessary to complete saturation; this would indicate directly the drying properties of the air.

It is obvious that, as in very cold regions the absolute humidity of the air may be very low but its relative humidity high, in warm places the air may contain much moisture but have low relative humidity. In arid climates high temperature is combined with lack of moisture. In the cold of winter watery vapor may form only one-thousandt't part of the weight of the air, while in the heat of summer it may increase to 
one-twentieth part. $\left({ }^{1}\right)$ As the surface of the ground and vegetation cool at night by radiation, the contiguous layer of air deposits its moisture when its temperature falls below that of saturation, or the deze point of the air. Meteorological tables show a diurnal variation in the relative humidity varying as a rule from a maximum in the early morning to a minimum in the afternoon.

Although the vapor of water is specifically lighter than air its distribution is practically lifted to the lower atmosphere. About half the watery vapor lies below the altitude of 6,500 feet and nine-tenths below 21,300 feet. $\left({ }^{8}\right)$ This depends both on the surface origin of moisture and on the rapid cooling with ascent. The extraordinary temperature reactions of water form not the least of the properties which, as Henderson $\left({ }^{18}\right)$ shows, distinguish this medium from all others in the universe. The latent heat of steam is said to be greater than that of any other known vapor. The atmospheric vapor absorbs and renders insensible much of the solar heat; conversely, when the air is cooled beyond the point of vapor saturation, water is precipitated and the heat energy which had been used in its vaporization is set free to warm the air. The agent is thus an automatic equalizer of air temperature. A gram of water in being converted into steam at $100^{\circ} \mathrm{C}$. would absorb enough heat to raise about 536 grams of water from $\mathrm{o}^{\circ}$ to $\mathrm{I}^{\circ} \mathrm{C}$. This measures the cooling effect of evaporation.

The intensity of cold produced is dependent on the rate of evaporation; when this is very rapid, as in a spray of ethyl chloride applied to the skin, the temperature of the surface from which evaporation occurs quickly falls below the freezing-point. Though the amount of watery vapor which can be sustained in a unit volume of air is nearly indifferent to the number and pressures of gases already present, evaporation proceeds more rapidly as barometric pressure is lowered. "Boiling begins in water, as in other volatile liquids, when its temperature is raised so high that the pressure of its saturated vapor equals the pressure of the air." ( ${ }^{1}$ ) At sea-level, under a pressure of $760 \mathrm{~mm}$. Hg., water boils at $212^{\circ} \mathrm{F}$. With every 555 feet of ascent above sea-level the boiling-point falls approximately one degree and thus furnishes a crude means for measuring altitude. As the air in contact with the surface from which evaporation occurs becomes charged with moisture, it may be up to the point of saturation, further evaporation is proportionately retarded according to a familiar physical law, and the heat of the body is retained within its surface. A breeze which removes the water-laden coat and replaces it with dry air may enable evaporation to proceed at its maximum for the temperature existing.

From all the preceding it may be rightly inferred that in climates 
having low humidity there tends to be a great range of temperature between the heat of day and the cold of night.

The meteorologist calculates the saturation percentage of moisture in the air by comparing the temperature registered by the dry- and wet-bulb thermometers contained in an instrument known as the psychrometer. The bulb of one thermometer is covered with muslin which is moistened with water. Before taking an observation the instrument is rapidly whirled through the air so as to remove the film of moist air about the wet bulb. As the temperature registered by the wet-bulb thermometer is lowered in proportion to the rate of evaporation, and this is determined by the tension of aqueous vapor in the air, the relative humidity of the air can be deduced by comparison of the readings of the two thermometers. As will be seen later, the records of the wet-bulb thermometer have peculiar physiological value, since this instrument more nearly imitates the physical condition of the surface of the human body than does the ordinary thermometer.

Altitude. The attributes of altitude depend upon the barometric pressure. "The action of gravity on the gases of the atmosphere causes them to exert a pressure on the surface of the earth. The actual pressure at any given place is directly due to the elastic pressure (molecular bombardment) of the gases present, and is distributed among them according to their volume percentages at that particular place, but the sum-total of these several partial pressures per unit area in the open atmosphere, as measured by the barometer, is always just equal to the combined weight of all the gases in a vertical column of unit crosssection above this area." $\left({ }^{1}\right)$ The average atmospheric pressure at sealevel is that of a column of mercury $760 \mathrm{~mm}$. high and the weight borne by each square inch of surface is about 14.7 pounds.

Air is a somewhat complex mixture of gases to which adventitious elements may be added in industrial communities. Disregarding the important but variable factors of aqueous vapor as well as certain rare gases that occur in minute quantities, the composition of pure dry air is represented in the accompanying table, taken from Moore's work:-

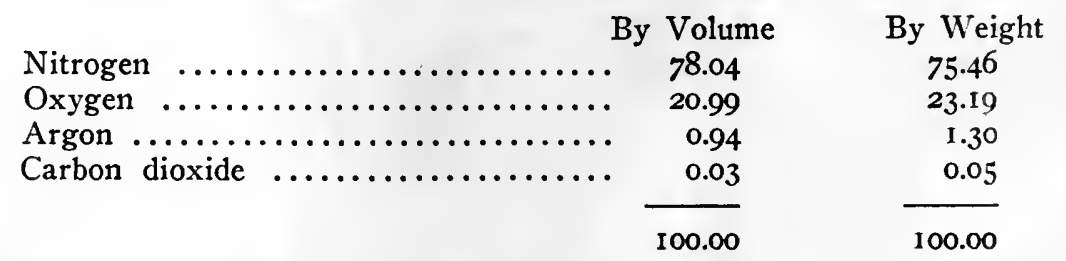

The relative proportions of these gases remain practically the same in the open everywhere.

With increase of elevation above the sea there is a continuous 
diminution of atmospheric pressure. A barometer reading $760 \mathrm{~mm}$. ( 29.92 inches) at sea-level, would register $168 \mathrm{~mm}$. (6.6r inches) at an elevation of six miles. At an elevation of 16,000 feet, the highest inhabited altitude, atmospheric pressure has about half its value as at sea-level. As will be seen later, the physiological effects of altitude are chiefly dependent on the diminution in the tension of oxygen, but the physical effects are due to lowered temperature and to diminution in the absolute humidity of the air. Dry air falls in temperature about I degree F. for every 183 feet of ascent. When the air is moist this rate is disturbed by the latent heat released from condensing moisture.

The effects of altitude upon humidity are complicated by the fact that warm moist winds striking the side of a mountain range flow up the slope and, chilled by the cold, may precipitate their moisture, thus leading to an actual increase of rainfall up to the level of 6,000 to 7,000 feet in middle latitudes. Accordingly one side of a mountain range may be very moist and the other very dry.

The moving air, robbed of its moisture, crosses the mountains and descends as a warm, dry wind known as föhn in Europe and as chinook along the Rocky Mountains. Pure, dry air is nearly diathermanous. Aqueous vapor has great capacity for absorbing and making latent the radiant energy of the sun; therefore, the intensity of insolation varies somewhat inversely as the absolute humidity of the air.

It is said that the atmosphere absorbs, on the average, about 76 per cent of the incident solar energy; on a cloudless day about one-half the energy reaches the surface of the earth, but when the air is cloudy nearly all is reflected or absorbed. In spite of intense insolation, mountain peaks harbor perpetual snow because radiation outward keeps pace with insolation inward. The interesting chapter of physics dealing with the specific reactions of the atmosphere towards various wave lengths of radiant energy cannot be opened here, but it is important to observe that the increase of insolation in high altitudes includes the shorter or "chemical" rays as well as the longer or "heat" rays. The physical characteristics of high climates, then, depend chiefly on the increased insolation upon and radiation from the earth, the great difference in temperature between sun and shade, and between day and night.

It is hardly necessary to observe that the purity of the air as regards the suspension of dust and other foreign matter rapidly improves with altitude. The historic experiments of Pasteur $\left({ }^{23}\right)$ which proved the absence of bacteria from the air of the high Alps made a fundamental advance in knowledge.

Electricity and Radioactivity. It may be surmised that climatic variations in electric potential have important relations to the vital reactions 
of forms of life, but nothing definite can be said about the matter. It is said that the atmosphere is usually electropositive as compared with the earth, that the open air is positive to that within dwellings, and that during rainstorms the air charge becomes negative. The allotropic change of oxygen into ozone, which occurs in electric discharges, is of uncertain import. As will be pointed out later, it is possible that the nervous system is specifically affected by radium emanations proceeding from the earth, as suggested by Grabley. $\left({ }^{24}\right)$

Grabley points out that the ions of the atmosphere arise chiefly from the radium emanations which stream from the earth in quantities determined by the nature of the soil and by its texture; the emanations being more abundant from a porous soil and diminished when the pores are filled with water. The radioactivity increases, accordingly, with elevation above sea-level. Atmospheric ions are attracted to and precipitated upon bodies that are negatively electrified. Grabley found that, when a sensitive person was insulated and negatively electrified on a day of high emanation, after a time he became very uncomfortable. To reach this effect demands the combination of high emanation and strong negative potential. The conclusion was drawn by Grabley $\left({ }^{24}\right)$ that much of the "nervousness" experienced by some persons before storms or in certain climatic environments is dependent on electric conditions. On the other hand, Durig $\left({ }^{25}\right)$ declares that there is no demonstrable connection between the ionization or electric potential of the air and physiological processes.

\section{BIOLOGICAL CLIMATOLOGY}

Climate and Vegetation. Climatology from the viewpoint of plant life presents a field of great human interest and of many involved problems. Other conditions, as soil and humidity, being favorable. the existence of vegetation depends upon the temperature of a locality. Plants do not grow until a temperature of about $43^{\circ} \mathrm{F}$. is reached. The longer the season during which the thermometer registers above this point, the more abundant and varied the vegetation. Of two places having the same mean temperature one may be barren and the other verdant according as the temperature remains continuously slightly below $43^{\circ} \mathrm{F}$. or fluctuates widely above and below this point. Nothing is more striking than the selective influence of temperature on the character of plant life as evidenced by the distribution of vegetation in high latitudes and on mountain slopes.

It is usually taken for granted that verdure-covered soil and forestation markedly modify local climates. Vegetation is thought to cool the 
soil by increasing evaporation and radiation, and the humidity of the air is greater in the neighborhood of forests. Deforestation has been held responsible for increase of flood waters in certain regions and for aridity and barrenness of soil in others. This view is combated by Moore $\left({ }^{28}\right)$ and evidently demands renewed investigation. It is probable that vegetation is primarily an effect and not a cause of rainfall. The necessity for checking conclusions from observation by application of the experimental method is apparently too little realized in this field of investigation. The relations of the soil itself to the growth of plant life are admirably set forth in the monograph by Cameron $\left({ }^{27}\right)$.

The relations of external temperature to human physiology. Temperature, the dominant factor in meteorological phenomena, becomes of paramount importance as a regulator of physiological processes. A fundamental classification divides animals into two great groups of coldblooded and warm-blooded forms. In the former division the individuals have a body temperature which rises and falls with that of the environment, being usually slightly elevated above that of the surrounding medium. In the latter group the body temperature has a fixed level which is fairly maintained whatever the fluctuation of external temperature. It is obvious that such a constancy of body heat predicates an exquisite exercise of physiological adaptation and it is necessary for us to inquire into the mechanism of heat control in the warm-blooded animal in order to appreciate the influences of climatic change. The following discussion owes much to Lusk's admirable chapter on the Regulation of Temperature $\left({ }^{28}\right)$.

The autonomy of animal temperature is due primarily to katabolic chemical changes within the body, chiefly involving oxidation. Practically, heat production in health may be regarded as the result of the combustion of the carbon and hydrogen of the food with the oxygen of the air to form $\mathrm{CO}_{2}$ and $\mathrm{H}_{2} \mathrm{O}$. The protoplasmic activity of every living cell demands' a specific temperature whose chief source is in the oxidations of the $\mathrm{C}$ and $\mathrm{H}$ supplied in the food. There may be a variation of heat production and a chemical regulation of body temperature by alteration of the amount of oxidation in a unit time. It is well established that the principal source of animal heat is oxidation within the fixed tissues whose activities are under control of the nervous system. In an animal under the influence of curare, which prevents the passage of motor impulses to the skeletal muscles, the body temperature fluctuates with that of the environment as in a cold-blooded animal. It is said also that in a deeply anesthetized subject there is a similar failure in temperature regulation.

Equally important with the chemical regulation of heat production 
within the body is the physical regulation of heat loss from its surface. The climatic environment exerts its influence largely through its effect on the mechanism for the loss of heat. The animal body loses heat from the skin, from the lungs, by warming the ingesta, and by loss of heat in excreta. Neglecting the last two relatively unimportant factors, heat is lost to the body from the skin and lungs somewhat in the following proportions:-

1. By radiation and conduction from the skin ...... 74.00 per cent.

2. By evaporation from the skin $\ldots \ldots \ldots \ldots \ldots \ldots \ldots$. 15.00 " "

3. By warming the expired air ............. "4.00" "

4. By vaporization of water from the lungs ...... " 7.00 "

It is obvious that the relative activity of these various channels for the loss of heat varies enormously under different meteorological conditions. The important matter from our viewpoint is that every change in temperature or humidity of the air calls out a coördinating response from a physiologic mechanism and the nicety of the adjustment is shown by the fact that in a fasting, resting animal the body temperature remains normal under a wide range of external temperature and humidity.

Although oxidations and the production of heat are increased in the presence of cold this chemical regulation of body temperature seems to be preferably avoided when the more purely physical means for the loss or conservation of heat prove adequate. With rise of external temperature the blood suffuses the skin, increasing loss of heat by radiation and conduction, the sweat glands become active, and the evaporation of perspiration becomes of extraordinary refrigerant value. Studies made on a man without sweat glands showed that in summer even slight muscular exertion raised his temperature to $104^{\circ}$ or $105^{\circ} \mathrm{F} .\left({ }^{29}\right)$ On the other hand, the eighteenth-century experiments of Blayden and Fordyce showed the extraordinary efficiency of evaporation from the skin. They found "that the effect depended on the humidity of the air. Thus, after remaining fifteen minutes in a damp room heated to $54.4^{\circ} \mathrm{C}$. $\left(129.9^{\circ} \mathrm{F}\right.$.) the temperature of the mouth and urine was $37.8^{\circ} \mathrm{C}$. (100 ${ }^{\circ} \mathrm{F}$.), but a similar exposure in a dry room heated to I $15.5^{\circ} \mathrm{C} .\left(239.9^{\circ} \mathrm{F}.\right)$, to $126.7^{\circ} \mathrm{C} .\left(302^{\circ} \mathrm{F}\right.$. $)$, and in which beefsteaks were being cooked by the heat of the air, did not raise the temperature of the body above the normal." $\left({ }^{30}\right)$ In a section on ventilation, as later will be shown, a new importance has recently been given to the factors controlling evaporation from the skin. The well-known fact that as a sphere increases in size its expanse of surface becomes relatively less than its weight would lead to the deduction that in small animals there 
must be found either a lower body temperature or an increased production of heat to offset the increase in this avenue for its dissipation. Rubner and others hold that the chemical activity of heat production in an animal bears a very definite relation to its expanse of surface. It is said that "an infant produces 90 calories per kilogram in twenty-four hours; an adult 32 calories." $\left({ }^{28}\right)$ The relation between heat production and extent of body surface has been formulated by Rubner into a law, according to which metabolism is proportional to the superficial area of an animal. In an adult man under normal conditions each square meter of body surface gives off about 39.7 calories per hour. "The organism, therefore, preserves the tropical temperature of its cells at the expense of a metabolism which is proportional to the skin area of the individual." $\left({ }^{28}\right)$

In order to carry on its cellular activities every organism must give rise to a.certain amount of kinetic energy in a unit time. The amount of energy thus required is least in an animal which is at rest, fasting, and surrounded by an atmosphere having a temperature of $30^{\circ} \mathrm{C}$. $\left(86^{\circ} \mathrm{F}\right.$.) to $35^{\circ} \mathrm{C}$. $\left(95^{\circ} \mathrm{F}\right.$.). The energy required is modified by food, temperature, and muscular exertion.

This minimal requirement of vital energy is known as the basal metabolism of an individual and is determined by the heat given off per unit of surface while the subject is resting before the morning meal. The heat thus radiated can be very exactly measured when the subject under observation sojourns within a calorimeter, and the method is known as direct calorimetry. Practically the same result as to the evolution of energy in the body is attained by calculation from the amount of oxidations occurring in the tissues. The data for such an estimate consist in the weight of oxygen absorbed and the weight of carbon dioxide given off in respiration, and the weight of urinary nitrogen excreted by the kidneys in a given time (Lusk, 28, p. 62). These quantities being known, the heat of combustion and its source in the body can be calculated easily. This is known as indirect calorimetry.

The lower animals regulate the loss of heat under varying external temperature to a remarkable degree by adjustment of their coats of hair or feathers, whose contained component of air is the chief source of warmth, without alteration of the intensity of metabolism. In man the same regulation is artificially achieved by means of clothing whose pliyșical attributes engage an important field of hygiene. Although under ordinary conditions the constancy of body temperature seems to be maintained preferably by mechanical regulation of loss of heat from the surface, chemical regulation, involving a change in metabolism, plays 
the part of a reserve which may instantly supply deficiency in the physical adjustment of heat loss. Thus, Rubner and others showed that, for the human subject, baths, even of duration of five minutes or less, may according to their temperature radically modify metabolism as proved by alteration in the amount of $\mathrm{O}_{2}$ absorbed and $\mathrm{CO}_{2}$ given off in respiration. A cold bath, especially a douche, increases greatly the oxidations of the body; thus, a douche at $16^{\circ} \mathrm{C} .\left(60.8^{\circ} \mathrm{F}\right.$.) increased by about fifty per cent the weight of $\mathrm{O}_{2}$ inspired and $\mathrm{CO}_{2}$ expired. The extraordinary effect of the fleeting douche is probably largely due to a reflex excited by the cold water falling on the skin. The increased metabolism persists for an hour or more after the bath.

The aim of the therapeutic application of cold water is not so much the mechanical abstraction of heat from a patient as the establishment of what is indefinitely called a "physiological reaction," which results in an amelioration of the pathologic symptoms. The physical basis of such a reaction may lie in the metabolic changes which might be apprehended by the application of direct and indirect calorimetry. A bath of $35^{\circ} \mathrm{C}$. $\left(95^{\circ} \mathrm{F}\right.$.) has no effect on metabolism; this is the "sedative bath " of the hydrologist. With higher temperatures of the water it is said that metabolism is again increased, possibly because the tissue cells are warmed and their chemical activities accelerated. Rubner found that in a hot bath of $44^{\circ} \mathrm{C}$. (III. $2^{\circ} \mathrm{F}$.) there was a marked increase in respiratory movement and in the gas exchange. Such baths are much affected by the Japanese. $\left({ }^{28}\right)$

The physiological relations of humidity of the air. The familiar fact that evaporation of water occurs at a rate inversely as the tension of water vapor in the air predicates a close connection between humidity and the regulation of heat loss through evaporation from the surface. We have seen in a previous section how humidity of the air modifies the sun's temperature through its great absorptive power for heat, and how at night, when the earth cools by radiation, the dew precipitated sets free its heat that had been latent and the high tension of the aqueous vapor remaining obstructs cooling by evaporation. Even more conspicuous are the effects on consciousness of the modification of physiological processes associated with varied humidity of the atmosphere. As pointed out by Huggard $\left({ }^{7}\right)$, " while humidity moderates the temperature of the air, it intensifies the chilling action of cold and the oppressive influence of heat. Dry air, a little above the freezing-point of water, is not felt to be nearly so cold as moist air of the same temperature; while warm air, if dry, is pleasant, but if saturated with moisture oppressive and enervating." These effects depend upon alteration, induced by change in temperature and humidity, of the relative importance of the 
various means through which heat is removed from the body. When the air is dry and moderately warm, heat leaves the skin chiefly through radiation and evaporation. When the air becomes charged with vapor at the same temperature, both radiation and evaporation are hindered and heat tends to accumulate in the body. Commonly, air which is "moist" falls in temperature. Now the loss of heat by conduction assumes the more important rôle, and heat continues to be removed at increasing rate with lowered temperature and increasing saturation. The regulation of heat loss by radiation and evaporation is, as pointed out by Huggard, the more physiological process, since through these factors the body regulates its own output. When moist air becomes cooled, although the body heat is conserved by constriction of the skin vessels and inactivity of the sweat glands, the loss of heat still continues through conduction. Warm moist air when lowered but two or three degrees in temperature may not excite adequate vasoconstriction in the skin and the accelerated conduction by the air may, by excessive removal of heat, cause a decided chill.

It will be rightly inferred from the foregoing that the air temperature as indicated by the ordinary thermometer very inadequately expresses the conditions affecting loss of heat from the skin. In warm weather the relative humidity of the air determines the rate of cooling by evaporation of perspiration and in cold weather the absolute humidity determines loss of heat by conduction. Therefore, the temperature as shown by the wet-bulb thermometer, whose registration arises somewhat proportionately with the relative humidity, more nearly indicates the air temperature conditions as they affect the regulation of heat loss from the body. One of the most interesting functional coördinations in physiology is that existing between the psychic state and the regulation of the thermic apparatus. A sense of well-being subtends efficient action of the mechanism for regulation of the temperature of the body. When that efficiency is impaired in any way the body temperature rises or falls, as the case may be, and more or less acute discomfort is felt. It may be suggested, in passing, that this psychic reflection of the state of physiologic coördination was obviously, like the pain sense, developed because it was necessary to the protection of the mechanism whose reactions it pictures.

In brief, air temperature as felt, or the so-called sensible temperature, depends upon humidity, especially relative humidity, as much as upon absolute temperature of the air. As pointed out by Glassford $\left({ }^{31}\right)$, Harrington $\left({ }^{32}\right)$, and others, the courses of isotherms as determined by records of the dry- and wet-bulb thermometers, respectively, are far from parallel. In a dry climate evaporation from the wet bulb increases 
with the heat of summer, so that the sensible temperature of a place departs widely from that which would seem proper to the latitude. The summer heat as felt at E1 Paso, Texas, is not greater than that felt in Iowa several hundred miles to the north.

Physiological influence of air movement. Winds. While wind finds its physical origin in heat, it in turn is a most important accessory factor in the modification of temperature sensations. Though Durig $\left({ }^{25}\right)$, in his congeries of paradoxes on physiological climatology, declares that he and Zuntz found no influence to be exerted upon metabolism by either temperature or variation of wind movement, the greater weight of evidence appears to be in the direction taken by our previous discussion.

Nothing is more certain than the dominant physiological importance of the physical factors introduced with the play of air movement upon the surface of the body. A breeze, in general, accelerates loss of heat from the skin by removing its humid envelope and facilitating the evaporation of perspiration. The temperature and humidity of the wind are important details which may determine its specific physiologic effect. It has been well said that "wind makes a good climate better and a bad one worse."

Frankenhauser $\left({ }^{33}\right)$ states that a light breeze at $20^{\circ} \mathrm{C}$. $\left(68^{\circ} \mathrm{F}\right.$.) has as strong a cooling effect as still air at $2^{\circ} \mathrm{C} .\left(35 \cdot 6^{\circ} \mathrm{F}\right.$.). According to Rubner $\left({ }^{34}\right)$ air movement does not become perceptible until it reaches a velocity of 0.4 to 0.5 meters (nearly two feet) per second. Movements of less than this rate, he showed, may still exert considerable influence on the loss of heat from the body.

Leonard Hill $\left({ }^{35}\right)$, in his experiments on ventilation, made use of a chamber of three cubic meters capacity, with glass windows and fitted with an electric stove and fans. Eight men were crowded into this space. Before long the discomfort became unendurable; then the fans were set in motion and the disagreeable sensations were at once relieved, only to return when the fans came to rest.

Ventilation. It has been shown that the temperature and humidity of the environment are controlling conditions in the heat regulation and, therefore, of the metabolism of the body. Of late years these factors have been given a dominant position in the hygiene of ventilation. It is common knowledge that the sense of well-being of a person may become modified radically in going from the open air into a closed and particularly a crowded apartment. The recent development of tuberculosis therapeusis, which emphasizes the importance of a life in the open to the upbuilding of "physiological resistance," has especially given practical importance to the determination of the causes which biologically 
distinguish pure free air from that which is confined and contaminated with human exhalations.

It is not necessary to again summarize the interesting literature upon this subject $\left({ }^{36}\right)$. Two views have prevailed in explanation of the deleterious effects of close air, one ascribing them to chemical and the other to physical influences. The early belief that hygienic deterioration of rebreathed air could be accounted for by its increased content of $\mathrm{CO}_{2}$ was succeeded by the conception that animal exhalations, especially from the lungs, when respired act as poisons upon the body. In recent years, this theory also has been generally abandoned as result of the demonstration by many physiological experimenters that all the characteristic sensible effects of closed air could be produced or removed at will by manipulation of the physical factors of temperature and humidity. According to this view, the various morbid sensations that are experienced by one occupying a crowded and ill-ventilated apartment may be attributed to disturbance in the temperature regulating apparatus of the body through an environment of still air whose temperature and humidity interfere with the normal escape of heat. It has been shown that persons can abide in comfort and perform mental tasks with normal facility in a closed space in which the air has become excessively fouled by rebreathing, provided only that the temperature is kept cool and the humidity low.

The sensory response to a rapid development of the conditions found in close air is immediate and imperative, but when these conditions are introduced gradually, as in experiments by the New York State Commission on Ventilation $\left({ }^{37}\right)$, persons may carry on sedentary avocations for prolonged periods without realizing any discomfort or loss of facility. But there appeared to be a gradual loss of vital energy and loss of appetite for food, and the conclusion was reached that even with the correction of temperature and humidity there appears to be something about close air which militates against physiological well-being.

We divell upon this subject because there is danger that the medical mind, according to its habit, will go too far in its application of the important discoveries that have disclosed the physiological relations of temperature and humidity. It is the way of Nature to excite pain and discomfort to obtain immediate voluntary correction of conditions which threaten disaster to the economy.

This sensory disturbance is actuated by acute interference with the mechanism for the regulation of body temperature. The facts by no means disprove the chemic noxiousness of rebreathed air. On the contrary, the teachings of immunology are full of suggestions that human health demands pure air uncontaminated with organic effluvia. Rosenau, 
indeed, is said to have succeeded in sensitizing guinea-pigs to horse serum by exposing them to the atmosphere of horses, and the writer $\left({ }^{38}\right)$ has desensitized guinea-pigs, which had been made sensitive to horse serum, by keeping them for a few hours in a horse stable; in fact, in some animals reactions, which probably indicated the process of desensitization, occurred within a few moments of exposure. We may conclude that while the sense of comfort is of fundamental use in determining the efficiency of ventilation, reason demands that in practice we avoid the contingency that comfort may mask the operation of insidious poison.

The physiological influence of high altitudes. Elevation above sea-level introduces a new element in our consideration of climate, that of diminished atmospheric pressure. We have already seen that the meteorological conditions of sea-level are modified on the mountain height. Here there is less moisture in the air, stronger insolation, greater difference between the temperature in sun and shade, and, it is said, increase in radioactive emanation; but the outstanding fact is the fall in barometric pressure which, $760 \mathrm{~mm}$. or $30 \mathrm{in}$. $\mathrm{Hg}$. at sealevel, declines approximately $\mathrm{I} \mathrm{mm}$. $\mathrm{Hg}$. for every 40 feet or one inch per I,OOO feet of ascent. The highest permanent human habitations are at an elevation of about I6,000 feet, where the pressure of the atmosphere is about half that at sea-level. It is well known that in subaqueous construction work, men may remain safely in caissons under pressure of three or four atmospheres. But experience showed that too rapid decompression led to serious symptoms or even fatal results caused by the evolution in the central nervous system of gases, which had been dissolved in the blood under high tension. Formerly a somewhat similar mechanical explanation of the physiological effects of high altitudes was current, but the view is erroneous. Mountain elevations are too low and ascent of them too gradual to admit of evolution of free gas by decompression. Whether accidents suffered by aviators may sometimes be due to decompression is subject to investigation, but it is highly probable that even here the ascent is always sufficiently gradual to permit pari passu the adjustment of internal gaseous tension. The rate of descent frequently ventured by aviators is such, however, as to suggest that the flyer must often at the end of a rapid drop find himself in an atmosphere whose tension is considerably above that of his dissolved gases.

An experienced aviator $\left({ }^{39}\right)$ records that he had never suffered any physiological disturbance in the heights, but that once, on reaching the ground after a very speedy descent, he had promptly fainted. He also astutely points out the radical lack of similarity between the subjective state of a person flying at a high altitude and that of one who sits in 
a pneumatic cabinet and attempts to realize the physiological effects of a similar barometric environment.

It is not expedient to analyze here the rapidly accumulating literature on aviation from which much of value may be expected in the future. The most important task for the medical physiologist at present is to invent and apply methods which will distinguish those candidates for aviation whose physiological complex will quickly adjust itself to the conditions encountered at high and varying elevations. If one who never expects to make a flight may venture an opinion, the writer is impressed with a radical defect in all schemata of examination known to him in failing to take definite cognizance of the evidence for blood ptosis in candidates for aviaton.

Crampton $\left({ }^{40}\right)$ and others have shown that, with weak vasomotor control of the splanchnic arteries and with a flabby abdominal wall, the blood settles in the abdominal vessels when one assumes the erect posture, depriving the brain of its normal circulation. Such a condition is evidenced crudely by a considerable excess of the systolic blood pressure found in the recumbent over that shown in the standing posture. Great increase in heart rate or decrease in pulse pressure in the erect posture points in the same direction. It is not rare to find organically normal persons who find it difficult to stand without fainting while a blood pressure observation is being taken. In the writer's experience the psychic factor may be most prominent in the loss of control.

It is not intended here to review the voluminous literature treating of the physiological reactions dependent upon lowered barometric pressure. It will be assumed that the contention of Haldane $\left({ }^{12}\right)$ and his coworkers is correct, that the characteristic physiological changes found at high elevations are primarily dependent on the diminished oxygen tension of the air.

This does not preclude an element of influence, discovered and strongly emphasized by Mosso $\left({ }^{10}\right)$, in the lowered tension of carbon dioxide in the body at very high elevations. Paul Bert $\left({ }^{9}\right)$, forty years ago, predicted and later proved that the oxygen-carrying power of the blood increases at high altitudes, and he correctly drew the conclusion that this was a physiological adaptation to the lowered oxygen pressure of the air. Most observers since then have found in man and animals an increase in the proportion both of the red corpuscles and hemoglobin of the blood at elevations upwards of a mile above sea-level. It will be assumed in this article that augmentation of oxygen carrying power of the blood through increase in hemoglobin and red corpuscles is a constant and characteristic reaction to lowered barometric pressure, and that the stimulus to the physiological response consists in the lowering of the 
partial pressure of oxygen in the air. The views of those who deny or offer other explanations for the facts as claimed may be found in the literature cited.

The important work of Loevenhart $\left({ }^{42}\right)$ and his coworkers clearly indicates that it is not the variation of the total barometric pressure but only of its oxygen content that induces the blood changes. These physiologists constructed a ventilation chamber in which animals (rabbits, white rats, and dogs) could be kept for days at a time, either under reduced barometric pressure or at atmospheric pressure with a reduced percentage of oxygen. They conclude that "A decrease in the oxygen tension of the respired air obtained by reducing the oxygen concentration at atmospheric pressure or by reducing the barometric pressure stimulates the bone marrow and increases the erythrocytes and hemoglobin in the circulating blood. . . . From five to seven days is required for the increase in the blood count to become very marked, but the maximum increase requires a longer exposure. The increase in erythrocytes and hemoglobin is absolute and not relative. We have been able to increase the total hemoglobin per kilo in rats 43 per cent. In order to produce these effects the oxygen pressure in the respired air must be reduced, at least, to approximately 14 per cent of an atmosphere. The optimum pressure for increasing the oxygen capacity of the blood is apparently not far from Io per cent of an atmosphere." When the atmosphere of the chamber contained the normal concentration of oxygen (20.8 per cent) or even 16 per cent oxygen, the changes in the blood count were insignificant. When the oxygen is reduced to I4 per cent, marked increases in the blood count occur within the week."

The following table represents the percentage increase in erythrocytes and hemoglobin in rabbits kept in the chamber approximately a week under normal atmospheric pressure but with variable percentages of oxygen.

\begin{tabular}{cccc} 
& & \multicolumn{2}{c}{ Blood change } \\
Oxygen & Number of & Erythrocytes & Hemoglobin \\
per cent & Experiments & per cent & per cent \\
20 & 7 & 2.7 & 9.7 \\
I6 & 4 & 4.6 & -1.9 \\
I4 & 8 & 15.2 & 17.4 \\
I2 & 5 & 14.4 & 16.8 \\
I0 & I 5 & 18.5 & 26.7
\end{tabular}

Results in the same direction were obtained when the reduction in oxygen tension was secured by lowering the total barometric pressure. It may be added that the reduction of oxygen pressure to $I 7$ per cent of the total atmospheric pressure at sea-level corresponds to an altitude 
of $2,020 \mathrm{~m}$. (6,627 feet); oxygen pressure of I4 per cent of an atmosphere corresponds to an altitude of $3,500 \mathrm{~m}$. (I I, 483 feet); oxygen of 12 per cent is equivalent to an altitude of $4,730 \mathrm{~m}$. ( 15,5 I 8 feet), and Io per cent to an altitude of $6,060 \mathrm{~m}$. ( 19,877 feet).

The scientific value of physiological studies at low barometric pressure lies in the light they shed upon certain phases of metabolism. It has long been common knowledge that the hemoglobin of the blood has an affinity for oxygen that leads to the formation with it of a chemical compound, oxyhemoglobin, which is characterized by the fact that the union is possible only when a certain oxygen tension persists in the medium surrounding the hemoglobin. When a vessel containing blood is placed under the receiver of an air-pump, dissociation of the oxygen from the hemoglobin begins when the partial pressure of the oxygen in the atmosphere of the receiver is reduced to about $60 \mathrm{~mm}$. $\mathrm{Hg}$; t that is to say, when the total pressure has fallen from $760 \mathrm{~mm}$. to about $300 \mathrm{~mm}$., or to considerable less than half the pressure at sea-level. This is a barometric reading which, approximately. is found at an altitude of $5,500 \mathrm{~m}$. ( 77,000 feet). It is obvious that if dissociation of oxyhemoglobin occurs in vivo under these conditions it would be impossible for the venous blood in the lungs to take up oxygen at altitudes exceeding 17,000 feet.

It may be said that the partial pressure of oxygen at which the gas may be induced to enter into chemical union with hemoglobin, conditions which determine association or dissociation, is altered by circumstances. Thus, high temperatures favor dissociation; the lower the temperature the more oxygen is taken up at the same oxygen pressure. An important modifier of the affinity between hemoglobin and oxygen is the reaction of the blood; in the condition of "acidosis," a constant effect of stress due to altitude, oxygen is given off more freely, and this, as pointed out by Barcroft $\left({ }^{43}\right)$, is an important adaptation to low barometric pressures.

The facts that have been presented prepare us for the conclusion arrived at experimentally by Haldane and Smith $\left({ }^{44}\right)$ that "diffusion alone does not explain the passage of oxygen from the air of the pulmonary alveoli to the blood" and make it easier to accept the demonstration by Haldane and his colleagues, in their great work on Pike's Peak (altitude 14, IOo feet), that the passage of oxygen from the lungs to the blood may be a result of active secretion on the part of the cells lining the alveoli. Their conception, briefly put, is that, under ordinary conditions of atmospheric pressure, gaseous diffusion in the lungs is sufficiently rapid to supply the needs of the body, but when the supply of oxygen is sufficiently lessened, as by ascent above sea-level, the protoplasm of the alveolar cells reacts and manifests a function which till then 
has been in abeyance, the active absorption of oxygen and its transfer to the circulation. It is surmised that the immediate cause for the stimulation of the alveolar epithelium under deficient oxygen supply lies in the accumulation within the circulation of easily oxidizable metabolic products.

A constant feature of life at low barometric pressures is a diminution in alkalinity of the body fluids, resulting in the lowering of the threshold exciting-level of alveolar $\mathrm{CO}_{2}$; the resulting amplification of respiratory ventilation greatly raises the alveolar $\mathrm{O}_{2}$ tension. "Without a great lowering of the threshold alveolar $\mathrm{CO}_{2}$ pressure, life would be quite impossible at very great altitudes. . . . It seems probable that even at moderate altitudes the oxygen supply to the body during rest is dependent to an appreciable extent on the activity of the lung epithelium." $\left({ }^{12}\right)$

It may be that a similar explanation, namely, inability of the lung epithelium to develop the power to secrete oxygen, holds good for the not infrequent fatalities which occur, apparently from edema of the lungs, among tubercular patients who are imprudent in exercise after ascent to moderate elevations, a subject which was discussed in the opening section of this article (p. 66). Haldane also points out that the stimulation of respiratory epithelium induced by low oxygen tension in the air is in itself a suggestion that lowered barometric pressure, through improving the nutrition of the lungs, might be expected to prove salutary to the subjects of pulmonary tuberculosis. According to the authors we are considering there are three physiological adaptive changes induced by sojourn at high altitudes. "These are: (I) a rise in arterial oxygen pressure; (2) a fall in alveolar $\mathrm{CO}_{2}$ pre ure and corresponding rise in alveolar oxygen pressure; (3) an increase in the percentage and total amount of the hemoglobin in the blood." These conditions are developed gradually to a maximum in the course of several weeks; they then remain constant in the same environment, but gradually give way to the original state on a return to lower altitudes.

The object of this discussion has been to show that at very high altitudes, I4,000 feet or over, the living body enters at once upon adaptive changes which must involve every active cell but which affect most prominently the respiratory function. The result is both anatomical hyperplasia of certain tissues and physiological acceleration and modification of many reactions. As pointed out by Haldane and his colleagues, the completion of these adaptive changes at any altitude denotes the acclimatization of the individual for that level.

On Pike's Peak "after acclimatization the resting arterial oxygen pressure had risen to about $35 \mathrm{~mm}$. Hg. above the alveolar oxygen 
pressure, whereas at or near sea-level the resting arterial oxygen pressure is no higher than the alveolar oxygen pressure." This rise in $\mathrm{O}_{2}$ pressure is due to the secretory activity of cells lining the lung alveoli, and "is the most important factor in acclimatization. . . The alveolar carbon dioxide pressure required to excite the respiratory center gradually fell within about a fortnight to about two-thirds of the normal value at sea-level, with a corresponding increase in the volume of air breathed and a rise in the alveolar oxygen pressure which would otherwise have existed on Pike's Peak; this being a second important factor in acclimatization. . . . With very hard work, however, the aeration of the arterial blood became defective, and there was, in consequence, a still further lowering of alveolar carbon dioxide pressure for the time and corresponding increase of hyperpnea:

for short and considerable exertions the amount of air breathed might amount to three times the quantity breathed in similar exertions at sealevel. The per cent of hemoglobin increased for several weeks on the Peak and reached in various acclimatized persons I $_{5} 5$ to $\mathrm{I}_{54}$ per cent of Gowers-Haldane. The number of red blood corpuscles increased parallel with the hemoglobin." $\left({ }^{12}\right)$

It is easily seen that a mechanism whose adaptation is perfect under conditions of bodily rest may easily show signs of overstrain when muscular exertion multiplies the demands for oxidation in the organism. The process of acclimatization emphasizes the importance of training for the development of the inherent powers of living matter for the accomplishment of new and unusual tasks. Zuntz ( $\left.{ }^{11}\right)$ and his coworkers were able to show, by metabolism studies, an extraordinary increase in the machine efficiency of an Alpine guide as a result of his summer's work.

Acute failure in physiologic adaptation to the strain of lowered barometric pressure is apt to be signified by the development of a syndrome known as "mountain sickness," which is marked by prostration, mental and physical, by breathlessness, cyanosis, loss of appetite, and nausea, the symptoms of which are precipitated or accentuated by muscular effort. It is worth noting that the literature of aviation appears to be free from descriptions of any physiological disturbance comparable to mountain sickness, as resulting from high flights. An obvious explanation is suggested by the excess of muscular exertion put forth by the mountain climber as compared with the aviator. Haldane and his colleagues, probably correctly, ascribe the development of mountain sickness to deficiency of oxygen. To the writer this first cause seems reasonably to lead off a series of events which readily explain the symptoms encountered. Thus, cardiac power must suffer from the 
diminished oxidation while the cardiac load is increased by the exaggerated respiration; the result is back pressure from the overloaded right heart and congestion of the abdominal viscera which, associated with an acute acidosis, provokes the clinical syndrome. In confirmation of the view that cardiac weakness is a prominent factor in mountain sickness is Kronecker's $\left({ }^{45}\right)$ statement that persons with weak hearts or impeded pulmonary circulation suffer very readily at comparatively low altitudes.

If instead of studying life at great elevations where excessive physiological adjustment is necessary to permit of normal existence, we begin at sea-level and note the appearance of signs of vital stress with ascending altitude, we find, as should be expected, a rather broad zone in which the influence of lowered barometric pressure is of varied prominence. Thus, few healthy people, transported from the plains to the elevation of 5,000 feet and remaining at rest, are at all conscious of a change of environment. However, an amount of muscular exercise which at sea-level would produce no distress would now cause breathlessness and untoward acceleration of pulse. After a few weeks of residence the same exertion fails to produce signs of incoördination; that is to say, the "field of physiological response" of the individual has been enlarged and there is good reason for believing that the vital factors involved in this change are identical with those so obviously concerned in the adaptations to the conditions of the highest altitudes.

While it is obvious that elevations approximating 17,000 feet, at which gas exchange in the lungs has lost the physical aid of that partial pressure of oxygen necessary to complete its combination with hemoglobin, must demand a vastly greater output of physiological effort to secure adaptation to the environment than is required at lower levels, it is hardly doubtful that the essential processes of adaptation are the same as at lower levels. We will assume that a person resting comfortably at an elevation of 10,000 feet, on indulging in strenuous physical exercise, instantly transports himself into the physiological condition which would correspond to a state of comparative rest at a much higher elevation. In our study of the temperature regulation of the body it was pointed out that the body temperature could be kept constant under widely different atmospheric temperatures by merely physical regulation of the loss of heat by the skin. When the limits of physical adaptation are surpassed, the intensity of metabolic combustion is varied to conserve the constancy of cellular warmth. Wholly similar appears to be the analogy of physiological reaction to lowered oxygen tension; only on falling below a certain critical tension in the alveolar oxygen is there definite excitement of metabolic reaction. 
One of the most important results established by Zuntz $\left({ }^{11}\right)$ and his coworkers was the effect of lowering of barometric pressure on protein anabolism. While, under ordinary conditions, changes of weight in the adult are due solely to fluctuations in the hydrocarbon moiety of the body, at even moderate elevations above sea-level there is an actual laying on of protein tissues, and this process continues for a time after descent to the plains. It is said that the same modification of metal)olism occurs at the seashore.

Although life at high altitudes appears to involve no special change in arterial blood pressure, we should expect that the increased ventilation of the lungs, especially during muscular exertion, would lead to a notable increase of blood aspirated into the right heart, inducing overstrain of that organ when it is incapable of compensation. The writer has studied such a course of events, resulting in tricuspid insufficiency, in the person of an unacclimatized person who indulged imprudently in mountain exercise. For many years he has seen frequent clinical evidences of right heart overstrain which seemed to have relation to the conditions involved in ascent to the moderate elevation of one mile above sea-level. It is obvious that, in subjects of cardiac debility, such overstrains become of serious consequence.

Mitral stenosis is the valvular lesion which predominantly calls for strength in the right ventricle; and there is clinical support for the view of Babcock $\left({ }^{46}\right)$ that this is the one cardiac lesion which contraindicates high altitude. In a paper already cited $\left({ }^{42}\right)$ various investigators are quoted to the effect that a congestion of the lungs attends sojourn in high altitudes, and that, as determined by the electrocardiograph, there is hypertrophy of the right ventricle in such environment.

The preceding discussion must make it clear that for the invalid high-altitude climates are to be regarded as therapeutic agencies of great potency, equally capable of accelerating healing or hurrying disaster. The fundamental biological property of such environments is to stimulate and modify metabolism by deprivation of the most important element in nutrition-oxygen. When the physiological powers can respond to the whip, the body gains in strength as under no other condition. That the vital organs may have a fair chance to adjust themselves to the burdens, direct and indirect, imposed by lowered barometric pressure the clinician must insist on complete physical and mental rest for every case of disease, until adequate acclimatization to the altitude is secured. When we consider, in addition, the effects upon the organism of increased insolation, great difference between the temperatures of day and night, sun and shade, drying of the skin and mucous membranes, we are impressed with the range of psychic and 
physiological stimuli to which the newcomer into high altitudes is forced to adjust himself.

\section{ACTUAL CLIMATES IN HEALTH AND DISEASE}

It hardly can be doubted that the human organism responds to every kinetic energy acting upon it. Most of the stimuli produce no obvious reaction but are none the less important though, to borrow a term from the psychotherapeutist, they may be surmerged in the subconscious. We have seen how the maintenance of a certain physical state of temperature within the body demands a constant balancing of physiological reactions to compensate for the slightest environmental change. We have seen how economical of effort is Nature in accomplishment of the purpose, using the dermal mechanisms for the dissipation of heat before employing her more profound powers for changing heat production. Assuming, as we have done, the truth of Haldane's epoch-making conception of oxygen secretion by the pulmonary alveolar cells, we find evidence of indefinite magnification of specific functions under certain climatic conditions. We found that acclimatization, at least to high altitudes, consists in the hyperplasia of certain cells and an increased efficiency of function, no doubt having a morphological basis by which adaptation is secured. It is no mere symbolism to say that a person who attempts a task beyond his strength fails or becomes exhausted because he is unacclimatized to that task. A course of training may so develop his powers as to make easy accomplishment of the task.

The training concept lies at the basis of most successful therapeusis. We know empirically that all disordered organs tend, in general, to right themselves best the less the demand for their functional activity. The doctrine of cellular rest is the salient law for the treatment of active tuberculosis, uncompensated heart disease, nephritis, and diabetes mellitus. When a diseased organ is forced to become functionally active there results an incoördination analogous to the mountain sickness of one newly arrived at the mountain top; attempt to exercise, and the result is intensified in proportion to the limitation of the organic powers. On the other hand, the great benefit that accrues to the body and its powers as a result of training is an indication that the therapeutic ideal in respect to the sick should be not rest but exercise; exercise, however, whose duration and intensity always fall well within the "field of physiological response" enjoyed by the living tissues. There must be an optimum of stimulation for every living cell which both develops best its machine efficiency and promotes anabolism. It is the intricate problem of the clinician to coördinate external agents with the internal reactions. 
These considerations seem to demand discussion here because they underlie the rational application of medical climatology. Our study of climate thus far has involved its influence only upon the healthy organism. When the body becomes diseased the nature of the biological effect of climatic change is not qualitatively altered, but it may be quantitatively greatly magnified owing to the limitation in the powers of physiological adaptation. A study of the geographical distribution of disease $\left({ }^{47}\right)$ would seem to show that the endemicity of various disorders is based upon the relations of certain physical factors of climate, as temperature and humidity. But, as was pointed out at the beginning of this article, infective diseases, at least, are concerned with climate only as this tends to conserve the living agents through which the pathogenic virus is transmitted. Just recently have sanitarians been able to show that "tropical diseases" are no essential attribute of the torrid zone and have opened a rich field to the uses of medical climatology. Even where the seasonal agency in the development of disease seems clearest, as in the causation of pneumonia, profounder study gives more prominence to the importance of other factors, as intimacy of contact between the sick and the well, and the immunological status of the person exposed as determined by personal history, present and past.

A thorough biological knowledge of climate would inivolve a study of metabolism by both direct and indirect calorimetry and through a wide range of functional activity. It would involve also a measurement of all those compensatory physiological changes, such as the varied distribution of the blood, which elude the calorimeter; and above all it would take account of the psychic state which is the resultant of innumerable factors, social and material. Such a knowledge is being slowly evolved, but as yet the climatologist must be guided chiefly by the empirical data of experience.

It must be confessed that except for the drastic tests thrown upon the organism in very high altitudes, the physiologic effects of climatic change have for the most part eluded scientific experimental observation. Thus, Loewy, Müller, Cronheim, and Bornstein $\left({ }^{48}\right)$ found that at seaside, as compared with inland, resorts there was no special change in respiratory exchange, pulse rate, body temperature, or other functions, and the same result rewarded Zuntz and Durig on a trip from Germany to the Canary Islands and return.

Major W. P. Chamberlain $\left({ }^{49}\right)$ has published, in behalf of the U. S. Army Board for the Study of Tropical Diseases, a varied series of observations on large groups of soldiers and of natives in the Philippine Islands. No foundation whatever was found for the postulates of Major Woodruff $\left({ }^{50}\right)$ that blond individuals fare badly in the tropics 
as compared with brunettes, that the intense insolation was a cause of neurasthenia and other nervous disorders, or that the wearing of orangered underclothes to cut off the actinic rays was of any special benefit. He concludes, "It is doubtful if the actinic component of the sunlight is a factor in tropical morbidity and deterioration." About 7,000 observations on the pulse rate and systolic blood pressure in nearly 1,500 persons showed no radical departures from similar data as determined at home, though the pulse rate averaged slightly above 72 per minute. A study of the blood showed a normal count for the red corpuscles and possibly a slightly lowered percentage of hemoglobin. The so-called "tropical anemia" he regards as a secondary anemia due to infection.

The failure of the more or less mechanical methods of investigation to disclose definite biological differences in the effects of widely diverse climates must not be taken as proof that such differences do not exist. Herein the truth is more likely to be reached by trained clinical observers until the scientific experimenter is able to apply his tests closer to the molecular source of physiological function. As Gordon $\left({ }^{51}\right)$ points out, for example, it is an important practical conception that the ocean front is a bad site for the case of pulmonary tuberculosis while it possesses distinct remedial value for the case of so-called "surgical tuberculosis." It would be vain to inquire now the reason, which one day biochemistry will doubtless give. In this connection light perhaps could come to us by consideration of the conditions of "acidosis" which is accompanied, if not caused, by a diminution of the alkali reserve of the body. "A vast array of physiological reactions and of pathological symptoms can flow out of disturbance of the acid-alkali balance of the blood with little or no impression on its $\mathrm{H}$-ion concentration, which is the final index of its chemical reaction. Other methods of study are required to measure these slight variations which may be of great significance.

Pulmonary Tuberculosis. It has been the main effort of this essay to help clear away the confusion that still clouds the medical concept of climate. Possibly no other disease has borne such a burden of therapeutic misuse as that which from earliest times has been supposed to find its chief remedial agent in climate; namely, Pulmonary Tuberculosis. The physician who refers his patient to a new climate, too often as a last resort, and who has not well-founded confidence in the future behavior of that patient and in the reliability of his patient's new medical adviser, abandons every principle of treatment on which his practice has been built up. Climatic environment is but one remedial condition whose action may be modified radically by incidental circumstances. The history of hygiene is prolific in examples of the rise and fall of reputations of places as health resorts. Let fame pronounce a locality as 
curative for tuberculosis, and at once invalids flock there and, ere long, that place becomes a hotbed of the disease, and dangerous for well people to visit. There has been no deterioration of the climate, but the lack of sanitary care of the agents of infection has proved that geographical location is but a minor factor in determining the origin and course of the disease. None experienced in the care of tuberculosis deny the preëminent importance of the patient's morale for the mastery of his disorder. The nostalgia of one who is ostracized from home is fatal to the necessary courage. All municipalities which have a reputation for the amelioration of tuberculosis have to meet the problem of caring for poverty-stricken consumptives who have emigrated from various parts of the country with barely more financial means than sufficient to pay the railway fare. The charitable organizations of Denver, at least, have been burdened greatly by this class of cases. It would be an ideal climate which could compensate for these deficiencies.

It would be tedious to rehearse the splendid advances which so largely have disclosed the real essentials to the cure of tuberculosis and have made home treatment of the disease reasonably successful; but both theory and experience combine in the doctrine that there arc preferable climates for tuberculosis. Physiological reaction is the biologic resultant of climatic change, and physiological reaction is the curative force in tuberculosis. It is the physician's task to estimate the patient's powers from his signs of reaction, of which none is so common as a rise of body temperature when adaptation fails. We hear much of the curative effect of graduated physical exercises. The writer has long been forced to believe that the most necessary acquisition of the practitioner in tuberculosis is a comprehension of the indications for and methods of securing ungraduated rest. The superlative importance of rest for the patient with fever is well emphasized by Lawrason Brown at Saranac Lake, elevation about I,200 feet. An experience of many years at one mile above sea-level more and more has impressed upon the writer that all tubercular invalids having unstable temperature need careful supervision of their energy output. It is exceedingly common to find that the minute muscular exertion, which causes the mouth temperature to rise a fraction of a degree after one day, will, if continued, have a cumulative effect and result within a few days in a demonstrable increase in the area and intensity of the disease.

When we recall the constant play of mechanisms for the regulation of body temperature it is manifest that an excess of the normal heat betokens a disturbance of metabolism which must divide perilously the forces of resistance. Both theory and practice agree in the indication for that degree of rest for the patient on which students of nutrition 
are wont to found their estimates of the basal metabolism. Certainly no more remarkable or frequent experience meets the clinician than that of a case of active pulmonary tuberculosis, turbulent with the signs of threatened dissolution, subsiding within a few days into a manageable condition under the mere application of the principle of rest. The clinician familiar with tuberculosis in Colorado sometimes finds in a newcomer with the disease an experience such as the following: A young man, whose lungs are clear except for a small area of apparent dry consolidation below one apex, feels well and maintains a normal temperature while in bed. Allowed to get up once a day for toilet purposes, there is a progressive rise of temperature and at the same time moist sounds ramify throughout the neighborhood of the original lesion and even extend to the other side. There is every auscultatory evidence of sudden extension of the disease, but with the enforcement of complete bed rest the signs of moisture disappear within a day. In a recent case of my own this experience has been repeated three times within as many weeks.

It seems very improbable that the change within a few hours of the auscultatory signs of dryness to moisture and the reverse, wholly conditioned on slight physical exertion, can be due to variations in tubercularization. The possibility of an intercurrent infection in such cases only emphasizes the importance of rest. I have interpreted these experiences as results of edema, affecting preferably previously diseased areas of the lungs, and their proper apprehension as a result of overexertion in the unacclimated person is of great practical therapeutic importance and full of suggestion as to the intimate relations between the circulation and respiration. The same explanation doubtless accounts for the fact that the Denver physician commonly finds, in newly arrived pulmonary invalids, much more extensive signs of lung disease than are described by their previous medical attendants.

One may perhaps explain these results best on the basis of Haldane's hypothesis of oxygen secretion by the alveolar epithelium of the lungs, a function which is strained by every output of exertion and which, when overstrained, leads to immediate metabolic disorder of vital mechanisms. In such cases, in my experience, the urinary examination for acetone usually has shown this substance to be in excess and a brief course of alkaline remedies, soda bicarbonate or citrate, appears to aid recovery.

Guided by the crude objective signs of temperature and heart rate and the subjective symptoms of fatigue, the pulmonary invalid may be expected to thrive best under that mode of treatment and in that environment wherein his output of energy or physiological reaction is greatest without abnormal disturbance of body temperature, pulse, or sense of 
well-being. These principles are elaborated because they seem to give a rational basis for the choice of climate.

Although, as in any remedial system, the individual reaction of the patient is of paramount importance, we should expect from the foregoing that moderately cold climates would be more remedial for tuberculosis than hot regions; that variable temperatures would prove more salutary than equable warmth; that the humidity of the air should not be so great as to sensibly interfere with the radiation of heat in warm weather or lead to its insidious loss by conduction in cold, that the gently moving open air would be superior to calm; that the abundance of sunshine might safely be regulated according to its psychic effect. It is believed that these deductions must accord with the experience of those clinicians who have practical knowledge of tuberculosis.

The well-founded belief in the remedial efficacy of moderately high altitudes for many cases of tuberculosis finds its justification and its limitations in the conception that has been set forth. There is reason to believe that the higher the altitude the greater the metabolic disturbance with a given output of energy. According to our theory those patients will recover most surely and quickly who can respond to the demands of an appreciable altitude without pathologic reaction. Webb and Williams $\left({ }^{53}\right)$ found that the lymphocyte corpuscles of the blood increase absolutely and relatively on ascending from sea-level. At Colorado Springs, an altitude of about 6,000 feet, they discovered that the prognosis in pulmonary cases improved somewhat proportionately with the rise of lymphocytes. Webb and Williams make the pregnant suggestion that this is a specific reaction to altitude by which the formation of especially tuberculocide corpuscles is stimulated.

It is obvious that cases of very active disease or those running a constant fever while at rest are probably not suited for altitude treatment. Many attempts have been made to classify patients according to climatic adaptability. Barlow $\left({ }^{54}\right)$ states that most cases of pulmonary tuberculosis from seventeen to forty years of age should be sent to a high, dry climate; that children and persons over forty should be kept at or near the coast.

Heart Disease is perhaps the sorriest reproach of medicine today. Sufficient data are already at hand which, if properly applied, might be expected to greatly reduce the incidence of this class of disorders. The prophylactic ideas of Catron $\left({ }^{55}\right)$ should be inculcated in the minds of medical students and laymen. In another place I have ventured to draw a close analogy between pulmonary tuberculosis and heart disease from the viewpoints of pathology, clinical history, and treatment $\left({ }^{56}\right)$. An appreciation of the essentials in the care of the cardiopath is founded on 
the principles of rest and exercise; these already have been discussed. No adequate treatment of so broad a theme can be attempted here. The maximum efficiency of the debilitated organ, in the absence of acute infection or toxemia, is to be developed by gently graduated exercises whose demands of energy must fall far short of the extreme reserve power of the heart. Theory and experience combine in recommending moderate altitudes, 5,000 or 6,000 feet, for believing that lowered barometric pressure exerts its influence chiefly on the right side of the heart, tending to its overfilling, probably through increase in the aspiratory force of the lungs; the coincident hyperoxygenation of the blood must be expected to favor nutrition of the organ. Such a strain upon the chambers of the heart leads normally to their hypertrophy and increase of power. No valvular disease, except advanced mitral stenosis, no myocardial involvement in which fair reserve power remains, no degree of arteriosclerosis contraindicates residence at a moderately high elevation above sea-level. But let the heart become overstrained, let compensation for a lesion be broken, let an active infection or widespread myocardial degeneration demand the minimum possible of exercise, and altitude is distinctly contraindicated and often cannot be endured by a patient who ventures upon the slightest physical exertion.

In such a situation the manifest therapeutic indication would seem to be to increase the barometric pressure for the sake of its oxygen component. Likewise important from the theoretical standpoint are the temperature and humidity of the air. The physican in Colorado is prone to refer his cases of ill-maintained cardiac compensation to the coast or valleys of southern California, so often with such happy results as to create a confidence in the specific efficacy of that region to ameliorate the conditions of cardiac overstrain.

Nephritis and disorders involving functional deficiency of the kidney demand for their treatment the application of the same principles which guide the therapeusis in tuberculosis and heart disease; namely, such a degree of rest or reduction in functional burden, as will not exceed the reserve power of the organ. Christian $\left({ }^{57}\right)$ and his coworkers have cogently demonstrated the inadequacy of diuretics to whip up efficiently the failing renal powers. The practitioner knows full well that his successful treatments have involved bed rest and management of the circulation, skin activity, and diet. There is a good deal of desultory clinical evidence that even moderately high altitudes may add greatly to the burden opposed to a weakly acting kidney. It is not uncommon to see the signs and symptoms of renal inefficiency clear up or ameliorate rapidly in patients sent from Colorado to southern California. San Diego appears to be an especially salutary resort. The writer has been 
impressed with the clinical evidence of circulation failure as an explanation of the renal inefficiency. When the right heart is inadequate we may assume an increase in the venous blood pressure which must add greatly to the secretory difficulties of the kidney. Unfortunately we are far from possessing the data from which we might deduce the relations of climate to renal activity.

Abderhalden and others hold that in high altitudes the transpiration of watery vapor is in excess and that the blood thereby becomes abnormally concentrated. It would seem that in this respect the effect of lowered barometric pressure would be the same as that of therapeutic sweating and favorable to the patient except for concomitant unfavorable conditions.

Carter $\left({ }^{58}\right)$. was unable to determine any geographical parallelism between climate and Bright's disease and was forced to conclude that the important determining factors in the distribution of the disorder are diet and infection. A most competent and careful observer, who is a medical officer in the Base Hospital at Camp Logan, near Houston, Texas, tells interesting details of life in that warmest of all camps. Noting the excessive perspiration that marked every exertion, this physician measured his input of fluid, which was taken in the ordinary manner as needed, and found that it amounted to more than a gallon, while the output of urine was but thirty ounces in twenty-four hours. This was in summer. In winter with an air temperature above freezing it was almost impossible to keep warm in bed, so insidious was the loss of heat by conduction to the humid atmosphere. At Houston the mean temperature and relative humidity at 8 A.M. for January are about $54^{\circ} \mathrm{F}$. and 84 per cent. For July the corresponding data are $8 \mathrm{I}^{\circ} \mathrm{F}$. and 84 per cent.

Rheumatism and Gout are grouped together because of the inextricable union of the symptoms of the infective and the metabolic disorders. The difficulties that have confronted us already in distinguishing between the conditions which determine the physiological resistance against the implantation of infection and those which favor physiological reaction against acquired infection, are especially prominent in this field. $\left({ }^{14}\right)$

We know that the victim of pain, whether of rheumatism or gout, is benefited by the external application of heat, and experience confirms the deduction that the appropriate patient should seek a dry, warm, equable climate. Acute articular rheumatism is apparently a comparatively rare disease in Colorado, but subacute rheumatism, marked by disseminated pains and morbid sensations, which nowadays always leads to the search for "focal infections," is exceedingly common. In one such sufferer, long under the eye of the writer, there is an astonishing 
relief from the subacute pains with every journey toward sea-level, whether in winter or summer. It can hardly be doubted that the primary change here is one of metabolism.

Balfour $\left({ }^{50}\right)$, in his work on the Senile Heart, writes, "Venous congestion, as it is usually termed, has long been recognized by all physicians since the days of Galen as the first condition essential to the formation of the gouty diathesis." It has been assumed above that physiological reaction to altitude tends to relative increase of venous blood pressure and would, therefore, presumably accentuate the circulatory conditions favoring development of the lithemic state.

The Relations of Climate to the Mind and Nervous System. It has been made obvious, perhaps, that climate change may provoke reactions of every physiological function, but a mere mechanistic view of the influence of climate would fall far short of representing the reality. The modification of the mental state following a judicious "change of scene" is probably the most important factor in remedial climatology. For the sick this is a field of psychotherapy which seems to offer a rich opportunity for development and for the well it is a potent source of prophylaxis.

Medical meteorologists have been accustomed, indeed, to classify climates on a psychological basis as " stimulating," "sedative," " relaxing," " tonic," etc. It is generally conceded that the stimulating effects of climate increase with elevation above sea-level, as typified in the region of the Rocky Mountains, while the sedative influence eharacterizes areas of equable, moderate temperature, and a fairly uniform humidity, such as are found in southern California and in Oregon west of the mountain range. It seems especially important that the nervous reactions to high altitudes be considered, since harm as well as good can result from a sojourn at even moderate elevations according to the mode of behavior of the immigrant.

I can do no better than quote from a former essay on this subject $\left({ }^{60}\right)$. " The late Dr. J. T. Eskridge lived in Colorado for many years and made a special study of the climatic relations of nervous phenomena. Eskridge is careful to distinguish the nervous influences of high altitudes according as they occur in 'acclimated' or 'unacclimated' persons. For nine out of every ten healthy persons who come to Colorado and do not almost immediately begin to overexercise at the higher altitude no unpleasant effects are produced. If physical exercise be freely and indiscriminately indulged in, the person soon becomes restless. To keep quiet then is difficult and irksome, but to continue the exercise increases the nervousness and restlessness. The legs feel tired and heavy, and numb and tingling sensations with pains in the joints are often experienced. 
The muscles become sore and painful and cramping of the calf muscles is suffered from at night. Sleeplessness follows, and this, with the restlessness, makes the night almost unendurable. The severer symptoms are experienced only by the nervous and unacclimated persons who disregard advice and exercise too much. . . . For persons advanced in years and for nearly all invalids there is but one rule,- - keep comparatively quiet at first and when moderate exercise is begun always stop short of the point of decided fatigue. For the majority of persons, especially for the consumptive invalids, sleep is more easily obtained, more continuous, and more refreshing in Colorado than in the Eastern States.

"Eskridge thought that cases of insomnia from venous stasis or passive hyperemia of the brain sleep well in Colorado, but that insomnia caused by organic cerebral disease or active hyperemia of the brain is made worse. "The general impression that a prolonged residence in Colorado has a tendency to produce simple nervousness and finally sleeplessness, I think, is correct. At least I have advised many to take a trip to low altitude for a few weeks once a year. . . . These periods of relaxation have been especially advised for the overworked, mentally or physically, and for the nervous or hysterical.' According to Eskridge persons who are nervous as a result of malnutrition or overwork do well in Colorado because of improvement in the general condition, but those of inherent nervous temperaments do badly, and some who are not considered nervous become so after long residence in Colorado. 'Hysterical subjects do better at sea-level than in Colorado unless the hysterical manifestations are due to depressed states of health that are relieved by residence in Colorado. .. . The same may be said of neurasthenic subjects, except that some of the causes of neurasthenia are more commonly removed by a residence in Colorado than those of hysteria. The ideal life for those afflicted with migraine is a frequent change of climate, from Colorado to sea-level, living at least two-thirds of the time at low altitudes. . . . Choreic patients should not be sent by choice to Colorado for treatment. . . . I have been unable to observe any marked difference in the frequency, course, and results of organic disease of the nervous system here from what I found to hold in Philadelphia."

\section{BIBLIOGRAPHY}

I. MOORE, W. L.: Descriptive Meteorology, 1910.

2. VON HUMBOLDT, ALEXANDER: Travels, Translated by Ross, 1884.

3. WILLIAMS, C. T.: Aerotherapeutics, 1894 .

4. SOLLY, S. E.: Medical Climatology, 1897.

5. WEBER, F. P., and HINSDALE, G.: System of Physiological Therapeutics, Cohen, Vol. III. 
6. HANN, J.: Handbook of Climatology, Translated by Ward, 1903.

7. HUGGARD, W. R.: Handbook of Climate Treatment, I906.

8. HINSDALE, GUY: Atmospheric Air in Relation to Tuberculosis, Smithsonian Misc. Collections; also in Trans. Amer. Climatological and Clin. Assoc., 1914, XXX, 305.

9. BERT, PAUL: La Pression Barométrique, 1878 .

Io. MOSSO, ANGELO: Life of Man on the High Alps, Translated by Kiesow, I 898 .

I I. ZUNTZ, N., LOEWY, A., MÜLLER, F., CASPARI, W.: Höhenklima und Bergwanderungen, 1906.

I2. DOUGLAS, C. G., HALDANE, J. S., HENDERSON, Y., SCHNEIDER, E. C.: Physiologic Observations Made on Pike's Peak, Phil. Trans. of Roy. Soc., I9I3, Ser. B, CCIII, I85.

13. FOSTER, MICHAEL: Textbook of Physiology, 3rd. ed., I879.

14. KRAUSE, A. K.: Amer. Review of Tuberculosis, 1918, II, 102.

15. HUNTINGTON, ELLSWORTH: Civilization and Climate, 1915.

I6. DEXTER, E. G.: Weather Influences, 1904.

17. LOEB, JACQUES: The Mechanistic Conception of Life, Igr2.

18. HENDERSON, L. J.: The Fitness of the Environment, 1913.

19. OSBORN, H. F.: The Origin and Evolution of Life, 1917.

20. MACKENZIE, JAMES: Diseases of the Heart, 1908.

21. PHILliPS, W. F. R.: Climate, Buck's Reference Handbook of Med. Sc., I901, Vol. III.

22. Weather Forecasting in the U. S., Weather Bureau, U. S. Dept. Agric., I9I6.

23. Life of Pasteur, Translated by Devonshire, I9II.

24. GRABLEY, P.: Medizinische Klinik, I9r3, IX, r 335.

25. DURIG, A.: Wiener klin. Wochenschr., I9rI, XXIV, 619.

26. MOORE, W. L.: The Influence of Forests on Climate and on Floods, Gov. Print. Off., I910; also, discussion in Trans. Amer. Climatological Assoc., 19ro, XXVI, 46.

27. CAMERON, V. K.: The Soil Solution, I9r.

28. LUSK, GRAHAM: The Science of Nutrition, I9I7.

29. ZUNTZ, N., quoted by Howell, Textbook of Physiology, 1907.

3o. PEMBREy, M. S.: Animal Heat in Scharfer's Textbook of Physiology, I898, Vol. I.

31. GLASSFORD, W. A.: Fourth Report, Colo. State Board of Health, 1894, 212.

32. HARRINGTON, M. W.: Sensible Temperatures, Trans. Amer. Climatological Assoc., I893-94, XX, 368.

33. FRANKENHAUSER: Münch. med. Wochenschr., I9II, LVIII, I42I.

34. RUBNER: Arch. f. Hygiene, 1905, L, 296; quoted by Lusk.

35. HILL, L., ROWLANDS, R. A., WALKER, H. B.: Jour. of Physiology, I9I0-II, XLI, III.

36. SEWALL, HENRY: Forchheimer's Therapeusis of Int. Dis., 19I4, I, 245. SEWALL, HENRY: Musser and Kelly Practical Treatment, 1917, IV, 93.

37. Amer. Jour. Public Health, igi5, V.

38. SEWALL, HENRY: Arch. of Int. Med., I9r4, XIII, 856.

39. OVINGTON, E. L.: Jour. Amer. Med. Assoc., I914, LXIII, 419.

40. CRAMPTON, C. W.: New York Med. Jour., igi3, XCVIII, gr6; also, Med. News, 1905, LXXXV, 529. 
41. SEWALL, HENRY : Amer. Jour. Med. Sc., I9I6, CLI, 491.

42. DALlWIG, H. C., KOLLIS, A. C., LOEVENHART, A. S.: Amer. Jour. Physiology, 1915, XXXIX, 77. LOEVENHART, A. S.: Arch. Int. Med., I9I5, XV, 1059.

43. BARCROFT, JOSEPH: The Respiratory Function of the Blood, I914, 278.

44. HALDANE, J. S., SMITH, J. L.: Jour. of Physiology, I896, XX, 497.

45. KRONECKER: Bergkrankheit, 1903, quoted by Huggard $\left({ }^{7}\right)$.

46. BABCOCK, R. H.: Trans. Amer. Climatological Assoc., I899, XV, 159.

47. HIRSCH: Geographical and Historical Pathology, New. Syd. Soc. Translation, I886.

48. Editorial, Jour. Amer. Med. Assoc., igi2, LVIII, I449.

49. CHAMBERLAIN, W. P.: Philippine Hour. of Sc., I9I I, VI.

50. WOODRUFF, C. E.: Effect of Tropical Life on White Men, 1905; also, New York Med. Rec., 1905, LXVIII, 1005.

5I. GORDON, WILLIAM : Lancet, I9I3, I, I643; I7I5.

52. BROWN, LAWRASON: Jour. Amer. Med. Assoc., I912, LVIII, 1678.

53. WEBB, G. B. and WILliAmS, W. W.: Trans. Nat. Assoc. Study and Prevention Tuberculosis, 1909, V, 231.

54. BARLOW, W. J.: Jour. Amer. Med. Assoc., I9I I, LVII, 1426.

55. CATRON, RICHARD: The Prevention of Valvular Disease of the Heart, 1900.

56. SEWALL, HENRY: Jour. Amer. Med. Assoc., I9I7, LXVIII, I604.

57. CHRISTIAN, H. A.: Arch. Int. Med., i9i6, XVIII, 606.

58. CARTER, W. S.: Med. News, 1904, LXXXV, 917.

59. BALFOUR, G. W.: The Senile Heart, I894.

60. SEWALL, HENRY : Musser and Kelly Practical Treatment, I9I I, $5^{87 .}$ 
, 


\section{THE \\ OXFORD MEDICINE ADVANCE PAGES}

EDITED BY

HENRY A. CHRISTIAN SIR JAMES MACKENZIE

\section{Contributors Volume I}

LEWELLYS F. BARKER

FRANK BILLINGS

WILLIAM T. BOVIE

RICHARD C. CABOT

HENRY A. CHRISTIAN

CHAS. BENEDICT DAVENPORT

EUGENE F. DU BOIS

FREDERICK P. GAY

LAWRENCE J. HENDERSON

A. WALTER HEWLETT

GUY HINSDALE
WALTER B. JAMES

WILLIAM B. JOHNSTON

SIR JAMES MACKENZIE

JOHN J. MACKENZIE

ELMER V. MCCOLLUM

SIR WILLIAM OSLER

FRANCIS W. PEABODY

LEONARD G. ROWNTREE

HENRY SEWALL

DONALD D. VAN SLYKE

WILLIAM H. WELCH

PART 2

Volume I

NEW YORK

OXFORD UNIVERSITY PRESS

AMERICAN BRANCH: 35 WEST 32 ND STREET 
COPYRIGHT, 1919 BY THE

OXFORD UNIVERSITY PRESS

American Branch 
CONTENTS OF PART II, VOLUME I-OXFORD MEDICINE

Chapter

III. Pathological Physiology and Its Relation to Internal

Medicine-Albion Walter Hewlett . . . . 109

Chapter

IV. Hydrotherapy-Guy Hinsdale . . . . . . ${ }^{\text {I72 }}$ 



\section{PATHOLOGICAL PHYSIOLOGY AND ITS RELATION TO INTERNAL MEDICINE}

\section{By Albion Walter Hewlett}

Anatomy, the science that deals with structure, is divided into normal and pathological anatomy, according as the structure studied is normal or diseased. In a similar manner, physiology, the science that deals with function, may be divided into normal and pathological physiology, according as the functions studied are healthy or are disturbed by pathological processes. Unfortunately, however, the term physiological is frequently employed in the sense of normal, and in this sense it is contrasted with pathological. Thus the expression pathological physiology is an awkward one because in a certain sense the two words are contradictory. When the expression is employed, it is therefore necessary to keep in mind the fact that the term physiology is used in this connection to indicate the science of function without reference to whether this be normal or perverted. Other designations for the science that deals with alterations of function in disease might be suggested. Functional pathology, for example, indicates without ambiguity the scope of such a science; but it is doubtful if this or similar expressions will replace the older designation, the medical profession having become accustomed to the use and meaning of the term pathological physiology.

The study of functional changes in disease is as old as medicine itself, for symptoms are in the main but alterations in function that are appreciated and described by the patient. Moreover, the usual bedside observations of the physician deal largely with evident alterations in function. The study of disturbed function in disease has been greatly assisted and extended, however, by the introduction of instruments and methods of precision. By these means the accuracy of clinical observations can be checked and new information can be obtained which would otherwise escape observation. An early example which illustrates the value of an accurate method is the use of the clinical thermometer. Our present knowledge of infectious diseases is based largely upon data supplied by this instrument. In more recent years a 


\section{IIO}

\section{PATHOLOGICAL PHYSIOLOGY AND MEDICINE}

considerable number of new physical, chemical and biological methods have been used for studying the functional disturbances in disease. A description of these is beyond the scope of the present article; but we may mention, among the physical methods, the bloodless estimation of arterial blood pressure, the study of cardiac function by means of venous tracings and electrocardiograms, and the study of gastrointestinal functions by means of the X-ray. Among the chemical methods we may mention those that have been used for determining alterations in the composition of the urine, the blood and the respired air. Among biological methods may be noted those used for studying the changes that follow the introduction into the body of foreign proteins and particularly of those proteins or protein-like substances which are contained in or are elaborated by the infectious microorganisms.

These newer methods for studying the functions of the body in health and in disease have brought about important advancements in modern medicine. They have supplied data on the changes in function that occur in particular diseases, and in this way they have aided in the diagnosis, the prognosis and the treatment of these diseases. At the same time the application of more accurate methods to the study of perverted function has increased our understanding of pathological processes in general. They have helped to show how the original cause of a disease may lead to changes in structure and function, they have made more clear the relation that exists between structural and functional deviations from the normal, and they have furnished to the physician more rational explanations for the symptoms and signs that are observed during the usual clinical examination.

In its broader aspects pathological physiology is the science that - describes and seeks to explain all functional deviations from the normal that may occur in the animal body. As a medical science its problems are restricted to those changes in function that occur during the natural diseases of man. In so far as it aims to describe and to classify the functional changes in individual diseases it is a descriptive and special science. In so far as it seeks to explain these functional changes and to relate them to one another, to the causes of disease, to the structural alterations present and to the more obvious signs and symptoms, it is a general and rational science. In the present chapter the endeavor will be made to present and to illustrate certain principles and general applications of pathological physiology, leaving the discussion of more special problems to the chapters dealing with the various diseases. 


\section{RELATION OF PATHOLOGICAL PHYSIOLOGY TO PATHOLOGICAL ANATOMY}

In directing attention to the functional as contrasted with the structural changes during disease it is not our intention to convey the impression that the former are of greater importance than the latter. In the end the two sciences supplement each other; but since they approach pathological processes from different points of view and employ different methods of study, each may be treated as a more or less independent unit.

Anatomical studies often furnish an obvious explanation for the symptoms and signs of disease; but this is not always the case. In some instances, although anatomical changes are present, their relation to the disturbances of function is not an obvious one; in other instances no constant anatomical changes can be found. The manner in which structural alterations produce symptoms is frequently less simple than would appear at first sight. Why, for example, should the lodgment of a calculus in the ureter give rise to the exquisite pain of renal colic? Is this pain due to an injury of the mucous membrane, is it due to a local stretching of the ureter about the calculus, or is it due to distension and to muscular contractions behind the obstruction? Pathological anatomy furnishes no answer to these questions. Again, the intestinal ulcers present in typhoid fever offer a ready explanation for the important complications of intestinal hemorrhage and intestinal perforation, but neither these nor the other anatomical changes in this disease throw any light upon its characteristic incubation period, its fairly constant temperature curve or the loss of weight that usually occurs. Thus we see that even where well-defined anatomical changes accompany a disease, there may yet exist a gap between these and the most important clinical manifestations. Frequently this gap can be bridged only by physiological methods of study.

In other cases anatomical changes are the result and not the cause of the associated disturbances of function. In chronic arterial hypertension, for example, the most striking and most common alteration found at autopsy is cardiac hypertrophy; yet this appears to be, in large measure, a result of the increased work thrown upon the heart by the high blood pressure, and it does not explain the latter. Similarly the abnormal deposits of glycogen in diabetes and the abnormal deposits of urates in gout are manifestations rather than causes of these general metabolic disorders.

Finally we have an important group of diseases and of symptom complexes where anatomical changes are either not demonstrable, or 


\section{I2 PATHOLOGICAL PHYSIOLOGY AND MEDICINE}

where, if demonstrable, they are plainly secondary to the functional alterations present. Among these are the so-called functional neuroses, viz., psychasthenia, neurasthenia and hysteria, certain psychoses such as manic-depressive insanity, and certain cases of cardiac palpitation, of cardiac irregularity, of gastric hyperacidity, of cardiospasm, of constipation, of diabetes insipidus, and of a number of other conditions usually described as neuroses of one or another organ.

It is true that in the last analysis all disturbances of function must be capable of explanation in terms of physical or chemical changes in the body cells and fluids. Anatomical investigations have repeatedly shown that conditions which have been classed among the functional diseases possess in reality an anatomical basis. The Stokes-Adams syndrome, for example, which is characterized by cerebral attacks associated with a very slow pulse, is now known to be frequently caused by definite structural changes in the bundle of muscle fibers that connects the cardiac auricles with the ventricles. It does not follow, however, that all disorders, now classed as functional, will ultimately be found to show definite anatomical changes. Various drugs produce marked alterations in function without primary anatomical change, and there is every reason to believe that analogous alterations in function without primary changes in structure may result from internal disturbances in the chemistry of the body cells and fluids.

This group of diseases and of symptom complexes in which no anatomical changes in the affected organs can be demonstrated is usually described as nervous or functional. As we have just pointed out, in certain of these conditions structural changes will ultimately be found which will remove them from this category and will place them among the so-called organic diseases. Others are due to demonstrable alterations in the physical or chemical constitution of the body cells or fluids, and this group will likewise grow as our knowledge of biochemistry and biophysics increases. A third group of diseases, however, will always remain in which no changes will have been demonstrated in the structure or in the chemical or physical condition of the affected organs.

By what name shall we designate this group of diseases and of symptom complexes for which no structural or chemical causes are known? They are frequently classified as neuroses or as nervous disorders. In so far as the diseases thus described depend upon functional changes in the nervous system or are due to nervous reflexes from some other part of the body, there is no objection to this classification. It should be remembered, however, that functional disturbances without a known anatomical cause may result not only from a disturbed innervation but 
also from undemonstrated structural or chemical changes. To group all functional disturbances without apparent cause among the neuroses is not merely a confession of ignorance but it cloaks this ignorance in a nomenclature that is misleading and that obscures the outlook on what may be the true nature of the conditions present. Cardiac palpitation, for example, is at times of nervous origin, as when it accompanies an evident psychic disturbance, such as fear. At other times it accompanies toxic conditions, such as hyperthyroidism, or it depends upon changes in the strength of the heart muscle. If cardiac palpitation be classed as a neurosis we are apt to lose sight of these important possibilities. Similarly, changes in the amount of hydrochloric acid in the gastric contents may be due to nervous causes, either psychic or reflex; but they may also be due to an altered chemistry of the secreting cells in the stomach, dependent upon local changes in these cells or upon changes in the body at large. Here again it is not advisable to speak of these conditions as neuroses. These and similar disorders are in fact functional disturbances for which no anatomical or other cause has been demonstrated. In this restricted sense they may be called functional diseases. Such a designation allows us to approach a functional disease with no bias as to its possible cause, be this nervous, structural or chemical. Such a nomenclature has furthermore a practical advantage in that the physician who diagnoses a condition as functional should understand that in so doing he has left open the problem as to its nature. In practice it not infrequently happens that the functional changes first noted are found eventually to depend upon some intoxication or infection, upon some disturbance of the internal secretions or upon some structural change that has escaped early observation.

Intensive study of the functional disturbances that occur in disease has thrown light upon various problems that are of immediate interest to the practitioner. They have aided in the interpretation of phenomena commonly observed at the bedside and have thus broadened our knowledge of the significance of symptoms and signs of disease. They have furnished new methods of diagnosis, new rules for prognosis and new indications for treatment. Finally they have thrown light upon many minor complaints and upon the earlier stages of many serious diseases, both of which have been neglected by pathological anatomy for the reason that the latter science has to deal mainly with the late and final stages of organic disease. From the standpoint of therapy this last knowledge has been particularly helpful, because therapeutic measures promise success in proportion as the earlier stages and milder types of disease are recognized and understood. For all these reasons the study of disturbed function in disease has become one of the chief centers 


\section{I14 PATHOLOGICAL PHYSIOLOGY AND MEDICINE}

of interest for those physicians who are anxious to follow and to assist the advancement of our knowledge of internal diseases.

\section{GENERAL METHODS OF STUDY}

The problems of pathological physiology have been attacked in two main ways. In the first place patients themselves have been studied, using the methods employed in physiology, in biochemistry and in immunology. In the second place animals have been similarly studied after they have been made the subjects of pathological changes. Each method of study has its advantages and each has its limitations. In the following paragraphs these general advantages and limitations will be discussed without attempting to describe in detail the various methods used.

The study of patients by the methods employed in physiology, in biochemistry and in immunology presents the obvious advantage that we are dealing with conditions that are of immediate clinical interest;viz., disturbances of function in human disease. At the same time, physiological studies on living human beings impose upon us this limitation, that the methods used must be harmless to the objects of study. To develop and utilize accurate methods that may be applied without harm to patients is one of the main problems of our science, and its rapid advance in recent years has been due largely to the discovery of just such methods. We are not permitted, for example, to insert a canula into a human artery for the purpose of estimating the arterial blood pressure; yet an approximate estimate of this pressure can be made by using a pressure cuff on the arm, and the data thus obtained have proved of great practical service. Similarly we cannot record the contractions of the auricles and ventricles by attaching levers to the exposed human heart, but we can recognize the effect of these contractions and determine their sequence either by recording the venous pulse in the neck or by recording with the string galvanometer the electrical changes which accompany each cardiac contraction. The latter method, indeed, by indicating in what direction the contraction waves are spreading through the heart muscle, gives information which cannot be obtained by direct registration of the mechanical movements of the auricles or ventricles. Disturbances in the chemistry of digestion have been studied by analyzing the gastric contents, the duodenal contents and the feces. The motor functions of the gastrointestinal canal have been studied not only by determining the pressure changes in the accessible extremities of this canal, but by observing and recording with the X-ray the form of its parts after the ingestion of material that is 
opaque to this ray. The presence of albumin in the urine has furnished information concerning the condition of the kidneys, while other changes in the urine have proved of great value in the study of metabolic disturbances. The total metabolism of the body has been accurately determined not only by measuring the heat losses from the body with a calorimeter but by chemical analyses of the products of combustion eliminated in the expired air and in the urine. Finally detailed studies of the blood have yielded results of far-reaching importance. Through such studies we have become familiar with alterations in the number and kind of its formed elements, and in certain infections we have been able to demonstrate the infecting organisms. We have learned furthermore that as a result of infection the blood serum often shows characteristic alterations in its content of antibodies, such as antitoxins, agglutinins, precipitins, opsonins, and substances that fix complement in the presence of the foreign (infectious) material. Finally new methods have been devised for determining, in such small quantities of blood as easily may be withdrawn from the superficial veins, the content of sodium chloride, of carbonates, of glucose, of urea, of uric acid and of other non-protein nitrogenous constituents. The percentage of each substance in the blood serum is not only of importance in itself, but the relation between this percentage and the amount excreted has furnished evidence of the functional efficiency of the particular excretory organ concerned.

These methods of study, which, owing to their harmlessness, can be applied to patients, possess the further advantage that they can be used repeatedly in a given subject. It is therefore possible to follow the pathological changes thus observed from day to day or from hour to hour, and to show the relation of these changes to other transient manifestations of the disease. A further advantage of such methods lies in the fact that the interpretation of the results obtained is not complicated, as it is in many animal experiments, by the effects of anaesthesia and operation.

On the other hand, physiological studies on patients, restricted as they are by the necessity of avoiding danger to the subject, frequently fail to solve problems of disturbed physiology which might be settled easily by operative experiments on animals. Studies on patients are also limited by the clinical material available and the results of such studies must be interpreted with due regard to the various complicating factors which enter into each particular illness. In other words, the data obtained in the clinic rarely permit that strict control of conditions which characterizes the experimental method. In animal experiments. on the other hand, a much wider range of methods may be used; and 


\section{II6 PATHOLOGICAL PHYSIOLOGY AND MEDICINE}

observations may be indefinitely repeated with variations in the experiments so that a definite answer may be given to a definite question. Normal physiology has solved most of its problems by recourse to animal experiments, and the results thus obtained for the most part have been transferred easily to man because we know that on the whole very slight differences exist between the normal physiology of man and the normal physiology of other mammals.

The study of functional changes in animals after the artificial production of pathological processes has yielded results of great value in the interpretation of clinical problems. It is only necessary to recall such important contributions as the production of diabetes by removal of the pancreas, the production of tetany by removal of the parathyroid glands, the experimental production of valvular lesions in the heart and the experimental infection of animals with pathogenic mircroorganisms. The data thus obtained by the methods of experimental pathology are not only accurate, but their interpretation is relatively simple because various factors can be controlled by altering the type of experiment.

Nevertheless, conclusions drawn from observations on animals after the artificial production of disease cannot be transferred to the interpretation of human ailments in the same way that the results of functional studies on normal animals have been incorporated in human physiology. In the latter case we assume that the physiological processes of man and of the higher animals are for the most part identical. In the former case this additional question must always be met:-to what extent does the experimental disease induced in animals parallel a similar disease which occurs naturally in man? It is true, for example, that human diabetes frequently is accompanied by demonstrable lesions of the pancreas, but we are not yet certain that all cases of diabetes are of this origin. Similarly, tetany invariably follows the complete removal of all parathyroid tissue, but tetany frequently occurs in man when no parathyroid changes are demonstrable. Valvular disease in man is. commonly associated with infectious processes in the heart muscle, and these muscle changes, which are absent in experimental heart lesions, may dominate the clinical picture. Thus we see that the results of experimental pathology, important as they are, cannot be transferred unreservedly to clinical problems. On this very account, however, experimental pathology has proved a stimulating incentive to clinical research; for each advance in experimental pathology necessitates a restudy of the corresponding human disease in order to determine to what extent the new knowledge is applicable to diseases that occur naturally in man.

Light also may be shed on the functional alterations in human 
disease by applying the methods of physiology to the study of diseases that occur naturally among animals. Researches of this type on infectious diseases, and especially on tuberculosis, have been made; but aside from these, few natural animal diseases have been studied by physiological methods. Beyond doubt such studies are destined to advance general pathology and to throw light upon specific clinical problems, because it is possible to use methods on sick animals that cannot be used on patients. Chronic auricular fibrillation, for example, was first demonstrated to the eye when the heart of a horse suffering from this irregularity was exposed by opening the chest. The study of diseases occurring naturally in animals, like the study of human disease, is limited by the available clinical material and by the available laboratory facilities. Furthermore, the results obtained must be interpreted with due regard to the various factors that complicate every natural disease. For these reasons the study of perverted physiology in the natural diseases of animals cannot reach its full development until we have animal clinics which in size and facilities for research are comparable to the modern medical clinic with its attached laboratories.

\section{THE NERVOUS CONTROL OF VISCERAL FUNCTIONS}

Disturbances of function in an organ may be secondary to pathological changes elsewhere in the body. Viewed from a functional standpoint, the organs are far from being independent units, for the activity of each is being constantly influenced by a variety of external conditions. This external control is exercised in part through the nerves supplied to the organ, and in part through the chemical and physical properties of the blood which comes to it.

The nervous control of body functions has been studied extensively, because a common physiological method of investigating the activities of any organ has consisted in noting the changes which occur when its nerve supply is either stimulated or interrupted. Nearly all parts of the body receive nerve fibers, and the functional activities of nearly all organs are influenced by the impulses carried in these fibers. Particularly is this true of the voluntary muscles. These muscles contract on stimulation of any portion of the motor paths which extend from the cortical centers to the muscles themselves. Conversely the voluntary muscles become paralyzed when either the cortical centers or the motor paths are destroyed or are seriously damaged by injury or disease.

The various glands and involuntary muscles receive their nerve supply from the so-called vegetative or autonomic nervous system. This consists of two subsidiary nervous systems which differ from each 


\section{II8 PATHOLOGICAL PHYSIOLOGY AND MEDICINE}

other in their origin, in the course of their fibers, in their effects upon the visceral functions and in their reaction to drugs.

The first of these subdivisions of the vegetative nervous system is known as the sympathetic system. Its proximal fibers emerge from the thoracic and lumbar portions of the spinal cord by way of the anterior nerve roots, leave these in the rami communicantes and terminate about nerve cells in the sympathetic ganglia which lie in the neck, the chest and the abdomen. From these ganglia a second set of nerve fibers proceeds to the various glands and smooth muscles.

The second subdivision of the vegetative system is known as the parasympathetic or craniosacral autonomic system. Its fibers leave the cranial and sacral extremities of the cerebrospinal axis and are found in the oculomotor nerves, the trigeminal nerve, the vagus nerve and certain pelvic nerves, particularly the nerves erigentes. The craniosacral autonomic fibers, found in these nerves, terminate about ganglion cells which usually lie in close proximity to the structures supplied by the secondary fibers coming from the ganglia.

The origin, distribution and physiological actions of the chief subdivisions of the vegetative nervous system are shown in the accompanying diagram and table. It will be noted that in most instances a single organ receives fibers from both the sympathetic and the craniosacral autonomic systems. When this is the case the two frequently exert a mutually antagonistic action. For example, stimulation of the autonomic fibers in the oculomotor nerve causes myosis through contraction of the circular muscle fibers in the iris, whereas stimulation of the cervical sympathetic causes mydriasis through contraction of the radial fibers in the iris. Similarly stimulation of the vagus slows the heart rate, whereas stimulation of the sympathetic fibers to the heart, the cardiac accelerator, increases this rate. Stimulation of the vagus increases the tone and contractions of the intestines, while stimulation of the splanchnic nerves causes relaxation. In other instances the sympathetic and the craniosacral autonomic nerve impulses to a given organ influence its activities in a given direction but in different ways. Thus the submaxillary gland pours out a viscid secretion after sympathetic stimulation and an abundant clear secretion after stimulation of the chorda tympani. Finally certain glands and smooth muscles, among which are many blood vessels, appear to be innervated from only one subdivision of the vegetative nervous system.

Much has been learned through studying the effects produced by certain drugs which exert a selective action upon one or other of the chief subdivisions of the vegetative nervous system. Atropin and related drugs depress the craniosacral autonomic terminals. After the 
$118^{a}$ 


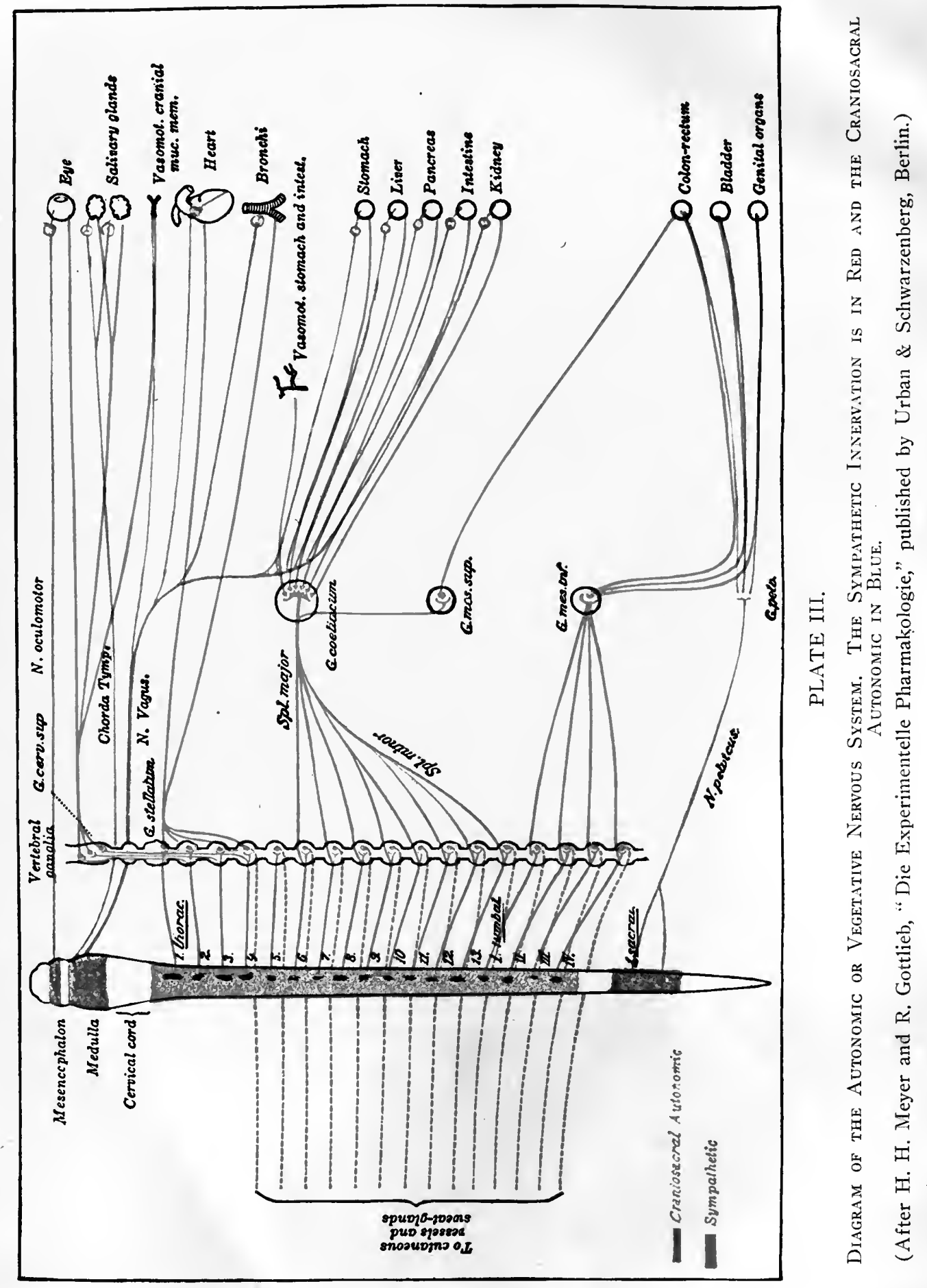


NERVOUS CONTROL OF VISCERAL FUNCTIONS

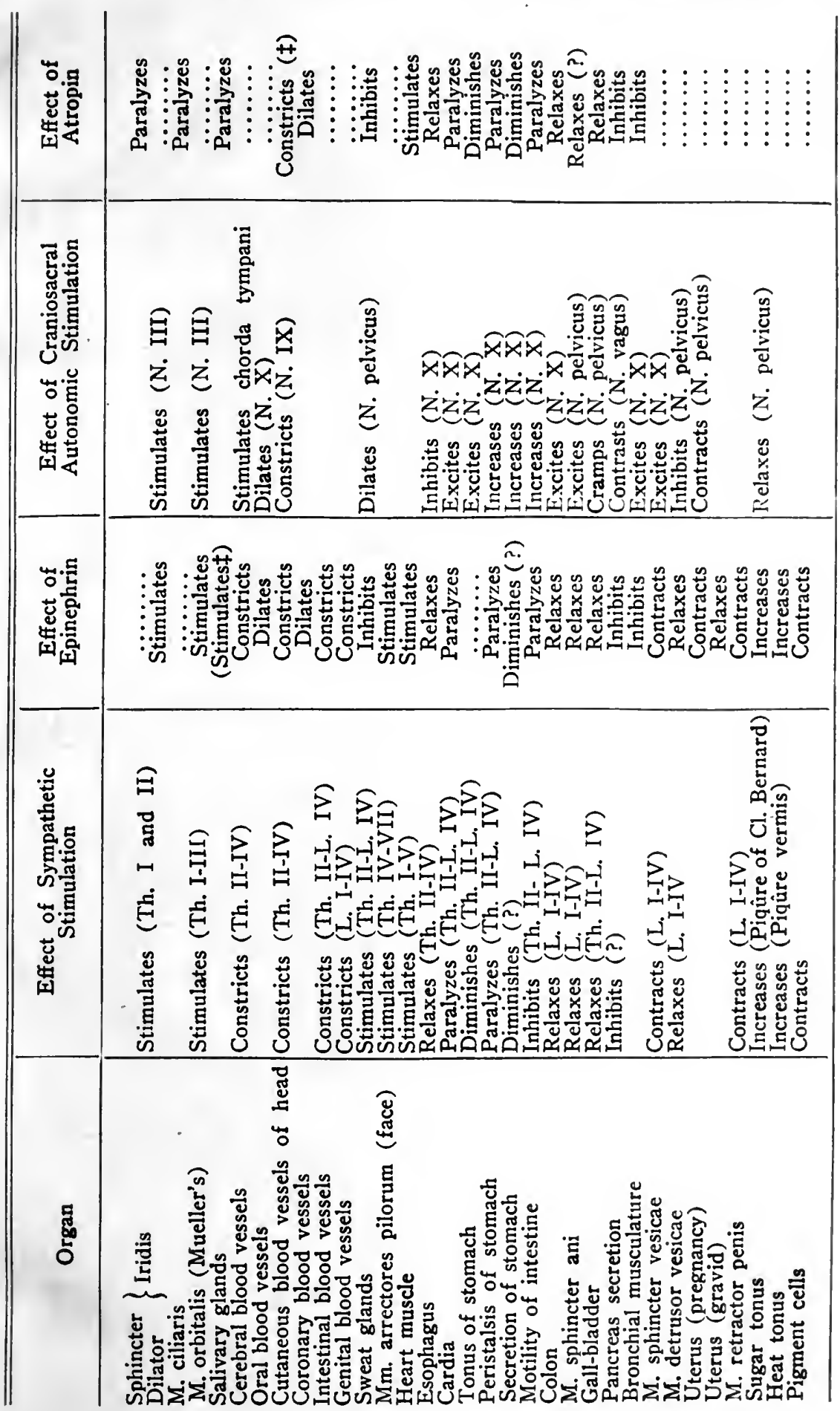


administration of atropin, the pupils dilate and the heart rate increases, while bronchial and intestinal spasms may be relieved. On the other hand, the sympathetic terminals are stimulated by large doses of epinephrin. The blood pressure is raised, sugar is discharged from the liver, and under proper experimental conditions the pupils dilate, the heart rate is accelerated and the intestines are relaxed.

There is then abundant physiological evidence for the belief that the functions of the internal organs may be influenced through nervous channels. Yet these functions are by no means so dependent upon nervous control as are the activities of the voluntary muscles. While the activities of the latter are governed almost exclusively by nerve impulses, the internal functions are to a certain extent independent of extrinsic nerve control. We know, for example, that the heart continues to beat when all of its extrinsic nerves are severed, that the arterioles will in time regain tone after being isolated from their extrinsic nerve supply, and that analogous relations hold with regard to most of the other internal organs. It seems evident therefore that nervous influences, while they direct and modify the activities of the internal organs, are not absolutely essential for these activities.

This view, that the internal functions are influenced by but are not entirely dependent upon nervous influences, is borne out furthermore by clinical evidence. Nervous disturbances of the internal functions are seldom fatal. A number of conditions that threaten life and that were formerly thought to be of nervous origin, have now received a different interpretation. Thus the cardiac death which may follow diphtheria is no longer attributed to a vagus paralysis but to myocardial disease; the Stokes-Adams syndrome is usually associated with demonstrable structural alterations in the heart muscle; and a number of serious conditions without evident structural cause, such as the pernicious vomiting of pregnancy, are now believed to be caused by toxic substances rather than by nervous reflexes. In general the prognosis of internal disorders that result from abnormal nervous impulses is a good one, in so far as life is concerned.

Nevertheless, even though nervous impulses rarely cause internal disorders which threaten life, they frequently play an important rôle in producing and modifying the symptoms of both minor and major ailments, and occasionally they may be the determining cause of a serious train of symptoms. Faintness or syncope may follow emotions, the fall in blood pressure being produced by vagus inhibition of the heart or by relaxation of the arterioles. The fall of blood pressure in the form of shock which immediately follows a grave injury likewise appears to be of nervous origin, and it may threaten life. Excitement raises the 
blood pressure, and in susceptible individuals this may provoke a cerebral hemorrhage or an anginal seizure. It seems quite possible indeed that the frequent repetition of such psychic rises of arterial pressure plays a rôle in producing certain forms of chronic arterial hypertension. In animal experiments the thyroid gland can be stimulated to activity by sympathetic impulses, and, according to Cannon, a continuation of such impulses over a considerable period of time produces a condition analogous to Graves' disease. Clinical observations indicate that in man this disease may follow prolonged nervous strain or even a single violent emotional disturbance. Vagus stimulation causes a spasm of the bronchial muscles, and the spasm present in asthma may at times be due to reflexes through the tenth nerve or to the toxic stimulation of its terminals in the bronchi. Changes in cardiac rate and rhythm, notably sinus irregularity and partial heart block, frequently result from vagus impulses. Finally a variety of gastrointestinal disturbances, such as cardiospasm, pylorospasm and gastric hyperacidity, are at times due to psychic or reflex nervous causes.

What initiates the impulses in the vegetative nerves that lead to functional disorders in the internal organs? In the first place they may be initiated by changes in the medullary or other centers from which the nerves arise. The bradycardia associated with meningitis or increased intracranial pressure, for example, is due to stimulation of the vagus center in the medulla. Medullary injury may also cause a discharge of glycogen from the liver, a sudden change in blood pressure or an alteration in respiration such as that seen in meningitis (Biot's breathing).

In the second place nervous alterations in the visceral functions may be initiated by changes in the higher cerebral centers. The major emotions, fear and anger, are accompanied by alterations in the cardiac activity, by changes in the blood pressure, by hyperglycemia and by depression in the secretory and motor functions of the gastrointestinal tract. As a rule these physiological alterations disappear when the emotion subsides. Occasionally, however, they may, in susceptible individuals, provoke more serious consequences, such as anginal seizures, cerebral hemorrhages and hyperthyroidism. Continued emotional strain is believed by many to be a cause of chronic arterial hypertension and diabetes mellitus.

In the third place the innervation of the internal organs may be disturbed by reflexes from other parts of the body. Reflex vomiting may follow changes in the semicircular canals, as in seasickness, or it may result from abdominal disease. The initial stages of traumatic shock appear to be caused by reflexes from the site of injury. Gastric 
hyperacidity and pylorospasm are frequently associated with disease elsewhere in the abdominal cavity. After the removal of a chronically inflamed appendix or of some other diseased organ, the gastric symptoms may disappear.

Finally the functions of an internal organ may be disturbed by toxic substances which act upon the peripheral portion of its nerve supply. Many drugs affect the vegetative nerves in this manner, and it seems probable that similar effects will be disclosed when the various toxic processes that may develop within the body are better understood.

We have pointed out that certain drugs act in a selective manner upon one or other of the two main subdivisions of the vegetative nervous system. This selective action may be explained by assuming that all of the nerve structures affected possess some chemical peculiarity in common. For example, the fact that atropin paralyzes the craniosacral autonomic terminals in a variety of organs indicates that all the terminals affected contain certain chemical substances which are not present in other parts of the nervous system. Similarly the fact that epinephrin stimulates the sympathetic terminals suggests that these possess a common chemical structure which is not present elsewhere. This specific susceptibility to certain drugs indicates therefore that each subdivision of the vegetative nervous system is in a sense a distinct functional unit.

From this it follows that one might expect to encounter pathological processes in which one or other subdivision of the vegetative nervous system was specifically affected. Eppinger and his associates have indeed described two major symptom complexes of this type. One of these is characterized by an overactivity of the craniosacral autonomic nervous system and the other by an overactivity of the sympathetic nervous system. The first has been called vagotonia, the second sympathicotonia. An overactivity of the craniosacral autonomic system might be expected to manifest itself by contracted pupils, bronchial spasm (asthma),, bradycardia, sinus irregularity of the heart, partial heart block, gastric hyperacidity, pylorospasm, and intestinal hyperperistalsis and spasm. Overactivity of the sympathetic nervous system, on the other hand, might be expected to cause dilated pupils, protruding eyeballs, thyroid and adrenal hypersecretion, rapid heart, increased blood pressure, diminished tone and lessened peristalsis in the gastrointestinal tract and hyperglycemia with a tendency to alimentary glycosuria.

While the conception of such vegetative systemic neuroses is logical and attractive, little success has in fact attended the effort to establish disease complexes of this type. Widely distributed nervous alterations 
of function are rarely of such a character that they can be attributed to a single type of change in either the craniosacral autonomic or the sympathetic nervous systems. The symptoms of exophthalmic goitre, for example, are characterized by hyperactivity in many branches of the sympathetic system (exophthalmos, tachycardia, alimentary glycosuria), but the relaxed peripheral vessels and the tendency to diarrhoea do not conform to this type of system disease. The same is true of most neuroses which involve a number of organs. As a rule both subdivisions of the vegetative nervous system appear to be involved or a single subdivision may appear to be overactive at one place and underactive at another. Pure examples of widely distributed system disease of the vegetative nerves are exceedingly rare. To describe the others as mixed types of vegetative systemic disease is merely an acknowledgment that they do not conform to the fundamental conception of a disease which affects in a given manner numerous subdivisions of either the sympathetic or the craniosacral autonomic systems.

\section{THE CHEMICAL CONTROL OF FUNCTIONS}

In addition to the control of body functions which is exercised through the nerves there is a further control which is exercised through the blood. By means of the blood there is normally maintained about the cells a nearly uniform chemical reaction and osmotic pressure together with a nearly uniform concentration of various substances, such as oxygen, carbon dioxide and sugar. These blood constants are of great importance for all tissues, but they hardly have a directive influence upon specific functions comparable to that exercised by the nerves. Such an influence is, however, exercised by the substances that have come to be known as hormones and internal secretions.

The term hormone, or chemical messenger, was used by Starling in discussing chemical substances which are elaborated by one organ and which act in a specific manner upon the functions of another. When the mucous membrane of the duodenum or upper jejunum is treated with weak acid or with soap, and the filtrate thus obtained is injected into the blood stream, a more rapid flow of pancreatic juice occurs. The active substance thus obtained from the intestinal mucous membrane is called secretion. An analogous hormone, which stimulates the flow of gastric juice, has been obtained by treating the pyloric portion of the stomach with meat extractives and other substances. Of pathological changes which may result from alterations in these particular hormones we know nothing at the present time.

The substances known as internal secretions enter the blood from 


\section{I24 PATHOLOGICAL PHYSIOLOGY AND MEDICINE}

glandular organs and have a specific but widely distributed action upon the body tissues. Internal secretions are formed not only by the ductless glands, such as the thyroid, the parathyroids, the pituitary and the adrenals, but they are also formed by some glands which likewise elaborate an external secretion. Among glands with such a double function are the pancreas and the testes.

Internal secretions may be demonstrated by two methods. In the first place, the active substance may be found in the blood which leaves the gland. For example, the concentration of epinephrin in the blood leaving the adrenal has been shown, under certain experimental conditions, to be greater than its concentration in the blood elsewhere in the body. In the second place, excision of the gland may be followed by certain symptoms which can be relieved or prevented if the gland is successfully transplanted to some other part of the body or if the gland substance is taken by mouth. By this method it has been shown that the pancreas, the thyroid, the parathyroids, the pituitary, the adrenals and the sex glands furnish to the body certain substances which are essential to its normal development or well-being.

The pathological changes which follow partial or complete excision of these glands from animals are similar to the clinical conditions in man which result from improper development, extensive disease, or operative removal of the corresponding glands. In contrast with these clinical conditions which depend upon the diminution or absence of an internal secretion (hypofunction), we encounter certain well-defined clinical pictures which appear to be due to the overactivity of one or other of the glands producing an internal secretion (hyperfunction). In the latter, operative removal of a portion of the gland may result in an amelioration or cure of the symptoms. The operative successes in exophthalmic goitre and in acromegaly, for example, indicate that these diseases are caused by an excessive or an abnormal activity of the thyroid or pituitary glands respectively. Finally it is possible that qualitative changes in the internal secretions may occur and that such changes may produce pathological alterations in the body. Although the occurrence of qualitative alterations in the internal secretions has not been demonstrated beyond question, it seems probable that they do occur, and that such dysfunctions may explain certain clinical pictures which evidently depend upon alterations in the internal secretions but which are not clearly the result of either hyperfunction or hypofunction.

The various symptom complexes which characterize disturbances in the individual internal secretions will be discussed under the special pathology of the organs in question. It may be pointed out here, however, that the internal secretions influence particularly the nervous 
system, the metabolism and the body growth and development. Furthermore they exercise a considerable influence upon one another.

(a) Nervous effects. Hyperthyroidism is characterized by mental restlessness, by nervous instability, and by a group of symptoms which are due largely to overactivity in the sympathetic nervous system. In myxedema an opposite group of symptoms occurs. Extirpation of all parathyroid tissue leads to the nervous hyperirritability characteristic of tetany. Epinephrin, which is an active principle of the adrenal gland, possesses a special affinity for the sympathetic nerve terminals. In large doses it stimulates these terminals, while in small doses it may exert a contrary effect. Castration of the male alters his psychic characteristics. The eunuch is reputed to be less ambitious, more indolent, more treacherous and more cowardly than the normal male. Finally the menstrual function in women is frequently accompanied by emotional disturbances and by psychic alterations, while the natural or artificial menopause is accompanied by a variety of nervous phenomena.

(b) Metabolism. In exophthalmic goitre the total metabolism is increased; in myxedema it is diminished. In the former disease there is a tendency to alimentary glycosuria, while in the latter the alimentary tolerance for sugar is said to be increased. The internal secretion of the pancreas is essential for sugar combustion in the body, and it likewise influences the glycogenic function of the liver. Extirpation of the parathyroid glands appears to diminish the calcium content of the blood and nervous system. Finally obesity is common in diseases of the endocrine glands. It frequently occurs in certain types of pituitary disease, in eunuchs, in women after the natural or artificial menopause, and in certain cases where the thyroid secretion appears to be deficient.

(c) Body growth and development. One of the most remarkable effects of the internal secretions is that exerted upon the growth and development of the body. This effect is seen particularly in diseases of the pituitary gland. When the anterior lobe of this gland is overactive during the period of growth it leads to excessive bodily growth, gigantism; when overactivity occurs during later life, it causes the enlargement of the facial structures, the feet and the hands which characterizes acromegaly. An underactivity of the same portion of this gland appears to be responsible for the small stature present in Fröllich's syndrome and related conditions. The eunuch is tall beyond the average owing to the length of his lower extremities. The cretin shows a stunted growth. Adrenal tumors in childhood have been accompanied by a prematurely rapid growth. Finally excision of the thymus gland from animals is said by some to retard growth. 


\section{I26 PATHOLOGICAL PHYSIOLOGY AND MEDICINE}

(d) Interrelationship of the internal secretions. Numerous and complex relationships have been noted between the functions and structures of the various endocrine glands. The normal sexual functions are largely dependent upon a normal condition of the other internal secretions. Lack of sexual development and absence of the secondary sexual characteristics occur in cretinism and in certain pituitary diseases, such as Fröhlich's syndrome. Menstrual disturbances may accompany thyroid disease in women. Sexual precocity has been noted in connection with pituitary disease, pineal tumors and adrenal tumors. The pituitary gland enlarges after thyroidectomy and during pregnancy. The thyroid may swell during menstruation and during pregnancy. The adrenals enlarge during experimental hyperthyroidism, and many symptoms of exophthalmic goitre indicate a stimulation of the sympathetic nervous system, such as might result from the excessive liberation of epinephrin from the adrenal glands.

Such complex interrelationships, of which we have named only the best established, often make it difficult to determine which gland is responsible for certain symptoms in diseases of the endocrine glands. For example, the changes in hairiness which frequently accompany pituitary disease may be a direct result of the altered pituitary function, or they may be an indirect result of this alteration owing to the secondary changes in the sexual functions.

\section{AUTOINTOXICATIONS}

Pathological states may result from an intoxication by various substances which are formed in the body. Aside from disorders of the internal secretions, we may name as causes of such autointoxications (I) a failure to eliminate normal waste products, (2) an incomplete or improper combustion of certain compounds within the body, and (3) the formation and retention of toxic bodies whose nature and origin are still obscure.

(I) Retention of waste products. Since the kidneys are the chief excretory organs of the body, it is particularly in renal disease that one might expect to find pathological changes which are due to the accumulation of normal waste products. Complete anuria, whether caused by excision of the renal tissue, by blocking of both ureters or by extensive kidney disease, leads to a characteristic train of symptoms. After a few days the patient loses his appetite and becomes apathetic and irritable. The blood pressure rises moderately. Drowsiness and muscular twitchings gradually develop and death supervenes after a period of from seven to fourteen days. Since the above symptoms follow excision of all renal tissue and are therefore not the result of poisons absorbed 
from altered kidney substance, it follows that they presumably result from an accumulation in the body of compounds that are normally excreted in the urine. Examinations of the blood during anuria have shown that urea and other non-protein nitrogenous substances do in fact accumulate gradually in the blood and that their concentration reaches a high level before death ensues. Notwithstanding this, we are still uncertain concerning the precise cause of the symptoms in prolonged anuria. Animals may be killed by the intravenous injection of urea, but the blood concentration necessary to produce this result is rarely reached during anuria; and so far as we know similar relations also hold with respect to the other non-protein nitrogenous compounds whose concentration in the blood during fatal anuria has been determined. Death as a result of anuria must therefore be attributed either to a prolonged intoxication by the simpler urinary substances, or else to the retention or new formation of other more toxic substances of whose exact nature we know nothing at the present time.

In chronic nephritis with extensive destruction of renal tissue, the blood concentration of urea and of other non-protein nitrogenous compounds not infrequently rises far above the normal, and it is known that when this rise becomes marked there is apt to supervene a group of symptoms which are in many ways analogous to those that occur during anuria. Muscular asthenia, mental apathy, drowsiness, headache and muscular twitchings characterize this intoxication, which has been called the asthenic type of uremia. The ingestion of large quantities of urea by mouth causes marked prostration in normal individuals, and it is possible therefore that the accumulation of urea in the body contributes to the symptomatology of anuria and of the asthenic type of uremia. Yet no very exact relationship exists between the severity of uremic symptoms and the concentration of urea or of other substances that have been determined in the blood, and patients usually die long before the blood concentration of urea has attained the level that causes death when this compound is injected into animals. In asthenic uremia, as in anuria, therefore, the fatal outcome appears to depend either upon a prolonged intoxication with the simpler urinary constituents or upon the formation and retention of some more toxic substances whose character is not yet known.

The form of uremia that is characterized by general convulsions is much less common than the asthenic form, and it is usually not accompanied by any significant increase in the concentration of urea or of the total non-protein nitrogen in the blood. In this form of uremia, particularly, the symptoms appear to be caused by small quantities of some very toxic substance. Foster has indeed isolated from the blood of 


\section{28}

such patients a compound which is highly poisonous for laboratory animals.

The concentration of uric acid salts in the blood is usually increased during serious renal diseases, and this increase is said to precede the rise in the concentration of urea and of other non-protein nitrogenous wastes. The concentration of uric acid salts in the blood is also greater than normal in most cases of chronic gout, but in the latter disease there is little if any rise in the concentration of urea or other wastes. This high concentration of uric acid compounds in the blood of gouty patients is not accompanied by an increased elimination in the urine, nor is there any proof that the gouty individual forms more uric acid or destroys less than the normal. For these reasons it seems probable that in gout the accumulation of uric acid salts in the blood is due to insufficient elimination of these salts by way of the kidneys. It is by no means certain, however, that this retention of uric acid is the sole cause of the deposits of urates in the cartilages and other tissues which characterize the anatomical picture of gout. On the one hand, some patients suffering from this disease have shown no increased con- centration of uric acid compounds in the blood. On the other hand, we know that the concentration may be equally high in chronic nephritis and in leukemia without the occurrence of the characteristic urate deposits. It would appear therefore that the pathological disturbance in gout is not due solely to a retention of uric acid compounds in the body, but that there is in addition some change in the tissues or fluids which favors their deposition in susceptible tissues.

Bile escapes into the blood and lymph streams whenever there is an obstruction of the common bile duct, of the hepatic duct, or of numerous small ducts in the liver itself. Among the symptoms of obstructive jaundice, the cutaneous itching and slowing of the heart rate appear to be due to an autointoxication from retention of the bile salts or the bile pigments. The coagulation of the blood may be somewhat delayed from a similar cause. Certain other symptoms which frequently accompany jaundice, such as slight fever, malaise, a marked hemorrhagic tendency and the serious group of symptoms known as cholemia, appear to be caused not by an autointoxication with retained biliary constituents but by a coincident infection or by metabolic changes which follow diffuse damage to the parenchyma cells of the liver.

The possibility of an autointoxication from the absorption of injurious compounds in the large intestines has given rise to much discussion. Is such an autointoxication responsible for the symptoms of chronic constipation? On this question opinions differ. Some have maintained that chronic constipation causes an autointoxication which 
may lead to a variety of functional disturbances and of anatomical changes. Others believe that autointoxication plays little if any rôle in producing the symptoms of which patients with chronic constipation complain. At the present time this question is not definitely settled. It is true that a sense of well-being usually follows a fecal evacuation. This occurs so promptly, however, that it cannot possibly be attributed to the reduction of an autointoxication. It seems to result rather from the motor phenomena associated with defecation, together with the subsequent lessening of tension in the rectum and lower colon. The influence of mechanical changes in the lower bowel upon the psychic centers may be compared with the effect of certain gastric contractions that cause the sensation of hunger.

Chronic constipation produces widely varying symptoms in different individuals. Some persons, who habitually defecate at intervals of several days, experience no unpleasant symptoms; whereas others never feel comfortable unless at least one "satisfactory" movement occurs each day. Many appear to be obsessed with the fear of poisoning from an overloaded colon. We may therefore safely attribute some of the manifestations of chronic constipation to mechanical changes in the lower bowel and some to the mental attitude of the patient toward his condition.

Nevertheless the prevalent opinion that autointoxication plays a considerable part in producing the symptoms of chronic constipation cannot be lightly dismissed. Headache, lassitude, easily induced fatigue and nervous irritability are symptoms that in themselves suggest a toxic origin, and they may be relieved when chronic constipation is corrected. Concerning the substances which may produce such symptoms in chronic constipation we have no definite information. Indol and skatol have been incriminated at times, but they appear to be relatively innocuous and the symptoms in constipation do not parallel the elimination of derivatives of these substances in the urine. More recently it has been shown that highly toxic amins can be derived from the aminoacids which are formed during the intestinal digestion of proteins. The suggestion that these toxic amins may at times be formed in and absorbed from the intestines, and that they may be responsible for the symptoms of chronic constipation or of other intestinal disorders is attractive; but as yet we know nothing of the occurrence of these toxic amins in clinical conditions.

(2) Autointoxication from improper combustion. In the animal body the proteins, fats and carbohydrates are catabolized into relatively simple end-products, mainly carbon dioxide, water and urea. During this process various intermediary products are formed. Autointoxica- 


\section{I30 PATHOLOGICAL PHYSIOLOGY AND MEDICINE}

tion may result either when the catabolism of certain substances is arrested in an intermediary stage or when catabolism pursues an abnormal course and thus gives rise to abnormal intermediary products.

Proteins are broken down by a series of hydrolytic cleavages into their constituent aminoacids and a number of interesting anomalies with regard to the final combustion of these aminoacids are presented by those individuals who show cystinuria (failure to metabolize cystin), diaminuria (failure to metabolize lysin and ornithin normally) and alcaptonuria (failure to metabolize phenylalanin and tyrosin normally). These alterations in the intermediary metabolism rarely lead to serious symptoms, but they illustrate how certain aminoacids may fail to burn to their normal end-products, and they suggest the possibility that other disturbances of this type might lead to the formation of more toxic bodies which would injure the health of the individual.

Of the autointoxications that are produced by improper combustion the best understood is that which leads to diabetic coma. This condition is due to the accumulation in the body of the so-called acetone bodies, particularly diacetic acid and beta-oxybutyric acid. These substances are derived in the main from an incomplete combustion of fat in the animal body. The fatty acids derived from fats contain a long chain of carbon atoms whose number is a multiple of two. These fatty acids are normally burned by oxidation in the so-called beta position and by a subsequent splitting off of two carbon atoms at a time. In this way a succession of simpler acids is formed, each of which contains two less carbon atoms than its predecessor. Inasmuch as the original fatty acid contained an even number of carbon atoms the number of these is eventually reduced to four. In this way butyric acid is formed during the combustion of fat in the animal body. Oxidation of butyric acid takes place as usual in the beta position (betaoxybutyric acid). In diabetic acidosis the normal combustion is arrested at or near this point, and beta-oxybutyric acid with its oxidation product, diacetic acid, tends to accumulate in the body. The autointoxication produced by the accumulation of these substances is due mainly to their acid character, and it does not differ essentially from the intoxication produced when inorganic acids are administered to certain animals. It is possible, however, that diabetic coma is due in part to the character of the acids that are present in the body and that even the neutral salts of beta-oxybutyric acid are in a measure toxic.

Of other intoxications produced by the accumulation of well-defined normal or abnormal intermediary products of metabolism we know but little. It is certain that lactic acid may be increased in the blood and urine in conditions of imperfect oxidation (violent exercise, asphyxia, 
carbon monoxide poisoning), and in such conditions it may produce the increased respirations characteristic of acid accumulations in the body. The increase of sugar in the blood and tissues will account for certain manifestations of diabetes. Finally aminoacids may be increased in the blood and urine in diffuse hepatic disease; but we know nothing of their relation to the toxic symptoms which are at times observed in this condition.

(3) Autointoxications from complex unidentified substances. The autointoxications thus far described are caused mainly by the accumulation in the body of relatively simple chemical substances which are known to be formed during metabolism and which are either endproducts of combustion or are normal or slightly abnormal intermediary products. A number of other intoxications arise from the formation of more complex substances of whose exact nature we have no definite knowledge at the present time. We have already seen that the symptoms of asthenic uremia may be due in part to other substances than the common urinary wastes, while the symptoms of convulsive uremia are probably due to complex and highly toxic compounds.

By cholemia is meant a group of toxic symptoms which occasionally terminate diffuse diseases of the liver, such as acute yellow atrophy or advanced hepatic cirrhosis. Prominent among the symptoms of cholemia are those referable to the nervous system, viz., muscular twitchings, irritability, somnolence and coma. That these symptoms are not due to the presence of bile in the blood or tissues is definitely established, for complete obstruction of the common bile duct with intense jaundice may exist in man for months without causing any serious toxic symptoms. The types of hepatic disease that are prone to terminate in cholemia are often accompanied by metabolic changes which depend upon an altered function of the hepatic cells. Among these is an excess of aminoacids in the blood and urine. Yet there is no reason for assuming that aminoacids cause the intoxication in cholemia, and this condition is therefore attributed to some more complex substance or substances which result from damage to the hepatic cells.

Acute hemorrhagic pancreatitis is a rapidly fatal disease. The symptoms are evidently the result of an autointoxication, for the disease may be produced experimentally by aseptic injuries to the pancreas, as by injecting bile into the pancreatic duct. The cause of this intoxication is not known. It seems probable that it is not due to an escape of pancreatic ferments into the body. On the other hand, the pancreas after removal from the body will become highly toxic for animals, and the symptoms of acute hemorrhagic pancreatitis probably result from a disintegration of the pancreatic tissue in situ. 


\section{I32 PATHOLOGICAL PHYSIOLOGY AND MEDICINE}

Intestinal obstructions involving the duodenum and the upper jejunum are serious and often rapidly fatal. In spite of much experimental study the exact cause of death in such conditions is not settled, but evidence has been brought forward to show that the symptoms are due in part to the absorption of highly toxic substances from the damaged mucous membrane of the affected bowel.

Finally toxic symptoms may follow the injection into the blood stream of a variety of substances which are ordinarily regarded as nontoxic. Among these are agar solutions, colloidal silver solutions, solutions of proteins, such as milk and even the animal's own blood after it has been withdrawn and allowed to stand for a few minutes in a syringe. Following such injections, there may occur a sharp febrile reaction or the symptoms of anaphylactic shock. These effects, which are much the same no matter what material has been injected into the veins, probably result from change in the animal's own blood, and they are therefore only in a sense due to an autointoxication.

The exact poisons which cause the various intoxications just enumerated are not known. They seem, however, to be derived in some way from the proteins of the affected tissues. Several of these conditions have been attributed to the formation of proteoses or similar compounds. Since the number of proteoses is almost endless, such a designation merely indicates the general nature of the supposed poison and does not identify it. In discussing intestinal autointoxication it was pointed out that highly toxic amins may be derived in vitro from the aminoacid building stones of the protein molecules. It is possible that similar toxic amins might be derived from proteins in the body, although there is no evidence to support such a conjecture.

\section{THE BODY CONSTANTS IN DISEASE}

A noteworthy characteristic of all higher animals is their ability to surround the body cells with relatively constant conditions, which are influenced but little by fluctuations in the outside world, by variations in the amount and kind of food taken, and by changes in the tissue activities. Among these relatively constant conditions surrounding the body cells may be mentioned the temperature, the chemical reaction, the molecular pressure and the concentration of certain substances in the blood, notably glucose, sodium chloride and hemoglobin. The adjustments which permit the tissues to work under conditions that ensure proper warmth, food supply and chemical reaction seem favorable for the development of the more complex physiological functions. At least we know 
that, generally speaking, the body constants are more numerous and are maintained with greater precision in the higher animals. The body temperature in lower animals, for example, varies according to the external temperature, whereas in birds and animals it is relatively independent of this temperature. In man, furthermore, it is more nearly constant than it is in most other mammals.

Various factors both in health and in disease tend to alter the body constants, but such alterations are prevented or lessened through definite physiological adjustments. Two types of pathological disturbance in the maintenance of the body constants are therefore possible. In the first an unusual strain is thrown upon the regulatory mechanism, in the second this mechanism itself is altered. When, for example, an excessive amount of acid is introduced into the body, the compensatory mechanism usually acts so perfectly that the reaction of the body fluids is hardly altered. The main demonstrable effect is an alteration in the various compensatory factors which serve to maintain the normal chemical reaction of the body fluids. Similarly when the body is exposed to heat or to cold, there occurs a striking change in the mechanism which controls heat losses, long before there is any appreciable alteration in the body temperature. In fever, on the other hand, the temperature rises, not because there is any extraordinary production of heat, or any unusual change in the ability to dissipate heat, but because the mechanism that normally regulates the body temperature has been deranged.

\section{THE CHEMICAL REACTION OF THE BODY}

One of the most important constants in the body is the chenical reaction of its fluids. This reaction is very slightly alkaline, so slightly alkaline indeed that it may be considered almost neutral. This slight alkalinity is maintained despite the constant production of acid substances in the body. Carbonic acid is formed from the combustion of proteins, fats and carbohydrates, sulphuric acid is formed from the combustion of proteins, and phosphoric acid is formed from the nucleoproteins and lecithins. Furthermore, variations in the amount and composition of the food cause variations in the acid or alkaline character of the end-products of metabolism. Despite the constant formation of acid substances and despite variations in the food taken, the neutrality of the body fluids remains practically unaltered. The physiological mechanism which maintains the reaction in the normal individual is the same as that which preserves the patient from a dangerous change of reaction when excessive quantities of acid are formed in the body as a result 


\section{I34 PATHOLOGICAL PHYSIOLOGY AND MEDICINE}

of disease. Among factors that maintain body neutrality under normal and abnormal conditions are the following:

(I) The buffer action of the blood salts. Sudden and marked fluctuations in the reaction of the body fluids are prevented by the presence of certain salts, especially the carbonates, and to a less extent the phosphates. If a few drops of any strong acid are added to distilled water, they produce a marked change in reaction, because strong acids are readily dissociated and yield a large number of the free hydrogen ions which cause acidity. If, on the other hand, a few drops of this same acid are added to a solution containing carbonates or phosphates, the resulting change in reaction is extremely slight, owing to the so-called buffer effect of these salts. The changes which occur under these conditions may be represented by the following formulae:

$$
\begin{aligned}
& \mathrm{HCl}+\mathrm{NaHCO}_{3}=\mathrm{NaCl}+\mathrm{H}_{2} \mathrm{CO}_{3} \\
& \mathrm{HCl}+\mathrm{Na}_{2} \mathrm{HPO}_{4}=\mathrm{NaCl}+\mathrm{NaH}_{2} \mathrm{PO}_{4}
\end{aligned}
$$

It is apparent from these formulae that when hydrochloric acid is added to a solution containing the slightly alkaline carbonates and phosphates, the strong acid is converted into a neutral salt and the weak' carbonic acid or the weakly acid salt, monosodium phosphate, is set free. These yield but few hydrogen ions by dissociation, and consequently they cause a very slight change in the chemical reaction of the solution. This instructive experiment illustrates how the carbonates of the blood act as a buffer, which prevents any marked change in the blood reaction, even when excessive quantities of acid substances are formed in the body. A similar buffer effect is exerted by other blood substances, among which are the proteins.

(2) The elimination of acid substances. Excesses of acid in the body may be eliminated or neutralized. Elimination takes place either through the lungs or through the kidneys.

The respiratory center is exceedingly sensitive to slight alterations in the hydrogen ion concentration of the blood. It is stimulated to increased activity by a reduction of alkalinity that is so slight as to be difficult to demonstrate by physico-chemical methods. As a result of this stimulation, larger volumes of air are breathed, the pulmonary alveoli are better ventilated, the percentage of carbon dioxide in the alveoli is reduced, and this finally leads to a reduction of carbonic acid in the blood and other fluids. Thus a very slight change toward acidity in the reaction of the blood leads to an increased elimination of carbonic acid, and the loss of this acid tends to restore the normal reaction, whenever an excess of acid has reduced the carbonate reserve in the blood. 
When incombustible and non-volatile acids are present in the body, their salts are eliminated mainly through the kidneys. The kidneys likewise excrete an acid urine and this acidity tends to increase when there is an increased formation of acids in the body. We do not know just how the kidney excretes acid, but it is probably excreted largely as monosodium phosphate, the salt which contributes most to the acidity of the urine. However this may be, the excretion of a more acid urine is one of the means by which an excess of acid is eliminated from the body.

(3) Neutralization of excess acid. An excess of acid in the body is usually partly neutralized by an increased production of alkaline substances. The additional alkali results from a greater fixation than usual of the ammonia that is normally formed during the combustion of proteins and that is normally converted almost entirely into urea. The excess of acid tends to fix this ammonia and to be neutralized by it. It is indeed a general rule that any marked increase in ammonium salts in the urine indicates an increased formation of acid substances in the body. In diabetic acidosis, the quantity of ammonia in the urine furnishes an approximate measure of the degree of acidosis, provided alkalies, such as sodium bicarbonate, have not been administered by mouth.

\section{ACIDOSIS}

These various mechanisms which serve to regulate the chemical reaction of the body fluids are so perfectly adjusted that this reaction hardly varies. Even in the condition known as acidosis no great change necessarily occurs in the normal reaction of the body fluids. Indeed it appears that an actual acidity throughout the body is incompatible with life.

Acidosis is due to the formation or retention in the body of an excess of non-volatile acid substances. Through the chemical and physiological reactions just described, the blood carbonates are in part decomposed and their quantity lessened, the respiratory center is stimulated with a resultant augmentation of breathing, carbon dioxide is pumped

- out of the lungs and out of the blood, some of the ammonia which would otherwise be converted into urea is fixed by the excess of acid, and finally there is usually an increase in the total amount of acid excreted in the urine. Through these adjustments the chemical reaction of the body fluids remains almost constant, despite the excess of acid formed or retained in the body. Acidosis is therefore characterized not so much by a marked reduction in the normal alkalinity of the body fluids as by those changes which serve to neutralize and to remove the 


\section{I36 PATHOLOGICAL PHYSIOLOGY AND MEDICINE}

acid excess, and in this way to maintain the normal chemical reaction. The tests for acidosis, ordinarily employed, seek to demonstrate these compensatory adjustments, particularly the lowered blood carbonates, the lowered carbon dioxide tension in the alveolar air and the increased excretion of ammonium salts in the urine. In some instances, as in diabetic coma, the acids responsible for the condition can be found in the urine and blood. In other instances they are not known.

One interesting and important deviation from the normal in acidosis seems to depend upon the reduction of carbonates in the blood. When normal individuals take from five to ten grams of sodium bicarbonate by mouth, the urine becomes alkaline. Patients with acidosis, on the contrary, may take many times this amount before the urinary reaction changes. Apparently the reaction of the urine is not changed until the carbonate deficit in the blood has been replenished. The quantity of sodium bicarbonate that must be administered in order to change the reaction of the urine is sometimes described as the tolerance to sodium bicarbonate or to alkali. It is a simple and satisfactory method for measuring acidosis.

It has been pointed out that two types of disturbance in maintaining the body constants may occur. Either an unusual strain may be thrown upon the regulatory mechanism or that mechanism may itself be altered. With respect to acidosis, an unusual strain is thrown upon the regulatory mechanism whenever unusual quantities of acid are formed in or are introduced into the body. The effect of introducing a large quantity of incombustible acid, particularly hydrochloric acid, has been studied experimentally on animals. No similar condition occurs naturally in man because large quantities of acid or large quantities of food which yield an acid residue are not ordinarily taken by mouth.

Acidosis from the formation of an excess of acid within the body is the classical type of this disorder. The best known and best studied example of this type is that which complicates diabetes. This is due to the formation of large quantities of beta-oxybutyric and diacetic acids within the body.

The acid-base equilibrium of the body may also be disturbed by alterations in the physiological mechanism which controls this equi- librium. We have seen that the chief factors here concerned are the blood carbonates, the elimination of carbon dioxide by the lungs, the excretion of an acid urine and the fixation of ammonia derived from protein catabolism. So far as we know, depletion of blood carbonates occurs only as a secondary manifestation of acidosis.

The elimination of carbon dioxide through the lungs depends in part upon the sensitiveness of the respiratory center to the reaction of the 
blood and in part upon the pulmonary mechanism for eliminating this gas. The latter includes the ventilation of the pulmonary alveoli, the passage of carbon dioxide through the alveolar walls and the rate of blood flow. If the respiratory center were abnormally sensitive to hydrogen ions in the blood, the pulmonary ventilation would be increased, carbon dioxide given off and the blood made slightly more alkaline. If the sensitiveness of the center were lessened, the opposite effects would be produced. We know that such changes in the irritability of the respiratory center may be produced by drugs. Morphine, for example, lessens its irritability and caffeine is said to increase its irritability. Of analogous changes produced by disease we know little.

A deficient elimination of carbon dioxide may be caused by pulmonary or circulatory disease. The resulting acidosis differs from other types, in that the retained acid, being carbonic acid, does not lead to a reduction in the blood carbonates. This type of acidosis is partly responsible for the dyspnea associated with certain pulmonary and circulatory disorders.

Renal disease and particularly chronic nephritis with retention of nitrogenous wastes may be accompanied by acidosis. In such cases the blood carbonates are reduced, the alveolar tension of carbon dioxide is reduced and the tolerance for sodium bicarbonate is increased. The ammonia in the urine, however, is not increased. Howland and Marriott have shown that in this type of acidosis there is an increase in the blood phosphates which appears to be due to an inability on the part of the kidney to excrete the normal amount of phosphates in the urine. Since the excretion of acid by the kidney, probably in the form of acid sodium phosphate, is an important means of preserving the normal reaction of the body fluids, the retention of phosphates offers a possible explanation for the acidosis of advanced renal disease. A similar retention of phosphates has been noted in some acidoses complicating infantile diarrhoeas. The acidosis complicating advanced renal disease is peculiar in that the ammonium salts in the urine are not increased. By some this has been attributed to an inability on the part of the diseased kidney to excrete these salts; but since Addis and Barnett have shown that the administration of acid phosphates by mouth to normal individuals causes no increased excretion of ammonia, it seems equally probable that the absence of an increased excretion of ammonium salts in the acidosis of advanced nephritis is due to a failure on the part of acid phosphates to fix the ammonia derived from proteins. 


\section{THE REGULATION OF THE BODY TEMPERATURE}

One of the important body constants is the body temperature. Heat is continually being lost from the body through conduction, radiation and evaporation, and it is continually being liberated within the body by reason of the metabolic processes. In warm-blooded animals the relation between heat loss and heat production is so adjusted that the temperature is maintained at a constant or nearly constant level. Even during violent exercise, when the heat production is increased four or more times the normal, there is usually but a slight rise in temperature, and this disappears shortly after ceasing the exercise. Evidently a corresponding increase of heat losses, mainly from the warm and moist skin, has rid the body of the excess heat liberated. Similarly when the body is exposed to heat or to cold, its temperature usually alters but little. Marked changes in heat losses are prevented by changing the amount of perspiration and the blood flow at the body surface so as to meet the external conditions. During exposure to cold, furthermore, the body is protected, if need be, by the increased liberation of heat that accompanies heightened muscular tension and active shivering.

The adjustments which maintain a constant body temperature occasionally are subjected to such conditions that the normal temperature can be preserved no longer. On the one hand, prolonged exposure to cold may lead to a reduction of body temperature and eventually to freezing. On the other hand, prolonged exposure to moist heat or prolonged exercise when heat elimination is restricted may lead to a marked or dangerous rise of the body temperature.

The increased body temperature which accompanies most infections is not due to extraordinary alterations in either the production or the elimination of heat. During a continuous fever the heat production is moderately increased, but this is less than the increase in many cases of exophthalmic goitre and is far less than the increase during moderate exercise. Nor is there any striking inability on the part of the body to lose heat by conduction, radiation or evaporation, for when the febrile patient is exposed to heat or to cold he reacts in much the same manner as does the normal individual. The cause of the febrile temperature appears to be an abnormal adjustment between heat formation and heat loss. The nervous centers which normally control the body temperature appear to be so deranged, or one may say, so stimulated, that they regulate the body temperature at a new and abnormally high level. The adjustment at this new level usually is less perfect, however, than the normal adjustment, for in fever the body temperature is altered more readily than

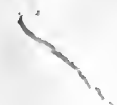


in health by such disturbing factors as exercise, cold baths and antipyretic drugs.

The chills and sweats which accompany sudden rises and falls of fever may be explained on the assumption that the heat regulating center finds itself out of harmony with the body temperature. The chill which follows prolonged exposure of a normal individual to slight cold may be attributed, in part at least, to the discrepancy between a normal center that has been stimulated and a normal body temperature. In either case there is thrown into action the mechanism which raises the body temperature to a higher level, viz., increased heat production by shivering and retention of heat by constriction of the peripheral vessels. In an analogous manner the sweat, in normal individuals following violent exercise, is due in part to the discrepancy between a normal heat center and a heightened body temperature, while the sweat in fever during a critical fall of temperature is due to the discrepancy between a heat center that is no longer stimulated and the elevated body temperature. In both cases the temperature falls because a more rapid blood flow at the body surface and a profuse sweat increase the heat losses. The febrile temperature and the rises or falls of this temperature may therefore be explained in large part by assuming that in fever there is an alteration in the central mechanism which normally controls the temperature of the body.

There is abundant evidence that when this regulatory mechanism is normal considerable changes in heat production may occur without affecting materially the body temperature. We have seen, for example, that during exercise the production of heat may be several times the normal; with only a slight rise of temperature. Among pathological conditions, the heat production is increased most markedly in exophthalmic goitre, in which disease it may exceed the normal by eighty per cent. or more. Yet the body temperature in this disease is raised but slightly, if at.all, because the excessive heat production is accompanied by a corresponding increase in heat elimination. The warm moist skin, so characteristic of severe cases of Graves' disease, serves to rid the body of the excess heat.

\section{THE MAINTENANCE OF BODY WEIGHT}

The body weight is not constant in the sense that the body temperature or the reaction of the body fluids is constant. Indeed, we are more apt to be struck with the differences of weight in different persons or with changes of weight in a given person, forgetting that for each individual there is a weight which may be regarded as normal for him at the 


\section{0}

\section{PATHOLOGICAL PHYSIOLOGY AND MEDICINE}

time and which he tends to maintain under varying circumstances. The breeder knows that certain strains of stock tend naturally to be fat, whereas other strains tend naturally to be thin. Similarly the physician knows that no small effort is required to alter the weight of his patients. The factors which tend to maintain the weight at a given level in health and to alter it in disease may therefore be discussed from the point of view that there is in each person a tendency to maintain a certain average weight. Variations may be due on the one hand to factors which overcome this tendency. On the other they may be due to changes in the tendency itself, an alteration in the regulatory mechanism.

Generally speaking, the body weight may be due solely to variations in its water content. In normal individuals changes in weight from this cause are usually transient and limited to a few pounds. During violent exercise, however, five to ten pounds may be lost, mainly from profuse sweating, and most of this loss is subsequently made good by drinking more liquid. In disease, striking and prolonged alterations in the weight may occur from a change in the water content of the body. Thus in Asiatic cholera the loss of fluid causes a considerable reduction of the body weight, while in edema many pounds may be gained. Considerable water may indeed be retained in the body without the appearance of edema, for it has been noted in nephritis that a gain of from ten to fifteen pounds in weight usually precedes any demonstrable pitting of the skin.

The present discussion will be restricted to those changes in body weight which depend upon gains or losses in the energy-containing constituents of the body. Energy is supplied to the body by the food ingested. The potential energy contained in the material absorbed from the gastrointestinal tract is liberated during metabolism and eventually leaves the body, mainly as heat. If the amount of energy available to the body in the food absorbed be greater than the amount lost from the body, the excess is stored in the tissues in the form of combustible material, as proteins, carbohydrates or fats. The capacity of the body to store proteins and carbohydrates is limited, however, so that any considerable storage of excess energy is accomplished by a deposit of fat. On the other hand, if the energy liberated during metabolism exceeds that which is available in the food absorbed, the deficiency is supplied by a combustion of energy-containing material stored in the body. In the long run this means a consumption of fat.

Even when considerable variations occur in the intake of food or in the rate of combustion in the body, the weight of a given individual usually varies only moderately. When, for example, a young man of sedentary occupation enters the army, his rate of combustion increases 
from approximately 2400 to approximately 3600 calories per day, on account of the increased muscular activities. Nevertheless his weight rarely falls to any considerable extent. Similarly when the change is made from an active to a sedentary occupation, the body weight may increase for a time, but it usually becomes stationary at a level only moderately higher than before. These facts are the more remarkable when we recall that even a slight discrepancy between the intake and loss of energy will, if continued, eventually produce a marked change in weight. For example, a daily intake of 200 calories (two glasses of milk) over and above the daily energy losses will in a year's time lead to a gain of over twenty pounds in weight. It is apparent that some mechanism in the body must ordinarily operate to prevent continued gains or losses in weight whenever a change in the habits of diet or exercise tend to alter the equilibrium between the intake and loss of energy-containing material.

Such an adjustment between the two factors may be accomplished (I) by an alteration in the rate of combustion to conform with the intake, (2) by an alteration in the food intake to meet the needs of the body, or (3) by a combination of both methods.

It is well known that there is a temporary increase in combustion following the intake of food. This effect is most marked after proteins, less marked after fats, and least of all after carbohydrates. In man the ingestion of 200 grams of dextrose or the ingestion of 60 grams of protein increases the fasting metabolism by about 12 per cent. (DuBois). A large meal given to a dog may double its fasting metabolism. According to Graham Lusk, this increased heat production following food is due to an increase in the cellular activity. In the case of carbohydrates and fats, the increased activity is attributed to the greater concentration of food about the cells, while in the case of proteins it appears to result from stimulation of the cells by certain products of protein catabolism.

While this transient increase of metabolism after a meal has been well established, there has been much more uncertainty concerning the effect of a continued excess or a continued deficiency of food upon the metabolic processes. In other words, do the cells adjust their activities in a more or less permanent manner to meet the condition of bodily nutrition? So far as a continued deficiency in the diet is concerned, we now know that this may indeed lead to a lessened metabolism. During complete starvation the rate of metabolism per unit of body weight is moderately reduced. Of more immediate interest is the fact that during a prolonged restriction of diet there occurs a very considerable reduction in the rate of metabolism. This has been observed during 


\section{I42 PATHOLOGICAL PHYSIOLOGY AND MEDICINE}

the rigid dietary restriction that is now practical in the treatment of severe types of diabetes. It has also been observed during the prolonged undernutrition of otherwise healthy persons, particularly among those peoples who, during the great war, have been subjected to partial starvation. Such persons not only lose weight, but their rate of metabolism per unit of weight is considerably reduced. It is evident therefore that in response to a continued restriction in the amount of energy taken in the food there may be a reduction in the metabolic activities of the tissues.

There are reasons also for believing that the rate of body metabolism may become accelerated in consequence of a very liberal intake of food. At least it has been observed during the fattening of cattle that in the later stages of this process the maintenance requirements increase disproportionately to the increment in weight (Armsby and Fries).

The adjustment between the intake and the consumption of energycontaining material is principally accomplished, however, by altering the intake of food to meet the metabolic requirements of the body. With a free choice of food, the intake is governed by hunger, by appetite and by the sense of repletion. These sensations vary with sudden changes in weight. If the diet is restricted for a few days, the lost weight is rapidly regained because more food is eaten. If, on the other hand, a series of attractive meals induces the individual to eat more than usual, the few pounds gained are usually lost when he returns to the original diet, because there is a disinclination to eat the customary amount.

In normal individuals the weight appears to be maintained primarily through this adjustment of the food intake to meet the body needs. The adjustment of the rate of metabolism to correspond with the food intake probably becomes an important factor only under unusual conditions.

Loss of weight. The body fat is reduced whenever the amount of energy contained in material absorbed from the intestines is insufficient to cover the energy requirements of the body.

The intake of energy may be lessened because of a poor appetite, as happens in fevers, nervous disorders and diseases of the abdominal viscera. The intake may also be lessened because, as in gastric ulcer, there is a fear of pain or distress after meals. Finally absorption of nutriment from the intestines may be deficient. This condition is far less common than is popularly supposed, yet it does occur in extensive intestinal disease, and also where no pancreatic juice enters the intestines. In all of these conditions losses of weight are common.

An overrapid combustion of body substance is the rule in exoph- 
thalmic goitre, where the rate of metabolism may exceed the normal by 50 to 80 per cent. Muscular activity, restlessness, or insomnia likewise increase the consumption of body material. These conditions frequently cause a loss of weight because the intake of food does not keep pace with the increased metabolism. If the appetite and digestion are good, however, the intake of food may be increased sufficiently to prevent any loss of weight.

In diabetes mellitus combustible material (glucose) is lost in the urine. Here again the effect upon the body weight depends largely upon the appetite. If this be good and if the disease be mild, the body weight is frequently well preserved. If the diabetes be severe, however, it becomes difficult to maintain a good state of nutrition.

The loss of weight during infectious diseases is due only in part to a more rapid consumption of body substance. It is true that the resting metabolism in fever is on the average about 30 per cent. greater than the normal resting metabolism. If, however, we compare this with the patient's metabolism under conditions of ordinary activity, we find no great discrepancy. Even a sedentary occupation increases the daily metabolism by about 20 per cent. above the metabolism in bed, while more laborious occupations increase it by 40 to 70 per cent. In the latter case it is obvious that the loss of weight during an infectiousi disease cannot be attributed to any increased combustion within the body, but is due to the loss of appetite that accompanies every infection. By forcing food the loss of weight may be prevented and typhoid patients may actually become fat during the fever.

Obesity. As we have explained, fat is deposited whenever the energy intake in the food exceeds the energy requirements of the body. Immoderate eating on the one hand and indolence on the other are recognized causes of obesity.

It must be confessed, however, that some individuals appear to gain weight despite a moderate intake of food and despite an average amount of exercise. One gets the impression that in such individuals the rate of combustion in the body is less than normal and that in this way food material is conserved and is converted into fat. Admittedly such individuals usually conserve their energy, in that they sleep well, are of an even disposition, do not worry and do not make unnecessary movements. Aside from this conservation by the avoidance of unnecessary muscular activity, however, there is no accepted proof that the rate of metabolism in the obese individual differs from the normal in any essential particular. The resting metabolism, the liberation of heat after eating and the liberation of heat after exercise have all been studied, and none has been proven to lie definitely outside normal limits. If the 
rate of metabolism in certain obese individuals be indeed less than the normal, the difference is certainly far less than the difference between persons who follow different occupations, such for example as the tailor . and the carpenter.

The difficulty which leads to obesity seems to lie not so much in a primary reduction of metabolism as in a failure to adapt the intake of food to the needs of the body. When a normal individual indulges himself at the table there follows a lessened appetite for subsequent meals. In the individual who is laying on fat, the normal control of the food intake appears to be disturbed in such a manner that he is constrained, day after day, to eat an amount of food which exceeds the body requirements. The excess need not be great. Even a discrepancy that is so slight as to escape detection by any available method of study will in time lead to a marked gain in weight. In most cases the person, having attained a certain degree of obesity, tends to maintain this level in much the same manner as the normal individual maintains his normal weight. Indeed, the tendency toward obesity in some instances appears comparable to the tendency after typhoid toward gaining flesh until the original level of weight has been reached.

Since we know little of the factors which govern the food intake and which adapt this to our body needs, the disturbance in this adaptation underlying obesity is likewise unknown. We may point out, however, that the tendency to obesity is frequently inherited, and that it is also common in certain disorders of the internal secretions as well as in metabolic disorders, such as mild diabetes and gout.

\section{THE CHEMICAL COMPOSITION OF THE BLOOD}

When one considers how the blood serves as a carrier within the body-receiving and distributing new supplies, redistributing material to various tissues, and carrying wastes to the excretory organs-one wonders that its chemical composition varies so little. The regulation of this composition is effected in part through an interchange with the tissues and in part through the activities of the excretory organs.

Concerning the conditions which regulate the interchange between the blood and tissues little is known. As an example of this interchange one may cite the maintenance of a nearly constant percentage of glucose in the circulating blood. Whether glucose be absorbed from the intestinal tract or whether it be introduced directly into the blood stream, the rise in blood concentration soon subsides. This subsidence is due in part to an increased combustion of sugar, in part it may be due to an excretion of sugar by the kidney, but in the main it is due to a deposi- 
tion of glycogen and related substances in the liver and other tissues. Conversely, when carbohydrates and other glucose-formers are not present in the diet, the concentration of glucose in the blood falls only slightly, because the supply is replenished from glycogen deposits and other sources in the body.

We assume that this adjustment between the blood sugar and the glycogen or other deposits of energy in the tissues is governed by the activity of certain tissue cells. Certain of these cellular activities are susceptible to nervous and chemical influences. Violent emotion, irritation of a certain portion of the medulla, stimulation of the splanchnic nerves or injection of epinephrin, any of these may lead to the transformation of liver glycogen into sugar, and in this way give rise to a hyperglycemia.

The concentration of glucose in the blood is further influenced through the activities of the kidneys. Normally this concentration is about .09 per cent. and normally little if any sugar escapes into the urine. If, however, the blood concentration rises, so as to exceed about o.I6 per cent., noteworthy quantities of sugar pass into the urine.

The regulation of blood sugar therefore depends primarily upon its combustion and an interchange with the tissues. Excretion through the kidneys occurs only under exceptional conditions. Various attempts have been made to explain why the renal cells excrete practically no sugar when the blood concentration is normal and excrete it in quantity when its concentration passes a certain point. The condition is perhaps comparable with the activity of cells which store glycogen, for the latter also remove glucose from the blood when its concentration is increased. They do this, however, when there is only a slight hyperglycemia, whereas the renal cells become active only when the hyperglycemia is relatively marked.

The renal barrier to the passage of glucose into the urine is not a fixed one. A number of poisons may alter this barrier, the most effective being phlorhizin. This drug causes a marked glycosuria, even though the concentration of sugar in the blood is normal (renal diabetes). In other instances, it fails to be excreted even though a moderate hyperglycemia is present.

The concentration of sodium chloride in the blood, like the concentration of glucose, is dependent in part upon its deposition in the tissues and in part upon its excretion through the kidneys. In the case of sodium chloride, however, a rise of concentration in the blood is sometimes prevented by an increase of water in the blood, thus producing an hydremia. Furthermore sodium chloride is incombustible and all that enters the body eventually must be excreted, mainly through the kidneys. Hence 


\section{I46 PATHOLOGICAL PHYSIOLOGY AND MEDICINE}

there is a constant passage of sodium chloride into the urine. Yet the rate of excretion is not determined so much by its absolute concentration in the blood as by the excess of this concentration above a certain level. If this level be approached, either because the individual abstains from taking salt in his diet, or because, as in pneumonia, salt is deposited in the tissues, then the excretion of sodium chloride by the kidneys almost ceases. Why the kidney should be almost impervious to a certain concentration of sodium chloride in the blood and quite sensitive to any rise above this level is not known, but the condition appears to be analogous to the relation that exists between the concentration of glucose in the blood and its excretion in the urine.

\section{THE MARGIN OF SAFETY IN PHYSIOLOGICAL PROCESSES}

The engineer who plans a bridge first calculates how strong it must be in order to carry any reasonable load. In actual construction, however, this calculated strength is increased several times so as to provide an ample margin of safety. Thus built, the bridge is able to carry a much heavier load than was originally planned, if this should become necessary through a change in the traffic. The additional strength may likewise prove invaluable should any portion of the material prove defective or should it become defective with age.

In most physiological processes a similar margin of safety is provided. The various functions are, for the most part, capable of accomplishing far more than they are usually called upon to perform. On account of this provision they are prepared to meet the strain of an emergency. On this account also they may still be able to meet the ordinary demands of the organism even after they have been damaged by disease.

The methods used for estimating the functional capabilities will be discussed later. Though the methods are often difficult in practice the underlying principle is fairly simple. It consists in subjecting the function tested to a definite and fairly difficult task or to a series of increasingly difficult tasks and then noting what tasks or what portions of the tasks given are accomplished. The functional capacity of the heart, for example, is roughly tested by determining how much exercise can be taken without producing marked dyspnea or other evidence of cardiac strain.

If the maximum capacity of any function lessens, then the margin of safety, or difference between the maximum capacity and the usual requirements, is likewise reduced. At first, the individual is hardly conscious of this reduction. With a further lessening of the functional 
capacity, there comes a time when the person, though able to carry on his usual duties, finds himself unable to accomplish unusual tasks with the customary ease. The margin of safety then has become appreciably reduced. A further reduction in the functional capacity leads eventually to a condition in which even the ordinary or the lowest possible demands upon the function cannot be accomplished.

Thus we see that a minor reduction in functional capacity may place no noteworthy restriction on the individual, a more marked reduction may cause him to restrict certain activities only, while a still more marked reduction may make him an invalid. Not the least interesting of these conditions is the second. There are, in every group of individuals, a certain number who by reason of disease or constitutional endowment belong to this group. Such persons may feel well so long as they restrict themselves in certain directions, as, for example, in diet, in exercise or in excitement. Because of these limitations such individuals tend to drift into the lighter occupations. If they are taken out of this relatively favorable environment and placed under conditions suitable for the average vigorous individual, as happens for example when they are drafted into the army, then their functional deficiencies, hitherto largely latent, become a source of symptoms. Some develop cardiac complaints such as -breathlessness, precordial pain or palpitation on exercise, some become nervous and apprehensive, while still others develop digestive disturbances. An environment to which the normal individual readily accommodates himself proves in one way or another too rigorous for persons of this substandard class.

The factors which provide a margin of safety in physiological processes are varied. In the first place most organs are capable of accomplishing far more work than is usually required of them. After excising part of an organ, for example, the residue frequently continues to do the work formerly done by the whole, presumably because its cells are now working more actively. This fact has been demonstrated time and again. It is well known, for example, that diabetes invariably follows complete excision of the pancreas. If about one-third of the gland is left in place, no disturbance in the sugar metabolism can be demonstrated. If one-fourth of the gland is left, there is likewise no obvious disturbance, although according to Allen the tolerance to subcutaneous injections of glucose is diminished. The margin of safety has been demonstrably reduced, but the function still suffices to meet the ordinary requirements. If only one-sixth of the gland is left, there is usually a mild, transient diabetes. Finally if only one-tenth is left, severe diabetes occurs. Thus we see that three-fourths of the pancreas can be removed without causing any disturbance in sugar metabolism under normal conditions. In other 


\section{I48 PATHOLOGICAL PHYSIOLOGY AND MEDICINE}

words, the function of the pancreas which influences the combustion of glucose has a margin of safety which amounts to at least 300 per cent. above that necessary to accomplish the work ordinarily required.

Similar relations hold with respect to various other organs. Half of one kidney can do the work that is usually done by two. Three of the parathyroid glands may be removed with impunity, although removal of the fourth results in tetany. A large part of the thyroid can be excised without causing myxedema. Over half of the small intestines can be excised from dogs with almost no effect upon the digestion and absorption of food, unless by using a special diet an unusual strain is placed upon the intestinal function. One lung may be excised or thrown out of function.

Such evidence as this shows that many organs are capable of performing far more than is usually required of them. It is this which makes possible the surgical excision of a kidney, the removal of most of the thyroid gland together with three of the four parathyroids, the resection of a long piece of intestine or the resection of a lobe of the lung. It is this margin of safety which accounts for the fact that extensive localized disease in a gland may lead to no serious loss of function. In the liver and in the kidneys, for example, there may be large tumors with no failure of function. Functional disturbances in these organs are more commonly due to diffuse diseases of the parenchymas.

The second factor which provides a margin of safety in certain physiological processes is the fact that a given function may be performed by more than one organ. Not only are many organs paired, but the same or a similar function may be performed by organs which in other respects differ widely from one another. For example, glycogen can be stored in other cells than those in the liver. During digestion, the food is acted upon by a number of juices which supplement one another. Starch-splitting ferments are present in both the saliva and pancreatic juice and even when both these are absent starch digestion occurs in the intestinal tract. Protein cleavage can be accomplished by both the gastric juice and the pancreatic juice. Of these the pancreatic juice plays the more important rôle. Its absence from the intestines leads to a considerable loss of nitrogenous material in the stools, whereas a lack of gastric juice usually has little or no effect upon the amount of protein absorbed. Indeed many individuals of middle or advanced age have gastric achlorhydria without suffering from digestive symptoms. Nevertheless such individuals cannot be considered normal, for their margin of safety in digestion has been reduced. Food reaching the small intestines is not so well prepared as usual, and we know that a certain proportion 
of those with achlorhydria suffer from intestinal symptoms, particularly if the food be coarse or if poor teeth interfere with its proper mastication.

The third factor which makes for safety in physiological processes is the hypertrophy and increased functional activity which sometimes follows exercise of a function. It is well known, for example, that muscular exercise causes hypertrophy of the voluntary muscles used and that disuse leads to their atrophy. In the former case the muscles become stronger, while in the latter case they become weaker. Analogous relations appear to hold with respect to the heart muscle. Exercise increases the weight of the heart relative to the weight of the body. Animals which habitually take a great deal of exercise, such as the deer, the hare and birds, have very large hearts; race horses and coursing dogs have hearts which are considerably heavier than the average for their species; and men who habitually take considerable exercise also have relatively large hearts. When such individuals exercise violently they show less dyspnea than the average normal, presumably because their hearts and respiratory apparatus are capable of doing more work. Valvular disease and chronic arterial hypertension cause cardiac hypertrophy, largely through increasing the work of the heart muscle. This hypertrophy not only enables the heart to do more easily the extra work: imposed by these conditions but it furnishes a margin of safety which may be called upon in emergencies. Unfortunately, however, valvular disease as well as chronic arterial hypertension are frequently accompanied by conditions which damage the myocardium and this lessens the capabilities of the heart muscle.

The removal of a large portion of an organ is, in certain instances, followed by hypertrophy or regeneration of the remainder. This has been observed in the case of the liver, the thyroid, the adrenal cortex, the kidney, etc. The cause of this increase in the size of the glandular residue is not clear, although it seems probable that it is partly due to the increase in the functional activities. In other words, the increase in size may be the result of an increased exercise of function.

When part of the tissue of an organ is injured by disease, more work is thrown on the remainder. This may lead to a regeneration or hypertrophy of that remainder. When, for example, there is an extensive destruction of the hepatic cells through toxic or infectious influences, the remaining parenchyma cells show a marked regenerative activity. Somewhat similarly a diffuse damage to the heart muscle throws more work upon the relatively intact fibers and this may lead to their hypertrophy. Thus it is that cardiac hypertrophy may follow a primary damage to the heart muscle, and in this way the strength of this muscle presumably 
becomes greater than it would have been had no such hypertrophy succeeded the primary damage.

A number of other physiological adjustments tend to compensate for a primary injury and thus to preserve the margin of safety. $A$ loss of red corpuscles, for example, is followed by increased activity on the part of the erythroblastic tissues. The red marrow in the bones increases in extent, and immature red cells become more numerous in the marrow and in the circulating blood. A somewhat similar reaction takes place when the supply of oxygen is restricted from any cause. In marked cyanosis from congenital heart disease or advanced pulmonary emphysema, in life in high altitudes, in carbon monoxide poisoning where hemoglobin is rendered useless for respiratory purposes, and in certain intoxications which damage the red cells or interfere with internal respiration, there frequently occurs an increased activity of the erythroblastic tissues with an increase in the total number of red corpuscles and total quantity of hemoglobin in the body. These changes make it possible for the blood to transport the usual amount of oxygen to the tissues without increasing the average rate of blood flow.

\section{THE EFFECT OF STRAIN}

We have cited instances in which exercise has increased the functional capacity of an organ. Unfortunately increased demands upon a function do not always have this beneficial effect. Where the demands exceed the functional capacity, harm may follow. In normal individuals this seldom happens; for, as we have seen, the margin of safety is normally so wide that the. functional capacity seldom is overloaded. In disease, however, the work demanded may easily exceed the capabilities of a damaged function and the resulting strain may produce harmful effects.

We may take the voluntary muscles as an example. A normal person who exerts himself to the limits of his strength or to the limits of his endurance rarely does himself harm. If, however, certain muscles are weakened by disease, then it is not difficult to put an excessive strain upon these muscles or to fatigue them excessively, and in such a case they may become weaker than they were before. For this reason exercise of muscles that have been partially paralyzed from any cause must be undertaken gradually and with care.

In the case of a normal heart it is doubtful if harm ever results from violent or prolonged exertion. If the heart muscle is damaged, however, violent exertion may cause acute dilatation, while less violent exercise that is continued despite warning symptoms may lead to a gradual decline in the strength of the myocardium. 
In diabetes the ability to burn sugar in the body is reduced. If the patient disregards this limitation of function and by improper diet permits an excess of sugar to be present in his body, the ability to burn sugar tends to become reduced still further. If, on the other hand, the patient refrains from those articles of diet which are readily converted into glucose and thereby lessens the strain on a weak and overburdened function, then the ability of the body to burn sugar tends to improve. By this method of treatment cases of diabetes that at first have been classified as severe later have become mild or moderately severe.

In nephritis the ability of the kidneys to excrete sodium chloride sometimes is lessened. In some of these patients an overburdening of this function by the ingestion of much salt in the diet has been followed by a further reduction in this renal function.

From these examples it is evident that excessive demands on a weak function frequently cause harm. Not only is the extra work not properly done and the individual more or less inconvenienced or harmed by the failure to meet the demands, but the functional capacity, already below normal, tends to be reduced yet more as a result of being asked to do more work than it can accomplish.

\section{PATHOLOGICAL PHYSIOLOGY AND THE INTERPRETA- TION OF CLINICAL PHENOMENA}

Internal medicine is gradually becoming more dependent upon the fundamental sciences of physiology, pathology and bacteriology. Not only have these sciences furnished to clinical medicine many methods - which are useful in the diagnosis, prognosis and treatment of disease, but they have exerted a marked influence upon clinical thought. The discoveries of the diphtheria bacillus, the diphtheria toxin and the diphtheria antitoxin have not alone supplied new methods of diagnosis and treatment but they have given us a better conception of the disease itself. We now define diphtheria in terms of its etiology rather than in terms of throat changes. We now know that most manifestations of the disease are caused by the absorption of a soluble toxin, and that recovery from the infection usually results if this toxin is neutralized before it becomes fixed to the body cells. In other words, we think of diphtheria mainly in terms of bacteriology and immunology. Other fundamental medical sciences have exerted a similar influence upon medical thought. According to the nature of the disease and according to our knowledge, we are learning to interpret the phenomena encountered at the bedside in terms of etiology, altered structure or perverted function. 


\section{I52 PATHOLOGICAL PHYSIOLOGY AND MEDICINE}

Several instances have already been cited where physiology has aided in explaining clinical manifestations. The febrile chill and the febrile sweat indicate an effort suddenly to raise or to lower the body temperature. The small radial pulse during a chill is part of the vascular constriction which affects all vessels at the periphery of the body. The warm moist skin in severe forms of Graves' disease is necessary in order to rid the body of the excess heat liberated in this disease. Gains or losses of fat result from a difference between the amount of energy available in the food absorbed and the average rate of metabolism in the body. Acidosis increases the respiration.

Other examples will now be cited in order to illustrate how the physiological point of view may assist greatly in the interpretation of clinical phenomena.

The cardiac arrhythmias. The heart muscle, like living tissue in general, is sensitive to external stimuli, its most obvious response to a stimulus being a contraction. In addition, it has the property of initiating contractions spontaneously and of conducting contractions from their point of origin to other regions of the heart. These fundamental properties of the heart muscle appear to depend upon the continual accumulation of unstable chemical compounds, which decompose during each contraction. At least we know that immediately after a contraction the fundamental properties of the heart muscle are greatly lessened, and that at this time the muscle neither initiates spontaneous beats nor contracts when stimulated or conducts impulses. This is the so-called refractory period that follows each contraction.

Although any portion of the cardiac muscle inherently is capable of initiating contractions, this property ordinarily is exercised only by that part of the muscle which possesses the most rapid spontaneous rhythm. The contractions initiated at this point, which is known as the pacemaker, spread to all other parts of the muscle and discharge the unstable material that has been accumulating in them. The pace-maker of the heart is usually located in the sinus node, a group of special cells lying at the junction of the vena cava with the right auricle. The impulse initiated in this region spreads first to the auricles and causes them to contract. From there it is conducted to the ventricles by way of the His bundle, the latter being the sole functional connection between the auricles and ventricles. The upper portion of this bundle is a single trunk, while below it divides into two primary branches which supply the right and the left ventricles respectively. These primary divisions give off finer branches which eventually terminate in the Purkinje fibers lining the inner surface of the ventricular muscle.

For a detailed description of the cardiac arrhythmias the reader is 
referred to the special chapter on this subject. Here, however, we wish to emphasize the point that an understanding of these arrhythmias is based upon a knowledge of the physiological properties of the heart muscle just enumerated.

Certain of the cardiac arrhythmias originate in the normal pacemaker of the heart, and are therefore known as the sinus arrhythmias. Irregularities of this type are usually due to changes in the influence normally exerted by the vagus nerve upon the pace-maker. Normally this influence is fairly uniform; but in certain conditions, particularly in young and neurotic individuals, the vagus influence fluctuates markedly. During the respiratory cycle or owing to changing emotions and variations in attention, considerable degrees of cardiac irregularity may be produced from this cause.

A second group of arrhythmias are caused by an imperfect conduction of the contraction impulse over the heart. Disturbances of conduction occur most frequently in the His bundle or in its branches. When conduction in the whole bundle is impaired there may result (I) an unusual delay between the auricular and ventricular contractions, (2) a failure of certain impulses to cross the bundle with a resulting omission of ventricular beats (partial heart block), or (3) a complete functional break between the auricles and ventricles (complete heart block, auriculoventricular dissociation). In this last condition the ventricles usually beat slowly and regularly, their rhythm being determined by impulses arising in the main stem of the His bundle. When conduction is impaired in the primary or secondary branches of the His bundle, the ventricles beat regularly but the path of conduction through the ventricular muscle is altered. This may cause an alteration in that portion of the electrocardiogram which corresponds to the ventricular contraction. Alterations of this character are of considerable clinical significance because they indicate changes in the heart muscle. These cannot be recognized by any. other method.

A third group of cardiac irregularities are those which result from premature contractions, which originate in some portion of the heart muscle other than the normal pace-maker. Occasional beats of this sort (extra systoles) may be initiated in either the auricles or the ventricles. A rapid succession of such beats usually originates in the auricles and gives rise to the conditions known as paroxysmal tachycardia and auricular flutter.

The fourth common group of cardiac arrhythmias is that in which the auricles, though motionless in a gross mechanical sense, show throughout their extent multitudinous incoordinated contractions of separate muscle bundles. This is known as auricular fibrillation. In such a condition 


\section{I54 PATHOLOGICAL PHYSIOLOGY AND MEDICINE}

the upper end of the His bundle is continually bombarded by numerous stimuli of varying intensity. Of these only a small proportion reach the ventricles, and those that succeed in traversing the bundle reach the ventricles at irregular intervals. Consequently, in auricular fibrillation, the ventricular rhythm is absolutely irregular, in the sense that there is no fundamentally regular rhythm that may or may not be interrupted by premature or by dropped beats.

To these commoner forms of cardiac irregularity one might add a considerable number of less common varieties, all of which, however, can be interpreted in terms of functional changes in the myocardium. The recognition of these types of irregularity is important in practice because the different types differ in their etiology, in their pathological anatomy and in their response to drugs. For the accurate identification of the various cardiac arrhythmias, instruments of precision are necessary; but the physician who is familiar with the subject can recognize most of the irregularities with fair accuracy during an ordinary clinical examination. For this new knowledge he is indebted to physiological studies on the normal and damaged heart.

Visceral pain. Knowing that severe pains are common in visceral disease, one is at first surprised on learning that most internal organs are insensitive to touch and to mechanical injury. The gastric mucous membrane may be cut, pinched, or pricked without producing any subjective sensation. The serous coverings of the lungs, stomach, and intestine may be touched or injured without pain. How then are the pains of visceral disease produced?

In the first place the parietal portions of the pleura and peritoneum, unlike their visceral portions, are quite sensitive to pain. This difference between the two has been repeatedly demonstrated in the case of the peritoneum during laparotomies under local anesthesia, and in the case of the pleura by touching different parts of this membrane with a wire that has been introduced through an aspirating needle. Involvement of the sensitive parietal surfaces accounts for the pain in acute pleurisy and in local or general peritonitis. Pleural or peritoneal adhesions may also cause pain by dragging on the sensitive parietal membrane.

The second type of visceral pain is that which arises from the hollow organs. This pain is frequently cramplike in character and is exemplified by intestinal colic, renal colic and biliary colic. Beyond doubt, however, other and less severe pains possess a similar origin. We know from experience that renal and biliary colic usually follow some sudden obstruction of the ureters or the cystic duct. Gradual obstruction may cause no pain. Thus in malignant obstruction of the common bile duct, 
the gall bladder is frequently much dilated although the patient has never experienced pain. Since infection of the passages may be present without pain and infection may be absent when pain is intense, it is evident that infection, while it may favor the production of this type of pain, is not essential for its occurrence. An interesting form of renal colic and one that sheds light on its cause is that which can be produced by the rapid and forceful injection of fluid into the renal pelvis through a ureteral catheter. In this instance the pain is obviously produced by the sudden distention of the renal pelvis which may perhaps be combined with a reactive spasm on the part of the smooth muscle lining its walls. The view that contractions of the smooth muscle play a part in the pathogenesis of such pains gains support from observations that have been made on the cramps which occur in intestinal and pyloric obstruction. In these conditions the motor activities of the stomach and intestine proximal to the obstruction can sometimes be observed through the abdominal wall, and it has been noted occasionally that each cramplike pain coincides with a violent tonic spasm of the distended viscus. Other forms of intestinal colic, such as that caused by lead poisoning, may be of a similar nature, except that here the organic obstruction is replaced by functional spasms which oppose the onward progress of the intestinal contents.

The pains that accompany gastric and duodenal ulcers were at one time likened to those that result from irritating a wound on the surface of the body. This analogy is probably incorrect, for there is reason to believe that gastric ulcers are insensitive both to the acid and to the food found in the stomach. Aside from pains produced by peritoneal involvement, gastric and duodenal ulcers appear to cause pain through some disturbance in the motor activities of the stomach and particularly through a muscular spasm at or near the pylorus. If this explanation be correct, one may readily understand how ulcer pains may be independent of the degree of gastric acidity, how they may occur when the stomach is empty or nearly empty (hunger pains), and how they may be stimulated by motor disturbances in the stomach that are not due to a primary gastric disease but to reflexes from disease elsewhere. In many instances the removal of a diseased appendix has relieved pains which resembled those caused by gastric ulcer.

Variations in the muscular contractions and muscular tone of the stomach and intestines may produce a number of sensations which differ from pain in the ordinary sense. Thus the sensation of hunger, a gnawing or aching sensation roughly localized in the epigastric region, is due to gastric contractions. Moreover, as we have already pointed out, the sense of well-being that immediately follows a fecal evacuation is 


\section{56}

probably due to lessened distention of the lower bowel, combined with changes in the tone and with other activities of this muscle.

Besides the two types of visceral pain, produced either by changes in the serous membranes or by spasm or distention of the hollow organs, there are probably other forms of pain arising within the body. At the present time, however, we have no clear conception of their nature.

Pain caused by disease of an internal organ is not always localized by the patient in the region of his disease. It is well known, for example, that the pain of renal colic frequently radiates to the corresponding testicle and to the anterior and lateral aspects of the thigh, that the pain of biliary colic frequently extends to the right scapular region, and the pain of angina pectoris frequently involves the left shoulder and the median aspect of the left arm and forearm. Disease of an internal organ may also be accompanied by an area of cutaneous hyperalgesia and by muscular spasm or weakness which may or may not overlie the affected organ. These referred pains and cutaneous hyperalgesias, together with some alterations in the voluntary muscles, are caused by changes in the portion of the spinal cord which receives centripetal impulses from the diseased organ. These impulses on entering the cord cause an irritation of various nerve elements in the affected region. As a consequence of this irritation, pain is felt or hyperalgesia is demonstrable along the peripheral distribution of those sensory nerves which enter the affected segment of the cord, and spasm or weakness may occur in muscles innervated from this same region.

These changes and especially the occurrence of pain in distant parts of the body are of considerable diagnostic significance. It is known, for example, that nerve impulses from the peripheral portions of the diaphragmatic pleura are carried to the lower thoracic cord. Since the lower thoracic nerves supply the abdominal wall, inflammations of the peripheral diaphragmatic pleura may cause pain, cutaneous hyperalgesia and muscular spasm on one side the abdomen, thus simulating a surgical disease within the abdomen. When the central portion of the diaphragmatic pleura is irritated, pain in the neck on the same side, either above the clavicle or over the anterior part of the trapezius muscle, is experienced. The afferent fibers from this central portion of the diaphragmatic pleura appear to travel upward in the phrenic nerve, entering the cord at the level of the third, fourth and fifth cervical segments. Since cutaneous nerves from the neck also enter the cord at this same level, the pain in diaphragmatic pleurisy is frequently referred to the supraclavicular region, and when neck pains occur in connection with pleural disease one may suspect an involvement of the central portion of the diaphragm. 
In order to illustrate the different types of pain that may come from a single organ, let us consider briefly the pains of appendicitis. These pains are frequently due to involvement of the neighboring peritoneum. In this case the pain and tenderness are usually localized over the site of the disease. If the appendix occupies some unusual position in the abdominal cavity, such pains and tenderness will also occur in an unusual position. But appendicular pains may also arise from an obstruction of the lumen of the appendix with acute distention of its tip, thus giving rise to the so-called appendicular colic. In such a case the location of the pain and muscle spasm is governed not by the position of the appendix within the abdomen but by its central nervous connections. Since the latter do not change with changes in the position of the appendix, it may happen in this type of disease that the pain, hyperalgesia and muscle spasm are most marked in the lower quadrant even when the appendix occupies some unusual site in the abdomen, such, for example, as the lower left or the upper right quadrant.

Before leaving the subject of visceral pain it may be well to point out that this pain together with the associated hyperalgesia, being due to changes in the nerve centers, may be stimulated by lesions that are located in these centers or their adjacent nervous connections. Particularly is this true of lesions involving the spinal ganglia or the posterior nerve roots of the cord. In herpes zoster and in tabes dorsalis, therefore, we frequently encounter pains which resemble those of visceral disease.

Intoxication by foreign proteins. In discussing the autointoxications, it was pointed out that many of these appear to be caused by products which arise within the body from protein decomposition. That proteins may, in one way or another, give rise to toxic symptoms is shown in the effects produced by injecting foreign proteins into animals or men. These effects are of clinical interest, not only because solutions that contain proteins are frequently injected for therapeutic purposes, but because symptoms analogous to those produced by such injections have been observed to follow the inhalation of protein-containing dust, the ingestion of certain foods and the invasion of the body by infectious microorganisms.

The first type of foreign protein intoxication to which we wish to call attention is the febrile reaction that frequently follows the injection of a foreign protein solution directly into the blood. This reaction begins one or two hours after the injection and is characterized by a chill followed by a high but transient fever. During the chill the pulse becomes small and rapid, and at times there is cyanosis with general prostration. Very occasionally death follows such an injection. In medical practice this febrile reaction is encountered most freqently after 


\section{I58 PATHOLOGICAL PHYSIOLOGY AND MEDICINE}

intravenous injections of therapeutic sera, of suspensions of dead bacteria and of gelatine solutions. In the early days of arsphenamin therapy, a similar reaction frequently complicated intravenous injections of this drug, but since care has been taken to use properly distilled water in making up the solution this reaction has become much less common. The earlier reactions after arsphenamin were apparently due to minute traces of organic matter in the water used for preparing the solution.

The febrile reaction after intravenous protein injections is nonspecific. All persons are liable to the reaction and a variety of proteins have produced it. Indeed it is not quite certain that proteins are essential for its production. Similar effects have followed the intravenous injection of other colloidal solutions, such as those made from agar or colloidal silver, even when these were supposed to contain no protein. It is possible, therefore, that this reaction depends upon the colloidal nature of the solution rather than upon the chemical constitution of the protein used.

In its clinical features, the febrile reaction following the intravenous injection of a foreign protein resembles the febrile paroxysm of malaria or of marked sepsis. The malarial paroxysm coincides with sporulation of the parasites. At this time many young parasites together with the detritus from formerly infected red cells become free in the blood. The form of sepsis which most frequently gives rise to violent chills is that in which there is a septic venus thrombosis. Here the chills are supposed to coincide with the escape of infectious material from the venous thrombi into the general circulation. Both in malaria and in septic venous thrombosis, therefore, the paroxysms may be attributed with considerable probability to a sudden escape of foreign protein material into the general blood stream.

Subcutaneous injections of a foreign protein seldom cause the acute and violent symptoms that occur after intravenous injections. The effects produced also vary with the individual and with the kind of protein used. The best studied of these effects are those which follow the injection of horse serum, the so-called serum disease. This occurs in the majority of all who have received large doses of horse serum (IOO cc. or over). In the mild form of serum disease, the principal manifestation is urticaria; in the more serious forms, there are in addition joint pains, fever and edema. If the individual has never before received an injection of horse serum, these symptoms usually occur in from eight to ten days after the injection. If he has received injections more than two weeks previously the incubation period is shortened.

Disease manifestations may also be caused by foreign proteins which have gained access to the body through natural channels, particularly 
through the respiratory and digestive mucous membranes. Such cases are uncommon and they usually occur only in individuals who show an abnormal sensitiveness to the particular protein which causes the disturbance. Animal experiments demonstrate that such an abnormal sensitiveness may be produced by a previous injection of the protein in question. Thus the first injection of horse serum into a guinea pig causes no symptoms, whereas the second injection given after an interval of about two weeks causes sudden death by asphyxia, owing to a violent bronchial spasm. In dogs the second injection causes an acute circulatory collapse.

Among the diseases of man which result from abnormal sensitiveness to foreign proteins we may mention cases of urticaria and other skin rashes which follow the ingestion of certain foods, hay fever which is caused by the lodgement of certain pollens upon the mucous membranes of the upper air passages, and asthma, bronchial spasm, which in certain instances follows the inhalation of pollen or other protein-containing dusts, the eating of certain foods, the injection of certain proteins or the acquiring of certain infections of the air passages. The sudden death which occasionally follows the subcutaneous injection of horse serum is due to an acute bronchial spasm similar to that which kills the guinea pig after a second injection of horse serum.

Just why certain individuals are sensitive to a given protein is not altogether clear. From animal experiments we know that sensitization may be acquired by the injection of a given protein at some previous time. We know also that in man a previous injection of horse serum modifies the reaction to subsequent injections in that serum sickness develops more promptly and the local reaction about the point of injection is usually more marked. We know also that persons infected with tuberculosis acquire an unusual sensitiveness to tuberculin. On the other hand the sensitiveness to a specific protein which characterizes hay fever, certain asthmas, certain skin rashes and some gastrointestinal disturbances has not in most instances been preceded by an injection of the offending protein. The occasional sudden death after a subcutaneous injection of horse serum usually has followed from a previous entrance of the protein into the body through natural channels, such as the mucous membrane lining the digestive or respiratory tracts. Even though this be the case, there must be some underlying defect which permits such individuals to become sensitized, while others living under similar conditions remain unaffected. Two additional facts favor the view that the sensitization which develops in those who have never received a protein injection must depend upon some fundamental defect in the organism. In the first place the tendency to such diseases as asthma 
and hay fever is frequently inherited. In the second place a given individual is frequently sensitive to more than one protein. We must assume, therefore, that in man sensitizativeness to given proteins not infrequently depends upon some individual predisposing factor. This may possibly act through permitting proteins to enter the body without being broken down.

The study of the changes that follow the entrance of a foreign protein into the body has given us a clearer conception of such diseases as asthma, hay fever and urticaria. It has helped also to explain certain manifestations of the infectious diseases, for in these the organisms that gain entrance to the body play the rôle of foreign proteins. Thus the incubation period of an infection finds its counterpart in the incubation period of serum disease and in the time which must elapse before the guinea pig which has received one injection of a foreign protein becomes sensitive to a second. In serum disease as well as in the protein sensitization of the guinea pig, time is required before the changes in the body occur which cause it to react to the foreign protein in such a manner as to produce symptoms. The incubation period of many infections probably is due likewise to the time required to elaborate a set of reactive substances in the body. The changes in temperature which occur during infections are also comparable to those which result from the introduction of foreign proteins into the body. Finally the circulatory collapse occasionally seen during infections finds a counterpart in the circulatory changes which follow the second injection of a foreign protein into the dog.

\section{FUNCTIONAL METHODS IN DIAGNOSIS}

We have pointed out that the functional changes during disease have been studied in part by applying biological methods of study to patients. Such investigations not only have broadened our horizon of medical knowledge but they have added new means for the differentiation of diseases. Any method which can be used for studying functional changes in man eventually may become useful in diagnosis. The practical utility of such a method is determined in part by its reliability in differentiating one pathological condition from another and in part by the ease with which it can be performed. If we view these physiological methods from the standpoint of their value in practical diagnosis, therefore, we have (I) methods of study which yield no information of diagnostic value which cannot be obtained by other and simpler methods, (2) methods which yield diagnostic information but which by reason of their complexity can only be carried out in special laboratories and 
by specially trained workers, and (3) methods which yield diagnostic information and can without difficulty be used in the office or at the bedside.

Of physiological methods that have proved useful in diagnosis we shall name only some of the more important. Those which depend upon the use of physical instruments include the determination of body temperature by the clinical thermometer, the bloodless determination of arterial blood pressure, the registration of the contractions of the auricles and ventricles by means of the venous pulse and electrocardiogram, and the electric tests of paralyzed muscles. Those which depend upon the use of chemical analysis include examination of the urine, especially for albumin and sugar, the determination of urea and carbonates in the blood, the determination of hydrochloric acid in the gastric contents, and the estimation of protein and fat losses in the feces. Among biological methods are the complement fixation reactions, the agglutination tests and the determination of individual sensitiveness to a given foreign protein.

Such functional diagnostic methods do not necessarily give information concerning the site and character of the anatomical lesion. Changes of blood pressure, for example, may be due to an altered activity of either the heart or the blood vessels, and these structures may or may not show anatomical changes. Cardiac irregularities rarely permit one to diagnose a specific alteration in a particular part of the heart muscle. The presence of albumin in the urine or an excess of urea in the blood may follow a variety of renal lesions. The correlation of the amount of acid in the gastric contents with anatomical changes in the gastric mucous membrane has proved difficult and uncertain. Finally biological methods in general give information concerning the altered reaction of the body as a whole and rarely locate structural alterations in a specific region.

Nevertheless, even where functional changes cannot be interpreted in terms of specific alterations of structure, they may yet be of considerable value in classifying disease. Chronic arterial hypertension, for example, appears to be a well-defined entity, which leads to ventricular hypertrophy and which frequently is complicated by cardiac insufficiency, by renal disease or by cerebral hemorrhage. Gastric achlorhydria is usually a chronic disorder, and it may cause certain gastric symptoms and may lead to intestinal derangements. Acidosis and diabetes represent general metabolic disturbances in which definite anatomical changes are frequently absent-a fact that lessens in no way the importance of their recognition in practice.

The diagnostic nomenclature chosen for such conditions as these 
should, we believe, indicate the alteration in function wherever the anatomical changes are doubtful or unknown. In other words, an accurate functional diagnosis is better than an inaccurate anatomical diagnosis. Diabetes mellitus, for example, is universally accepted as a satisfactory diagnosis. Rarely is anything gained by conjectures as to the exact pancreatic lesion which may or may not be found at autopsy. Similarly a diagnosis of chronic arterial hypertension is usually preferable to a diagnosis of sclerosis of the finer arterioles, even though the latter may represent the physician's conception of the underlying change. In like manner gastric hyperchlorhydria and gastric achlorhydria are definite conceptions which lose rather than gain by indiscriminate attempts to express them in anatomical terms-unless indeed there is further evidence upon which to base an anatomical diagnosis. Admittedly such functional diagnoses leave unanswered both the question of etiology and the question of structural change. If either of these be known, it furnishes a more satisfactory basis for classifying disease. But where both are unknown or where in a particular case they are uncertain, no advantage is gained by substituting for a term that indicates a definite functional change another term that may be misleading.

One of the important problems in diagnosis is the estimate of damage to a particular function. How much work can a given organ do without signs of failure? If it be diseased, how well does it carry on its functions under ordinary or unusual requirements? We have pointed out that most healthy functions are protected from strain by a considerable margin of safety. They are able to do more than usually is required of them. For this reason the total functional capacity usually must be reduced considerably before there is any actual failure in the performance of the ordinary physiological tasks. We have, therefore, to consider (I) the estimate of the degree of failure to meet ordinary! rquirements, and (2) the detection of a restricted margin of safety, where the deficiency becomes apparent only when unusual demands are made upon the function.

When the failure of a function to meet the ordinary demands of life causes well-marked symptoms, the severity of these symptoms under varying conditions may enable tus to estimate the degree of functional impairment. In the case of the heart, for example, the amount of dyspnea and of other cardiac symptoms, when compared with the work demanded of the heart by muscular exercise, furnishes one of the most trustworthy guides for estimating the degree of cardiac insufficiency. Shortness of breath even when at rest in bed, shortness of breath when up but quiet, and shortness of breath only after ascending stairs or going up an incline are the rough standards by which we measure different 
degrees of cardiac insufficiency in most types of heart disease. The exact site and nature of the lesion is usually of less importance than the loss of cardiac efficiency as measured by these rough tests.

In other instances the impairment of a function can be demonstrated and measured, even when few if any symptoms are present. In diabetes mellitus, for example, the symptoms are often indefinite or even absent. The severity of the condition is commonly measured not by the symptoms but by the amount of sugar-forming food that may be taken without the appearance of glycosuria. Using this standard, the following classification of diabetic patients is commonly used: (a) mild, if considerable protein and at least 60 grams of starch can be taken daily without glycosuria, (b) moderately severe, if considerable protein and less than 60 grams of starch can be taken daily without glycosuria, and (c) severe, if glycosuria persists even though all carbohydrates are eliminated from the diet.

Other functional disturbances are measured more directly. The acid secreted by the stomach is estimated by determining the concentration of acid in gastric contents that have been removed at a standard interval or series of intervals after a standard meal. The ability of the stomach to discharge its contents is estimated by removing the contents at certain intervals after a standard meal, or by observing with the X-ray what proportion of an opaque meal remains in the stomach at various times after its ingestion. Chronic arterial hypertension is measured directly, care being taken to exclude the influence of emotions and other factors which disturb the blood pressure. The leak in aortic insufficiency is believed roughly to parallel the fall in diastolic pressure, when other causes for a low diastolic pressure, such as infections, are excluded.

Renal disease is usually recognized by the presence of albumin and of casts or other formed elements in the urine. At times renal disease interferes with the excretion of one or more of the normal urinary constituents. If urea is not properly eliminated its concentration in the blood rises. Since such a rise increases the rate of elimination, an equilibrium becomes established between the urea formation in the body and the urea elimination in the urine. Hence in this form of renal insufficiency there is an increased concentration of urea in the blood together with the excretion of normal amounts in the urine. Various attempts have been made by Ambard and others to express this relation between the urea concentration in the blood and the rate of its elimination in the urine as a mathematical formula, so as to give a numerical expression for the efficiency of urea excretion. Whatever view may be taken of the assumption that the renal functions work with mathematical precision, a formula of this type which shows the relation between 


\section{I64 PATHOLOGICAL PHYSIOLOGY AND MEDICINE}

the blood urea and its excretion should give a better index of renal efficiency than does the blood urea alone. Most clinicians who have used both believe that in practice the former is at times more valuable than the latter.

The retention of urea in renal insufficiency is associated with the retention of other non-protein nitrogenous compounds. Even a slight urea retention is said to be accompanied by a rise in the concentration of uric acid in the blood, while marked retentions of urea are accompanied in addition by a rise in the blood creatinin.

Renal edema is accompanied by a retention of water and of sodium chloride in the body. The ability of the kidneys to excrete these substances does not parallel the ability to excrete urea; for renal edema may occur with no increase in the blood urea, and a marked retention of urea may occur without edema. These two functions may be damaged disproportionately in nephritis. The cause of renal edema is not entirely clear. We know, however, that in certain patients with nephritis edema may be made to disappear by restricting the intake of sodium chloride, and that it may be provoked again by taking an excess of this salt in the diet. Similar effects have occasionally been observed during the administration and withdrawal of sodium bicarbonate, which has been given for the purpose of combating acidosis. In such cases, the kidneys are apparently unable to excrete all of the salt taken, and the excess retained in the body leads to a secondary retention of water. Partly on account of this water retention, the concentration of sodium chloride in the blood rises only slightly, differing in this respect from the marked rise of urea concentration when there is a serious retention of the latter.

A failure to excrete the chlorides taken in the food does not necessarily indicate an insufficiency of this renal function. We know that in pneumonia the reduction of chlorides in the urine is accompanied by a low concentration of this salt in the blood, due apparently to its deposition in the tissues. The relation between the blood concentration and the rate of excretion remains normal, thus indicating that the low chloride output in pneumonia is not due to a renal insufficiency.

It is evident from what has been said that the study of patients by physiological methods has helped to define the kind and severity of the functional changes present. In addition to methods for studying the existing functional changes in disease, a number of methods have been used in which the functional capacity is estimated from the response to an artificial demand on the function. These are the so-called functional tests. They are useful not only in cases of marked functional insufficiency but also where there is merely a reduction in the margin of safety; for by demanding some unusual performance, a reduction in 
the margin of safety may be detected. Two types of demand upon a function may be made. The first consists in a task which is similar in character to the task normally performed by the organ. The second consists in a task which though unlike that usually performed by the organ can be shown to measure its capacity to accomplish the usual tasks or can be shown to indicate some type of structural change.

It is not our purpose to enumerate and describe the considerable number of functional tests that have been proposed from time to time. We merely wish to point out the principles involved in certain of the more valuable tests.

In the case of the heart, the usual functional test consists in determining the amount of work that can be done within a given time without producing evidence of cardiac insufficiency. Among the exercises recommended are climbing stairs or inclines, hopping on one foot and lifting weights. Standardization of the exercise chosen is of course necessary. The chief difficulty in these tests, however, appears to lie in the selection of functional changes that best indicate a slight degree of cardiac strain. Among the criteria that have been suggested are changes in the size of the heart, a rising blood pressure shortly after the exercise and a continuation of the rapid pulse rate for some minutes after stopping the exercise. The first of these has proved difficult to determine and is of little help even when the X-ray is used. The last two are of some value in judging a slight cardiac strain. Equally good perhaps is the general condition of the patient after completing an exercise, particularly with respect to breathlessness, exhaustion and other cardiac symptoms. With reliable criteria for detecting slight cardiac insufficiency, this method of determining the cardiac response to graded exercises should indicate the degree of functional impairment, not only in patients with marked insufficiency but also in those whose cardiac capacity for work has been but slightly reduced.

We have pointed out that in diabetes the severity of the disease is estimated from the ease with which sugar can be made to disappear from the urine on reducing those articles of diet that give rise to sugar in the body. The question sometimes arises whether an occasional trace of sugar in the urine indicates an incipient diabetes mellitus. In order to answer this question it has been proposed to introduce an excessive quantity of glucose into the body through the mouth or by injection, and then to determine whether the concentration of sugar in the blood rises to an unusual degree or whether an excess is excreted in the urine. Such tests do not yield an unequivocal answer with regard to the sugarburning ability of the body, because an excess of sugar in the blood is removed by storage rather than by increased combustion. Nor in prac- 
tice have they been of great service, in separating mild and transient glycosuria of incipient diabetes from the glycosurias that result from some disturbance in the storage mechanism.

Numerous functional tests have been applied to the kidneys. In some the rate of excretion is measured after normal urinary constituents have been introduced into the body. For example, if urea is taken by mouth, its subsequent rate of elimination may be used to measure this renal function. Water and salt elimination have been measured in a similar manner, or several substances may be given at one time and the excretion rate of each determined. Such tests are subject to this possible error, that the substances taken are either not promptly absorbed from the intestinal tract or that after absorption they are deposited in the tissues to a varying degree. Despite this possible fallacy, renal tests of this type have been standardized and they appear to be valuable indicators of slight changes in the renal functions.

The second group of renal tests are those in which substances, more or less foreign to the body, are introduced into it and their elimination in the urine subsequently determined. Lactose, potassium iodide and various dyes have been used in this manner. In order to be of value such tests should give information concerning the common renal functions, or they should indicate specific structural changes in the kidneys. By far the most useful of these tests is the excretion of certain dyes, particularly phenolsulphonephthalein. We know that a marked inability to excrete this dye indicates, with few exceptions, a corresponding inability to excrete urea. On account of its simplicity and on account of the important information given by this test, it has become the most widely used of all functional kidney tests.

A number of methods have been proposed for testing the functional capacity of the liver. For example, phenoltetrachlorphthalein is excreted in the bile, and it has been proposed to measure the hepatic function by injecting this dye and then estimating the amount that appears in the feces. Other hepatic tests depend upon the ability of the liver to remove various sugars from the blood. In such tests a considerable quantity of the sugar in question is taken by mouth, and its concentration in the blood or its elimination in the urine is used to measure hepatic function. Tests of this type have been made with various sugars. Those made with glucose have not aided in the diagnosis of liver disease, apparently because other organs than the liver play a rôle in removing excess glucose from the blood. Similar tests with levulose and galactose have been more successfut, and alimentary levulosuria or alimentary galactosuria usually indicate some defect in the hepatic functions.

Hepatic insufficiency is rare in local disease of the liver, such as 
tumor or abscess; while it is relatively common in diffuse hepatic disease, such as advanced cirrhosis and catarrhal jaundice. When we remember that the margin of safety in the liver is a wide one it is easily understood that a tumor or abscess will rarely destroy enough tissue to lead to insufficiency, whereas any disease that affects all of the parenchyma cells may cause the above tests to be positive.

\section{PATHOLOGICAL PHYSIOLOGY AND THE TREATMENT OF DISEASE}

It is interesting to recall how the various fundamental medical sciences have influenced our attitude toward therapy. Pathological anatomy has furnished the basis for most surgical treatment. The draining of pus, the removal of foreign bodies and of diseased tissue, and the cutting or dilatation of strictures, all these are surgical procedures which depend upon a knowledge of the location and character of the structural changes. Upon therapy in a medical sense, however, pathological anatomy has exercised a depressing influence. One immediately encounters the question, what medicine can remove this or that structural change, and the negative answer is responsible for the therapeutic nihilism that has prevailed among those trained in the anatomical school of pathology. Trust in the healing powers of nature and help these by nursing and by hygienic measures is the familiar advice of this school.

With the study of infectious organisms, however, a more hopeful outlook dawned for the treatment of diseases due to this cause. Attempts to kill the infectious agents by chemical substances or by specific antisera, attempts to neutralize their poisons and attempts to discover and to remove local infections that might be the cause of chronic inflammatory changes elsewhere in the body have led to many brilliant advances in modern therapy.

In what way has therapy benefited from the physiological point of view? If we omit from consideration the use of antitoxins, vaccines and similar measures, there remain two main lines of treatment that are based upon the functional conception of disease. The first of these depends upon the principle that weak functions should be protected from strain by lessening the demands made upon them. The second consists in the correction of functional changes, so far as this is possible, whenever we are unable to remove the cause.

The demand upon a weakened function should if possible be kept within its capacity for performance. If this be done, the function, even though impaired by disease, can carry on the work required of it under the new and restricted conditions. Moreover, a weakened function tends 


\section{I68 PATHOLOGICAL PHYSIOLOGY AND MEDICINE}

to become less capable when it is overburdened, whereas it tends to regain some of the lost ground when it is rested.

This principle of suiting the work to the capability of an individual has a wide range of application. The moron, for example, with less than the average intelligence, is a failure if he is forced to compete with average persons in pursuits that demand average intelligence. Once his mental limitations are recognized, however, and tasks adapted to his native intelligence are assigned to him, he may become a useful though lowly member of society. The person who by reason of nervous instability breaks down under the strain of an active life often recovers during prolonged rest and may be able to resume work under less trying conditions.

In the treatment of cardiac decompensation, bodily rest, which reduces the work of the heart to a minimum, is of first importance. By lessening the requirements made upon it, the damaged heart is often able to restore a disordered circulation to conditions that approximate the normal. In favorable cases, moreover, the functional capacity of the heart increases during this rest. Exercises which formerly induced cardiac symptoms may now be carried on with no unfavorable effects.

The modern treatment of diabetes mellitus is based on a similar principle. The dietary restrictions are not carried out solely in order to reduce the excess of sugar in the blood and to stop losses of sugar in the urine. They are recommended in part because we know that if the strain on the sugar-burning function be removed, the patient's ability to burn sugar often increases. Hence we find that after a strict dietetic régime the diabetic patient can burn quantities of sugar which formerly escaped combustion; and after such treatment, patients who at first appeared to be suffering from a serious form of diabetes have improved to such an extent that they may be classified among the moderately severe or mild cases.

In nephritis the ability of the kidneys to excrete certain of the normal urinary constituents may be lessened. That a reduction in the intake of such constituents may lead to good results in practice is amply attested by the brilliant success which sometimes follows the reduction of salt in the diet of those suffering from renal edema. Such a restriction in the diet not only may make it possible for the kidney to excrete more sodium chloride than is taken in the food, but it may lead to an absolute improvement in the ability of the kidney to excrete this salt.

The removal of strain from an overburdened function has therefore become a recognized and important therapeutic procedure. We are much less certain concerning the value of the exercises that are sometimes prescribed for the purpose of strengthening a weakened function. Be- 
yond doubt, our first duty is to remove strain. Having accomplished this, the question presents itself whether exercise below the point of strain will improve the function. So far as diabetes and renal disease are concerned, we have no justification in practice for attempting to work the damaged function up to but short of the point at which we suspect a mild insufficiency will develop. Experience indicates that it is a safer rule in these diseases to reduce the work required to the lowest point consistent with general health. With regard to the heart, experience seems to indicate that in the milder cardiac disturbances, exercise. which causes no symptoms is usually harmless and may often be of benefit. Certain it is that the recognition of a cardiac lesion is in itself no indication for the absolute rest that is necessary to reduce the work of the heart to the lowest possible level.

With regard to the type of therapy that aims to correct functional changes, the simplest case is that in which a substance necessary to the welfare of the body is not present in sufficient quantity and can be effectively given with the food or by injection. In myxedema, in sporadic cretinism and in cachexia strumapriva, there is a deficient amount of thyroid secretion in the body, and brilliant results have been obtained by giving thyroid substance to such patients. Organotherapy has obtained no results comparable to this in the treatment of other diseases that depend upon deficient internal secretions. Nevertheless, ovarian and corpus luteum extracts have been used, in some cases with apparent benefit, in the treatment of symptoms associated with menstrual disturbances; and occasional reports of the successful use of other tissue extracts for deficient internal secretions have appeared.

When digestive disturbances are due to the absence of a digestive secretion, they are sometimes benefited by giving a substitute for this secretion. In gastric achlorhydria, the administration of dilute hydrochloric acid not infrequently has been followed by a lessening of gastric and intestinal symptoms. When no pancreatic juice enters the intestines, the serious loss of proteins and fat in the stools may be greatly lessened by taking pancreatic substance with the food. Preparations of bile have been given with apparent benefit for the digestive disturbances that accompany an absence of bile from the intestine.

After acute losses of blood and in circulatory collapse, where the quantity of blood appears insufficient to fill properly the vessels, the addition of fluid to the body, either by transfusion of blood, by the intravenous injection of suitable liquid or by the administration of saline solution per rectum, often has saved life. The beneficial effects of iron upon certain anemias, particularly those resulting from repeated small hemorrhages, appears to be due, in some cases, to a replenishment of 
the store of this element in the body. Certain goitres are believed to be caused by a continued lack of iodin compounds in the diet, and it is possible that the not infrequent reduction in the size of goitres during the administration of iodin compounds is due to the replacement of this deficit.

There are a number of diseases which result from the continued use of diets that are deficient in some organic element other than the usual proteins, fats and carbohydrates. Of these diseases the best example is beriberi, which usually occurs in those whose diet has consisted almost wholly of polished rice. This disease is preventable and frequently is cured by using a liberal diet or by merely adding rice polishings to the former diet. Scurvy belongs to this same class of diseases. It can be arrested and cured by adding fresh vegetables to the diet. Pellagra, also, appears to result from a food deficiency. Finally certain types of edema which have occurred during the great war and which disappeared when the diet was improved, particularly by adding fat, are believed to belong to this group of diseases that are caused by the absence of some necessary element in the diet.

The chemical products of excessive glandular activity can rarely be neutralized by medicine. Nevertheless, in the treatment of gastric hyperchlorhydria the excess acid may be neutralized by giving the alkaline carbonates and oxides. Such alkaline treatment not only lessens the symptoms of hyperacidity, but it may assist in healing a gastric ulcer by reducing the corrosive action of the gastric juice. In general acidosis, also, the administration of sodium bicarbonate serves to replenish the depleted carbonate reserve in the blood, and it may relieve the symptoms of acidosis.

Drugs are usually given, not in order to remove the causes of disease, but in order to improve certain functional disturbances which are present. The activities of the voluntary muscles and glandular organs are altered by a number of drugs. Some of these act directly upon the parenchyma cells, while others influence these cells through the medium of their vegetative nerve supply. Drugs of this class are widely used in medical practice. Digitalis, for example, improves the contractile power of the heart muscle, and it is employed in all types of cardiac insufficiency. In addition it lessens the conductivity of the His bundle. This latter action has a practical bearing, because on the one hand it may be disadvantageous to increase an existing partial heart block, while on the other it is of great advantage to slow the very rapid and irregular ventricular action which is often present in auricular fibrillation. Indeed, the most striking improvements following the administration of digitalis are observed in this last group of cases. Paroxysms of asthma are due 
to a spasm of the bronchial muscles, and drugs which relieve this spasm either through paralysis of the vagus supply (atropin and related drugs) or through stimulation of the sympathetic supply (epinephrin) often abort the paroxysms. The nitrites relax the arteries and arterioles, and they are often of signal benefit in relieving attacks of angina pectoris, especially when these are associated with heightened blood pressure. The arteries and arterioles are constricted by epinephrin administered intravenously and by subcutaneous injections of tyramine. These drugs have been used with some benefit in the treatment of acute hypotension. The motor functions of the large intestines are very susceptible to drug action, the constipating effect of opium and the cathartic action of many other drugs being well known.

Another group of drugs in common use alter the functional activity of central or peripheral nervous structures. These are widely used for the relief of headache and other pains for the production of anesthesia, for the reduction of general nervousness and in order to produce sleep.

It may be admitted that the ultimate object of therapeutic endeavor extends beyond the relief of symptoms and the correction of altered functions. It consists in the removal of the cause of a disease and in the restitution of the tissues to such a condition that they will function normally. This ideal is approached in many surgical procedures as well as in the treatment of certain infectious diseases by drugs that damage or kill the invading organisms without injury to the host. In the treatment of many other diseases, however, we are still far from attaining this ideal, either because we do not know the cause, or because the cause, though known, cannot be removed by any known means, or because irreparable changes have already taken place in the tissues. In all such cases, our main therapeutic objective is the correction or the improvement of functional disturbances. By accomplishing this we at least render the patient more comfortable during the course of his illness, and at best we restore him to a useful place in society, where he is able to accomplish much despite his deficiency. 
CHAPTER IV

\section{HYDROTHERAPY}

\section{By Guy Hinsdale}

HydRotheRAPY is a very ancient method of treatment, but only comparatively recently has it been constituted as a science. With latter-day equipment it has become one of the most effective of all measures known for combating disease. Hydrotherapy has the advantage over medicinal or, specifically, drug therapy, that it is applicable to those in good health as well as to those in poor health, very often with admirable effect on the sense of well-being, enhancing mental as well as bodily efficiency. Water and air and places properly chosen and judiciously utilized are of untold value and have been described, advocated and used since the time of Hippocrates, whose work on Airs, Waters and Places is a medical classic. The early beginnings of hydrotherapy have been referred to in work of the author $\left({ }^{1}\right)\left({ }^{2}\right)\left({ }^{3}\right)$ published during the last ten years, and the history of its past need not be reviewed again at this time.

With others we have urged a wider use of waters both externally and internally, and have advocated utilizing the rich natural resources which are afforded in America, resources such as have been used for years abroad. Hydrotherapy and balneology go hand in hand. Hydrology is perhaps a better term, as it includes the use of water whether used internally or by baths in mineral springs or at the seaside, or pure as it comes from a public water supply. $\left({ }^{4}\right)\left({ }^{5}\right)$ Whether we bathe at the seashore or at spas, or take a course of baths at hydrotherapeutic establishments in cities, it is of prime importance to know the probable effects of such applications on the individual, whether well or the subject of disease; not forgetting that vast group who occupy an intermediate stage between good health and actual illness, a class that furnishes probably the greater proportion of visitors at our spas and health resorts. Without them many of these would close their doors.

It will be convenient to note three phases of treatment from the standpoint of temperature in relation to the body: First, plus applications of heat; secondly, plus applications followed by minus; thirdly, minus applications. (Fortescue Fox, q. v.) It will be noticed that there 
is no provision for heat after cold; it would reverse the rational order and check the cultivation of the reactive powers of the body-powers subject to development and training just as muscular or mental faculties can be trained. This power of response to applications of heat and cold and the production of secondary consequences in the entire organism are, as Fox well says, the key of hydrotherapy. Baruch lays great stress on the same principles under the name of neurovascular training. We speak of baths and associated measures as educational; they draw out and develop dormant powers just as education, in the usual sense, draws out the mental and moral faculties.

How is one to estimate in advance the reactive power of the body to heat and cold, disregarding for the present the effect of the pressure of associated douches and manipulations, the time element, or the psychic effects which attend measures new to the patient? Even in health our reactive powers vary; the time of day makes a difference. One does not, for instance, perspire as readily on rising from sleep as he does at the end of the day, or after exercise and mental activity. A bath before a meal may be safe and proper, but within an hour or so after luncheon or dinner, or when greatly fatigued, it very likely would . be injurious. In disease, too, these variations are commonly noted.

In balneological treatment, as at mineral springs, painful reactions often follow a very mild bath; gouty and rheumatic joints and even the soft tissues redden, swell and become painful so that, unless forewarned, the patient may be discouraged. Experience at any of our spas teaches that such reactions commonly attend the mobilization of exudates, and that this activity foretells the ultimate dispersion and elimination of the materies morbi. We may give some warning at the outset that the subject of gout or rheumatism may be worse before he is better and that processes adopted for his cure must not be judged by their immediate effects. We must instill hope for an ultimate recovery when all these trying ordeals will have been forgotten.

The psychic influences of hydrologic treatment and the necessity for courage and persistence on the part of the patient, and no less on the part of his medical attendant, are prime factors in the cure if it is to be successful. The mental attitude of the patient should be hopeful, and we usually find it so. Obviously it is a disadvantage in any cure to be burdened with affairs of business or to be led into social dissipations. We consider it decidedly detrimental in our modern resorts that long hours should be spent in watching by day the fluctuations of the stock market and by night the flickering screen with exciting scenes of warfare, crime and vice. The Roman visitor in search of health as he approached the baths of Caracalla read these famous lines: 
Curae vaccuus hunc adeas locum

Ut morborum vacuus abire queas

Hic enim non curatur qui curat.

\section{"Light of heart approach the shrine of health So shalt thou leave with body freed from pain For here's no cure for him who's full of care."}

Some patients seeking hydrotherapeutic treatment are very apprehensive and they already may have had unpleasant experiences at home and abroad. It will be well with each patient at the outset to inquire whether there are any idiosyncrasies as to heat and cold, or to any other features of bathing, so that we may at least consider these and proceed cautiously. One cannot tell in advance always what the reaction will be; the best we can do will be to examine the heart and consider its muscle and its valvular action, to measure the arterial tension, diastolic and systolic, and to note the state of the skin, the general muscular tone and the mental temperament, and then adopt moderate methods, advancing, if necessary, to those more positive and introducing after two or three days, if the case warrants, such associated measures as massage, Zander exercises or exercise out of doors. Of course we are now speaking of cases of chronic disease, or at least of patients who are able to be about. Acute febrile diseases and their hydrologic treatment occupy quite a different field of hydrologic practice and their needs and limitations will have to be met accordingly.

The advantage of hydrotherapy is that it is flexible, adjustable to individual needs, that it brings out inherent recuperative powers, employs the simplest element in the physician's armamentarium, that it can be employed universally and has stood the test of time far beyond all other measures.

\section{SPAS}

Balneology includes not only the internal administration of waters, but also bathing in mineral springs. These uses of water are almost always associated, and different observers from time immemorial have attached different values to these two therapeutic methods. At most spas both modes are utilized. Their combination in a given case usually gives the best result. At resorts like Saratoga the internal use of the spring waters takes precedence, while at Mount Clemens, Michigan, and White Sulphur Springs, West Virginia, baths chiefly are relied upon. At Hot Springs, Virginia; French Lick, Indiana, and Aix-les-Bains and Vichy in France, and at most British Spas, the combined method prevails. 
It is rather remarkable that in the three great resorts for the treatment of gout and rheumatism in the United States these affections have been treated with great success for many years with baths of widely different character. Take, for example, the analysis of waters at Hot Springs in Virginia and in Arkansas and the cold waters of Mount Clemens, Michigan. These waters are totally different from each other in chemical analysis and molecular concentration. If we assume that approximately equal results are achieved by a course of bathing in waters so dissimilar, the chemical constituents of which do not permeate the uninjured skin, we cannot avoid the inference that the chemical constituents of these different springs are not per se the active therapeutic agents. That certain baths should be used for a case of syphilis and the same for a case of gout with good result ought to suggest that the hydrotherapeutic or balneologic methods employed are more potent than the fact that the waters employed have a special chemical analysis. The differentiation of mineral springs for therapeutic purpose has some practical basis, but we are inclined to question whether it has not been carried too far, especially in European practice. Such a problem cannot be settled offhand or decided arbitrarily. It is well known that the French for many years have insisted on specialization in balneological practice, and their best men, such as Bardet, Durand-Fardel and Albert Robin, strongly uphold the importance of the chemical constitution of the springs.

France is wonderfully rich in mineral springs, and they have been utilized since the time of the Roman occupation, when Vichy, Plombières, Mont Dore and Aix-les-Bains were highly prized for their curative baths. The sick and wounded in the Allied armies are now afforded spa treatment, just as the soldiers of Napoleon were treated, or as the Roman soldier was bathed and cured twenty centuries ago.

There is special need now, particularly in America and England, of more definite knowledge of the French spas and their individual adaptation to particular affections. To begin with, they are either under government ownership or under strict government control. Since 1800 the Commission des Eaux Minérales has had the official direction or oversight of all French mineral spring resorts. In I912 the Institute of Hydrology and Climatology was founded and is attached to the chair of medicine of the Collège de France. It maintains five laboratories, including those of physical hydrology, thermal and climatic hygiene, physical and hydrological chemistry, hydrologic and climatic therapeutics and general hydrology. The whole field is covered and is a part of the national administration. 
In French resorts the treatment, and consequently the specialization, is based directly on the composition and physical properties of the different springs, the associated physical measures and the various methods of applying the waters being considered merely adjuvants and not the primary influence in the cure. There is, consequently, in France, a distinct specialization according to the properties of the mineral water, with special appliances for the rational employment of the waters and complementary means systematically adapted to the therapeutic indications of the resort. Thus medicinal applications of the water as mineral water are reinforced and completed by the employment of the necessary physico-therapeutic agents in view of the predominant specialization. The specialization of spas is due in part to the knowledge and exertions of local doctors and attendants; their special studies and skill in practice have added to their popular repute in the treatment of particular affections, with the result that in the course of time a certain reputation is acquired and recognized by a grateful clientele. This is no small factor, and whatever the cause of the specialization, it is a real condition and not a theory with which we are concerned.

However, just as we are relegating gradually to the background the idea that there is a special medicine for every disease, so we do not hold, nor do the French hold, that in spa treatment we must limit a given water to the treatment of any single disease. We can often treat successfully several groups of affections at the same resort, as we are accustomed to do quite generally in America. Nevertheless, among these affections there is always one more amenable than the others to the particular course of treatment and, according to Bardet, that should be taken as the type of the specialization. Durand-Fardcl, and after him Robin, distinguished the dominant note referred to, relegating to a second rank the ordinary applications, and lastly admitting certain secondary indications.

In regard to sulphur waters, for example, herpetic disease and catarrh of the air passages are accorded first place, and in the secondary category are lymphatism, rheumatism, chlorosis, syphilis and scrofula, and finally to chronic affections of the uterus and catarrh of the urinary tract and dyspepsia. $\left({ }^{6}\right)-\left({ }^{11}\right)$

The chemical classification of the French springs is as follows:

Sodic Sulphide-High temperature: Ax, Luchon, Eaux-Bonnes, Barèges, Amélie-les-Bains, Cauterets, La Preste, Challes, Le Vernet, Saint Sauveur.

Calcic Sulphide-Saint Honoré, Allevard, Enghien.

Sodic Chloride-La Mouillière, Besançon, Dax, Salies-de-Béarn, Biarritz, Salies-du-Salat. These are rich in salt. Others containing 
2,000 to 16,000 parts per million are Luxeuil, Bourbonnes-les-Bains, Bourbonne-Lancy, Bourbonnes-l'Archambault, Balaruc, Uriage.

Sodic Bicarbonate-These contain heavy metals and may be arsenical; Vichy, Châteauneuf, Vals, Le Boulon.

Calcic Bicarbonate-Alet, Foucade, Ganties, Pouges, Saint Galmier, Saint Alban, La Malou.

Bicarbonate Chloride, Arsenical-La Bourboule, Royat, Mont Dore, Saint Nectaire, Vic-sur-Cère.

Bicarbonated Chloride, Sodic and Magnesic-Chatel-Guyon.

Sodic and Magnesic Sulphate-Montmirail.

Sulphated, Mixed-Brides-les-Bains, Saint Gervais, Miers, Cransac.

Calcic Sulphate, Cold-Vittel, Contrexéville, Martigny. Hot-Bagnères-de-Bigorre, Capvery, Barbazan Hammam R'Irha.

Ferrous Sulphate-Rennes-les-Bains.

Radio-active Waters-Aix-les-Bains, Dax, Néris, Plombières, Ussat. Muds-Dax, Saint Armand, Barbotau, Balaruc.

\section{Therapeutic Classification}

Vichy-Diseases of the liver.

Chatel-Guyon, Plombières-Diseases of the intestine.

Pougues-les-Eaux, Vals-Diseases of the stomach.

Mont Dore-Asthma.

La Bourboule-Dermatoses, adenopathies.

Royat, Bourbon-Lancy-Arterial hypertension.

Aix-les-Bains, Bourbonne-les-Bains, and Bourbonne-l'Archambault - Articular rheumatism.

Luxeuil-Gynecological affections.

Evaux-Amenorrhea.

Néris-les-Bains-Neuralgia, neuropathies.

Uriage--Syphilis.

Evian-les-Bains, Vittel, Contrexéville, Martigny, and CapveryArthritis, bladder and kidney diseases.

Saint Nectaire-Albuminuria.

La Malou-Locomotor ataxia.

Bagnoles-de-l'Orne-Phlebitis.

Brides-les-Bains-Obesity.

Ussat-Exophthalmic goitre.

Saint Christau-Psoriasis.

Forges-les-Eaux-Chlorosis and anemia.

Luchon, Cauterets, Eaux-Bonnes, and Amélie-les-Bains-Respiratory diseases.

Saint Amand, Barbatau, Dax and Balaruc-Arthritis. 
La Bourboule and Mont Dore in France have been selected as "leave areas" for members of the American Expeditionary Forces. These are mountain resorts in the department of Puy-de-Dome, on the banks of the Dordogne River, in the valleys of the Auvergne Mountains, in the center of France. La Bourboule has an elevation of 2,780 feet and Mont Dore, four miles further up the Auvergne River, has an elevation of 3,440 feet above sea level. These places have been celebrated since the days of the Romans for their hot springs.

The waters of $\mathrm{La}$ Bourboule are classed as sodic chloride, bicarbonated, and contain an appreciable amount of arseniate of sodium. They range from $118^{\circ} \mathrm{F}$. to $130^{\circ} \mathrm{F}$. in temperature. The waters of Mont Dore are about one-third as strong in alkaline salts, and the arsenic content is very much less; they also contain some bicarbonate of iron.

The La Bourboule waters are employed for drinking, for baths and douches, and for pulverization and inhalation. This last is accomplished by allowing the water to drop from a height into the center of the bathroom so that some of it is inhaled at the same time as the vapor. The patients remain for about half an hour seated in the inhalation chambers dressed in large bath towels or dressing gowns. A foot bath is often prescribed to be taken while inhaling. This method of inhalation and "pulverization," which is not uncommon in France, is not employed as far as we know in America, although there is some approach to it in the natural steam caves of the Arrowhead Hot Springs of California.

The waters used in this manner have favorable effect on affections of the respiratory system. The arsenic and iron are of undoubted therapeutic value. The patients who patronize these resorts have always included many with chronic laryngitis and bronchitis, and Mont Dore has a special reputation for the treatment of asthma, particularly when complicated with chronic bronchial catarrh. The mountain climate and very attractive surroundings, together with excellent accommodations, will go far to restore our soldiers, and particularly any who have suffered from gases on the battle front. Owing to the altitude there will be good opportunities for winter sports, such as skiing and snowshoeing, although these places have not had as great vogue as winter resorts as those of Switzerland.

British and American waters have also been classified on a chemical basis, but the therapeutic application has nowhere been so closely differentiated as in the case of the French springs. In the case of British spas the following has been suggested: 


\section{THERAPEUTIC CLASSIFICATION}

Harrogate-Biliousness and gout.

Bath-Anemia and nervous debility.

Tunbridge Wells-Anemia.

Droitwich-Gout, nervous debility and neuralgia.

Cheltenham-Gout.

Leamington-Diseases of the liver.

Llangammarch-Nervous debility.

Malvern-Neurasthenia and asthma.

Woodhall-Rheumatism.

Buxton-Sciatica.

Shanklin-Eczema.

\section{EFFECT OF COLD BATHS ON METABOLISM}

Tissue changes brought about by cold baths are caused by an effect on the innervation of the muscles; in fact, all combustion processes in the body are referable to the muscles. A simple hot-air bath may have little effect, but a series of hot-air or hot-water baths increases nitrogen elimination, urea elimination keeping pace with the excretion of nitrogen, while uric acid also is excreted in greater quantity.

Elaborate studies of these metabolic changes have been made by many observers in connection with ordinary hydriatric procedures, the half-bath, the Scotch douche, etc. It is interesting to note that Hippocrates states that the temperature elevation which occurs in connection with most acute infectious diseases is, within limits, remedial in purpose and effect. It apparently follows that temperature-elevating baths may be beneficial in aiding resistance to infection, especially when followed by a short cold bath, by favoring the production of alexins and antitoxins. It seems to us, however, that cold baths are better, practically, in the infectious fevers-witness the brilliant results obtained from the Brand treatment of typhoid fever.

The effect of the carbonated bath on metabolism would naturally be expected to be most pronounced, consisting, as it does, of solid and gaseous elements in large quantities. Improved assimilation and tissue metabolism is evidenced by formation of fresh muscle substance. In the carbonated bath the area of the dilated heart is diminished, but its power and true volume are enhanced. Superfluous fatty tissue and inflammatory residual products disappear, effete products are eliminated, and a loss of weight is commonly noted at the outset. Soon substantial evidences of constructive metabolism begin to be noted, and 
continue long after the patient ceases to take the course of treatment provided in accordance with this system of hydrotherapy.

The acceleration in tissue metabolism, aided as it probably is by osmosis, promotes the absorption of exudates in the joints and pelvic and serous cavities. Carbonated or effervescent baths are among the most efficient means of abolishing the residues of rheumatism and gout, of serous inflammations, such as pleurisy and pericarditis, and of inflammatory conditions of the adnexa of the female genital organs.

Turkish baths tend toward producing a gain in weight, but if the heating process is shortened, and cold water is applied liberally in connection with the plunge, the subject loses because the loss of heat has to be made good by increased catabolism in the tissues.

Short, cold shower-baths increase heat elimination from 25 to 66 per cent., even more if mechanical friction be carried out, and the flow of respiratory air is increased nearly three times in the half-bath. Increased vigor is undoubtedly the result of the procedure, but it should be remembered that if the water be at the higher temperatures, as from $80^{\circ}$ to $90^{\circ} \mathrm{F}$. $\left(26.7^{\circ}-32.3^{\circ} \mathrm{C}\right.$.), a corresponding increase in the friction is required to produce good circulatory reaction. By raising the temperature of the water and communicating heat to the body, distinct effects on metabolism are produced. Carbon dioxid is exhaled at a much more rapid rate, and this is also true of the application of cold water.

Short, cold baths, in which actual heat loss is small, cause a fairly marked increase in chemic metabolism just like the longer ones, only there is a less heat deprival, due directly to the movements which the baths set up. The heat production due to muscular activity exceeds and obscures the catabolism due to cooling, or in practice renders it quite negligible, except under very unnatural conditions. It is impossible to determine the extent of this increased catabolism accurately, because no method can be devised which will deal with muscular activity alone. Shivering and active movements in connection with cold baths are thus natural aids in the regulation against cold; they produce heat, favor and call forth chemic regulation, and usually fully compensate, or may overcompensate the heat loss.

Cold, per se, reduces the oxidizing capacity of animal tissues. Warmed tissues have a much higher oxidizing capacity. In order to maintain a constant normal temperature, we are compelled in the presence of cold to call more vigorously than usual on our heat-regulating powers, so as to increase our oxidative activity. Cold applications have a good moral effect in cultivating will power. While the effect of such stimulations may be, for a time, unpleasant, particularly at the 
outset, the patient soon feels their benefit and is encouraged to continue them. Encouragement, suggestion, firmness, and kindness from the attendant and the personal interest and presence of the physician will do much at this stage to secure the coopperation and confidence of the patient and his continuance in the prescribed method of treatment. Physicians do not always realize the mental anguish which some poor creatures undergo when they begin a new and, as it appears to them, a formidable course of hydrotherapy. Some patients are hopeful and confident, others are apprehensive and distrustful. These latter must be made to do something besides sit around bemoaning their condition, and bathing is useful in occupying their time; they derive at the same time the benefit of the baths in the way of improved circulation and improved processes of metabolism. The baths take the patient completely out of himself for the time being, and though the patient does not usually like them, he feels that something active is being done for him. In different cases a good deal of personal persuasion is required until a reasonable attitude is assumed by the patient toward the prescribed treatment. Of course, in institutions a better control of the patient obtains than in treatment in private practice. At the McLean Hospital at Waverley, Massachusetts, Dr. Tuttle had a patient who was possessed with the delusion of demoniacal possession, and after realizing that he was being improved wrote this: "Your baths are excellent to reduce cerebral excitement. You can't fight the devil with fire, he is in his element there, but he is mortally afraid of cold water."

Undoubtedly, cases will present themselves in which it is impossible even for those of experience to foretell what the effect of hydrotherapeutic measures will be. Especially is this true in disorders of psychic origin, or when patients are sent by physicians who have been familiar with their history, to other physicians who may not know the idiosyncrasies and peculiarities of the patient thus despatched to a sanitarium or health resort. Patients must be studied individually in the light of a full letter of advice, and time must be taken, with moderate procedures, to arrive at a proper estimate of the best course to follow. Not infrequently neurasthenic patients wish to discontinue ireatment after the first or second bath has been taken. In many neurasthenic women a feeling of exhaustion comes on a short time after the treatment and persists the remainder of the day. This is usually regarded both by physician and patient as evidence that the hydrotherapeutic procedure has been too strong, but, as this temporary exhaustion develops after the mildest treatments, and as it almost invariably disappears after the second or third treatment, the sensation of weakness is undoubtedly due in the great majority of cases to the nervous strain of the visit to 
the institution. The surroundings are new and the appliances are strange and somewhat formidable in appearance. All these factors act to exhaust the patient.

In using a powerful agent like hydrotherapy to modify physiological and pathological processes, and the no less important psychic states, we should remember that the whole effect of baths of every description is founded on the power of reaction possessed by the tissues. Sometimes this is very limited at first, but it can be increased in a remarkable manner. As Fox well says, the power of response of tissues to applications of heat and cold, and the production of secondary consequences in the entire organism, are the whole key to hydrotherapy. "This reaction in different individuals and in different maladies, and even at different times in the same individual and in the same malady, is a most variable quantity. It follows that in practice two things are above all necessary; in each case first of all is determination, so far as may be, of the reactive power, which usually can only be made by cautious experimental methods; and, secondly, a nice adaptation of the bath or application to this individual power. A dormant or defective reactive power can be educated and strengthened to a remarkable extent so that as treatment progresses stronger and stronger stimuli can be employed."

"There is in every case a point at which heat or cold ceases to act as a stimulant, that is, to evoke a healthy reaction, and beyond which effects of quite different order are produced. The first order of effects is helpful; the second order injurious and depressive. The critical moment is sometimes a point of temperature, but more often a point of time for the application of the same stimulus." As Fox well says, the crux of bath treatment consists in the ascertainment and observance of this law, and it is more difficult because the danger point, following the reactive power, varies from time to time in the same individual and in the same malady. To meet these conditions practically, Dr. Simon Baruch devised his plan of neurovascular training which he has taught and practiced for many years. (For description of this see page 212.)

\section{THE EFFECT OF HOT BATHS ON METABOLISM}

In the case of hot baths there is no mechanism, corresponding to the increased oxidation by which lack of heat is compensated for, whereby the organism can reduce its heat production or decrease its metabolism. When exposed to heat or when heat loss is prevented, the body temperature is governed only by physical regulation; that is, it is a question of evaporation. If this is checked, the body temperature neces- 
sarily rises. It can still go on rising for a short time after the end of a hot or vapor bath, because the thoroughly heated skin must for a time continue to act as though it were still in the hot bath. This, however, is adjusted in the course of an hour or two, and afterward a slightly subnormal temperature and a slight secondary rise has been noted. This fall after hot baths might be expected to be greater than it is, considering the fact that the skin is left in such a highly vascular state.

Hot baths, without the usual water-drinking, diminish the urine, since they promote sweating. Cold baths increase the amount of the urine temporarily. This, however, is usually quickly compensated for. The increased secretion depends on the raised blood pressure due to cold. Experiments show that as much as one quart of sweat may be lost in a prolonged hot-air bath, and it can be readily understood that this abstraction of fluid must affect the entire circulation of blood and lymph, and undoubtedly aids in the elimination of morbid products. The abnormally high concentration of blood present in renal insufficiency can be reduced by sweating, and in these cases the normally low nitrogen content and the molecular concentration of sweat is thereby considerably increased. The increased secretion of sweat is always sought in the elimination of various poisons, including lead, mercury, and bacterial toxins.

\section{CONTRAINDICATIONS TO BATHS}

A positive contraindication to such measures as the carbonated bath, for instance, is any acute or subacute condition of the heart, and, on the other hand, the loss of compensation, as in chronic heart disease. In aneurysm much harm can be done. It is highly necessary to satisfy one's self that these conditions do not exist before prescribing the more stimulating measures. Palpation and percussion of the heart and the use of the sphygmomanometer are good routine methods before prescribing hydrotherapy.

\section{EFFECT OF BATHS ON THE RATE OF THE HEART AND ON THE BLOOD}

The most striking results on heart rate are noted under the influence of the hot-air bath followed by cool douches. In the hot-air cabinet at a temperature of $170^{\circ} \mathrm{F}$. $\left(76.66^{\circ} \mathrm{C}\right.$.) the author has noted a rise in rate from about 70 to 120 per minute, and as the douche was cooled to $65^{\circ} \mathrm{F}$. ( $18.33^{\circ} \mathrm{C}$.), the pulse fell rapidly. A drop of 60 beats inside of five minutes or less has been noticed, and, as the patient was rubbed, the rate rose to a little above normal. Such changes in the rate and slight changes in the rhythm should be expected. They illus- 
trate the quick response which the circulatory system makes to the stimulus of water applied in this manner.

There is, as it were, a conflict between the center and the periphery for the possession of the blood, and the influence of hydrotherapy in promoting a rapid interchange is doubtlessly more significant than any particular change in the ratio of its various histologic elements.

In general procedures in which thermic and mechanical stimuli of not too intense a type are applied to the entire surface of the body, with very few exceptions, there is not merely a diminution of the leucocytes, but an increase of the number of the red blood cells when a test is made from the finger tip or the ear. The hemoglobin may also be increased. Moderate procedures are thus favorable for anemic patients. Warm packs and compresses tend to increase the leucocytes and diminish the red cells.

\section{EFFECT OF BATHS ON BLOOD PRESSURE}

Every hot or cold douche calls forth an increase of blood pressure, paradoxic as it may seem. Cold baths contract the capillaries of the skin, there is a slower but more vigorous contraction of the heart and consequent rise of pressure. Baths between $95^{\circ}$ and $104^{\circ} \mathrm{F}$. $\left(35^{\circ}\right.$ and $40^{\circ} \mathrm{C}$.) produce a primary rise, a secondary fall below normal, then a rise. Baths above $104^{\circ} \mathrm{F}$. $\left(40^{\circ} \mathrm{C}\right.$.) increase the pressure and also the pulse rate, the pressure remaining high. In baths accompanied by mechanical excitation the pressure is augmented, but returns easily to normal.

Carbonic acid baths between the temperatures of $84^{\circ} \mathrm{F} .\left(29^{\circ} \mathrm{C}\right.$.) and $89.6^{\circ} \mathrm{F}$. $\left(32^{\circ} \mathrm{C}\right.$. $)$ do not lower a pathologic hypertension. Above the latter figures there is a marked fall, which, however, tends to rise even above the original tension after the bath; but this secondary increase is not permanent.

It follows that in the application of an agent which affects the blood pressure so noticeably care should be taken not to aggravate any state of the system that cannot bear such an influence. We should carefully note any weakness of the heart or blood vessels, so as to avoid any accident, for undoubtedly accidents have occurred through neglect of this precaution. Longevity is a cardiovascular question. To a majority death comes primarily or secondarily through this portal. For that reason the greatest care should be exercised at all times, but especially after middle life, and in those using alcohol and tobacco to excess, to see that the circulatory organs are preserved and not weakened by sudden and unusual strain. The blood pressure should be carefully recorded in all cases. 


\section{EFFECT OF COLD AND HEAT UPON RESPIRATION}

The cold douche produces at first short, gasping respiratory movements, a sudden cold stream under high pressure producing the most marked effect. A full cold bath produces after the first few moments slow, full and deep respiratory movement. The cold douche or spray accelerates the respiratory rate. Dry heat retards the respiratory gaseous interchange, increases the rate of respiration, but at the same time renders it less efficient and deep.

During a hot bath, although the rate is increased, the respirations gradually become deeper. The skin's action is increased, but there is a corresponding elimination through the lungs of much moisture and toxic material.

\section{PHYSIOLOGIC EFFECT OF THE CONTINUOUS BATH}

Captain Strecker's observations of the body temperature, pulse rate and respiration, recorded over a period of six months in the continuous bath, showed practically no variations from the normal. The blood pressure is not essentially influenced. There is some experimental and considerable empiric evidence to prove that the continuous bath acts as a mild hypnotic agent, and that, unlike drugs, it induces physiologic fatigue without the sacrifice of mental capacity. It is extremely likely that the excretory function of the skin and kidneys is stimulated. This is an exceedingly important consideration, as a large percentage of mental patients, for at least a week following admission, present problems principally of checked secretional activity.

\section{PRESCRIPTIONS FOR HYDROTHERAPY}

Success will never be obtained in hydrologic practice unless physicians know how to order and take the trouble to write out definite prescriptions for this form of treatment. Verbal directions or vague orders and a virtual consignment of patients to bathing attendants are justly open to criticism. Bath prescriptions should be as exact as those for drugs.

The prescription should state some of the following points:

I. Method to be employed.

2. Time of day.-Sedative baths as a rule should be taken at night before retiring or in the afternoon. At spas sedative baths can rarely, if ever, be given at night, inasmuch as the bathing establishments are not open and the attendants could not be expected to work so late; but in household practice sedative measures may usually be given at a later hour. Stimulating baths, that is, those involving colder 
applications, are usually best taken in the morning. However, a great deal depends on the time of year and what it is desired to accomplish. It is very important to forbid all bathing within one and a half to two hours after the close of a meal.

3. The temperature and quantity of the water.

4. The duration of the bath or douche.

5. The pressure of the douche ordered.

6. The nature and duration of the compress or pack ordered.

7. The special form of douche, friction or exercise used to assist reaction.

8. The length of time subsequently to be devoted to rest.

9. The number of treatments and time interval.

Io. General directions as, for example, avoiding bathing for at least an hour and a half after meals.

In regard to temperature, we may designate the following ranges for the full bath: Cold, $50^{\circ}$ to $75^{\circ} \mathrm{F}$.; tepid, $75^{\circ}$ to $95^{\circ} \mathrm{F}$. $\left(24^{\circ}-35^{\circ} \mathrm{C}\right.$.) ; warm, $95^{\circ}$ to $104^{\circ} \mathrm{F}$. $\left(35^{\circ}-40^{\circ}\right.$ C. $)$; hot, $104^{\circ}$ to $114^{\circ} \mathrm{F}$. $\left(40^{\circ}-\right.$ $45.6^{\circ} \mathrm{C}$.).

\section{TYPHOID FEVER}

The systematic use of water in cases of ship fever or typhus apparently dates from the time of Dr. Robert Jackson, who used warm baths, followed by cold affusions, about I774. Dr. Wright, who practiced in the West Indies, applied the same treatment to himself and a companion suffering on shipboard about $\mathrm{r} 777$. In I797 James Currie, an English ship surgeon, advocated strongly the use of cold baths in all cases of fever, and practiced it successfully on shipboard. He used affusions of salt water. His writings influenced Nathan Smith, of New Haven, who practiced it in 1798 , very shortly after its introduction by Currie. In 1802 Dimsdale published in London "An Account of Cases of Typhus Fever in which the Affusion of Cold Water has been Applied in the London House of Recovery." During that time typhus and typhoid were not differentiated.

Credit also is due to Dr. Hiram Corson, of Montgomery County, Pennsylvania, who for more than fifty years treated typhoid fever, as well as other infectious diseases with high temperature, by means of cold baths. This remarkable man, who was graduated from the University of Pennsylvania in 1828 , practiced cold water bathing notwithstanding the lack of any general sympathy at that time with a method which has since become popular, and he obtained success even with all the disadvantages attending country practice.

It is a mistake in treating fevers by hydrotherapy to apply very low 
temperatures, very slight mechanical stimuli, and only for a short period. Such applications, according to Winternitz, raise the temperature instead of reducing it. Long cool, not cold, baths, rather intensive mechanical stimuli, tranquil rest under adequate covering after such a bath, and its repetition at the proper time are the necessary factors. By paying attention to the body temperature alone the physician is apt to err by too frequent and too cold baths in the severe infectious diseases. In this manner nervous disturbances may be engendered. By longer intervals, slightly warmer water, and longer baths these complications are avoided.

In typhoid fever a very dicrotic and rapid pulse is more important as an indication for repeating the baths than a high temperature. A very rapid pulse with a still vigorous heart calls for wet packs, repeatedly changed, which in case of a slow pulse and signs of weakness on the part of the heart would be a serious blunder. The behavior of the blood vessels is the guide for the choice of the temperature of the bath and for the length and degree of the mechanical stimulation. When the vessels display a paralytic tendency, the most energetic thermic stimulation is demanded with avoidance of much mechanical stimulation of the skin. This may be accomplished by brief dips and douches of quite cold water.

Important guides besides the thermometer. While it seems necessary to formulate certain rules as to tubbing, especially in hospital practice, it must not be forgotten that there are important guides besides the thermometer, for, as Gilman Thompson says, this instrument only records the average or temporary balance of heat gain and heat loss. Excessive heat production, if accompanied by proportionally rapid heat loss, may give a low thermometric record, and yet the obscure abnormal metabolism producing the increase in heat may be working great havoc within the body. This fact may in some degree account for the exceptionally rapid emaciation in a certain class of cases in which the thermometric record remains low throughout; for heat production in the body is mainly due to chemic processes, its loss to physical process. During the Franco-Prussian War a large number of fatalities from typhoid fever were reported in a series of cases in which the temperature did not reach $102^{\circ} \mathrm{F} .\left(38.8^{\circ} \mathrm{C}\right.$. $)$.

Rubbing. The intense involvement of the nervous system in severe cases is shown by the early headache and disturbance of special senses; by restlessness, insomnia and delirium; by subsultus, tremor, and the whole train of familiar symptoms. It is here that sudden cold and vigorous rubbing are demanded. Indeed, rubbing may be the keynote to the whole system of tubbing. For the full use of rubbing Thompson 
prefers a full bath tub, so that the patient may float and all surfaces of the body be easily accessible.

Enthusiasm for hydrotherapy should not lead one to deprive the patient of sufficient rest, and except in cases of a temperature protracted in the neighborhood of $105^{\circ} \mathrm{F} .\left(40.6^{\circ} \mathrm{C}\right.$. $)$, it is best to omit at least one of the three hourly baths at night, and if desirable to give a mild hypnotic. The number of baths given in a single case may* reach the extraordinary number of 175 . This record was made by Kinnicutt in a case of such exceptional gravity and hopelessness that the question of discontinuing the baths was discussed, but it was decided to continue them. The patient made a good recovery.

It cannot be too often reiterated that the main object of the cold bath in typhoid fever or in any other febrile disease is not the reduction of temperature. The antipyretic quality of the cold bath, cold affusion, or cold spray is entirely subordinate to the general effect on the nervous system, the heart, the lungs, the blood, and its circulation. The cold bath cannot be considered alone, without the all-important friction, the "sine qua non" of the cold bath. Even in such a serious affection as sunstroke, in which it is highly essential that body temperature be reduced, the good effect of the use of cold water in the form of sprinkling and cold affusions is accomplished only by the vigorous stimulation of the nerve centers, inducing an added power to withstand the toxemia.

The good effects of the bath in typhoid fever are seen not only in the reduction of excessive temperature, but also in a clearing of the intellect, a lessening of the stupor, a disppearance of the muscular twitching, a general tonic influence upon the nerves, and especially upon the heart and lungs, in an improvement of all of the bodily functions, in the promotion of sleep, and finally, in a reduction of the mortality.

The hydriatric treatment of typhoid fever may be employed at any stage of the disease and at any period of life. The best results are naturally secured the earlier in the attack the patient is placed under treatment. Not all children bear the full cool bath well. In patients past sixty it is best to use the gradually cooled bath, the water being at first about $10^{\circ} \mathrm{F}$. $\left(3.4^{\circ} \mathrm{C}\right.$.) lower than the temperature of the body, and gradually reduced in the course of from twenty to thirty minutes to $68^{\circ} \mathrm{F}$. $\left(20^{\circ} \mathrm{C}\right.$. $)$. The only complications that interdict the continuance of the baths are intestinal perforation, peritonitis and free hemorrhage. Experience shows, however, that complications are, in general, less common in those treated according to this plan than in those treated by any other method. $\left({ }^{12}\right)$ A common practice in typhoid fever is to apply an ice-cap to the head when the patient's temperature reaches IOI $^{\circ} \mathrm{F}$. $\left(38.3^{\circ} \mathrm{C}\right.$.); cool sponging when it reaches 
$102^{\circ} \mathrm{F}$. $\left(38.9^{\circ} \mathrm{C}\right.$. $)$; and a tub bath at $70^{\circ} \mathrm{F}$. $\left(2 \mathrm{I} . \mathrm{I}^{\circ} \mathrm{C}\right.$.) when the patient's temperature is $103^{\circ} \mathrm{F}$. $\left(39.5^{\circ} \mathrm{C}\right.$. $)$ or over.

Cold sprinkling. Sprinkling is a good substitute for the bath and is often better borne; it certainly is more easily carried out and has many advocates. The head of the bed is first raised 10 to 12 inches from the floor. Three boards as long as the bed is wide are placed crosswise under the mattress to keep it from sagging. A rubber sheet covered with a linen or cotton one is placed under the patient, whose head, wrapped in a cool wet turban, rests on a pillow. The night dress is removed, and the water, at a temperature Io or I2 degrees cooler than would ordinarily be used in the bath, is applied from a sprinkling pot or from an irrigating apparatus provided with a large rose nozzle. Active friction is kept up. Some practitioners use a dry sheet over the patient and sprinkle this with ice water using friction. The water should come from a height of 2 or 3 feet and should be directed chiefly to the abdomen and lower extremities. The surplus water should be allowed to drain into a pail or tub. The patient is then wrapped in a dry sheet, covered with a blanket, and rubbed. The process is repeated as in the directions for the bath.

Cold pack. The wet cold pack is another substitute, but its application is more exhausting to the patient than sprinkling, for it has to be renewed more frequently, Dr. Anders finds this method, however, of great use in children.

Hot sponging. This substitute for bathing sometimes yields excellent results when the patient is very nervous, or when for any other reason baths cannot be given. The water should be at about $\mathrm{IIO}^{\circ}$ or I $12^{\circ} \mathrm{F}$. ( $43.3^{\circ}$ to $44.4^{\circ} \mathrm{C}$.) ; in another receptacle containing water at about $100^{\circ} \mathrm{F} .\left(37.8^{\circ} \mathrm{C}\right.$.) stands a two-ounce bottle of grain alcohol. Sponge the limbs separately, and then the body for ten minutes with the water, and finish with a quick general rubbing with the warm alcohol. This is an excellent method, especially for children. The temperature of the water and the alcohol may be lowered and its strength varied as judgment and experience with the patient may indicate. The alcohol sponge may be tempered to suit the feelings of the patient, and the strength may vary from 25 to 95 per cent.

Much of the benefit of hydrotherapy is attributed to the greatly increased excretion of toxins. One of the errors which most of us are still laboring under is our attitude with reference to the presence of fever, and a failure to recognize the remedial character of baths apart from their influence on temperature. Attention has been called by our best clinicians to the fact that either a local or general fever de- 
velops in practically all infectious processes, failing to develop only in those cases in which the infection is so overwhelming that the organism does not have an opportunity to protect itself. If an animal be given a moderate infection it speedily recovers if fever is permitted to occur, whereas if fever is prevented, it dies. Dr. Hare $\left({ }^{13}\right)$ has pointed out that, if fever is prevented by the use of antipyretic measures, infections prove fatal, and from this we learn the important fact that in the vast majority of instances the physician should not attempt to modify fever, but rather to regard it as a helpful ally, and as a manifestation which will help him materially in gauging the severity of the illness and the ability of his patient to combat it; still when the fever becomes a hyperpyrexia, exceeding $103^{\circ}$ to $104^{\circ} \mathrm{F}$. $\left(39.5^{\circ}\right.$ to $40^{\circ} \mathrm{C}$.), for a considerable time, it may become a symptom that requires attention. This view, at first sight, may seem to be in opposition to the well-recognized value of the employment of the cold bath in typhoid fever, but in reality no such antagonism exists, because it has been proved that the use of the cold bath in typhoid fever actually increases the production of heat in the body, and probably at times actually raises the temperature of the internal organs, at least for a few minutes. In other words, the use of the cold bath in typhoid fever, to use a simile, opens the draughts and increases oxidation processes-that is, it increases the production of heat, although at the same time a large amount of heat is abstracted from the body by its exposure to cold. To express it differently, the metabolic changes which are produced by the increased oxidation processes associated with the cold bath aid the system materially in combating the infection, not only the heightened temperature, but the metabolic changes, as just pointed out, being protective in their nature. We believe not only that the febrile process is an action beneficial to the organism, but that it is intimately associated with the development of protective substances to combat the injurious agencies which have invaded the body.

\section{HEAT STROKE}

Hydrotherapy for heat stroke does not differ materially from that applicable in typhoid fever. Cool water with vigorous friction in the bath, or douches or sponging with cold or ice water are greatly employed. Parkes first suggested local ice water enemata, and these were very successfully used in the British Army. They were found to be especially useful in those severe cases in which the circulation of the blood through the skin is often so defective that the blood cooled at the surface is not exchanged rapidly enough to reduce the temperature of the heated blood of the internal organs. Ice applied to the head and 
neck gives good results, especially when the high temperature endangers the integrity of the brain. Cold, whether applied in this manner, or by baths, douches, sprinkling, or sponging, corrects the disordered temperature regulation produced by excessive heat.

In case a patient is found with a temperature of $103^{\circ} \mathrm{F} .\left(39.5^{\circ} \mathrm{C}\right.$.) or over, he should be placed in a tub of tap water as cold as it runs; this will be in the neighborhood of $70^{\circ} \mathrm{F}$. in summer in our cities. This is usually quite low enough. Vigorous friction is employed by from two to four persons to the entire body in the tub for ten to fifteen! minutes. As the body yields its heat the water should be maintained at the initial temperature by adding iced water or ice itself. Rectal temperatures should be taken every two minutes. While the reduction of temperature is not the chief end sought, it accompanies a better circulatory equilibrium, enhances a good reaction and thereby favorably affects the nervous system and the heart.

The tendency in the past has been toward the overuse of cold, and the comparative neglect of friction, causing a paralysis of the cutaneous muscles and cyanosis. The stimulant effect of cold has been compared to the effect of other stimulants, as for example, alcohol. If too little is used it is negative; if we use too much we may get an atonic hyperemia and harmful results. For that reason ice packs are never permissible and should be condemned. If the patient's temperature should fall to subnormal, non-alcoholic cardiac stimulants are to be given freely, an ice-bag placed at the head and chipped ice should be given by the mouth. External heat should be applied to the trunk and extremities, using great caution that no damage is done to the skin. Of course a recurring high temperature calls for a renewed use of cold.

Sprinkling from a hose attached to a watering pot is an excellent measure, and with a cot protected by a mackintosh may be used where tubs are not available, and indeed may be better than tubbing. Not much help is required and very little water. This is a desideratum in military and country practice, in the Orient, in tenements and indeed almost everywhere. On shipboard sprinkling can be carried out easily on deck and may be supplemented by affusions from dipper and bucket. Stokers suffer not infrequently from heat stroke in port or at sea; men at work in sugar refineries, steel and iron furnaces, as well as on city streets or in the fields, are equally liable, and apparatus for prompt treatment should always be at hand.

\section{SCARLET FEVER}

As a routine measure the patient should be sponged twice a day with water over the entire body. The water may be cold $60^{\circ} \mathrm{F}$., cool $75^{\circ} \mathrm{F}$., 
or lukewarm $90^{\circ} \mathrm{F}$., depending on the bodily temperature; afterwards the skin is oiled with cocoanut oil or cold cream. It is more tactful to begin with the warmer and use the cooler water later. In case adenitis occurs local cold compresses should be applied at the very first sign and repeated at hourly intervals. It is a good preventive measure. High temperatures demand cool sponging and packs. There should be no hesitation about using cold in scarlet fever or in any disease of childhood with high temperature, whenever fever causes restlessness, loss of sleep and rapid pulse. No definite rules can be formulated, but cases must be judged individually: Sponging may be instituted at $103^{\circ}$, $104^{\circ}$ or $105^{\circ}$ as the patient seems to be affected by the fever. One part of alcohol may be added to three parts of water at about $80^{\circ} \mathrm{F}$. The water after being applied should be allowed to evaporate from the skin surface.

If sponging is very annoying it is better to use some other method. Dr. Kerley advises the cool pack in this case and prepares it as follows: -A rubber sheet being used to protect the bed sheet, a large bath towel or some thick, soft absorbent material should be used; muslin, linen or any thin material does not answer as well. Slits are cut in the towel large enough for the arms to pass through, and the towel is folded around the body, enveloping only the trunk and buttocks. The pack should not extend below the middle of the thighs. This leaves the arms and the greater part of the lower extremities free. A hot water bag, carefully guarded, should be placed at the feet and the patient covered with a blanket of medium weight. The towel is moistened with water at $95^{\circ} \mathrm{F}$. This higher temperature is necessary at first, in order not to frighten the patient, as sudden cold is apt to do, and also to avoid shock. In two or three minutes, the towel, without being removed, is again moistened with water at $90^{\circ} \mathrm{F}$., later with water at $85^{\circ} \mathrm{F}$., and still later at $80^{\circ} \mathrm{F}$. When the temperature of the water reaches $80^{\circ} \mathrm{F}$., it is better to hold it at this point for half an hour; then the patient's temperature should again be taken. If at the beginning his temperature was $105^{\circ} \mathrm{F}$., and now shows but slight or no reduction, the temperature of the water with which the towel is moistened should be reduced to $70^{\circ} \mathrm{F}$. or, if necessary, even to $60^{\circ} \mathrm{F}$. The patient throughout need not be disturbed, except to turn him from side to side to wet the towel with water of the desired temperature, this being one of the advantages of the pack over a bath tub or sponging.

For the first hour or two in a pack the temperature of the patient should be taken every half-hour. When it is reduced to $102^{\circ} \mathrm{F}$., the pack should be removed, for, if it is continued longer, too great a reduction may take place. If it rises again rapidly to $105^{\circ} \mathrm{F}$. or 
higher, it is as well to keep the patient in the pack continuously. The degree of cold necessary, in the individual case, to keep the temperature within safe limits will soon be learned. Kerley kept in a pack for seventy-two hours a boy four years old with a lobar pneumonia. In this case a continuous pack of $70^{\circ} \mathrm{F}$. was required to keep the temperature at $104^{\circ} \mathrm{F}$. or slightly lower. The towel or other material employed should not be used for more than six hours, when it should be changed for a fresh one. Another reason for taking the temperature frequently is that early in the attack we do not know how it will be affected by the continued cold applications. In some children it is very readily influenced, and in such a case collapse might follow a very sudden reduction of the temperature. In cases readily controlled, the pack may be necessary for only one-half hour or an hour, at intervals of three or four hours. An ice-bag may with advantage be kept at the head when the patient is in the pack.

No attempt should be made to treat children in the same manner as adults. The average child will probably do better if left undisturbed with a temperature below $103.5^{\circ} \mathrm{F}$., but for pyrexia the cool pack may be necessary as previously described. In most cases we may use sponging with tepid water with a little alcohol added if the child is not dried after its use. This should be done for fifteen, twenty or thirty minutes every three or four hours, unless it irritates the child. In this case the cool wet pack may be used, as it is a less exciting measure. The effect of sponging or of packs should be noted, if possible personally by the physician, as children differ remarkably in their behavior, and it encourages the patient and the parents if he supervises these applications, especially at the start.

\section{DISEASES OF THE HEART}

There is a wide divergence of opinion as to the effect of baths in heart disease. The effect of hot and cold baths on the rate of the heart and the arterial pressure has been referred to already. The use of complex baths combining a thermal influence with the addition of certain salts, such as sodium chloride and calcium chloride and a greater or less amount of free carbonic acid gas, in which a great impression is made on the circulatory and the nervous systems, involves the most interesting physiologic problems. To begin with, the subjects for treatment presumably have some serious alteration in the structure of the heart or blood vessels. Whether it is a valvular disease, a cardiac muscular degeneration, an arterial sclerosis, the more dangerous nervous disturbances of the heart, neurocirculatory asthenia or the D. A. H. so common in military practice, we have in hydrotherapy a powerful 
agent which, in selected cases, may accomplish a great deal of good. On the other hand, there are undoubtedly great dangers often unrecognized in treating cardiac patients. Sudden death in the bath or shortly afterward is occasionally reported in the daily press and may happen at the best regulated spas. Deaths from apoplexy, from embolism or from angina pectoris are most to be feared and, unfortunately, cannot always be foreseen.

In all spas there is a class of patients who persistently have overworked their brains, overfilled their stomachs, overstimulated their hearts, indulged in all sorts of excesses, notably tobacco, and the problem of restoring them to health is exceedingly great, if not beyond the power of any medicine or any physician. However, with intelligent coöperation symptoms can often be relieved.

For cardiac cases we have been accustomed in these later years to rely on some form of carbonated or effervescent bath associated with what are known as resisted movements. Some think that these graded exercises are more important than the baths. At any rate cardiac patients are not, as a class, favorable subjects for voluntary exercise. Obviously the decompensated cardiac cases are unsuitable for the usual hydrotherapeutic measures, at least until compensation may be established. In these patients all diagnostic means and all measures for estimating the functions of the heart and of arterial and venous systems need to be used in forming a judgment as to both selection of suitable cases and estimation of results of treatment.

Much discussion has concerned the question whether a dilated heart, for example, can be reduced in size by baths. Probably it can, and eminent clinicians in England and America are on record very positively as to the value of carbonated baths in reducing the area of a dilated heart. It takes skill in delimiting the boundaries, especially in the absence of instruments like the orthodiagraph; but careful records of the point of maximum impulse and the boundaries under skilful auscultatory percussion will give encouraging evidence, if the treatment is well adapted to the case in hand. As Sir William Osler says: "There is no question that the bath in suitable cases will alter the position of the apex beat, and that it lessens the area of cardiac dullness; this means that it diminishes the dilatation of the heart."

The literature of the subject is voluminous and has been notably enriched by the works of Bezley Thorne, Satterthwaite, Anders and Camac. Sir James Mackenzie, in his monograph on Diseases of the Heart, has recorded some of his personal experiences with these baths and offers some well-merited criticisms. $\left({ }^{22}\right.$ ) (For a fuller discussion of their views and the methods employed see the author's 
"Hydrotherapy," ( ${ }^{14}$ ) with illustrations of a system of resistant movements.)

The good effects are due to the fact that the mineral and gaseous constituents of the bath excite the skin and stimulate sensory nerves. At the same time the pulse rate is consistently lowered and, as a rule, the blood pressure is raised. Carbon dioxid baths are of undoubted value in the cardiac neuroses but probably are never followed by improvement when the heart muscle is markedly weakened. The best results are obtained in cases of enfeebled, relaxed, dilated hearts, with or without murmur, following prolonged or exhausting disease, and in cases of cardiac inadequacy following severe muscular effort.

Even in incurable organic disease good results may be obtained in mitral insufficiency with dilatation. There is a point sometimes when carbon dioxide baths may afford timely aid, namely, when compensation is maintained with difficulty, when blood pressure is low and needs an adequate and safe physical stimulus.

When the pulse is taken just before and after the bath of from ten to fourteen minutes, the temperature of the water in a series of ten ranging from $9 \mathrm{I}^{\circ} \mathrm{F}$. to $84^{\circ} \mathrm{F}$., the heart rate may be lowered in each bath as the following shows.

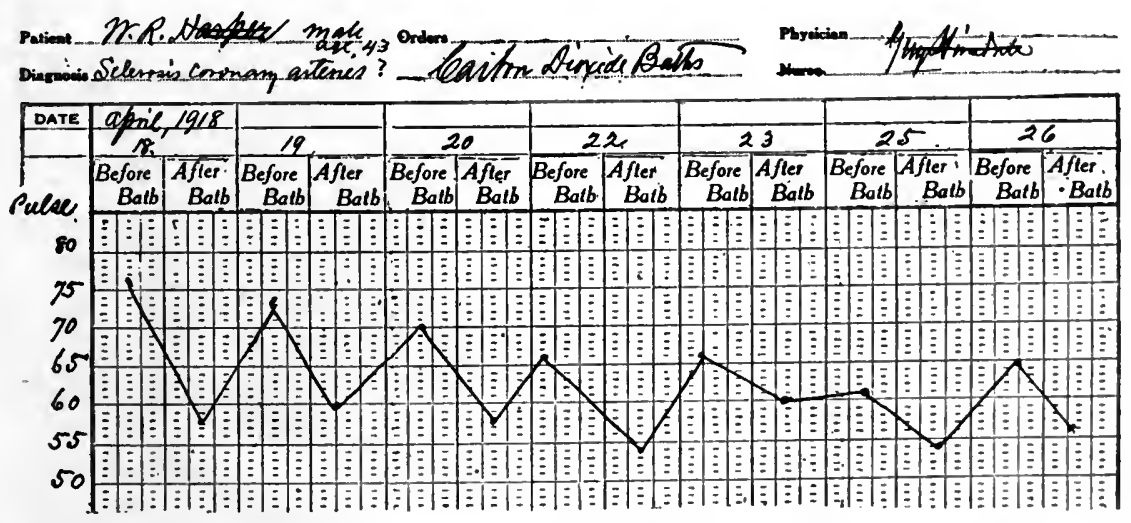

Fig. 1.-Reduction of Pulse-rate during Carbon Dioxide Baths.

The blood pressure may rise from ten to twenty $\mathrm{mm}$. of mercury and, as has been demonstrated in a series of ten baths, make a more or less permanent gain of twenty points. If there is a fall of blood pressure after a bath, as occasionally happens, it may be that the bath is too strong for the particular individual and some correction of the elements of the bath, its temperature or duration, should be made. 
It has been suggested that the tonic effect on the heart is brought about by a stimulation of the vagus nerve so as to alter cardiac movements and benefit myocardial nutrition, just as mild gymnastic exercise increases the tone of the voluntary muscles. $\left({ }^{15}\right)$

Baths containing carbonic acid gas with sodium chlorid and calcium chlorid have been used extensively during the last thirty years at various localities in Europe and America. In France there are thermal carbonated natural baths at Vichy (Grand Grille), Royat, ChatelGuyon and Mont Dore. In America there are certain localities where baths of this type are given, but with this difference, that the waters are either naturally cold and must be heated to the required temperature or, where carbonated thermal springs are available, the mineral constituents do not include a sufficient amount of the chlorides to present an exact counterpart. Thus at Saratoga Springs, New York, the carbonic acid gas and saline constituents are present in sufficient amount but the waters must be heated artificially. At Glen Springs, Watkins, New York, where special arrangements are made for treating cardiac disease, the cold saline waters are heated and carbonic acid gas is added artificially. At Byron Hot Springs in Contra Costa County, California, are united very closely the three elements of temperature, saline and gaseous contents required. Excellent results are obtained, however, by adding whatever is missing to make up a natural deficiency. So it is that in England and America we have come to rely on artificially produced carbon dioxid baths. These can be afforded by mixing in a tub of forty gallons of water varying quantities of chlorides, chiefly sodium chloride, and by adding some form of acid, and finally, when a stated quantity of sodium bicarbonate is added, we have generated instantly all through the bath free carbonic acid gas.

There is a choice of acids. It was deemed convenient some years ago to use cakes of acid sodic sulphate dissolved in the bath before the addition of the bicarbonate. This was made easy by firms who furnished packages containing the acid in cakes and the required bicarbonate in bags with instructions to use the chlorides as may be desired. Other packages contained a bottle of formic acid and bags of bicarbonate of soda. Others used plain hydiochloric (muriatic) acid and double the weight of sodium bicarbonate. We have used the formic acid extensively, but the recent excessive prices make its further use almost prohibitive.

The following scheme for ten carbonic acid baths may be used as a working guide for treatment in a case of slight cardiac insufficiency, using forty gallons of water: 


\begin{tabular}{|c|c|c|c|c|c|c|c|c|c|c|}
\hline \multirow{2}{*}{$\frac{\text { Temp. F. }}{95^{\circ}}$} & $\mathrm{HCl}$ & $\begin{array}{c}\text { Soda } \\
\text { Bicarbonate }\end{array}$ & $\mathrm{NaCl}$ & \multicolumn{2}{|c|}{ Duration } & \multicolumn{5}{|c|}{ Remarks } \\
\hline & 400 c. c. & $800 \mathrm{gm}$. & n....... & 10 & $\min$. & Dry & ub; & rest & $1 / 2$ & hour. \\
\hline $94^{\circ}$ & $500 "$ & 1,000 & $\ldots \ldots \ldots$ & II & “ & “ & “ & “ & “ & “ \\
\hline $93^{\circ}$ & $600 "$ & 1,200 “ & 4 kilos & 12 & “ & “ & “ & “ & “ & “ \\
\hline $92^{\circ}$ & $700 "$ & $1,400 "$ " & $6 "$ & I2 & “ & “ & “ & “ & “ & “ \\
\hline $91^{\circ}$ & $800 "$ & $I, 600 \quad$ “ & $8 "$ & 12 & “ & “ & “ & “ & “ & “ \\
\hline $91^{\circ}$ & 900 “ & $I, 800$ “ & $10 \quad "$ & 12 & “ & “ & “ & “ & “ & “ \\
\hline $90^{\circ}$ & 900 “ & 1,800 “ & I0 " & 12 & “ & “ & “ & “ & “ & “ \\
\hline $90^{\circ}$ & $900 “$ & 1,800 & I0 & 12 & “ & “ & “ & “ & “ & “ \\
\hline $89^{\circ}$ & $900 “$ & 1,800 “ & 10 & 12 & “ & “ & “ & “ & “ & “ \\
\hline $89^{\circ}$ & 900 “ & 1,800 “ & 10 & I2 & “ & “ & “ & “ & " & “ \\
\hline
\end{tabular}

Omit the bath every third day.

It is best to have the bicarbonate sewn up in two gauze bags of several thicknesses so that effervescence will not take place too rapidly. This favors the diffusion of the gas in the water and there is not such violent ebullition as when sodium bicarbonate is fully exposed. During the first five minutes the bags are to be gently moved about while the patient lies quietly in the tub, placing them first near the arms, then near the hips and then near the legs. At the end of five minutes the effervescence commonly ceases and the patient lies quietly until the full time has elapsed, when he is taken out, quickly dried and placed in bed with a hot water bag of moderately warm water at his feet. There is no massage and absolute quiet should be observed. A halfhour is the minimum for rest. An hour is often better, and if the patient is in his own bed at home so much the better.

The pulse should always be noted and recorded before the bath and after the patient is put to bed. Observations of blood pressure while of great interest to the doctor are usually made too much of by the patient. I do not believe it good practice to cultivate an undue interest in blood pressure measurements. Patients with cardiac disease are usually nervous and unduly elated or depressed by the doctor's reports. It is better to leave the everyday record for use in some case in which the mental attitude is more of the stoical type and never to push blood pressure tests when the patient is apprehensive and likely to dwell on his symptoms. 
A word should be said about the apparatus sometimes used for distributing carbon dioxid in the bath. Kny-Scherer's apparatus is a practical one and consists of a cylinder of compressed or liquefied carbonic acid and a mixing chamber to which the cylinder is connected. A pipe leads from the mixing chamber to a perforated frame of piping placed in the bottom of the bath tub. It is obviously impossible to lead the gas directly from the cylinder to the tub. By adjusting valves the gas is gently introduced and one cylinder suffices for several baths.

In cases of disordered action of the heart great use has been made recently in England and France of the sedative pool bath. Fox in his book ${ }^{16}$ ) shows by a chart given to him by Dr. F. Radcliffe the favorable effect on the pulse rate in soldiers treated for D. A. H. by pool baths given daily for thirty minutes at $92^{\circ} \mathrm{F}$.

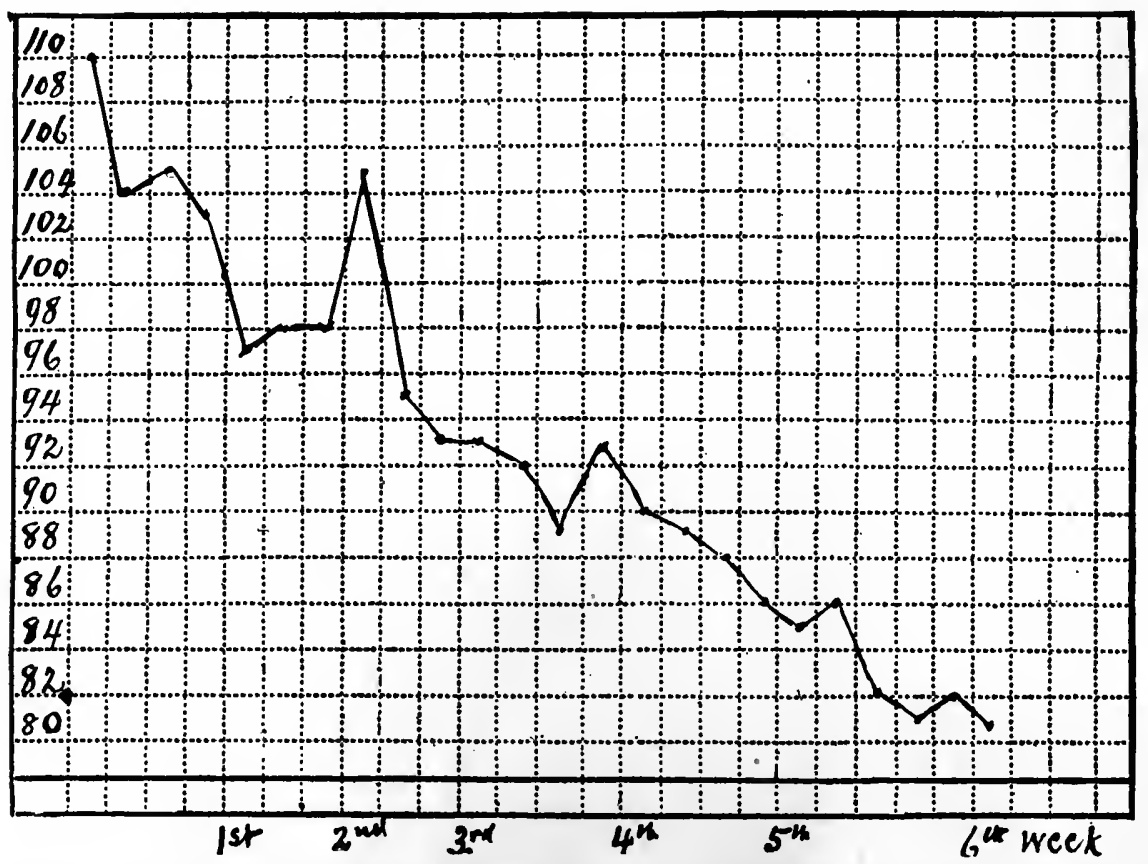

Fig. 2.-Average Pulse-rate of Ten Cases of Disordered Action of the Heart Treated at Heaton Park Command Depot by the Sedative Pool Bath.

\section{OBESITY}

Hydrologic measures are not sufficient in the reduction of weight unless associated with a régime including diet and exercise. The combination of measures properly balanced will in most cases accomplish good results, as all practitioners at mineral water spas well know. The visitors at such resorts include a notable proportion of these cases, and 
they demand suitable treatment. Experience proves that a régime should not be entered into lightly or unadvisedly, but carefully, and with respect to individual requirements and capabilities. Most of these subjects are in middle life, are of sedentary habit and consequent muscular weakness. The heart should be carefully observed at the start and all through the course of treatment. If baths are ordered a daily record should be made of weight and also notes of the heart action; and from time to time the blood pressure should be recorded. Few go to hospitals for the reduction of weight but many go to spas. The further they go and the more sacrifices they make to undergo the cure, the more conscientious they are in adhering to discipline.

We believe that hot water bathing yields better results than cold baths or salt water bathing. The latter more surely stimulates the appetite and there is not the same degree of loss through perspiration.

\begin{tabular}{|r|r|r|r|r|r|r|}
\hline H.E.S. & & & & & & \\
\hline & & & & & & \\
\hline & Oct. & 1917. & & & & Nov. \\
\hline & 16 & 19 & 22 & 24 & 29 & 4 \\
\hline 259 & & & & & & \\
\hline 256 & & & & & & \\
\hline 255 & & & & & & \\
\hline 254 & & & & & & \\
\hline 253 & & & & & & \\
\hline 252 & & & & & & \\
\hline 251 & & & & & & \\
\hline 250 & & & & & & \\
\hline 249 & & & & & & \\
\hline 248 & & & & & & \\
\hline 249 & & & & & & \\
\hline 246 & & & & & & \\
\hline 245 & & & & & & \\
\hline 244 & & & & & & \\
\hline 243 & & & & & & \\
\hline 242 & & & & & & \\
\hline
\end{tabular}

Fig. 3.-Reduction of Weight by Hot Baths and Packs.

After hot full baths followed by hot blanket packs applied to the entire body, there is often a loss of one or two pounds, as determined by, careful weighing before and after treatment. Some of this loss is replaced inside of twenty-four hours, but there is a gradual downward 
tendency in the weight curve that encourages the patient to persevere as shown in the chart. At the end of three or four weeks ten or twelve pounds may be safely lost in persons weighing in the neighborhood of two hundred pounds.

\section{CHRONIC INTERSTITAL NEPHRITIS}

It is preemminently in the case of nephritis in which cardio-vascular changes have preceded the actual changes in the kidney that baths will do most good. These patients complain of circulatory disturbances, gastric disorders, gradual loss of strength, and anemia. The urine has a low specific gravity, is abundant in quantity, and deficient in urea. Albumin and hyaline casts are present. Patients of this type may have a high arterial tension, but this fact need not debar them from the use of baths under proper precautions. Carbon dioxid baths may be employed in cases of this type. At the outset it is best to use salt baths containing only a small amount of carbon dioxid. Permanent lowering of arterial pressure has been noted after this method of treatment.

The associated drinking cures at spas are highly beneficial in these cases, particularly when alkaline, alkaline calcic, or magnesium sulphate waters are available with little or no sodium chlorid in their composition. Sulphur springs are useful in these cases, the water being applied both externally and internally. Physicians practicing at sulphur spas claim that great benefit is derived from the baths through their stimulating effect, nutrition is improved and perverted metabolism is corrected. The stimulating effect of this class of waters upon the skin also tends, to a certain degree, to improve the tone of the circulation.

In cases of marked arteriosclerosis, baths should not be given except with the utmost care, but in cases of high tension without arteriosclerosis it is rather common to observe patients in whom there is a high nervous tension. It is in these cases that the full warm bath for eight or ten minutes, followed by a rain bath for five or six seconds, or a fan douche, will prove eminently useful by allaying nervous irritability.

In treating chronic nephritis, hot applications are preferable to cold, the latter producing a peripheral contraction of the blood vessels before the ensuing dilatation. In chronic nephritis the reaction may fail altogether owing to a lack of vascular tone or from changes in the heart muscles or in the peripheral arteries. For reducing vascular pressure hot moist applications are more lasting in their effect, as deeper vessels become dilated. 


\section{CHRONIC RHEUMATISM}

Chronic rheumatism is essentially a fibrositis or an inflammatory hyperplasia of the fibrous tissue of the muscles, joints, and ligaments. It is brought about by exposure to cold, damp and wet weather, sudden changes of temperature from hot to cold, or an attack may date from predisposing causes, such as injuries to limbs. An acute traumatic arthritis may eventually become a " rheumatic joint." Irritating toxins from the intestinal tract or from an acute gonorrhea may result in as so-called general acute articular rheumatism due to their specific poisons. Focal infections are well known as a cause of arthritis.

The value of thermal springs in the treatment of chronic articular rheumatism and the arthritides is recognized the world over. The chief factors are the systematic application of heat, the manipulation practiced in the baths, and the consequent improvement of the circulation in the parts affected.

The patient for whom such treatment is deemed suitable is placed in a full bath of $102^{\circ}$ to $104^{\circ} \mathrm{F}$. $\left(38.9^{\circ}\right.$ to $40^{\circ} \mathrm{C}$.), with his head wrapped in a turban wet in cold water. An attendant rubs the limbs and the body while the patient is in the bath, applying movements, graded in force, to the affected joints. In patients accustomed to hot bathing, it is possible to begin with a temperature of $104^{\circ} \mathrm{F}$. $\left(40^{\circ} \mathrm{C}\right.$.), which in most cases is quite agreeable. After eight or ten minutes of the full bath the patient is dried and laid on a couch where he is closely packed in a hot dry sheet and enclosed in three or four double blankets. Here he remains for ten or even twenty minutes, after which he is rubbed vigorously for five minutes with alcohol. On the fourth day the bath may be omitted and afterward the baths may include a preliminary hot douche at $103^{\circ}$ or $104^{\circ} \mathrm{F}$. $\left(39.3^{\circ}\right.$ or $40^{\circ} \mathrm{C}$.), applied all over the body excepting the head, the anterior chest, and the abdomen. The elements of this bath are naturally adjusted in time and in force to the needs of the patient as may be determined by his medical adviser. Cool sponging or a cold douche frequently is employed after the pack. General massage is also employed in suitable cases some time during the day, but most usually not directly after the bath, as after the bath the patient should rest quietly for an hour.

The rationale of this method lies in the better circulation established around the joints and in the increased mobility of all the tissues involved. Exudates in the fibrous investments of joints, in the sheaths of tendons, and in the muscular tissue create more or less disability, and, if untreated by external agencies, tend toward increased pain and stiffness, if not toward fixation of the limb. In the case of spinal arthritis, 
even more serious fixation and deformity are likely to ensue, and hence the usefulness of general physiologic measures like hydrotherapy and massage.

In a systematic course of bathing the secret of success lies in a judicious education of the patient to bear heat and manipulation in an increasing gradation. Mild measures at the outset induce confidence and make possible later a degree of manipulation, depending upon the special characteristics of the case in hand, that otherwise would not be secured.

After a full bath in warm or moderately hot water, e. g., at $104^{\circ} \mathrm{F}$. $\left(40^{\circ} \mathrm{C}\right.$.), followed by a hot dry pack, there is relaxation with sweating. A subsequent alcohol rub is then refreshing and produces a tonic effect. This is the main feature of the treatment at the Virginia Hot Springs.

It is well known that when limbs are immersed in water less pain is elicited on active or passive movements than when the same movements are practiced in the usual atmospheric medium. This fact is taken advantage of in these cases to the fullest extent, constituting one of the advantages of the full bath. In the bath the action of the skin is made better by the friction, which should always be insisted on; it becomes more pliable and free from all excretory matter. Both the superficial and the deep blood vessels of the part, too, are enabled to take up and carry off any exudates or infiltrates, and thus relieve the restrictive influences on the motion of the structures involved, and the impairment of a proper interchange of circulation. Therefore, we adopt every means calculated to improve nutrition.

Secondary muscular atrophy, which is commonly observed in chronic joint disease, may be forestalled if treatment be started early and massage judiciously used in connection with hydrotherapy.

After three or four baths there is usually a febrile painful reaction in the affected parts. This return of tenderness and swelling is discouraging to the patient. He should be warned in advance of it, and should be comforted by the thought that it is a favorable indication of the ultimate relief to be derived from the methods employed.

Although the method previously described is the one usually-adopted by the author, another method may be chosen and will give excellent results. Patients who have had the baths and packs for a time may be given a short series of cabinet baths and douches. The prescription would read as follows: Electrical cabinet to perspiration. Circular douche $106^{\circ}$ reduced to $90^{\circ} \mathrm{F}$. $\left(32.2^{\circ} \mathrm{C}\right.$.) and fan douche $90^{\circ} \mathrm{F}$. reduced to $60^{\circ} \mathrm{F}$. ( $15.6^{\circ} \mathrm{C}$.), twenty seconds, 20 pounds. Massage for the affected joints and muscles, fifteen minutes.

Fomentations are useful in muscular rheumatism. Compresses well 
wrung out of hot water at about $140^{\circ}$ to $150^{\circ} \mathrm{F}$. $\left(60^{\circ}\right.$ to $65.6^{\circ} \mathrm{C}$.), or even hotter, should be applied. Compresses lose heat so quickly that the actual temperature of application is much less, and by making cautious attempts to place them a fairly high degree of heat can be borne. After the fomentations have been applied and re-applied for ten, fifteen or twenty minutes the patient is washed off with water at $70^{\circ} \mathrm{F}$. (2I.I ${ }^{\circ} \mathrm{C}$.), with friction, and dried. The addition of magnesium sulphate will enhance the value of the compresses. After this treatment the patient should rest for an hour on a bed or lounge.

The acute arthritic outbreaks which so often follow spa treatment depend upon the thermal action of the mineral baths. Similar attacks follow the use of plain hot-water baths devoid of mineral properties. A severe attack of gout has been brought on by taking a hot bath soon after dinner. Hot-air baths in cases of suppressed gout may determine an acute attack. These acute phenomena are usually interpreted as preparing the way for the elimination of uratic deposits. Though painful, the ultimate result is beneficial; the absence of any reaction in a case of gout after the adoption of thermal baths does not augur well for the benefits to be derived from the treatment.

\section{INSANITY}

Hydrotherapy has proved of great value for the insane. James Currie, $\left({ }^{17}\right)$ the Liverpool surgeon, recorded in 1796 several cures of maniacal patients by means of cold baths which he had previously used with success in typhus. Prolonged neutral baths for promoting quiet and sleep in nervous and mental affections were advocated over a hundred years ago in France, and over seventy years ago by Dr. Isaac Ray, in his day the leading American alienist. After his return from Europe, Ray wrote, $\left({ }^{18}\right)$ "Nothing seems to be so much relied upon, especially in France, for subduing inordinate excitement, as the warm bath protracted for an hour or two."

In the early stages, before the patient is admitted to a hospital, skin eruptions due to the use or misuse of bromides will be commonly met with in the insane. It is here that much can be done not only for the bodily comfort but for the cure of moderate cases.

When epilepsy coexists with insanity unusual care will be required to avoid accidents. The sudden onset of an epileptic attack may result in injury from a fall against the apparatus or a burn from incandescent lamps unless they are well guarded. So also in the continuous bath accidental drowning and fatal scalding have occurred, and where several insane patients are taking the continuous baths they have sometimes tried to drown each other or commit suicide in that way. Every pre- 
caution must be afforded and constant watchfulness on the part of the attendants is required.

Notwithstanding the difficulty of handling this class of patients, it is well recognized that baths are of great value, and all the best institutions for their care rely to some extent on this form of treatment. It could be extended with advantage to the majority of patients, not merely to the excited and melancholic. A swimming pool for the insane would be of doubtful utility unless shallow; but showers, a control table and cabinet baths, in which heavy wire glass replaces the ordinary glass in the doors, are a necessary part of the equipment. The use of douches from time to time ought to be provided. A great deal could be accomplished with fomentations to the abdomen and spine, followed by ice rubbing. In this way a physical as well as a mental impression is made, and a judgment may be formed as to which method is likely to give the best result for continued use.

In some institutions selected groups of patients are formed in line at some convenient hour and stand under large overhead showers, descending or "rain" douche. These, by the way, should be arranged so as to avoid discharging water directly on the head; the rosettes should be adjustable and inclined at an angle so as to shower the neck and shoulders. If desired the head can be lowered for an initial wetting. The douche room should be provided with a railing to keep the line in order and to give support for the hands of patients who feel the need of it. This daily bath with suitable appliances has a wholesome effect on a large class of mental cases, and it can be given to large numbers with very few attendants and with economy of warm water. The temperature should be at about $100^{\circ}$ to $104^{\circ} \mathrm{F}$. $\left(37.8^{\circ}\right.$ to $40^{\circ} \mathrm{C}$.) to start with, and lowered in the course of four or five minutes to $90^{\circ}$ or $80^{\circ}$ F. $\left(38^{\circ}\right.$ or $27^{\circ} \mathrm{C}$. $)$. In summer the terminal temperature may be lowered as directed by the physician.

Those who have much to do with the insane usually speak highly of the cold wet sheet pack; the hot blanket wet pack; the incandescent electric light or steam heated hot air bath, followed by circular (needle) douche, jet, Scotch. and fan douche; and, in the excited states especially, the continuous bath.

What do we expect to accomplish with continuous baths? In the first place the action of the skin is improved and a general hygiene is ensured. Secondly, and no less important, is the remedial element of rest. This is accomplished par excellence in a continuous bath. New patients should be invited into the tub, not forced into it. They are to be encouraged by the example of other patients. They may be allowed to run about a little at first until their confidence is obtained and per- 
haps their curiosity aroused. It is not best to use force, but the best practice is probably to use more tact, so that no restraining hammock will be required. Thirdly, is the psychic effect. Nothing is more hopeless than to see large numbers of insane sitting about their wards, or perhaps confined to rooms, hour in, hour out, day after day, with no provision for occupation. These patients are like the maimed and halt and the blind of old who sat waiting for the angel to trouble the waters. Hydrotherapy, and especially a measure which occupies an entire day, is a Godsend to these afflicted. It reacts favorably upon the patient and, incidentally, no doubt provides a stimulus to the attendant and the doctor as well, tending to break up a deadly routine common in insane asylums.

One attendant can usually look after two or three patients. The tubs are specially constructed with a large exit, so that fecal matter may be carried away quickly. Patients eat and sleep in the tubs, and as a rule take to this amphibious life remarkably well. The room should have low windows, so placed that while in the tubs they may look out of doors, if possible, on some attractive landscape and not on blank walls. A fountain or an aquarium would go far to interest the patients and mitigate monotony.

In the tub there should be a floating thermometer as well as the ordinary thermostat placed so as to indicate the desired temperature; attendants should observe both and see that the temperature is constant. This should be from $98^{\circ}$ to $99^{\circ} \mathrm{F}$. $\left(36.7^{\circ}\right.$ to $37^{\circ} \mathrm{C}$. $)$ in winter, and $96^{\circ}$ to $97^{\circ} \mathrm{F}$. $\left(35.6^{\circ}\right.$ to $36^{\circ} \mathrm{C}$.) in summer.

There should be a rubber pillow attached at the head of the tub; a toilet should be at hand so that patients who are able to do so may use it conveniently. Screens between the tubs may be used for appropriate cases. Even in so-called first-class institutions the odor prevalent in the rooms of the "violent ward" is horrible; a naked maniac lying on a leather couch day and night is filthy, and no time should be lost in getting such patients into the continuous bath where the air can be kept pure and the floor clean. It is simply a matter of physical equipment and competent attendants. There is an opportunity in America to design and build a bath house for the insane on more artistic, attractive and hygienic lines, with much greater capacity, with single rooms for the more violent and provision for small groups of those quiet and tractable. Such an equipment may exist, but we have not seen it.

At the McLean Hospital at. Waverley, Massachusetts, the excited cases are given continuous baths. These are not used at night. There is no restraint while in the tub, but attendants are employed to insure the patients remaining in the water. At the Philadelphia General Hos- 
pital (Blockley), where eight continuous baths were installed twelve years ago, restraint is used when deemed necessary. Here the patient rests on a swinging hammock; the arms and feet are restrained with a canvas restraint sheet held in place to hooks below the margin of the tub.

The cases at Blockley have included cases of acute, chronic and recurrent mania; excited types of dementia praecox; maniacal episodes of epilepsy; dementia paralytica and senile dementia; alcoholic insanity and insanity of chorea. We have elsewhere recorded the history of some of these cases while they were under the care of Dr. W. W. Hawke of the Philadelphia Hospital $\left({ }^{19}\right)\left({ }^{20}\right)$ and Dr. B. R. Logie of Washington, $\mathrm{D}$. C. There are now hundreds of continuous baths in use in the United States.

Captain E. A. Strecker, M. C., U. S. Army, formerly of the Pennsylvania Hospital for the Insane, Dr. J. Allen Jackson of the Philadelphia General Hospital, Insane Department, and Dr. B. R. Logie, formerly of the Government Hospital for the Insane, Washington, D. C., have published most favorable accounts of these baths. $\left({ }^{21}\right)-\left({ }^{26}\right)$ Dr. Strecker's observations are based on an experience of over three years in treating about three hundred patients in the continuous baths, exhibiting various kinds and degrees of mental excitement. In contrast to the use of moist packs which are unsatisfactory because of the resistance which they offer to the motor restlessness common in the apprehensive confusional states, the continuous baths have a distinct advantage, in that they rely solely on water, a non-resistant medium, which favors rather than retards excretion, and whose action approximates the physiologic steps between the production and onset of normal fatigue and sleep, in that it is gradual and sedative.

In Strecker's experience the manic phase of manic-depressive insanity responds to the continuous bath in more than half of all cases; others have noted that the excitement is allayed only during the treatment time and when suspended continues as before.

In dementia praecox the baths are employed mainly for the relief of the episodic excitements of the catatonic form, in which they were moderately successful, and to combat various vasemotor disturbances, in which their effect was highly beneficial. Not only was cyanosis improved, but the tendency to coldness and sweating of the extremities, oiliness and increasing coarseness of the skin texture were lessened. In paresis the baths were found valuable not only in controlling the disturbed periods, but also in maintaining an active healthy condition of the skin which, especially in the late stages of the disease, is prone to trophic degenerations. The most unfavorable cases for continuous baths were deemed to 
be those in the apprehensive states which occur in the involutional and presenile depressions and the senile dementias.

The entire class of toxic and infection psychoses would prove eminently suitable for treatment with continuous baths. The proportion of these cases is doubtless higher in private institutions and may equal ten per cent. of the total number of admissions. We may mention, for example, the toxic psychosis due to alcohol; such patients should be afforded more generally the advantages of the continuous bath; the restlessness and delirium and all the other phases of psycho-motor excitement have been most successfully treated by means of the continuous bath. These cases demand some method of rapid and free elimination to reduce the toxicity and afford sleep so as to prevent exhaustion. Bromides, chloral and morphia, as well as the various coal-tar derivations, are used freely in alcoholic cases and are notoriously ineffective and dangerous; it is commonly believed that these patients can stand much larger doses than would be proper in other conditions and there is a corresponding abuse of drugs.

At the Boston Psychopathic Hospital ten unselected patients with delirium tremens were treated in the continuous baths with the addition of moist packs and eliminatory measures without a single fatal result. This is a remarkably good showing and has led to its adoption in the Lambert-Towne treatment to combat the marked restlessness which commonly makes its appearance on the second day.

At the Government Hospital for the Insane (Saint Elizabeth's), Washington, there are over three thousand patients. Hydrotherapy has been used for twenty years, but at the present time consists principally of cold wet sheet packs. The continuous bath is used in excited cases, but only to a very slight extent, as there are not more than three or four of these baths installed. The cold wet sheet pack is given for a period of 35 to 45 minutes during the morning for the white patients and in the afternoon for the colored. The requisites in order from the bottom upward are: A table, mattress, pillow covered with white rubber, sheet, rubber sheet, two blankets and two sheets wrung out of cold water. The patient who should be warm, if necessary by placing in the cabinet, is laid on the couch and quickly enveloped in the wet sheet, well packed in the blankets and allowed to remain until time for the warm shower. This, beginning at $106^{\circ} \mathrm{F}$., is gradually lowered to about $60^{\circ}$, after which the patient is quickly rubbed while standing and then dresses to return to his room. 


\section{NEURASTHENIA, HYPOCHONDRIASIS AND SHELL SHOCK}

Physical measures are much more useful in neurasthenia than drugs. About forty-five years ago Weir Mitchell discovered a practical method of treating this disease by means of rest, massage, electricity and seclusion, and he gave instructions for hydrotherapy in appropriate cases.

The drip sheet was his favorite method, applied at night; but later both he and the late Dr. John K. Mitchell used cabinet baths with needle, jet, and Scotch douches, especially during the later periods of treatment, when seclusion was over and the patient was able to go to the douche room. Dr. Mitchell also used hot and cold applications in alteration to the spine. The application of the hot sponge should be followed by a much briefer cold sponge to the spine and the periods of application should not occupy more than a few seconds.

Neurasthenics have to be treated with great moderation and no extreme measures should be adopted. At Hot Springs, Virginia, the full tub baths followed by the hot, dry, blanket packs are found to be suitable for neurasthenics of the apprehensive, excitable type not too much physically weakened. However, sedative measures should not be continued too long, and before many days have passed a change is usually made to the more tonic type of bath, using the cabinet and needle and hot douches, followed by an alcohol rub. The same method is applicable to most hypochondriacs. The psychic effect of baths, varied from time to time from the sedative form to the more stimulating type, is a recognized aid in these patients. The baths in and of themselves are valuable in promoting a better circulation. They favor appetite and are usually followed by better sleep. They occupy the patient's mind and interrupt habits of introspection and despondency, and when given amid agreeable surroundings by agreeable and skilful attendants they yield good results. The additional advantage of a good climate, whether at the sea or in the mountains, must not be lost sight of. That is why such resorts as Atlantic City, Old Point Comfort, the Virginia Hot Springs and the White Sulphur Springs in West Virginia are eminently suited to this class of patients.

At Virginia Hot Springs there is also a full equipment of Zander apparatus for graded passive and active exercise, so that another therapeutic measure can be added when more active out-of-door exercise is not admissible.

Nervous shock following surgical operations or severe injuries has long been treated by surgeons by means of artificial heat, but during the last five years a new condition of shock has presented itself, and it is one that has never been observed so extensively in any previous war. It 


\section{NEURASTHENIA, HYPOCHONDRIASIS, SHELL SHOCK 209}

is a neurosis, and rightly or wrongly it is commonly known as shell shock. It is one of the very serious calamities of modern warfare. The peculiarly exhausting and long-continued nervous stress due to trench life and trench fighting, the high explosives, bombs, the impending gas and flame attack, the death of comrades and other terrible scenes, make the stoutest heart afraid. Much has been written about the shell shock problem. One of the best and most vivid descriptions is by Dr. M. Allen Starr, of New York, $\left({ }^{27}\right)$ who has analyzed the various underlying conditions. They are not always the same. There may be actual damage to the nerve centers; there may be exhaustion and disintegration; there may be merely a temporary suspension of brain activity. The final result will vary in accordance with these various conditions, thus determining the different lines of treatment. Rest amid happy, pleasant surroundings, diversion, occupation and good nutrition bring recovery in a large proportion of cases. It is a question largely of re-education of the will and muscles and the suggestion of the hope of a good recovery that must be afforded. To aid this there are special hospitals provided with the proper equipment for hydrotherapy, electrotherapy and other physical measures conducted by a trained personnel, skilful and sympathetic. The continuous baths as previously described may be used, but it is found more convenient to group those patients in the sedative pool baths, accomplishing the same results for the large numbers undergoing treatment. These are very much like the piscines of the French spas.

In traumatic shock there is a lowering of arterial tension, due to a greater or less degree of vasomotor paralysis, and this should be taken note of in continuing any hydrotherapeutic treatment. Hot baths and packs would therefore not be in order, but the neutral or indifferent bath which may be prolonged without any special effect on the blood pressure. The study of the blood pressure is the best means for determining the degree of shell shock. The pressure should be always noted in hydrotherapy and will give valuable evidence in all kinds of cases.

According to Professor Sims Woodhead, who has treated large numbers of patients at the Command Depot at Tipperary, the pool bath is used principally for shell shock cases, many of them doing exceedingly well, though a few, who seem lethargic, do better under a hot douche, followed by an almost cold douche or spray. Only exceptional cases fail to derive benefit from the pool bath at an indifferent temperature. Most of the patients complain that they feel tired and sleepy afterward; this, however, is deemed desirable and every man is sent to bed for an hour's rest. They gradually form the habit of sleeping, which becomes 
more and more marked as time goes on, and with this the dream becomes a less marked feature.

Captain Edward Ryan, of the Canadian Army Medical Corps, who had ten years' experience at the Rockwood Hospital for the Insane, using continuous baths with success, says that at the Ontario Hospital at Orpington he used these baths in patients with shell shock and records some interesting cases. Among them was one who was admitted deaf and mute and was quite depressed. After two weeks in the continuous bath at $92^{\circ}$ to $96^{\circ} \mathrm{F}$. he recovered hearing and voice, gained in weight and made a good recovery. Another case was admitted with marked excitement; this patient was also deaf and mute, was very irritable and would jump at the slightest touch. His recovery was attributed to the bath. $\left({ }^{28}\right)$ Experience with these baths at the Red Cross Clinic in London shows an almost invariable reduction of the pulse rate in cases of shell shock, with improvement of sleep and relief of headache. The temperature of the body, which is usually subnormal in cases of tachycardia and shock, is raised a degree and a half by sedative pool baths, with a coincident reduction of pulse.

For insomnia, especially in functional nervous states, cold wet packs may be employed. The early shock is followed by a reaction which affects the whole circulation and the peripheral nervous system.

Disturbances of the peripheral vasomotor system and central nervous system can be corrected by baths and packs unaccompanied by much internal medication, and they afford a good method of checking and curing the use of habit-forming drugs. In using baths for insomnia it is very important to apply constant active surface friction. In the case of packs this may not be so important, as reaction is usually more readily obtained. When using baths and packs for insomnia it is obviously necessary to give them late in the day or in the evening if possible.

\section{TUBERCULOSIS}

For the prevention of tuberculosis in children, tonic hydrotherapy is no doubt useful; but cold water bathing should be approached cautiously and with tact, as most children dread it, and any sudden or unlooked for shock will frighten the child. It is best to cultivate a reaction by imperceptible gradations of temperature downward, using a bath thermometer. Attempts to fortify the system must be modified according to the vigor of the patient. A child under size and under weight, and all children under two years, should be spared a temperature much below the body heat.

Whenever the feet after a bath or washing do not warm as quickly as 
the rest of the body they should be rubbed with warm water, or a mixture of alcohol and warm water, until a good reaction sets in.

Sea bathing and sunning on the sand are excellent prophylactic measures when gradually employed and not carried to extremes. The exposure of the limbs and the partial exposure of the body through thin bathing suits to the action of the sun and air develops tan and increases the tone and natural resistance of the skin. It has a salutary general effect on the system and constitutes the best safeguard known against tuberculous disease, whether in the bones, the glands or the lungs. Sea bathing may thus be employed as a preventive measure.

Where the disease is established, especially in the lungs, surf bathing would be dangerous, but tub baths with sea-water rubbing would be beneficial in many cases. Salt tubs may be used. Cloths or the bare hand, after being wet in warm water, are dipped in finely ground salt and the whole body rubbed. Salt water artificially made in somewhat greater strength than sea water may be used.

At the Adirondack Cottage Sanatorium, Dr. Lawrason Brown states in a private communication to the author that some of the patients throughout the winter take cold sponges and some cold plunges, but he does not advocate a plunge into such cold water as flows from the faucets in winter, in the neighborhood of $40^{\circ} \mathrm{F}$. $\left(4.5^{\circ} \mathrm{C}\right.$.). His patients are treated usually along the lines laid down in his chapter on the treatment of pulmonary tuberculosis in Osler and McCrae's " Modern Medicine," but such measures are not advised, except in a very moderate form, if there be heart disease, aneurysm, or marked arteriosclerosis.

The bath should be taken in a room never below $55^{\circ} \mathrm{F}$. ( $12.8^{\circ} \mathrm{C}$.), and the lower half of the body should be kept clothed, while the upper half of the body is being cleansed with water at $100^{\circ} \mathrm{F} .\left(37.8^{\circ} \mathrm{C}.\right)$. According to Dr. Brown, in special cases full baths at $110^{\circ} \mathrm{F}$. may be advised. All cold sponging in the morning should, if possible, be preceded by a hot cleansing sponge. Cold water, $40^{\circ}$ to $60^{\circ} \mathrm{F}$. $\left(4.5^{\circ}\right.$ to $15.6^{\circ} \mathrm{C}$.), should then be applied with a sponge to the neck, to the front and back of the chest, and to the arms for from one to two minutes. The body is then dried with a coarse towel. In cases unaccustomed to a morning bath, it will be best to use tepid water at $80^{\circ}$ to $100^{\circ} \mathrm{F}$. $\left(26.7^{\circ}\right.$ to $37.8^{\circ} \mathrm{C}$.), gradually reducing the temperature each day until the lower temperatures are reached. In other cases it is best to begin with sponging one arm, then on the following day both arms, and so on until the body to the waist has been sponged. Alcohol rubs or salt rubs may be substituted in special cases. For more vigorous patients the cold plunge at $50^{\circ}$ to $70^{\circ} \mathrm{F}$. ( $10^{\circ}$ to $2 \mathrm{I} . \mathrm{I}^{\circ} \mathrm{C}$.) may be permitted, or the cold shower, or the full cold sponge while standing in warm 
water. In winter these measures are to be modified if they increase nervousness. These morning baths and sponges, however, are in no sense a substitute for a bath twice a week in water $100^{\circ}$ to $105^{\circ} \mathrm{F}$. $\left(37.8^{\circ}\right.$ to $40.6^{\circ} \mathrm{C}$. $)$, with a free use of soap.

In febrile cases great care should be taken to avoid any exhausting hydrotherapeutic measure. Dr. Brown advises a salt sponge in the morning or in the evening, followed by an alcohol rub, possibly limited to the back. This refreshes the patient and induces sleep. If the patient be weak, alcohol may be added to the salt water. If the sponges fail to reduce the temperature sufficiently, an ice rub may be given or a cold pack to the trunk. An ice bag over the heart frequently quiets the nervous system. If patients fail to react or feel chilly, it will be well to try dry rubbing, and in subsequent days sponging with warm water, gradually reducing the temperature. A little food or hot drink should be given before the morning bath. In sponging with salt water, onehalf ounce of salt to the quart of water will be sufficient.

For the tuberculous soldier who is able to be about, Colonel Frank Billings lays stress on the use of shower baths, and particularly the alternating hot to cold or hot to cool overhead shower bath. This form of bath can be applied to the most delicate individual who is not febrile and without shock, because with a primary overhead shower, not directed to the skull but to the shoulders, the cold can be gradually applied; and the end of the shower is also hot, so that reaction is perfect. This flushes the blood without exciting the patient and without exhaustion.

From personal observations of military and civil hospitals for the tuberculous, it would appear that not enough attention is given to simple hydrotherapeutic measures. Tuberculous patients need daily " neurovascular training" as well as simple physical exercise. This training should be commenced with ablutions, then followed with affusions, dripping sheet and wet pack gradually made of lower temperature. The apparatus required should be very simple, and we should urge some treatment along these lines in all hospitals for the tuberculous.

Dr. Baruch has formulated this system of " neurovascular training," founded on his experience at the Montefiore Home in New York, in a paper presented to the National Tuberculosis Association in 1912, and we heartily endorse it, as it is the product of a large experience. Needless to say, it has a much wider application than in the field of tuberculosis alone.

Graduated neurovascular training. Cardinal rule.-All water applications below $90^{\circ} \mathrm{F}$. must be made with friction and on rising from bed. 
First stage. Ablution.-Avoid extremities below knee and elbow. (a) Begin with $90^{\circ}$ with saturated linen cloth or gloved hand. (b) Pass rapidly over successive parts. (c) Dry thoroughly. (d) Dress and exercise in open air in ambulant cases. (e) Reduce temperature of water daily two or more degrees until $60^{\circ}$ is reached.

Second stage. Affusion.-(a) Patient stands in water at $105^{\circ}$. (b) From a bucket of water at $85^{\circ}$ water is dipped and thrown over back, each shoulder and chest. (c) Dry, dress and exercise in open air. (d) Reduce water temperature daily $5^{\circ}$ or more to $60^{\circ} \mathrm{F}$. (e) If reaction is good proceed to third stage.

Third stage. Cold rub.-(a) Patient stands in water at $105^{\circ} \mathrm{F}$. (b) A linen sheet is wrung out with water at $80^{\circ}$, decreased daily. (c) Damp sheet is wrapped over body snugly. (d) Patient is actively rubbed over sheet until sheet is warm. (e) Dry, dress and exercise in open air. (f) Reduce temperature of water $5^{\circ}$ to $10^{\circ}$ daily to $50^{\circ}$ if reaction is good. Then proceed to drip sheet.

Drip sheet. (a) Patient stands in water at $105^{\circ} \mathrm{F}$. (b) Upper edge (12 inches) of linen sheet is gathered into folds. (c) Sheet is dipped in water at $80^{\circ}$. (d) The dripping sheet is held by attendant while patient wraps himself into it by turning the body. (e) Friction and slapping with flat hands for two minutes. (f) Pour water at $70^{\circ}$ over shoulders. Rub and slap again until the sheet feels warm. (g) Repeat this over successive parts of body, rapidly. (h) Sheet removed. Patient dries, dresses, and exercises. (i) Reduce water temperature daily until $50^{\circ}$ is reached.

In small sanatoria and in private practice the douche may be replaced by the drip sheet.

It should be borne in mind that it is not the object of any hydrotherapeutic measure to produce in a tuberculous subject any degree of tissue change. A hot air bath or a tub of hot water should not be employed excepting for the purpose of enhancing reaction, and this presupposes a very short application. If such a measure causes perspiration, it should be shortened or omitted entirely.

\section{OCULAR AFFECTIONS}

Sweating processes, either with or without the use of jaborandi, are called for in many ocular affections. These include inflammation of the sclerocornea, of the uveal coat, acute and chronic, of the chorioretina and of the optic nerve. Its greatest value is in the chronic inflammation associated with exudation in large amount in the uveal coat, with secondary involvement of the tissues immediately adjacent.* After the

* The author is indebted to H. F. Hansell of Philadelphia. 
stage of atrophy and connective tissue change nothing can be hoped for. In cases where mercury is used a much larger dosage is possible when baths and sweating packs are used during the course of treatment. All oculists know the value of these measures. The sweats may be continued for as much as one and a half hours daily, and the mercurial inunctions carried to the point of saturation in specific cases. In some cases mercury and iodides used alone may fail, but in connection with sweating processes become eminently successful.

The writer has treated patients for Dr. de Schweinitz by the combined method with highly gratifying results, although the progress seemed at times exceedingly slow. Vitreous opacities and irido-choroiditis are particularly suitable for this form of treatment, and when combined with residence in an invigorating mountain climate these rather exhausting measures can be borne fairly well. Much, however, can be accomplished in well-equipped city hospitals, as Hansell's recorded cases $\left({ }^{2 \theta}\right)$ clearly prove.

One of Hansell's cases occurred in a woman who had become infected with syphilis. The usual secondary symptoms followed, and she was placed under mercury and potassium iodid. One year after infection iridocyclitis had developed in the right eye and two weeks later in the left eye. Vision at the time of examination was: R., counting fingers at one foot. L., 2/200; total posterior synechiae and vitreous opacities in both. No view of the fundus of either eye could be obtained. One week after commencing the sweats and mercurial inunctions the synechiae were almost all broken off, the vitreous had regained to a great extent its transparency and vision had improved to $20 / 70$ in each eye. She later was able to read and sew, and could see practically as well as before. In this case, notwithstanding the intelligent and continued treatment by mercury and iodid, the disease was not only not eradicated but not even held in abeyance. Seven sweats, each one and a half hours in duration, and mercurial inunctions carried to the point of saturation, saved the patient from a blindness that had been thought incurable.

In cases of pneumococcus infection of the cornea, heat, when applied by means of nickel-plated brass thermophore points at about $15 \mathrm{O}^{\circ}$ to $152^{\circ} \mathrm{F}$. for one minute, has been found very useful. The ulcers of the cornea are treated first in the upper temporal surface for one minute and then in the lower nasal surface for one minute. $\left({ }^{30}\right)\left({ }^{31}\right)$

\section{SKIN DISEASES}

There is no doubt that, in cases in which the entire integument is involved, baths constitute the most convenient, and in many cases, the most efficient mode of treatment. In a universal, chronic, squamous 
eczema it constitutes the best method of hastening desquamation, of softening the skin and in allaying, in great part, the intolerable itching which is present. The same may be said with equal truth concerning cases of subacute eczema. In such cases it will be found that a cool bath is the best, and it is still more grateful if some alkali like borax or bicarbonate of soda has been added to the water. In others again flaxseed, corn meal, bran or some other demulcent will add much to the comfort of the patient. The flaxseed, meal and bran should be boiled in bags of gauze and the resulting gelatinous liquid added to the bath. The skin is softer, less irritated and, consequently, better prepared for whatever medical treatment may be ordered. In ichthyosis we find that the same reasons hold as also in psoriasis; but in both of these sapo viridis must be used in order to insure the removal of the scales. In those skin cases which are to be looked upon as universal, baths are absolutely necessary in order to obtain any effects on the cutaneous disorder.

Although it is true that water is a veritable poison in acute eczema, it was found that in severe cases of generalized chronic eczema and other chronic squamous diseases the continuous bath was attended by success. In specially obstinate cases patients have spent months, and even years, in the continuous bath.

Severe types of pemphigus are greatly palliated by the use of the continuous bath. In the case of psoriasis there is no prospect of success unless, in conjunction with bathing, special attention is paid to the underlying problems connected with food metabolism in the adoption of a non-nitrogenous diet. Cold baths are probably harmful, and this includes sea bathing, but the tonic action of sea air and sunlight may favor the general health; the sunlight, however, should not be intense.

Warm baths favor the maceration and drainage of the skin, and the longer they are the more pronounced the effect. Bran baths used on alternate days with sulphur baths are sometimes advised. The generally refractory nature of psoriasis is reflected in the widely differing views held regarding all forms of treatment. $\left({ }^{32}\right)$

\section{HYDROTHERAPY IN MILITARY AND NAVAL HOSPITALS}

Hydrotherapy is of great value in military practice. The medical departments of all the armies recognize this, and provision is made for various measures such as showers, baths, sedative pools, and whirlpool baths for both acute and chronic cases. The physical treatment and training of disabled and discharged soldiers and sailors is now and will be for years to come a serious and urgent problem. Success in this 
direction means the prevention of an army of cripples. In Great Britain and on the Continent of Europe there have been organized a large number of hospitals providing hydrotherapy with associated installations for electrotherapy, mechanical treatment and massage. The same problems are now presented to American surgeons, and an equipment similar to that now in successful operation in Europe will be demanded. It is a wellrecognized fact that in a great majority of cases physical treatment should be provided as an integral part of re-education of the wounded and that the degree of progress made ought to be checked up by accurate measurements and tests.

A hydrotherapeutic unit should be attached to each general military hospital and especially to the reconstruction hospitals. In order to assure greater efficiency it is desirable that this hydrotherapeutic unit should be standardized both as to general plan and equipment. Such plans have been submitted to the Surgeon General of the United States Army and have been adopted on a scale which will be a credit to the country. This equipment is now in actual use at Fort McHenry, General Hospital No. 2; at Colonia, New Jersey, General Hospital No. 3, and at Walter Reed Hospital, Washington.

An immense advantage may be taken from experience already obtained in France and England. All the old measures have been utilized, the old bathing resorts have been filled with soldiers, but some very valuable new measures have been devised and used with remarkable success.

Hydrotherapeutic apparatus has been going through an evolutionary process during the last twenty-five years, and it is well recognized that in America it has attained a remarkable degree of perfection, far beyond that found in England and the Continent, but, on the other hand, excepting in a few centers, it has not been used in America to anything like the same extent and with the same intelligence that has marked the European practice of hydrotherapy and balneology. We are gradually remedying that, and the lessons from the present war will be learned and acted on.

In London the Red Cross Clinic for physical treatment of disabled officers was opened in Great Portland Street in 1916 and has provided treatment for the officers who are patients in the various hospitals of London. It is financed by the British Red Cross Society. A similar equipment is installed at several convalescent camps and military hospitals, especially the Military Orthopedic Hospital at Shepherd's Bush, Heaton Park, Manchester, and at the Army Hospital at Netley.

Besides the usual equipment for giving tonic douches, Scotch or alternating douches, needle douche, etc., there are two special features 


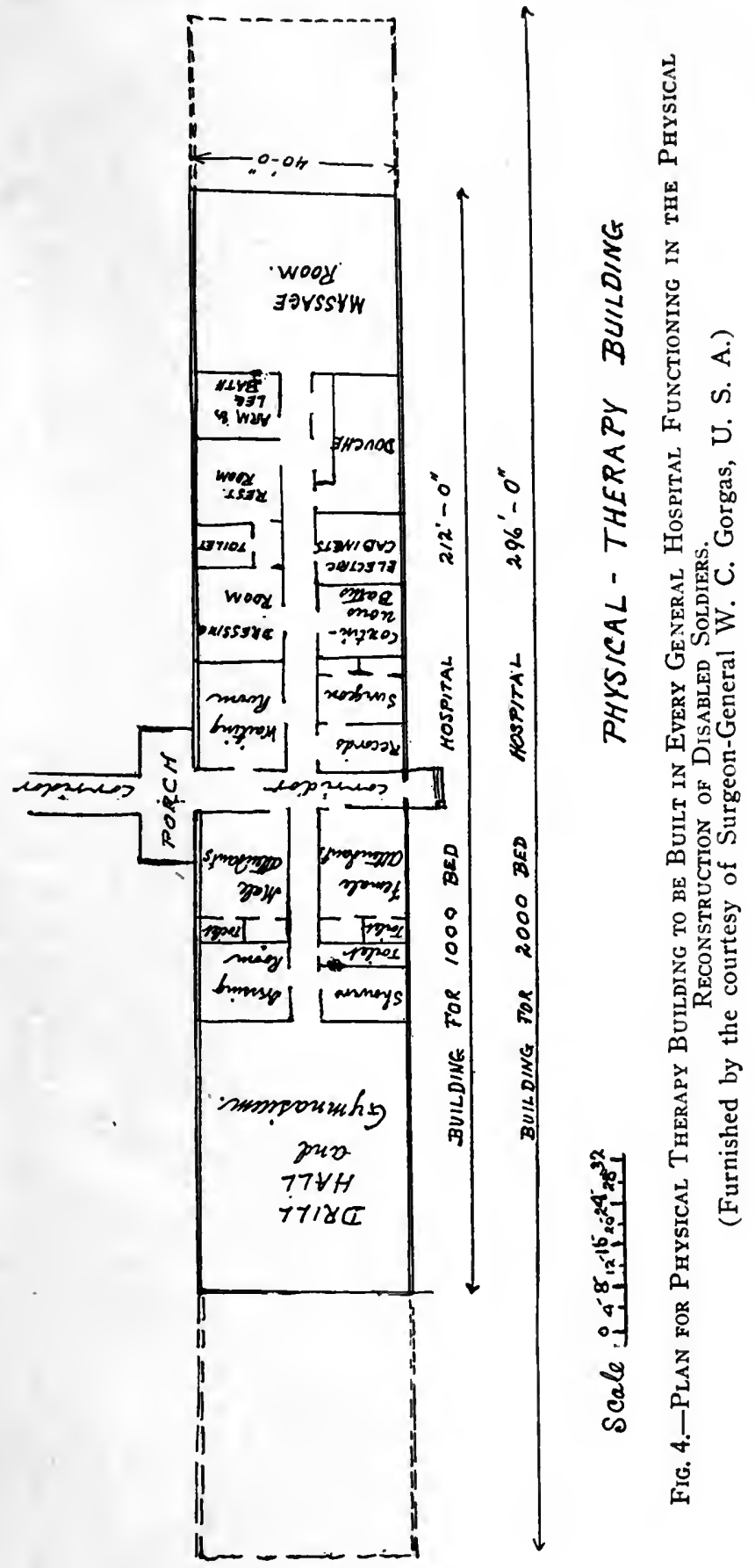


not as yet generally known in America. These are the "whirlpool baths" and "sedative pool baths."

\section{WHIRLPOOL BATHS}

Whirlpool baths are used in both French and English hospitals and depend for their usefulness on a rotary current, of a strength that can be varied at will and can be directed to any part of the limb. The arm or leg is placed in a local bath of running water (balnéation à l'eau courante). The temperature is hyperthermal, ranging from $104^{\circ} \mathrm{F}$. $\left(40^{\circ} \mathrm{C}\right.$.) to $\operatorname{II} 5^{\circ} \mathrm{F}$. $\left(46^{\circ} \mathrm{C}\right.$.). Starting with the lower temperature it is gradually raised and the bath may be prolonged to fifteen, twenty or thirty minutes as may be indicated.

In case of wounded limbs, especially where there are trophic lesions resulting from prolonged suppuration, chronic edemas, swellings of the periarticular tissues, fractures about joints, and in case of painful and adherent cicatrices, the whirlpool baths are very useful. They prepare the way for massage and passive movements and render them easier and less painful. Ten minutes in a whirlpool bath will prepare the limb better for passive movement than a still bath of three times the duration.

Such applications produce extreme vaso-dilatation and an increased arterial circulation. In addition to these familiar effects, which are well seen in the treatment of stiffness and fatigue fever by hyperthermal baths, it is believed that "whirlpool baths," as they may be called, have a special action due to the movement of the water. The hydro-massage, added to their high temperature, appears to have a marked sedative action in relieving pain and promoting the lymph circulation, diminishing the effusions and swellings of soft parts. This form of bath therefore accelerates the retrogression of sub-inflammatory conditions. Whirlpool baths are now given in the hospitals referred to before manual or mechanical treatment.

Hitherto the desired swirl has been obtained by means of a water service from a tank fixed at a height of fifty feet or more, or by the use of mechanically driven paddles fitted in the bottom of the baths after the manner of a turbine; but in the improved system the water pressure is independent of this. It is derived from an electrically driven centrifugal pump forcing the water at great pressure through tubes around the room in which the baths are placed; the baths are fed from these tubes by means of jets so arranged as to create a rapid swirl, the force of which can be controlled by means of a speed regulator attached to the motor pumps. The water for the baths is heated in a small calorifier which can be worked from an existing steam circulation, or by a gas-heated boiler, as may be most convenient. 
A constant temperature is maintained in the baths by fitting an automatically acting thermostatic valve on the calorifier. The water on leaving the baths is returned by gravitation to the calorifier, this continuous circulatory action effecting a great economy in fuel, as the water once heated, a very small amount of fuel is necessary to maintain the temperature. The only waste of water occurs when the system is emptied and refilled, which may be done as often as directed. These baths are made of appropriate design for the local treatment of arms, legs and feet.

\section{THE POOL BATH}

The pool as used in the English military hospitals is designed for sedative treatment especially in the cases of shell shock. In this the patients sit immersed up to the neck for hours at a time, and the capacity is sufficient to accommodate from four to sixteen men. The water for the pool can be heated from the same boiler as that used for the whirlpool baths, as by means of a second calorifier with separate thermostatic valve attached two distinct services of hot water each of different fixed temperatures can be maintained from the same boilereach service adapted for the different kind of treatment.

In the case of the pool there is usually fitted an attachment for injecting air into the water by means of a motor-driven air compressor. This may also be applied to the local baths and where it has been tried it is believed to have distinct advantages.

The cost of installation designed for twelve baths for local treatment in England is $\mathfrak{E}_{400}$, or $\$ 2,000$; when packed for shipment and ready for re-erection in another country it would be $£_{340}$, or $\$ 1,700$. The pool would be more economically built at the place in which it is to be installed.

We hope that never again there will be need to devise means to combat the effects of poisonous gases on the battlefield, but it is of historic interest to know how baths were employed to counteract their terrible effects in the recent war. The chemical warfare service used squadrons of traveling hot bath trucks in the treatment of soldiers suffering from German mustard gas. At several points behind the American sector these bath trucks were held in readiness to start instantly whenever the Germans started a gas bombardment.

The most important part of the treatment for mustard gas is a hot bath as soon as possible after the gas touches the skin. Before the inauguration of the "hurry-up" bath outfits, gas sufferers had to be transported to the nearest hospital with facilities for bathing. This sometimes meant a delay of several hours when every minute was 
precious. Under the new arrangements the "bath trucks" hurried toward the front upon receipt of a telephone message announcing the beginning of a gas bombardment. The trucks were rushed up as close to the firing line as possible, and within a few minutes were ready to begin operations.

Each truck carried a 2,500-gallon tank of water and an instantaneous water hater. Other automobiles carried a demountable bath pavilion and the necessary piping. The work of putting up a tented pavilion and making the water connections was a matter of only a few minutes. More than twenty soldiers could bathe simultaneously in the tent. As the gas sufferers were brought in the attendants undressed them and dipped the clothing in a solution of lime and water. Even the helmets and shoes were immersed in the solution. Meanwhile the sufferers were quickly sprayed with liquid soap and taken into the showers for fifteen seconds. Then other attendants scrubbed the gassed men and sprayed their eyes, noses and throats with a solution to counteract the burning and irritation of the poison. The men were then given clean clothing and sent to an evacuation hospital. The attendants wear oilskin one-piece suits with tight-fitting headpieces. During a recent test 48 men were bathed in two and one-half minutes.

\section{APPARATUS AND METHODS FOR HYDROTHERAPY}

At nearly all the health resorts and in hospitals and hydrotherapeutic establishments, there is a special equipment consisting of tubs, a sitz bath and a control table with arrangements for circular or needle, jet, steam and fan douches. This apparatus has been fully described in several works on hydrotherapy and the description need not be repeated here. $\left({ }^{33}\right)-\left({ }^{38}\right)$

On application to the manufacturers much detailed information and illustrations of the various cabinets, tubs, control tables and general equipment can be obtained. Those in general use in the United States are made by the J. L. Mott Company of New York; the New York Hydrotherapeutic Apparatus Company, 66 West 7oth Street, New York, and James B. Clow and Sons, 534 South Franklin Street, Chicago.

The control table is the largest single item of expense but is usually necessary for quick and accurate work. For practice in private much can be accomplished with ordinary tub baths, packs, drip sheets, foot tubs, sitz tubs and the various portable cabinets now supplied by dealers. More is accomplished naturally by experienced attendants. Physicians are not themselves adept in carrying out these procedures, and a good 
attendant understands the niceties so requisite for the comfort and confidence of the patient.

It is impossible to describe all the measures adopted in our institutions. The list has grown to an unnecessary length. Neither have we been able to include all the affections amenable to hydrologic treatment; but we have mentioned some of the diseases and will now refer to some of the more important types of treatment.

It would be highly desirable if there could be some standardization of hydrotherapeutic measures. This has been attempted in other fields as, for example, electrotherapy. It is not wholly impossible to follow standard methods without subjecting patients to thoughtless routine treatment. It is better to understand and practice a few of the time-honored bathing procedures than to disregard them all because charlatans have in the past recognized their usefulness. In English, French and American health resorts a genuine attempt has been made for many years to apply rational hydrotherapy, and physicians at these resorts have made an earnest attempt to establish it on a scientific foundation.

In case the patient is not sent to a health resort or sanatorium there are bathing establishments in American cities where tonic bathing is afforded. In Boston the Medical Baths were established fifteen years ago by the late Dr. James J. Putnam, Dr. J. H. Pratt and others. In Philadelphia there are two similar institutions. In Chicago there is the Institute of Physiologic Therapeutics established in 1907 by Dr. William S. Sadler. These are all equipped with the necessary adjuncts for physical therapy. New York has several institutions such as the Neurological Institute, the Hydriatic Institute, the Astor, Biltmore and Plaza Baths. In London there is the Alexandra Institute of Baths and in Paris Dr. Darecq's hydrotherapeutic establishment, both of which have been noted for many years for their scientific methods.

\section{CONTINUOUS BATHS}

When the time of a warm bath extends from fifteen minutes to two hours, it is termed a prolonged bath; when it occupies more than two hours, it is usually termed a continuous bath.

The tubs used for continuous baths are somewhat different from ordinary bathtubs; they are provided generally with temperature regulators and especially large outflow pipes. They are also provided with an extra-sized roll rim under which are hooks to attach the restraining sheets and hands should these ever be required; a cushion is provided for the head and a hammock is arranged so as to give support to the body. The temperature of the water is usually kept at $98^{\circ} \mathrm{F} .\left(36.7^{\circ} \mathrm{C}\right.$. $)$. 
The J. L. Mott Company of New York and Clow and Company of Chicago make an excellent model of porcelain enameled iron tub for continuous baths. We have described the use of these baths in mental cases, but they need not be confined to this class. They have been used successfully in surgical and dermatological practice. It is recorded that Paulus of Aegina used them in the seventh century under the reign of Heraclius, the Byzantine emperor. Paulus had patients operated on for hernia, especially children, whom he submerged in warm water for seven consecutive days in order to prevent inflammatory symptoms.

Among surgical affections which have been successfully treated during the last forty years are severe burns. Excellent results have been noted in the relief of pain and the avoiding of infection, lymphangitis, pyemia, etc.; suppuration was slight, new skin rapidly formed and the scars were better than under other treatment.

Among skin diseases pemphigus vulgaris, bedsores, psoriasis and chronic eczema have been favorably influenced by continuous baths. In cases of bedsores developing in patients with spinal cord disease the baths have been found to help both the disease and the bedsores. Confluent smallpox has been treated in this manner, thus controlling the fever of suppuration and modifying the scars. Gangrene of the skin, gangrenous buboes, and phagedenic chancres may be included among conditions suitable to such treatment.

Among nervous diseases such as meningitis, various paralyses, obstinate sciatica, hysteria, and alcoholism there are countless cases that should be treated with continuous baths. The good results may be summed up as follows:

First, they act as a sedative upon the nervous system.

Second, they influence the circulation by diverting blood from the inner organs toward the skin.

Third, they favor the nutrition and healing processes in the skin.

Fourth, they augment heat withdrawal.

Fifth, they accelerate metabolism.

In formidable diseases like pemphigus this bath relieves pain and reduces fever although the ultimate result in these cases is as a rule fatal.

The chief requisites for the treatment room are ample space, sufficient light and air, and mechanical facilities for the shutting off of sound communication with the outside world. In the Pennsylvania Hospital for the Insane there are two large sunny bathrooms, each containing extra-sized tubs, and equipped with heavy plate glass double windows and doors and ventilating devices. With the door and windows closed, the rooms are practically sound proof. The temperature of the 
$222^{a}$ 


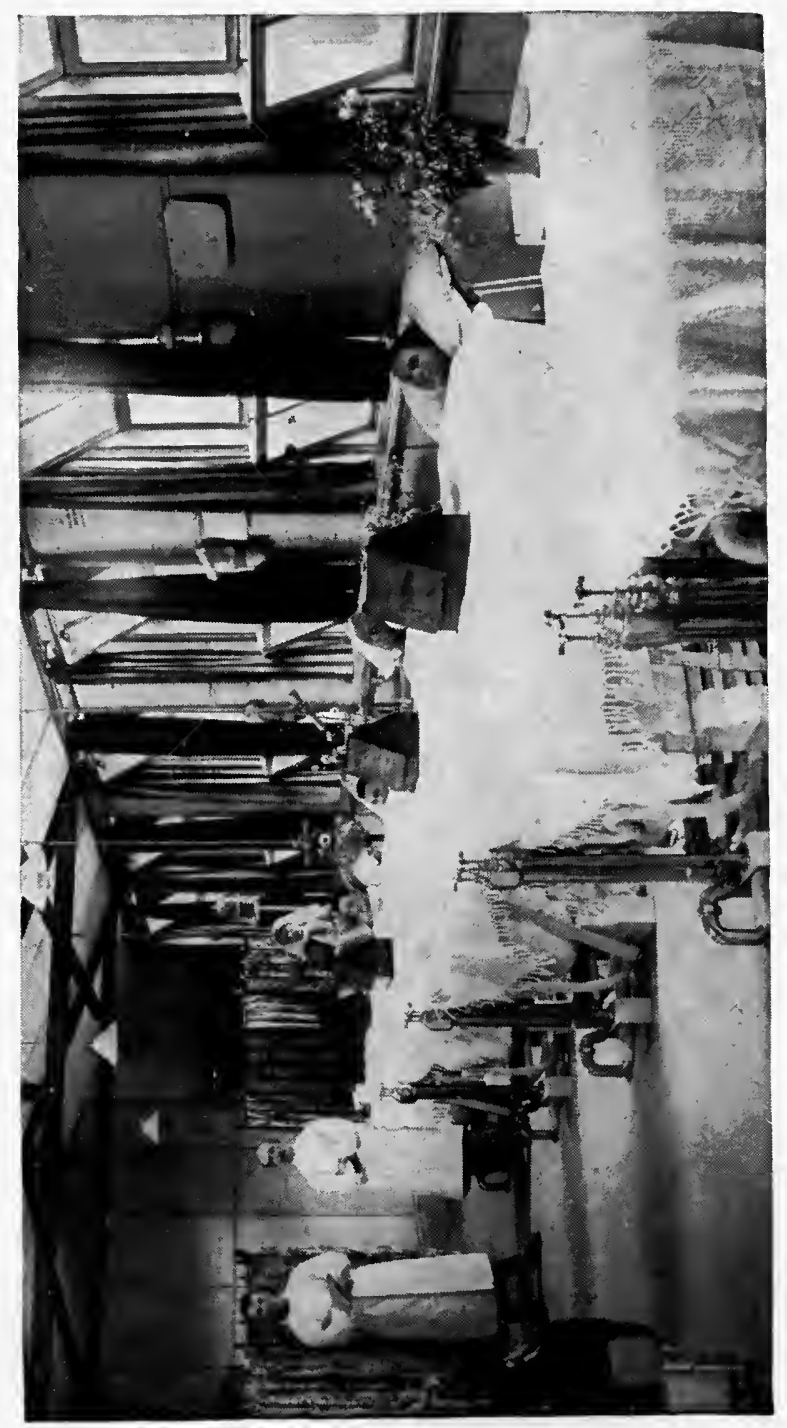

总焉

它焉里 . की

눙용

要势

运穿

En

कै

证牙

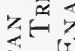

is

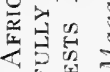

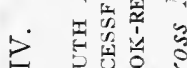

(1)

ज.

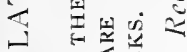

D

然里

佥负

$\geqslant$ 密

安密记

落舟

究要

论䁬

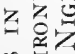

元

政

的穵是

尔趽

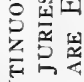

3 
water is controlled with a control table, and the nurse, who is in constant attendance, takes half-hour readings from an ordinary bath thermometer. The patients are clothed in a loose-fitting one-piece garment, permitting great freedom of movement. No restraint whatever is used in the tubs. There is an observation window facing the hall through which every part of the bathroom can be readily seen. An electric button enables the attending nurse to signal for assistance in case of emergency.

The continuous bathroom of the future will probably be developed along somewhat more esthetic lines. Since certain patients are under treatment for many hours, days and sometimes weeks, the room must necessarily be used not only as a bath, but also as a living apartment. The introduction of books, magazines, a few games and puzzles, and possibly a picture or two on the walls, will perhaps help to modify its rather monotonous appearance. There is also a distinct need for more individual bathrooms, in which certain types of patients, who are either annoying to others or are themselves easily disturbed, may be placed under treatment. Partial segregation may be accomplished by the use of screens.

\section{RULES FOR CONTINUOUS TUBS IN FORCE AT THE PENNSYLVANIA HOSPITAL, DEPARTMENT FOR MENTAL AND NERVOUS DISEASES}

I. Each morning before using baths fill them with the hottcst water obtainable and record the temperature. Telephone to a physician if the temperature is over $120^{\circ}$. This always insures a warm tub to start with.

2. Fill the tub with water at body temperature before the patient is allowed to get in. Never fill a tub with the patient in it. $\left.99^{\circ}\right)$.

3. The water in the tubs should be at body temperature $\left(98^{\circ}\right.$ to

4. Test the temperature every thirty minutes by placing your arm in the tub water and by taking the reading from a thermometer in the tub.

5. After adjusting the temperature of a tub full of water, shut off the intake of water for about thirty minutes, then gradually adjust the temperature if necessary. Do not keep a continuous stream going.

6. Notice when a patient gets her ears filled with water. Thoroughly dry the ears when the patient is taken from the bath.

7. Do not use mechanical help to keep patient in tub.

8. Do not leave the room while patients are in the baths.

9. When patient leaves the bathroom provide clothing and especially slippers, to avoid exposure to cold. 
Under these rules no accident possibly can happen during continuous baths. Fatal accidents have occurred elsewhere, and Dr. William A. White of the United States Government Hospital for the Insane has called attention to these accidents which have happened where restraint was used.

\section{SITZ BATH}

Three changes. Cold cloth to head. Two sitz baths should be used. Temperature ranging from $106^{\circ}$ to i $18^{\circ}$, reducing to $60^{\circ}$ or $55^{\circ}$, according to patient's condition. The following temperatures give very good results: I I ${ }^{\circ}, 4$ minutes; I $16^{\circ}, 6$ minutes; $65^{\circ}, 4$ minutes. The feet always should be placed in a foot bath, the temperature of the latter ranging from $102^{\circ}$ to II $5^{\circ} \mathrm{F}$. The medium is found between $110^{\circ}$ to II $4^{\circ}$. The patient is to be entirely covered with a sheet, at the same time covering foot tub and bath.

\section{PERINEAL DOUCHE}

Temperature, $-100^{\circ}$ to $\mathrm{IIO}^{\circ}$. Gentle pressure. Douche stool is to be covered with towel laid in circular form; sheet is to be placed around patient. Duration of treatment, about five minutes.

\section{THE SHEET BATH AND THE DRIP SHEET}

Both these measures involve the use of cold water to the entire body and require only sheets for their application, but in the first case they are employed with the patient in the recumbent position, while the drip sheet must be used when the patient is standing. The sheet bath is used for an antifebrile effect when we wish to avoid the fatigue accompanying a full bath, and it has, incidentally, a more decided antipyretic action. It may be applied in acute disease and especially when tubs are not available. It would therefore have an obvious advantage in military practice as well as in rural districts. The following are Dr. Baruch's instructions.

The bed is prepared with a rubber sheet over which a blanket is laid and several linen sheets. A basin and a tub of water at a temperature from $50^{\circ}$ to $80^{\circ} \mathrm{F}$., according to the effect desired, should be at hand. A cup of ice water and a sponge will also be required. A sheet is placed in the tub and partly wrung out and rapidly placed on the bed. The head and face of the patient are bathed with ice water, and a wet turban having been placed on his head, he is laid on the sheet and directed to hold both arms above his head. The upper left border of the sheet is now brought close under the left axilla and laid across the front of the chest, reaching beyond the axillary line of the right side, while the lower 
portion is placed over the pelvis and the edge tucked in between the thighs and legs. The arms are brought down and placed alongside the body, from which they are separated by the intervening sheet. The right portion of the sheet is now carried across the body, above and below, enveloping the arms and shoulders and lower extremities. The right upper border of the sheet is firmly drawn over the left shoulder and securely tucked underneath the heels. In this manner the patient is snugly enveloped in the wet sheet and no uncovered parts of the body lie in apposition. The arms may, in feebler patients, be left out altogether and simply bathed during the process.

The first impression will be a shock to the peripheral nerves, caused by the sudden contact with the cold wet sheet and a deep gasping and a little shivering follow. In febrile cases especially these are readily overcome by the patient's temperature aided by the manipulations of the bath nurse, who, with outstretched hands, gently but firmly and gradually sweeps over the wet sheet, passing over the entire body successively. Small parts of the body should be rubbed in this manner until they warm up. So soon as any part of the body becomes thoroughly warmed, water from $50^{\circ}$ to $60^{\circ}$, according to the condition of the patient, is poured from a cup or squeezed from the sponge over the body and rubbing resumed. These gentle but firm passes or friction are alternated with the pouring on of small quantities of cold water until the entire body feels cooled or the patient shivers a good deal. Friction thus prevents all objectionable features of cold baths and permits the renewed applications of cold water on the warm part of the sheet to maintain the cooling effect. There need be no fear that patients with a temperature of $103^{\circ}$ or over will take cold: The patient may be put to bed covered up in the sheet and blankets and allowed to remain a half-hour. $\mathrm{He}$ will possibly go to sleep.

The drip sheet, as we have said, is far more suitable for the chronic forms of disease and is given in the standing position. The temperature of the room should not be less than $70^{\circ} \mathrm{F}$. The patient stands in a foot bath of about twelve inches of water at $100^{\circ} \mathrm{F}$. A sheet dipped in water at $75^{\circ} \mathrm{F}$., daily, or less frequently, reduced until it reaches $60^{\circ}$, is placed over his shoulders and back as follows:-The left upper border of the sheet is held by the left hand while the right gathers the right border into folds. The sheet is now dipped into a bucket of water from which it is taken dripping and applied under the right axilla of the patient. Pressing the sheet firmly to his side with the right arm the patient is directed to turn and thus envelop himself in the wet sheet. When the entire body is thus covered, the sheet is tucked in around the neck and the lower border is wrapped around the legs. The attendant 
now makes rapid passes over the sheet, up and down the back, sides and lower extremities with the outstretched hand, occasionally slapping the surface to increase mechanical irritation. A basin of water from ten to fifteen degrees below the temperature of the sheet water is poured over the head and shoulders two or three times at short intervals. This is alternated with frictions for from five to ten minutes. The sheet is now rapidly withdrawn. The skin becomes decidedly hyperemic and this effect shows more intensely as the process is repeated. The patient now steps upon a woolen rug and is thoroughly dried. This is followed by friction with a warm sheet or towel which increases the hyperemia of the skin. There may be some fatigue but the patient feels refreshed. If there is much fatigue the process should be shortened in the next application, but as a rule fatigue is slight and the patient should be encouraged to take a walk afterwards.

The drip sheet should have a wider use. It is very simple and effective. Its value was highly appreciated by Weir Mitchell in many nervous affections such as melancholia, hysteria and hypochondriasis, and by many physicians in institutional practice; but as it is susceptible of various modifications, and as effects vary accordingly, experienced attendants naturally get better results. Two to five minutes, according to Dr. Baruch, suffice for a tonic affect in most cases, while for an antipyretic effect, fifteen to twenty minutes may be required.

\section{COMPRESSES}

The abdominal compress for conveying heat is one of the most comfortable of all hydrotherapeutic measures. Three or more changes should be made at intervals of three minutes. A bath towel is first placed over the abdomen; a fomentation, wrung dry from very hot water, wrapped in a piece of woolen blanket to retain the heat is placed in position and covered well with blankets. In applying. compresses to the arm or leg great care should be taken, particularly with the heels, so as not to burn, as in that region there may be lack of proper temperature sense. Always finish with an ice or cold water rub.

A strong revulsive effect can be produced by removing the fomentation or compress at the end of the periods and rubbing with ice. When fomentations are used on the trunk, finish by rubbing with ice commencing at the lower part of the spine and then up and down each side twice. Dry with towels and renew the fomentation if necessary for a period of fifteen to twenty minutes. 


\section{HOT TRUNK ENVELOPING PACK}

We first lay a dry blanket across the couch, folded crosswise, sufficiently wide to reach from the axilla to the pubes; then a blanket previously wrung as dry as possible from boiling water is placed over the first. This should be four inches narrower than the first. Another dry blanket is to be placed over this and a sheet is to be placed over the third blanket. A bath towel should be placed over the sheet so as to protect the hips, as in many cases they need extra protection.

The patient is then to be wrapped snugly from one side and then the other, placing a cold compress or turban to the head. The feet must be made warm. After the time for the duration of the pack has expired, a cool spray should be applied or cool sponging where a spray is not convenient. Sheets should not be used instead of blankets, as they are not nearly so efficacious when heat is to be applied.

In using cold packs sheets may be used; for the final cold application previously referred to we may substitute a tepid shower or an alcoholic rub.

Cold trunk compresses are indicated in inflammatory disorders of the abdominal and pelvic organs and in all varieties of hemorrhage from those organs. Cold may be maintained by a Leiter coil of rubber tubing carrying cold water.

Hot packs are of great value in case of suppression of urine and abdominal pain from gastritis, gastralgia, and in biliary, renal and intestinal colic. It should be noted, however, that when they are removed the damp blankets are relatively cool, whereas when the cold wet sheet of the cold pack is removed it is decidedly warm. This is a point in favor of the cold pack and is due to the activity of the compensatory heat-regulating function not observed when hot blanket packs are used.

\section{FOMENTATIONS}

These are applied so as to cover an area two or three times that of the affected part, using blanket flannel folded in five or six thicknesses. The flannel already folded is dipped into very hot water and it must be wrung very dry. Care must be taken that the fomentation does not burn, particularly with paralytics, diabetics, the feeble and the insane; in delirium and collapse; in children and elderly people. Sensitive parts, in particular bony prominences such as the hips, clavicles, etc., must have extra protection with dry flannel. A cold compress should always be applied to the head. Finish with cold, either ice, wet-hand rub, or wet towel.

Effects: Primary-Dilates the surface vessels, emptying the deeper blood vessels, and relieves pain in the affected parts. 
Secondary-Sedative, lowers the vessel tone and diminishes vital activity.

Third-Lessens sensibility of the cutaneous nerves and relieves in reflex related parts.

The following is Dr. Curran Pope's graphic description of the application of hot compresses. ( ${ }^{38}$ )

"I will now simplify my apparatus by limiting it to a basin, two towels, and a tea-kettle filled with boiling water. The majority of hot applications fail for two reasons. In the first place the application is too wet, and in the second the hot application is too cold. Hot applications should range anywhere between $125^{\circ}$ and $165^{\circ} \mathrm{F}$. (51.7 $7^{\circ}$ and $73.9^{\circ} \mathrm{C}$.). You cannot handle them with your hands; in fact, they are too hot for you to pick up and hold at all. If you put these applications on the patient's skin too wet they blister. If you put them on only warm you lose two-thirds of the benefit of the treatment.

"Suppose that we are going to make a hot moist application or fomentation to the pelvis, where we want all the heat we can get. It is best to use a Turkish towel or a small piece of blanket. If you can't get that, get a piece of an old flannel petticoat. Take this and place it right in the center of your towel. Now you have everything ready, and the mistress of the house brings in the tea-kettle full of boiling water. If you were to place it on the patient without previous preparation you will burn her. Place around the hips, pelvis, and lower abdomen a folded blanket, so applied that the ends overlap in front. Now rub the surface to be covered by the fomentation with vaselin. (The use of vaselin in this case does not warrant its use when dry heat at much higher temperature is used, as in "baking.") This prevents maceration or softening of the superficial epithelial layers. Having poured the boiling water over the flannel that lies in the water so hot that you have to keep back, two persons commence twisting the opposite ends of the towel and keep twisting until all or nearly all the water has been removed. We have in this towel, we will say, a piece of flannel at a temperature of $165^{\circ} \mathrm{F}$. $\left(73.9^{\circ} \mathrm{C}\right.$.), and what does the nurse now do? She picks up the towel containing the flannel, goes to the bedside, rapidly unrolls the towel, and places the hot flannel in place upon the bare skin. If the patient howls, lift it up for a second, and then put it back again. Just as soon as the patient can tolerate the high temperature, the fomentation is covered by the blanket, which is pulled as tight as possible to prevent the entrance of the air. There is very little risk of burning if plenty of vaselin has been used." 


\section{VICHY DOUCHE}

This douche is given with the patient in the horizontal position on a marble slab. The water descends in a fine spray from three roses attached to a movable bracket. This is so arranged as to be conveniently drawn down to within twelve to fourteen inches of the body. The water under pressure should be at $106^{\circ} \mathrm{F}$. for from five to eight minutes. Then the temperature may be gradually lowered while the body and limbs are thoroughly shampooed.

\section{SWEDISH SHAMPOO}

Shampoo the body thoroughly with soap, then use spray, then Vichy douche with thorough rubbing of the entire body, then finish with five minutes Swedish movements of the arms and limbs. Take the patient to the douche room, use needles one minute $105^{\circ} \mathrm{F}$. with hot and cold douche over spine, legs and arms, $100^{\circ}$ and $80^{\circ}$. Dry and give oil rub, then spray with alcohol in an atomizer and use talcum powder. Time of treatment thirty minutes.

\section{RUSSIAN BATH}

Give patient full tub bath at a temperature of $98^{\circ}$ for eight minutes, take patient to shampoo room, give bran rub, use spray, then Vichy douche with friction massage. Use Swedish movements of arms and limbs for two minutes. Take patient to douche room, use steam jet one-half minute, then give shower one minute, temperature $100^{\circ}$ to $70^{\circ}$. Dry and give alcohol rub, then spray the body with alcohol in an atomizer and use talcum powder. Time of treatment thirty minutes.

\section{SALT MASSAGE}

Place the patient on a marble slab, use spray over body, and give friction with rubber glove for five minutes, then give a good salt rub, use spray; then Vichy douche with rub while douching, and give Swedish movements to arms and limbs for two minutes. Take to douche room, use steam spray one-half minute. Use needles one minute, then give hot and cold douche over spine and limbs. Give good dry rub, then oil rub, spray the body with alcohol in an atomizer and use talcum powder. Time of treatment thirty minutes.

\section{SALT RUB}

Have patient stand in a foot bath at a temperature of $102^{\circ}$ to $115^{\circ}$. Moisten fine salt. Procedure.-To arms, chest and back and legs. simultaneous movements. Wet the part first, previous to applying the 
salt. Method.-Commencing at the shoulder, coming down to hand. Chest and back, commence by wetting all over trunk, rubbing simultaneously. Legs, commence at thigh, downward to foot. Pressure even, modified according to patient. Gently over sensitive parts and bony prominences. Immediately followed by spray. Duration two to three minutes. Also called "Salt Glow."

\section{BRAN RUB}

Similar to the above excepting that bran is used instead of salt.

\section{OIL RUB}

This is applied in turn to the arms, chest and abdomen, legs and back. A good cold cream or mineral oil may be used. Apply the lubricant with a long stroke from wrist to shoulder, returning with four rotary sweeps. Do this three times. Beginning at the tips of the fingers, give heavy passes to palm and back of hand. Raise the arm upright, then with the hands encircling the arm give heavy passes from wrist to axilla, sweeping the outer hand over point of shoulder. Glide back. Do this three times. Centrifugal strokes from shoulder to wrist, very light, three times. Apply lubricant to chest and abdomen with long strokes up center, returning with four rotary sweeps down the sides, covering whole surface. Do this three times. Stroke downward over the sternomastoid muscles three times; from ears to shoulder three times. Stroking with thumbs and thenar surfaces give heavy stroking from median line, outward, over ribs and abdomen, three at each point, advancing downward to the abdomen, stroking up over sternum, out to elbow, coming back with a firm stroke on the arms and down the sides. Do this three times. Apply lubricant to legs with a long stroke from ankle to hip, returning with four rotary sweeps. Do this three times. With the leg extended apply heavy centripetal stroking simultaneously to the side of the foot with the fingers and to the sole with the palm; three times. Stroke the dorsum of the foot three times. Rotary strokes to the heel; three times. Leg flexed, one hand on knee, with the other give heavy friction to the calf of the leg, ankle to knee, then with both hands encircling the limb with thumbs anterior and meeting, treat the thigh from knee to hip, covering the anterior surface and sides; three times. Long centrifugal strokes from hip to toes; three times. Lubricate the back with long strokes up the center, with four rotary sweeps down the sides, including the hips; three times. Strokes down the back of the neck; three times. Strokes from ear to shoulders; three times. Transverse wringing of whole back, three times in each area from neck to hips. Starting at the sacrum pass up each side of the spine with a zigzag 
movement applied with the tips of the fingers, then out to the elbows, coming back with a firm stroke on the arms and down the sides; three times. Tranverse friction with alternate strokes across the hips, right to left, covering the surface systematically; three times. Stroke from the median line outward over the flesh parts, covering the whole surface; three times. Rotary movement starting at the median line, describing large circles with one hand on each side and covering the whole surface. Light stroking up center and back and down to the sides; three times. Alternate strokes the whole length of the spine; six times.

\section{MOLIERE BATH}

This is used at some institutions for the treatment of neurasthenic cases. It consists of an electric cabinet bath provided with incandescent lamps and also an electrical attachment with electrodes. The current passes from the sponge electrode at the back to electrodes on the soles of the feet. The lights are turned on and perspiration ensues during the bath of ten to twenty minutes. There is a door in the back of the cabinet to admit of arranging the electrodes. This procedure is followed by a needle douche of two minutes at $106^{\circ}$ to $108^{\circ}$ reduced to $90^{\circ}$; and this is followed by an alcohol rub.

\section{MUD BATHS; PEAT BATHS}

The use of mud baths and peat baths has been practiced for thousands of years in Europe and they were highly prized by the Romans. At Acqui, in northern. Italy, not far from Genoa and Milan, there are hot sulphur springs which were mentioned by Pliny. The baths consist in the application of hot fango, a mud-like substance impregnated with organic matter and with the salts of the mineral waters. The temperature of the mud when applied is about $122^{\circ} \mathrm{F}$. $\left(50^{\circ} \mathrm{C}\right.$.), or considerably above that usually employed in mineral baths. This mud is of volcanic origin and is used at Abano, Battaglia and Valdieri in Italy, in Dax and Saint Amand in France, and at Arrowhead Hot Springs in California. At Buxton, England, there are peat baths; the latter contain both organic and inorganic matter and are applied like immense poultices. Both mud and peat baths are continued much longer than the usual baths. They may last for an hour or an hour and a half when applied only locally. They are specially useful in neuralgia, gout, rheumatism, arthritis and lumbago. The fango of Battaglia is a soft, grayish brown, plastic substance about the consistency of butter, without odor. It is first applied at about $\mathrm{I} 10^{\circ} \mathrm{F}$. $\left(43.3^{\circ} \mathrm{C}\right.$.) for ten minutes; in subsequent applications the temperature and duration is increased until $125^{\circ} \mathrm{F}$. $\left(51.7^{\circ}\right.$ C.) and a duration of twenty, thirty or forty minutes is reached. A 
more moderate temperature is required where the entire body is enveloped.

The fango has been exported from Italy to England and America for this purpose. At Arrowhead Hot Springs in California the mud was originally used by the Indians. They dug a shallow hole, put in leaves and laid the patient on these and then covered him with the mud. The whole marsh near Arrowhead Springs and Palm Hot Springs, close by, is impregnated with minerals; the hot waters emerge from countless vents through the peaty material of the tule marsh.

As now applied, the mud is passed through large vats and over a year is required to give it the proper character. This process of "curing" destroys all organic matter and leaves the finished mud nearly aseptic, containing pure carbonaceous matter as well as the associated salts, chiefly sodium bicarbonate, calcium bicarbonate and sodium sulphate in higher concentration than the waters of the springs. They have a wonderful effect in softening the skin, in relieving pain and reducing inflammatory conditions about joints.

A special cot is covered with a large canvas; mud is spread on this canvas a little wider and longer than the patient. On lying down he is then wholly or partly covered with mud as the case may demand. The sides and ends of the canvas are then brought up over the covering of mud, thus enclosing the patient in a solid mud pack. The degree of heat is $\mathrm{IIO}^{\circ} \mathrm{F}$.

While the patient is taking the pack cold cloths are placed on the head, and cold drinks given if desirable, the patient usually remaining in the pack for about fifteen minutes. When the time is up the canvas is folded back and the impurities have come pouring out with the perspiration, the elimination induced by the heat and chemical action of the mud and water being marvelous.

The patient then goes to a warm shower to remove the last traces of the mud; from there to a warm tub and back to a warm shower, which is gradually cooled to the proper degree. The patient is then rubbed dry and placed on a cot in the cooling room, where he can take a quiet nap and rest for half an hour or more before dressing. The mud pack is also used for a single limb or any portion of the body desired for local treatment. Mud baths are also given at Mudlavia, Indiana, U. S. A.

\section{SEA BATHS}

Sea baths are employed more than any other kind of bath, and yet they are not very much employed as a distinctly therapeutic measure. At Atlantic City and at Coney Island as many as one hundred thousand 
may bathe in a single day at the height of the season, and at countless places on the Atlantic Coast this is the principal pastime. It is rather rare for anyone to take ocean baths under the direction or guidance of a physician. No competent person is usually in attendance. It is almost impossible to keep track of the time elapsing, and few have any idea of the temperature of the water.

The conditions of ocean bathing are by no means constant. The temperature of the water changes almost from day to day, especially when the wind makes a notable change. Preceding or during a heavy northeast or easterly storm on the Atlantic Coast, the strong winds drive the surface water toward the shore, with the result that in summer the temperature rises and there is excellent bathing. As the storm clears away and the wind blows fresh from off shore, the surface water is blown out to sea, and colder water from greater depths takes its place. Thus there may be a fall of eight or nine degrees Fahrenheit in twentyfour hours. When the wind is west or northwest, we may expect cold bathing on the North Atlantic Coast. At the coast resorts of Nova Scotia and eastern Maine, the water is too cold for enjoyable bathing except for the robust. On the westerly or southwesterly coast of Maine, and thence around the New England Coast, there is excellent summer bathing at a temperature from $60^{\circ}$ up to $70^{\circ}$. In Long Island Sound and on the south shore of Long Island, and on the New Jersey Coast, higher temperatures are reached after July I5th. At Manhattan Beach, Long Branch, Spring Lake, Atlantic City and Cape May, and at innumerable intermediate points, excellent bathing is the feature of summer life. In winter we may have good bathing at Palm Beach, where the temperature of the water during the latter part of January, February and March is $70^{\circ} \mathrm{F}$. It is the same at Nassau during January, but rises slightly during February and March. Sea bathing is quite popular during these months. On the Pacific Coast the water temperature at San Diego is above $70^{\circ}$ from June 15 th to September 30 th, and usually reaches a maximum of $74^{\circ}$ or $75^{\circ}$ during the latter part of July and August, after which it rapidly falls until a minimum of $54^{\circ}$ is reached December I 5 th. The water is frequently warmer than the air. Ocean bathing also varies greatly in regard to the force of the waves. Surf bathing in cold water calls for greater activity in meeting the onrushing waves, and the reaction is usually quickly established. For those who do not swim, the quieter bathing of protected bays, devoid of surf, is not so safe, because the bather does not know how to make proper physical effort; hence he is more quickly chilled and fails to enjoy the bath, or at least fails to get sufficient exhilarating and refreshing exercise.

Aside from the danger of being carried beyond one's depth by un- 
known currents or the common danger on some parts of the New Jersey Coast of getting into channels of deep water on returning shoreward, a rather frequent source of trouble, there is the danger of remaining in the bath too long and of failing to react properly afterward. On entering the water there is a profound impression on the circulation. The temperature may be thirty or forty degrees below that of the body, and the stimulus to the nerve centers is great. The superficial vessels are immediately contracted, and the abstraction of heat from the extensive surface calls immediately for greater heat production. The momentary shiver on entering the water should promptly give place to warmth and a sense of reaction. When the skin circulation is poor, as it is commonly in those inherently feeble or weak from illness, or in those of advanced years, sea bathing should be avoided at least until better conditions obtain. In any case of heart disease, great care should be exercised; but, as is well known, cold bathing, when properly used, tends to strengthen the dilated and weakened heart. Persons with cardiac lesions should have friends or attendants near, and take no risks.

Complete immersion is a sine qua non of safety; it tends to equalize the circulation and hasten reaction. Women especially dislike to wet their heads, as it takes so long to dry their hair; but with closely fitting rubber bathing caps the hair may be kept dry and the head and neck are sufficiently cooled. Sea bathing should not be indulged in when the body is greatly overheated; but a good warmth is to be desired, and no time lost so as to get chilled by strong winds before going into the water. The chief safeguard in the water is to keep moving. A tennis ball in the surf promotes activity and adds to the pleasure of the bath. Of course, no one should bathe shortly after a meal. Two hours should be allowed for digestion. One should not bathe when greatly fatigued. The bath in the quiet of one's room will then be safer and more refreshing.

The writer has taken ocean baths each summer for many years, but has rarely seen ill effects in those who bathe regularly. Children who go in and out, and sun themselves on the sands, and return again to the water sometimes show bad effects; but most young people acquire a well-tanned skin in summer, and they become so accustomed to sun and water that they seem to bear those transitions with wonderful ease. The author saw a case of appendicitis in a boy of ten, fever and pain developing on the evening of the day on which he bathed in the surf. Forty hours after the bath the operation disclosed free pus in the abdominal cavity. In this case, which fortunately terminated in recovery, no other exciting causes could be assigned, and it was believed that imprudent bathing had brought on the attack. 
No fixed rule can be made as to the proper duration of an ocean bath. This will vary from four to twenty minutes, according to the bather's age, physical condition, and the temperature of the water. No one who has pulmonary disease or has recently had pneumonia, pleurisy, endocarditis, peritonitis, appendicitis, or acute Bright's disease should bathe in the ocean. It is stated on good authority that sea baths increase the elimination of urea. On that account we might suppose, a priori, that sea bathing at moderate water temperaures would be beneficial in cases of moderate chronic nephritis. Care should be exercised, however, that the body should not be exposed to cutting winds. Women should not bathe during the menstrual period, certainly not in the ocean. Strange as it may seem, this rule is occasionally disregarded.

Sea bathing is useful in anemia, when not too prolonged, in neurasthenia and hypochondria, when not associated with too much debility, in nervous dyspepsia, chronic constipation and obesity.

\section{BIBLIOGRAPHY}

I. HINSDALE, GUY : Hydrotherapy, W. B. Saunders Company, Philadelphia, I9IO.

2. Practical Treatment, Edited by Hare, Lea \& Febiger, Philadephia, Igro.

3. Modern Treatment, Edited by Musser and Kelly, W. B. Saunders Company, Philadelphia, IgIo.

4. FOX, FORTESCUE: Outlines of Medical Hydrology, Trans. Royal Society of Medicine, I9I I, I, 63.

5. FOX, FORTESCUE: Medical Hydrology, University of London Press, I9I3.

6. BARDET: Revue hebdomédaire, July I4, I9I7.

7. ROBIN, ALBERT: Revue scientifique, June I6 and 23, I9I7.

8. ROBIN, ALBERT: Le Monde Médical, I917, XXVII, r6I.

9. GLENARD, ROGER: La Presse Médicale, I9I8, XXVI, 225.

I0. DURAND-FARDEL, MAX: Bulletin de l'Académie de Médicine, I890.

I I. DURAND-FARDEL, MAX : Lyon Médical, I892.

12. ESHNER, A. A:: Some Considerations of the Treatment of Typhoid Fever, Medical News, I896, LXIX, 315.

13. HARE, H. A.: Therapeutic Gazette, I910, XXVI, 168.

14. HINSDALE, GUY: Hydrotherapy, W. B. Saunders Company, Philadelphia, I910, pp. 134-148.

15. WEBER, SIR HERMANN, and WEBER, PARKES : Climatotherapy and Balneotherapy, London, 1907, pp. 333-334 and 408-409.

I6. FOX, FORTESCUE: Physical Remedies, Wm. Wood and Co., New York, 1917.

17. CURRIE, JAMES : Medical Reports on the Effect of Water, Cold and Warm, as a Remedy in Fever, Cardell and Davies, Liverpool, 1797.

18. RAY, ISAAC: American Journal of Insanity, I846, II, 289.

19. HINSDALE, GUY: Hydrotherapy, W. B. Saunders Company, Philadelphia, I910, pp. 168-178. 
20. FOSTER, G. W.: American Journal of Insanity, I898-99, LV, 639.

2I. STRECKER, EDWARD A.: The Modern Treatment of Insanity, Musser and Kelly's Practical Treatment, W. B. Saunders Company, Philadelphia, I9I7, IV, 926.

22. MACKENZIE, SIR JAMES: Diseases of the Heart, Oxford University Press, I9I 3 , 3rd edition, p. 365 et seq.

23. Jour. Am. Med. Assn., r9I7, LXVIII, I796.

24. GREGG, DONALD: The Treatment of Deliria in General Hospitals for the Insane.

25. Contributions from the Psychopathic Hospital, Boston, I9r3, pp. 65-67.

26. JACKSON, J. ALLEN : Hydrotherapy in the Treatment of Mental Diseases, Jour. Am. Med. Assn., 1915, LXIV, 1650.

27. STARR, M. ALLEN : Scribner's Magazine, r9I8, LXIV, 183.

28. FOX, FORTESCUE: Physical Remedies, Wm. Wood and Co., New York, I9I7.

29. HANSELL, H. F.: Penn. Med. Jour., 1907.

30. SHAHAN, WILLIAM E.: Further Study of the Effects of Heat on the Eye, Jour. Am. Med. Assn., I9I7, LXVIII, 1969.

31. SHAHAN, WILLIAM E.: Effect of Heat on the Eye, Jour. Am. Med. Assn., I9I6, LXVII, 4 I4.

32. MONTGOMERY, D. W.: Psoriasis: the Value of Baths and of Maceration in its Treatment, Jour. Am. Med. Assn., I9I2, LIX, 1520.

33. HINSDALE, GUY: Hydrotherapy, W. B. Saunders Company, Philadelphia, I9IO.

34. BARUCH, SIMON : Hydrotherapy, Wm. Wood and Co., New York, 3rd edition.

35. ABBOTT, G. K.: Hydrotherapy, Loma Linda, California, IgI2.

36. DIEFFENBACH, W. H.: Hydrotherapy, Rebman and Co., New York, 1909.

37. KELLOGG, J. H.: Rational Hydrotherapy, F. A. Davis Co., Philadelphia, 1904.

38. FOX, FORTESCUE: Medical Hydrology, University of London Press, Igr3.

39. POPE, CURRAN : Kentucky Medical Journal, I908, VII, 722. 


\title{
THE
}

\section{OXFORD MEDIGINE ADVANCE PAGES}

\author{
EDITED BY \\ HENRY A. CHRISTIAN SIR JAMES MACKENZIE
}

CONTRIBUTORS VOLUME I

LEWELLYS F. BARKER

FRANK BILLINGS

WILLIAM T. BOVIE

RICHARD C. CABOT

HENRY A. CHRISTIAN

CHAS. BENEDICT DAVENPORT

EUGENE F. DU BOIS

FREDERICK P. GAY

LAWRENCE J. HENDERSON

A. WALTER.HEWLETT

GUY HINSDALE
WALTER B. JAMES

WILLIAM B. JOHNSTON

SIR JAMES MACKENZIE

JOHN J. MACKENZIE

ELMER V. MCCOLLUM

SIR WILLIAM OSLER

FRANCIS W. PEABODY

LEONARD G. ROWNTREE

HENRY SEWALL

DONALD D. VAN SLYKE

WILLIAM H. WELCH

PART 3

PART 3

VOLUME I

NEW YORK

OXFORD UNIVERSITY PRESS

AMERICAN BRANCH: 35 WEST 32 ND STREET 
COPYRIGHT, I9:8

BY THE

OXFORD UNIVERSITY PRESS

American Branch 
CONTENTS OF PART III, VOLUME I-OXFORD MEDICINE

\section{Chapter}

V. The Treatment of Disease-Sir William Osler . . 237

Chapter

VI. The Living Causes of Disease and How They ActJohn J. Mackenzie . . . . . . . . . .

\section{Chapter}

VII. Eugenics in Relation to Medicine-Charles B. Davenport . . . . . . . . . . . . . . . . 299

\section{ChAPTER}

VIII. Focal Infection-Frank Billings . . . . . . 319 



\title{
CHAPTER V
}

\section{THE TREATMENT OF DISEASE}

\author{
By Sir William Osler
}

\section{I}

As true today as when Celsus made the remark, "The dominant view of the nature of disease controls its treatment." As is our pathology so is our practice; what the pathologist thinks today the physician does tomorrow. Roughly grouped, there have been three great conceptions of the nature and treatment of disease.

$A$. For long centuries it was believed to be the direct outcome of sin, "flagellum Dei pro peccatis mundi," to use Cotton Mather's phrase, and the treatment was simple - a readjustment in some way of man's relation with the invisible powers, malign or benign, which had inflicted the scourge. From the thrall of this "sin and sickness" view man has escaped so far as no longer, at least in Anglo-Saxon communities, to have a proper saint for each infirmity. Against this strong bias towards the supernatural even the wisdom of Solomon could not prevail; was not the great book of his writings which contained medicine for all manner of diseases and lay open for the people to read as they came into the temple removed by Hezekiah lest out of confidence in remedies they should neglect their duty in calling and relying upon God? And the modern book of reason, which lies open to all, is read only by a few in the more civilized countries. The vast majority are happy in the childlike faith of the childhood of the world. I am told that annually more people seek help at the shrine of St. Anne de Beaupré, in the Province of Quebec, than at all the hospitals of the Dominion of Canada. How touching at Rome to see the simple trust of the poor in some popular Madonna, such as the Madonna del Parto! It lends a glow to the cold and repellent formalism of the churches. In all matters relating to disease credulity remains a permanent fact, uninfluenced by civilization or education.

$B$. From Hippocrates to Hunter the treatment of disease was one long traffic in hypotheses; variants at different periods of the doctrine of the four humors, as dominated by some strong mind in active revolt 
it would undergo temporary alteration. The peccant humors were removed by purging, bleeding, or sweating, and until the early. years of the nineteenth century there was very little change in the details. To a very definite but entirely erroneous pathology was added a treatment most rational in every respect, had the pathology been correct! The practice of the early part of the last century differed very little from that which prevailed in the days of Sydenham, except, perhaps, that our grandfathers were, if possible, more ardent believers in the lancet.

$C$. In the past fifty years our conception of the nature of disease has been revolutionized, and with a recognition that its siltimate processes, whether produced by external agents or the result of modifications in the normal metabolism, are chemico-physical, we have reached a standpoint from which to approach the problems of prevention and cure in a rational way. Let me indicate briefly the directions in which the new science has transformed the old art.

In the first place, the discovery of the cause of many of the great scourges has changed not only its whole aspect, but, indeed, we may say, the very outlook of humanity. No longer is our highest aim to cure, but to prevent disease; and in its career of usefulness the profession has never before had a triumph such as we have witnessed in the abolition of many fearful scourges. Great as have been the Listerian victories in surgery, they are but guerrilla skirmishes, so to speak, in comparison with the Napoleonic campaigns which medicine is waging against the acute infections. These are glorious days for the race. Nothing has been seen like it on this old earth since the destroying angel stayed his hand on the threshing-floor of Araunah the Jebusite. For seventeen years Cuba, once a pest-house of the tropics, has been free from a scourge which has left an indelible mark in the history of the Englishman, Spaniard, and American of the New World. Today the Canal Zone of Panama, for years the graveyard of the white man, has a death rate as low as that in any city of the United States. In the island of Porto Rico, where many thousands have died annually of tropical anemia, the death rate has been cut in half by the work of Ashford and others. But, above all, the problem of life in the tropics for the white man has been solved, since malaria may now be prevented by very simple measures. These are some of the recent results of laboratory stadies which have placed in our hands a power for good never before wielded by man.

Secondly, a fuller knowledge of etiology has led to a return to methods which have for their object, not so much the combating of the disease germ or of its products, as the rendering of conditions in the body unfavorable for its propagation and action. How fruitful in prac- 
tical results, for example, have been the new views on tuberculosis! Not that the discovery of the bacillus itself modified immediately our treatment of the disease, but, as so often happens, a combination of circumstances was responsible for the happy revolution-the recognition of the widespread prevalence of the infection, the great frequency with which healed lesions were found, and the knowledge of the importance of the character of the tissue soil, led to the substitution of the open-air and dietetic treatment for the nauseous mixtures with which our patients were formerly drenched. We scarcely appreciate the radical change which has occurred in our views even within a few years. Contrast with a recent work on tuberculosis one published thirty-five or forty years ago. In the latter the drug treatment takes up the larger share, while in the former it is reduced to a page or two. And it is not only in the acute infections that the use of the "non-naturals," as the old writers called them, has replaced other forms of treatment, but in diet, exercise, massage, and hydrotherapy, we are every day finding out the enormous importance of measures which too often have been used with greatest skill by those outside or on the edge of the profession.

Thirdly, the study of morbid anatomy combined with careful clinical observations has taught us to recognize our limitations, and to accept the fact that a disease itself may be incurable, and that the best we can do is to relieve symptoms and to make the patient comfortable. The relation of the profession to this group, particularly to certain chronic maladies of the nervous system, is a very delicate one. It is a hard matter, and really not often necessary (since Nature usually does it quietly and in good time), to tell a patient that he is past all hope. As Sir Thomas Browne says, "It is the hardest stone you can throw at a man to tell him that he is at the end of his tether," and yet, put in the right way to an intelligent man it is not always cruel. Let us remember that we are the teachers, not the servants, of our patients, and we should be ready to make personal sacrifices in the cause of truth, and of loyalty to the profession. Our inconsistent attitude is, as a rule, the outcome of the circumstances that of the three factors in practice, heart, head and pocket, to our credit, be it said, the first named is most potent. How often does the consultant find the attending physician resentful or aggrieved when told the honest truth that there is nothing further to be done for the cure of his patient! To accept a great group of maladies, against which we have never had and can scarcely ever hope to have curative measures, makes some men as sensitive as though we were ourselves responsible for their existence. These very cases are "rocks of offense" to many good fellows whose moral decline dates from the rash promise to cure. We work by wit and not by witchcraft, and while 
these patients have our tenderest care, and we must do what is best for the relief of their sufferings, we should not bring the art of medicine into disrepute by quack-like promises to heal, or by wire-drawn attempts to cure in what old Burton calls "continuate and inexorable maladies."

Fourthly, the new studies on the functions of organs and their perversions have led to most astonishing results in the use of the products of metabolism, which time out of mind physicians have employed as medicines. Pliny's "Natural History" (Bohn, London, I855-57, vol. ii, 29I) is a storehouse of information on the medicinal use of parts of animals or of various secretions and excretions. Much of the humbuggery and quackery inside and outside of the profession has been concerned with the use of the most unsavory of these materials. The seventeenth century pharmacopeias were full of them, and in his oration at the Hunterian Society, 1902, Dr. Arthur T. Davies has given an interesting historical sketch of their use in practice. Modern metabolic therapy represents one of the greatest triumphs of science. The demonstration of insufficiency of the thyroid gland is a brilliant example of successful experimental inquiry, and as time has passed the good results of treatment in suitable cases have become more and more evident. Before long, no doubt, we shall be able to meet, in the same happy way, the perverted functions which lead to such diseases as exophthalmic goitre, Addison's disease, and acromegaly; and as our knowledge of the pancreatic function and carbohydrate metabolism becomes more accurate we shall probably be able to place the treatment of diabetes on a sure foundation. And it is not only on the organic side that progress has been made. Important discoveries relating to the metabolism of the inorganic constituents, such as those relative to acidosis, have opened a new and most hopeful chapter in scientific medicine.

But the best of human effort is flecked and stained with weakness, and even the casual observer may note dark shadows in the bright picture. Organotherapy illustrates at once one of the great triumphs of science and the very apotheosis of charlatanry. One is almost ashamed to speak in the same breath of the credulousness and cupidity by which even the strong in intellect and the rich in experience have been carried off in a flood of pseudo-science. This has ever been a difficulty in the profession. The art is very apt to outrun or override the science, and play the master where the true rôle is that of the servant.

And, lastly, we have advanced firmly along a new road in the treatment of diseases due to specific microorganisms, with the toxic products of which we are learning to cope successfully. The treatment with antitoxins and bacterial vaccines, so successfully started, bears out the truth of that keen comment of Celsus: "He will treat the disease 
properly whom the first origin of the cause has not deceived." We are still far from the goal in some of the most important and fatal infections, but anyone acquainted in even slight measure with the progress of the past twenty years cannot but have confidence in the future. Considering that the generation is still active which opened the whole question, we cannot but feel hopeful in spite of disappointments here and failures there. But in our pride of progress let us remember cancer and pneumonia. The history of the latter disease affords a good illustration of the truth of the remark of Celsus with which I began. Year by year the lesson of pneumonia is a lesson of humility. For purposes of comparison statistics are not available, but it is not likely that the great masters from Galen to Grisolle lost a larger number of cases than we do. Pneumonia has always been, as today, a dreaded and a fatal clisease. For one thing let us be thankful. We have had the courage tc abandon the expectorant mixtures, the depressants, the cardiac sedatives, the blisters, the emetics, the resulsives, the purges, the poultices, and, to a great extent, the bleedings. Surely our forefathers must have killed some patients by the appalling ferocity of their treatment, or to have stood it the constitutions of those days must have been more robust. We still await, but await in hope, the work that will remove the reproach of the mortality bills in this disease. I say reproach because we really feel it, and yet act justly, for who made us responsible for its benign or malignant nature? We can relieve symptoms, but we must find the means which will, on the one hand, limit the extension of the process, loosen the exudate, minimize the fluxion, control the alveolar diapedesis, and, on the other hand, diminish the output of the toxins, neutralize those in circulation, or strengthen the opsonic power of the blood. But someone will say, Is this all your science has to tell us? Is this the outcome of decades of good clinical work, of patient study of the disease, of anxious trial in such good faith of so many drugs? Give us back the childlike trust of the fathers in antimony and in the lancet rather than this cold nihilism. Not at all! Let us accept the truth, however unpleasant it may be, and with the death rate staring us in the face, let us not be deceived with vain fancies. Not alone in pneumonia, but in the treatment of certain other diseases, do we need a stern, iconoclastic spirit which leads, not to nihilism, but to an active skepticism-not the passive skepticism born of despair, but the active skepticism born of a knowledge that recognizes its limitations and knows full well that only in this attitude of mind can true progress be made. I hope to live to see a true treatment of pneumonia. Before long we should be able to cope with the products of the pneumococci; it may indeed come within the list of preventable diseases. 
II

Along these five lines the modern conception of the nature of disease has radically altered our practice. The personal interest which we take in our fellow creatures is apt to breed a sense of superiority to their failings, and we are ready to forget that we ourselves, singularly human, illustrate many of the common weaknesses which we condemn in them. In no way is this more striking than in the careless credulity we display in some matters relating to the treatment of disease. Recently the Times had an editorial upon a remark of Bernard Shaw that the cleverest man will believe anything he wishes to believe, in spite of all the facts and textbooks in the world. We are at the mercy of our wills much more than of our reason in the formation of our beliefs, which we adopt in a lazy, haphazard way, without taking much trouble to inquire into their foundation. But I am not going to discuss, were I able, this Shavian philosophy; but it will serve as an introduction to a few remarks on the Nemesis of Faith which in all ages readily overtakes doctors and the public alike. Without trust, without confidence, without faith in himself, in his tools, in his fellowmen, no man works successfully or happily. For us, however, it must never be the blind questioning trust of the devotee, but the confidence of the inquiring spirit that would prove all things. But it is so much easier to believe than to doubt, for doubt connotes thinking and the expenditure of energy, and often the disruption of the status quo. And then we doctors have always been a simple, trusting folk! Did we not believe Galen implicitly for I,500 years and Hippocrates for more than 2,000? In the matter of treatment the placid faith of the simple believer, not the fighting faith of the aggressive doubter, has ever been our besetting sin.

In the progress of knowledge each generation has a double laborto escape from the intellectual thralls of the one from which it has emerged and to forge anew its own fetters. Upon us whose work lay in the last quarter of the nineteenth century fell the great struggle with that many-headed monster, Polypharmacy-not the true polypharmacy which is the skillful combination of remedies, but the giving of manythe practive of at once discharging a heavily loaded prescription at every malady, or at every symptom of it. Much has been done and an extraordinary change has come over the profession, but it has not been a fight to the finish. Many were lukewarm; others found it difficult to speak without giving offense in quarters where on other grounds respect and esteem were due. As an enemy to indiscriminate drugging. I have often been branded as a therapeutic nihilist. That I should even venture to speak on the subject calls to mind what Professor Peabody of Harvard 
remarked about Jacob Bigelow, that, "for his professorship of Materia Medica he had very much the same qualifications that a learned unbeliever might have for a professorship of Christian theology. No other man of his time had so little faith in drugs." I bore this reproach cheerfully, coming, as I knew it did, from men who did not appreciate the difference between the giving of medicines and the treatment of disease; moreover it was for the galled jade to wince, my withers were unwrung. The heavy hands of the great Arabians grow lighter in each generation. Though dead, Rhazes and Avicenna still speak, not only in the Arabic signs which we use, but in the combinations and multiplicity of the constituents of too many of our prescriptions. We are fortunately getting rid of routine practice in the use of drugs. How many of us now prescribe an emetic? And yet that shrewd old man, Nathanial Chapman, who graced the profession of Philadelphia for so long, used to say, "Everything else I have written may disappear, but my chapter on emetics will last!" How much less now does habit control our practice in the use of expectorants? The blind faith which some men have in medicines illustrates too often the greatest of all human capacitiesthe capacity for self-deception. One special advantage of the skeptical attitude of mind is that a man is never vexed to find that after all he has been in the wrong. It is an old story that a man may practice medicine successfully with a very few drugs. Locke had noticed this, probably in the hands of his friend Sydenham, since he says, "You cannot imagine how far a little observation carefully made by a man not tied up to the four humors ... . would carry a man in the curing of diseases, though very stubborn and dangerous, and that with very little and common things and almost no medicine at all." Boerhaave commented upon this truth in a remark of Sydenham "that a person well skilled in cases seldom needs remedies." The study of the action of drugs, always beset with difficulties, is rapidly passing from the empirical stage, and this generation may expect to see the results of studies which have already been most promising. It is very important that our young men should get oriented early in this matter of drug treatment. Our teachers used to send us to the works of John Forbes ("Nature and Art in the Cure of Disease," J. Churchill, London, I857), and to Jacob Bigelow ("Nature in Disease," Ticknor and Fields, Boston, 1854), for clear views of the subject. A book has been written by Dr. Harrington Sainsbury, the well-known London physician and teacher ("Principia Therapeutica," E. P. Dutton \& Company, New York, 1907), which deals with these problems in the same philosophic manner. It opens with a delightful dialogue between the pathologist and the physician. He lays his finger on the weak point of the pure morbid 
anatomist who thinks of the lesion only, and not enough of the function which even a seriously damaged organ may be able to carry on. The book should be in the hands of every practitioner and senior student. Some of you may have heard of the lecture-room motto of that distinguished pathologist and surgeon, and the first systematic writer on morbid anatomy in the United States, S. D. Gross: "Principles, gentlemen, principles! principles!!" And it is upon these fundamental aspects that Dr. Sainsbury dwells in his most suggestive work, which I would like to see adopted as a textbook in every medical school in the land.

And we are yet far too credulous and supine in another very important matter. Each generation has its therapeutic vagaries, the outcome, as a rule, of attempts to put prematurely into practice theoretical conceptions of disease. As members of a free profession we are expected to do our own thinking; and yet the literature that comes to us daily indicates a thraldom not less dangerous than the polypharmacy from which we are escaping. I allude to the specious and seductive pamphlets and reports sent out by the pharmaceutical houses, large and small. We owe a deep debt to the modern manufacturing pharmacist, who has given us pleasant and potent medicines in the place of the nauseous and weak mixtures; and such firms as Parke, Davis \& Company, of the United States, and Burroughs \& Wellcome, of England, have been pioneers in the science of pharmacology. But even the best are not guiltless of exploiting in the profession the products of a pseudoscience. Let me specify three items in which I think the manufacturing pharmacists have gone beyond their limit and are trading on the credulity of the profession to the great detriment of the public. The length to which organotherapy has extended (not so much on the American side of the water as on the European continent) beyond the legitimate use of certain preparations is a notorious illustration of the ease with which theoretical views place us in a false position. Because thyroid extract cures myxedema and adrenalin has a powerful action, it has been taken almost for granted that the extract of every organ is a specific against the diseases that affect it. This forcing of a scientific position is most hurtful, and I have known an investigator hestitate to publish results lest they should be misapplied in practice. The literature on the subject issued by reputable houses indicates, on the one hand, the pseudo-science upon which a business may be built up, and, on the other, the weakminded state of the profession on whose credulity these firms trade. A second most reprehensible feature is the laudatory character of literature describing the preparations which they manufacture. Foisted upon an innocent practitioner by a traveling. Autolycus, the preparation is 
used successfully, say, in six cases of amenorthea; very soon a report appears in a medical journal, and a few weeks later this report is sent broadcast with the auriferous leaflets of the firm. Some time ago a pamphlet came from $\mathrm{X}$ and Company, characterized by brazen therapeutic impudence, and indicating a supreme indifference to anything that could be called intelligence on the part of the recipients. That these firms have the audacity to issue such trash indicates the state of thraldom in which they regard us. And I would protest against the usurpation on the part of these men of our function as teachers. Why, for example, should $\mathrm{Y}$ and Company write as if they were directors of large genitourinary clinics instead of manufacturing pharmacists? It is none of their business what is the best treatment for gonorrhea-by what possibility could they ever know it, and why should their literature pretend to the combined wisdom of Neisser and Guyon? What right have $Z$ and Company to send on a card directions for the treatment of anemia and dyspepsia, about which subjects they know as much as an unborn babe, and, if they stick to their legitimate business, about the same opportunity of getting information? For years the profession has been exploited in this way, until the evil has become unbearable, and we need as active a crusade against pseudo-science in the profession as has been waged of late against the use of quack medicines by the public. We have been altogether too submissive, and have gradually allowed those who should be our willing helpers to dictate terms and to play the rôle of masters. Far too large a section of the treatment of disease is today controlled by the big manufacturing pharmacists, who have enslaved us in a plausible pseudo-science. The remedy is obvious: give our students a firsthand acquaintance with disease, and give them a thorough practical knowledge of the great drugs, and we will send out independent, clearheaded, cautious practitioners who will do their own thinking and be no longer at the mercy of a meretricious literature which has sapped our independence.

Having confessed some of our weaknesses, I may with better grace approach the burning question of the day in the matter of treatment. An influenza-like outbreak of faith-healing seems to have the public of both continents in its grip. It is an old story-the oldest, indeed, in our history-and one in which we have a strong hereditary interest, since scientific medicine took its origin in a system of faith-healing beside which all our modern attempts are feeble imitations. Lincoln's favorite poem, beginning "We think the same thoughts that our fathers have thought," expresses a tendency in the human mind to run in circles. Once or twice in each century the serpent entwining the staff of Æsculapius gets restless, untwists, and in his gambols swallows his tail, and 
at once in full circle back upon us come old thoughts and old practices, which for a time dominate alike doctors and laity. As a profession we took origin in the cult of Esculapius, the gracious son of Apollo, whose temples, widespread over the Greek and Roman world, were at once magnificent shrines and hospitals, with which in beauty and extent our modern institutions are not to be compared. Amid lovely surroundings, chosen for their salubrity, connected usually with famous springs, they were the sanatoriums of the ancient world. The ritual of the cure is well known, and has been beautifully described by Walter H. Pater in "Marius the Epicurean" (Macmillan, New York, 1907). Faith in the god, suggestion, the temple sleep and the interpretation of its dream were the important factors. Hygienic and other measures were also used, and in the guild of secular physicians which grew up about the temples scientific medicine took its origin. No cult resisted so long the progress of Christianity; and so imbued were the people with its value, that many of the practices of the temple were carried on into the Christian ritual. The temple sleep and the interpretation of its dreams were continued long into the Middle Ages, and, indeed, have not yet disappeared. The popular shrines of the Catholic Church today are in some ways the direct descendants of this Æsculapian cult, and the cures and votive offerings at Lourdes and St. Anne are in every way analogous to those of Epidaurus.

As I before remarked, credulity in matters relating to disease remains a permanent fact in our history, uninfluenced by education. But let us not be too hard on poor human nature. Even Pericles, most sensible of men, when on his deathbed, allowed the women to put an amulet about his neck. And which one of us, brought up from childhood to. invoke the aid of the saints and seek their help-which one of us under these circumstances, living today in or near Rome, if a dear child were sick unto death, would not send for the Santo Bambino, the Holy Doll of the Church of Ara Cceli? Has it not been working miracles these four hundred years? The votive offerings of gold and of gems from the happy parents cover it completely, and about it are grateful letters from its patients in all parts of the world. No doll so famous, no doll so precious! No wonder it goes upon its ministry of healing in a carriage and pair, and with two priests as its companions! Precious perquisite of the race, as it has been called, with all its dark and terrible record, credulity has perhaps the credit balance on its side and in the consolation afforded the pious souls of all ages and of all climes, who have let down anchors of faith into the vast sea of superstition. We drink it in with our mother's milk, and that is indeed an even-balanced soul without some tincture. We must 
acknowledge its potency today as effective among the most civilized people, the people with whom education is the most widely spread, yet who absorb with wholesale credulity delusions as childish as any that have ever enslaved the mind of man.

Having recently had to look over a large literature on the subject of mental healing, ancient and modern, I have tried to put the matter as succinctly as possible. In all ages and in all climes the prayer of faith has saved a certain number of the sick. The essentials are first a strong and hopeful belief in a dominant personality, who has varied naturally in different countries and in different ages. Buddha in India, and in Japan, where there are cults to match every recent vagary; Æsculapius in ancient Greece and Rome; our Saviour and a host of saints in Christian communities; and lastly, an ordinary doctor has served the purpose of common humanity very well. Faith is the most precious asset in our stock-in-trade. Once lost, how long does a doctor keep his clientèle? Secondly, certain accessories-a shrine, a grotto, a church, a temple, a hospital, a sanatorium-surroundings that will impress favorably the imagination of the patient. Thirdly, suggestion in one of its varied forms-whether the negation of disease and pain, the simple trust in Christ of the Peculiar People, of the sweet reasonableness of the psychotherapeutist. But there must be the will-to-believe attitude of mind, the mental receptiveness-in a word, the faith which has made bread pills famous in the history of medicine. We must, however, recognize the limitations of mental healing. Potent as is the influence of the mind on the body, and many as are the miracle-like cures which may be worked, all are in functional disorders, and we know only too well that nowadays the prayer of faith neither sets a broken thigh nor checks an epidemic of typhoid fever.

What should be the attitude of the clergy, many of whom have been drawn into the vortex of this movement? I feel it would be very much safer to hand over this problem to us. It is not a burden which we should ask a hard-working and already overwrought profession to undertake or to share. It might be a different matter if it were really a gift of healing in the apostolic sense, but we know this was associated with other signs and wonders at present conspicuous by their absence. Then think of the possibilities of self-deception-of the saintly Edward Irving and the gift of tongues; of Monsieur de Paris, the French priest, and the miracles at his tomb, to the truth of which two fine quarto. volumes, with "before and after" pictures, attest! The less the clergy have to do with the bodily complaints of neurasthenic and hysterical persons the better for their peace of mind and for the reputation of the Cloth.' As wise old Fuller remarked, Circe and Esculapius were: 
brother and sister, and the wiles of the one are very apt to entrap the wisdom of the other.

III

It adds immensely to the interest in life to live in the midst of these problems which concern us so closely. We must meet them with an intelligent cheerfulness, in the full confidence that the Angel of Bethesda never stirred the waters without happy results. It is for us to see that the soldiers we are training for the fight against disease, bodily and mental, are well equipped for the battle; and let me briefly, in conclusion, indicate how I believe we should teach the art-the management of patients and the cure of disease. To know how to deal with disease is the final goal, to reach which the whole energies of the student should be directed. We all recognize that it is in the out-patient departments and in the wards-I wish I could add in the homes of the general practitioners-that he must get this part of his training, not in an elaborate course of lectures on the properties and action of drugs. In the congested curriculum it is by no means easy to find the proper amount of time for this, the most essential part of his education. But as we learn the futility of the lecture room as an instrument of teaching men the Art, so, I think, we shall gradually be able to adapt the courses so that plenty of time may be given to the practical study of the treatment of cases under skilled direction. We should take over to the hospital of the school the whole subject known in the curriculum as therapeutics. The composition of drugs, the method of their preparation, and the study of their physiological action should be taught in practical classes in the pharmaceutical laboratories. In the out-patient departments and in the wards much more systematic practical instruction should be given how to treat disease and how to manage patients. If we could only get the students for a sufficiently long period in the hospital, what helpful courses could be arranged in the senior years! Certain aspects of the subject must be ever kept before the assistants * and the students, considered, perhaps, by different men associated with the clinic according to the special capacity of each one. The fundamental law should be ingrained that the starting-point of all treatment is in the knowledge of the natural history of a disease. Typhoid fever, tuberculosis, pneumonia, and, where possible, malaria, should be used for this important lesson, and in the everyday routine observation of cases the student would learn to know the course of the disease, its obvious features, the

* A post-graduate course in medical pedagogy would be most helpful organized by five or six of the large colleges and conducted by them in rotation with teachers selectcd from the different schools. Many able young fellows take years to acquire methods to which they might be introduced in a six month' course. 
complications likely to arise; and he would be taught how to discriminate between the important and the unimportant symptoms of a case. This work should form the very basis of his course in medicine, and it should be accompanied by a seminar to take the place of set lectures, in which the features of all the common diseases would be discussed.

The hygienic and dietetic management of patients has now come to be such a prominent part of the work of our hospitals that the student may become acquainted with the open-air treatment, the various modifcations of diet suitable to different diseases, and the use of massage, electricity, and other physical agents. But too often he is allowed to pick up this information in a haphazard, irregular fashion. One assistant of the clinic should be detailed to see that every member of the class knows, for example, how to arrange the open-air treatment for $\mathbf{a}$ tuberculous patient, and how to supervise the diet of a diabetic case. The student should prepare personally the various nutritive enemata, and be able to give the different kinds of massage, and I would have him thoroughly versed in all branches of hydrotherapy. A serious difficulty is that nowadays the nurse does a great many things that it is essential the medical student should know how to do-the administration of hypodermics, the giving of a cold pack, etc.

Much more attention should be paid to the important subject of psychotherapy. It is not every teacher who has a special gift for this work, but if the professor himself does not possess it, he should, at any rate, have sense enough to have an assistant familiar with and interested in the modern methods. How many of our graduates have been shown how to carry out a Weir-Mitchell treatment or to treat a patient by suggestion? The student should be taught that the very environment of a well-managed clinic is in itself an important factor in psychicis treatment. A Philadelphia friend once jokingly defined my practice at the Johns Hopkins Hospital as a mixture of hope and nux vomica, and the grain of truth in this statement lies in the fact that with many hospital patients once we gain their confidence and inspire them with hope, the battle is won.

And lastly, from the day the student enters the hospital until graduation, he should study under skilled supervision the action of the few great drugs. Which are they? I am not going to give away my list. A story is told that James Jackson, when asked which he considered the greatest drugs, replied: "Opium, mercury, antimony and Jesuit's bark; they were those of my teacher, Jacob Holyoke." "Yes," replied his interlocutor, "and they were those of Holyoke's master, James Douglas, in the early part of the eighteenth century." Mine is a much longer one! The student should follow most carefully the action of 
those drugs the pharmacology of which he has worked out in the laboratory. He should be sent out from the hospital knowing thoroughly how to administer ether and chloroform. He should know how to handle the various preparations of opium.* Each ward should have its little case with the various preparations of the ten or twelve great drugs, and when the teacher talks about them he should be able to show the preparations. He should study with special care the action of digitalis on the circulation in cases of heart disease. He should know its literature, from Withering to Cushney. It should be taken as the typical drug for the study of the history of therapeutics-the popular phase, as illustrated by the old woman who with it cured the Principal of Brasenose; the empirical stage, introduced by Withering in his splendid contribution, a model of careful work of which every senior student should know; and the last stage, the scientific study of the drug, which he will already have made in the pharmacological laboratory. He should day after day personally give a syphilitic baby inunctions of mercury; he should give deep injections of calomel and he should learn the history of the drug from Paracelsus to Fournier. He should know everything relating to the iodides and the bromides, and should present definite reports on cases in which he has used them. He must know the use of the important purgatives, and he should have a thorough acquaintance with all forms of enemata. He should know cinchona historically, its derivatives chemically, and its action practically. He should study the action of the nitrites with the blood pressure apparatus, and he should over and over again have tested for himself the action, or the absence of action, of strychnine, alcohol, and other drugs supposed to have a stimulating action on the heart and blood vessels. While I would, on the one hand, imbue him with the firmest faith in a few drugs, "the friends he has and their adoption tried," on the other hand, I would encourage him in a keenly skeptical attitude towards the pharmacopeia as a whole, ever remembering Benjamin's Franklin's shrewd remark that "he is the best doctor who knows the worthlessness of the most medicines." You may well say this is a heavy contract, and one which it is impossible to carry out. Perhaps it is with our present arrangements, but this is the sort of work which the medical student has a right to expect, and this is what we shall be able to give him when in his senior years we give up lecturing him to death, and when we stop trying to teach him too many subjects.

*Sydenham obtained the appellation "Opiophilos" (Ogle); and the best practitioner is the man who knows best how to use "God's own medicine," as it has been called. 


\section{CHAPTER VI \\ THE LIVING CAUSES OF DISEASE AND HOW THEY ACT}

\section{By John J. Mackenzie}

INFECTIOUs diseases in man may be caused by a great variety of living organisms, belonging to both the plant and animal kingdoms. These organisms may live either on the surface of the body or within it, and may produce the phenomena of disease in a variety of ways. Organisms living upon the surface of the body are spoken of as ectoparasites. Their disease manifestations are as a rule local, but the presence of large numbers of them may give rise to more general symptoms. Those organisms which live within the body are called endoparasites. They live either in the alimentary canal, pulmonary tubes, genitourinary apparatus or, the strictly endoparasitic forms, within the tissues themselves.

The groups to which human parasites belong are as follows:

Vegetable organisms causing disease:

1. Bacteria.

2. Streptothrix.

3. Higher fungi.

Organisms of uncertain biological relationship causing disease:

1. Spirochetal forms.

2. Filterable viruses.

Animal organisms causing disease :

1. Protozoa:

$\begin{array}{cl}\text { Plasmodroma } & \begin{array}{l}\text { Mastigophora } \\ \text { Rhizopoda } \\ \text { Sporozoa. }\end{array} \\ \text { Ciliophora } & \begin{array}{l}\text { Ciliata. } \\ 251\end{array}\end{array}$


2. Metazoa :

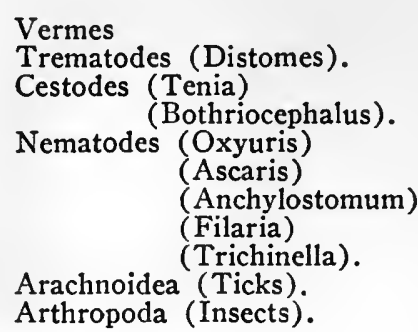

\section{PORTALS OF INFECTION}

The first problem in regard to parasitic disease is how the parasite gains entrance into the body.

The infant at birth is normally free from parasitic organisms; the contents of the alimentary canal as well as the surface of the body is sterile. It has been shown experimentally that the invasion of the alimentary canal by bacteria, which takes place within a few hours of birth, is not necessary, as was at one time supposed, to the proper function of the canal. On account of its universal occurrence, however, the body has become adapted to this invasion, and throughout life bacteria are normal inhabitants of the canal. They are numerous in the mouth, comparatively few in the acid gastric contents; they increase in numbers from the bile papilla to the ileocecal valve, and are very numerous in the large bowel. They live here, as far as we know, without producing disease of the body, but it must be remembered that Metchnikoff $\left({ }^{1}\right)$ was convinced that that universal disease of animals-old age-was primarily due to the absorption of bacterial products from the large bowel. Adami $\left({ }^{2}\right)$ and others have also shown that bacteria may in health enter the portal circulation and reach the liver, or be carried by leucocytes into the lymphatics. Adami has suggested, under the term subinfection, a very definite rôle to these otherwise harmless saprophytes. It must be remembered also that a modern school of surgeons, basing their views primarily upon the teaching of Metchnikoff, but supported by radiological studies of the movement of the contents of the bowel, have related a large number of symptoms to the absorption of the products of bacterial decomposition in the bowel and have elaborated surgical methods to combat the condition.

The tissues of the body are well protected against the invasion of parasitic organisms. The external surface is covered by an almost impenetrable protection in the stratified layers of the epidermis, specially thickened on the palmar and plantar surfaces where the wear and tear is more severe. Normally, as Welch $\left({ }^{3}\right)$ was the first to establish definitely, 
there is a great bacterial flora of the immediate skin surface, and the elaborate methods of surgical technique in skin disinfection point to the difficulties of reaching this flora so as completely to destroy it. But this epidermal defense line is not everywhere equally efficient; the hair follicles, sebaceous and sweat glands, are all points where the bacteria of the skin may find portals to the deeper tissue. For the more highly organized parasites these portals do not seem to be necessary. The different parasitic fungi find conditions favorable to growth in the epidermis. The embryos of anchylostoma rapidly penetrate the unbroken skin (Loos ${ }^{4}$ ). Even spirochetal forms are able to do the same. This has been especially shown by Japanese observers $\left({ }^{5}\right)$ in the case of spirochaete icterohaemorrhagica, the cause of epidemic jaundice, and this also explains its rapid spread in damp mines and in the trenches of the present war. The occurrence of certain forms of extragenital chancre, or the existence of syphilitic infection without primary sore, points to a probable power of the treponema to penetrate the skin, so that there seems some probability that all spirochetal forms have this ability to penetrate the unbroken skin. Friction of the skin may lead to introduction of such pathogenic bacteria as staphylococcus. This is demonstrated not only by the characteristic position in which furuncles develop, but Garre $\left({ }^{6}\right)$ and others have actually produced these lesions by rubbing virulent staphylococcus into the skin. Ordinarily, however, an actual break in the surface is necessary before the organisms penetrate to the deeper tissues. These breaks may be the result of direct trauma, accidental or surgical in origin, or may be produced by insect bites, in the latter instance the insect being the carrier of the infection. An important form of penetration through the skin, which may be mistaken for penetration by insect bite but which is really due to self-inflicted abrasions, has recently been demonstrated in trench fever. Here the virus is in the bodies of the lice which have fed upon cases of the disease. It is also present in the excreta of the lice. It has been shown conclusively by Bruce's commission $\left({ }^{7}\right)$, and confirmed by American observers $\left.{ }^{8}\right)$ in France, that the infection passes in through the abrasions produced by scratching as well as through the bite of the infected louse. French observers claim to have demonstrated the same fact in the transmission of typhus and relapsing fever. There is a possibility that the bacillus of bubonic plague may penetrate in the same way.

The epithelial lining of the alimentary canal is also a protective mechanism, although not so perfect as that of the skin. In the mouth cavity and nasopharynx we have an area which is continually exposed to bacteria introduced from without in food, drink, and air. At the same time the accumulation of food about teeth forms masses of rich 


\section{LIVING CAUSES OF DISEASE AND HOW THEY ACT}

culture media for the growth of bacteria. Over the greater part of this area there is an efficient protection in the layers of stratified squamous epithelium, whilst the secretions of the serous and mucous glands have a mild but distinct bactericidal action. In the mouth itself, however, is found one of the most important gateways for infection in the whole body. The tooth when it erupts is covered with a layer of altered epithelium which is called the enamel cuticle or Nasmyth's membrane. This at the enamel margin is continuous with the epithelial reflexion of the subgingival pocket; thus at first there is no break at the tooth of the epithelial barrier. Wear and tear soon removes Nasmyth's membrane from the crown of the tooth, but it may remain for a long time covering the neck. The subgingival epithelium, on the other hand, is specially exposed to injury from the lodgment of food débris, traumatism from food particles, toothbrushes and toothpicks, and especially from deposits of tartar. This is most marked just at the point where the gingival epithelium is continuous with the enamel cuticle. It is probable that no individual reaches maturity without the development of epithelial defects at this point. But even in childhood the tissue abuut the tooth may serve as a point of entry for bacteria; during the loss of the deciduous teeth and the eruption of the permanent set, there are many opportunities for the entrance of bacteria, especially when, as often occurs, the milk teeth are badly decayed. The results of such defects are the lodgment of foci of infection which form the basis for the development of the various forms of pyorrheal infection which become so common in later life. Aside from the influence of these infections upon the tooth and its alveolus, their existence is of very great importance as foci from which pathogenic bacteria may pass to the deeper tissues. In addition to infection breaking through the epithelium of the subgingival pockets, the tooth presents another path of entrance for bacteria when the pulp becomes infected. Recent study, especially radiological examinations of the tooth, show that small foci of subacute infection may be situated about the apical foramina. These may give rise to strikingly few local symptoms, and their minute size and slight local influence might lead one to regard them as of no importance; yet cultures made from these areas practically always reveal the presence of streptococci, and their radical treatment and removal results in such striking improvement in subacute streptococci infections elsewhere in the body, as for instance the joints or heart valves, that one is forced to regard them as seed-beds for the continuous infection of the tissues generally. There is even a strong probability that more obscure forms of disease, such for instance as pernicious anemia, may owe the entrance of the causative organism or its toxins to foci in the alveolus. 
Equally important are the tonsils as gateways of infection. The structure of the crypts which affords lodgment of débris favoring the growth of bacteria, as well as the thinness or defective character of the epithelium at the bottom of the crypts, permits invasion of the tonsillar tissue and the establishment of foci of infection which may seed the deeper tissues. Clinical observations have for a long time established the close connection between subacute and chronic tonsillar infections and such more serious conditions as joint, cardiac, and renal inflammations. An interesting suggestion from the Mayo clinic $\left({ }^{8}\right)$ is that many chronic tonsillar infections are not primary in the tonsils but are secondary to infections about the teeth.

From the upper nasopharynx and accessory sinuses, organisms may find a pathway either to the tissues generally or to the surface of the brain. The meningococcus and the organism of anterior poliomyelitis apparently penetrate to the central nervous system by this path.

The respiratory tract is defended normally by its epithelium, assisted materially by the mucous secretion and the activities of its ciliated cells. An interesting observation upon the breakdown of the epithelial defense in the respiratory tract is that of Mallory's $\left({ }^{10}\right)$ in whooping cough. Here he shows the actual formation of a culture of the organism upon the surface and between the cilia. This superficially irritative action of the organism probably explains the clinical characters of the cough. The respiratory epithelium is not to be compared in efficiency with that of the mouth or pharynx, but it must be remembered that in normal breathing the air impinges upon so many moist surfaces and is so sharply deflected from one to the other that by the time it reaches even the main bronchi it must be deprived of bacteria.

In the gastrointestinal tract the epithelium forms the first line of defense, but it is strongly supported by the specific secretions of the intestinal glands and by the antagonistic action of the normal bacterial flora of the intestine. In the bowel itself it is questionable whether the natural surface defenses are at all adequate for the protection of the underlying tissue, or whether the tissue does not accept a minimal invasion as normal and meet it by the more complicated humoral and cellular activities after invasion has taken place. The studies of Adami and his co-workers have fully established the importance of the liver in this regard.

In the alimentary tract, as in the genitourinary apparatus, it is evident that although the normal epithelial defense is normally adequate, yet it may easily break down. An important factor in the prevention of the invasion of parasites through the epithelium is the preservation of the normal movements of the contents of the various tubes. The 


\section{LIVING CAUSES OF DISEASE AND HOW THEY ACT}

bile is a fluid in which bacteria will grow either in vitro or in vivo, yet normally it is uninfected, although it is difficult to understand how bacteria are prevented from entering through the ampulla of Vater. As soon, however, as interference with the flow occurs infection may promptly appear. Similarly about the meatus of the urethra there is an abundant growth of bacteria, yet the urethral canal and the bladder remain sterile, but if interference with the flow of urine occurs, infecting organisms are likely to ascend and to set up infections and inflammation of the bladder. Even when the bladder has become seriously infected, for a time the infecting organisms may not be able to ascend the ureters to the kidneys.

\section{PARASITISM AND VIRULENCE}

One organism may live in the tissue of another and still not be regarded as a true parasite. In certain cases two organisms living together may confer distinct benefit upon one another; they are mutually helpful. In other cases an organism may live within another and derive advantage and protection by such a habitat, yet it may in no way affect its host. This condition is known as commensalism. The bacillus coli, a common inhabitant of the human intestine, is an example of such a commensal, and many other bacteria in the gastrointestinal tract might be regarded as commensals. When, however, we speak of a parasite we mean a living organism which not only derives advantage from its host, but by its presence inflicts definite injury on the host. Instances of such parasites are the various parasitic worms, the parasitic protozoa, and the pathogenic bacteria. Among the higher animal parasites, especially the parasitic worms, parasitism is an essential condition of their life. They cannot exist in other than a parasitic condition. The same is true of the majority of the protozoal parasites of man and animals. Among the bacteria and vegetable parasites there are all degrees of parasitism. Some bacteria, as for instance bacillus coli, may live as harmless commensals in the large bowel, but under certain conditions, especially when it escapes into the tissues, or invades the peritoneal cavity or the bladder, it may show the characters of a true parasite. Other bacterial organisms, as for instance the gonococcus or the meningococcus, may be regarded as strict parasites, since they only grow in the animal tissues. It is true that by imitating conditions of the body in food material, temperature, etc., we can induce many of them to grow in the laboratory, and then they behave like non-parasitic organisms, or simple saprophytes. With the elaboration of bacteriological methods it has been possible to force more and more of the strictly pathogenic organisms 
thus to lead a saprophytic existence, but they remain nevertheless true parasites. On the other hand, Thiele and Embleden ( ${ }^{11}$ ) by gradually accustoming strict saprophytes to the conditions of the animal body, by a system of education in fact, have succeeded in converting such a harmless saprophyte as bacillus mycoides into an organism pathogenic for animals.

Virulence-that is, the power of a parasite to infect a host-is a condition which depends upon a complicated interrelationship of host and parasite. In any given instance it may be difficult to say how much the establishment of an infection may be due to absence of protective mechanism on the part of the host, or to the presence of special infecting powers on the part of the invader.

In infection by the higher animal parasites, such as the worms, it is probable that parasitism is so well developed that there is no marked protection by the host, once it is exposed.

The embryos of trichinella probably resist any protective mechanism in the gastrointestinal tract, and even when the blood stream is invaded the only indication of protective reaction on the part of the host is in the eosinophilia which is so characteristic. How this multiplication of eosinophils, which occurs in all worm infections, acts-whether against parasite or its products-we do not know. That it indicates a reaction which is ever successful in protection seems doubtful. It is only after the trichinella embryo becomes encysted in the muscle fiber that normal tissue reaction eventually brings about its slow destruction.

Among the bacterial parasites, on the other hand, relationship of host to parasite is so complicated that it is sometimes difficult to differentiate the two factors. A maximum virulence in a bacterial organism would be where a single cell is capable of establishing an infection. This is a condition rarely met with except in laboratory experiments. Sometimes in severe epidemics it may be seen at the beginning of the outbreak, as for instance in certain outbreaks of bubonic plague. In many epidemic outbreaks of infectious disease the incidence of morbidity and mortality seems to follow a definite curve. On account of a higher mortality percentage the earlier cases may seem to indicate more virulence in the infecting organism than those later in the epidemic. But Koch $\left({ }^{12}\right)$ many years ago pointed out that, for water borne diseases like typhoid or cholera, where in an explosive outbreak a whole population is simultaneously exposed to the organism, only a certain percentage acquire the disease; the only explanation of this fact would be a varying immunity, natural or acquired, in the population. On the other hand, where epidemics occur in a crowded population, as in camps and barracks, they are apt to show much greater virulence, and this is prob- 
ably explained by the rapid transfer of the infecting organism from individual to individual without intervening saprophytic existence. How much this variation is due to changes in the parasite, or changes in the immunity of the host, is often difficult to decide. Undoubtedly the continuous passage of bacteria from host to host without intermediate saprophytic existence frequently results in enhancing virulence, but quite frequently only enhances it up to a certain degree.

Pasteur $\left({ }^{13}\right)$, showed that the street virus of rabies, that is that obtained from the rabid dog, would at first require three weeks to kill a rabbit. But continuous passage through the rabbit shortens the incubation period until, after a number of passages, the infected animal died in between seven and eight days-that is, the stage of fixed virus was reached. The interesting point is that no matter how many generations of rabbits are used after the stage of the fixed virus is reached, the incubation period and fatal termination are not altered. They may vary by a few hours, but one cannot reduce them further. Here we have an instance of virulence for one animal steadily increasing until a fixed incubation period is reached. On the other hand, Pasteur showed that passage of the rabies virus through monkeys lengthened the incubation period, and there is evidence that the fixed virus of rabbits shows diminished virulence for dogs and man.

It is an old observation that the longer an organism is cultivated upon laboratory media the more it suffers in its infecting power, that is, its virulence.

Perhaps the most striking demonstration of this has been made by Webb, Williams, and Barber $\left({ }^{14}\right)$. By an ingenious method which enabled them to pick up individual anthrax threads, they were able to show that a single thread taken from the blood of a mouse which had just died of the disease would suffice to infect another mouse, whereas after a few hours' cultivation in the incubator, it required several threads to infect. Similar observations have been made upon many different bacteria. It may in fact be regarded as a law that pathogenic organisms steadily lose virulence during saprophytic life.

In the tissues of the host, however, the organism is subjected to influences which must materially affect its virulence. Observations upon pneumococci isolated at various stages of a lobar pneumonia seemed to show a diminution of virulence until the approach of the crisis, and this possibly may happen in other cases, but more commonly the effect of continuous growth in the host tissues is to enhance virulence.

A natural or an acquired immunity to pathogenic bacteria has been shown to be due to complicated cellular and humoral processes in the host tissue. An account of these phenomena does not belong here, and 
they will be discussed fully in another chapter, but it is easy to see that these conditions of immunity are important determining factors in the invasion of the host tissues by bacteria. There is, however, another side of the question which is equally important. Welch $\left({ }^{15}\right)$, in his Huxley lecture, first suggested that we must assume that the infecting organism may undergo changes of the nature of an immunity to the protective elements of the host. The result would be that virulence, or infecting power on the part of an organism, would be the resultant of two factors-on the one hand the degree of immunity of the host, on the other the degree of immunity of the parasite. Among the parasitic worms it is probable that the first factor is small in amount, the second large, consequently infection is easily established. Among the bacteria, on the other hand, both factors may vary. Natural or acquired immunity to a bacterial infection is probably always relative, not absolute, consequently quantitative and especially qualitative variations in the infecting organism may or may not determine the infection. It would be among the qualitative changes in the pathogenic bacteria that we must look for the true explanation of virulence.

We now have a great deal of evidence in regard to the nature of these qualitative changes. In many cases there is a distinct morphological difference between virulent and non-virulent examples of the same organism. This morphological difference consists in the possession of a distinct capsule, a sort of swelling of the outer layer of the bacterial cell. Many pathogenic bacteria normally possess such a capsule, for example the pneumococcus or the bacillus of Friedländer. The relation of capsule formation to virulence is seen in a variety of ways. Virulent bacteria leading a saprophytic life upon culture media, tend to lose virulence and at the same time to lose their capsule. Capsulated organisms like Friedländer's bacillus, which retain their capsule under saprophytic conditions, also retain their virulence. Organisms which never show a capsule in culture media may constantly show it in the animal tissues. Again Eisenberg $\left({ }^{18}\right)$ has pointed out that virulent organisms in the tissues may not show a distinct capsule, and yet morphologically there seems to be evidence of a change in consistency and thickness of the outer cell membrane. There is evidence also that agglutinins do not act so well upon capsulated as upon non-capsulated organisms. Direct evidence of the influence of the capsule as a protective mechanism for the bacteria is given by Gruber and Futaki $\left({ }^{17}\right)$, who state that leucocytes avoid capsulated anthrax threads whilst they actively phagocyte those destitute of a capsule. Where no visible difference can be made out between virulent and non-virulent strains there may be evidence of a difference in the behavior of the leucocytes. Rose- 
now $\left({ }^{18}\right)$ has shown that virulent pneumococci are not so readily phagocyted as non-virulent. In. regard to other protective mechanisms of the body similar facts have been observed. Walker $\left({ }^{19}\right)$ found that typhoid bacilli, grown in typhoid immune serum, became inagglutinable.

Besides changes, morphological or otherwise, in virulent bacteria which render them immune to the protective mechanisms of the body, it has been shown that they produce substances which directly attack and neutralize these protective mechanisms. Denys and Vandevelde $\left({ }^{20}\right)$ many years ago showed that staphylococcus pyogenes aureus produced a substance which they called leucocidin, which directly paralyzes and kills the leucocytes. Bail $\left({ }^{21}\right)$ extended these observations and maintains that virulent organisms produce a definite substance, which he terms aggressin, and that this destroys the protective cells and substances of the body fluid. The important point in regard to Bail's view is that the aggressins are not formed in vitro but are only produced when the organism invades the body. Wassermann and Citron $\left({ }^{22}\right)$, whilst admitting the importance of aggressive substances for the establishment of an infection, maintain that they do not differ from the ordinary toxins of bacteria and that they may be formed in vitro. Recently Rosenow $\left({ }^{23}\right)$ has returned to this question in his studies on pneumococcus, and he claims to have shown that substances which he obtained from virulent pneumococci but cannot obtain from avirulent strains, favored infection and especially inhibited phagocytosis. To this substance he gives the name virulin.

From all these facts it is evident that Welch was correct in his view as to pathogenic bacteria developing what we may call an immunity to the host defenses.

Not only can pathogenic organisms defend themselves against the humoral and cellular protective mechanism of the body but they may acquire an immunity to the drugs which are used therapeutically to destroy them. Ehrlich $\left({ }^{24}\right)$ was the first to show this in connection with trypanosome infections. In experimental trypanosomiasis he found that certain aniline dyes (trypan red, trypan blue) and certain arsenical preparations (atoxyl) would on injection cure the animal. He found, however, that if he did not give sufficient of the drug to kill all the parasites, from those which remained a new race developed which resisted the action of the drugs; they became as he designates it, giftfest. Clinically the fact has been observed in the relationship of malarial organisms to quinine or of the treponema pallidum to various antisyphilitic remedies. It is indeed so well established as to have a very important bearing on therapy. 


\section{MANNER OF DISEASE PRODUCTION BY LIVING ORGANISMS}

Living organisms when parasitic within the animal body may cause damage in a variety of ways. It is conceivable that the mere presence of the parasite might give rise to damage, and if they are present in very large numbers they might cause great damage. A good example of this is seen in trichinella infection. When the number of infecting organisms is large there may be gastrointestinal symptoms, due to their presence in the bowel. When their number is not large this stage may pass without symptoms. When the embryos invade the blood stream their presence is revealed by the leucocytosis and especially by the eosinophilia. The characteristic myositis is due to the reaction in the damaged muscle produced by the invasion by the worm. Actual trauma is produced by the parasite penetrating into the muscle fiber. Metabolic products from the parasite are in part responsible for the local symptoms, and the products of disintegration of the damaged muscle cells themselves contribute to the picture. All these factors play a part, and it is almost impossible to disentangle one from the other. When the worm has become quiescent and subsequently dies, it acts as an ordinary foreign body, and the changes which supervene in the muscle in no way differ from those which one sees when any foreign body is imbedded in the tissues. Williams $\left({ }^{25}\right)$ has shown by routine examinations at postmortems that cases are found after death with the parasites in the muscle in which clinical histories gave no record of symptoms during life.

In the infection of the body with the taenia echinococcus, the echinococcus cysts may reach such a size as to cause disease symptoms by their mere presence; this is especially true where the brain is invaded when relatively small cysts may set up serious symptoms, but even in the abdominal organs the abnormal size of the cysts may mechanically interfere with the functions of the body. But aside from the mechanical effects of such a parasite as echinococcus, the existence of an eosinophilia and the presence of a positive complement deviation reaction show that metabolic products of the worm pass into the circulation and give rise to reaction in the blood: In other cases of worm infection the influence of the secreted poison may play a very large rôle in the manifestations of the disease. In bothriocephalus latus infection, where the worm is lodged in the small bowel, the profound anemia, which is hardly distinguishable from pernicious anemia, has been shown by Faust and Tallquist $\left({ }^{20}\right)$ to be due to a lipoid hemolytic agent secreted by the worm.

In uncinariasis also, where the most striking symptoms are a profound anemia combined with definite nervous disturbance, the cause 
seems not to be the simple destruction of blood by the parasite, nor the occurrence of repeated hemorrhages from the attack of the worm upon the duodenal mucosa, but the toxic substances secreted by the parasite.

A curious example of the varied effects of a parasite is seen in filaria infections in man. The filaria embryos may swim freely in the blood stream, reaching in many cases enormous numbers. Manson $\left({ }^{27}\right)$ estimated the number in one case of nocturna infection at three hundred to six hundred per drop of blood, or for the whole body, at between forty and fifty millions. It seems difficult to believe that these parasitic embryos produce no symptoms by their presence, yet, in the majority of cases, they seem to be simple commensals without any reaction upon the part of the blood or the tissues. The adult worm, on the other hand, produces very serious forms of lymphangitis and lymphadenitis resulting in the striking types of tropical elephantiasis.

In malaria we have a type of infection in which it is equally difficult to unravel the relative influences of the number of the parasites, the products of the parasitic metabolism, and the catabolic products of the patient. In severe cases of malignant tertian malaria, especially of the cerebral type, an examination of the brain at post-mortem shows the capillaries literally stuffed with the parasites. Ewing $\left({ }^{28}\right)$ has described a similar condition of the capillaries of the heart in a case showing chiefly cardiac symptoms. Here unquestionably the presence of enormous numbers has had a direct mechanical effect. In confirmation of this is the remarkable effect of intravenous quinine in a case of severe cerebral malaria. Undoubtedly the anemia and melanemia are due to the direct destruction of blood corpuscles and hemoglobin. In severe tropical infections the number of corpuscles per cubic millimeter may drop a million in twenty-four hours. How far the typical temperature chart is due to toxins of the organism, or to the liberation into the blood of the remnants of the infected corpuscles, it is difficult to decide. It is directly caused by the sporulation and breaking out of the spores into the blood stream. Ruge has noted that if one compares two cases of simple tertian in one of which the parasites all ripen within two to four hours, the other in six to eight hours, the former will give the sharper rise in temperature, reaching a higher point with more severe general symptoms. It is very probable that the recent studies of Novy $\left({ }^{28}\right)$ upon anaphylaxis provide the most acceptable explanation of the malarial pyrexia.

When we come to study bacterial diseases we realize at once that the effect of mechanical interference, due to the presence of the bacteria in the blood stream or the tissues, becomes very much less. Even in anthrax, which was long held up as an example of the mechanical effect 
of the flooding of the blood with organisms, we see that the chief causes of the clinical symptoms are the products of bacterial metabolism and not the mere presence of the organism.

It was natural that when our attention became focused upon bacteria: as the causative agent in a variety of diseases, we should look to the metabolic products of their growth for the explanation of the symptoms of poisoning. As severe poisoning was known to result from the eating of foods which had undergone putrefaction, the first place in which these poisons were sought was in putrefying organic matter, especially organic matter of a proteid nature. When pure cultures of pathogenic bacteria were isolated these were allowed to grow in proteid-containing culture media and the products of their growth isolated, studied, and compared with similar substances obtained from putrefying meat, fish, etc. From all these sources there were isolated a group of chemicals: of basic nitrogenous character which were closely related to the vegetable alkaloids, and which were consequently spoken of as animal alkaloids, and to which Selmi $\left({ }^{30}\right)$ in 1878 gave the name ptomaines. For a time ptomaines were assigned an important rôle in the production of the symptoms of bacterial disease, but the fact that many of them were not toxic, that they were not specific products of specific pathogenic bacteria, but appeared everywhere in the putrefactive breakdown of the protein molecule, and the fact that they could not be demonstrated in the infected animal body, led us to realize that they play but a minor rôle in the production of symptoms. But although ptomaines are not now regarded as important in bacterial disease, putrefaction of organic material, such as sausages, canned vegetables, etc., may, when due to one specific group of bacilli (B. botulinus), give rise to a poison which is extremely virulent when ingested and absorbed. The chemical structure of this poison has not been closely determined because of the ease with which it breaks down when exposed to the necessary chemical manipulation. Its. chief interest lies in the fact that it is produced outside the body by the saprophytic growth of an anaerobic bacillus; that the same poison may be produced by this organism from a variety of proteins, both animal and vegetable; and especially in the fact that the poison when absorbed through the alimentary tract produces a definite specific train of symptoms which enables one to recognize the disease, whether it occurs in man or in animals.

Further study of the catabolic products of pathogenic bacteria, when grown in culture media, showed that some of these produced similar specific poisons which reproduced the symptoms of the actual infection. This was shown first in the case of diphtheria and tetanus. No matter what the medium in which diphtheria bacilli or tetanus bacilli are placed, 
if they grow, they will produce their specific poison, and for a given strain of organism the more luxuriant the growth the more poison produced. Since these poisons could not be isolated chemically, as the ptomaines could, they have been given the general name of toxins. Their discovery in the case of diphtheria and tetanus led us to hope that specific poisons of a similar character would be found for all pathogenic bacteria. The early literature of the subject, following Roux and Yersin's $\left({ }^{31}\right)$ discovery of diphtheria toxin in 1889 , is filled with accounts of attempts to find such toxins. Carefully elaborated experiments by Pfeiffer $\left({ }^{32}\right)$ upon the toxin production of the cholera spirillum brought out the fact that in a cholera culture nothing was so poisonous as the bodies of the organisms themselves, and that the poison within the cell of the cholera organism was so labile that any severe treatment in killing the organism resulted in weakening the toxin. This poison did not pass into solution in the culture fluid but remained attached to or part of the cell protein. As a result of this work and its extension to other organisms there developed the view that specific bacterial poisons were either exotoxins, those which passed into solution in the culture fluid, or endotoxins, those bound up with the bacterial proteins. The exotoxins are evidently all closely related in chemical constitution; they are very labile and they have this in common that they have antigenic properties, that is, when injected into the animal body they generate a specific antitoxin. Toxins with this antigenic character are not peculiar to bacteria; some have been obtained from plant cells, such as ricin from the seeds of ricinus communis, abrin from those of abrus precatorius, or from animals, as the various types of snake and insect venoms. Among the many pathogenic bacteria only a few produce definite exotoxins. Diphtheria and tetanus have been mentioned, also bacillus botulinus. Besides these, bacillus pyocyaneus, the bacillus of symptomatic anthrax, possibly the anthrax bacillus itself, and bacillus of dysentery. Most interesting among recent exotoxins discovered are those produced by certain of the anaerobes causing gas gangrene, discovered and used for antitoxin production by Carrol Bull $\left({ }^{33}\right)$ of the Rockefeller Institute.

The successful treatment of a bacterial infection by a so-called antitoxic serum must not, however, be taken to indicate that the corresponding crganism produces an antigen of the nature of an exotoxin. The work of the Rockefeller Institute has resulted in the production of a serum for the treatment of epidemic cerebrospinal meningitis, which is sometimes spoken of as an antitoxic serum. It is likely, however, that the success of this serum is due not to antitoxic characters but to its opsonic power-that is, its power to sensitize the organisms so that 
more active phagocytosis may result - and to distinct bactericidal activity leading to an extracellular solution of the organism. In antipneumococcus serum also the action is probably chiefly an opsonic one.

Although exotoxins are so important in those infections where they have been found, yet, as stated above, there are many infections in which the toxin is so closely combined with the bacterial cell that it is only liberated when the cell disintegrates. The antigenic activity of the endotoxins is much more complicated than that of the exotoxins. When the endotoxins are liberated in the tissues, there are formed either specific sensitizing substances (amboceptors of Ehrlich) which, when combined with the complement, or alexin, of the blood plasma, lead to the solution of the bacteria; or bacterio-tropic substances, or opsonins, are formed which lead to a more active phagocytosis of the specific organism by the leucocytes. In regard to the chemical nature of these endotoxins there still reigns considerable doubt. One view, which was first enunciated by $P$ feiffer, is that these endotoxins are preformed in the bacterial cell ready to be liberated when the cell breaks up. Another view which has gained ground in recent years is that of Vaughan $\left({ }^{34}\right)$, which would represent the endotoxin as a split product, resulting from the action of enzymes in the serum upon the bacterial proteins, liberated by the death and disintegration of the bacteria. Vaughan has shown that not only bacterial proteins but all proteins when subjected to the action of an alcoholic soda solution are broken up into a toxic and an atoxic moiety. By use of the toxic product Vaughan has been able to imitate by injection into animals the different types of fever characteristic of bacterial infections.

Friedberger $\left({ }^{35}\right)$, in a long series of investigations, has shown that bacterial cells, both pathogenic and non-pathogenic, will yield, upon exposure to the enzymes of serum, toxins which are similar in character and action to the anaphylatoxins obtained from other protein antigens. As a result of these observations it may be that the endotoxins do not exist preformed in the bacterial body. In fact the whole phenomena of bacterial intoxication have been brought into line with the observations upon anaphylaxis.

The subject of anaphylaxis will be discussed fully in another chapter, but the explanation of the action of bacteria upon the body is so closely bound up with anaphylaxis that it is impossible to discuss them apart. Two views have been held as to the production of anaphylactic intoxication, one being the view of Vaughan, that when bacteria or other foreign proteins are introduced into the blood stream, they are broken up by the action of specific or other enzymes so as to liberate what Vaughan calls the toxic moiety of the protein molecule. This toxic moiety is the 


\section{6

real anaphylatoxin. The other view is that introduction of the foreign protein brings about such a change in the blood plasma that the normal enzymes of the blood digest normal plasma constituents and thus liberate anaphylatoxin. The most important recent work is that of Novy ${ }^{38}$ ) and his associates. They seem to have proved conclusively that the second view is the correct one. There seems evidence from their observations that the constituent giving rise to anaphylatoxin is related to the fibrin-forming elements of the blood, and there is a relationship between anaphylaxis and blood coagulation. Novy's most interesting observation is that such a non-toxic substance as agar can produce anaphylactic shock if proper precautions are taken to bring the agar colloid into a proper state of dispersion. To quote Novy: "It is a rather remarkable fact that a substance apparently as inert as agar should be almost as toxic as the cholera vibrio, and possibly twenty times as toxic as the tubercle bacillus, and sixty-two times as toxic as vibrio metschnikovi. The effects produced by the agar are the same, and yet one cannot speak of an agar endotoxin.

"This fact goes to show that the so-called toxicity of the cholera vibrio, as well as that of the tubercle, typhoid, and dysentery bacilli, pneumococcus, trypanosomes, etc., is of the same order as that of agar. The substance of the invading organism is not a poison, neither is it broken up into a poison by enzymatic action, but the whole cell or its fragments, like agar, kaolin, peptone, etc., induce a change in the plasma which results in the production of anaphylatoxin. The more perfect the comminution of such matter, the more toxic does it seem to be."

Rosenau and Anderson ( ${ }^{37}$ ) were the first to suggest that the incubation period of an infectious disease represented the period of time necessary to elapse before the body reached the stage of sensitization to the antigen. In confirmation of this it has been shown that in producing bacterial anaphylaxis one dose of the antigen is not sufficient, but that the most certain method is by daily small doses. This observation is just in line with the conditions which we assume to obtain during the period of incubation.

That anaphylatoxins may be produced by bacterial proteins is certainly true. That these poisons can cause the most varied types of febrile reaction according to the method of administration is also true. Vaughan has shown this with his split products, and Friedberger in his observations upon bacterial anaphylaxis; but that all the clinical and pathological phenomena of bacterial infection are explained by a theory of bacterial anaphylaxis seems very doubtful. There are too many differences between the manifestations of different infections to allow of their being explained by one cause. If we consider for a moment the reaction of 
the blood cells to infection we see that there may be great differences. The cells of staphylococci or streptococci when injected into the blood stream produce a pronounced leucocytosis. Many other organisms have a similar action; on the other hand, in man the typhoid infection is characterized by a leucopenia and, as Mallory ${ }^{38}$ ) was the first to show, in the tissues where the organism is lodged there is no accumulation of polymorphonuclear leucocytes but an active proliferation of the endothelial cells of the lymph sinuses. The same is true for the paratyphoids and for Danysz' rat virus. Here there is evidence of specific differences in endotoxins which cannot be explained on the hypothesis of a splitting of the bacterial protein into a toxic moiety by the action of enzymes, a toxic moiety which is common to all proteins. It is much more likely that in all bacterial infections a variety of poisonous substances play a part different in quantity and quality in each different infection. In some, as for example tetanus, the exotoxin determines practically the whole intoxication; in diphtheria the exotoxin also plays - the chief part, but here endotoxins are probably responsible for the local necrotizing activity of the bacillus on the mucous membrane. In others, as for instance typhoid or cholera, the exotoxins may be present in but relatively small amounts, whilst the chief rôle is played by endotoxins, either specific or as the result of splitting of the bacterial protein.

The exact method by which the body cells are damaged by bacterial toxins has given rise to a very great deal of discussion. That a combination of poison and cell protoplasm must take place is self-evident. Tetanus toxin in the blood of a susceptible animal rapidly disappears. On the other hand, in the blood of an animal which is not susceptible, and therefore cannot develop tetanus, it may circulate unchanged for days. The whole basis of Ehrlich's side-chain hypothesis of immunity rests upon the assumption of an anchoring of the toxins to elements of the cell protoplasm. The nature of the combination of protoplasm and poison is, however, still a matter of doubt ; there is considerable evidence in favor of a solution of the poison in the lipoid cell constituents. But the physical solution of poison in cell constituents can only explain the first step in the process; the further steps are those which lead to chemical destruction of cellular elements with a resulting interference with cell and tissue function. There are considerable variations in the action of different toxins upon the body. The tetanus toxin shows a special affinity for the nervous system, and apparently must spread to the nerve cell by its axon process. The botulism toxin also has an affinity for the central nervous system, but whether the specific symptoms of botulism, which point to bulbar involvement, are due to the direct action of the poison upon the bulbar centers or to changes which are secondary 


\section{LIVING CAUSES OF DISEASE AND HOW. THEY ACT}

to the peculiar thrombi which form in the vessels of the brain, has not yet been discovered. The diphtheria toxin has a special affinity for the heart muscle and for certain of the peripheral nerves. In rabies and in anterior poliomyelitis we may assume also that the poison has a special affinity for the central nervous system, although in addition the virus itself is found in these tissues. Pasteur pointed out that the path of the rabies virus to the brain is by the nerve fiber and not by the blood stream. Flexner and Amoss $\left({ }^{39}\right)$ have shown the same thing in regard to anterior poliomyelitis; whereas a fraction of a cubic centimeter of this virus injected into the brain of a monkey will produce the disease, five hundred times as much injected into the blood stream will not infect the animal nor produce immunity. If the meninges are damaged, however, the virus passes from the blood stream to the nervous tissues and the disease develops. This perhaps is the most striking example we have of specificity of attack by organisms and their toxins.

The typhoid toxin especially stimulates the proliferation of the endothelial cells of the lymph sinuses and the blood sinusoids. The toxin of the cholera vibrio not only causes the destructive changes in the wall of the small bowel, but when absorbed, it is excreted by the kidney and may lead to serious damage of that organ. Flexner and Sweet $\left({ }^{40}\right)$ showed that the special lesions of the bowel in dysentery were due, not to the absorption of the toxin from the lumen, but to its excretion through the glands of the large bowel. As a rule one may say that the bacterial toxins are not absorbed through the bowel. The most interesting exception to this rule is in botulism where the only way in which the poison reaches the system is through the mucosa of the intestine.

Pathogenic bacteria when they attack the body may remain strictly local or may spread widely through the tissues. The best example of a local infection is seen in tetanus, where the organism remains at the point of introduction, elaborating there its exotoxin which spreads, not by the blood stream, but by the nerve trunks, ultimately reaching the central nervous system and then causing the development of the typical symptoms. Diphtheria is always cited as an example of a local infection. It is true that as a rule it remains localized on the mucous membrane which it attacks, and the symptoms are due to the circulating exotoxin, but on the mucous surfaces or on the surfaces of open wounds it may spread to neighboring areas, and instances are recorded in which the organism has been found in such internal organs as the spleen. Other organisms may tend to remain more or less local in the tissues but spread by contiguity. A very good example of this is in erysipelas. Here the streptococcus is lying in the tissue spaces of the corium; a section of the advancing margin of the erysipelatous tissue will show the organism in the spaces confined to 
a very narrow band. To obtain cultures from a case of erysipelas the material must be taken from this margin, further back the organisms are dead or have disappeared. In staphylococcus infection of the skin there is the same local lodgment of the organism and the same tendency to spread by continuity of tissue. But in these infections, as in many others-whether due to increased virulence of the organism or diminished immunity of the host-the extension may be by the lymphatics to the regional lymphatic glands. There are thus developed metastatic foci of infection. In some infections this path is very clearly shown. In bubonic plague the infection, when it enters through a flea-bite or an abrasion of the skin, promptly spreads to regional glands, giving rise to the typical buboes of the disease. In tuberculosis, especially in experiments on animals, a local infection is followed by establishment of the disease in the regional glands. A further step occurs when the infection, having passed through the lymph glands, reaches the blood stream. When we find the organism actually in the blood stream we may speak of the condition as a septicemia. But as a rule the term septicemia has been confined to the more serious forms of blood infection with streptococci or staphylococci, whilst the term bacteriemia is used for the more general condition. This question of the presence of pathogenic bacteria in the blood stream has assumed a different aspect with the development of the technique of blood culture. In typhoid fever we have a very good example of this. When the typhoid infection is established, it apparently begins as a bacteriemia, and in the first week of typhoid, with proper technique we can always recover the organism from the blood. With the development of the protective mechanism of the body the bacteriemia disappears, and the organism is caught and held in metastatic foci in the body. Such metastatic foci are seen in the roseolar eruption of typhoid, and the local lesion in the lymphoid elements of the bowel and mesentery are of the same nature. Again, when a relapse occurs it is always heralded by a return of the organisms to the blood stream and a renewed bacteriemia.

In lobar pneumonia, although the infecting organisms are mainly confined to the lung, yet in many cases there is a true bacteriemia. Cole's $\left({ }^{41}\right)$ observations would tend to show that the more severe the case the more the organisms are found in the blood. In meningococcus infections also, one can frequently obtain the organism from the blood by blood culture. In a frank septicemia, due to the streptococcus, there is no difficulty in obtaining the organism by blood culture, but in many streptococcic infections of a subacute character this can be done only if special methods are used. Apparently a few organisms are present which the ordinary methods would not reveal. Whether the condition is to be 
compared to that found in a true septicemia, that is an actual growth of the organism in the blood stream, or whether it is due to the continual shedding of viable organisms, for example from an infected heart valve, is still unsettled. At any rate all degrees between the two extremes may occur. Libman $\left({ }^{42}\right)$ and his co-workers were the first to show that in certain types of subacute endocarditis, due to non-hemolytic streptococci, one could always, with proper technique, obtain cultures from the blood, and this not once but repeatedly over long periods of time. In fact today the significance of bacteria in the blood stream is not nearly as seriously regarded as formerly. With some organisms the invasion of the blood stream may be only temporary. In tuberculosis the ordinary spread of the infection is by continuity of tissue or by the lymphatics, but where a large blood vessel wall becomes invaded by the organism a secondary invasion of the blood may result. Here, however, the organism apparently is carried along passively in the blood stream, and is immediately picked out and deposited in the capillary areas, giving rise to the metastatic foci spoken of as miliary tubercles. As a rule only the intensely virulent organisms can give rise to such a bacteriemia as may be spoken of as a septicemia, such for instance as the virulent hemolytic streptococci, the gonococcus, sometimes staphylococci or the bacillus of plague. Some organisms do not seem to invade the blood stream at all, as for instance the cholera organism or the bacillus of dysentery.

One of the most striking effects of parasites, especially bacterial parasites, is the production of fever. For a very long time the fever of an infectious disease was regarded as harmful in itself. It was not until the work of Liebermeister $\left({ }^{43}\right)$ that this idea gave place to a more truly biological view which regarded fever as one of the reactions of the body against infection, which it is true may be so severe as to cause damage in itself, but which in its genesis and course must be regarded as a protective character.

It is obvious that the primary factor in fever is the skin reaction which prevents heat dissipation. Calorimetric study shows that though there is an increased heat production, this is moderate in amount and not nearly so large as in muscular exercise. If then the body is provided with a complicated reflex mechanism which deliberately forces the temperature up, it seems self-evident that it must have value. In the laboratory it is a well-recognized fact that the rate of a chemical reaction is accelerated according to a definite law with every increase in temperature. It would seem probable upon a priori grounds that the same law would hold in the body. In the tissues, however, there is the complicating factor that when the temperature rises above a certain optimum, the cell protoplasm itself begins to suffer, and consequently we see that an excessively high 
temperature, a hyperpyrexia, may bring injury in its train. The work of Rolly and Meltzer $\left({ }^{44}\right)$ has shown that up to a temperature of $4 \mathrm{I}^{\circ} \mathrm{C}$. phagocytosis is increased in activity, also that agglutinins and bactericidal substances are produced much more rapidly than in control animals at normal temperature.

The relationship of fever production to infection is very clear in some diseases. The malaria pyrexia is coincident with the liberation of the malarial schizonts into the blood plasma. In relapsing fever, the rise in temperature is always associated with the appearance of the spirochaete in the peripheral blood stream. Experimentally, as stated above, Vaughan $\left({ }^{45}\right)$ has succeeded in producing every different type of fever by the introduction of bacterial and other proteins into the blood stream in proper doses. Fever in fact seems to be an anaphylactic phenomenon, and when not excessive, it is of definite value in aiding the tissues to combat the infection.

\section{DISEASE PRODUCTION BY BACTERIA}

Bacterial parasites, as pointed out in the general discussion, produce disease in a great variety of ways, and it is possible only to instance types of their action.

The first important group is that in which an exotoxin is formed and the infection remains strictly local. The bacillus of tetanus is perhaps the best type of this group. The organism is a minute motile rod which produces a terminal spore slightly larger than the diameter of the bacillus, so that the sporing form is drumstick shaped. It is a strict anaerobe. It is always introduced into the tissues by trauma. Its natural habitat is in the soil, and especially in soil contaminated with manure. It is on this account that tetanus has been such a serious infection in the early part of the present war, where the fighting was in the richly manured areas of Flanders and northern France. The tetanus spores alone, if washed free of toxin, are apparently incapable of producing the disease. In fection is favored by ragged wounds which are accompanied by necrosis of tissue, by the presence of other organisms, and especially by the presence of dirt and foreign material carried into the wound. The organism remains localized in the wound, and the toxin which is formed there passes by the nerve paths to the central nervous system. The exact chemical nature of tetanus exotoxin is unknown; it is so labile that the chemical manipulation necessary for its isolation tends to destroy it, but when in its most concentrated form it probably represents the most poisonous substance known.

- It is not a single poison, but probably contains several constituents. 


\section{LIVING CAUSES OF DISEASE AND HOW THEY ACT}

There is evidence that its action upon the nervous system is due to a component called tetanospasmin, whilst there is another constituent, tetanolysin, which attacks the red blood corpuscles. In spite of the intensely poisonous action of the tetanus toxins there are surprisingly few visible anatomical changes. The Nissl granulations of the anterior horn cells show evidences of degeneration, and there are changes in the nuclei of these cells.

The typhoid bacillus may be taken as a type of organism which is introduced into the body by the alimentary tract. It is an aerobic organism, motile, non-spore-bearing, and does not produce an exotoxin. It undoubtedly gains entrance into the body through contaminated food, especially water and milk. Once within the alimentary tract the bacilli apparently penetrate through the wall of the small bowel. At an early stage of the disease the blood stream is invaded, so that there is a characteristic bacteriemia which gradually disappears as the metastatic foci of the disease develops. These are situated chiefly in the lymphadenoid apparatus of the abdominal area. Mallory $\left({ }^{46}\right)$ was the first to show that the characteristic change in the Peyer's patches, the mesenteric lymph glands, and other organs, is a proliferation of the endothelial cells of the lymph sinuses. The anatomical changes in the intestine are entirely traceable to this process. In addition to the lymphadenoid changes there are other evidences of toxemia in the degenerative changes in the heart muscle and even the striated muscles. One peculiar character of the typhoid bacillus is the tendency it has to become localized in the gall bladder. Here it may live for years, sometimes without causing much 'pathological change. But from this situation it is continually shed into the bowel, and the infection of the feces is maintained for very long periods. These cases are the so-called typhoid carriers, and it is through these carriers that the disease is maintained and spread through the population.

An important group of bacteria have been called the pyogenic group on account of the predominant purulent character of the tissue reaction. This group is exemplified by the staphylococci, the streptococci and other forms of pathogenic cocci. It would be difficult to say which one of the organisms of this group may be regarded as most important from the standpoint of human pathology, but perhaps the streptococcus plays a larger part in human disease than any other. This globular, Gram-positive organism, growing in chains of varying length, is not only the cause of such infections as erysipelas, abscesses, septicemias, endocardial infections, acute arthritis, but it shows a tendency to invade the tissues as a secondary infection when the resistance has been lowered by some other primary disease. As Flexner $\left({ }^{47}\right)$ first pointed out, it is the most frequent terminal infection in any exhausting disease and in old age. Its rôle as a 
secondary infection in scarlet fever is so constant and so great as to lead some authors to believe that it is the actual cause of this disease. In pulmonary tuberculosis its significance is very great. In the recent study of broncho-pneumonias of camps, following measles, it has been found to be by far the most important organism. The classification of the different forms is still in a somewhat chaotic condition, and it may be said that we do not yet positively know whether we have to do with one or several species. Indeed, Rosenow $\left({ }^{48}\right)$ would maintain that we have to do with a pleomorphic organism including also the pneumococcus, in which appropriate cultural methods may convert one form into another. The length of the chains, the form of growth in bouillon, the fermentation of sugars, or the action on hemoglobin have all been used to separate the streptococcus into groups. Possibly the most important differential character is the action on hemoglobin. As a rule the more virulent strains are all hemolytic, whilst the less virulent ones, typified by streptococcus viridans, produce a peculiar green color upon blood agar or have no effect upon hemoglobin. Classifications of the streptococci have been made by Andrews and Horder $\left({ }^{49}\right)$, by Holman $\left({ }^{50}\right)$ and others, based upon biological character, but the most interesting recent attempt is that of Kinsella and Swift $\left({ }^{51}\right)$. The basis of their classification is the complement fixation reaction, and they bring out certain interesting biological relationships. These authors show that the division of the streptococci into hemolytic and non-hemolytic strains corresponds in a very striking manner to the immunological data, and most probably indicate definite difference in invasive and pathogenic character. As tested by the complement fixation method the hemolytic group is homogeneous, whilst the non-hemolytic group is heterogeneous. But on the other hand the non-hemolytic group may be divided into two subgroups, one of which approaches the hemolytic whilst the other does not. It is a most interesting observation. One concludes from Kinsella's discussion that it may be biologically fundamental, and that all strictly pathogenic organisms are more or less homogeneous whilst they are surrounded by a large number of related strains of low pathogenicity and heterogeneous in their immunity reactions.

It must not be assumed, however, that the non-hemolytic streptococci of the viridans group are negligible as pathogenic organisms. As the factors in focal infections about the teeth, tonsils, gall bladder, appendix, they are probably responsible for many of the subinfective conditions, and consequently may be of the utmost importance in longstanding, subacute, and chronic human disease. Whilst the infections with the more virulent types of streptococcus produce a reaction which is characterized by the presence of polymorphonuclear leucocytes, that is, is 


\section{LIVING CAUSES OF DISEASE AND HOW THEY ACT}

purulent, the viridans forms are very apt to show a plasma cell or round-celled infiltration. Very little is known definitely in regard to the toxins of the streptococci. Quite recently Clark and Felton $\left({ }^{52}\right)$ have succeeded in obtaining a filterable exotoxin from virulent strains of hemolytic streptococci by growing them upon rabbit's blood diluted with Locke's solution. It was destroyed by heating to $50^{\circ} \mathrm{C}$.; an antitoxin could be produced; it seemed to have aggressive characters, as they found in one experiment that one-fifth of the lethal dose of the toxin increased the rabbit's susceptibility so that one-fortieth of the lethal dose of living culture caused death. The toxicity of the less virulent strains is slight; possibly on this account they do not call forth an active resistance from the defenses of the body with the result that they produce infections which are very chronic in character and not readily eliminated from the tissues.

Closely related biologically to the streptococci is the pneumococcus or diplococcus lanceolatus. This organism is also distinctly pyogenic in the character of the reaction it calls forth in the tissues, but in addition to the leucocytic exudate the pneumococcus is characterized in lung infection and, to a considerable degree, in infection of the serous cavities, by the heavy fibrinous exudate which it produces. As the cause of lobar pneumonia and a common cause of infection in other parts of the body, it is one of the most important of human parasites. The recent work of the Rockefeller Institute, the South African Institute for Medical Research, and other similar institutions, has shed a flood of light upon this organism, and we may feel that only now are we beginning to understand the pathogenesis of pneumococcus infection. The first important step has been the demonstration that in spite of the well-marked cultural and morphological character of the pneumococcus, it nevertheless represents a series of groups of types which have different immunological reactions. Although in the gross anatomic picture of lobar pneumonia there is, so far as we know, no marked difference in the tissue reaction to the different types, yet the differences in the intensity of clinical symptoms and in mortality in infections due to different types show that there must be differences in the reaction of the body. The common occurrence of a pneumococcus in the saliva, known since the early observations of Pasteur and Sternberg, led us to look upon pneumonia largely as a form of self-infection, due to changes either in resistance of the host or invasive power of the organism. Dochez and Gillespie's $\left({ }^{53}\right)$ discovery that this salivary form, their type IV, is a heterogeneous group giving rise to only a smaller percentage of pneumonias with a distinctly lower mortality would indicate that we must look upon the spread of the disease as due to the more parasitic types, 
especially type I and type II. This must lead to a better understanding of prophylaxis and epidemiology. On the other hand, Lister $\left({ }^{54}\right)$ in South Africa has now determined the existence of as many as eleven strains, three of which are identical with the American strains I, II, and III. The incidence of these strains and the mortality caused by them does not coincide with American observations, and it is possible with systematic typing of pneumococci in large centers we may have to revise some of our ideas. The importance of the heterogeneous type IV as a mixed infecting organism is shown by observations at Camp Sherman in the recent influenza epidemic, in which eighty per cent. of the pneumonias were due to type IV organisms. It may be, of course, that the heterogeneous type IV may contain forms identical with some of Lister's South African types, and that the influenza epidemic created the conditions favorable for them to invade the tissue. The present pandemic of influenza has brought out one interesting point in regard to the reaction of the lungs, not only to pneumococci but to the different strains of streptococcus. Our own experience has been that at necropsy there was a great similarity in the gross appearance of the influenzal bronchopneumonias, yet one never knew what organism bacteriological study would reveal as the predominant form. The virus of epidemic influenza, whatever it may be, seems to have extraordinary aggressive character's, which so lowers the resistance of the lung tissue to these other organisms that whichever one happened to be introduced was able to produce the same hemorrhagic broncho-pneumonia.

Pneumococcus lung infection is unquestionably a disease of cities and crowded places, the organism spreading from preëxisting cases or healthy carriers. South African observations showed that natives who had recently arrived at the mines and were placed in crowded compounds were most susceptible, and Gorgas $\left({ }^{55}\right)$, in a report upon pneumonia in the Rand mines, points out that the same high incidence occurred among laborers in the canal zone until they were removed from crowded barracks and compounds and encouraged to live in separate huts.

The bacillus of tuberculosis is an organism which plays an exceedingly important rôle in human pathology. Owing to the peculiar chemical nature of its cell membrane, it stains with difficulty, and once stained, resists strongly the action of decolorizing agents, such as acids. On account of this peculiarity it, and related forms, are spoken of as acidfast organisms. This peculiar resistant character of the bacterial cell protects it also against the ordinary humoral and cellular defenses of the body, and the tissue reacts to the presence of the organism by a proliferation of the cellular elements to form the minute anatomical structure called a tubercle. The tubercle consists of fusiform or 


\section{LIVING CAUSES OF DISEASE AND HOW THEY ACT}

branched cells with vesicular nuclei, poor in chromatin, which are called epithelioid cells. Among the epithelioid cells one usually finds one or more multinucleated giant cells with the nuclei arranged about the periphery of the cell. The periphery of the tubercle always shows more or less infiltration with small round cells. The origin of these essential elements of the tubercle has been a matter of controversy among pathologists and is not even yet settled. It seems likely, however, that the epithelioid cells are similar in origin to the large mononuclear cells of granulation tissue, and that the giant cells are formed by a fusion of individual cells to form a syncytial phagocyte which is necessary for the destruction of the resistant organisms. The tubercle is in fact a special form of granulation tissue, only differing from ordinary granulation tissue in the absence of blood vessels. At first the tubercle is barely visible to the eye and is translucent, but it rapidly develops an opaque yellowish center which is due to a coagulation necrosis of the central portion. This coagulation necrosis, or caseation, is an important part of the tuberculous process, as it accounts for the extensive destruction of tissue which results from the infection with the bacillus. The toxins of the tubercle bacillus are apparently chiefly endotoxins with relatively low toxicity. The combination of a strongly resistant cell envelope with slow toxin liberation probably accounts for the fact that the inflammatory reaction in infection with this organism is chiefly proliferative and not exudative in character. It probably also accounts for the fact that when the organisms are not numerous the process is very chronic and tends always to a healing by connective tissue formation or by encapsulation. The epithelioid cells, like the giant cells, may be regarded as phagocytes; if they are not destroyed by the caseation they ultimately function as fibroblasts. The method of action of the round-celled infiltration is not understood, but they are essential, as it has been shown that if the lymphocytes of the body are destroyed by X-radiations of an infected animal the development of the infection is much more rapid. In certain tissues, as for instance in the lungs, infection by the bacillus of tuberculosis is almost always complicated by the presence of mixed infecting organisms which, by stimulating exudation and by the elaboration of toxins, bring about a more complicated anatomical and clinical picture.

Similar acid-fast bacilli produce tuberculous conditions in coldblooded animals, in birds, and in lower mammals. The first two do not produce infection in man, but some of the tuberculous infections of childhood have been shown to be due to the introduction of the mammalian type of bacillus through the milk of tuberculous cows. 


\section{DISEASE PRODUCTION BY HIGHER FUNGI}

Disease-producing organisms among the higher fungi are not so numerous or so important as the bacterial forms. Three subgroups of these fungi are represented, viz., the streptothrix forms, the blastomycetes, and the hyphomycetes.

The streptothrix forms most closely resemble the bacteria, in fact such bacteria as the tubercle bacillus and the diphtheria bacillus sometimes show branching which is thought to indicate a relationship to the streptothricaceae. The most important human example is the actinomyces or ray fungus. There are probably several varieties of this organism; it is much more common in animals than man (lumpy jaw of cattle). It seems likely that the organism is normally saprophytic, growing upon grain, grass, and similar vegetable matter. In order that infection may be established, this irritating vegetable substratum (barley beards, for instance) must be introduced into the tissue. As a consequence of this the infection is commonest about the mouth. It has also been found primary in the region of the cecum and appendix. Commonly the disease remains more or less local, spreading by continuity of tissue, but it may become generalized, and the lung may be invaded. The infected area shows a very moist granulation tissue with small pockets of pus and with a general purulent infiltration. In the pockets of pus and in the unhealthy granulation tissue are the small colonies of the fungus, with the characteristic radiating clubbed ends of the mycelial filaments, which gives it the nane of ray fungus.

An interesting group of infections are those due to yeast-like forms, the so-called blastomycoses. Blastomycotic infections seem to be more common in America than elsewhere. There are two forms which are evidently distinct, although the literature shows a tendency, on the part of some writers, to regard them as identical. In the eastern portion of America, and in the Middle West, there occurs a form of dermatitis with the development of a granulomatous lesion in which one finds a yeast-like organism with distinct budding of the cells of the parasite. The organism is found in miliary abscesses or sometimes in the tuberclelike giant cells of the granulation tissue. Although as a rule the infection is confined to the skin, general infection may result and especially the lungs may be involved. In the lungs the lesions closely resemble a tuberculous infection, but are perhaps of a more purulent character. In a particular district of California, the San Joaquin Valley, there occurs another blastomycotic infection which may occur in the skin, but shows a special tendency to involve the lungs. The disease is particularly fatal. 


\section{LIVING CAUSES OF DISEASE AND HOW THEY ACT}

The lesions are also granulomatous, but have a greater resemblance to tuberculosis and are less purulent. In the lesion the parasites are large doubly-contoured cells without any evidence of yeast-like budding but with many of the cells showing the formation of endospores. On account of this endosporulation the organism was at first thought to be a coccidium and is now called the coccidiodes immitis. Both organisms can be cultivated and both, but especially the coccidiodes, give rise to mycelium in cultures. This latter organism grows best at body temperature, according to Brown and Cummins $\left({ }^{56}\right)$, whilst the other organism grows best at room temperature. The first form, if not too advanced, reacts to the administration of iodides, whilst iodides have no effect upon the San Joaquin Valley disease.

The highest group of fungi, the hyphomycetes, contain a number of species pathogenic for man. As a rule these fungi occur only as ectoparasites of the skin, but in this situation they may show an obstinate parasitism which is difficult to overcome. In the infected areas of the skin the mycelium and spores of the fungi are found between the epithelial cells, and especially in the hair and hair follicles. The result of the growth is to lead to superficial disturbances of the skin. Secondary invasion by pyogenic organisms may readily take place, so that suppurative processes, due to these organisms, may complicate the condition.

More rarely hyphomycetes may invade the deeper organs, especially the lungs, and give rise to very serious infection. These lung mycoses are almost always due to a species of aspergillus.

\section{DISEASE PRODUCTION BY SPIRAL ORGANISMS}

The first microorganisms which were seen were the spiral organisms of the mouth described by Leeuwenhoek in 1692. Curiously enough the first pathogenic microorganism discovered and described was also a spiral form, the spirochete of relapsing fever, first seen by Obermeier in 1868 . In spite of this early knowledge of spiral forms, it is interesting that one of the most widespread and most assiduously studied of human diseases, syphilis, was only discovered to be due to a spiral organism by Schaudinn and Hoffmann in 1905. The biological relationship of these organisms has not yet been definitely established, but the majority of investigators consider that they should be classed with the protozoa and not with the bacteria.

The treponema pallidum, the cause of syphilis, is the most important organism for human pathology among the spiral forms. Its first description by Schaudinn and Hoffmann $\left({ }^{57}\right)$ in 1905 was quickly con- 
firmed by other observers; with the development of a special technique for its demonstration in the tissues, by Levaditi $\left({ }^{58}\right)$, its intimate relationship to the lesions of the disease became clear. Most important also was the demonstration, by Noguchi $\left({ }^{59}\right)$, of the organism in the brain and cord in paresis and tabes, thus finally settling the position of these so-called parasyphilitic diseases. In I9IO Noguchi $\left({ }^{60}\right)$ succeeded in cultivating the treponema.

We now know that every lesion of syphilis, no matter what the stage of the disease, is caused by the actual presence of the treponema. It may be present in extraordinary numbers, as in the liver and other tissues of the syphilitic fetus, or it may be so rare as to require the most accurate technique and infinite patience to demonstrate it, as in gummata, in syphilitic aortitis, or in the central nervous system, but it is always present.

This persistence of the parasite in the lesions of the disease, and especially the presence of the organism in the tissues for long periods of time without active evidence of the disease, so-called latent syphilis, has led to great confusion in the interpretation of its clinical course. The habit of clinically dividing the course of the disease into the primary, secondary, and tertiary stages has tended to give a false view of its natural history and should be abandoned.

Syphilis begins as a local infection, the primary sore. In this primary infected area we find proliferative changes; there develops a little mass of granulation tissue in which the predominant cells are plasma cells and larger mononuclear granulation tissue cells. The newly formed blood vessels are surrounded by heavy mantles of these round cells, and.as a result of the pressure of these cells, together with the local toxic action of the treponema, superficial necrosis and ulceration occurs. From the primary lesion the organisms rapidly spread to the system generally, at first involving the local lymphatics and the regional lymphatic glands, which show the same proliferating mononuclear elements. . The treponemata do not lie in the cells but between them, and often in relation to the perivascular collections of round cells. In the later stages of the disease the lesions are all characterized by similar proliferative activity, modified by the local character of the tissues in which they occur. The most typical granulomata are the gummata which develop in the later stages and which show a peculiar coagulation necrosis. The most interesting recent study of the presence of treponemata in syphilitic lesions is that of Warthin $\left({ }^{61}\right)$ In a routine search of organs by the Levaditi method, he discovered a surprising number of cases in which the organism's were demonstrated, although clinically syphilis was not suspected. In these late cases he places the organs con- 
taining active lesions with demonstrable treponemata in the following order-aorta, heart, testes, adrenals, pancreas, central nervous system, liver, and spleen.

The treponema is easily demonstrated in the primary lesion, but as the disease progresses it is more difficult to find. Direct experiment, however, has shown that even in these late stages it is just as virulent, and when introduced into animals reproduces the primary sore. It would seem as if the long persistence of the infection in the tissues has developed in them a change in character of the reaction. It may be also that in syphilis we have a particularly good illustration of Welch's suggestion of an immunity in the infecting organism, so that the treponemata of the late stages of the disease represent a survival of resistant organisms, an immune race against which the tissues react by a more elaborate proliferative process.

\section{DISEASE PRODUCTION BY FILTERABLE VIRUSES}

There is a large group of organisms which produce disease in man and animals which are so minute that they are barely visible under the microscope or are ultramicroscopic. These organisms, on account of the fact that they are able to pass through the pores of porcelain filters small enough to hold back all ordinary bacteria, are spoken of as filter passers or filterable viruses. Many of these are probably bacteria, but others probably belong to the protozoa, although one can only surmise their biological relationships. In quite a number of diseases the more obvious and more easily isolated bacteria which are often present as mixed infecting organisms have been at first mistaken for the true etiological agent, and it was only by a more refined technique that the filter passer was discovered.

One of the best examples of a filter passer is the virus of yellow fever. It illustrates the point just made. In the early study of yellow fever several bacterial organisms were isolated, and for a time each was considered the cause of the disease. It was not until the epoch-making investigations of the American Commission in Cuba that the true etiology was discovered. The work of Reid, Carrol, and Agramonte $\left({ }^{62}\right)$ presents perhaps one of the most perfect examples of a complete etiological demonstration which immediately bore fruit in the practical sanitation of the tropics. The virus of yellow fever was shown by these investigators to be a filter passer, which is only present in the blood during the first three days. They also demonstrated that the organism is transferred from person to person by the stegomya mosquito, just as malaria is transferred by the anophalines. As in the case of malaria also, the mosquito is not 
immediately infective. The virus must remain in the stegomya at least fourteen days before the bite of the insect can transfer the disease. Reasoning by analogy with malaria we may assume that a change in the virus takes place in these fourteen days, and that this is possibly in the nature of a sexual cycle. If this is true, then the yellow fever virus must be regarded as a protozoan. The effect of the yellow fever virus upon the tissues has been the subject of much discussion. It has the characters of a hemorrhagic septicemia, but there is apparently an exceedingly virulent toxin which accounts for the extreme degeneration of the cells of the liver, kidney, and heart muscle. The American Commission showed that the virus was only present in the blood for the first three days. Corresponding to this period is the primary pyrexia. On the third day the temperature falls, but in many cases rises again in a second pyrexial period. This secondary fever is due to the invasion of the tissues by secondary infecting bacteria which most probably come from the intestine.

Another filter passer which is of great importance in human pathology is the organism which causes acute anterior poliomyelitis. This organism, which was first discovered by Flexner $\left({ }^{63}\right)$ and his co-workers of the Rockefeller Institute is not ultramicroscopic; it can be seen as a minute globoid body just on the border of visibility, and it has been cultivated. From its appearance it has been thought to belong to the bacteria. Indeed, Rosenow $\left({ }^{64}\right)$ has maintained that this organism is but one form of his pleomorphic group of streptococci. But the investigations of the Rockefeller Institute show that it has biological characters which are quite different from ordinary bacterial parasites. In fact in many points there are resemblances to another filter passer, the virus of rabies. This organism shows a peculiar affinity for the central nervous system. It is easily transmitted to monkeys by intracerebral or subdural inoculation. On the other hand, it does not readily infect these animals if it is injected into the blood stream. Flexner has shown that O.I cc. of an emulsion of a virulent cord will surely infect a monkey when introduced into the central nervous system, whilst $50 \mathrm{cc}$. of the same virus injected into the blood stream fails to infect. This intravenous virus not only does not establish an infection, it also fails to call forth the protective mechanism of the body, that is, it produces no immunity. One must conclude therefore that it remains in the circulation as an absolutely inert organism. When, however, the meninges and choroid plexus are irritated by the injection subdurally of so bland a substance as normal saline, the virus migrates from the blood stream to the nervous tissues and infection is established. The lesions produced in the tissues by the virus have been studied in the first instance in man. The experimental infection of monkeys has enabled us to follow the sequence of events in the develop- 


\section{LIVING CAUSES OF DISEASE AND HOW THEY ACT}

ment of the pathological changes. The vessels of the pia and those passing into the anterior fissure of the cord show hyperemia which spreads to the gray matter of the cord and brain. Following the hyperemia there is a cellular exudate, at first of polymorphonuclear leucocytes and later of mononuclear cells, so that ultimately the small vessels become surrounded by a mantle of small mononuclear cells. The nerve cells, especially those of the anterior gray matter, rapidly show evidence of degeneration in protoplasm and nucleus. These degenerating cells are ultimately absorbed by mononuclear neurophages, and the areas damaged become replaced by neuroglial tissue. Besides the lesions of the central nervous system, the lymphadenoid tissue of the body generally may show areas of necrosis and proliferation of endothelial cells.

Among the other filter passers which cause disease in man are the virus of variola and vaccinia, the virus of rabies, the virus of trachoma and of molluscum contagiosum. These particular forms of filter passers apparently all occur as parasites in epiblastic tissue or its derivatives (ganglion cells in rabies). In these cells the presence of the minute parasite brings about a reaction, in which plastin substance is extended from the cell nucleus and forms a mantle about the organism. On account of this mantle-like investment of the parasite, Prowazek $\left({ }^{65}\right)$ has suggested the name chlamydozoa for the group. Mallory $\left({ }^{66}\right)$ has described chlamydozoa-like structures in the epithelial cells of scarlet fever.

There is evidence that the cause of the common coryzal cold is due to such a virus, and it is possible that epidemic influenza may be caused by a filter passer. The latest filterable virus to be demonstrated is that causing trench fever, a disease which has been so important a cause of army sickness in the present war. The report of the American Red Cross Commission $\left({ }^{67}\right)$ established completely the ultramicroscopic and filterable character of this virus. It has also established the methods of transmission of the virus by the body louse. On account of the slight mortality from trench fever and the failure so far to infect animals, we know very little of the pathological changes produced in the tissues by this virus.

\section{DISEASES CAUSED BY PROTOZOA}

Many forms of protozoa pass the whole, or the greater part, of their life as parasites. Of these only a relatively small number are parasitic in man. Those which are parasitic in lower vertebrates and invertebrates are of interest to human parasitology because of the greater ease with which they can be studied, consequently from a knowledge of their life history considerable light may be thrown upon the human forms. 
Some of the protozoa found in the human alimentary canal are not true parasites but commensals. In regard to others, doubts still exist as to whether they are commensals or whether they may not sometimes cause disease. Finally we have a number which are strictly parasitic.

Among many important protozoal parasites there is a peculiar form of alternation of generations. Part of the life cycle, usually the asexual portion, is passed in man or some other vertebrate, whilst the other part, usually the sexual, is passed in a biting insect. Experimentally, it is possible by transferring infected blood from vertebrate to vertebrate to transmit the infection. Under natural conditions this cannot occur, and the intervention of insects is necessary. The insects acquire the infection by biting and sucking the blood of an infected person. In the insect, sexual reproduction occurs. When it is completed, the insect host, in feeding upon a new individual, injects the sporozoit along with the secretion of its salivary glands. In many protozoan infections the full life history of the organism in the insect carrier or vector is known. In others we can only conclude that a sexual cycle occurs, since the insect is not able to transmit the disease until a certain number of days has elapsed after it acquires the virus. The insect carrier or vector consequently is necessary for the transmission of many of these protozoan parasites, and the destruction of the carriers becomes an important problem in preventive medicine.

It is not necessary to give a complete classification of the protozoa here, as this may be found in any textbook on zoology, but a brief synopsis will help to understand the relationships of the forms.

The protozoa are divided into two main groups, the plasmodroma and the ciliophora. The former group includes organisms which possess flagella, the mastigophora, those which possess pseudopodia, the rhizopoda, and those which are destitute of organs of locomotion, the sporozoa. The ciliophora are provided with cilia more or less differentiated as locomotive organs.

The most important and most numerous human parasites are found among plasmodrome protozoa, and they are distributed among all three classes, the trypanosomes in the mastigophora, the entameba in the rhizopoda, and the malarial organism in the sporozoa.

\section{TRYPANOSOMIASIS}

The trypanosomes are all parasitic. They live in the blood of different vertebrates where they pass through an asexual reproduction, and in the intestine of biting insects, where the sexual cycle is completed. The most important human parasite is trypanosoma gambiense, the cause of 


\section{LIVING CAUSES OF DISEASE AND HOW THEY ACT}

African sleeping sickness. The organism is slender, $16-30 \mathrm{u}$. in length, I.5-2 u. in breadth; there is a small undulating membrane, a wellformed flagellum at the anterior end; the posterior end is either sharp-pointed or rounded, depending upon the state of contraction of the protoplasm. It is found in the blood, in the lymph glands, and in the cerebrospinal fluid of the infected individuals. It passes through its sexual reproduction in a species of tsetse fly, glossina palpalis. In the infected human host it produces at first an irregular remittent type of fever. At the same time the lymph glands, especially those in the cervical area, become enlarged and remain so throughout the duration of the disease. Gradually there develop evidences of the invasion of the central nervous system; various peripheral manifestations may occur such as neuralgias, hyperesthesias, cramps and muscular tremors, and transitory incoördinations. Then there slowly develops a somnolence or lethargy with profound emaciation and wasting of the muscles, passing finally into deep coma before death. In a small proportion of cases there may be definite mental symptoms, sometimes even maniacal attacks. The duration of the disease is long, usually more than a year, and in many cases it probably lasts for a number of years. Its incubation period is very doubtful; it is usually recognized by the irregular fever and the swelling of the cervical lymphatic glands. In the quarantining of areas of Rhodesia against the introduction of sleeping sickness from Central Africa, the lymph glands are punctured and the material stained and examined for organisms. The most important pathological changes are those found in the brain. Everywhere the capillaries of the cerebral cortex are surrounded by a mantle of small round cells, usually plasma cells. The same infiltration is seen in the pia. Although the change is most marked in the brain, yet throughout the body, in the spleen, in the heart and lymph glands, there is the same proliferation and infiltration of lymphocytes and plasma cells. In tissues where the condition has existed for a longer time there may be fibroblastic proliferation and actual connective tissue new formation. As Mott $\left({ }^{68}\right)$ has pointed out, the change in the essential elements of the central nervous system are not primary but secondary to the perivascular and interstitial changes.

In northern Rhodesia another trypanosome has been found. $T$. rhodesiense, which is closely related to $T$. gambiense but is probably transmitted by another vector than glossina palpalis.

In Brazil in 1909 Chagas $\left({ }^{68}\right)$ discovered a new trypanosome parasitic in man. This form, called trypanosoma cruzi, is widespread in certain provinces of Brazil. Children become infected in the first year of life. These either die from the acute disease, or the disease passes over into a chronic type, as described by Chagas. The acute disease 
shows a continuous fever with slight morning remissions, a distinct increase in volume of the thyroid, a peculiar change in the skin of the face (a sort of dry crepitation), general lymph gland swelling, a swelling of liver and spleen, and often meningeal symptoms. In the more chronic stages in older children there is thyroiditis with development of a pseudomyxedema. The facies become dulled and stupid, the skin pale and cyanotic. A general glandular swelling develops and there is tachycardia as well as intestinal and nervous disturbances. The organism can be transmitted to laboratory animals, and a study of its development shows that it may occur as free swimming individuals in the blood or as endoglobular parasites in the red blood cells. Other cells of the body may be invaded also. In the endocellalar situation the parasite undergoes multiplication and resembles the parasite of kala-azar. The Brazilian observers have shown that it is transmitted by a species of bedbug, where it goes through its sexual cycle.

\section{LEISHMANIASIS}

Another important group of flagellate parasites is represented by the genus leishmania. These organisms exist in the human host as endocellular parasites, and undergo asexual reproduction there, whilst in cultures and in the intermediate insect host they appear as fusiform bodies provided with a single flagellum. Species of leishmania are the cause of three human diseases, viz., kala-azar, dumdum fever or tropical splenomegaly, most common in Ińdia, infantile kala-azar, or splenic anemia, seen in Africa and the Mediterranean basin, and the Oriental or Delhi boil, which occurs throughout the Tropics. These three diseases are believed to be due to three different species; in the tissues and in cultures the first two are much alike but differ in their pathogenic action upon animals. In all three diseases the organism is parasitic upon endothelial cells. In kala-azar the spleen is enormously enlarged and the endothelial cells of the sinusoids contain enormous numbers of the parasite. The infection is by no means confined to the spleen, but endothelial cells in the liver, bone marrow, lymphadenoid tissue, lung, and intestine contain them. The organism multiplies asexually by cell division within the cell until the cell becomes so crowded with them that it dies and breaks down, setting them free in the blood stream. These free parasites immediately penetrate fresh cells and thus the disease progresses. The organisms are sparingly present in the blood stream but may be found in polymorphonuclear phagocytes which have picked them up after liberation from the endothelial cells. All the clinical phenomena of the disease can be directly traced to the destruction of the endothelial elements all over the body. 


\section{LIVING CAUSES OF DISEASE AND HOW THEY ACT}

In the Oriental boil the parasite remains chiefly in the skin, giving rise to a characteristic granulomatous lesion, but here also the cells invaded are the large mononuclear and endothelial cells.

There seems no doubt that in Indian kala-azar varieties of bedbugs are responsible for the transmission of the disease. The infantile variety of the Mediterranean is probably transmitted by fleas, and in regard to the Oriental boil the actual intermediate host has not been demonstrated.

\section{PIROSOMIASIS}

Under this genus are found a number of minute parasites which occur chiefly in the red blood cells of domestic and wild animals. The first form described was that recognized by Smith and Kilborne $\left({ }^{70}\right)$ as the cause of the Texas cattle disease. Since that time a number of additional species have been described, each species being peculiar to one species of vertebrate. The typical piroplasma is a minute oval or pear-shaped body occurring either singly or in pairs, or sometimes in groups of four, within the erythrocyte. The infected red cells are rapidly destroyed with a resulting hemoglobinemia which gives rise to a striking hemoglobinuria and jaundice. All forms of pirosoma are transmitted by species of ticks; within the bodies of these carriers the parasite apparently passes through a sexual reproduction which takes a considerable time. For instance, in certain cases the tick larva sucks blood from the infected animal but is not able to transmit the disease until the insect has passed through a complete metamorphosis and reached its full development, a period of several weeks.

As yet no human diseases have been found due to typical pirosoma infection, but there are forms which may be related. In certain districts in Peru there occurs an endemic fever called oroyo fever which is characterized by a rapidly progressive and pernicious anemia with characteristic degenerative changes in liver, spleen, and other organs. In the red blood cells of the oroya fever cases are minute rod-shaped organisms resembling but much smaller than pirosomata. In addition to the red blood cells the endothelial cells of the capillaries become infected and crowded with the parasite. This organism has been named bartonella bacilliformis, and quite possibly may belong to the same group as pirosoma. Its insect carrier has not been discovered.

In the Rocky Mountain area of North America there occurs in isolated districts a disease called Rocky Mountain spotted fever. This has been shown to be transmitted by the wood-tick dermatocentor. In the blood of Rocky Mountain fever cases Ricketts $\left({ }^{71}\right)$ found a minute rod-like organism. Wolbach $\left({ }^{72}\right)$ has confirmed Ricketts' discovery and 
has demonstrated that the parasite occurs not in the red blood cells but in endothelial cells, and especially in the smooth muscle fibers of the smaller vessels. Here it causes destruction of tissue, and the anatomical lesions of the disease are traceable to this destruction. This organism has been regarded by some as a piroplasma, but this is probably incorrect, and according to Wolbach it represents an entirely new genus of organism.

\section{MALARIA}

The most important human protozoan parasite is that which causes malaria. Perhaps more than any other organism it has had an influence upon the humn race and human migrations. The ability of man to live and work in great areas of the tropical world is determined to a large extent by his ability to combat the malarial parasite. Certain authors have maintained the thesis that the decadence of Greece and Rome was due to the activities of malaria. At any rate the depopulation of many cf the Mediterranean areas, such as the Roman Campagna or southern Macedonia, is almost entirely due to this disease. Perhaps no protozoan parasite has been so completely studied, nor is there one whose life history is so exactly known. It is usually classed with the haemosporidia of the group of sporozoa, and is represented by three forms-the tertian, quartan, and malignant tertian or tropical. The organism in its asexual stage attacks the red blood corpuscle, and by the majority of authors is believed to lie within the red cells. The intracellular position of the parasite has been doubted by some investigators and recently Mary R. Lawson $\left({ }^{73}\right)$ has again taken up this question. She believes that it is always extracellular, clinging to the corpuscle about the small corpuscular hillock which it draws out. She also thinks that it may migrate from corpuscle to corpuscle, thus destroying many more red cells than if each organism remained intracellular up to the stage of sporulation. This view is not yet generally accepted, but Lawson's photomicrographs and drawings are very convincing. It has been repeatedly noted that the degree of anemia by no means corresponds to the amount of the infection. The anemia progresses also during the afebrile stage. Poech $\left({ }^{74}\right)$ has shown that in a case which showed an average of 9,500 parasites per cubic millimeter the number of corpuscles dropped a million per cubic centimeter in three days. This can only be explained on the hypothesis of a toxin acting upon uninfected corpuscles, or on that of migrating parasites. The former view is the one which has been most generally accepted. In regard to the existence of a toxin there is no actual evidence, but in its favor is the relatively greater toxemia in the malignant tertian than in the simple tertian or quartan. The sexual 


\section{LIVING CAUSES OF DISEASE AND HOW THEY ACT}

reproduction takes place in species of anophiline mosquitoes. Within the insect stomach fertilization takes place, the resulting ookinete migrates through the wall of the stomach and appears as a round body about the size of a red blood cell. It rapidly increases in size and its contents break up into a number of spheres in the periphery of which a multitude of sickle-shaped sporozoites are formed, these break out into the body cavity of the mosquito and make their way to the salivary glands from which they are injected into the next victim when the mosquito bites. About fourteen days are necessary for the sexual cycle, but a definite air temperature is necessary before the cycle can be completed, and this is higher for malignant tertian than for simple tertian and quartan.

\section{INTESTINAL AMEBIASIS}

One of the most important protozoan infections in man is that due to entamaeba histolytica, the cause of amebic dysentery. This parasitic ameba is a large organism 20 u. -40 u. in diameter, with a granular entoplasm and a very distinct, clear, transparent ectoplasm. It passes into a resting stage in the form of cysts in which four nuclei are seen, and when these germinate four small amebae emerge from the cyst. In the intestine it invades the wall of the large bowel, apparently directly migrating to the bottom of the crypts of Lieberkiuhn, bringing about a necrosis of the epithelium and passing down into the submucosa. In the submucosa it rapidly multiplies and gives rise to deep ragged ulcers with overhanging margins. From the submucosa it may wander into the capillaries reaching the liver, and there producing the characteristic tropical liver abscess. The action of the organism has been studied in cats, which are susceptible to the disease, and it has been shown that the infection takes place through swallowing the encysted form. In man it is probably spread by human carriers and flies.

\section{INTESTINAL FLAGELLATES}

The intestines of most animals contain flagellated protozoa. In the majority of cases these are mere commensals and have no pathogenic significance; however, many authors are inclined to attribute pathogenic activity to certain species on account of their occurrence, sometimes in enormous numbers, in the intestine and their association with forms of diarrhea and even dysentery. Two forms-trichomonas intestinalis and lamblia intestinalis-especially have been regarded as pathogenic. Since the Gallipoli campaign of the present war a third form-tetramitus Mesnili-has been blamed for producing forms of diarrhea. These 
three flagellates have undoubtedly been found in large numbers in cases of diarrhea and dysentery in Gallipoli, Egypt, and Salonica, but so far no definite evidence has been offered of their pathogenic activity. Lamblia intestinalis is possibly the only one which may be seriously suspected. It inhabits the duodenum, and each organism is attached to an epithelial cell by a cuplike depression on its under surface. Wenyon $\left({ }^{75}\right)$ states that in rabbits infected with lamblia he has found them within the intestinal glands, and in certain forms of diarrhea which he has seen recurring in lamblia-infected patients he has found flakes of mucus swarming with the parasites. Graham $\left({ }^{76}\right)$, in a study of dysenteries of the Salonica area, believes that all forms of mild diarrheas in Salonica in which flagellates were found in numbers were really due to mild bacillary dysentery. Dobell $\left({ }^{77}\right)$ also believes that the pathogenic character of any of the intestinal parasites remains unproved.

\section{INTESTINAL CILIATA}

The only species of ciliophora which is important in human pathology is balantidium coli. This form, which was one of the earliest discovered in human feces, is now believed to be responsible for one type of tropical dysentery. The most recent studies of balantidium colitis are those of Strong and Musgrave and E. L. Walker $\left({ }^{78}\right)$ upon cases occurring in the Philippines. It is an insidiously developing dysentery in which ultimately extensive chronic ulcerations of the large bowel may resılt.

\section{DISEASE PRODUCTION BY VERMES}

A large number of parasites belonging to the class vermes occur either constantly or occasionally in man. The majority of these resemble protozoan parasites in that man is only one of the hosts, and for a complete life cycle of the organism other vertebrate or invertebrate hosts are necessary. Even in those which do not require an intermediate host a longer or shorter period of development takes place outside the human body. As a rule the invasion of the human body is by the alimentary tract, but this is not universally true. The larvae of anchylostomum can penetrate the unbroken skin. It has also been shown that the larvae of schistosomum japonicum can penetrate the skin. In many cases of worm infection the larvae of the parasite lie encysted in the tissues of the intermediate host, and the human host becomes infected when this food is eaten in an uncooked or insufficiently cooked condition, the larva being set free by digestion and developing into the adult worm. In other cases man is the intermediate host and the para- 
site is present in the larval form, the adult worm being in a lower vertebrate. Because of these peculiar differences in the paths of worm infections diseases caused by worms are liable to show distinctly local distribution, depending partly upon the distribution of the intermediate host, partly upon the food, habits, or other life conditions of the exposed population. Thus in Japan, China, and Korea there are several diseases due to trematode or fluke-worms which are not met elsewhere in the world. Schistosomum hematobium is peculiarly prevalent in Egypt and adjacent parts of Africa and Asia, and occurs less frequently in other parts of the world.

Infection with various forms of adult tapeworms (tenia and bothriocephalus) depends upon the eating of uncooked meat or fish, and therefore depends for its distribution on food habits; the same is true of trichinella infection. The echinococcus disease in man which depends upon the presence of the larval form of a tapeworm, which in its adult form infects the dog, often shows a striking limitation of distribution. It is, for example, common in Iceland, and when Icelandic immigrants came to Canada and the United States the disease began to appear in their settlements, and it has remained endemic in these settlements.

On the other hand, in the case of a parasite like the hookworm, which has no intermediate host but develops in moist soil and water where the temperature is favorable, the distribution is world-wide, depending upon temperature conditions. In this disease also, life habits of the population are a determining factor, since we see that in hookworm infection, in regions where it occurs, it comes to be confined to such classes of the population as are specially exposed to infected mud and water, viz., mine workers, tunnel workers, brickmakers, and those who go barefooted.

Parasitic worms are divided into two large classes-platyhelminths or flat worms, and hemathelminths or round worms. The first class is divided into two large subclasses, the trematodes or fluke-worms and the cestodes or tapeworms.

As a type of trematode or fluke-worm infection, perhaps the most important and interesting is the schistosomum hematobium, which gives rise to what is most commonly spoken of as bilharzia disease. The disease is especially common in Egypt, but occurs throughout the African continent and the adjacent islands. The adult worms inhabit man, the larval development takes place partly in certain fresh water mussels. The path by which the larva enters the human host has not been clearly demonstrated, but it has been shown that the closely related Japanese form can penetrate the skin, and it is probable that this is the path of infection in the Egyptian form. The larva, once it has entered the 
body, invades the portal vein and its branches, and there reaches sexual maturity. Apparently the presence of the adult worm in the portal system does not in itself cause disease. The adult worms at first in the main portal vessels work their way against the blood stream to the root vessels, especially those of the pelvis. Here they lodge and the female produces her eggs. All the pathological changes are due to the presence of the eggs. These are produced in enormous numbers, and they block the capillaries of the rectal mucosa, the mucosa of the bladder, and ultimately escape into the tissues. As a result of their presence in the tissues, acute, subacute, and chronic inflammatory reactions occur in the mucous membrane, giving rise to areas of necrosis and ulceration with finally the development of papillomatous, or polypoid, growths which may ultimately undergo malignant change. As a rule the liver is not seriously involved, and though the adult worms and ova may invade the lungs, there is no serious pulmonary involvement. The ova escape to the outside in the urine and feces, which become infected by the breaking down of the mucosal growths. In the closely related Japanese disease, due to another species, the pathological changes are mainly in the intestines, liver, and spleen.

As a type of cestode or tapeworm infection we may take two examples. Bothriocephalus latus passes its larval stage in fish, the embryo penetrating the tissue of the fish and there becoming encysted; when this food is eaten uncooked by man the larvae develop into adult worms which are attached to the mucosa of the intestine. About twenty-four days after eating the infected fish the eggs begin to appear in the human feces. In man the worms, if few, may cause little trouble or only slight gastric disturbances, but if numerous they may give rise to all grades of anemia, in some cases indistinguishable clinically from a pernicious anemia. This anemia has been shown to be due to a lipoid substance produced by the worm which is actively hemolytic. Bothriocephalus is an excellent example of a worm infection dependent upon the habits of the infected population. The area of its occurrence is limited, but where it exists it is relatively common. Another type of cestode infection in man is the taenia echinococcus. This worm is very small, not more than $6 \mathrm{~mm}$. in length, and is parasitic only in the intestine of the dog. The ova escape with the feces of the dog. If these ova gain entrance to the human alimentary canal they develop into embryos which penetrate the wall of the intestine, invade the lymphatics or blood vessels, and are carried to the tissues, where they lodge and undergo further development. This development consists in the formation of cysts sometimes cf enormous size, so called hydatids. In man, daughter and granddaughter cysts may form with many embryonic taenia heads within 


\section{LIVING CAUSES OF DISEASE AND HOW THEY ACT}

them, showing in fact a form of asexual reproduction. They cause disease by their mere presence, but in addition there is evidence of systemic disturbance, and the blood serum of an infected individual gives a positive complement fixation reaction with the cyst fluid as an antigen.

The disease also occurs in sheep and cattle, and where the disease occurs in man, one always finds a very close association between man, dogs, and the domestic animals-as, for instance, in the sheep ranches of Australia or among Icelandic farmers.

There are a number of important hemathelminths or round worms parasitic in man. As a type, perhaps the most interesting and certainly the most widespread is the anchylostoma duodenale, or hookworm. Indeed this infection is probably responsible for as much human disease as malaria, and is as important an economic factor in the communities where it exists as malaria. The observations of the Rockefeller sanitary commission show that it is world-wide in its incidence, occurring between $36^{\circ}$ north latitude and $30^{\circ}$ south latitude. This commission estimates a total morbidity for the world of some $900,000,000$, a figure which is more than half the estimated total population of the world. In the tropical areas it attacks the great mass of the population. India is believed to have between $180,000,000$ and $250,000,000$ infected. In some tropical countries up to 90 per cent. of the working population has been found to be suffering from hookworm. In the tropics it is a disease of the whole population; in its northern and southern limits where it occurs in the temperate zones it is limited to certain classes, such as mine workers, tunnel workers, or brick workers.

The hookworm is a nematode worm. Two different forms occur in man-anchylostoma, which occurs throughout the eastern hemisphere, and necator, the form which occurs in America. The adult worms are quite small, the male from 8-i2 $\mathrm{mm}$., the female slightly larger. The mouth of the parasite is provided with a powerful sucker which enables it to attach itself to the intestinal mucosa; when attached it sucks in the mucosa into its esophagus, and by means of its chitinous teeth opens up the blood vessels.

There is still doubt as to the method of nutrition of the worm. Leichtenstern $\left({ }^{79}\right)$ thought that it nourished itself from the blood plasma and not from the corpuscles, as he found unaltered corpuscles escaping from the anus of the parasite. Looss $\left({ }^{80}\right)$, on account of finding remnants of epithelium in the intestine, thought that it fed upon the mucosal tissue, and as in feeding it frequently changes its situation, there would result repeated damages to the mucosa from which secondary hemorrhage could occur. Both their views are attempts to explain the profound anemia produced by such a small parasite. Unless active treatment is 
adopted the worm is able to exist for a very long time in the bowel. Cases are noted where the worm has been found six and even eight years after the patient has removed from possible areas of reinfection.

The ova of the parasite escape to the outside in the feces of the patient. If the feces remain moist, exposed to the air and at a sufficiently favorable temperature (the optimum temperature is about $25^{\circ}$ C.), the embryos develop in the ova; after a few days they escape as free living larvae. These larvae are very active, and by their movements escape from the fecal mass into relatively cleaner water, where they become surrounded by a flexible capsule within which active movements persist for a long time. Until the parasite attains the larval stage it cannot infect a new individual.

The larva is able to penetrate the new host by two paths. It may pass into the alimentary canal in contaminated food or liquids, where it loses its capsule and reaches maturity in the small bowel, or if the fluid containing the encysted larvae comes in contact with the skin, the larvae break out of the capsule and penetrate the hair follicles, ultimately reaching the connective tissue from which they enter either the small blood vessels or the lymphatics. From here they pass to the right heart, and so to the pulmonary capillaries, from which they break out into the alveoli, ascend the bronchioles, bronchi, and trachea to the esophagus, and thus reach the alimentary tract. From the penetration of the skin to the appearance of ova in the feces takes a long time, in many experimental infections as long as ten weeks has elapsed.

The most characteristic clinical manifestation of hookworm infection is the profound anemia. It is still uncertain whether this is entirely due to actual loss of blood from the bite of the parasite, or whether there is a toxin secreted, similar to the bothriocephalus toxin, which contributes to the blood destruction. There is a pronounced increase of the eosinophiles in the blood, and Boycott and Haldane $\left({ }^{81}\right.$ ) have shown that in experimental infections this occurs before the eggs appear in the feces. These authors consider an eosinophile count of over 5 per cent. in suspected hookworm disease as of great importance for diagnosis.

\section{DISEASE PRODUCTION BY TICKS AND INSECTS}

The large and important group of the arthropoda (spiders, mites, ticks, insects) contain many human parasites. Many of these, however, are blood-sucking forms, and man is only one of the hosts from which they obtain their food. In attacking the host, they inject into the puncture secretions of the salivary glands which probably have an anticoagulating effect upon the blood of the host, thus facilitating its 


\section{LIVING CAUSES OF DISEASE AND HOW THEY ACT}

removal. But in addition to this action these secretions invariably are toxic and cause more or less irritation. In susceptible individuals this irritation may be very great, and even general symptoms of poisoning may develop. Sometimes excessive numbers of biting insects, as for instance in persons badly infested with body lice, may give rise to serious general trouble. As a rule, however, the importance of these biting arthropods is due to their acting as carriers for other human infections, especially those due to pathogenic protozoa. In a few cases it is still doubtful whether the illness following the bite is due to the insect or to some yet unknown virus. For example, in southern British Columbia a species of dermatocentor is believed to cause paralysis in sheep and sometimes in children. The close connection between the bite of the tick and the onset of the symptoms, as well as the disappearance of these symptom's when the tick is removed, suggests the possibility of the action of some toxin.

A large number of arthropods are true parasites, burrowing into the tissues either as larvae or adult forms. The most widespread of these is the itch mite (sarcoptes scabiei), a strict parasite, which spends its whole life in the tunnels which it excavates in the skin. The tissue damage and the reaction is purely local, but in extensive and neglected cases, as for instance among soldiers on active service, the areas may serve as portals for the entrance of pathogenic bacteria which sometimes produce very serious conditions.

Another mite (demodex folliculorum) is a common parasite on the hair follicles and sebaceous glands of the face. It may cause so little trouble as to be regarded almost as a commensal, but sometimes there is obstruction and inflammation of the sebaceous glands. Borrell $\left({ }^{82}\right)$ has made some interesting observations upon the relationship of demodex infection and skin cancers, and suggests that the mite may transmit an unknown cancer virus. A much more plausible explanation is that the demodex gives rise to a persisting chronic inflammation which may ultimately lead to the malignant change in the gland epithelium.

Finally among the flies, certain species have acquired the habit of laying their eggs in open wounds, in the nostrils and ears of vertebrate hosts including man. When the eggs hatch the larvae may cause serious destruction of tissue. They are specially attracted by foul and discharging wounds, or to the nose in cases of ozena. Other forms are even' more parasitic, laying their eggs beneath the skin. These forms occur only rarely in man but are fairly common in domestic animals. This invasion of the skin by fly larvae is spoken of as myasis externa, whilst the more rare occurrence of fly larvae in the intestinal tract is called myasis intestinalis. 


\section{BIBLIOGRAPHY}

I. METCHNIKOFF, E. I. I.: The Prolongation of Life. G. P. Putnam's Sons, 1908.

2. ADAMI, J. G.: Jour. Am. Med. Assoc., 1899, XXXIII, 1509.

3. WELCH, W. H.: Am. Jour. Med. Sciences, I891, CII, 439.

4. LOOSS, A.: Centralblatt f. Bakteriologie, I90I, XXIX, 733.

5. INADA, R., and others: Jour. Exp. Med., 1916, XXIII, 377.

6. GARRE, : Quoted by Neisser, Kolle and Wassermann, Handbuch der Path. Mikroorgan, 1912, IV, 386.

7. BRUCE, D., and others: British Med. Jour., 1918, I, 354.

8. STRONG, R. P., and others: Trench Fever-Report of Medical Research. Committee American Red Cross, Oxford Press, 1918.

9. ROSENOW, E. C.: Jour. National Dental Assoc., 1918, V, II3.

10. MALLORY, F. B.: Jour. Med. Research, I912-13, XXII, II5.

II. THIELE, F. H., and EMBLEDEN, D.: Zeitsch. f. Immunitätsforschung, 1913, I Teil, XIX, 643.

I2. $\mathrm{KOCH}, \mathrm{R} .:$ Zeitsch. f. Hyg., I 893, XV, 89.

13. PASTEUR, L., and THUILLIER, L.: Comptes Rendu de l'Acad. de Science, I883, XCVII, II63.

14. WEBB, G. B., WILLIAMS, W. W., and BARBER, M. A.: Jour. Med. Research, 1909, XV, I.

15. WELCH, W. H.: British Med. Jour., 1902, II, I,I05.

16. EISENBERG, P.: Central. f. Bak., I907, XLV, 638 .

17. GRUBER, M., and FUTAKI, K.: Muenchener Med. Woch., I906, LIII, 249.

18. ROSENOW, E. C.: Jour. Inf. Diseases, 1906, III, 683.

19. WALKER, E. W. A.: Jour. Path. and Bact., 1903, VIII, 1903.

20. DENYS, J., and VAN DE VELDE: La Cellule, I896, XI, 357.

21. BAIL, O.: Arch. f. Hygiene, 1905, LII, 272, and 1905, LIII, 302.

22. WASSERMANN, A., and CITRON, J.: Deutsche Med. Woch., 1905, XXXI, 573.

23. ROSENOW, E. C.: Jour. Inf. Diseases, I907, IV, 285.

24. EHRLICH, P.: Gesammelte Arbeiten zur Immunitätsforschung, Berlin,. 1904.

25. WILLIAMS, H. U.: Jour. Med. Research, I90I, I, 64.

26. FAUST, E. ST., and TALLQUIST, T. W.: Arch. f. Exp. Path. u. Pharm., 1907, LVII, 367.

27. MANSON, P.: Tropical Diseases, London, I907.

28. EWING, J.: Jour. Exp. Med., I90I-05, VI, I19.

29. NOVY, F. G.: Jour. Inf. Diseases, 1917, XX, 499-854.

30. SELMI, F.: Ptomaine od Alcaloidi Cadaverici, Bologna, 1878.

3r. ROUX, E., and YERSIN, A.: Ann. de l'Inst. Pasteur, I889, II, 273.

32. PFEIFFER, A.: Zeitsch. f. Hygiene, 1892, II, 239.

33. BULL, G. B., and PRITCHETT, I. W.: Jour. Exp. Med., I917, XXVI, I19.

34. VAUGHAN, V. C.: Protein Split Products in Relation to Immunity and Disease, Philadephia, I913.

35. FRIEDBERGER, E.: Zeitsch. f. Immunitätsforschung, I9II, IX, 369; 19I I, $\mathrm{X}, 215,362,453 ; 1911, \mathrm{XI}, 389$. 


\section{LIVING CAUSES OF DISEASE AND HOW THEY ACT}

36. NOVY, F. G.: Jour. Inf. Diseases, I9I7, XX, 499.

37. ROSENAU, M. J., and ANDERSON, J. F.: U. S. Public Health and Marine Hospital Hygienic Laboratory Bulletin, 1906, No. XXIX, and 1907, No. XXXVI.

38. MALLORY, F. B.: Jour. Med. Research, I897-98, II, I 50.

39. FLEXNER, S., and AMOSS, H. L.: Jour. Exp. Med., I9I4, XIX, 4II, and I9I4, XX, 249.

40. FLEXNER, S., and SWEET, J. E.: Jour. Exp. Med., I906, VIII, 514.

4I. COLE, R., and others: Monograph of the Rockefeller Inst. for Med. Research, Oct. I6, 1917, No. 7 .

42. LIBMAN, E.: Trans. Assoc. Am. Phys., 1912, XXVII, 157, and 1913, XXVIII, 309.

43. LIEBERMEISTER, C.: Handbuch der Path. und Therapie der Fiebers. Leipzig, I875.

44. ROLLY, F., and MELTZER, K. S.: Deutsche Arch. f. klin. Med., 1908, $\mathrm{XCIV}, 335$.

45. VAUGHAN, V. C.: Trans. Am. Assoc. Phys., I9i I, XXVI, I9I.

46. MALLORY, F. B.: Jour. Med. Research, I897-98, II, I 50.

47. FLEXNER, S.: Jour. Exp. Med., I896, I, 559.

48. ROSENOW, E. C.: Jour. Am. Med. Assoc., I9I4, LXIII, 903.

49. ANDREWES, F. W., and HORDER, J.: Lancet, I906, II, 708.

50. HOLMAN, W. L.: Jour. Med. Research, I9I6, XXXIV, 377.

51. KINSELLA, R. A., and SWIFT, H. F.: Jour. Exp. Med., I917, XXV, 877, and 19I8, XXVIII, I69.

52. CLARK, A. H., and FELTON, L. D.: Jour. Am. Med. Assoc., 19I8, LXXI, I048.

53. DOCHEZ, A. R., and GILlESPIE, L. J.: Jour. Am. Med. Assoc., I9I3, LXI, 727.

54. LISTER, F. S.: South African Institute for Medical Research, Johannesburg, I9I4.

55. GORGAS, W. C.: Jour. Am. Med. Assoc., I9I4, LXII, I885.

56. BROWN, P. K., and CUMMINS, W. T.: Arch. Int. Med., I9I 5, XV, 608.

57. SCHAUDINN, F., and HOFFMANN, E.: Arb. a. d. Kais. Gesundheitsamte, I905, XXII, 527.

58. LEVADITI, M.: Comptes Rend. Soc. Biol., I905, LIX, 326.

59. NOGUCHI, H., and MOORE, J. W.: Jour. Exp. Med., I9I3, XVII, 232.

6o. NOGUCHI, H.: Jour. Exp. Med., IgI I, XIV, 99.

6r. WARTHIN., A. C.: Am. Jour. Med. Sciences, I9I6, CLII, 508.

62. REED, W., CARROL, J., AGRAMONTE, A., and LAZEAR, J. W.: Phila. Med. Jour., I900, VI, 790.

63. FLEXNER, S., and LEWIS, P. A.: Jour. Am. Med. Assoc., I909, LIII, 1639 .

64. ROSENOW, E. C., TOWNE, E. B., and WHEELER, G. W.: Jour. Am. Med. Assoc., I916, LXVII, I202.

65. PROWAZEK, S.: Arb. a. d. Kais. Gesundheitsamte, r905, XXII, 535.

66. MALLORY, F. B.: Jour. Med. Research, 1903-4, X, 483 .

67. STRONG, R. P., and others: Trench Fever-Report of Medical Research Committee American Red Cross, Oxford Press, I9I8.

68. MOTT, F. W.: Sleeping Sickness, Report, Royal Society, No. 7, 1906. 
69. CHAGAS, C.: Arch. f. Schiffs u. Tropenhyg., I909, XIII, I 20.

70. SMITH, T., and KILBORNE, F. L.: Report Bureau Animal Industry, 1897, VIII-IX, I77.

71. RICKETTS, H. T.: Jour. Am. Med. Assoc., I909, LII, 379.

72. WOLBACH, S. B.: Jour. Med. Research, 1916, XXXIV, I2r.

73. LAWSON, M. R.: Jour. Exp. Med., I9I3, XVII, 324.

74. POECH, R.: Zeitschr. f. Hyg. u. Infectionskrankheiten, 1903, XLII, 563.

75. WENYON, C. M., and O'CONNOR, F. W.: Human Intestinal Protozoa in the Near East, London, 1917.

76. GRAHAM, D.: Lancet, I918, I, 51 .

77. DOBELL, : Report Medical Research Committee National Health Ins., November, 1916.

78. STRONG, R. P., and MUSGRAVE, W. E.: Bull. Johns Hopkins Hosp., I901, XII, 31. STRONG, R. P.: Bureau of Gov. Lab., 1904, No. 26. WALKER, E. L.: Phillippine Jour. Science, I9I3, VIII, 333.

79. I.EICHTENSTERN, O.: Deutsch. Med. Wochensch., 1885, XI, 537; 1886, XII, 237 ; 1887 , XIII, 565.

8o. LOOSS, A.: Centralb. f. Bakteriologie, I90I, XXIX, 733.

8I. BOYCOTT, A. E., and HALDANE, J. S.: Jour. of Hyg., I904, IV, 73.

82. BORRELL, A.: Annals de l'Inst. Pasteur, 1909, XXIII, 97. 
CHAPTER VII

\title{
EUGENICS IN RELATION TO MEDICINE
}

\author{
By Charles B. Davenport
}

IN this article the subject-matter is grouped under three heads:

I. General statement about heredity;

II. Inheritance of special human traits, particularly disease and defects;

III. Applied eugenics.

\section{GENERAL STATEMENT ABOUT HEREDITY}

In order to understand the relations of genetics, or the science of heredity, to medicine, it is necessary to be clear on the biological significance of heredity. In the two-cell stage of a developing starfish egg, if the two cells be separated from each other, each will undergo a complete course of development and eventually produce a young starfish with all of the specific characteristics of the particular kind of starfish which produced the elements that went to the fertilization of the egg. This property of a fertilized egg, or of any piece of an organism that is capable of further development, to reproduce the specific form to which it belongs is heredity. A child of two Caucasian parents, or say of two Scandinavian parents, has a trunk, two arms and two legs, a head covered with light brown hair, practically hairless skin, and facial features of such and such form because it develops from Scandinavian protoplasm 4 This is heredity. Practically everybody believes in heredity. The two white parents who would be appalled at having a black-skinned child believe in heredity. The person who invests ten cents in a package of seeds marked "double, variegated petunias" has a deep faith in heredity. The only limitation to universal belief in heredity is in respect to its application to particular cases. If a man has his forefinger cut off by a hay knife, we recognize that this peculiarity which he carries through his later life is not due to heredity. If a man is born with four fingers and belongs to a family in which for generations numerous persons have been born with only four fingers instead of five, the special student of genetics will emphatically pronounce his peculiarity to be hereditary. If, however, a single case appears in a family of a child 
born with a finger which has been constricted, or perhaps entirely lost off before birth, we are in more doubt as to whether this is a case of uterine accident, constriction of the developing finger by adhesion to embryonic membranes, or whether there is in this case a genetic, hereditary factor. We are familiar, also, with striking instances of members of the same family who have not been in close contact and who, nevertheless, show similar gestures, idiosyncrasies of speech, or special gifts. Popularly these idiosyncrasies are recognized as hereditary; but when one member of the family is feeble-minded, or has epileptic fits, or shows a lack of control over actions such as makes it necessary to remove him from society, then a great difference of opinion arises as to whether these conditions are, or are not, hereditary. In regard to all such cases, however, we have no right vehemently to deny or vehemently to insist that the results are or are not hereditary - that is, due to some genetical impulse or factor. The proper attitude is to investigate and find out whether a factor of heredity be present or not.

\section{NATURE OF HEREDITY AND ENVIRONMENT}

The popular method of expressing the antithesis between qualities which are wholly accidental or environmental and those which are genetical is formulated in the expression, "environment and heredity." And it is commonly assumed that many a striking trait about which there may be discussion is to be accounted for either on the ground of heredity or of environment, and that these explanations are mutually exclusive. The situation, however, is rarely so simple as this. Whether a developing starfish egg shall develop into a starfish or not depends upon the presence of numerous favoring environmental circumstances. If some of these be removed the characteristic traits of the starfish may not reappear. The same thing is true of the developing human egg; in order to produce a normal human being the necessary environmental favoring factors must be present. If some of them are absent new and peculiar traits will arise in the resulting individual. These differences are commonly supposed to be sufficiently accounted for by the peculiarities of the environment; but this is not at all the case. If I plant a gourd seed in a manure pile. it will grow into an enormous vine and produce huge fruit, and this result, we may say, is produced by the extraordinary conditions offered by the manure pile; but if I plant the seed of a huckleberry in the manure pile, and it sprouts at all, it will grow very poorly, although exactly the same environment seems to be afforded to the huckleberry shrub as to the gourd vine. The difference in the end-result in the two species, however, is due to the fact that in the one case the extraordinary conditions afforded are such as to incite 
development in the protoplasm of the gourd vine, whereas in the second case the same conditions inhibit the growth of the huckleberry shrub. The end-result, then, of excessive growth on the one hand or of inhibition of growth on the other, is due not merely to the environment but to the nature of the protoplasm upon which the environment acts. Thus we see that what effect a given peculiar environment shall have upon the organism depends upon the genetical hereditary nature of the stuff of which the organism is made. Thus environment and heredity have the relation to each other of action and reaction, of stimulus and response; and the end-result of most environmental effects upon an organism depends quite as much upon the nature of that organism as it does upon the nature of the environmental peculiarity. Hence it comes about, that rarely can we say properly that a given peculiarity in development or in end-result in adult form and function is purely environmental. Nor can we often say whether in producing a given result heredity or environment has played the greater part. Nor can we hope to figure out just how much of a rôle has been played by heredity and how much by environment. We can only hope by research to show for particular cases just what effect is produced upon a particular race by a particular environment.

Recognizing, however, that environmental conditions are usually tremendously complex and that, for the most part, the average individual during his development is subjected to a lot of environmental influences that are, on the average, about the same, we then look first of all to the genetical factors for an explanation of the peculiarities of development or behavior of the individual. However, on the other hand, we must never forget that these peculiarities may be the organism's reaction to some peculiarity in the conditions under which it has developed. From this point of view the problem of heredity, as popularly understood, and as it comes to the student of genetics is this: is the peculiarity that this individual shows due primarily to a peculiarity in his genetical constitution or is it due primarily to some peculiar environmental circumstances to which he has shown this peculiar specific reaction. To such specific questions we are in many cases able to give an answer, and in the following account an attempt will be made to indicate the answer which has been reached in the case of many of these peculiarities, especially peculiarities of some functions and those in relation to disease.

\section{SKETCH OF MECHANISM OF HEREDITY}

(a) General considerations. It is clear that all which passes from the parents to the child is a pair of germ cells. The fertilized egg, 
only about a millimeter in diameter, contains the potentialities, even to minutest detail, of the man that may develop out of it. It is inconceivable that a homogeneous particle of this size could move with precision and definiteness to so complicated an end as the adult structure with its thousands of parts and millions of cells. The fertilized egg must on the contrary have a complicated organization within it which determines the course of development and forces it to proceed along welldefined roads. The studies of cytologists early revealed a complex mechanism in the egg, and their attention was soon directed to the behavior of the chromosomes. Recent studies in genetics, associated with the names of Professor T. H. Morgan and his pupils, have demonstrated (what Weismann concluded on speculative grounds) that the details, at least, of the adult structure are determined largely by substances lying in these chromosomes. And, indeed, there is good evidence that substances lying at particular points along the course of the chromosomes play a particular rôle in the production of specific organs.

Modern studies in genetics, moreover, point clearly to the conclusion that the course of human embryological development is what it is because of the different substances arranged in serial fashion along the course of the chromosome. If some of these substances were other than they are, then there would be produced, perhaps, an organism, but it would not be a man. Man, as we know him, is the visible expression of the substances in the chromosome. Man has not determined the nature of the substances in the chromosome; the nature of the substances in the chromosome has determined man. In just the same way the substances in other kinds of chromosomes have determined a pig, a snake, a jellyfish, or a sponge. From this point of view the history of the evolution of the animal kingdom is the history of the changes that have occurred in the material of the chromosome. It seems probable that these changes are largely due to the very nature of the chromosomal material, and so there is good reason for believing that the nature of that material in the primitive organism determined, to a large extent, the nature of the animal and plant kingdoms that have been derived from it, and have determined largely the details of forms of plants and leaves and roots and flowers and locomotor appendages and hair and brain. From this point of view the anatomical and histological analysis of a man is merely a study of the visible end-result of the workings of the substances that have through various spontaneous or mutative processes in the history of chromosomal evolution come to lie in the human chromosomes.

(b) Method of inheritance of particular traits. If two individuals having precisely the same germ plasm should marry, their off- 
spring would be exactly alike and like their parents. This hypothetic situation is, however, never realized. The chromosomes, germ plasm, that we call human, exist in hundreds of different forms, and these hundreds of kinds have intermingled so repeatedly that any given germ plasm is different from any other. Consequently practically all human matings are hybrid matings. The consequence of this is that the germ cells of the next generation that arise some time after the fertilization of an egg, are practically all dissimilar, and when a union of these dissimilar cells occurs with the dissimilar germ cells of a consort, the offspring are all different from each other and, of course, unlike their parents. If one of the parents belongs to a race most of whose external characters are very dissimilar to those of the race to which the mate belongs, then a dilemma appears as to what the children will be like. Empirically we find that if the father is short in stature, has curly and black hair, deep brown eyes and swarthy skin, and belongs to a race with these characters, while the mother is tall and blond and straighthaired, blue-eyed and fair-skinned, and belongs to a race which has these characteristics, then the offspring will be in these respects much more like the father than the mother. They will be short and have curly and dark brown hair, dark eyes, and swarthy skin. The reason for this is that all these traits from the paternal side are due to positive determiners in the germ plasm, whereas the absence of these traits in the children is due to the absence of such determiners in the maternal germ plasm. The offspring receive determiners for these different traits, to be sure only in single doses, and consequently they show, though in perhaps diluted condition, the traits that belong to the paternal race. If in turn two such hybrid children, produced by such a mating, should marry, then their offspring will be very variable. Some will have brown eyes and some blue, some curly hair and some straight, some swarthy skin and some blond, some will be tall and others short in various combinations. These end-results in the children are due to the dissimilarities in the germ plasm which has been brought about by the hybrid matings.

This, then, is the first law of heredity: that characters due to positive determiners in the germ plasm are dominant over characters due to absence of such determiners. The characters due to the absence of determiners recede from view and are said to be recessive. Emphatic warning must be given at this point, however, that all characters are not due either to a single determiner or to its absence. Many characters are due to two or more determiners, and these may behave independently in heredity. Thus the simple formula that holds for the presence of a determiner and its absence does not hold for these multiple 
determiners mated with the absence of one, two, or more of their elements. But, in general, where a character can be said to be due to the absence of the determiners that cause its opposite, then if both parents lack the character, it is absent in the germ plasm of both of them, and all of their children will lack the character. It is for this reason that the offspring of pure blue-eyed parents have only blue eyes and the offspring of two typical feeble-minded parents are all feebleminded.

In addition to the simple cases of heredity due to the presence or absence of determiners in the germ plasm, there is a group of traits that depend upon the determiners found in the germ plasm of one sex only. Such determiners are located in the same chromosome as that which determines sex. In man the sex determiner lies in an extra chromosome found in the egg cells and not in the sperm cells. The consequence is that the germ plasm of a woman contains samples of the determiners of every one of the chromosomes of both the father and mother, but the germ cells of a man will lack the determiners that were found only in his father's sex chromosome. Thus the mother may transmit certain traits which her father showed-traits that are carried by the sex chromosome-whereas the father cannot transmit such traits as his father showed. Among traits whose determiners are located in the sex chromosome are color blindness, hemophilia, and optic nerve atrophy. We shall speak of such traits as sex-linked traits.

In addition, there are certain traits the determiners for which are carried in both sexes equally but that do not appear ordinarily except under the stimulus of the secretions of the sex gland. Such are, for example, the beard in man and the large spurs and comb of the cock. The hen does not lack determiners for large spur or large comb. This may be demonstrated by grafting a testis into a young hen; large spurs and comb and male coloration soon make their appearance under the stimulus of hormones derived from the male gland. Characters whose appearance is controlled by the secretions of the germ glands may be called sex-limited traits.

Thus we see that the studies of the last decade or two upon heredity have demonstrated that it is, at the same time, much more definite and much more complex than had been anticipated. Much is still to be learned about the inheritance of traits in man. The near future will, no doubt, show that just as color blindness and sex production are linked in the sex chromosome, so other traits are linked in others of the dozen pairs of chromosomes in the germ cells of man. But of the traits thus associated in the chromosome, other than the sex chromosome, all will be found to be inherited alike through the maternal and the paternal lines. 
The determination of the association of determiners in the twelve chromosomes is one of the alluring fields of research for the future.

\section{INHERITANCE OF SPECIAL TRAITS, PARTICULARLY DISEASES AND DEFECTS}

\section{INTRODUCTORY REMARKS}

The abnormal traits that man shows fall into a number of categories. Some of them are defects due to imperfect development, others are the symptoms of disease for which, in some cases, a parasitic organism has been determined, and for others chemical peculiarities of the body seem to be responsible, such, for example, as diseased conditions due to abnormal internal secretions, or certain diseases of metabolism, such as alkaptonuria. Without, however, attempting to classify the different causes of the abnormal conditions shown, we may consider them in order, by groups of organs chiefly concerned.

One explanatory remark, however, may be ventured. Because it has been demonstrated that there is a particular parasitic microorganism responsible for the particular disease, it does not follow that the particular symptoms shown by the diseased individual are solely dependent upon that microorganism and its activities. In a great epidemic, like that of influenza which has recently covered the globe, we find individuals in the same house, even in the same family, who, though they clearly have the disease, show very different symptoms. One can hardly think of the parasitic organisms as differing in virulence in such cases; rather the human beings in which they are developing differ in their resistance and reactions to the germ. Indeed, as every farmer knows, the harvest of his planting is determined not only by the seed put into the soil, but also by the qualities of the soil itself. Similarly, the symptoms that a microorganism will induce in the body depend not only upon the particular physiology of the microorganism, but also upon the soil in which it grows, namely, the chemical constitution of the individual. With these general remarks the different diseases and defects found in man may be briefly considered with special reference to the part that heredity plays in inducing or modifying them.

\section{GENERAL DISEASES}

(a) Typhoid fever. As is well known, typhoid fever or enteric fever is induced by the bacillus typhosus. The symptoms shown by the individual, however, in whom the bacillus typhosus is developing are 
greatly varied. As Flexner has said, "Typhoid fever is a protean disease, whether considered in its chemical, its pathological, or its bacteriological aspects." As is well known, certain individuals tend to show especially the skin symptoms, others the intestinal symptoms, and others the circulatory ones. In some persons the bacilli are harbored persistently and maintained at a low rate of development; such are the carrier cases of typhoid. That the difference in symptoms depends in part upon the constitutional differences in the person is further shown by the fact that in colored persons the death rate from typhoid fever is twice as great as in whites. Moreover, if organisms are introduced into the bodies of different species of animals, there is a difference in the result, depending upon the specific nature of the host. For example, certain long-tailed monkeys of Panama have been infected with and died from smallpox, whereas other species, such as the orang-outang and macacus, have been experimentally inoculated with the disease without showing symptoms. Now the difference between human beings is of the same kind as, though naturally much less than, the difference between the white man, for example, and the orang-outang. Just as the two species, man and orang-outang, differ vastly in their reactions, so may we expect to find, as we do, human beings differing. There is indeed some reason for believing, from data gathered at the Eugenics Record Office, that some families are characterized by a high resistance to typhoid feverlow temperature, slight intestinal disturbance, little rash,- -while in other families there is a high mortality rate from this disease.

(b) Diphtheria. There is a racial, that is an inherited, difference in the reaction of different persons to diphtheria. As is well known, negro children are comparatively unaffected by diphtheria.

(c) Tuberculosis. This widespread and highly fatal disease does not affect all individuals alike. The white race in America, especially the old New England stock, is said to be now highly resistant to tuberculosis, though formerly the disease was very fatal to it. Races which have only recently come into contact with whites, such as the North American Indian, the negro, the dwellers in the Pacific Islands, and others, are extraordinarily non-resistant to this disease. Also among white persons resistance to the disease varies greatly. In the files of the Eugenics Record Office are numerous instances of large families in each of which ten or more individuals have died of the disease; on the other hand, it was an easy task to find records of ten families, comprising a total of 104 individuals who have died and none of whom, though full-grown, had died of or indeed shown symptoms of tuberculosis.

(d) Pellagra. This disease, which in our country is most widespread in the Southern States, is alleged by some to be "caused" by a 
microorganism, and by others is believed to be due to eating spoiled corn. But, whatever its "cause," a study that has been made by the Eugenics Record Office (Bulletin No. I6 [1916]) abundantly demonstrates that in different families the disease runs very different courses. Thus in some of the families, out of three or more individuals affected not one will show mental symptoms. On the other hand, in certain families five or more blood relatives will show extreme mental symptoms. Again, single families will show a marked prevalence of intestinal symptoms without eruptions of the skin. Finally, certain strains are characterized by skin troubles chiefly. Indeed, the conclusion has been drawn that in their reaction to pellagra we have to recognize "skin-susceptible biotypes, mouth-susceptible biotypes, intestinal-susceptible biotypes, and nervous-susceptible biotypes, and other biotypes that are resistant in all of these respects, or in two or more of them taken together."

(e) Cancer. The question of heredity of cancer has been much debated. It has been investigated statistically, demologically, by the method of family history, and by experiment. It must be admitted that the method of mass statistics has yielded no positive information. Thus Arthur Hunter, in analyzing family histories of cancer by the method of mass statistics, has concluded that there is no evidence of inheritance of this disease. On the other hand, positive results have been obtained by students of detailed family histories, as in the studies by Isaac Levin (1912), Alfred Scott Warthin (1913), and others. The studies of I. P. Lyon on the towns of Plainfield and Edmeston, New York, have revealed an inbred rural community in which there is a high incidence of cancer. Again, we have experimental evidence that in mice different strains are unlike in their resistance to inoculability of cancer. Many strains have shown, through a large number of generations, complete immunity to transplanted tumors, while in other strains the transplanted tumors grow rapidly to a fatal termination. This result has been secured by Tyzzer of Harvard, by Maude Slye, and many others; it is in fact generally recognized. C. C. Little, working with Tyzzer, has indeed attempted to show the exact method of inheritance of the immunizing factor. Finally, studies that have been made upon tumors, some of which pass rapidly into malignant tumors after surgical interference, have demonstrated in the clearest way the fact and method of inheritance of such tumors. The résumé of some of these cases is given in the Eugenics Record Office Bulletin No. I9 (1918), in a review of the literature of multiple neurofibromatosis. The tendency to this latter disease is clearly dominant. However, particular families show variations in the symptoms that accompany the disease-that is, in the amount of pigmentation, in the number of the tumors, and in their tendency to 
develop into sarcomata. Other multiple tumors that are inherited in the same way as neurofibromatosis are multiple telangiectases and polyadenomata of the rectum. Other skin disturbances are likewise inherited as dominant traits, such as epidermolysis bullosa, angioneurotic edema, and persistent hereditary edema. The inheritableness of the preceding traits cannot be denied, since the evidence of such inheritance is voluminous and sufficient. Some of it has been well set forth by Osler (I888) and Blumer ( 1892 ). All of these skin tumors may on occasion pass into malignant sarcoma. Their inheritableness, consequently, is proof of the inheritableness of the "cancerous" tendency in some -men.

$(f)$ Pernicious Anemia. This constitutional defect has been repeatedly shown to run in families, as, for example, by Patek (I9II) and Bartlett (I9I3). The exact nature of the genetic basis for the disease is not known, nor has the method of inheritance been formulated, so that one cannot predict its recurrence in the progeny of affected individuals.

(g) Diabetes. This constitutional disorder has been shown by numerous studies to have an hereditary factor, for example, by Gossage (1908), by Williams (I9I7), and others.

\section{SKIN DISEASES}

A large number of skin defects, passing under the terms keratosis psoriasis, ichthyosis, tylosis, have been shown to depend upon hereditary factors. In some cases the tendency is a dominant one. Likewise peculiarities of the hair often show hereditary factors. The darker pigmentation is dominant over the lighter pigmentation. The abnormal form known as monilithrix is dominant. The tendency to curliness dominates over its absence. The liability to have spots in the hair of the head of a lighter color than the remainder is a positive tendency. It has been shown, by Dorothy Osborne, that the tendency to early baldness is inherited as a male character. Defects of skin glands, either in excess or deficiency, and of the nails and of the teeth, have been shown to have an hereditary basis. The occurrence of moles, like that of skin tumors, tends to run in families. Marked differences in the reaction of skin to wounds is noted in the larger anthropological races. Thus, negroes tend to form ridges, so-called keloid tumors, from scar tissue.

\section{SKELETAL SYSTEM}

In the formation of the skeletal system of cartilage and bone there are clear hereditary factors at work, though we are far from having a complete knowledge of the rôle that they play in all cases. It is known, 
however, that the defect called achondroplasia, which is characterized by shortness of the legs (particularly the proximal segments) and small feet, has one or more hereditary factors which behave in dominant fashion, that is, seem to act as inhibitors of the normal development. This conclusion is supported in papers by J. H. Porter (I907), G. Franchini and M. Zanasi (I9IO), O. Eicholz (I9I0), G. A. Wagner (1913), and E. H. Ehrenfried (I9I7). Other distrophies of the bones which behave in dominant fashion are described by O. Crouzon (1915) and by many authors in the case of osteopsathyrosis, or brittle bone. The latter condition has been described in Bulletin No. I4 of the Eugenics Record Office. In this Bulletin all of the available family histories have been reproduced.

One of the most remarkable of the hereditary traits connected with the skeletal system is that of anchylosis of the phalangeal joints. This has been repeatedly described, among others by George Walker (I9Or), by K. Morgenstern (I913), by Harvey Cushing (1916), by H. Drinkwater (I9I7), and by J. H. F. Wilgress (19I8). Cushing's case is a remarkable American example traced through seven generations, and Drinkwater's was traced through fourteen generations. In this defect, as in the related defect of brachydactylia, there is a dominant factor. A similar factor seems to be present also in many exostoses, of which one of the most remarkable families is that described by N. M. Percy (I9I5).

Even in the formation of the carpal bones hereditary factors govern. This has been well brought out by J. W. Pryor, who by means of radiophotography has traced the order of development of the carpal bones. $\mathrm{He}$ finds this to vary in different individuals, though in one and the same family there is apt to be a close resemblance in the sequence of occurrence.

The tendency to irregularity in the development of the digits is another well-known hereditary quality. This has been extensively studied in poultry and the lower mammals, where the method of inheritance is precisely the same as in man, namely, there is a dominant factor which interferes with the normal, precise, definite number of digits formed out of the paddle at the tip of the embryonic limb. Recent extensive examples of polydactyl families are those of J. W. Brandeis (I9I5), S. R. Ayyar and J. W. Cornwall (1916). That this dominant factor is not merely one which induces an extra digit is indicated; it is one which interferes with precise morphogenetic processes. In cases of syndactylism and reduction of the normal number of digits there is also a dominant factor present. Examples of rather full records of syndactyl families are those of Karl Schlatter (I9I4), J. G. Edwards 
(1916), H. B. Robinson and W. H. Bowen (1919). It may be added that this topic has been monographically treated in Memoirs of the Galton Laboratory of Eugenics, Part 6.

Particular bones may be absent, as for example the patellae, in the cases described by E. M. Little (1897) and A. C. D. Firth (1912); also the clavicles may fail to be developed, as in the case described by G. Carpenter ( 1899 ).

Even the details of form of hands and feet are controlled by hereditary factors. The tendencies toward crooked fingers and double joints are hereditary. Even the relative length of the first and second digits of the foot and the third and fourth of the hand are characteristic of families. Also a tendency toward left-handedness.

That the form of the head is racial is well known, and details of size and proportions of the head are notoriously found in families.

\section{MUSCULAR SYSTEM}

Just as the details of number, of attachments, and of form of muscles is determined by the hereditary factors in the germ plasm of normal individuals, so even slight deviations from the normal condition of muscles is determined by corresponding peculiarities in the germ plasm. It would be wearisome to go through the list in an exhaustive way. Attention may, however, be called to a few extensive families. One of these is described by Paul Hänel (I890) in the case of muscular atrophy; another by Theodore Thompson (1908-09) in the case of hand muscles; and another by A. S. Hamilton (I9II), in the case of a family with muscular atrophy of the Charcot-Marie-Tooth type, and again by the Longs (1913) and by Souquez (1914). Interesting family histories of Thomsen's disease (congenital myotonia) have been described by Arthur Biart (1908) and J. P. Sedgwick (I9I0) and several others. The nervous control of muscular action depends upon hereditary factors, as the large literature on hereditary ataxia indicates. We have also families showing hereditary tremor as described by C. L. Dana (1887), by Hamaide (1892), by Schmaltz (1905), by C. B. Craig (19i6), and others. Finally small muscular deviation such as produce face dimples show clear dependence upon hereditary factors.

\section{NERVOUS SYSTEM}

Above all other systems of organs the control of the nervous system by hereditary factors is of the greatest moment to human society and to the progress of civilization. For the constitution of the nervous system, combined with the reaction of the nervous system to internal secretions and to other bodily conditions, determines conduct, behavior, and to a 
large extent the interaction of man on man and race on race. These hereditary nervous factors determine instincts and emotions, aspirations, and the control or absence of control of instincts and, consequently, the individual's fitness as a social being.

That the development of the brain with its accompanying intellectual capacity is determined by the absence of one or more factors that make for normal development has been shown again and again in the innumerable studies that have been made upon the feeble-minded, particularly in the United States. Also the tendency toward epileptic convulsions of the degenerating type that manifests itself usually at adolescence has been demonstrated to be similarly due to some defect of a factor that normally makes for nervous strength (Eugenics Record Office Bulletin No. 4, I9I I).

Of the different types of functional insanity there is much reason for believing that dementia praecox depends upon the absence of some factor essential to nervous vigor. On the other hand, in the case of manic depressive insanity the factors involved are more complex. There is reason for thinking that the lack of control that shows itself in great emotional output and excitability is due to the presence of some factor which inhibits that self-control, while depressions are due to the absence of certain factors that are essential to calmness under ordinary circumstances. That alcoholism, sex offense, and many other kinds of antisocial behavior are due to hereditary factors which seem to interfere with the normal inhibitions is perfectly clear from the facts which are on record, even though the analysis of the factors involved has not been completed. In the case of the nomadic trait, which is found in vagrants as well as sometimes in persons of wealth and culture, there is much evidence that this is inherited as a sex-linked trait (Bulletin, Eugenics Record Office, No. I2, I915).

Among the more strictly nervous diseases the history of Huntington's chorea has been perhaps more completely worked out than any other. It has been possible to trace this disease in certain of our families through ten generations, and to show the way in which the germ plasm carrying the defect has migrated from Southern New England and Long Island to upper New York State, Vermont, Ohio, Michigan, Wisconsin, Kansas; Nebraska, California, Oregon, and other parts of the United States (Eugenics Record Office Bulletin No. I7, 1916).

On the numerous special nervous diseases such as migraine, speech defect, and numerous paralyses of special organs it will not be necessary to dwell. Suffice it to say that when these are specially studied nearly all reveal the presence of clear hereditary factors. Even the tendency to form tumors upon nerves as seen in neurofibromatosis (Von Reckling- 
hausen's disease) is inherited as a simple dominant (Eugenics Record Office Bulletin No. 19). Among the great families through which run these defects of the nervous and inhibitory systems may be mentioned that studied by H. Lundborg in the province of Blekinge, Sweden (I9I3), the Jukes family of New York State, first described by Richard L. Dugdale in $1875-77$ and brought up to 1916 by A. H. Estabrook (Carnegie Institution of Washington, Publication No. 240); the Hill Folk and the Nam Family (Eugenics Record Office Memoirs Nos. I and 2), the Dack Family by Mrs. Finlayson (Eugenics Record Office Bulletin No. 15), and many others.

Not only those abnormalities in the nervous system and its output which society regards as defects, but also those other nervous and mental peculiarities which are commonly spoken of as special gifts, show the hereditary factor. Though this is not a matter primarily of medical interest, still attention may be called, in passing, to the evidence of inheritance in the factors that make great fighters, great mathematicians, great musicians, great writers, painters, explorers, missionaries, divines, and the rest.

\section{SENSE ORGANS}

Ever since Alexander Graham Bell published his "Deaf Variety of the Human Race" (Memoir of the National Academy of Sciences, I883), it has been clear that certain forms of deafness depend upon hereditary factors. It has, however, been obvious that congenital deafness is a complex thing. An advance has been made by the studies of J. Kerr Love, who has distinguished sporadic congenital deafness and the deafness occasioned by syphilis. The latter is more of an accident in its distribution in the family, while the former seems to depend upon the absence of an hereditary factor.

The eye is subject to scores of defects in the course of its development and the hereditary factors present in many of these have long attracted the attention of ophthalmologists. Several bibliographic works on hereditary eye defects have been published, of which that of Clarence Loeb (1909) may be referred to. Some of these traits are inherited as dominant, others as recessive, and still others (like atrophy of the optic nerve) are sex-linked.

\section{ALIMENTARY SYSTEM}

The hereditary factors in this system seem, except some of those marvelously complex ones involved in the mouth and teeth, to have been little studied. The striking family trees of harelip and cleft palate belong rather to the category of defects of development of the face. 
There are those who have found hereditary factors in the tendency toward inflammation of the appendix, and since this must be conditioned among other things by the form and connections of the appendix there is no inherent improbability in the conclusion. Two large family trees have been published by R. Forschheimer (I9OI). Hereditary jaundice, also known as chronic family jaundice, has been repeatedly described. One of the most extensive trees is that published by C. A. Elliot and A. B. Kanavel (I9I5). No doubt a tendency toward intestinal stasis also runs in families.

\section{RESPIRATORY SYSTEM}

That there are hereditary, or racial, factors present in the mucous membranes of the nasopharynx there can be no doubt. Among colored persons there is relative resistance to those diseases that enter the body through these portals which are so ill defended among whites. Adenoids, tonsillitis, and diphtheria are much less common in our colored population than in whites despite the superior sanitation, on the whole, of the latter race in this country.

As regards the pneumonias experience makes it quite clear that the organisms responsible for these diseases are normally present in the body. When these organisms develop tremendously in the body and show the dreaded symptoms it is because the body is no longer able to keep down the microorganisms. This is especially the case after an attack of measles or of pandemic influenza. They seem to inhibit the production of or to destroy the protective agents of the body. Now the evidence from family records is complete that in certain families pneumonia attacks almost every member, whereas in other families the disease is quite unknown. We have to conclude that in the latter case the protective factors which give a natural immunity are more effective than in the former.

\section{Io. CIRCULATORY SYSTEM}

The heart and blood vessels, the blood itself, and the secretions of the internal glands are all markedly under the control of hereditary factors. Repeatedly children are found to be more or less cyanotic from birth, with imperfect development of the valves of the heart, and this condition has been reproduced in many other members of the family. A good example is given by O. Bourwinkel (I9IO). There is reason also for suspecting that the tendency toward degeneration of the walls of arteries depends again on constitutional factors.

That condition of the blood known as hemophilia, which depends upon insufficient formation of the clotting elements, has been repeatedly shown 
to run in families, usually in a sex-linked fashion. In typical cases the males alone are affected, and in such cases it is usually the mother's father or brothers rather than the other ancestors who shows the same trait. The tendency toward epistaxis (nosebleed) and the formation of telangiectases are closely interrelated and, again, show clear heredity.

Among internal secreting glands there are frequently found irregularities of functioning which are clearly genetical. Such is the case, for example, with enlargement of the spleen, Claude Wilson (1890). To this category belong all those cases of abnormal secretions of the thyroid which induce cretinism, simple goiter, and exophthalmic goiter. Here belong also many defects of development, such as infantilism, gigantism, acromegaly, and some types of dwarfism.

\section{EXCRETORY AND REPRODUCTIVE SYSTEMS}

Disorders of metabolism are among the most striking functions that are controlled by hereditary factors. As to the numerous cases of alkaptonuria, cystinuria, hematuria, and the rest it is not worth while to go into detail. There is evidence that hereditary factors are present in the production of urinary calculi.

The imperfect development of the external genital organs, a form of infantilism, properly belongs under the category of internal secretions. The evidence for hereditary modifications of them is abundant. The same is true of cryptorchism.

\section{SUMMARY}

The above review of diseases and defects shows sufficiently that the hereditary factors present must always be looked for even though these diseases may never occur without the presence of a particular microorganism. For the microorganisms cannot be regarded as the sole and effective cause of these diseases or defects. We must believe that the constitutional factors prepare the soil and the nature of the soil determines the nature of the harvest-that is, the symptoms-which the seed sown upon it will produce. The medical man who neglects in his consideration of diseases and defects the hereditary factors will never succeed completely in accounting for the phenomena with which he has to deal. Heredity is not something occasional and special. Heredity determines the very nature of the organism, both the normal organism and the organism that deviates from the normal. Only pathologists who are willing to admit that there is a disease apart from the diseased organism can decline to consider the man apart from the parasite that is one of the factors in producing disease. 


\section{APPLIED EUGENICS}

Since the development of the physical, mental, and emotional traits of man is determined primarily by heredity, it follows that reasonable human beings should act in accordance with this knowledge. Our social difficulties are largely due to the presence in our population of feeble-minded, of paranoiacs, of those lacking social instincts, of those with little control over the emotions. Our present methods of dealing with these social disturbers are various. Sometimes we pity them, give them alms, and let them go; sometimes we punish them with severity, incarcerate them, and even flog them. Sometimes we point the finger of scorn at them and heap social obloquy upon them. Sometimes we segregate them for a longer or a shorter period. Thus we may keep feeble-minded children in a "training" school until they reach the age of twenty-one and then send them back upon the community, or we may lock up the paranoiac for a few years under conditions where he has a chance to develop his wrong-headed social universe and then release him to carry out his own system to its logical conclusions. By releasing such anti-social individuals at a time when the reproductive urge is strong we permit the reproduction of such traits. Now that we realize that all of these difficulties are primarily due to reproduction of inherently defective germ plasm it seems desirable to exercise some control over this reproduction.

All States of the United States and most countries of the civilized world have long recognized their right and duty to attempt to control matings. Thus we have laws against the marriage of feeble-minded, epileptic and insane, cousins and representatives of different races (Eugenics Record Office Bulletin No. 9); but these laws have primarily a legal import and, moreover, they are inadequately enforced. Much to be desired is a humane, sympathetic, intelligent, and at the same time effective control over matings such as is, indeed, exercised in some countries much more satisfactorily than in ours. Over one-fourth of the States of the Union have law's prescribing sterilization in some cases as a punishment or, more properly, as a eugenic measure (Eugenics Record Office Bulletins Nos. IOA and IoB, I9I4).

Since the health and happiness of a nation depend so much upon the predominance of the physically, mentally, and emotionally fit stock, the State may well inquire into the relative fertility of the most effective and the least effective strains. At this time throughout civilized countries, and particularly in America, through the voluntary limitation of the size of families, the most successful stock is not reproducing itself in anything like the proportion of the less successful stock. The sons of 
Harvard University have only about 0.8 of a son on the average, while the daughters of Wellesley have even a smaller proportion of daughters. There are those who have urged that the dissemination of so-called "birth control" information might be relied upon to diminish the relatively vastly greater fertility of the less effective stock, and it is even alleged that this would tend to increase the number of children in the more effective stock. This conclusion is far from being demonstrated, however, and it would seem desirable rather to encourage the more effective stock to have larger families than to extend more widely the principles of restriction of reproduction. Such stimulus might be given on the one hand by appealing to higher ideals and, on the other, in economic ways, by reducing taxation and inheritance levies in proportion to the number of children in the family.

A predominance of the fit will not be maintained merely by increasing the number of offspring but also by increasing the number who survive to marry, and in turn become progenitors. An intelligent society will therefore do its utmost to encourage the survival of its fittest strains and will be more concerned therein than in securing the survival of the children of unsocial strains. The strong appeal made by a certain social worker to philanthropists to provide him with funds to diminish the mortality rate of the children of prostitutes might very well have failed to arouse an enthusiastic response. Any control, moreover, that society may exercise over the matings, fertility, and survival of its native stock may well be rendered ineffective if no attention is paid, in a country like ours, to a control over immigrants.

In response to purely commercial demands our ancestors introduced into North America hundreds of thousands of jungle folk from the heart of Africa and scores of thousands of convicts from the prisons of London, and we have lured many of the most suggestible from the least developed countries of Europe to build our railroads and dig our canals without giving a thought as to the part that their progeny would play as components of our social body. Only comparatively recently have we begun to sort out the feeble-minded, insane, and criminalistic. The sorting has, however, been very ineffective, as witness the hundreds of criminals that Germany successfully exiled to America, to whom and to the descendants of whom some of the vilest crimes committed in the country can be traced. Our American population may still be preserved if a control be exercised over immigration which is directed first toward maintenance of an increasing proportion of good hereditary traits in our American population and only secondarily toward securing cheap labor.

Recognition of the fact of heredity does not render unnecessary 
efforts that have been made toward education and moral and religious culture. Even plants, to yield their best fruit, must be cultivated, and the innate traits of children may be repressed by a bad environment. Eugenics, however, teaches that it is as futile to try to train the feebleminded boy into a scholar as it is to try by cultivation to make a golden Bantam variety of corn into a giant. Our efforts toward education will be more effective when we recognize first that children are all different, and when we seek, secondly, to develop to the utmost those germs of desirable traits that they possess, and, thirdly, to repress undesirable tendencies. So also in matters of health the physician must recognize that all of his patients are different, and he must urge therefore different hygienic training in accordance with individual needs. It is sometimes said that eugenics is a medical matter, and so it is indeed, but it is also a social matter of the highest import. It is for physicians and those interested in social welfare and development to unite in applying the principles of eugenics to the advancement of the State.

A fact like heredity which affects so profoundly every part of the body and determines so intimately a man's relation with his fellows is certain to have many practical applications. One of these which the medical man most often will be called upon to practice is that of the determination of unknown relationships for legal purposes. With the knowledge of heredity that we already possess, it is often practicable to assert that a given child cannot have had two given persons as his father and mother. It is often more difficult to prove by the facts of heredity that a given person is the son of a particular man, and still more difficult to assert which one of several possibilities is the actual father of a given child. However, in some cases this can be done with assurance, and with the progress of knowledge of inheritance of traits the range of cases in which a definite answer can be given will be greatly increased.

By means of the facts of heredity also in the near future we may hope to advise concerning the choice of a profession. Specifically, we may answer the question whether a given boy would probably succeed as a physician or surgeon. It is necessary in such cases to consider not only the parents and close relatives on each side, but above all to consider the juvenile traits that have appeared. Corporations interested in the choice of executives have been urged to consider the family history of candidates.

To the psychiatrist a knowledge of family history of patients is of vast importance. E. E. Southard, of Boston, has stated that he would hardly diagnose with confidence a case of manic depressive insanity without a knowledge of the family history. At the present time, indeed, a 
large part of the more prominent institutions for the insane, feebleminded, and epileptic of the eastern United States have maintained engenics field workers whose function has been to study family histories of patients in order to secure data for diagnosis, prognosis, and explanation of conduct, and for an understanding with and a sympathetic attitude by the parents or the family of the patient.

Lastly may be mentioned an organization in this country which has as one of its functions to assist institutions in securing trained investigators of family history-the so-called "eugenics field workers." The Eugenics Record Office, Cold Spring Harbor, Long Island, New York, a department of the Carnegie Institution of Washington, has trained about 150 eugenical field workers, mostly young women who are college graduates, and during the past nine years has supplied these on a coöperative plan in considerable number to institutions. That is, the institution during the first year pays the traveling expenses and maintenance of the field worker while the Eugenics Record Office pays her salary. The Eugenics Record Office also serves by supplying data concerning families which have already been investigated by eugenical field workers so as to avoid an unnecessary duplication of time and expense. It thus acts as a clearing-house of eugenical data. At the same time it is carrying on investigations and publishing results with the aim of establishing general principles that may be universally applied. 


\section{CHAPTER VIII}

\section{FOCAL INFECTION}

\section{By Frank Billings}

Focal infection * may be defined as a systemic or local disease due to infectious microörganisms carried in the blood or lymph stream from a focus of infection. A focus of infection is a localized or circumscribed area of tissue invaded by pathogenic microörganisms and may be either primary or secondary. By primary focus is meant the principal or first infected tissue area from which the pathogenic agents gain entrance into the blood or lymph stream to cause systemic or organic disease. By secondary focus one refers to a localized or circumscribed tissue area invaded by pathogenic microörganisms carried in the blood stream or in the lymph channels from the primary focus; from the secondary foci the pathogenic agents may be ultimately carried in the blood or lymph stream to more distant sites. A focus of infection may be acute or chronic. An acute focus is of recent origin, and is usually manifested by local inflammation. A chronic focus is of long duration, and may be symptomless. A chronic focus may become acute in character through trauma, new microbic invasion, and other factors.

\section{SITE OF PRIMARY FOCI OF INFECTION}

Primary foci are located usually in tissues communicating with cutaneous or mucous surfaces. The most usual sites of foci are about the head. They occur in the mouth, pharynx, nares and its accessory sinuses, the middle ear, and the mastoid cells. Dental caries associated with pyorrhea dentalis and alveolaris; faucial tonsillitis and sinusitis; nasopharyngitis involving any or all of the accessory sinuses; suppurative otitis media leading to mastoiditis and venous sinus thrombi are often neglected conditions.

* The clinical and laboratory investigations made at Rush Medical College during the last fifteen years by many clinical and laboratory workers have done much to establish the principles of focal infection.

"Focal Infection." The Lane Medical Lectures, by Frank Billings. D. Appleton \& Company, 1916. 
The larynx, trachea, and bronchial tubes are not so frequently sites of foci of infection, but bronchiectatic cavities and ulcers of the mucous membrane of the respiratory tract may be the source of bacteriemia and of infection of the lymph glands of the mediastinum. Ulcers of the esophagus, stomach and intestinal tract, especially certain types of enteritis; cholecystitis; appendicitis; and the secondary mesenteric thrombophlebitis and lymph gland involvement which may occur with any of the above-named infections of the gastrointestinal tract are not unusual examples of sites of foci in the abdomen. Intestinal stasis due to morbid anatomical conditions may lead to invasion of the intestinal walls, lymph channels, and blood stream by pathogenic agents. The rectum is not an unusual site of foci of infection in the form of ulcers and infected venous thrombi associated with hemorrhoids. The genitourinary tract is not an infrequent site of foci of infection in the form of infected thrombi of the venous sinuses of the uterus, especially after labor and abortion; salpingitis; seminal vesiculitis; prostatitis; ulcerative cystitis; and obstructive pyelitis. Subcutaneous abscesses, even those involving the nails of the fingers and toes, may be a source of systemic infection. Wounds of the skin, even trivial punctured wounds and abrasions of the skin when infected, are often serious sources of general disease.

The lymph nodes proximal to the primary focus may become secondarily infected and then may serve as additional depots of the infectious agents. These secondary lymph node foci may persist and continue to be the source of systemic infection after the spontaneous disappearance or operative eradication of the primary focus. This explains the not unusual persistence of systemic disease after the eradication of the apparent primary focus.

\section{ETIOLOGY OF FOCI OF INFECTION}

The causes of the primary localized tissue infection are practically the same as the etiology of general infectious diseases. The principles involved embrace the exposure of the susceptible host to pathogenic microörganisms. Susceptibility of the host may be increased by general debility caused by acute or chronic illness, old age, over-fatigue both mental and physical, by lowered temperature, by exposure to cold, by alcoholic abuse, drug addiction, starvation, squalor, and by other causes. The susceptibility of the host is increased also to local tissue infection by the presence of overgrowth of lymphoid tissue of the upper air passages, the pharyngeal tonsils and adenoids. This overgrowth of 
lymphoid tissue is not uncommon in the upper air passages of children. Poor individual hygiene with lack of cleanliness of the mouth is productive of carious teeth, infected gums, and pyorrhea alveolaris.

The natural immunity of the host may be unimpaired by any of the factors named and yet he may suffer from local tissue infection because of the great virulence of the infectious agents to which he is exposed. The host may expose himself unduly and irrationally, as for example in indiscriminate sexual intercourse so productive of venereal diseases, with not infrequent resultant complications which are essentially local tissue involvement-typical foci of infection.

The opportunities for infection of the host are as numerous as the character and type of pathogenic agents which abound on the skin and the mucous surfaces of men and animals and in the saliva, sputum, and nasal secretions, and in the excretions of the body. Indiscriminate human intercourse in domestic, social, public, educational, and business relations; and the use of food and drinks infected in various ways, are examples of modes of infection.

\section{CHARACTER OF THE MICROÖRGANISMS WHICH CAUSE FOCI OF INFECTION}

The pathogenic agents which cause foci of infection and are usually found therein are, streptococci (s. hemolyticus; s. viridans; s. rheumaticus; s. mucosus and other strains); micrococcus catarrhalis; pneumococci; bacillus mucosus capsulatus; staphylococci (aureus and albus); meningococci; tubercle bacilli; colon bacilli; gonococci; spirochaeta pallida; endamoeba buccalis; fusiform bacilli; bacillus Welchii; diphtheria bacilli; tetanus bacilli; bacillus proteus; bacillus pyocynaneus, and others.

Foci about the mouth and nose may contain pure cultures of any one of the streptococci, pneumococci, staphylococci, and of some other microörganisms, but as a rule there is a mixed infection of the invaded local tissue. Tubercle bacilli may be found in a focus of infection of the tonsil, but are more constantly found in the secondarily involved lymph nodes. Fusiform bacilli are not infrequently associated with acute types of alveolar abscess. Non-spore-bearing bacilli Welchii, presumably parasitic in type, are frequently found in morbid tissues. The usual habitat of the gonococcus is in the genitourinary tract. The colon bacillus apparently assumes pathogenic qualities in a mixed infection, as is not uncommonly found in appendicitis, cholecystitis, and infection of the urinary tract. Bacillus proteus and bacillus pyocyaneus are sometimes apparently active factors in infection of the urinary tract. 


\section{SUSCEPTIBILITY TO FOCAL INFECTION}

The incidence is great of acute local tissue infection (focus of infection) about the head in the form of tonsillitis, sinusitis, and suppurative otitis media. Associated with these acute types of local infection there is often evidence of systemic infection manifested by more or less febrile reaction and general discomfort of short duration. For example, acute tonsillitis is of common incidence. Much local distress is associated with fever and often with great general discomfort. An acute nasal infection is usually associated with involvement of one or more of the accessory nasal sinuses with more or less febrile temperature and general debility. It is rational to presume that the character of the invading pathogenic microörganisms which cause a brief inflammation of the local tissue and a transient systemic disturbance, are not very virulent, or the degree of the resulting bacteriemia is not great, or finally that the natural defenses of the body are sufficient to overcome the invaders in a short period of time. On the other hand, some peculiar pathogenicity of the microöganisms may result in distinct damage to the distant tissuesjoints, endocardium, kidney, or other organs. This phase of focal infection embracing organic disease due to a special pathogenicity of the morbid agents which may be termed a selective tissue affinity or tropism will be more fully dealt with under systemic diseases.

The susceptibility of individuals to systemic infection from chronic foci is governed by the same factors enumerated under the etiology of foci of infection. At the same time one must recognize that the incidence of chronic foci of infection is far greater than the acute types. Chronic types are frequently sequential to acute forms and in many individuals the chronic type is a primary incident. In this connection it should be noted that neglected chronic foci are a constant menace because they are often left without treatment and continue as a rule over a long period of time. At some period of their existence the immunity of the host, which is never absolute and always relative, may be diminished by the conditions already enumerated, or at any time the pathogenic microörganisms may take on an increased degree of virulence through trauma or other factors. Thus the existing immunity may be overcome and systemic infection occurs.

It must be borne in mind too that chronic foci of infection may serve in the sense of a prophylactic vaccination and thus arouse the natural defenses of the body against the microörganisms which at any time may invade the blood stream and lymph channels from the focus. We can appreciate too that this constant source of stimulation may result sooner or later in exhaustion and an inefficient elaboration of the 
body defenses. Therefore, in addition to the factors which may increase the susceptibility to infection, namely old age, exhaustion from previous disease, and mental and physical fatigue, exposure to extreme cold and wet, starvation and squalor, alcoholic and drug addiction, there may be added the "negative phase" effect of constant vaccination from the focus and a resulting exhaustion of the body defenses.

Because of the frequent incidence of chronic foci of infection which are unattended with outspoken evidence of bodily disturbance, many physicians fail to recognize their etiologic relation to systemic disease. An extensive personal experience justifies the statement that many minor ills and numerous conditions erroneously designated rheumatism, malaria, autointoxication and the like, are often etiologically related to existing foci of infection somewhere in the body.

\section{DIAGNOSIS OF FOCI OF INFECTION}

The evidence of carious teeth and of dental and alveolar pyorrhea is usually manifested by oral inspection. Alveolar abscesses, chronic in type, may exist without carious teeth and without discomfort to the patient. Radiography properly applied and interpreted often will disclose surprising evidences of serious apical root infection when the condition is totally unsuspected because of the normal appearance of the teeth and the mucous membrane of the gums. In the presence of infection about the edges of the gums and especially in the presence of carious teeth, the infectious agents may be disclosed by microscopic examination of the pus obtained from the undermined gum tissue or from cultures made from this source. Bass and Johns $\left({ }^{1}\right)$ have confirmed the statement of Barrett $\left({ }^{2}\right)$ and others that endamoebae buccalis are frequently found in the exudate of diseased gums. The endamoeba buccalis may have no primary etiologic relation to the disease, but no one will deny that the microörganism is a contributory etiologic factor in aiding streptococci and other pathogenic bacteria to invade the deeper tissues.

Chronic hypertrophic tonsillitis is frequently present in children and is readily recognized by an oral inspection. This type of tonsillitis is frequently a less dangerous site of a focus of infection than the small tonsil. The small tonsil may be smooth on its external surface because of previous attacks of tonsillitis and contain obstructed crypts and unsuspected abscesses. The stump of a tonsil, the remains of tonsillectomy, frequently sealed over by the scar, may also contain a dangerous focus of infection. Nasopharyngeal adenoids, most commonly present in children, may be recognized by the obstruction to nasal breathing, nasal voice, and by the insertion of the finger behind the palate. This over- 
growth of lymphoid tissue is probably not the usual site of a focus of infection, but is frequently etiologically related to middle-ear infection and to sinusitis.

Acute infection of the accessory sinuses is usually easily diagnosed because of the local distress. The chronic types may be recognized by transillumination of the face and by properly applied radiography. Diagnostic skill is required in the recognition of bronchiectasis, and is greatly aided by properly made X-ray plates. Diagnosis of foci of infection in the gastrointestinal canal involves the same measures applied in the diagnosis of chronic appendicitis, chronic cholecystitis, and of intestinal stasis due to abnormal anatomical conditions. Fluoroscopy with the bismuth or barium meal and enema and the addition of X-ray plates will greatly aid in the establishment of the diagnosis. The presence of rectal ulcers and of hemorrhoids, which may be attended with local infection, is easily recognized by means of oral inspection through a speculum or proctoscope. Salpingitis of the chronic type may be difficult of recognition except by the most expert specialists in the diseases of women. Prostatitis is easily recognized by a digital rectal examination, and massage of the organ will usually yield an exudate containing the infectious agents. Urinalysis, cystoscopy, ureteral catheterization, and, when necessary, the utilization of X-ray plates will enable one to make definite diagnosis of foci of infection of the bladder and kidney pelvis.

In attempting to establish the presence of the etiologically related foci of infection in a patient who suffers from focal infection, it is very essential that a complete and definite history of the patient shall be obtained. It is also requisite that all of the refinements of diagnosis should be utilized, and this usually requires the aid of specialists in the examination of patients. In doubtful conditions the patient should be placed in a hospital where a qualified medical and laboratory personnel and adequate facilities for diagnosis may be commanded.

\section{MODE OF DISSEMINATNION OF INFECTIOUS MICROÖRGANISMS FROM THE FOCUS OF INFECTION}

Hematogenous infection. The infectious microörganisms of the focus of infection readily reach the blood stream through the surrounding capillaries. The bacteria may be compared with emboli and are carried in the blood stream to the smallest blood vessels of the body. If the invading microörganisms are small in number and of low virulence, the defenses of the body overcome any harmful result of their presence [Kolle and Wassermann $\left({ }^{3}\right)$ ]. If they are virulent and in sufficient number, they may excite characteristic reactions in the tissues. At the 
point of lodgment in the smallest blood vessels, the specific tissue reaction will depend upon the virulence and character of the invaders. If very virulent, gangrene or necrosis of tissue or positive chemotaxis will result in a multiplication of leucocytes and plasma cells in the immediately infected area; if less virulent, their presence may result in local hemorrhage and a fibrinoplastic exudate with subsequent local connective tissue overgrowth.

Lymphogenous infection. The infectious microörganisms may pass from the focus of infection through the lymph channels to lymph nodes. Lymphogenous infection probably occurs coincidently with hematogenous infection. Kretz $\left({ }^{4}\right)$ has shown that lymph node invasion may serve as a source of protection to further systemic infection by holding the infectious microörganisms in a tissue environment which may render them latent and for a time harmless to the patient.

Systemic intoxication. The exotoxic bacilli of diphtheria and of tetanus excrete soluble toxins in the focus of infection with consequent systemic intoxication. It has been assumed that the systemic disturbance which may occur from a focus invaded by endotoxic pathogenic bacteria is due to intoxication. In this connection it has been suggested that the toxic material of the endotoxic bacterial invaders is released in the focus by autolysis of the dead microörganisms. It has also been suggested that toxic substances are elaborated in the focus of infection by a biochemical reaction excited by the presence of the microörganisms in the tissues involved in the focus.

This hypothesis may contain an element of truth, but it is more probable that the damage to systemic tissues is caused by the pathogenic microörganisms of the focus of infection carried in the blood and lymph channels to and lodging in distant tissues.

\section{FOCAL INFECTION AND ANAPHYLAXIS}

A focus of infection may be the site of the parenteral introduction of a foreign protein and the consequent anaphylactic sensitization of the body tissues. This foreign protein may be the bacterial protein of dead microörganisms, or the protein elaborated in the focus of infection by the reaction between the microörganisms and the local infected tissues. It is reasonable to assume that the individual sensitized by the parenteral introduction of the foreign protein through a focus of infection is subject to anaphylactic shock by subsequent periodical parenteral reintroduction of the foreign protein. The subsequent reinoculation with the foreign protein may be caused by a periodical activity of the focus of infection due to trauma, a "cold," or other factors. Expe- 
rience [Meltzer $\left({ }^{5}\right)$, Weil $\left.\left({ }^{6}\right)\right]$ has proved that bronchial asthma is often an expression of anaphylaxis due to the parenteral introduction of a foreign protein elaborated in a focus of infection located usually in the upper and sometimes in the lower respiratory tract.

\section{SYSTEMIC OR FOCAL INFECTION}

Systemic or organic disease, which may be caused by the hematogenous or lymphogenous invasion of tissues by pathogenic microörganisms introduced through foci of infection, may be acute or chronic. If the invading pathogenic agents are virulent and in sufficient number, the tissue reaction is severe and the systemic disturbance is relatively violent, i.e. acute. If the systemic tissue involved is limited in area the acute attack may subside without anatomical damage; or on the other hand a permanent morbid anatomical condition may remain with corresponding disturbance of function. If the invading pathogenic microorganisms are of low virulence the tissue reaction is less destructive and often so mild in character as to provoke much less bodily disturbance. Chronic foci of infection may serve as depots of constant or intermittent supply of pathogenic agents of low virulence to the blood stream. Repeated intermittent lodgment in the tissues of pathogenic microörganisms, even of low virulence, may finally cause serious anatomical lesions and greatly disturbed function. As evidence that certain systemic diseases may be etiologically related to foci of infection, a brief description will be made of a few morbid conditions. These general and local diseases will be dealt with only in reference to the etiology and to some extent in relation to the associated morbid anatomy and the resultant disturbance of function of the invaded tissues. In other chapters each disease mentioned here will receive full consideration by other writers.

Acute rheumatic fever is an infectious disease. The specific etiologic microörganism is a coccus, which, as variant strains, may assume the morphological characteristics of a diplo-, a micro-, or a streptococcus. The chemical and laboratory researches of Poynton and Paine $\left({ }^{7}\right)$ and the confirmatory work of Beattie $\left({ }^{8}\right)$, of Walker, of Ryffel $\left({ }^{9}\right)$, of Rosenow $\left({ }^{10}\right)$, and other investigators have conclusively established the specific pathological character of the diplococcus (micrococcus, or streptococcus) rheumaticus. It has been obtained and artificially cultivated from foci of infection in the tonsils, alveolar abscesses, infected accessory sinuses, abscesses involving the nails of the fingers and toes, infected lymph nodes, and other infected tissues; and has been cultivated also from the stools, the urine, the blood, and joint exudates of patients 
suffering from rheumatic fever. Its virulence is not great. It is not pyogenic and hence the tissue reactions aroused in man and experimental animals by its presence are never destructive. The mode of infection is hematogenous and the invaders which lodge in terminal blood vessels excite tissue reaction in the form of small local hemorrhages and serofibrinous exudate which in due time may result in connective tissue proliferation and plastic adhesion of serous surfaces. In our clinic Rosenow $\left({ }^{10}\right)$ was able to isolate the characteristic microörganism from the joint exudate of all patients in the first few days of the disease. This is explicable, probably, upon the ground that the pathogenic agents lodge in the terminal vessels of the subserous tissues of the joint. In the first days of the disease the microörganisms readily pass into the joint with the serous exudate; with the cessation of further exudation the migration of the bacteria into the joint ceases. Conditions within the joint are not favorable for their growth and multiplication; they die and disappear by autolysis; hence after a few days the joint exudate is apparently sterile.

The faucial tonsils are the most common site of foci of infection which may provoke an attack of rheumatic fever. The relation of tonsillitis to rheumatic fever has been noted for many years. Practitioners of past decades recognized certain types of tonsillar infection as rheumatic, and applied the remedies usually utilized in rheumatism in the treatment of the local throat infection. The tonsillar infection which is the source of rheumatic fever may be acute and may be a. coincident occurrence. More often the attack of tonsillitis occurs a week, or a month, or a longer period of time, before the onset of the rheumatic fever. The focus of infection in the tonsil may be so mild that the patient is unaware of it. It must be recognized then that the streptococci of the focus may attain, through unknown factors, a specific rheumatic pathogenicity; during this period of time, their presence in the tonsil may excite varying degrees of tissue reaction, indicated by acute, subacute, or only very slight local manifestations. In the temperate zone rheumatic fever occurs most frequently during the fall, winter, and spring, when changeable cold and wet weather prevails. The incidence of the disease is greatest in the young and in the more exposed male of all ages. The excess of lymphoid tissue in the pharynx of children is conducive to tonsillitis, nasopharyngitis, and sinusitis at the same seasons of the year. The prevalence of foci of infection is coincident with the increased susceptibility to general infection due to exposure to cold and dampness. In this connection the observations of Rosenow are significant. $\mathrm{He}$ found that cultures of the diplococcus. rheumaticus grew and thrived at a low temperature and that the exposure: 
to cold of animals, inoculated with the specific microörganisms, intensified the resulting tissue reactions.

An attack of rheumatic fever does not confer immunity to further attacks. Indeed the primary seizure seems to increase the susceptibility to the disease. Experience has proved that a thorough eradication of the apparent etiologic focus will usually prevent subsequent attacks of rheumatic fever. When the disease recurs after the removal of the suspected focus, it must be remembered that the real focus may have escaped recognition, or that more than one primary focus existed, or that a secondary focus had developed by hematogenous infection from the primary focus in distant local tissues, with conditions favorable for the multiplication of the specific infectious agents in the new site. There the natural tendency to parasitism may favor continued existence. During this period of time, new or increased pathogenicity may be acquired through agencies described under susceptibility to focal infection. The management of conditions due to secondary foci will be considered under treatment.

Endocarditis caused by the diplococcus rheumaticus may occur coincidently with the general systemic infection during the convalescent period or may occur as the sole clinical entity, especially in children. Endocarditis may cause so little systemic disturbance that it is not recognized until a later period, when the physician recognizes a valvular defect. These patients usually reveal a history of tonsillar infection. The incidence of endocarditis increases with the number of attacks of rheumatic fever and always in larger percentage in children. The morbid changes in the endocardium and valve leaflets are characteristic of the low virulence of the diplococcus rheumaticus, as evinced by the fibrinoplastic exudate in the form of small wart-like, fibrous nodes and adhesions of the surfaces of the valves. Rarely are the tissue changes destructive or ulcerative in character. Although the tissue reactions of rheumatic endocarditis are mild, the secondary sclerotic changes and consequent deformity of the valves are an irremediable and harmful sequel.

Myocarditis is a common incident in rheumatic fever, only recognized clinically when associated with cardiac incompetency. Myocarditis as an incident in the ambulatory patient, who has passed the period of the meridian of life, is in most instances an example of focal infection. Infectious bacteria, usually strains of streptococci of low virulence, are supplied to the blood stream from foci of infection located in the tonsils, dental alveoli, gall bladder, or elsewhere. Babcock $\left({ }^{11}\right)$ has confirmed the writer's observation that chronic cholecystitis is very frequently associated with evidences of chronic myocarditis. The tissue reactions of the myocardium in the form of islands of proliferative interstitial 
change are indicative of focal invasion of infectious agents of low virulence.

Rheumatic pericarditis may occur alone, or in association with endocarditis, or as an incident of pancarditis. It may occur, especially in children, unassociated with any other clinical evidence of rheumatism. The diplococcus rheumaticus may excite tissue changes productive of fibrinous or serofibrinous exudates within the pericardium.

Acute chorea is an infectious disease. It is closely related, etiologically and clinically, to rheumatic fever. It may be manifested coincidently with acute rheumatism. Endocarditis is a frequent coincident and pericarditis may occur with it. The seasonal and age incidence is the same as that of rheumatic fever. Recurrent or periodical attacks are characteristic. The symptoms express temporary disturbance of function of the nervous apparatus; usually ataxic movements, muscular weakness, mental disturbance, mutism, and other phenomena of varying degree of severity. The morbid tissue changes in the heart, of endocarditis clinically associated with chorea, are the same as in rheumatic endocarditis. The character of the tissue changes in the heart, the temporary disturbance of the functions of the nervous apparatus, and the usual recovery of the patient indicate etiologic agents of low virulence. It seems rational to assume, therefore, that acute chorea is caused by multiple cerebral bacterial embolism, by a strain of streptococci of low virulence. The embolic invasion of the brain, by these infectious agents, excites mild reaction in the tissues of but slight importance anatomically, but sufficient to provoke all of the motor and sensory phenomena, characteristic of chorea.

Thyroiditis, manifested by swelling and tenderness of the gland and by mild or severe evidences of thyro-intoxication, is a not infrequent clinical coincident of rheumatic fever. Vincent $\left({ }^{12}\right)$ and others have observed swelling and tenderness of the gland in from 50 to 80 per cent. of patients suffering from rheumatic fever. I have confirmed these statements, but the percentage of the incidence of thyroiditis was less than that noted by Vincent. Vincent ascribes the not infrequent tachycardia and mental excitement and the excessive sweating and tremor of acute rheumatism to thyro-intoxication. Acute thyroiditis may be associated with typhoid fever and other general infectious fevers.

Chronic thyroiditis may be a focal infection. In our clinic we have observed many instances of goiter with and without thyro-intoxication, in patients with coincident chronic tonsillitis and sinusitis and alveolar abscesses. After removal of the focus or foci of infection, resolution of the chronic thyroiditis usually occurred. With the subsidence of the goiter the toxic symptoms vanished. 
Acute and chronic iritis and iridocyclitis may be caused by strains of streptococci carried in the blood stream from foci of infection of the teeth, tonsils, sinuses, and other sites, as demonstrated by the experimental work and clinical observation of Rosenow $\left({ }^{13}\right)$ and of Irons and Brown $\left({ }^{14}\right)$.

Erythema nodosum may be a clinical coincident of rheumatic fever or may occur as a factor of the syndrome described by Osler. The syndrome, when complete, is manifested by polymorphic skin lesions; nodose erythema, local hemorrhage, and edema; and by iritis, arthritis, visceral crises, hematuria, and occasionally by endocarditis and pericarditis. The syndrome may be expressed by only two or three of the lesions enumerated. The clinical observations of the writer and the laboratory experiments of Rosenow $\left({ }^{15}\right)$ confirm the infectious character of the syndrome of Osler. The isolation of strains of streptococci from the excised skin lesions of patients and the production of like lesions in the skin of animals intravenously inoculated with the specific bacterial cultures has been successfully demonstrated by Rosenow $\left({ }^{15}\right)$. The removal of the apparent etiologically related foci of infection from patients suffering with the syndrome, in our clinic, has been followed by entire relief over sufficient periods of time to demonstrate satisfactorily the focal infectious character of the condition.

Herpes is due to lesions caused by trauma or infection of the ganglia of cranial nerves and of the posterior roots of the spinal nerves, followed by Wallerian degeneration. Herpes of the lips and face is coincident with pneumonia, cerebrospinal meningitis, and infection of the throat and nose. In our clinic Rosenow and Ofterdal $\left({ }^{16}\right)$ demonstrated the etiologic relation of foci of infection in the tonsils and dental alveoli to herpes by the production of herpes, labialis and zoster, in animals intravenously inoculated with strains of streptococci isolated from foci of infection of patients suffering from herpes.

Gonococcemia has its source in depots of the bacteria, in foci of infection located usually in the genitourinary tract. Virulent strains may produce ulcerative malignant endocarditis and acute destructive arthritis and purulent exudates. Bursitis and tenosynovitis are not infrequent clinical coincidents. Less virulent strains may cause non-destructive arthritis with serofibrinous exudate into the joint cavity. The fluid may be apparently sterile. The presence of alleged sterile joint exudate has been the authority of some writers for the statement that some types of gonorrheal arthritis are caused by the effect of toxins carried to the joint in the blood stream from gonorrheal foci of infection in the genitourinary tract. The more rational view is justified by the fact that the strains of gonococcus of low virulence are deposited as emboli in the end 
arteries of the subserous tissues. There they arouse, by virtue of their toxin, serofibrinous exudates into the tissues and the joint cavity. Doubtless gonococci pass into the joint and in the early stage of the disease may be demonstrated in the fluid with a proper technic. Later in the disease, as in rheumatism, the exudate is apparently sterile because the serous fluid is an unsuitable culture medium for the growth and multiplication of the gonococcus.

Malignant or ulcerative endocarditis is so characterized because of the tendency to destructive lesions of the valves and endocardium and also because of the high incidental mortality. The disease is usually acute. One type is characterized by chronicity. It is always secondary to preēxisting general or local infection. Old valvular scars and congenital deformities of the heart and big blood vessels predispose to the malignant infection of the endocardium and valves in the constant presence of bacteriemia. The disease may be a local complication of general infections like pneumonia, epidemic cerebrospinal meningitis, epidemic influenza, occasionally of typhoid fever and acute rheumatism, or it may occur with the bacteriemia emanating from foci of infection anywhere in the body due to the gonococcus, streptococcus hemolyticus, staphylococcus, and less frequently other infectious bacteria. The chronic type is due most often to a strain of the streptococcus viridans. Occasionally malignant endocarditis due to streptococcus viridans runs an acute course.

Chronic streptococcus viridans malignant endocarditis, first recognized by Schottmüller $\left({ }^{17}\right)$, under the name of endocarditis lenta, has been described and named by Osler $\left({ }^{18}\right)$ chronic infectious endocarditis, by Horder $\left({ }^{19}\right)$ infective endocarditis, by Libman $\left({ }^{20}\right)$ subacute bacterial endocarditis, and by Lenhartz $\left({ }^{21}\right)$ and others chronic septic endocarditis. The disease is characterized by a clinical course which in the early stages may be very mild. The patient is usually up and about, attending to the ordinary duties of life. More or less general debility, poor appetite, loss of weight and strength, chilliness and night sweats, and occasional cough may be interpreted as indicating tuberculosis, malaria, and the like. The progressive course and characteristic evidences of malignant endocarditis supervene with the fatal end sometimes as late as twelve to eighteen months. The most usual focus of infection which is the source of streptococcus viridans bacteriemia is pyorrhea alveolaris.

Acute appendicitis, the result of bacteriemia having its source in foci of infection, is regarded as the usual mode of infection of the appendix by Kretz $\left({ }^{4}\right)$, Canon $\left({ }^{22}\right)$, Ghon and Namba $\left({ }^{23}\right)$, Adrian $\left({ }^{24}\right)$, many French, English, and American clinicians, and this has been conclusively demonstrated by Rosenow $\left({ }^{25}\right.$ ) by well-directed clinical 
and laboratory investigations. The clinical coincidence of appendicitis with general infectious diseases, with attendant bacteriemia, has been noted by many observers. Adrian calls attention to the similarity of lymphoid tissue structure of the tonsil and appendix veriformis. $\mathrm{He}$ has noted the etiologic connection of tonsillar infection with appendicitis and coined the term. "anginal appendicitis" to express the relation. The invading microörganisms, reaching the appendix hematogenously, lodge in the small vessels of the wall of the organ. Dependent upon the number and virulence, the tissue reaction will be mild, severe, or even so destructive as to cause early gangrene and necrosis. The less virulent strains may excite local hemorrhages and serous exudate causing local edema, swelling of the organ, and partial or complete obstruction of the lumen of the appendix. This morbid anatomical result creates favorable conditions for the secondary mixed infection of the organ by microörganisms which have a normal habitat within the lumen of the bowel, namely colon bacilli and fusiform anaerobes. It is conceivable that conditions such as fecal stones and possible kink of the organ, which may cause obstruction of the lumen of the appendix, may establish conditions favorable for the primary invasion of the walls of the appendix by the saprophytic microorganisms usually found in the bowel. The saprophyte becomes a pathogenic microörganism because of the morbid anatomical conditions and other unknown factors. Mild appendicitis due to infectious agents of low virulence may disappear entirely or the infection become latent. Persistence of the original focus of infection in the tonsil, dental alveoli, or elsewhere, or the occurrence of a new focus may furnish the source of a bacteriemia from time to time and coincident recurrent appendicitis. The morbid anatomical condition of the appendix, due to previous attacks of appendicitis and the presence of foreign bodies within its lumen, may invite future invasion of the organ by infectious agents in the blood stream.

Cholecystitis is probably a hematogenous infection. Typhoid bacteriemia is a not uncommon cause. Cultures of strains of streptococci isolated from alveolar abscess, tonsillitis, maxillary sinusitis and from other foci, have caused cholecystitis in animals inoculated intravenously. A patient in our clinic had suffered from an acute tonsillitis a week preceding an attack of acute cholecystitis. At the surgical operation a small area of the fundus of the gall bladder was found infected. From this excised infected tissue Rosenow $\left({ }^{28}\right)$ isolated a strain of streptococci, which was morphologically and culturally similar to a strain isolated from the patient's tonsils. Each strain was intravenously injected into animals and produced focal cholecystitis. Similar positive experiments 
have been made with strains isolated from the gall bladder and coincident foci of infection of other patients. Focal infection of the gall bladder of animals killed a month or longer after intravenous inoculation with strains of streptococci obtained from infected pieces of the wall of the gall bladder at operations and also from foci of infections about the head have demonstrated focal chronic cholecystitis and the formation of gallstones. In the center of many gallstones obtained from human gall bladders Rosenow has isolated bacteria, usually strains of streptococci. Many of these strains were pathogenic and caused cholecystitis in the inoculated animals.

Circumscribed parenchymatous hepatitis is always focal in origin and the related streptococcemia, usually a pyogenic strain, has its source in infected foci of the rectum or in other regions from which the blood is returned through the radicals of the portal vein.

Acute gastric and duodenal ulcers have been produced in animals by Rosenow and Sanford $\left({ }^{27}\right)$ and others by the intravenous injection of strains of streptococci. The bacteria lodge in the terminal vessels of the submucosa. The resulting tissue reaction is productive of local hemorrhage and infiltration with plasma cells and leucocytes. The small area of overlying gastric mucous membrane becomes ischemic and devitalized and is readily digested by the gastric juice. If an artery is involved gastric hemorrhage may ensue. If the tissues of the edges and base of the ulcer are free of infectious microörganisms the ulcer may heal. On the other hand, if the tissues about the ulcer are infected by a persistence of the invading pathogenic agents the lesion remains unhealed and may become a typical chronic peptic ulcer: Rosenow has isolated strains of streptococci from the walls of chronic peptic ulcers of the human stomach excised at operation. These strains have produced focal infection of the wall of the stomach of animals inoculated intravenously. If killed within a few hours, an acute gastric ulcer was found; if killed after a few weeks autopsy revealed often chronic peptic ulcer.

Acute pancreatitis may be a focal infection. It has been produced experimentally by Rosenow $\left({ }^{28}\right)$ in animals, by the intravenous injection of streptococci obtained from the duct of Steno of patients suffering from epidemic mumps, and from foci of infection of the dental alveoli, tonsils, and other sites. The resulting degree of tissue reaction is dependent upon the virulence of the infectious microörganisms and varies from destructive lesions associated with necrosis and gangrene to mild reactions in the form of small hemorrhages and serofibrinous exudate. The destructive fulminating type requires emergency surgical interference, while the less serious focal infection may resolve spontaneously. Repeated focal infection of the organ with bacteria of low virulence may 
finally result in serious anatomical damage. If the islands of Langerhans are involved the consequent diminution or absence of the internal secretion of the gland may so reduce carbohydrate tolerance that diabetes mellitus supervenes. Opie $\left({ }^{29}\right)$ has demonstrated the occurrence of pancreatitis from infection extending from the infected gall bladder and ducts through the pancreatic duct to the body of the organ. Surgical drainage of the infected gall bladder and tracts has completely relieved the coincident chronic pancreatitis in many patients.

Acute osteomyelitis is often associated with infected compound fractures of the bones. It may be a focal infection. Foci of infection in the tonsils, dental alveoli, sinuses, or lymph nodes may be the source of virulent strains of streptococci, staphylococci or tubercle bacilli which may reach the medulla of the bone through the nutrient artery. External bruises and other injuries to the shaft may increase the susceptibility to local focal infection of the medulla of the bone.

Tuberculosis is a classic example of focal infection. In childhood tuberculous infection of lymph glands of the mesentery and mediastinum is a frequent occurrence. If the individual resistance to the invasion is sufficiently strong the gland infection becomes latent. Later in life under conditions favorable for the multiplication of the tubercle bacilli with coincident greater susceptibility of the host, these foci of infection may become active. Through lymphogenous and hematogenous routes, pulmonary, pleural, kidney, testicle, bone, and joint tuberculosis may occur. Tuberculous arthritis is usually, if not always, secondary to focal tuberculous osteomyelitis of the epiphysis.

Syphilis is a focal infection in the meaning of lymphogenous and hematogunous systemic infection from a focus of infection,- - the Hunterian chancre. Syphilis too is an example of pathogenicity with a special affinity for types of tissue; skin, bone, liver, cerebrospinal system, and other organs. This specific selective tissue pathogenicity may exist at the time the infection is received or may be apparently acquired later.

Spinal myelitis is conceded to be a type of focal infection which may result in insular sclerosis. A young man in our clinic had suffered for three years with progressive symptoms, indicative of multiple insular sclerosis of the spinal cord. Obviously infected tonsils were enucleated. The strain of streptococci isolated from abscesses within the tonsils were injected intravenously into two dogs. Both animals became ataxic and had partial loss of power in all, but most in the posterior, extremities. The autopsy of both animals revealed infection of the cord, with focal areas of hemorrhage and softening.

Glomerulonephritis is in many instances an example of focal infec- 
tion. Le Count and Jackson $\left({ }^{30}\right)$ have observed the renal changes in animals injected intravenously with strains of streptococci isolated from the tonsils of patients suffering from epidemic septic angina. The renal lesions were primarily of the vascular structures; glomeruli, intertubular vessels, and arcuate and interlobular veins. The tissue reaction consisted of an exudate consisting chiefly of lymphocytes and plasma cells. The autopsy of the animals killed within a few days revealed a notable tendency to repair of the acute glomerular lesion. Le Count and Jackson also noted that animals autopsied from fifteen to one hundred and eightysix days after inoculation showed chronic kidney changes corresponding with human chronic interstitial nephritis. Klotz $\left({ }^{31}\right)$ states that a type of acute interstitial nephritis, induced in animals by the intravenous injection of strains of streptococci, developed into renal sclerosis corresponding with chronic interstitial nephritis. Ophüls $\left({ }^{32}\right)$ concludes that chronic nephritis is usually the result of hematogenous infection. In our clinic strains of streptococci have been isolated from the infected tonsils or other apparent focus of infection of many patients suffering from nephritis whose urine was albuminous, more or less bloody, and contained casts and leucocytes. Injected intravenously into animals these strains have almost invariably caused glomerulonephritis, with bloody albuminous urine containing many casts. Furthermore many of these patients have speedily become apparently well after the eradication of the foci of infection, while others improved for a time but in later years developed evidence of typical chronic nephritis. There appears to be a peculiar pathogenicity manifested by an affinity for special tissues, possessed by certain bacteria. This is apparent in the clinical and laboratory experiments noted in reference to nephritis. Forssner $\left({ }^{33}\right)$ cultivated a strain of streptococcus hemolyticus in kidney broth containing pieces of kidney. Subcultures grown in the same media finally acquired an affinity for the kidney, as indicated by numerous lesions of the kidneys and the slight involvement of other organs of the inoculated animals. Strains of the microörganisms which had acquired the affinity for the kidney lost this peculiar quality when grown in ordinary bouillon. By cultivation again in the kidney extract medium the affinity for the kidney was regained.

Chronic infectious arthritis may be due to the specific microörganisms of typhoid fever, tuberculosis, gonorrhea, syphilis, and also to strains of streptococci. With the exception of traumatic chronic infectious arthritis, the condition is always typical of focal infection. Noninfectious arthritis due solely to faulty metabolism and degenerative changes from any cause is not considered here. The mode of infection 
of tuberculous and gonorrheal arthritis has been considered in preceding pages. There is usually no difficulty in establishing the diagnosis in these forms of arthritis if an adequate individual history including the character of the onset of disease is obtained. In gonorrheal arthritis a thorough examination of the genitourinary tract for foci of infection will often furnish confirmatory evidence. It must be remembered that gonorrheal foci may remain latent for years and then suddenly acquire increased virulence. In both diseases, suitably controlled von Pirquet skin and the complement fixation tests may be of diagnostic value. Typhoid spondylitis does not differ essentially from mild forms of the disease caused by strains of streptococci.

Chronic infectious arthritis [Billings $\left({ }^{34}\right)$ ] of the deforming type is usually caused by strains of streptococci of varying degree of low virulence. The microörganisms reach the joint through the blood stream and lodge in the terminal vessels of the periarticular tissues, of the subserosa, and of the branches of the nutrient artery which ends in the epiphysis. The tissue reactions are consistent with the degree of virulence of the infectious invaders: cellular infiltration blocks the small blood vessels, local hemorrhage occurs, and a serofibrinous exudate causes edema and swelling of the periarticular tissues and the synovial sac is more or less filled with serofibrinous fluid. The infection may be confined to the periarticular tissues or may be a simple synovitis or may occur as a panarthritis, usually termed osteoarthritis. Dependent probably upon the virulence of the invading infectious agents, including the number which primarily reach the joint tissues, the severity of the symptoms of the onset varies. If the virulence of the microörganisms is relatively high, the onset may resemble rheumatic fever with multiple arthritis. If the virulence is relatively low, the onset is often insidious and manifested by symptoms of slight involvement, usually of the periarticular tissues of distal joints. The severe types are progressive and the character of the morbid changes are designated hypertrophic or proliferative. The milder types are usually associated with morbid changes of a degenerative or atrophic character. Nichols and Richardson $\left({ }^{35}\right)$ have given an excellent description of the morbid anatomical condition of these joints. They state that the morbid changes, both proliferative and degenerative, cannot be differentiated etiologically. They suggest that the morbid changes are due to reactions excited by toxins. They suggest too that the source of the toxins may be from infectious invaders, or from faulty metabolism and other sources. Clinical observation shows that the involved joints of one patient may present examples of all types of arthritis: periarthritis, synovitis, osteoarthritis, and one or more joints may be typical examples 
of atrophic or degenerative changes, while other joints show evidences of hypertrophic or proliferative changes. This would indicate an etiologic agency, which causes various degrees of tissue involvement and dissimilar types of morbid tissue changes, because of varying doses of the etiologic factors. Long observation and study of these joint conditions. including clinical, laboratory, and post-mortem investigation, convinces the writer that the principles involved in focal infection afford a rational explanation of this vexatious problem. In most patients who suffer from chronic infectious arthritis it is usually possible to demonstrate foci of infection about the head or elsewhere in the body. Without harmful result to the patients, small pieces of morbid tissue may be excised from the synovial membrane, and other tissues and enlarged lymph nodes proximal to the joint may be obtained for the purposes of bacterologic and histologic study. With the isolation of infectious strains of streptococci from existing foci of infection and from the excised morbid tissues of the joint it is fair to assume an etiologic relationship between the focus and the joint infection. Accepting a hematogenous invasion of the joints by bacteria of low virulence, the embolic mode of infection, the tissue reactions embracing blocking of the blood vessels by cellular invasion, the presence of a serofibrinous exudate in the tissues and within the synovial sac, with the tendency to subsequent cellular proliferation and secondary sclerosis and contraction, conditions are produced which afford a rational explanation of the further progressive morbid changes. These primary changes involve the injury of the blood vessels and interfere more or less with the blood supply of the involved tissues. Repeated doses of the infectious microörganisms which the chronic condition affords increase the damage to the blood vessels and other tissues. A degree of ischemia of the joint tissues may follow which is consistent with starvation or malnutrition. This local condition is aggravated by the systemic malnutrition and lessened tone of the general circulation which is commonly found in these patients. Malnutrition leads to secondary metabolic changes in all joint structures, fibrous tissues, cartilage, and bone. With continuous and perhaps increased malnutrition the metabolism becomes retrograde in character, and promotes the change of the connective tissue group, tendons, ligaments, and cartilage, into bone. The character of the toxins, which is dependent upon the resistance of the invading bacteria, and the gradually increasing malnutrition of the joint tissues, may be the determining factors of the morbid anatomical conditions, i.e. hypertrophic or proliferative, atrophic or degenerative arthritis. The failure to eradicate foci of infection, the source of continued or intermittent bacteriemia, is one factor in the progressive character of chronic infectious arthritis, 
as well as other chronic infectious organic diseases. Persistence of the chief etiologic factor prevents recovery usually even when the most rational measures are applied to correct the joint conditions or to improve the general condition with the purpose of improving the resistance of the patient to disease. This neglect of proper treatment permits the disease to progress until retrograde metabolism and continued invasion of the infections have resulted in irreparable anatomic damage to the joints. Simultaneous invasion of the muscles and tendon sheaths with consequent contraction due to the focal sclerosis of the muscles aggravates the deformity due to the morbid condition of the joints. It should be noted that non-infectious morbid conditions of joints due to faults of posture, trauma, and the wear and tear of life, may increase the susceptibility to primary chronic infectious arthritis of the deforming type.

Muscular rheumatism, so-called, is usually a focal infection. Trauma in the nature of muscular sprain and exposure to inclement weather are not infrequent predisposing factors.

Chronic neuritis or perineuritis, especially involving the cervical plexus, the musculospiral and sciatic nerves, is frequently caused by foci of infection located in the head, gall bladder, and other sites.

Anaphylaxis may be clinically expressed as bronchial asthma, erythema and urticaria of the skin, and as physical debility with low blood pressure. The parenteral introduction of the bacterial protein or the protein produced by the microörganisms in the focus of infection may sensitize the patient. In our clinic many patients with bronchial asthma have been permanently relieved by the preliminary eradication of the apparent focus of infection, followed by attempts to desensitize the patient by the subcutaneous injection of antigen prepared from the dead microörganisms isolated from the focus of infection.

\section{TREATMENT}

In the treatment of focal infection the primary principle is to locate and eradicate the apparent focus of infection. This principle necessarily involves measures to prevent the occurrence of foci of infection. Prevention of foci of infection involves the application of the laws of general and individual hygiene. The eradication of foci of infection before the development of systemic focal infection occurs will conserve health and life. Children who present infected tonsils should receive efficient medical care or tonsillectomy should be practiced to lessen the incidence of rheumatic fever, chorea, and endocarditis in childhood. Dentists must accept the responsibility of the eradication of dental alveolar infection which is now recognized as a very common source of systemic focal infection. 
With the foci of infection removed, the obligation of the attending physician requires a management which will overcome the systemic infection, and which will preserve and if possible entirely restore the function of the organs involved. This involves the selection of a properly balanced diet, the use of hematinic and other tonics, good air, regulated passive and active exercise, the employment of hydro-, electro-, and mechanotherapy and play. For the purpose of mental diversion and also to restore function mental and manual work should be employed in a rational manner.

The writer has had an extensive experience in the employment of autogenous bacterial vaccines and antisera in the treatment of chronic infectious arthritis, streptococcus viridans malignant endocarditis, gonorrheal arthritis, and other focal infections, which justifies the opinion that the therapeutic use of these agencies is not warranted by the results observed. This conclusion is in accordance with Smith $\left({ }^{36}\right)$ and Pearce $\left({ }^{37}\right)$.

The intravenous injection of non-specific proteins in the treatment of chronic infectious arthritis has produced remarkable beneficial results as indicated by clinical reports made by many reliable physicians. At the same time the reaction to the injection is manifested by alarming symptoms and sometimes by such serious results that the conscientious physician will hesitate to take the responsibility of the employment of this method of treatment. Jobling and Petersen $\left({ }^{38}\right.$ ) have made extensive experiments upon laboratory animals, and they conclude that it may be possible in the near future to use intravenously the non-toxic portion of a foreign protein which will excite the mobilization of the helpful ferments of the body, as well as other body defenses, without the production of the painful, disagreeable, and even dangerous clinical phenomena which attend the intravenous injection of an unmodified protein antigen.

\section{BIBLIOGRAPHY}

I. BASS, C. C., and JOHNS, F. M.: Jour. Am. Med. Assoc., 1915, LXIV, 553.

2. BARRETT, M. F.: Dental Cosmos, I9I4, LVI, 948.

3. KOLLE, W., and WASSERMANN, A.: Handbuch der Path. Mikroörganismen, I903, I, 223.

4. KRETZ, RICHARD : Zeitschr. f. Heilkund., 1907, XXVIII, 296.

5. MELTZER, S. J.: Jour. Am. Med. Assoc., Ig10, LV, I021.

6. WEIL, RICHARD: Cinn. Lancet Clinic, 19I3, CX, 568.

7. POYNTON, F. J., and PAINE, A.: Lancet, I900, II, 86I ; Lancet, I9II, II, I 189 . 
POYNTON, F. J., and SHAW, W. V.: Trans. Path. Soc., London, 1904, LX, 126; Brit. Med. Jour., I902, II, 1414; Trans. Path. Soc., London, I901, LII, 248; Lancet, I902, II, 521 ; Brit. Med. Jour., 1904, I, III7; Lancet, I9I0, I, I524.

8. BEATTIE, JAMES : Edinb. Med. Jour., I904, LVIII, 122.

9. WALKER, E. W. A., and RYFFEL, J. H.: Brit. Med. Jour., 1903, II, 659.

Io. ROSENOW, E. C.: Jour. Inf. Dis., I9I4, XIV, 6I.

II. BABCOCK, ROBT. H.: Jour. Am. Med. Assoc., I909, LII, 1904.

12. VINCENT, H.: Compt. rend. Soc. de Biol., I907, LXIII, 389.

I3. ROSENOW, E. C.: Jour. Inf. Dis., I9I5, XVII, 403.

I4. IRONS, ERNEST E., and BROWN, E. V. L.: Jour. Am. Med. Assoc., I9I6, LXVI, I840.

15. ROSENOW, E. C.: Jour. Inf. Dis., 1915, XVI, 367.

I6. ROSENOW, E. C., and OFTERDAL, SVERRI.: Jour. Am. Med. Assoc., I915, LXIV, I968.

I7. SCHOTMÜLLER, H.: Münch. Klin. Wochenschr., I9ı, LVII, 880.

I8. OSLER, WM.: Quar. Jour. Med., I909, II, 2 I.9.

I9. HORDER, THOMAS J.: Quar. Jour. Med., I909, II, 289.

20. LIBMAN, E., and CELLER, H. L.: Am. Jour. Med. Sc., igio, CXL, 5 I6. LIBMAN, E.: Am. Jour. Med. Sc., I9I2, CXLIV, 313.

21. LENHARTZ, H.: Munch. Med. Wochenschr., I90I, XLVIII, I123.

22. CANON, DR.: Deutsch. Zeitschr. f. Chir., I908, XCV, 2I.

23. GHON, A., and NAMBA, K.: Beiträg. z: Path. Anat. u. z. Algem. Path., I9I2, LII, I30.

24. ADRIAN, C.: Mitteil, a. d. Grenzgeb. d. Med. u. Chir., I90I, VII, 407.

25. ROSENOW, E. C.: Jour. Inf. Dis., IgI5, XVI, 240.

26. ROSENOW, E. C.: Jour. Am. Med. Assoc., IgI4, LXIII, I835.

27. ROSENOW, E. C., and SANFORD, A. H.: Jour. Inf. Dis., I9I5, XVII, 219.

28. KOSENOW, E. C.: Jour. Am. Med. Assoc., I9r 5, LXV, 1687. Jour. Inf. Dis., 1916, XIX, 540.

29. OPIE, E. L.: Johns Hopkins Hosp. Bull., I90I, XII, I 82.

3o. LE COUNT, E. R., and JACKSON, LEILA: Jour. Inf. Dis., 1914, $\mathrm{XV}, 389$.

3I. KLOTZ, OSKAR: Trans. Assoc. American Physicians, I9I4, XXIX, 49.

32. OPHÜLS, W.: Arch. Int. Med., I912, IX, I56.

33. FORSSNER, G.: Nord. Med. Arkiv., I902, XXXV, No. 18, I.

34. BILLINGS, FRANK: Arch. Int. Med., I9I2, IX, 484.

Jour. Am. Med. Assoc., I9I3, LXI, 8Ig.

I1l. Med. Jour., I914, XXV, I4.

35. NICHOLS, EDWARD H., and RICHARDSON, FRANK L.: Jour. Med. Research, 1909, N.S., XVI, 149.

36. SMITH, THEOBALD: Jour. Am. Med. Assoc., I9I3, LX, 159 r.

37. PEARCE, RICHARD M.: Jour. Am. Med. Assoc., I9I3, LXI, 2115.

38. JOBLING, J. W., PETERSEN, W. F., and EGGSTEIN, A. A.: Jour. Exper. Med., 1914, XX, 32I, $45^{2}$ and 468, and 19I5, XXII, 401. 


\section{THE \\ OXFORD MEDICINE ADVANCE PAGES}

EDITED BY

HENRY A. CHRISTIAN SIR JAMES MACKENZIE

CONTRIBUTORS VOLUME I

LEWELLYS F. BARKER

FRANK BILLINGS

WILLIAM T. BOVIE

RICHARD C. CABO'T

HENRY A. CHRISTIAN

CHAS. BENEDICT DAVENPORT

EUGENE F. DU BOIS

FREDERICK P. GAY

LAWRENCE J. HENDERSON

A. WALTER HEWLETT

GUY HINSDALE
WALTER B. JAMES

WILLIAM B. JOHNSTON

SIR JAMES MACKENZIE

JOHN J. MACKENZIE

ELMER V. MCCOLLUM

SIR WILLIAM OSLER

FRANCIS W. PEABODY

LEONARD G. ROWNTREE

HENRY SEWALL

DONALD D. VAN SLYKE

WILLIAM H. WELCH

PART 4

VOLUME I

NEW YORK

OXFORD UNIVERSITY PRESS

AMERICAN BRANCH : 35 WEST 32 ND STREET 
COPYRIGHT, 1919

BY THE

OXFORD UNIVERSITY PRESS

AMERICAN Branch 
CONTENTS OF PART IV, VOLUME I-OXFORD MEDICINE

Chapter

IX. The Mechanism of Bodily Resistance to DiseaseFrederick P. Gay . . . . . . . . . . $34 \mathrm{I}$

Chapter

X. Medical Social Service as a Factor in the Diagnosis and Treatment of Disease-Richard C. Cabot . . . . 4I5

ChApter

XI. Environment: Its Bearing upon the Maintenance of Health and the Treatment of Disease-Walter B. James . . . . . . . . . . . . . . . $42 \mathrm{I}$

\section{Chapter}

XII. Radiations and Their Effects-William T. Bovie . . $43 \mathrm{I}$ ChAPTER

XIII. Acidosis and the Physico Chemical Equilibrium of the Organism-Lawrence J. Henderson 


\section{CHAPTER IX}

\section{THE MECHANISM OF BODILY RESISTANCE TO DISEASE}

\section{By FREDERICK P. GAY}

THERE is no more important knowledge to be obtained in medicine than an understanding of the way in which the animal body protects itself from disease. In the infectious diseases, where the most important body of knowledge of this sort has been obtained, it has been utilized in a most astonishing way in preventive and curative medicine.

Bodily resistance, insusceptibility, tolerance, or immunity to disease is as varied in mechanism and effectiveness as are the particular types of disease against which it is operative. For this reason it is necessary to begin this section which deals with resistance to disease with a certain consideration of nosology, or the classification of diseases.

\section{SOME CONCEPTIONS OF NOSOLOGY}

Disease may be defined in a general sense as any deviation from the normal condition in any of the functions or in the structure of the body. Such changes are inevitably referable to some injury. Nosology in its gradual evolution from superstition to present-day knowledge has suffered not only from the natural inhibition of ignorance, but from having been forced to serve practical purposes. The necessities of diagnosis for the humane purpose of alleviating individual suffering have obscured a strictly logical classification such as might have been more rapidly available in a more abstract science. The interest and attention of the physician has been held primarily by symptoms rather than causes of disease, and on the former he has first of all built his systems of classification. In spite of the fact that the understanding of bodily function has necessarily lagged behind knowledge of structure, the interpretation of symptomatology very properly has been largely physiological rather than anatomical. The humoral conception of disease of Hippocrates, although largely metaphysical, was based on observation and was essentially functional in essence; so too are the 
ideas of Themison (I24 B.C.), who would account for variations in symptomatology as due to degrees of " relaxation." Galen's (I3I-20I A.D.) association of disease types with the four elements, hot, cold, moist, and dry, must be viewed in connection with his rôle as the first experimental physiologist; it will be recalled that he produced aphonia in dogs by cutting the recurrent laryngeal nerve and demonstrated hemiplegia by hemisection of the spinal cord.

On the other hand, the more objective and readily acquired facts of structure which were collected in the development of anatomy naturally formed a framework on which a more mechanical though, for the time, more accurate grouping of diseases was based. This structural interpretation was initiated by the Alexandrian School, particularly under the influence of Erasistratus (280 B.c.), and was made real by the gradual development of pathological anatomy beginning with Morgagni ( 1682 ), followed by Baillie (I789), P. Brouissais (I772-I838), Laennec (I $78 \mathrm{I}-1826)$, and many others. Virchow (I82II902) $\left({ }^{1}\right)$, in uncovering the rich factoral realms of histopathology, served further to develop an essentially static aspect of disease, although he was himself well aware that the essential problem of pathology lies eventually in explaining the field of disordered function.

With the relatively recent, though rapid, development of bacteriology, immunology, the biochemistry of metabolism, and experimental physiology, it has become possible to consider disease in its initial, intermediate, and dynamic phases. As Allbutt $\left({ }^{2}\right)$ has well expressed it, "In the last quarter of the nineteenth century the conception grew clearer that morbid anatomy for the most part demonstrates disease in its static aspects only, and for the most part in the particular aspect of final demolition; and it became manifest as pathology and clinical medicine became more and more thoroughly integrated that the processes which initiate and are concerned in this dissolution were not revealed by the scalpel." There is danger, indeed, as he further points out, that the striking and impressive nature of structural change should isolate or emphasize the final act of nature and only suggest and often lead to an incorrect interpretation of the preceding moving drama in which we are primarily interested.

Modern nosology, whether viewed from the aspect of the pathologist or of the clinician, is a curious mixture of past schemes of classification. Perhaps the most fatal error of systematists in a biological science is that they should presume at any particular moment to present a complete series of facts. In metaphysics such complete systems are possible de novo and can be debated only on the basis of an interpretation of the terms employed. For this reason the nosology of Hippocrates, 
based on the four humors, blood, phlegm, yellow bile, and black bile, or the one of Galen, with his four elements, still might be defended and remain intact. With the increased appreciation of symptoms, both when subjective and particularly when objective, each additional fact is a potential disturber of any comprehensive classification.

Symptomatology has been the earliest and the most persistently pursued method of classification. Having first excited the interest and monopolized the attention of the physicians, it has, to some extent, paralyzed their initiative in seeking antecedent causes. Symptoms then, at first vaguely grouped and frequently with some idea of antecedent cause perhaps of supernatural origin, furnished the earlier bases of classification. The elaborate systems of taxonomic nosology begun by Sauvages and Linnaeus (I763), followed by Cullen (I764), Good (1823) $\left({ }^{3}\right)$, and others, were based on the rich accumulated experience of the ages in the finer distinction of symptoms, correlated with certain more or less accurate ideas of anatomical localization of disease processes.

The great development of morbid anatomy and histopathology in the nineteenth century led, as we have stated, to the recognition of the terminal aspects of many disease processes and the formation of a systemic classification of disease in accordance with the localization of lesions in some particular part or parts of the body. This association of definite changes of structure with a symptomatic history has been of the greatest value to clinical diagnosis. In the matter of classification, however, morbid anatomy has been found less complete than symptomatology; many diseases present no demonstrable lesions or present lesions that are common to several disease processes. There has been an added danger in the revelations of the structural changes of disease; the mind of the physician, impressed by the objectivity of the "silent moments in the pathological museum," has allowed his intellectual curiosity to be satisfied by the terminal and, in reality, the least important stage of disease. The initial and the transitory phases of disease are lost sight of in contemplation of the ruins; therapeutic nihilism arises when only the failures of human intervention are studied.

The greatest single body of facts contributed to medicine has been given by bacteriology. Nosology has benefited from bacteriology, not simply in the more precise grouping of the "Fevers" as "Infectious diseases," but in suggesting, by analogy, that the only correct method of classification of all diseases is on the basis of etiology. It is not surprising that in the earlier days of medicine, when few if any of the real causes of diseases apart from those of purely mechanical origin were manifest, the most objective aspect of disease, its symptomatology, 


\section{MECHANISM OF BODILY RESISTANCE TO DISEASE}

should either be regarded as the primary cause, or, at all events, should form the basis of classification. And again, with the still more objective demonstration of the anatomical localization of disease, it is understandable that the classification of diseases by systems should have been chosen as a firmer foundation. What does seem surprising is that, with a recognition that a rapidly growing group of diseases can be rigorously shown to be due to well-defined and specific primary, determining causes, no more attempts should have been made to classify all diseases on the basis of etiology. It seems unnecessary to argue that any group of correlated facts, irrespective of the sequence in which these facts are obtained, should finally be presented in chronological order. Although we may first have recognized that the disease general paresis is characterized by delusions of grandeur, habit disturbances, and the like, and later learn that it is accompanied in advanced stages by atrophy of the frontal convolutions, the final demonstration of treponema pallidum in these folds should suffice to include it finally in the category of infectious diseases rather than among the mental diseases or the diseases of the central nervous system.

Modern systems and treatises on both clinical medicine and pathology will be found to classify diseases by a combination of all three of the methods that have been outlined; on the basis of symptomatology, as in the so-called constitutional or metabolical diseases; on the basis of altered structure, as in the systemic diseases, such as those of the respiratory or gastrointestinal tract; and on the basis of etiology, as in the group of infectious diseases. The reason for this present heterogenous nosology is that no one of the systems will be found allinclusive. The etiology of many diseases is at present unknown, the lesions of many disease entities are non-specific, unlocalized, or not as yet apparent, and finally the symptoms may vary in the same disease or the symptom complex of several diseases may overlap. With the more recent rapid advances in exact knowledge, the tendency in clinical medicine has been to allocate more and more diseases to the etiological or to the structural group, with consequent diminution of the symptomatological group. In treatises on pathology, which consider disease from the more abstract and scientific viewpoint, diseases are dealt with largely either from the purely structural aspect of morbid anatomy or histopathology, or from the etiological aspect of bacteriology and parasitology. The term "pathology" is often misapplied when morbid anatomy alone is meant, or more properly subdivided into general pathology and systemic or special pathology. And again, even general pathology is frequently mishandled by the morphologist in discussing general types of cell degeneration or disorder, when, properly speaking, 
the growing data of disordered function, metabolic chemistry, and pathological physiology should be included.

\section{NOSOLOGY ON A BASIS OF ETIOLOGY}

There seems no doubt that with the gradual acquisition of a more or less complete group of facts in respect to the majority of diseases, we shall be logically forced to a nosology on an etiological basis. With the rearrangement of each disease in the sequential order of cause. course, and result will come a primary classification in respect to cause. That we are rapidly approaching some such rational classification is evidenced by at least two modern treatises on pathology. Krehl and Marchand $\left({ }^{4}\right)$ have arranged their discussion of General Pathology first on a basis of general etiology subdivided to include external and internal causes of disease. MacCallum $\left({ }^{5}\right)$, equally imbued with the importance of an etiological classification, apparently for the purpose of greater present inclusiveness has arranged disease on the basis of type of injury that initiates the process rather than the agent that produces such injury.

We believe it will become evident, as we have already suggested, that in considering the mechanism of bodily resistance and reaction the factors concerned are dependent on the nature and usually the identity of the etiological agent involved. Thus, in immunity to the infectious diseases with which we shall be most concerned, we must in each instance deal with an animate, exogenous agent of specific nature. In the case of drug tolerance, in those few instances where it is known to exist, we are dealing with the inanimate exogenous chemical agent. In describing, therefore, all the types and instances of bodily resistance to disease, with which we are properly concerned in this article, it is clear that we must offer ourselves as comprehensive a summary as may be of all recognized disease entities classified on the basis of etiology. It will be found of importance, too, to include as accurate a numerical estimate as possible of the number of recognized disease entities, included under each of the major etiological groups in our proposed nosology, in order to indicate the relative importance of these groups.

A tentative grouping of disease on the basis of etiology for the purpose of discussion of resistance is presented in Table I. 
TABLE I

A POTENTIAL NOSOLOGY ON THE BASIS OF ETIOLOGY

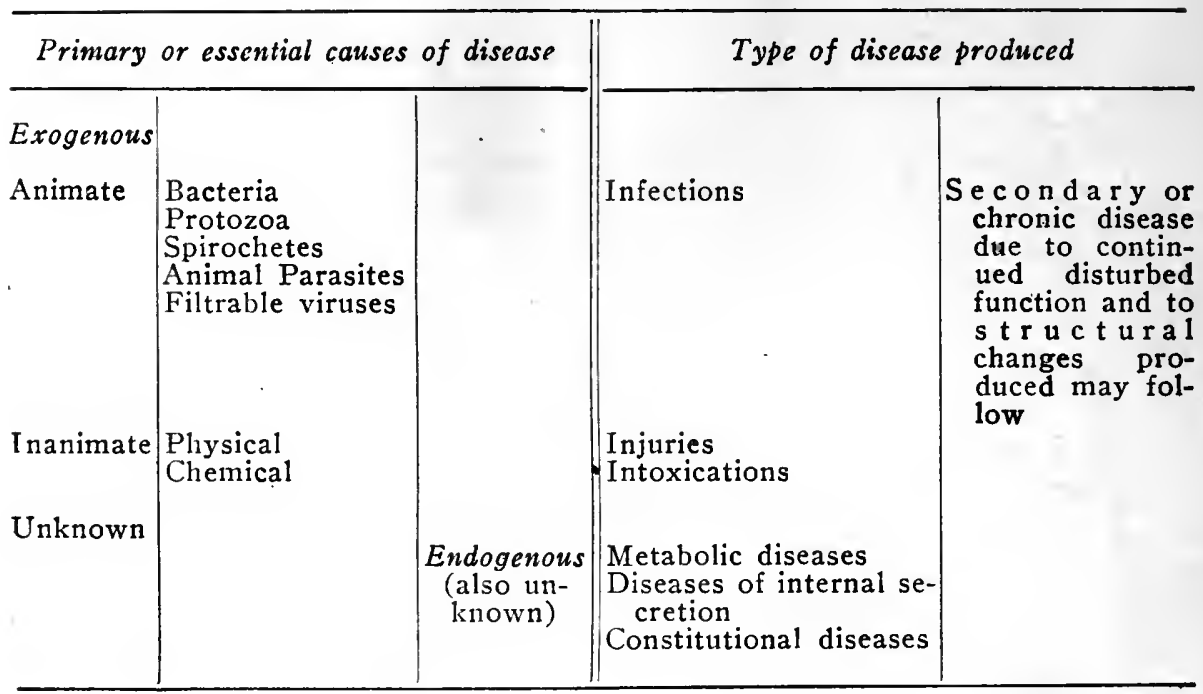

The table is arranged chronologically from left to right. As is indicated, we possess very definite information concerning a large group of diseases due to animate and to inanimate external agents. There exists another group of diseases, less than twenty-five per cent. of the whole number of disease entities as we shall see, concerning the cause of which we are essentially in ignorance. The smaller number of these unknown diseases are at present regarded as due to endogenous causes, inasmuch as they are characterized in their initial phases by definite alterations of function. It is by no means certain that these "endogenous" diseases are idiopathic; some of them are already suspected of being due to an external agent, and all of them eventually may be determined to be thus originated.

No account is taken in this classification of the indirect, secondary, or predisposing causes of disease, such as heredity. However important these accessory causes may be in determining the occurrence and extent . of some diseases, they do not represent the original, essential, and primary cause without which the disease does not occur.

Any presentation of a comprehensive list of recognized disease entities on the basis of the above simplified etiological chart lies outside our present interest and ability. An attempt has been made to separate the recognized diseases into the major groups, as outlined, in order to gain some suggestion of the relative importance of each etiological 
category. For this purpose two chief reference sources were employed. First, a recognized system of medicine, Osler and McCrae, Ist edition, 1907-1910, and second, the nomenclature list of diseases and injuries in the Manual of the Medical Department of the United States Army, Paragraph 455, edition of 1916 as corrected to April, I9I7.

Osler and McCrae's system was first employed to determine what correctly may be regarded as disease entities. The distinction here between a symptom complex and a true disease entity was not difficult. In the list from the Medical Department of the United States Army the determination is not so easy. For example, causes of death are included such as "Suicide by drowning," which in this particular case would naturally be included under Inanimate agents-physical. When "chronic" forms of disease already listed under an acute disease were given, they have been omitted, as also have such headings as "other diseases of the stomach not specified."

For uniformity, the etiological causes of disease have been given largely as accepted in Osler's "Practice of Medicine," and in Osler and McCrae's "System." De Schweinitz on "The Eye" and Stelwagon's "Diseases of the Skin" have been used. When two or more recognized causes of any disease are given the disease is listed under each cause.

In Table II are given the number and percentages of diseases separated on the basis of the etiological chart given in Table I, as determined from the references quoted.

TABLE II

ENUMERATION OF DISEASES ON THE BASIS OF ETIOLOGY

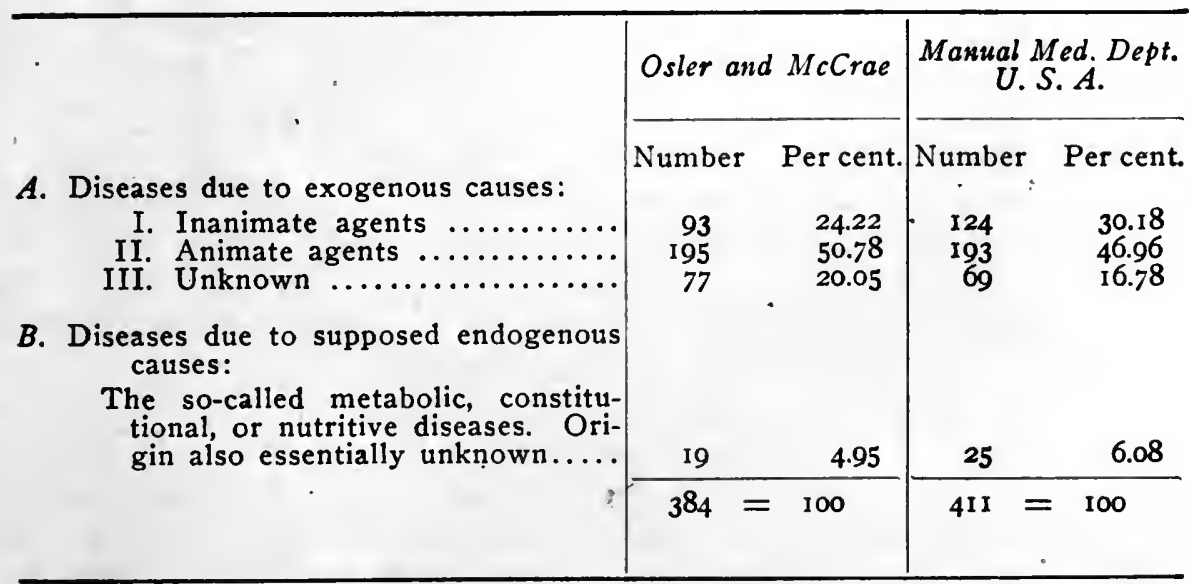




\section{MECHANISM OF BODILY RESISTANCE TO DISEASE}

These estimates were made at an interval of several years, and yet will be found in essential agreement. The relative numerical importance of diseases attributable to external animate agents, for the most part to bacteria, may seem surprisingly large. A further word of explanation would be well. In the case of each disease an effort has been made to trace etiology to its primary cause. Thus, mitral stenosis would be attributable to a previous acute endocarditis, and therefore to an acute infection of bacterial origin. A living agent has been reasonably proven in the majority of the diseases thus classified, although a few diseases have been included which by analogy are probably due to a similar cause. Thus of the 193 diseases listed under animate external agents in the Army list, I6I are certainly of this origin-as examples, typhoid fever, lobar pneumonia, pericarditis, trichiniasis, acute nephritis (the latter disease is also included among those due to chemical agents); 25 diseases are probably due to similar agents, scarlet fever, measles, pyorrhea alveolaris; only 7 are included as of "possible" animate origin-examples, dental caries, ulcer of the stomach, and Hodgkin's disease.

The relative vital importance of the different etiological groups of disease cannot, of course, be estimated on the basis of such an academic grouping as the number of disease entities. It may be of interest, to be sure, to estimate as we have done that approximately one-half of all the diseases we now recognize are due to infectious agents; it would be of far greater importance to learn whether a similarly large percentage of the actual instances of disease are due to the same agency. Reliable morbidity statistics are not however available, particularly when we consider minor ailments that seldom come to the attention of the physician. We have only the reportable diseases, which are all infectious in nature, and mortality statistics on which to base an estimate of the real importance of each group. It may seem a bit forced to trace deaths from Bright's disease back beyond the chronic or secondary disease which eventually is the cause of death, to an original infection which was really the starting-point of the trouble. And yet we are doubly justified both from the standpoint of scientific classification, as well as from the standpoint of prevention and therapy, as Sir James Mackenzie has so ably stated, in so doing. On such a basis it will probably also be found that nearly half the deaths may be traced to an infectious origin. For example, in the report of the State Board of Health of California for I9I I, there were 34,IO2 deaths listed, of which 13,567 , or $40 \%$, could be traced directly or indirectly to an animate exogenous agent.

So much for the classification of diseases on a simplified scheme of 
etiology. It makes possible the estimation of the relative number in each of the groups suggested, and has, we believe, demonstrated the preponderant significance of the infectious diseases. At all events, the classification affords a proper foundation on which to consider the various forms of reaction and resistance on the part of the host to which protection from disease is due. As already stated, these reactions will be found to vary in accordance with the etiological agent concerned.

\section{THE MECHANISM OF BODILY DEFENSE IN DISEASES OF UNKNOWN ETIOLOGY}

As we have seen, approximately one-quarter of the diseases with which we are familiar are due to as yet unknown causes. When we consider this group of maladies, many of them of considerable frequency and severity, we are struck with the importance of a knowledge of etiology from an aspect other than that of mere classification. It develops that coincidentally with our ignorance of essential causation there is an equal ignorance of the possible means of bodily defense. Consequently, from the practical standpoint we are unable to stimulate or intensify such natural defensive mechanisms as may exist or, in other words, to employ preventive measures such as those that may have proved so valuable in diseases of known etiology.

It may be suspected that, for each of these obscure maladies, an exogenous agent, animate or inanimate, will be found. Some of the diseases that have been temporarily included in this group, beri-beri, pellagra, scurvy, and rickets, are now either recognized as being due or suspected of being due to some food deficiency (avitaminosis) or food unsuitableness.

At present, the only further progress that we can report in ultimate analysis lies in segregation of a minority of the disease entities, some six per cent. of the whole in our tables, on the basis of demonstrable functional derangement during the progress of the disease. These would include the so-called endogenous, idiopathic, metabolic, or constitutional diseases. Further information has taught us that some of them are due to abnormal functioning of the organs of internal secretion; for example, Addison's disease (adrenal), goiter, cretinism and myxedema (thyroid), acromegaly, Fröhlich's syndrome, gigantism, diabetes insipidus, and certain forms of adiposity (hypophysis), diabetes mellitus (pancreas), tetany (parathyroid).

In another group of diseases there is a functional derangement affecting some anatomical system, as in the bone diseases, rickets, scurvy, arthritis deformans, osteitis deformans, senile osteoporosis, and osteo- 
malacia; or as in the diseases of the blood and blood-forming organs, chlorosis, pernicious anemia, hemophilia, paroxysmal hemoglobinuria, and the leukemias.

In diseases such as gout and obesity there is even less definite information, although they seem obviously constitutional or metabolic in nature.

In some twenty per cent. of all disease entities we possess little information beyond the symptom complex, and at times a lesion, on the basis of one or both of which the diagnosis is made. In this group of left-overs of unknown etiology there is little to point to a true origin within or without the body. Among them are included cancer, tumors in general, chronic articular rheumatism, multiple sclerosis, paranoia, angina pectoris, acute yellow atrophy of the liver, eczema, asthma, angioneurotic edema, Raynaud's disease, herpes, neurasthenia, dementia praecox, epilepsy, sprue, impetigo, and the like.

As we have already stated, there is no information of a definite sort on any mechanism which the body may have for defense against these diseases of unknown causation; it is probable, from the analogy of the infectious diseases, that knowledge of cause will precede knowledge of defense. There have been, to be sure, instances, as in smallpox, where the existence of a defensive mechanism was recognized and this knowledge made use of in a most practical and effective manner, in spite of ignorance as to cause. Most of the diseases in this "unknown" category differ from the acute infections in such a way as to make discovery of a protective mechanism more difficult; they are more insidious in onset and not readily suspected in their initial phases where resistance would probably be more evident. And again these diseases apparently do not tend to spontaneous recovery, so that their failure to recur in a given individual would point to some condition resembling acquired immunity.

Two instances may be mentioned in diseases of this class as illustrative of what may ultimately be shown to be defensive measures. It has been suggested to me by Prof. W. R. Bloor that the fat accumulation or adiposity which so frequently precedes diabetes mellitus (Joslin) $\left(^{6}\right)$, may represent, when some deficiency in the sugar-splitting mechanism begins to be felt, a more or less successful attempt at saving carbohydrates that would otherwise be wasted. The glycogen stores are first filled and then fat deposition occurs, both of which are eventually called on when glucose can no longer be utilized. The fact that the blood sugar concentration is higher in the diabetic than normal is further proof of an adaptive mechanism to prevent waste.

Another instance of a distinctly different sort, and resembling the 
phenomena of bacterial immunity, has been found in the experimental studies of the malignant tumors of mice. Different races of mice vary in their susceptibility to any given transmissible tumor. A second tumor fails to grow when a flourishing one has preceded it (atreptic immunity). The growth of a tumor in otherwise susceptible animals may be prevented by previous inoculations not only of tumor tissue but of any normal mouse tissue. The analogy was still further pursued by the author $\left({ }^{8}\right)$, who found that growing tumors of rats could be caused to retrograde by injecting tumor tissue and that in the early stages of this cancer growth (premetastatic stage) antibodies to the cancer cells could be demonstrated by the fixation reaction. These experiments should not be taken as indicating that cancer is a disease of infectious origin, a conclusion that would not be borne out by other facts.

These particular experiments must have been conducted under an unusually favorable set of conditions, for it must be admitted that they have not been readily confirmed by others who have tried to repeat them. They belong in the group of positive results which when properly controlled, as these experiments were, are more significant than numerous failures to repeat them, failures which are due to unavoidable misunderstandings of the conditions that are operative. Similar results have, however, been obtained by Lewin $\left({ }^{8}\right)$ and Taylor $\left({ }^{8}\right)$.

\section{DEFENSE MECHANISM IN DISEASES DUE TO INANIMATE EXOGENOUS AGENTS}

The external agents which produce disease may be divided into two general categories-physical and chemical in nature. There are distinct natural individual variations in resistance to both these classes of external disease agents, and these variations may be considerably intensified by repeated exposure to the injurious agent concerned. In the case of physical excitants this increased resistance is referred to as tolerance, habituation, acclimatization, training, and the like, as will be further specified. Increased resistance to chemical agents is usually referred to as tolerance or habituation.

\section{PHYSICAL AGENTS}

The physical causes of disease may be grouped in several categories: (1) traumatic or mechanical, (2) thermal, (3) luminous, and (4) electrical. Certain other groups may be recognized which usually combine certain of the properties of two or more of these already mentioned, such as, for example, the climatological causes of disease. 


\section{$35^{2}$ MECHANISM OF BODILY RESISTANCE TO DISEASE}

The traumatic or mechanical causes of disease include wounds of various sorts and pressure disturbances. In discussing these various agents we are not so much concerned here with the type and effect of the injury but with the mechanisms which may exist in the body for opposing them. A natural resistance to wounds exists in the skin which covers the exposed surfaces of the body and is more difficult to penetrate than some of the softer and more vital tissues. The presence of nerve terminals and of the sensation of pain accomplishes the definite purpose of giving rise to reflex muscular action of a protective sort. These reflexes, as is known, become distinctly more rapid as the result of physical training. The mechanism set in action by rage or fear, which Cannon $\left({ }^{10}\right)$ has so romantically and yet scientifically described, is obviously designed to protect the body against mechanical injury. A blow, actual or anticipated, gives rise to fear or anger which produces an increase in the secretion of the adrenal gland, and this in turn calls forth stored carbohydrates from the liver into the blood. The blood, by peripheral vasoconstriction, is sent directly to the muscles, thus supplying them with sugar and rendering their action in fight or flight more effective. Adrenalin, moreover, diminishes fatigue in muscles and increases the coagulation of the blood, so that in cases of a destructive wound there is less danger from bleeding.

Repeated injuries to the surface of the body, if not too severe, lead to a thickening of the epidermis in the form of callosities, and actually destructive injury leads to the processes of repair which are characterized primarily by the overgrowth of connective tissue (cicatrix) denser than epithelium and better able to protect the surface against future injury. The epithelial overgrowth on injured surfaces is often in excess and it may, in certain instances, lead to a tumor growth of possibly malignant nature. Such overgrowth must be regarded as the overdoing of a normal protective physiological function.

Foreign bodies, whether simply lying on the surface of the body or the outer reaches of the respiratory and alimentary tracts, are potential traumatic agents, either in themselves or as leading to secondary infections. The various reflexes such as sneezing, coughing, and vomiting are the first protective mechanisms designed to get rid of these foreign particles. When foreign bodies have penetrated beneath the superficial layers of the body, the phenomena of inflammation and particularly the formation of giant cells are all adapted to the purpose of isolating these extraneous agents and rendering them innocuous.

There are a number of secondary and of ten chronic diseases produced as the result of mechanical obstruction or distortions, that may occur to the mind as proper subjects for inclusion at this point. We 
refer to such abnormalities as valvular heart lesions, stricture of the urethra, atelectasis, "emphysema, pancreatic cirrhosis, and the like. They likewise give rise frequently to compensatory physiological mechanism, but are not included for elaborate discussion, as they do not come in the immediate picture of primary disease causes. The very fact that these recognizable secondary diseases are endogenous in origin leads one again to suspect that those maladies listed as "endogenous" are due primarily to some external agent.

Another set of physical agents are those dependent on changes or disturbances of atmospheric pressure. There are three definite diseases which illustrate the effect of pressure changes on the body: mountain sickness, produced by altitude or diminished oxygen pressure; caisson disease, produced by increased pressure with too rapid restoration to atmospheric pressure; and seasickness, which presumably is caused by the effect of variations of pressure in the semicircular canals of the ear. There is not, so far as we are aware, evidence of habituation or increased resistance to either seasickness or caisson disease. In the case of mountain sickness, however, there is a delicately adjusted protective mechanism at hand, as has been clearly described by Sewall $\left({ }^{11}\right)$ in a previous article in this volume. Mountain sickness due to altitude is brought about by the diminution in atmospheric pressure, with consequent decrease of oxygen tension, and is complicated by the effect of cold, and possibly, to a minor degree, by the effect of ultra-violet light (Hasselbalch and Lindhard) $\left(^{12}\right)$. The body rapidly responds to the lack of oxygen in a number of interesting ways. The red blood cells are rapidly increased, frequently to as high as seven and a half million in a few days, and the hemoglobin, designed to carry the oxygen, is also increased, but somewhat more slowly. Mountain sickness apparently is accompanied by an alkalosis of the blood, and to compensate for this the hydrogen-ion concentration, at first diminished, may increase above normal as a result of acclimatization to high altitudes (Sundstroem). The blood flow is increased, often as much as forty per cent., and remains slightly increased. The large mononuclear cells may increase up to twenty-five per cent., but for what purpose is not exactly known. Breathing likewise is increased in rapidity. The acclimatization to these high altitudes decreases the $\mathrm{CO}_{2}$ tension of the blood, and causes a relative rise in the alveolar oxygen pressure (Haldane).

The adaptive mechanism in aviators apparently lies almost entirely in the breathing and circulation (Schneider) $\left({ }^{13}\right)$. This acclimatization to high altitudes is the best instance available as showing the importance of training in adaptation to a new and at first harmful 


\section{MECHANISM OF BODILY RESISTANCE TO DISEASE}

environment; it is probable, however, that other less violent changes in environment bring into play similar mechanisms of adjustment.

In general, changes in climate are secondary or contributing causes to disease rather than primary causes, as in the particular instance of mountain sickness just quoted. Tropical diseases, for example, are not primarily due to the temperature of the tropics, but to the parasitic agents that live in the tropics. Light intensity and excessive heat, characteristic of tropical climates, do indeed give rise to definite diseases which will be considered under the next-or thermal-group of the physical causes of disease.

Too great heat or cold produces physical disturbances in the human and animal body which may be classified as definite diseases under certain conditions. The effects of heat are either local or general. Local heat when applied to any part of the body gives rise to burns of varying degree and intensity which, if sufficiently severe, lead not only to local necrosis but to the death of the individual. It is not in our province to consider the types of burns or the anatomical or functional disturbances produced by them. We find, however, that local tolerance can be produced by repeated exposure to excessive heat. This tolerance is evidenced in the thickening of the epithelium in the mouth of glass blowers and metal workers coincidentally with a lessened susceptibility of the nerve endings in these localities. Fuerst $\left({ }^{14}\right)$, who has studied this phenomenon experimentally, found that repeated heating of the guinea pig's ear to a temperature of $55^{\circ}$ led to a gradual thickening of the epithelium so that it reached from six to eight times its original depth. Coincidentally, exudation and the proliferation of giant cells were noted in the lower layers of the skin. This thickening of the epithelium finally became stationary; if, however, the heat is again raised to $55^{\circ}$ thickening continues to progress.

The general effects of increased temperature are evidenced by heat stroke or hyperthemia, and by insolation or sunstroke. Individual differences in resisting extreme heat are very marked, and repeated exposure, when the first exposure is not too severe, leads to habituation and resistance. This is evident in the resistance of coal passers and foundry workers, who come to resist heat that would be fatal for unaccustomed individuals. This increased tolerance to heat is due to some adaptative regulation of the heat mechanism the nature of which is not definitely known.

Other instances of the effect of excessive heat are complicated by the effect of the luminous rays, as in the exposure to tropical sunlight and in ordinary sunburn. Sunburn is a clear instance of a protective mechanism producing pigmentation which has the purpose of rendering 
the superficial layers of the skin better absorbents for the ultra-violet rays and thereby protecting the deeper and more vital layers. The almost perfect resistance of blacks to tropical sunlight has been shown to be due to a mechanism of this sort (Möller) $\left({ }^{15}\right.$ ).

A thickening of a pigmented epithelium may follow as in the disease xeroderma pigmentosum. In this condition, and more particularly in X-ray burns, the thickening of the epithelium, perhaps primarily for protective purposes, often leads to a malignant and invasive epithelium.

A somewhat similar though less accentuated adaptation to local and general cold has been shown by Nansen and other explorers who have been able to live at low temperatures and tolerate them. And again, repeated exposure of the skin to cold, when not accompanied by necrotizing lesions, leads to gradual thickening of the epithelium and greater resistance as in the case of local heat exposure. In general the heat-regulating mechanism of the body is less able to bear extreme cold than extreme heat.

\section{CHEMICAL AGENTS}

There is a group of well-known and often well-defined chemical substances against which varying grades of individual natural tolerance and of increased tolerance or habituation are evident. These conditions of tolerance suggest the natural immunity and acquired immunity produced by certain of the animate causes of disease, but may be separated from them by certain criteria. In the first place, the chemical substances here treated are non-protein, and therefore non-antigenic in nature. That is to say, they do not give rise to antibodies on injection into animals as do substances of protein nature, and the increased resistance to them is not due to the presence of such antibodies or antitoxins. The sharp separation between these two groups of protein and nonprotein chemical substances as antigens and non-antigens has been confused by the work of Abderhalden. He has described the production of ferment-like substances, having the specific property of splitting proteins, or carbohydrates, and produced by injection or natural absorption of non-antigenic substances such as gelatine, the polypeptids, and glucose. These ferments, however, are not specific and their increase following immunization is now questionable.

These non-antigenic chemicals differ from antigenic proteins in that they are crystalloids rather than colloids. They resemble proteins in that they are more toxic for warm-blooded animals than for coldblooded animals, and again differ from them in that they require no 


\section{MECHANISM OF BODILY RESISTANCE TO DISEASE}

incubation period, which is usually necessary with antigenic poisons. As a result of injection or application of certain of these chemical agents in non-fatal doses, a distinctly increased resistance may arise in the animal so treated. This resistance, although evident, is never so marked as in the case of the immunity acquired to antigenic poisons. On the other hand, a condition of hypersusceptibility, resembling in many respects a condition of anaphylaxis, to protein substances may be produced. Both of these conditions of habituation and hypersusceptibility may, as in the case of protein poisons, be specific in nature. There are some conditions in which the hypersusceptibility can be transferred from one animal to another by means of the serum, thus again resembling reactions to proteins. Conditions of local hypersusceptibility to drugs, like strychnine and atoxyl, have been noted and resemble the Arthus phenomena of local protein anaphylaxis. Symptoms of generalized anaphylaxis may follow a second dose of salvarsan. It has been suggested by Wells that these conditions may be due to a combination of the non-antigenic substances with the protein of the animal that is affected.

Another group of individual drug hypersusceptibilities or idosyncrasies should be noted here; they occur with substances like iodoform, corrosive sublimate, veronal, and morphine, and are demonstrable both locally and generally. Although transmitted by the serum of the susceptible individual, they apparently do not depend on previous sensitization and therefore do not require an incubation period, as do the instances of hypersusceptibility just mentioned.

Some of the best studied and interesting instances of chemical and drug tolerance have been in unicellular organisms. They have been well reviewed by Von Hausmann $\left({ }^{16}\right)$. Massart, for example, was able to habituate flagellates to normally intolerable concentrations of sodium chloride. Davenport and Neal were able to make Stentor tolerant to doses of sublimate that were four times the usual fatal dose. Ehrlich's experiments on drug-fastness are well known and of great practical significance. It was found that trypanosomes could be driven from the circulating blood in infected mice by a dose of fuchsin, although not all the organisms were killed. The trypanosomes would then reappear and could again be driven to cover; further therapeutic attempts were unavailing, as the organism had become fuchsin-fast. Similar results were found with atoxyl, trypan red, and other drugs. The fastness of the trypanosomes to a given dye is more or less specific and the interesting and unexplained fact is that it is transmitted for many generations.

Similar instances of drug-fastness have been observed with bacteria. 
Dansyz, for instance, trained anthrax bacilli to live in an appreciable concentration of arsenic. In this case the tolerance was definitely associated with the formation of the capsule, and capsule extract was found to neutralize the drug. Certain strains of yeasts have been raised by Effront in normally inhibiting solutions of ammonium fluoride. The tolerance here depends on the production of calcium that unites with the fluoride to form a harmless compound.

Wells $\left({ }^{17}\right)$ has discussed the means of defense against non-antigenic poisons. Nearly all harmful substances have their effect modified in the animal body by one or several chemical or physiological reactions. These natural protective mechanisms may be classified as follows:

(I) Rapid elimination-by diarrhea and vomiting, either before absorption of the poison, or even after absorption when the substances are excreted into the digestive tract.

(2) Deposition and fixation in single organs or tissues, particularly in the liver, which acts as a blood filter. This mechanism of fixation by tissues may be increased by repeated use of a poison. In the liver arsenic is fixed by the nucleus, and mercury by the globulins. Some of the alkaloids are combined with bile acids and some poisons combine with glycogen. The bones hold certain of the poisonous fluorides in relatively harmless form by combining them with calcium.

(3) Poisons may combine with substances formed or contained in the tissues, the resulting substances being less toxic than the poisons alone. Thus the lipoid-soluble narcotics are kept from the central nervous system by combination with tissue fats.

(4) Chemical alterations may be produced in the poison in ways that will be presently considered.

(5) Certain injuries may impair the absorption power of cells and these injuries may be produced either by the poison itself or some preexisting condition.

The chemical alterations in poisons which may take place differ first with the nature of the poison concerned, that is, with whether they are inorganic or organic. With the inorganic poisons there may take place a reduction, oxidation, or the splitting off of water, which latter condition includes the neutralization of acids and alkalies and the formation of albuminates and sulphides. With the organic poisons oxidation may take place, as when the fatty acid series are introduced into the body. A combination may occur with various chemical substances already in the body, and this may occur with or without preliminary oxidation. The principal of these substances which neutralize organic poisons are the following:

(I) Sulphuric acid, which unites with the phenols and cresols. 


\section{MECHANISM OF BODILY RESISTANCE TO DISEASE}

(2) Glycuronic acid, which combines with naphthol, thymol, camphor, chloral hydrate, and butyl chloral. acid.

(3) Glycocoll, which combines with benzoic acid to form hippuric

(4) Urea, which forms salts with the organic acids.

(5) Methane, which combines with the pyridine group and gives a methyl compound less toxic than the original alkaloid.

(6) Sulphur, split off from proteins, may combine with $\mathrm{CNH}$ and $\mathrm{CNK}$, converting them into much less toxic sulphocyanides.

(7) Bile acids form insoluble compounds with certain inorganic poisons, and also with some of the alkaloids like strychnine and quinine. In addition to these chemical combinations, a neutralization and dehydration of some organic poisons may occur.

With this outline of the general methods by which poisons are tolerated in the body, we may proceed to consider more particularly certain instances in which an increased tolerance or habituation occurs following repeated doses of well-known chemical substances. Whereas the original neutralization mechanism which we have outlined resembles natural immunity to bacteria, this increased tolerance or habituation resembles acquired immunity.

Morphine tolerance. The acquired tolerance to morphine evidenced by habitués is apparently due, to judge from Faust's $\left({ }^{18}\right)$ experimental work on dogs, to an increased ability to oxidize the drug. Faust found that about seventy per cent. of the morphine in normal animals is eliminated through the feces. In tolerant animals, however, the amount of morphine found in the feces decreases markedly and may eventually disappear, nor can it be detected in any other tissues of the body. The only obvious conclusion is that it has been destroyed in the body in some manner. Something like twenty-seven times the fatal dose of morphine may be taken by one who is tolerant to it, i.e. as much as $5^{1 / 2}$ grams a day, while 0.2 of a gram is usually fatal. It would seem that the simple destruction of morphine is not the only mechanism which renders it less fatal in habituated persons. Rübesamen $\left({ }^{19}\right)$ and Meyer and Gottlieb $\left({ }^{20}\right)$ found that the brain of morphine tolerant rats contains more morphine than the normal animal, and it would therefore seem evident that the brain tissue is actually more tolerant than normal. The symptoms produced by withdrawal of the drug, or morphine hunger, are another evidence of some cellular participation in the habituation.

Tolerance to digitalis and atropine. Clinical observations on the use of atropine to prevent night sweats in tuberculosis show that a distinct tolerance may be engendered by repeated doses. According to the work of Lhotak, as quoted by Doerr $\left({ }^{21}\right)$, digitalis is more readily 
broken up in tolerant rabbits than in normal ones. The resistance to this drug therefore apparently resembles morphine tolerance.

Alcohol tolerance. Although alcohol tolerance is evident in human beings where symptomatic effects can be clearly judged, it has not been experimentally demonstrated in animals. There is evidence to show. that alcolol is more rapidly absorbed from the intestines in habitués (Voltz and Deitrich) $\left({ }^{22}\right)$, and remains longer in the blood.. Further understanding of this tolerance does not seem to be at hand.

Arsenic tolerance. It may be that the tolerance of the so-called "arsenic eaters" has been somewhat exaggerated, but there seems little doubt that the habitués can consume from two to four times as much as normal individuals without harm. Experimentally in animals it has apparently been shown by Cloetta $\left({ }^{23}\right)$ that a tolerance to arsenic which can be produced in dogs is due to the failure of the intestinal mucosa to absorb the poison, since these animals are found as susceptible as normals when the drug is given subcutaneously. It is probable, however, that resistance to arsenic cannot be entirely explained on this simple basis. There would appear also to be increased tolerance in other cells. Adami and $\mathrm{McCrae}\left({ }^{24}\right)$, for example, have described an active intervention of the leucocytes in disposing of arsenic in the peritoneal cavity. It is also known that the nucleoproteins of the liver combine with arsenic.

Tolerance to other drugs such as cocaine, hasheesh, and nicotine seems definitely proved in human beings, but little is known as to the mechanism involved.

\section{BODILY DEFENSE IN DISEASES DUE TO ANIMATE EXOGENOUS AGENTS (IMMUNOLOGY)}

We have endeavored to point out the great importance of that group of diseases in which the causation is known, or assumed on reasonable grounds, to be due to infection by some external animate agent. Approximately one-half of all of the diseases with which we are acquainted, and so far as can be estimated one-half of the actual deaths, are referable to agents of this nature. There can be little question, then, as to the relative importance of the infectious diseases in any consideration of disease as a whole. The importance of the infectious diseases becomes even more manifest when we consider that it is in this group of diseases that we possess the most satisfactory, complete, and sequential group of facts. These facts not only have the advantage of completeness which renders their logical presentation possible, but have led in a number of instances to practical results of the greatest importance in diagnosis, prevention, and cure of disease. 
We find ourselves embarrassed by the facts that have been accumulated the last few years and which may be grouped in the justifiably separate science of immunology. These facts are so numerous, so recent, and so rapidly increasing as to make most difficult their presentation in a limited treatise of this kind designed to give a balanced idea of our present working knowledge. It will be our effort to emphasize the relation of the most important facts to one another rather than to mass all the important facts in any one or in several aspects of the question.

It would, perhaps, be more accurate to designate diseases or conditions which we purpose to discuss in this section under the heading of "Diseases due to foreign proteins." We shall find that although the infectious diseases, strictly speaking, are due in each instance to the entrance, extension, and multiplication of some living microscopic plant or animal, a bacterium, a spirochete, a fungus, a mould, or a protozoon, many of the conditions they engender are due simply to the fact that these exogenous agents are foreign proteins. Inanimate protein, as well as the living tissue from which it is derived, can produce disease and lead to reaction changes of immunity and hypersusceptibility.

It is rot in our province in this section to consider in any detail the animate causes of disease or their modes of infection. That has already been ably done elsewhere in this volume. In the presentation of our subject, however, it would be impossible, particularly in view of our development of it on the basis of etiology, not to have constant reference to the agents of disease, and the methods in which they act. It seems unnecessary in this place to consider in detail the evolution of our knowledge concerning the nature of infectious diseases in general. The earliest conception of "contagion" by which Frascator (1546) explained the direct transmission of diseases had the practical value of introducing measures of quarantine. The differentiation of certain of the infections as due to indefinite "miasms" was merely misleading. Neither of these ideas aided directly in pointing to the real agents concerned in producing the infectious diseases. The discovery of bacteria by Leuwenhoeck (1675) and their detection in wounds by Recklinghausen ( $187 \mathrm{I}$ ) led first to an idea that a single microorganism was responsible for all infections (Cohn), and this conception was gradually replaced by the demonstration of specific etiology as developed through the work of Pasteur and Koch. Our data remain most complete, and the results of specific diagnostic methods and specific therapy most effective, in the diseases due to the fission fungi or bacteria. It has been shown, however, that both local and general infection may be caused by minute organisms of other sorts: by protozoa, including both the rhizopods (amebiasis), the sporozoa (malaria), and the 
infusoria (relapsing fever, syphilis, sleeping sickness); by worms (bilharzia, filariasis, trichiniasis); by the moulds (ringworm, favus, sporotrichosis, blastomycosis); by the higher fungi (actinomycosis); by the as yet unidentified microorganisms called "filtrable viruses," or ultramicroscopic organisms (rabies, poliomyelitis, yellow fever); and finally, the undoubtedly microorganismal agents of disease of unknown nature (scarlet fever, measles, typhus fever, smallpox).

Both for technical reasons and because they are there most striking and effective, the defensive mechanisms of the animal body, the immunity phenomena, which we are here concerned in developing have been most completely worked out in connection with bacteria. It is known, however, that in type of response, at least, although frequently not in equal degree, the immunity responses to the other microorganismal agents are similar to those to bacteria. In other words, all pathogenic microorganisms partake in the general antigenic property of proteins. The degree of involvement of each disease has much to do with the extent of the response evoked. The skin diseases, for example, produced by the moulds lead to only slight immunity reactions in patients, although antibodies may be produced by animal inoculation. Of the diseases due to animal parasites those producing systemic disturbances with blood invasion are most likely to produce immunity phenomena (e.g. filariasis, trypanosomiasis, relapsing fever), whereas those that are more local in effect produce little or none (e.g. anchylostomiasis, amebiasis).

As already mentioned, the infectious diseases may be defined as due to the entrance, extension, and mutiplication of a living parasitic agent in the animal body. The entrance is effected by the parasite through the normal channels of contact with the external world, as the skin and the respiratory or gastrointestinal tract, or the parasites may be accidentally aided in reaching the body by mechanical wounds or abrasions. Such accidents are usual and designed in the case of protozoon diseases that are introduced into the blood by biting insects (malaria, trypanosomiasis). The need of a specific portal of entry for certain disease agents is known (malaria, gonococcus, cholera vibrio), whereas with other microorganisms it is not necessary. The various aids possessed by pathogenic microorganisms for overcoming the natural obstacles that represent the external means of defense of the host have been considered in the section on infection. A definite incubation period is known to be necessary before the manifestations of an infectious disease are evident. This incubation period is dependent on the rapidity of multiplication of the bacteria concerned and their pathogenicity, and perhaps also dependent to some extent on the 
elaboration of certain immunity substances in the body of the host (Vaughan) $\left({ }^{25}\right)$. The animate disease agents produce their harmful effects to some extent by simple multiplication, but more particularly by the production and elimination of certain poisonous substances, which must be taken to include not only the toxins properly speaking, but the foreign proteins of which the external agents are themselves composed. The problem of virulence or pathogenicity of bacteria as bearing on infection is of fundamental importance but will be discussed only as incidental to our subject.

The infectious diseases are characterized by a group of symptoms with certain general features and presenting in each particular disease other specific characteristics. These symptoms are both local and general, depending on the degree of penetration of the microorganisms concerned in producing the disease. We shall later see that the type and grade of immunity produced to any given microorganism varies markedly in accordance with the depth to which the foreign agent has penetrated in the body. The local symptoms of infections are inflammations of various types dependent on the nature of the invading microorganism and its toxins and on the particular tissue or tissues of the host that are affected. The most important general effects of an infection are fever, a disturbance in the leucocytes, usually as evidenced by their increase, and secondary results such as anemia, hemorrhage, and general nutritional changes. Most characteristic of all the changes in the host, as the result of an external animate agent, are the immunity reactions which have the result in various ways and in varying degrees of neutralizing the effects of the invading microorganism.

The altered condition produced by inanimate foreign proteins is somewhat more difficult to define. In chemical constitution these proteins are at times identical with some of the plant or animal parasites which, when alive, might produce disease, but they further include chemical substances derived from any harmless living animal or plant. They differ from the microorganisms which produce infections in that they are not living and therefore not able themselves to invade the body and to multiply within it. Their effect on the body is therefore due either to accidental contact, to artificial inoculation, or to some alteration that renders their assimilation in unchanged form from the gastro-. intestinal tract possible. These protein substances produce disease or abnormal conditions more frequently on their second or repeated introduction into the body in the condition known as anaphylaxis or hypersusceptibility, under which heading they will later be discussed. 


\section{THE NATURE AND SPECIFICITY OF ANTIGENS}

The phenomena which arise in the animal body following the introduction of the protoplasm of any foreign cell, whether plant or animal, are evidenced by an increased power of reaction, and are usually evidenced by the presence in the blood of antagonistic substances known as antibodies. Conversely, any substance capable of producing such antibodies in the animal body is known as an antigen. The only chemical substances that have certainly been proved to be antigenic are those characteristic components of protoplasm known as proteins. Some evidence has been offered to show that certain lipoids and at least two glucosides are antigenic, but it seems likely in the case of the lipoids that impure preparations were being dealt with (Wells). The two glucosides claimed by Ford $\left({ }^{26}\right)$ as antigenic are in the case of the one frcm Amanita phallades perhaps not a glucoside (Rabe) $\left({ }^{27}\right)$, and the apparently proved glucoside in Rhus toxicodendron was not found by Von Adelung $\left({ }^{28}\right)$ to be antigenic.

So far as we are aware, all proteins are antigenic provided they be in a colloidal state and soluble in the body fluids; that is to say, if a protein be hydrolyzed by ferment or chemical action to the state of polypeptids (Gay and Robertson) $\left({ }^{29}\right)$, or be coagulated, it loses its property of originating antibodies.

Closely linked with the antigenic property of proteins is the individuality of tissue as evidenced by the specificity of reaction between each antigen and its antibody. On the existence of a "specificity," used in its biologic sense, depends the proved etiological association of a given pathogenic bacterium with the peculiar disease that it produces. And again, the distinctive resistance produced by recovery from a bacterial infection or produced artificially by injection with a bacterial vaccine is specific in nature; it is evidenced by an antagonism against this particular foreign microorganism alone. The characteristic diagnostic reactions between antigen and antibody, of which we shall later speak in detail, depend for their accuracy on their essential specificity.

We possess little further information on the exact nature of specificity beyond the rigorously proved relations that have just been outlined. Protein chemistry is as yet unable to give us the key to the riddle of tissue differences; the delicacy of its methods are at present far outstripped by the biological reactions of immunity. Casein derived from cows' milk and from human milk are indistinguishable chemically, the protein complexes in horse serum and dog serum are not yet separable on the basis of molecular analysis, and yet in both cases immunity reactions by means of corresponding antisera will delicately differentiate 


\section{MECHANISM OF BODILY RESISTANCE TO DISEASE}

between each of the two apparently identical substances. Our present information of the chemical nature of specificity is fully and ably discussed by Wells, to whom the reader is referred for further information. We can treat of the matter here only in its most general aspects. In general, biological specificity is related to the species identity of protoplasm. The injection of a microorganism identified as species "A " into animal " B" gives rise to antibodies which produce characteristic reactions with any individual of species "A," but not with individuals of species " $C$ " or " $D$," unless species " $C$ " or " $D$ " be so closely related to "A" as to give partial or group reactions which by quantitative methods can be differentiated. In the same way the serum of animal " $E$ " injected into animal " $B$ " gives rise to antibodies that react only with the serum of species "E." Such antibodies, however, will be found to react not only with the serum of animal " $E$ " but with the tissue extracts of animal "E." In other words, antigenic specificity is based first of all on the protein identity of the protoplasm of the species concerned. A second possibility arises. It has been found that the protein constituent of the crystalline lens of the eye is apparently identical in all animals, indeed is more nearly identical than is the protein of the lens in a given animal with any other tissue in that animal. Consequently an anti-lens serum reacts with the extract of any lens irrespective of species, but not with other tissue extracts from the species furnishing the original lens. There is evidence also that the specificity depends not only on the chemical constitution of proteins, but on the physical arrangement of its molecules (Obermeyer and Pick) $\left({ }^{30}\right)$. Specificity can be removed from a protein by its degradation, and specificity can actually be produced by building up a protein by the reversible action of ferments, or by compounding non-antigenic with antigenic proteins [Gay and Robertson $\left({ }^{31}\right)$ ].

It is possible that the proportions and arrangement of the aminoacids in a protein are responsible for its specificity. An apparently simple substance like egg-white can be shown to contain at least five separate antigenic units (Wells). On the other hand, the antigen necessary to produce a hemolysin for sheep's cells was found by Forssmann $\left({ }^{\mathbf{3 2}}\right)$ to be contained in the tissues of several other animals. These examples serve to show the present complexity of a phenomenon which is as important as it is inexplicable. Fortunately the usefulness of the many reactions based on the reliability of biologic specificity are unaffected by an ignorance of its essential principles. The facts of specificity will find an apparent explanation, which has served to dull the curiosity of many, in Ehrlich's famous hypothesis. This side-chain hypothesis, however, has been found erroneous in its fundamental assumptions, and in 
its further detail may be regarded as a simple restatement of fact. We prefer, therefore, not to assist in the perpetuation of an ingenious but on the whole erroneous hypothesis by rehearsing it except in those connections where it has aided, usually through efforts to refute it, in stimulating the discovery of new facts of importance.

\section{TYPES OF RESISTANCE AND IMMUNITY TO THE INFECTIOUS DISEASES}

The animal body possesses an elaborate mechanism for preventing the entrance and extension of pathogenic microorganisms. Even when such an external agent of disease has gained a foothold in the host sufficient to produce local or general symptoms, the body continues to resist with more or less success its further advances. The evolution of an infectious disease has often and correctly been likened to a battle, the outcome of which depends on the balance established between the offensive and defensive forces. Recovery from such infections, although temporary or even permanently accompanied by some disability, may be signalized by a more or less durable and often an apparently absolute immunity against the particular infection concerned. It is the chance observation of such instances of natural resistance and of acquired immunity that led to successful attempts to increase the former and to produce the latter artificially. A few of these procedures succeeded through pure empiricism founded on shrewd observation and logical deduction; with the rapid and continually expanding knowledge of the microorganisms of disease, and of the bodily mechanism of defense against them, we have increased the instances, the safety, and the certainty of specific therapy of this sort.

\section{NATURAL IMMUNITY}

It is a matter of observation that certain infectious diseases characteristic of one of several animal species rarely or never occur in other animals. Animals of closely allied species have a certain community of susceptibility or insusceptibility to any given infection; they are less likely to suffer from a disease that is characteristic of animals that are remote from them in biological series. Such a condition of relative resistance is often referred to as a species immunity. In a general way the infections of cold-blooded animals are distinct from those of warm-blooded animals; for example, fish tuberculosis does not occur in warm-blooded animals, and anthrax and tetanus, which are characteristic diseases of warm-blooded animals, do not affect the coldblooded animals. Certain characteristic diseases of birds rarely occur spontaneously in mammals, for example, psittacosis; and the reverse is true, since anthrax does not occur in birds. And again the carnivora 


\section{MECHANISM OF BODILY RESISTANCE TO DISEA'SE}

and herbivora have each certain typical maladies; anthrax and tuberculosis do not affect dogs or rats, though very fatal to cattle. Bubonic plague does not occur in cattle.

The most interesting instances of species insusceptibility are in connection with many of the characteristic human diseases; the exanthemata, measles, and scarlet fever, also gonorrhea, syphilis, and typhoid fever, never occur spontaneously in other animals and can be produced only experimentally, and then in not quite characteristically human forms, in such animals as are the most commonly allied to man, the anthropoid apes.

Species immunity of this sort is rarely absolute and varies in degree with each particular case. It may be evidenced in one of three general ways: first, by the fact that the disease does not occur spontaneously in a certain animal species, but may be produced experimentally by inoculation; secondly, by the fact that when experimental infection is possible, relatively large doses are necessary to accomplish it; and finally, in some cases, relative resistance is indicated by the fact that the disease, which usually occurs in a systemic form, when introduced into the insusceptible animal, will produce only a local lesion, e.g. anthrax bacillus inoculated into dogs. We reserve a more complete description of the mechanism of the various types of immunity for a subsequent section, but at this point it may be stated that species immunity is due to variations in the temperature of the animal concerned, to variations in its metabolism, and frequently to a variation in the reaction in the intestinal canal when the normal portal of entry is through this tract.

\section{RACIAL IMMUNITY}

Racial immunity has been noted in several well-known instances where it has been found that although the species as a whole is extremely susceptible to a given infection, certain varieties or races remain relatively unaffected. It has been noted that Yorkshire swine do not suffer from erysipelas, and that Algerian sheep rarely die from anthrax.

An analogy to some extent applicable to racial immunity is perhaps the fact that certain races of mice are insusceptible to inoculations of malignant tumors, although here again we are not to regard malignant tumors as infectious in origin. These instances of racial immunity are probably due largely to differences in food and in hygiene.

Perhaps most interesting of all the forms of natural immunity is the relative resistance of certain individuals to an infection to which the majority may succumb. Such individuals have been noted since the time of Galen, and although exposed to the chances of epidemic infection, and even in many instances exposed by caring for the sick, they fail to 
contract the disease. These instances are apparently apart from the relative insusceptibility of people that are outside the normal age limit for the disease in question. They may, in some instances, be explained by better nourishment, better health, and perhaps also by certain differences in their blood, using this in an indefinite sense to indicate those protective substances which are normally there present. In general, however, individual immunity is probably not natural immunity at all, but is to be regarded as due, particularly when it is absolute, to an immunity acquired by recovery from an unperceived or forgotten attack of the disease in question.

\section{ACQUIRED IMMUNITY}

It has been noted, ever since diseases were identified and separated one from another, that individuals who had recovered from certain of the infections were thereafter not liable, or only rarely liable to a recurrence. This is true particularly in respect to the exanthemata, scarlet fever, measles, chicken-pox, and mumps, and also in the diseases plague, typhoid fever, cholera, smallpox, yellow fever, typhus fever, and anterior poliomyelitis. Instances of recurrence in these diseases may be cases of mistaken diagnosis or may be the exceptions which prove the rule. There are other diseases of an infectious nature in which the existence of a durable immunity is impossible or difficult to state. Such diseases are syphilis and probably also diphtheria. Tuberculosis, again, is a disease the occurrence of which in childhood is often regarded as producing insusceptibility under normal conditions for many years or for life. In other diseases, such as those produced by the pyogenic bacteria, and in gonorrhea, pneumonia, influenza, glanders, dengue fever, recurrent fever, tetanus, and erysipelas, there is at least no evidence of a durable immunity, but it is probable that in some of them an actual immunity does exist for at least an appreciable length of time, as is evidenced by the fact that antibodies are known to increase at the end and just after the disease and to be the cause of recovery therefrom, e.g. recurrent fever and pneumonia. The existence of a temporary and definite immunity following recovery from an infection is extremely difficult to appreciate and is often overlooked. In addition to a general immunity there is also evidence in some diseases of a local immunity, as evidenced by the fact that the disease is not likely to recur at the original point of infection.

The fortunate condition of immunity acquired by recovery from an infection has not only been noted since the time of the ancients but the fact made use of in a practical way. Individuals who have recovered 


\section{MECHANISM OF BODILY RESISTANCE TO DISEA'SE}

have frequently been used as nurses in hospitals where a given infection is prevalent, e.g. in plague epidemics, in smallpox, and in yellow fever. Various efforts have also been made to reproduce acquired immunity artificially by inducing the disease in healthy people either in a modified form or in such a way as to produce a local rather than general reaction. Metchnikoff $\left({ }^{33}\right)$ gives as an illustration the observations of a Portuguese traveler in Africa-Serpa Pinto-who found that the natives had discovered that they could protect themselves against the bite of venomous snakes by injecting small amounts of this venom mixed with resin under the skin. It is probable that the Hopi Indians in their snake dance, where they allow themselves to be bitten by rattlesnakes, have also utilized a method of immunization of some sort. The Moors, many years ago, protected cattle against pleural pneumonia by inserting a sword under the skin of a healthy animal after plunging it into the lung of an animal dead of the disease. The best instance of active immunization of this sort is, of course, the protection against smallpox that has been produced by the Chinese and East Indians since the eleventh century. "Variolization" was practiced either by inserting the scabs of smallpox pustules in the nostrils or by practicing arm to arm inoculation. In a healthy individual the disease produced by this form of inoculation was usually only local and harmless, but in certain instances unpleasant and even fatal results occurred. The method was introduced into England by Lady Montague, as is well known, in I78I, and practiced for a number of years until the introduction of vaccine by Jenner in 1798 .

Jenner's great discovery of the efficacy of vaccine was the result of shrewd observations and experiment, but the principle on which it rested was not appreciated until the work of Pasteur. Cowpox. (vaccinia) represents a modified or attenuated form of smallpox, although we have little more information regarding the actual nature of the virus concerned in these two diseases than had Jenner himself. It had frequently been noted that milkmaids who handled the teats of cows that were suffering from pustular eruption known as cowpox frequently acquired these pustules on their hands, and it was further observed that such individuals failed to contract smallpox when exposed to the chance of infection during an epidemic. Vaccination is superior to variolization because the vaccine is always available in fresh form. More important still, the harmlessness of the disease produced is assured, not only in so far as smallpox is concerned, but also in that the possibility of transmitting concomitant diseases in human beings is eliminated. It is not our purpose at this point to discuss the efficacy of vaccination. This will be considered at a later point. Attempts have been made to apply this 
principle to other diseases belonging to the same group, e.g. measles and scarlet fever, but no results have been obtained.

No further knowledge of protective inoculation or further instances of similar protection were discovered from the time of Jenner until the work of Pasteur on chicken cholera in 1879 . Pasteur, by a combination of chance and a flash of genius, found that if he allowed a pure culture of the organism that produced cholera to become attenuated, he could then inoculate fowls with such cultures without producing the disease but thereby protected them against infection with that microorganism. Pasteur recognized that he had simply rediscovered Jenner's principle of vaccination, and thereafter referred to his process by that name, which gives us a justifiable precedent for continuing the use of the term "vaccine" for preparations of bacteria modified in various ways and utilized in the prevention and cure of disease. By a similar method of vaccination Pasteur was able to prevent anthrax in sheep and later erysipelas in swine. It is the same principle which has further been employed in prophylactic immunization against typhoid fever, cholera, plague, and pneumonia in human beings. The next great step in the evolution of vaccination depended on Pasteur's discovery that one disease at least-rabies-could not only be prevented by previous vaccination, but owing to its long incubation period, could actually be cured, or at least prevented from developing symptoms, when treated sufficiently early. We shall later consider how the work of Wright ( ${ }^{\text {s4 }}$ ) and others led to the further evolution of the principles of therapeutic vaccination even after the symptoms of the disease are manifest.

\section{ACTIVE AND PASSIVE IMMUNITY}

We have summarized briefly the condition which constitutes what is known as natural resistance or immunity, and found that it varies relative to species, races, and individuals. Secondly, we have outlined the condition of acquired immunity which is produced either by recovery from a chance infection or else produced artificially. These forms of immunity are strictly the property of the individual or animal concerned. They have been produced in the case of acquired immunity by a certain reaction on the part of the animal, in other words, by an active participation by the animal in combating the infection concerned. We shall later consider in more detail the nature of this reaction and the characteristics of the active immunity which the animal body acquires, whereas here we are dealing simply with the practical expression of this immunity in so far as the fate of the animal is concerned. As we have already mentioned, the duration 
of active immunity acquired by recovery from an infection varies in the degree of protection it affords. Recovery from certain diseases would seem to protect for life in the majority of cases, whereas in other diseases the protection is very temporary or may actually be marked by no apparent increased resistance. It is probable, even in those diseases in which durable immunity is produced, that this durability should be qualified by saying that it is effective only in so far as chance infection or infection under ordinary conditions of virulence and dose are concerned. It is probable indeed that, in diseases like typhoid fever where the recovery seems to endure throughout the life of the individual, this is only true in cases under ordinary circumstances, but does not hold true under unusual circumstances. There are, furthermore, types of recovery connected with various conditions of relative immunity in which even the recovery is not complete. The condition that is known as " non-sterilizing immunity," for example in many of the protozoan infections such as malaria and sleeping sickness, is characterized by the fact that the antibodies produced are sufficient to kill the majority of parasites in the animal but leave a few resistant microorganisms which subsequently are able to repeat the infection.

Under usual conditions the active immunity acquired by recovery from a disease is more marked and durable than active immunity produced by artificial means. There is a distinct relation between the severity of the reaction produced on the one hand by the disease itself, and on the other hand by vaccination against the disease and the result produced.

Active immunity may be produced by employing the agent which produced the disease modified in various ways. In the first place, the clisease itself may be produced in modified form by utilizing the living virus which causes the disease under natural conditions. The old type of variolization against smallpox is an instance of immunization of this sort. By choosing animals when relatively insusceptible, or by localizing the point of the infection, this modified disease, under usual conditions, may be rendered sufficiently less dangerous than the natural infection. Living cultures of bacteria have in certain instances been used, as for example in the method of vaccination against cholera, in the process. originated by Ferran, who found that living cultures of this organism which normally produced infection through the intestinal tract could be used harmlessly for subcutaneous injections. The usual method, however, is to employ preparations of bacteria in some manner incapable of actually producing the disease for which they are responsible. This modification may be brought about by using cultures of diminished virulence as Pasteur did, and this diminished virulence may be obtained 
by attenuating cultures through age, or animal passage, by heat, or by desiccation (rabies). A very important advance was made by Salmon and Smith (1886), who found that they could actually use killed bacteria and still produce immunity. This latter method of a killed vaccine is now almost universally used for prophylactic purposes. Another means of modifying bacteria which not only renders them harmless but removes certain of their toxic effects is in the form of sensitized vaccines, that is to say, bacterial cultures, either living or killed, that have been treated by a corresponding immune serum.

Active immunity may be produced by injecting the modified preparations of cultures through various routes. In the case of human beings, injections are nearly always given subcutaneously for the purpose of prophylaxis, although in immunizing animals injections may be given into the peritoneum or directly into the circulation. Several injections are necessary and the degree of immunity produced usually varies with the number of injections, or at least with the total amount of bacteria that is given. When several injections are given, the intervals between them may vary markedly. The usual method has been to allow from a week to ten days between inoculations, but there is reason to believe that good immunity may be produced by inoculations at more frequent intervals, and even daily, with the additional beneficial result of producing the maximum protection afforded in a shorter space of time. Vaccines used for immunizing purposes must be given parenterally, that is to say, the undigested and intact protein must reach the remote portions of the body in order to produce the type of reaction necessary.

We have hitherto considered, by implication at least, only those forms of immunity designed to act directly upon the bacteria or microorganisms which produce disease. We know that bacteria produce harmful effects not'only by their own multiplication but through their toxins, and it is possible in the case of the more active of these toxins, as with those of the diphtheria and tetanus bacillus, to produce an antitoxic immunity by injecting the toxins themselves. Antitoxic immunity must then be contrasted with or serve to amplify antiinfectious immunity that we have hitherto discussed. The work of Charrin and Roger and of Roux and Chamberlain (1887) was the first to demonstrate the existence of soluble toxins that may be filtered from the growths of bacteria in fluid media. Roux and Chamberlain were unable, however, to produce an active immunity against the filtrate in the case of the diphtheria bacillus owing to its extreme toxicity and the great susceptibility of the animals they employed. In I89o Kitasato and Von Behring obtained better preparations of toxin from the tetanus bacillus and from the diphtheria bacillus, and were able, by modifying 
them by certain chemicals, to inject repeatedly small amounts of them in rabbits so that they became immune. They then made use of a discovery. that had been made two years previously by Richet and Hericourt, who found that in the case of actively immunized animals the serum may actually be used to transfer the protection against the same infection to a normal animal. This type of transferred immunity constitutes what we now-refer to as " passive immunity," inasmuch as the animal thereby protected is not actively concerned in elaborating those substances by which its life is preserved.' Passive immunity of this sort may be demonstrated by transferring the serum from animals immunized against bacteria (anti-infectious immunity) and also, as Von Behring showed, against the toxins of bacteria (antitoxic immunity). In considering the methods of producing active immunization, which we have just discussed, it should be noted that the number and amount of injections necessary to protect the animal itself against a toxin are less than in instances where it is desirable to hyperimmunize the animal for the purpose of utilizing its serum for passive immunity.

Passive immunity has both advantages and disadvantages as compared with active immunity. Its advantage lies principally in the fact that it can be immediately induced in a normal individual, while active immunity is a matter of days or weeks. It is inferior to active immunity in that it is never of quite so intense a degree and disappears more rapidly. Passive immunity to be most rapid and effective should be brought about in such a way as to bring the serum which transfers it to the point where it will do most good-in other words, to the major point of infection. In all general infections characterized by an invasion of the blood stream, either temporary or permanent, passive immunity is best effected by administering the serum directly into the circulation of the host. There are certain possible dangers in such administrations which will be considered when we come to speak of anaphylaxis. In localized infections the serum is also more effective when placed near the seat of infection, as for example in the therapy against cerebrospinal meningitis, where the remarkably successful results which have recently been attained by Flexner and others are due almost entirely to the fact that the serum was administered intrathecally.

\section{EARLY THEORIES OF THE NATURE OF IMMUNITY}

This brief outline of the development of our knowledge of immunity acquired by recovery from disease or produced artificially represents what may be termed the "empirical" state in our knowledge of im- 
munization. There followed naturally various explanations of the striking results that have been obtained, explanations largely philosophical in nature and which could not be expected to be ultimately explanatory in view of the limited information that was at that time available. Koch ( 1878 ) thought immunity was due to some variation in the condition of the blood, a rather noncommittal, though in its way accurate, statement. Fodor suggested further that these changed blood conditions might be due to the presence of certain antiseptic substances. The exhaustion theory of Pasteur (1880) explained the acquisition of a state of resistance following recovery from a disease as due to the fact that the first growth of the bacterium in the body had exhausted the nutritive substances suitable for the growth of that particular organism and that on second entry the same organism would find no suitable pabulum. This theory recently has been resuscitated in a somewhat different connection by Ehrlich in his atreptic theory of cancer immunity. There were several objections to this theory which Chauveau pointed out, and he in turn gave a theory which though opposite to the exhaustion theory was no more accurate. Chauveau explained immunity by the formation of harmful substances following the first injection which prevented the growth of the organism on its returning to the protected animal. It is to be noted that in both of these theories no allowance is made for the participation of the animal body in the process, which is now realized as the one essential factor in producing immunity. In certain of the other earlier theories this important reaction on the part of the host is provided for as in the theory of Nageli ( 1877 ), who thought that immunity was due to the successful struggle of the host for oxygen in competition with the bacteria. Grawitz in the same way, in his "excitation theory," thought immunity was due to an increased local assimilating power of cells, and Buchner (I883) added another point to this idea of cell participation in noting the importance of a local inflammatory reaction in protection.

\section{THE PHAGOCYTIC THEORY OF IMMUNITY}

Metchnikoff's phagocytic theory was the first experimentally substantiated and complete explanation of immunity. The protective cellular mechanism which he described remains the most important factor in bodily resistance to the infectious diseases as we understand it today. The beginnings of bacteriology in so far as human pathogenesis is concerned may be traced to the demonstration of bacteria in diseased tissues. It was further noted by several observers-Hayem, Birsch-Hirschfeld, and Koch-that these bacteria are frequently to be 


\section{MECHANISM OF BODILY RESISTANCE TO DISEASE}

found within the leucocytes, and in I874 Panum actually suggested that this inclusion might be a protective mechanism designed to get rid of invading microorganisms. The essential novelty of this explanation and its experimental proof is, however, to be credited first of all to Metchnikoff, who approached the subject from a novel and unbiased angle. Metchnikoff had been interested in the digestive function of the mesodermal cells of sponges. He found that leucocytes, which are also derived from the mesoderm, take up various particles and suggested that their function is primarily digestive. About this time Metchnikoff had the good fortune to hear a lecture by Cohnheim on "Inflammation" which represented the current view of the subjectnamely, that inflammation is a harmful manifestation rather than a beneficial process. It occurred to Metchnikoff that the object, or at all events the result, of inflammation was to destroy the bacteria that produce it, and that the leucocytes are intimately concerned in the process of destruction of invading microorganisms. He carried out a series of experiments, first by inserting thorns in the larva of the starfish (Bipinnaria), and later by infecting a small crustacean, Daphnia, with one of the blastomyces (Microsporon bicuspidata). In both of these instances he found that these foreign substances led to a diapedesis of white blood cells which more or less successfully surrounded and disposed of the harmful external agents. These fundamental experiments were then applied to higher animals and other types of bacterial infection and led Metchnikoff to the unequivocal conclusion that the leucocytes are the most important and perhaps the solely important factors which the body possesses for defending itself against the microorganisms of disease. This theory met with considerable opposition on the part of most pathologists, particularly the German pathologists, although Virchow from the first expressed a favorable opinion on the subject.

Let us take for granted, for the moment, that the activity of leucocytes is one important factor in explaining at least natural immunity, leaving the discussion of the obstacles which this theory has met with to a later development. With the importance of phagocytosis granted we may proceed to discuss its mechanism in more detail.

According to Metchnikoff the protective cells of the blood belong to two general groups which he has called microphages and macrophages. The microphages are the polymorphonuclear leucocytes which constitute from seventy-two to seventy-five per cent. of the white blood cells, and the macrophages include not only the large mononuclear leucocytes but the endothelial cells in the peritoneum in particular, the bone-marrow giant cells, and other less well-defined cells of the same 
type existing in various parts of the body. The distribution of these macrophages has been particularly emphasized recently by Evans $\left({ }^{85}\right)$, who by means of vital staining with various dyestuffs-isamin blue, for example-found there are various cells in certain fixed tissues of the body derived primarily from the endothelium and lining vascular channels which have the property of holding colloidal particulate matter. Such cells are not only the endothelial cells and giant cells but the "Kupfer" cells in the liver and the milky patches (taches laituses) of the omentum and the clasmatocytes. The function of the microphages, which as we know are primarily those cells concerned in acute purulent inflammations due to bacterial invasion, is to dispose of bacteria, whereas the macrophages take up red blood corpuscles and in fact any dead cells, including the dead microphages, both of the host and of foreign origin. The macrophages, moreover, are concerned directly in the more chronic type of bacterial infection as, for example, in leprosy, tuberculosis, actinomycosis, and protozoan parasites. The process of phagocytosis has been described by Metchnikoff and his pupils as occurring in three stages. In the first stage the bacteria and the leucocytes are brought together. At least they are brought together in those instances where the leucocytes are effective in making away with the bacteria, and this approach of the leucocytes is brought about by a process of positive chemotaxis. Chemotactic substances are eliminated by many bacteria in their growth, particularly by non-pathogenic bacteria which as a result of this positive chemotaxic influence are readily engulfed by the phagocytes. It may be shown, indeed, that bacteria of a given species will be found to vary in the nature of the substances they eliminate, as demonstrated by the action of these substances on leucocytes. Bordet $\left({ }^{36}\right)$, who was the first to demonstrate this phenomenon, showed that although an avirulent streptococcus attracts leucocytes, a virulent streptococcus repels them, or, in, other words, exerts a negative chemotaxis. The diapedesis of white blood corpuscles, one of the stages in inflammation and the formation of pus, is the most familiar instance of positive chemotaxis. Bordet further demonstrated that when virulent bacteria repel the leucocytes they do not actually destroy them or even paralyze their activity, for when he mixed cultures of harmless B. proteus and virulent streptococci together, it was found that the leucocytes readily approached and took up the proteus but remained at a distance from the streptococci. He found, moreover, that the toxins of bacteria are frequently associated with the negative chemotactic effect which they produce. Washed tetanus spores, for instance, are readily taken up by leucocytes, but tetanus spores accompanied by small amounts of their toxin repel leucocytes. In the case of the streptococci, negative 


\section{MECHANISM OF BODILY RESISTANCE TO DISEASE}

chemotaxis is also accompanied by the formation of a capsule about the organism which still further protects it from attack on the part of the leucocytes.

The second stage of phagocytosis covers the actual taking up or engulfing of bacteria by the leucocytes. From Metchnikoff's description one would regard this process as an active one on the part of the leucocytes which seem from his description not only endowed with ameboid motion but apparently possessed of almost intellectual functions. Recent studies on phagocytosis ascribe the engulfing function of the leucocyte, however, simply to a change in surface tension, a phenomenon of adsorption as first suggested by Pauli and later worked out by Bariakine. Phagocytosis does not mean the immediate destruction of the bacteria that have been enclosed within the leucocyte. They are seen, in most instances, finally to disintegrate, as shown by their separation into fragments and a change in their tinctorial reaction. The more resistant organisms like the tubercle bacillus may remain alive for considerable periods but finally become disintegrated within the leucocytes.

The guiding thought in Metchnikoff's conception of phagocytosis has been that it is a simple adaptation of a digestive process for a purpose that happens to be of great import to the animal, namely, its protection against foreign cells. The last stage in phagocytosis is the digestion of the bacteria that have been approached and engulfed by the leucocytes. Digestive ferments have been studied by Metchnikoff and others in connection with the various unicellular organisms, as in the ameba for instance, and it is found that leucocytes possess similar ferments. The nature of this ferment, although it has apparently been demonstrated by many investigators, remains even today somewhat unconvincing. Buchner found that by freezing and thawing leucocytes he could obtain an extract from them which would digest bacteria. Gengou found that an early leucocytic extract was more bactericidal than one which had been outside of the vessel walls for a longer period of time. He further thought he could obtain a different type of ferment from each of the two general categories of phagocytes that Metchnikoff described-a microcytase from polymorphonuclear leucocytes, and a macrocytase from the macrocytes or large mononuclear leucocytes. It seems unquestionable that the extract from leucocytes has certain digestive properties. It is more difficult to prove that they are of ferment nature if we understand that ferments must be destroyed at $56^{\circ} \mathrm{C}$. Opie, for example, found two different types of proteolytic enzymes demonstrable by their activity on coagulated blood serum. From polymorphonuclear leucocytes he obtained a substance designated as "leuco- 
protease" which acted only in an alkaline medium to digest blood serum and was destroyed at $100^{\circ} \mathrm{C}$. From mononuclear cells he obtained "lymphoprotease," active only in an acid medium and destroyed at between $55^{\circ}$ and $78^{\circ} \mathrm{C}$.

The best evidences of the fact that leucocytes can actually digest or destroy bacteria are the changes undergone by the bacteria within these cells and finally the obtaining of negative cultures from such leucocytes. We shall again return to a discussion of the importance of the mechanism of phagocytosis in correlating it with the developments of the humoral aspects of immunity. In conclusion, at this point it should be stated that according to Metchnikoff and his followers all of the destruction of bacteria and perhaps the neutralization of their toxins is directly or indirectly attributable to the action of phagocytes. In other words, all immunity is cellular in nature.

\section{THE HUMORAL ASPECTS OF NATURAL IMMUNITY}

Almost coincidentally with the early developments of Metchnikoff's theory of immunity there arose many observations that at first tended to disprove it and which led to the almost general rejection of Metchnikoff's work on the part of the German school. In 1875 Fodor and Wyssokowitch found that when bacteria were injected directly into the circulation of an animal, they were not eliminated through the kidney. In other words, they disappear somewhere within the body. The observations by Fodor in the following year showed that when living bacteria are mixed with freshly drawn blood, the clotted mixture becomes sterile. Nutall (I888) carried these observations further by showing that not only blood but pleural exudate has the property of destroying various bacteria, and he found moreover that this property is lost when the blood is heated to $60^{\circ} \mathrm{C}$. He took pains to emphasize that this destruction is extracellular in nature and therefore not the phenomenon that Metchnikoff described. Buchner (I89I) still further emphasized the humoral aspects of the controversy by showing that the bactericidal action of blood is due to the blood serum, and he attributed the activity of the blood serum to the presence of substances that he called alexins (Greek. $\dot{\alpha} \lambda \dot{\varepsilon}^{\xi} \xi \varepsilon \nu$, , to ward off, or protect"). These alexins were destroyed by heating to $55^{\circ} \mathrm{C}$. but were unaffected by dilution with normal sodium chloride solution at body temperature. They could be conserved for a considerable time by drying or by adding hypertonic ( $4 \%)$ salt solutions. Buchner regarded these alexins as enzymes or ferments. Daremberg in the same year described a similar destructive property for the red blood cells of one animal species by 


\section{MECHANISM OF BODILY RESISTANCE TO DISEA'SE}

serum of another animal species; this property was likewise destroyed by heating to $55^{\circ} \mathrm{C}$.

These observations on the properties of blood serum led to efforts to harmonize the cellular with the humoral theory. It was Hankin who first suggested that alexin which is present in shed blood serum is actually derived from the leucocytes, and the property of alexins simply represents an abnormal distribution of these ferments in the serum as a result of coagulation of the blood. We have already mentioned the more or less successful attempts to show that leucocytes contain a bactericidal ferment similar in nature to alexin, and it has also further been shown that the content of alexin in serum varies to some extent with the degree of leucocytosis and is increased with the period of time during which the serum is allowed. to come in contact with the clotted blood.

Here again crucial experiments, offered by Gengou to complete the harmonizing of humoral and cellular theories, have failed for various technical reasons of complete acceptance. To prove that alexin present in serum is derived from the leucocytes and in reality represents a condition which does not exist in the living body, it would be necessary to show that alexin does not occur in the blood plasma. Experiments apparently proving this point were submitted by Gengou. They have not, however, been generally confirmed, and cannot be accepted as definitely proving that circulating plasma contains no alexin. In our opinion there is no reason to assume either that alexins, or, as we shall later consider, antibodies, have their origin in the leucocytes alone as Metchnikoff would believe. We have simply to extend our conception of phagocytosis to the fixed tissue cells and also to believe in a wider distribution of antibodies irrespective of where the destruction of bacteria eventually takes place. The origin of alexin has also been sought for in certain of the fixed tissues, e.g. the thyroid and the liver, and apparently it has been shown to exist there.

\section{FACTORS CONCERNED IN NATURAL RESISTANCE AND IMMUNITY}

We have hitherto spoken of natural resistance and acquired immunity as synonymous. We must now anticipate the next step in our discussion of the mechanism of acquired immunity by explaining that resistance, properly speaking, refers to a non-specific protection, a form of protection equally operative against a great variety of relatively non-pathogenic microorganisms and brought about without the introduction, at least to any considerable extent, of the antibodies which by their nature are specific. Natural immunity to bacterial infection, prop- 
erly speaking, would be due first of all to the external measures of defense, some of which we have already mentioned. Such external defenses comprise the natural barriers of the skin and epithelial cells, the inaccessibility of the specific loci necessary for the growth of certain microorganisms, the reaction of gastric and intestinal fluids, and the like. Once the microorganism has penetrated the external barriers of defense we have then to consider resistance as due to, first of all, the response of phagocytes directly or indirectly to the bacterium in question. Secondly come the variations in the amount of alexin, whether within or without the phagocytes, that is available for the destruction of the bacteria. Natural immunity would differ from natural resistance only in the fact that in the first there are natural or normal antibodies present which have the property of uniting with the bacterium involved and rendering it more susceptible to the direct action of alexins or to phagocytosis.

On this supposition as to the operation of natural resistance and natural immunity, we realize that any natural process or intervention that raises the number of leucocytes either generally, or locally at the point of infection, and any intervention that increases the alexic power in the leucocytes or in the blood serum, increase resistance. Conversely, any process that lowers these substances will lower resistance. $\mathrm{Nu}$ merous observations tend to confirm this prevision. In the first place, it is well known that when an infection begins a marked local reaction and particularly one evidenced by the presence of leucocytes tends to prevent the further extension of the infectious process. Localized hyperenia and venous stasis, as in the Bier treatment, which tend to facilitate diapedesis of white blood cells in an infected region, aid in aborting infections. Hyperleucocytosis itself is not only a concomitant of many infectious diseases but is of prognostic import (e.g. pneumonia). The injection of leucocytic extract (Hiss and Zinsser) aids in curing localized infections. The effectiveness of the leucocytic extract is not due, we believe, as the originators once thought, to the alexins in the leucocytes administered, but rather to the stimulation of the leucocytes in the host which is brought about by the leucocytes simply because they are foreign proteins. We shall later have occasion to discuss the value of various foreign proteins in combating generalized infections such as arthritis and typhoid fever. Any substance, either Buchner's plasmin, a preparation of nuclein, typhoid vaccine, or albumose, may produce similar beneficial results.

Conversely, normal resistance may be lowered by numerous conditions which we suspect or actually know to have an effect upon the leucocytes or alexins. Thus general lowered conditions of nutrition 


\section{0

and social surroundings may be accompanied by a diminution in these essential factors. London showed that starving animals have less alexin than normal. Moro demonstrated similar results in bottle-fed infants. It should be stated, however, that most of the methods of determining the potency of the alexin in serum have been technically imperfect (Gay and Ayer). On the other hand, it seems definitely demonstrated that fatigue, cold, various chemical poisons, such as anesthetics, opium, phosphorus, and alcohol, all have harmful effects on the leucocytes.

\section{THE HUMORAL ASPECTS OF ACQUIRED IMMUNITY}

It is evident that natural immunity, at least, is due to the combined action of cells and certain properties that are present in the blood fluid, whether these latter properties are derived from the leucocytes or not. When we consider the factors concerned in acquired immunity, the importance of the fluids becomes still more strikingly evident. The great importance of certain of the factors in serum which we are about to discuss, however, does not controvert and perhaps enhances the importance of Metchnikoff's theory of phagocytosis, as we shall see later. It was evident that the observations by Kitasato and Von Behring concerning the passive transfer of antitoxic immunity did much to point out the importance of humoral factors. It may further be shown that a simple admixture of antitoxic serum suffices to neutralize the effect of its corresponding toxin. No intervention of cells is necessary to explain this type of immunity.

Lysins. Beginning with the intensive studies on cholera in 1892 , lysin, the first of a series of important properties or antibodies to be discovered in the serum of immunized animals, was described. Pfeiffer found that when guinea-pigs are immunized against cholera vibrios they are protected from these organisms not in virtue of any antitoxin thus formed, as he had at first supposed, but owing to the presence of certain substances in their serum which have the property of dissolving the infecting microorganisms. In his experiments with this destruction of the vibrios, bacteriolysis did not take place outside the animal body, but always did in immune animals, or when the serum was injected with the vibrios into the peritoneal cavity of normal animals. He therefore assumed that the serum contained some substance which is reactivated by the living endothelium. The true explanation of Pfeiffer's phenomenon was not given until the following year (1895) by Bordet, who found that the fresh serum of guinea-pigs, immunized against cholera, has itself the property of dissolving these vibrios in the test tube. This property is lost when the serum is kept for any considerable period of time, or immediately if the serum is heated for one-half hour at $55^{\circ} \mathrm{C}$. 
The property in this heated serum is immediately restored on the addition of a small amount of fresh serum from a normal animal. In other words, bacteriolysis is due to the interaction of two substances-a " preventive" substance, later called by Bordet a "sensitizing" substance, which is present in animals that have been artificially immunized, is specific in nature, and relatively thermostable; and a bactericidal substance which is identical with Buchner's alexin. This alexin is destroyed by heating to $55^{\circ} \mathrm{C}$., is in itself inactive, and is not increased by immunization. Bordet further showed in his experiments that although this lysis could be regularly produced with fresh anticholera serum outside the body, destruction of the vibrios is brought about by the phagocytes when they are injected directly into the circulation of an immunized guinea-pig. In other words, Pfeiffer's phenomenon is to a certain extent artificial and does not represent the actual condition operative in active acquired immunity. It does, however, emphasize a certain characteristic substance formed in the course of immunity. The fact that lysis in vitro is not readily demonstrable with any bacteria other than the vibrios still further emphasizes the unusual nature of the results described.

Though a partisan of the cellular aspects of immunity, Bordet continued to add the most significant facts for building up of the humoral phases. He found in 1898 that specific lysins, like the one described for cholera, were also produced by the injection not only of bacteria but of red blood cells. If an animal of species " A" receives injection of the red blood corpuscles of species " B," it forms a specific hemolysin that has the property of dissolving these cells. The study of this phenomenon of hemolysis has proved a most fruitful field of investigation, owing to the greater ease with which the results can be observed and analyzed. It resembles in all respects bacteriolysis; two substances are required, the alexin of normal serum plus the sensitizer produced as the result of immunization. Further facts elicited are that the sensitizer-or, as Ehrlich $\left({ }^{37}\right)$ later called it, the amboceptor-has the property of forming a union with the antigen which has given rise to it. This union is so firm that the complex resists washing. Bordet has conclusively shown that this union between sensitizer and red blood cells-in other words, between antigen and antibody-must take place before the alexin (Ehrlich's complement) which ultimately produces the destruction of the cells is operative. Ehrlich and Morgenroth gave a further demonstration of this fact in their experiments, which showed that if a fresh immune serum is added to red blood cells at $0^{\circ} \mathrm{C}$., the sensitizer still unites with the corpuscles, leaving the alexin in the supernatent fluid. This fact is detailed because it is the discovery of this 


\section{MECHANISM OF BODILY RESISTANCE TO DISEASE}

that led to a reaction of great diagnostic importance. Ehrlich and Morgenroth in further elaboration of the side-chain theory of Ehrlich, which would explain the origin and mode of action of antibodies, were led to assume the existence of innumerable complements or alexins. Bordet, who from the first believed in the unity of the alexin as a ferment-like substance which varies quantitatively rather than qualitatively in various animals, offered the following experimental proof of his contention: if cholera vibrios are treated with an inactivated $\left(55^{\circ}\right)$ anticholera serum they acquire a new property and become able to fix or remove the alexin from any fresh serum that is added to the mixture. This fixation incidentally destroys the vibrios, but the utilization of the alexin is proved by subsequently adding to such a mixture a combination of red blood corpuscles and inactivated hemolytic serum. If the alexin has been fixed no hemolysis takes place. This fixation of the alexin necessary to produce hemolysis by the combination of a bacterium and its antibody proved to Bordet that the hemolytic and the bacteriolytic alexins are identical, or, in other words, there is only one alexin. The importance of the reaction thus demonstrated lies in the fact that it serves to demonstrate the presence of either an antigen or an antibody when one of the two substances is known to be present. Bordet himself was the first to appreciate and utilize this fact, and two applications of it from Bordet's own work may here be given. It may be shown that a specific sensitizer is present in the serum of known cases of typhoid fever, for by adding the typhoid bacillus to such serum a combination is formed that fixes the alexin. No other bacteria will produce this phenomenon with typhoid serum and no other serum than that of a typhoid case will produce the reaction with the typhoid bacillus. The reaction, therefore, may be used in the diagnosis of typhoid fever. In the second example, Bordet suspected a certain coccobacillus that he had isolated from cases of whooping-cough to be the cause of the disease. He was able to demonstrate the etiological relation of this microorganism by showing that the serum of the individuals who were suffering from whooping-cough produced fixation with this bacillus and with no other organism isolated from their sputum. The serum of normal individuals did not give the reaction with this organism which is now recognized to be the cause of whooping-cough.

An enormous amount of work has been done in studying the exact mode of action of these lysins as representing part of the mechanism by which immunity is effective. It would not be possible for us even to summarize the main facts that have been demonstrated in these studies. We may simply point out the two main theories that have been used to explain the phenomenon observed, leaving those adaptations of 
the work that have already become of pr.mtical use for later discussion. Bordet has consistently emphasized the pinys.cochemical nature of the reaction that takes place between antigens and antibodies. According to him the action of a sensitizer upon a bacterium is similar to the process of dyeing, the sensitizer resembling the roordant tised in fixing the cloth. Union takes place in variable proportions in accordance with the concentration of tlie two substances, antigen and antibody, concerned in the reaction. It is similar to the adsorption phenomenon between colloids of opposing electrical charge and the reactions are characteristically reversible.

Ehrlich's theory, on the other hand, which has been elaborately built up on the original explanation that he offered for the union of toxin with antitoxin, is that these reactions are of a definite chemical nature resembling a simple reaction such as would take place between a strong acid and a strong base. They are governed by the laws of multiple proportions. Ehrlich's theory may be stated here in the briefest possible form. Antibodies are formed in the body, according to Ehrlich, by those particular cells which have an affinity for an'l unite with the antigen in question. It should be stated at this time tnat this assumption is based on no experimental evidence and wo: ${ }^{11}$ the 5 re. in our opinion, invalidate the rest of the theory. We do no. Wis to state that no evidence has been offered to prove this original hypothesis, but it has been most certainly unacceptable and for the most part disproved. Ehrlich's statement, that the union of a poisonous substance or antigen with a cell that it affects is similar to the absorption of a foodstuff, may probably be accepted as correct. Those particular substances in cells that unite either with foodstuffs or poisons are called receptors, and it is supposedly the formation of these receptors in excess after their injury and their extrusion from the cell that leads to their entrance into the circulation, where they are then to be regarded as identical with the antibodies. We do not wish to burden the reader further with a discussion of a theory which, although in ingenuity remarkable, is not, in our opinion, an essential explanation of the facts. It is at best only a restatement of facts rather than an explanation of them, as is evidenced by the fact that Ehrlich with an army of investigators behind him has for the most part contributed the experiments serving to uphold his theory and to refute the simple and logical explanation given by Bordet, who almost single-handed has contributed practically all the significant facts in the discussion. It is out of Bordet's work that the results of practical value have come irrespective of the merits of the discussion between him and Ehrlich. Bordet's theory has consistently been the heuristic one. 


\section{MECHANISM OF BODILY RESISTANCE TO DISEASE}

The essential differences of opinion between Bordet and Ehrlich may be briefly summarized as follows, $(a)$ whether the interaction of antigens with antibodies is chemical or physicochemical in nature, $(b)$ a discussion of the amboceptor nature of the antibody, that is to say, whether it is capable of uniting with the alexin as well as with the antigen, and (c) the multiplicity of the uniting of the complements or the alexin. A discussion of these differences has led to a minute examination of all the conditions under which specific lysins act. Many of the interesting facts described are as yet of significance only so far as the theory is concerned, and we may not with profit discuss them here too fully.

An antilysin to a hemolysin may be produced as Bordet was first to discover. This anti-antibody is composed of two factors, one of which acts upon the alexin (anti-alexin) and one of which acts upon the sensitizer (anti-sensitizer). He further showed (1903) that antisensitizers to any immune serum produced by, let us say, the guinea-pig will be formed by the injection of the serum of a normal guinea-pig into another animal like the rabbit. It is not necessary to employ guinea-pig anticholera serum but simply guinea-pig serum in immunizing a rabbit in order to produce a serum capable of protecting cholera vibrios from the guinea-pig anticholera serum.

These statements outline the properties and mode of action of the lysins as they are called. These lysins are present in the serum of actively immunized animals and in their sensitizing or amboceptor part represent the specific results of reaction to a foreign protein. Although the lysins can destroy bacteria in the test tube and apart from living cellular participation, it is by no means certain to what extent and in what way they are operative in producing the condition known as active immunity; that is a matter, however, that we shall discuss at a later point.

Proteolytic ferments in relation to lysins. It is by no means certain to what extent the lysins may be identified with the so-called "protective ferments." We have already noted that digestion of bacteria takes place within the leucocytes in the process of phagocytosis, and Pfeiffer's phenomenon is evidence that a similar destruction occurs in serum owing to the action of the enzymatic alexin or cytase. The digestion of a bland protein like serum or egg white renders it toxic, and such a hydrolized protein is no longer antigenic. The condition known as anaphylactic shock would seem to depend on the rapid formation of a substance which at least gives symptoms like those produced by the hydrolized protein. This led Vaughan to offer the ingenious theory that the sensitization to anaphylactic shock covers a period represented by the formation in the body of a specific ferment designed to 
split the particular protein used to sensitize. This theory was further upheld by the work of Vaughan and of Friedberger on the "anaphylotoxin" found during this shock, and also indicated by the work of Abderhalden on protective ferments. The explanation, however, is not so simple, and we are certainly not justified in regarding the lysins as identical with ferments. Abderhalden's protective ferments are almost certainly non-specific in character and are therefore not identical with the sensitizer. Are they, then, identical with the alexin? Not if we regard such ferments as being increased during immunization as does Vaughan, for we know that alexin is not so increased. The explanation of antiserum activity championed by Jobling and Peterson and by Bronfenbrenner is at present more satisfactory. Serum proteases are normally inhibited from acting by the presence of antiprotease. The addition of an antigen to an antiserum removes the antiprotease by a process of adsorption, leaving the protease or ferment free to act. Jobling has, however, presupposed a mobilization or an increase of ferments during sensitization or immunization which does not render the protease analogous to alexin. The toxic products liberated, moreover, come from the antiserum and not the antigen and so the analogy with Pfeiffer's phenomenon is again not complete. The question as to the importance of proteolytic ferments in immunity and their relations to the lysins is therefore still open.

Antitoxins. Toxic substances are produced not only by bacteria and plants but by foreign animal cells as well. We are here more particularly concerned with the bacterial toxins, but in addition to them should be mentioned similar toxic substances produced by some of the higher plants, as, for example, the active substances derived from the castor oil plant (ricin) and from the jequirity bean (abrin). The toxic substances of some of the pollens which give rise to hay fever are similar in nature, and all these substances resemble bacterial toxins in that they produce antitoxins. For completeness, there may be classified here also animal toxins such as snake venom and bee poison.

The poisonous substances in bacteria, or produced by bacteria, are of several varieties. They may simply be chemical substances, acids, alkalies, or nitrites, and still be responsible for some of the symptoms in certain bacterial infections. In addition, the proteins, of which the bacterial cells are in part composed, are toxic for most animals, and their nature will be considered more fully in a moment. The true, soluble exotoxins of bacteria are, however, their most characteristic products, although they have been described hitherto in connection with relatively few microorganisms. It is characteristic of these soluble toxins that they are freely produced in culture media during the growth 
of the microorganisms. Such toxins have been described in connection with the diphtheria bacillus, the tetanus bacillus, the bacillus of botulism, and the bacillus of symptomatic anthrax. The very fact that individual strains of each of these organisms vary in toxin production and that all varieties are dependent for maximum toxin production on a delicate adjustment of the culture medium suggests that other bacteria eventually may be proved to secrete similar bodies. It has, indeed, been shown in recent years that soluble toxins are produced under the right conditions by cholera vibrios, and apparently also by the dysentery bacillus and the typhoid bacillus. In addition to those exotoxins which we shall consider in more detail, bacteria of certain varieties are known to produce substances which readily dissolve red blood cells or hemotoxins as they are called. The bodies require no activation and should not be confused with the hemolysins. Leucocidins, destructive of white blood cells and distinct from the negative chemotactic substances we have already mentioned, have likewise been found and antibodies to them produced. The bacterial. "aggressins" are either identical or similar to one or more of these less well-defined toxic substances of bacterial origin. They are regarded as aiding the bacterial infection in some specific manner. It is not certain whether these recently mentioned substances are really soluble toxins, although they are present in even fresh cultures of the bacteria that produce them.

Another group of bacterial toxins is even less definitely known although their manifestations have long been evident. These are the endotoxins described by Pfeiffer to account for the death of choleraimmune guinea-pigs after the injection of too large a dose of cholera vibrios. Cultures from such animals are found sterile, the infection is overcome, although the animal dies from intoxication due to destruction of the cholera vibrios. It was characteristic of these endotoxins that they were developed only in the disintegration of bacterial bodies and that they did not give rise to antibodies, at least further immunization does not serve to protect the guinea-pig from them. This later criterion of endotoxins has been questioned by Besredka but on the whole is apparently true and, if so, there seems no reason to regard the endotoxin as anything more than the poison molecule of bacterial protein which, as we know from the work of Vaughan, is likewise devoid of antigenic property and therefore of specificity.

The true toxins are colloidal and, of course, protein in nature. They are specific as regards both origin and effect in the animal body. Each of them has particular affinity for some set of tissues or cells. True toxins require an incubation period before their effect is evident. They are thermolabile at $45^{\circ} \mathrm{C}$. and upwards, and are destroyed by 
acids and oxidases. The most characteristic effect which here interests us is the fact that the true toxins produce antitoxins, as has already been mentioned in connection with the work of Von Behring and Kitasato, who further showed that the antitoxins may be transferred in the blood serum of actively immunized animals. It is in connection with the formation of antitoxins in the body that Ehrlich's lateral-chain theory was first introduced. We know that the toxins affect certain tissues, as, for example, the cells of the central nervous system in the case of tetanus toxin. They are moreover extraordinarily active and a very minute amount is sufficient to produce symptomatic disturbances; $0.000,000,33$ gram of dried tetanus toxin, for example, will kill a guineapig of 300 grams weight. The susceptibility of different animals varies enormously, some being little affected; in other words, there is a definite natural species immunity to toxins. When a non-fatal dose of toxin is given to an animal, the animal recovers and can thereafter be given larger doses until its serum is found to contain antitoxins which neutralize the toxins not only in the animal body but in the test tube. This antitoxin cannot be regarded as a simple inversion of the toxin because one unit of toxin may produce 100,000 units of antitoxin (Knorr). It is known that the body cells react to form the antitoxins because it may be shown that after exsanguination and transfusion of an immunized animal, antitoxins reappear in the blood stream. Drugs like pilocarpin which stimulate cellular activity also cause increase in antitoxins. We have no further information as to where any particular antitoxin is formed; Ehrlich's receptor theory presupposes that antitoxin is formed in the cells that are effected by the toxins, but all efforts to prove this have failed, and indeed certain experiments exist, as, for example, Metchnikoff's experiments with antispermotoxin, which indicate that the antitoxin is certainly not formed in the cells that are acted on by the toxins.

Antitoxins are of great therapeutic value, the value depending absolutely on the strength of the actual product employed. For this reason a complicated but accurate method of measuring the exact potency of diphtheria and tetanus antitoxins has been devised by Ehrlich, Rosenau, and others. Such estimations must be carried out in guinea-pigs, and it is their analysis that has furnished the great stock of information on the nature and interaction of toxin and antitoxin. But although practical results of mensuration have been achieved, a difference of opinion has arisen as to the exact method by which the antitoxin neutralizes the toxin. According to Ehrlich the reaction is to be regarded as a straight chemical reaction as between a strong acid and a strong base. This necessitates the supposition of a number of toxins of varying 
toxicity and combining power in the toxic filtrate derived from the bacterium, and such substances or, better, such properties may apparently be demonstrated. On analysis, however, all the factors that Ehrlich has described in the toxin molecule can be accounted for by assuming that various degrees of attenuation take place in a single poison (Bordet). Bordet would explain toxin-antitoxin reactions as an adsorption phenomenon between two colloids of opposing electric charge. This is rendered probable by the demonstration that certain of these toxinantitoxin reactions are reversible. The neutralized or detoxicated mixture on injection into the animal body may be shown gradually to liberate small amounts of toxin, as is evidenced by the fact that such a neutral mixture, apparently containing no free toxin, will give rise to antitoxins.

Still another theory is the one of Arrhenius and Madsen which regards toxin-antitoxin combinations as like interactions between a weak acid and a weak base. This theory also accounts for the reversible nature of the reaction.

Agglutinins. Charrin and Roger (1889) found that when they added $B$. pyocyaneus to its immune serum small clumps of bacteria occurred instead of a homogenous suspension. Similar facts were noted with other sera and their corresponding microorganisms by Metchnikoff and by Issaef. In I895 Bordet in his work with anticholera vibrios, in vitro, noted an agglutination as well as the lysis that Pfeiffer had described. He found that this agglutination occurred with even small amounts of immune serum and suggested its use as a means of identifying the cholera vibrio. In the following year Gruber and Durham and Pfeiffer examined this reaction more carefully for its diagnostic value, and Widal and Grünbaum independently found that the serum of cases of typhoid fever actually has the property of clumping the typhoid bacillus in a characteristic manner. This agglutination reaction is now recognized to be of great diagnostic value not only in typhoid fever but in several other diseases. The phenomenon occurs whenever a small, though varying, amount of an immune serum or of the serum from certain instances of disease is mixed with the corresponding organism. The reaction is evidenced in the case of motile cultures first by their loss of motility and then by their collection in small clumps and a gradual settling of these masses to the bottom of the test tube. It occurs not only with living cultures of the organism but with those that have been killed by heat or chemicals. If the cultures are alive, they are not killed by the agglutinin in immune serum and may actually proliferate, but always in groups instead of as individuals. At times the clumping may be thread-like rather than spherical (Pfaundler's 
reaction). The reaction may be observed with small amounts of serum on a cover slip (microscopic method) or better in larger amounts of diluted serum in a test tube or watch glass (macroscopic method).

Bacteria differ in their ability to produce agglutinins both during the course of disease and as a result of immunization. Whereas such organisms as the typhoid bacillus, the cholera vibrio, and the plague bacillus give rise to agglutinins either during or following recovery from a disease, others, such as $B$. diphtheriae, $B$. anthracis, $B$. tetani, the pneumococcus, and some of the yeasts, produce agglutinins only when given repeatedly as in the courses of artificial immunization. Other bacteria, particularly those surrounded by a capsule, do not give rise to demonstrable agglutinins. In general it may be said that motile bacteria are more agglutinogenic than non-motile, and it has been suggested that separate agglutinins are formed to the flagella and to the bodies of such microorganisms. The reaction is used not only for the diagnosis of disease but for the identification of a suspected micruorganism, as may be proved by adding it to an anti-serum that has been produced by immunizing animals with a known culture of the bacterium in question. Agglutinins, moreover, are present in certain normal sera and there are normal agglutinins not only for bacteria, but for foreign red blood cells, as might be suspected by analogy with the work on the lysins. Immune sera may be produced by repeated injections of such potency that they agglutinate bacteria in dilutions as high as one part in a million.

Numerous theories of agglutination have been offered. The most essential facts are those given by Bordet, who regards the phenomenon as physicochemical in nature, and due to a process of molecular adhesion, as in the flocculation of inorganic or organic colloids. He demonstrated the fact that there were two phases in the reaction of agglutination. During the first of these phases the agglutinin unites with the bacterium and in the second phase, during which the presence of an electrolyte like sodium chloride is necessary, flocculation occurs. The reaction is reversible, as in the case of union of toxin with antitoxin.

The agglutination reaction is relatively but not absolutely specific, a property which is common to all immunity reactions. That is to say, whereas an antityphoid serum will agglutinate certain closely allied organisms such as $B$. coli and $B$. paratyphosus alpha, it always agglutinates the typhoid bacillus in highest dilutions. Saturation with one of the less specific organisms removes from the serum the property of clumping that organism but does not remove the property of clumping the typhoid bacillus. The diagnosis of a mixed bacterial infection, as with the typhoid bacillus and one of the paratyphoid bacilli, may occur. 
Such a case could be differentiated from an instance of group agglutination by saturation with each of the organisms in turn. In the case of mixed infections the agglutinin for the other organism is left behind.

Normal hemagglutinins may either be increased by immunization or may be produced in instances where they do not naturally occur. In addition to the agglutinins active against the red blood corpuscles of another species, in some animals isohemagglutinins occur, particularly in horses and human beings. The latter substances are of importance in the transfusion of blood, which has again become a common procedure. They are frequently paralleled by the occurrence of normal isohemolysins. In either case it is necessary to detect such dangerous and incompatible conditions before performing transfusions.

Precipitins. In 1897 Kraus found that when he added the filtrates of broth cultures of bacteria to their corresponding anti-sera, a cloudiness occurred. Two years later Tsistowitch and independently Bordet found that when an animal was given injections of the blood or the blood serum of another animal species, the immune serum produced has the property of producing a cloudiness with the antigenic serum. This cloudiness proceeded to the formation of a flocculent precipitate often in a few minutes that eventually fell to the bottom of the test tube. This phenomenon of precipitation is similar in most details to the phenomenon of agglutination and may be regarded simply as a clumping of protein molecules instead of organized cells. It was later discovered that other protein antigens such as egg albumin and milk give rise to. corresponding precipitins when injected repeatedly into animals of another species.

Wassermann and Schutze, and Uhlenhuth (I900) suggested the use of this reaction in the detection of human blood and, in later studies, of various muscle proteins. An extensive study has shown that the reaction, however, is not absolutely specific. Closely allied species may give group precipitin phenomena, but the value of the reaction for diagnostic purposes remains unquestioned and will be later considered in more detail. A further study of the precipitins has brought out many of the facts as to the physical aspects of the antigen-antibody reactions and has aided in establishing the essentials of specificity which we have already considered at an earlier point. The substance in the antigen which gives rise to the precipitins and unites with it in the reaction is known as the precipitinogen. The reaction is produced only when the two reacting substances are present in appropriate amounts. An excess of the precipitin, for example, or of the precipitinogen may give rise to a prezone at which point no reaction occurs. In this respect, the reaction resembles the zones of flocculation and of absence of 
flocculation, which occur between organic and inorganic colloidal compounds, as, for example, ferric hydrate and silicic acid. The stability of the colloids is changed by the ionic reaction and concentration of the added substances, so that they become less stable and flocculate out. Both antigens and antibodies may occur at the same time in the serum of an immunized animal without precipitation, which indicates the complexity of the reaction and the delicacy of the conditions which determine it.

Specific precipitation of this sort formed by an interaction of antigen and antibody has the property of fixing the alexin (Gay), as was also found to be true in the combination of antigen and sensitizer. This fixation reaction may also be used for the detection of either antigens or antibodies when one of them is known to be present.

Opsonins and Tropins. We have apparently for the moment lost sight of the importance of the leucocyte in immunity. It may be recalled that, according to Metchnikoff, leucocytes are the most important and essential factors in bodily protection against infections. There seemed little doubt in our discussion on natural immunity that phagocytes, and in addition certain ferment-like substances (cytases or alexins) which also may be derived from leucocytes, are responsible for the observed results. In acquired immunity it is evident that we have to deal not only with these substances that are normally present in the body, but with certain other substances known as antibodies that follow infection or immunization. We shall later see that there is evidence which indicates that even these distinctive and often very potent antibodies may be formed either by leucocytes or in those organs that produce leucocytes. There seems little question, however, that the powerful antibodies that we have discussed lie free in the serum. There is no definite evidence that they are artificially produced by the disintegration of leucocytes, and are not free in the plasma.

Metchnikoff was quick to interpret the interesting findings on antibodies in the light of his phagocytic theory. He first suggested that the action of antibodies was simply for the purpose of stimulating the leucocytes to the greater activity which he admitted existed in the case of acquired immunity. It was soon shown, however, by Bordet and by Denys and Leclef (1893) that although an antiserum, for example antistreptococcus serum, does increase the phagocytosis in an immune animal, or by transfer into a normal animal, the phagocytes are in no sense trained by immunization to greater efficiency. It was definitely shown that the serum affects the bacteria concerned in such a way that they are then more rapidly taken up by the phagocyte. We have then, in addition to what may be termed a simple or primary phagocytosis 
characteristic of normal immunity and fully described by Metchnikoff, a type of phagocytosis that may be called induced or secondary and depending on an antibody.

Induced phagocytosis as pointed out by Denys and Leclef was rendered more significant by the later work of Wright (1903) $\left(^{34}\right)$, Neufeld $\left({ }^{38}\right)$, and others. Wright found that there are a number of microorganisms which remain practically unchanged by the corresponding immune serum in the sense that they are not destroyed or dissolved by an immune serum, as is the case of the cholera vibrio. Such bacteria are the various cocci and the plague bacillus. Nor are these organisms to any considerable degree under normal conditions phagocytized. There is, however, a substance present in small amounts in normal serum which renders these microorganisms more readily taken up by the leucocytes. To this substance Wright gave the name of opsonin. $\mathrm{He}$ regarded the opsonin as a great determining factor in infections due to microorganisms of this group. He found, however, that this substance could be artificially increased by injections of the bacterium in question. By determining the potency of the opsonins and the demonstration of their increase following vaccination he developed a system of vaccine therapy, the significance of which we shall later consider. The same substances under the name of tropins have been described by Neufeld and his collaborators in various immune sera and there remains little question as to the facts concerned. To recapitulate, the opsonins or tropins are substances present in small amounts in normal sera and in large amounts in immune sera which have the property of rendering the specific microorganism against which they are active more readily phagocyted. There seems on further analysis little reason to regard the tropins as any different from the immune opsonins of Wright although they were at first so considered.

\section{A SUMMARY OF THE FACTORS CONCERNED IN IMMUNITY}

We have presented in the last few pages a very brief summary of the most important factors or properties that are associated in the animal body with the conditions of resistance, of natural and of acquired immunity to the infectious diseases. There are advantages as well as disadvantages in a presentation of this sort; the disadvantages are that the limits of space preclude the inclusion of essential evidence and of many facts which may eventually be judged more important than those given. The advantages that may be gained lie in an artificial orderliness in the facts presented that may not be fully warranted. We should fail of our purpose if we were not willing to risk an explanation of 
immunity as a whole, although it is realized that no such explanation can be ultimate. Certain questions constantly recur in reviewing the data of immunology and some of them may be answered with a fair degree of satisfaction, although it is realized that the answers may eventually be found incorrect.

What are the relations of the various antibodies that have been described to one another both in origin and function? To what extent does each of these antibodies aid in effecting the protection of which they are the manifestation? What is the relation of the antibodies present in the serum to the phagocytes and which, cells or fluids, are the more important in immunity? Do the cellular and humoral factors presented account fully for the manifestations of immunity?

There is this danger in discussing antibodies as such, that we should come to regard them as structural rather than functional. This again is the objection to Ehrlich's theory and its explanatory diagrams. What we have really been considering are the properties of immune serum and uncertain isolated bodies floating about in it. The danger is manifest when we find that certain antibodies with almost identical functions have been described as different rather than a single substance endowed with several functions or with a changeable function. To simplify the explanation of immunity we are justified in classifying antibodies or antibody activities on the basis of their chief function. We do no violence to the facts available if we divide all antibodies, as did Nicolle, into the "lysins" and the "coagulins." We should be forcing the matter, however, if we assume as he did that parallel bodies of each sort are formed in response to each antigen; he assumed, for instance, toxinolysins as well as toxino-coagulins. The function of the coagulins is to lock up the antigen and of the lysins to hydrolize or destroy the antigen. As examples of the coagulins we have the precipitins and agglutinins, and the antitoxins as ordinarily conceived, which immobilize and clump protein molecules or cells through their own single activity, whereas the lysins destroy protein molecules and cells and require for their complete action the addition of alexin or complement. The purpose or results of the lysins in protection is obvious; they destroy the antigen if it be a living microorganism, although, as we have seen, destruction and liberation of toxic protein molecules if sudden may result seriously for the host. The result of the coagulation is less evident; motile bacteria are immobilized, which may increase the ease of their phagocytosis, although when the agglutination is extreme-that is, in large massesit may actually protect mechanically the bacteria in the center of the mass.

It would seem as if the lytic antibodies are the more important in 
anti-infectious immunity. There are several different bodies described which have the main properties of the sensitizer of the lysins as we have described it; the important one, apart from specificity, is a property of uniting with the antigen and of so affecting it that the complex fixes alexin. We believe further that the opsonin or tropin is identical with the sensitizer and amboceptor as ordinarily described, although its activity is differently expressed. Sensitizer (amboceptor) and fixation antibody are identical; opsonin and tropin differ in potency only. Such an understanding of the tropin brings a complete harmony between the cellular and humoral aspects as we have seen them. A few demonstrated facts, a restatement of certain points already made, and a graphic representation of the varying degrees of protection alone are necessary to make this simplification clear.

It was originally assumed by Wright that the opsonin, the substance in normal serum which increases phagocytosis, is a single body that requires no reactivation, and is destroyed by heating to $55^{\circ} \mathrm{C}$. Chapin and Cowie (1906) have shown that it is really a dual body like the normal lysin. Another necessary prelude to our explanation is the fact that the increase of either sensitizer or alexin will make it possible to reduce the other substance and still produce the same result quantitatively (Morgenroth and Sachs). In the following expression of different grades of immunity we have to deal with a summation effect of varying amounts of two substances which, to recapitulate, are:

I. Alexin (complement or cytase). Ferment-like, thermolabile, non-specific, not increased by immunization. It is present in leucocytes and in -serum, probably not in plasma Has the property of combining with antigen-antibody.

2. Sensitizer (amboceptor; opsonin, tropin). Specific. May occur in small amounts in normal serum. Markedly increased by immunization. Thermostable $\left(55^{\circ} \mathrm{C}\right.$.) ; unites with antigen to form complex that attracts alexin whether within or without the leucocyte.

The different grades of protection may be expressed as follows:

I. Resistance in normal animals $=$ simple phagocytosis in vitro with washed leucocytes or in vivo in the plasma which contains no normal antibodies. Destruction of the bacteria takes place within the phagocyte where the alexin is situated.

2. Natural immunity $=a$. In vivo. Plasma contains a weak sensitizer or opsonin which combines with bacterium and renders it more attractive for the phagocyte or, in other words, forms a complex that combines with the alexin within the phagocyte which destroys it. 
b. In vitro. Serum contains weak sensitizer and alexin. Same attraction for phagocytes as (2), but result in greater destruction of bacterium within the cell owing to two doses of alexin, one within and one from without the cell. This corresponds to the conditions of Wright's opsonic experiment.

3. Acquired immunity $=$ Results essentially the same in vitro and in vivo. Plasma contains strong sensitizer or tropin which makes both engulfing and destruction of bacterium by alexin within the phagocyte rapid. Owing to the strength of the sensitizer destruction in vitro in presence of fresh serum (alexin) may take place extracellularly (Pfeiffer's phenomenon). Inactivated immune serum does not itself dissolve, but the attractiveness of bacterium treated with it for phagocyte is not appreciably lowered owing to the strength of sensitizer.

This explanation may serve to simplify the factors discussed and to show their actual relations to immunity as it occurs. It would appear that the phagocytes are ultimately responsible for the destruction of bacteria either by forming the alexin in serum or by containing it for intracellular digestion. Acquired immunity differs from natural immunity in the strength of those antibodies that direct and increase alexic activity.

But these factors combined do not suffice to explain all conditions of immunity. It does not explain why rabbit serum is strongly bactericidal for the anthrax bacillus although the animal succumbs readily to infection with it. It does not explain why the fresh serum of a rabbit highly immunized against the typhoid bacillus will not destroy this microorganism in vitro although the animal's tissues become sterile in twenty-four hours, contrary to a carrier condition that may be regularly produced in normal rabbits. And finally, it does not explain why typhoid recoveries with no demonstrable antibodies in their blood are protected from the typhoid fever, whereas the typhoid vaccinated may contract the disease in spite of a positive Widal reaction.

The last example points to an explanation by a process of exclusion, such instances having been attributed to a tissue immunity. As yet this expresssion means very little, but it is evident that the importance of this phase of immunity will grow. We already regard phagocytosis in a larger sense than did Metchnikoff, who emphasized the importance of the mobile phagocytes almost entirely. Evans has extended the conception of macrophases to the living cells of capillaries and a still further extension is possible. And in addition to the phagocytic function of 


\section{MECHANISM OF BODILY RESISTANCE TO DISEASE}

cells we must consider various cells as the loci of antibody formation. Although evidence points to the hematopoietic organs as a source of antibodies it is by no means certain that other and perhaps all tissues in varying degree may not produce them. Tissue immunity may be due not simply to a storage of antibodies but more likely to an increased ability to mobilize or to produce antibodies (Wassermann's "Umstimmung"). The continued residence in a cell of a changed reactibility for years or for life is somewhat difficult to comprehend but no more difficult to believe than the existence of memory traces in the brain. The local skin reactions provoked by an antigen as in typhoid and smallpox immunes are concrete evidence of a changed reactibility of this sort.

\section{ANAPHYLAXIS}

We come now to a consideration of a condition that is inextricably connected with immunity. This is the condition which has been successively named hypersusceptibility, anaphylaxis, allergy (changed reaction), and taraxy (disturbance). Although. instances of this unusual condition have long been recognized in disease and also in the process of immunization, no comprehensive idea of it existed until relatively recently. It had never been explained why animals that were being immunized with bacterial products began to waste away and die of what we were pleased to term cachexia. It was never understood why certain individuals are given symptoms of acute poisoning by eating some foods, such as eggs or strawberries. Other individuals show extreme local susceptibility to iodoform. Sufferers from hay fever and asthma are thrown into attacks resembling acute coryza or bronchial spasm under conditions which were only faintly appreciated. Finally there are the extraordinary group of symptoms known as serum sickness which followed the repeated doses, or sometimes the initial dose, of a foreign therapeutic serum, and which are manifest either by acute symptoms of vomiting, dyspnea, and collapse, or by delayed symptoms of joint pain, fever, and a rash.

We have seen how the study of the antibodies produced by the injection of harmless proteins finally came to explain much of the mechanism of the reactions of immunity that first attracted our attention in connection with bacterial proteins. The same history has been true in the development of our knowledge of anaphylaxis, only in the latter case the first well-studied instances were in connection with the harmless proteins. Isolated observations by Magendie (1839) and by Flexner ( 1894 ) were not appreciated as representing a widespread and fundamental condition in animals that may be induced by a previous adminis- 
tration of any foreign protein substance. It remained for Richet (1905) ( ${ }^{88}$ ), who was working with a poisonous protein of shellish, to show that dogs may be rendered more susceptible than usual to a second injection of this substance through having received a previous one. For this reason he gave the name of anaphylaxis to the condition as opposed to the condition of prophylaxis which ordinarily follows repeated injections of the protein under the guise of immunity, as we understand it. It may be stated at this point that increased susceptibility may be produced by any substance that gives rise to increased resistance, provided the dosage and intervals between injections is properly spaced. The exact relation, however, of an increased susceptibility to an increased resistance is by no means understood. It may be a parallel condition that is independent of the condition of immunity or, on the other hand, it may be simply a stage in the development of immunity. The best we can do at the present time is to outline the conditions under which anaphylaxis occurs.

Following the work of Richet two general phenomena were discovered in animals which illustrate directly the two general forms of anaphylaxis as we now understand them, a local form and a generalized form. In 1906 Arthus found that rabbits that had received several injections of horse serum finally responded to a subsequent injection by a local sterile necrosis at the point of inoculation. This evidence of local anaphylaxis never occurs in normal rabbits. An exactly similar condition has since been described in children who were given repeated doses of diphtheria antitoxin by Lucas and Gay $\left({ }^{40}\right)$. The second or generalized form of anaphylaxis was discovered independently by a number of observers in connection with a testing of diphtheria antitoxin. It was found that guinea-pigs that had received a minute amount of a toxin-antitoxin mixture were subsequently fatally intoxicated by injections of a relatively large amount of antitoxin or of normal horse serum. This form of anaphylaxis also occurs in human beings in the condition known as the accelerated serum reaction that may follow immediately on the injection of antitoxin or any foreign serum.

The study of these phenomena of generalized anaphylaxis in guineapigs and the analogous phenomena in other animals have given us most insight into the mechanism of the process in general. Certain of the more important facts should be outlined. A very small amount of a suitable protein, whether it be a bacterial protein and therefore somewhat toxic, or a harmless protein such as milk, serum, or egg white, will so alter the physiology of a normal guinea-pig that its reaction to a subsequent injection of the same substance becomes radically and often 


\section{MECHANISM OF BODILY RESISTANCE TO DISEASE}

fatally different. This specific sensitivity to the protein in question remains either for a long period of time or for the entire life of the animal. On receiving a second dose of the same protein an animal reacts characteristically, the rapidity of the reaction depending on the route chosen for administration of the protein and the amount of it. The animal shows symptoms of excitement followed by paralytic symptoms, which latter phenomenon is particularly significant in the form of a respiratory distress which often brings about death through asphyxia. Anaphylaxis is as specific as immunity. An animal sensitized to one protein reacts within group limits only to the same protein, and an animal may be sensitized to several proteins and react separately to each one of them in turn. When a protein is hydrolyzed to the point that it fails to produce immunity it likewise ceases to produce hypersusceptibility. Anaphylaxis as studied in the guinea-pig may be transferred by means of the serum, and is therefore also inherited by the first generation of the offspring. Death, which may occur within three to five minutes if the second dose is given intravenously, is preceded by the stormy symptoms that have been mentioned and at autopsy characteristic lesions are found (Gay and Southard) $\left({ }^{41}\right.$ ). These lesions are an insufflation of the lungs produced, as was subsequently shown by Auer and Lewis, by paralysis of the bronchial musculature, and secondly, minute hemorrhages in various parts of the body, particularly in the stomach, caused by fatty degeneration in the capillary endothelium. The phenomenon of anaphylactic shock, in the guinea-pig at least and in the dog, is primarily intracellular in nature, as was first pointed out by Gay and Southard and later confirmed by Weil, Manwaring, and others. Individual muscular organs of a sensitized animal, such as the heart and the uterus, may be shown to be more irritable than normal when subjected to a fluid containing the protein to which the animal is sensitive.

The extreme symptoms that occur in the guinea-pig are by no means equally prominent in other animals, and there are certain other criteria of anaphylactic shock, one or more of which may be found in practically all animal species that have been tried. These characteristic symptoms are a fall in the blood pressure, a fall in temperature, delayed coagulability of the blood, and, last of all, a falling in the alexin content of the blood serum. The mechanism on which the shock deperds, although intracellular in the guinea-pig and dog, is probably extracellular in origin in the rabbit.

Numerous theories have been devised to explain the facts already obtained, and they have been useful in leading to further experiments. The earlier theories of Rosenau and Anderson and others regarded the 
reaction as due simply to a condition of antigen and antibody. In the theories of Gay and Southard, Vaughan and Wheeler, and Besredka ( ${ }^{42}$ ) there is evidence which goes to indicate that the substance in a protein which gives rise to sensitization to that protein is not the same as the fraction of the protein that produces the intoxication. The idea is most concretely expressed in what may be regarded as the best of several proteoclastic-ferment theories of anaphylaxis, the one offered by Vaughan $\left({ }^{25}\right)$. Sensitization is regarded as originating or increasing the proteolytic ferment in the serum, and this ferment splits the protein rapidly on its second introduction into the animal body and discloses the poison molecule within it. This poison molecule may be artificially uncovered in vitro by splitting the protein with alkali alcohol. This theory is essentially corroborated by the experiments of Friedberger, who demonstrated an "anaphylatoxin" when the serum of a sensitized aninial was brought in contact with the antigen.

A further development of these ideas is expressed by the adsorption theories of anaphylaxis. These theories, although admitting the formation of a toxin under the conditions just noted, would attribute the substrate from which this toxin is obtained to the serum of the host rather than the antigen. This theory, which has been developed by the work of Keyser and Wassermann, by Bordet and Zunz, and by Jobling and Peterson, attributes the liberation of the toxic substances from the antiserum to an absorption of the antiferment in such serum, thereby allowing the ferment present in it to act upon its own protein. The latest theory (Taraxin theory) that has been offered to explain anaphylactic shock is the one advanced by Novy $\left({ }^{43}\right)$, who would explain the intoxication as due to a tautomerization of the blood proteins, by a process similar to blood coagulation. Protein cleavage is not affected according to Novy, but any substance, even distilled water, when added to serum may bring about this change in the arrangement of the molecules in the protein. Anaphylaxis is specific only in the sense that the combination of antigen and antibody acts best as a catalyzer to bring about this exchange of molecules.

Animals that recover from anaphylactic shock are thereafter for a period of time insusceptible to reintoxication by the protein to which they have been sensitized. They are spoken of as being in a condition of antianaphylaxis or in a refractory stage. The latter term indicates the fact that they eventually become sensitive again to intoxication. This condition of antianaphylaxis is of practical importance since it can be induced artificially by using relatively small amounts of the antigenic substance, with the advantage that the animal or individual may then be given any amount of the protein without harm. In the case of 
serum, antianaphylaxis (or desensitization) is produced by giving small amounts of the substance subcutaneously or by the rectum. When it is desired to administer large amounts of serum therapeutically directly into the circulation, desensitization can be accomplished by giving a very small amount of the substance intravenously, or simply by injecting the therapeutic dose very slowly. Hypersusceptibility to therapeutic serum, usually horse serum, should be tested for by means of a local reaction which will later be considered.

The phenomena of specific sensitization to proteins are already recognized as of great, although often not fully explained, importance to infection and resistance. The wasting process often referred to as cachexia which occurs in immunized animals and also in infectious diseases will doubtless eventually be explained as a type of chronic anaphylactic intoxication. A number of the symptoms or occurrences in the course of an infection have been explained on the basis of anaphylaxis. Thus fever is regarded by Vaughan as being due to the continual splitting of small amounts of the foreign protein of bacteria produced by the ferment engendered in the host during the incubation period in the infectious diseases. The incubation period itself is explained as being necessary for the formation of these ferments; until they have reached a certain maximum no symptoms of the disease itself occur, although the infecting agent has been resident in the body for some days. The crisis in fevers is explained as the sudden transition into a condition of antianaphylaxis due to a rapid or sudden splitting of a large amount of the foreign proteins followed by desensitization and cessation of the fever. Vaughan and Friedberger have actually produced fever by giving repeated small doses of harmless proteins and of bacterial proteins to rabbits. It should be repeated that bacterial proteins, as well as the harmless proteins that have been studied most, give rise to sensitization and anaphylactic shock, although with the small amounts of proteins in any ordinary dose of bacteria it is often difficult to demonstrate anaphylaxis experimentally.

Reference should again be made to the two general types of serum diseases described by Von Pirquet and Schick $\left({ }^{44}\right)$ as instances of anaphylactic phenomena in human beings. This serum disease is first a delayed form in which a characteristic syndrome comprising urticaria, fever, and joint pains occurs a number of days after administration of a therapeutic serum. These symptoms although annoying are not serious in nature. Secondly, the form of localized anaphylaxis characterized by necrosis and originally described by Arthus in rabbits may occur in human beings on the repeated injection of a therapeutic horse serum at short intervals. And thirdly, the guinea-pig type of 
acute anaphylactic shock may occur on the intravenous injection of foreign proteins, or even on subcutaneous inoculation when large amounts are given. This "accelerated" reaction, as it is called, is characterized by dyspnea, cyanosis, nausea, and vomiting, and at times by sudden death.

It is interesting to note the relation of this accelerated serum reaction to asthma; the symptoms in both conditions are practically the same. The most characteristic one, dyspnea and cyanosis, is caused by a paralysis of the musculature of the finer bronchioles. The analogy is even closer when we learn that even the first injection of a foreign serum may produce anaphylactic shock in asthmatics, which indicates that asthma represents a susceptibility to certain proteins, perhaps even a generalized susceptibility to a number of proteins. Much study has been made in recent years on the susceptibility of certain individuals to asthmatic attacks on the inhalation of the effluvia, or, more accurately speaking, the proteins from certain animals. Some individuals when brought in contact with horses, cats, or guinea-pigs, or with the hair of these animals, are thrown into violent asthmatic attacks. The susceptibility of these, individuals can be demonstrated by their local reaction when small amounts of the proteins from these substances are placed upon the abraded skin or injected intradermally. Of similar nature are the susceptibilities of individuals to foods of various sorts, particularly to shellfish, to the white of egg, as particularly noted in children, and to various berries. In these latter instances of food poisoning the anaphylactic shock attack is brought on not by inhalation but by ingestion of the substances concerned, and the symptoms may appear in the form of urticaria, which is characteristic of the delayed type of anaphylactic shock, or by acute respiratory distress and vomiting characteristic of the accelerated reaction.

The drug idiosyncrasies are typically anaphylactic in appearance. They are demonstrable both locally and generally. It is not easy, however, to correlate these conditions with true anaphylaxis on account of the non-antigenic character of the substances that give rise to them, unless we accept Wells's hypothesis of a combination with the proteins of the host.

\section{THE APPLICATION OF THE PRINCIPLES OF IMMUNOLOGY IN THE DIAGNOSIS, PREVENTION, AND TREATMENT OF INFECTIOUS DISEASES}

Interesting as are the facts of immunology viewed abstractly as constituent parts of a biological science, they become more compelling when viewed from the standpoint of their practical utilization. The guiding 
impulse that has provoked the enormous volume of work in this field during the past forty years has very properly been utilitarian. So strong indeed has this impulse been that it has very naturally led not infrequently to hasty and unwarranted conclusions. There is no branch of scientific medicine that has been so liable to exploitation and injudicious enthusiasm; no literature is more clogged with half-baked theories, unrealized expectations. The rewards have been actually so great that too many and too wide-reaching results have been expected. There is no reason why proper enthusiasm as to the possibilities of specific diagnosis and therapy should be in the least abated, but we may look forward to many years of patient endeavor before the ultimate realizable result is attained.

In the few remaining pages an effort is made to tabulate the practical results that have been obtained in the diagnosis, prevention, and cure of disease through the utilization of the principles of immunology that have been outlined.

\section{Diagnostic Reactions of Immunology}

The diagnostic value of the various phenomena that occur in connection with conditions of immunity depends on the production of the changed reaction, and frequently on an extracellular antibody formation representing this changed reaction, in the body of infected or immunized animals. The reactions depend on the specific property that antigens have of uniting with their corresponding antibodies. When either of these two substances is known to be present the existence of the other may be determined. We may therefore readily divide this discussion into those tests which depend on determining the presence of an antigen in the presence of a known antibody, and on the other hand the determination of the presence of an antibody when a known antigen is at hand.

\section{Tests for Suspected Antigen in the Presence of a Known Antibody}

In reactions of this sort the antibody is usually furnished by the serum of a hyperimmune or artificially immunized animal; in other words, an animal in which the antibody content has been raised beyond the point usually attained in simple recovery from disease. In this and the following subsections we shall make no attempt to describe the technical procedure necessary to demonstrate the substances that are being sought for. A description of the methods of procedure, the causes of error, the criteria of what constitutes a positive reaction and the like, will be 
found in recognized textbooks of immunity, notably in those of Zinsser $\left({ }^{45}\right)$ and Kolmer $\left({ }^{46}\right)$. We have space here simply to indicate the scope and the relative value of the different varieties of reaction that have been shown to be useful.

The identification of suspected bacteria is one of the important uses to which a known antiserum can be put. When microorganisms are isolated from the body in pure cultures, it is necessary to identify them not only by their cultural characteristic but ultimately by their specific property of reacting with a known immune serum. Thus in the case of typhoid fever or paratyphoid fever it is necessary not only that the organism isolated in the circulating blood or stools should conform morphologically and in its reaction on culture media to recognized typhoid or paratyphoid bacilli, but that it should become agglutinated in a characteristic manner by an antiserum that has been artificially produced by immunization with identified strains of the organism in question. In certain instances it may be possible to use serum from a case that is known to be suffering from the disease in question. The reactions between the suspected organism and the known immune serum that may be used are first of all agglutination reaction, and. second, the alexin fixation reaction, and in the case of the cholera vibrio Pfeiffer's phenomenon of bacteriolysis as produced either in the animal body or in the test tube. A precipitin reaction between a filtrate of broth culture and the corresponding immune serum may also serve as a diagnostic procedure.

The second useful application of reactions of this sort lies in the determination of the etiological relationship of an unknown organism that is suspected to be the cause of a disease, as shown by its reaction with the serum of patients suffering from that disease. We have already given an example of the usefulness of such a test in connection with the bacillus isolated from whooping-cough by Bordet and Gengou. These authors found that the coccobacillus gave a positive fixation reaction with the serum of whooping-cough cases, whereas controls with normal serum and with the patient's serum and other bacteria were negative.

Further use of tests of this sort lies in the differentiation of bacteria that are closely allied to one another. This type of test is useful not only for the purpose of classification but 'for the practical purpose of determining the exact variety of a given microorganism that may be responsible for an individual instance of disease. An example of the latter usefulness is the determination of the particular type of pneumococcus concerned in each case of lobar pneumonia, where such a determination is essential in order to establish the rigorously specific 
immune serum which alone will give results in therapy. Similar tests have also been applied to the type differentiation of the meningococcus for the same purpose.

A known immune serum may be used in a variety of other ways for the determination of suspected protein antigens. The various forensic tests are instances of the usefulness of reactions of this sort. The human origin of blood stains or semen stains may, as we have already intimated, be accurately determined by means of a precipitin reaction carried out by adding a known antihuman serum from the rabbit to a very great dilution of the suspected blood. The same reaction may also be used to detect the falsification of meat, as in the case of sausages that may be labeled pork but found to contain horse meat or other meat. It is further applicable in the detection of violation of game ordinances, as in the instance described by the author who could prove the presence of venison in the possession of a suspected individual who claimed that the meat was veal. The precipitin reaction is usually employed in tests of this sort. The reaction of alexin fixation as described by Neisser and Sachs is even more delicate for the purpose.

The diagnosis of any particular bacterial infection rests when possible on the isolation of the microorganism concerned from some local lesion or from the circulating blood. It is possible, however, in some diseases to demonstrate the presence of protein substances derived from the bacteria, even when the bacterium itself is not readily isolated. It may be shown, for example, that the precipitinogen of the pneumococcus is present in the urine and in the sputum of cases of pneumonia, and the variety of pneumococcus that is at hand may be determined by the precipitin test with the appropriate immune serum. The spleen of rats that have died of plague can be shown to contain protein of the plague bacillus by adding anti-plague serum to an extract of such organs. The diagnosis of anthrax in the organs of cattle that have died of the disease may be determined even when considerable decomposition has taken place by means of a thermo-precipitin test, that is to say, by demonstrating the presence of a precipitinogen that resists boiling. Antibodies may, in some instances, be demonstrated by their ability to evoke a condition of hypersusceptibility in animals, as, for example, in the anaphylactic tests for human blood which may be brought about by sensitizing the guinea-pig with a suspected blood stain and subsequently determining whether they are intoxicated or not by human blood serum. A similar reaction of this sort has been demonstrated by Force and Beckwith, who found that smallpox could be diagnosed differentially from chicken-pox by injecting small amounts of the pustular contents intradermally in rabbits that had been highly immunized against vaccine 
virus. In the case of true smallpox a characteristic skin reaction is produced, whereas chicken-pox produces no such result.

\section{Tests for Suspected Antibody in the Presence of Known Antigen}

Under this heading may be included not only the demonstration of an antibody in the serum of a diseased individual, but also the demonstration of certain changes in the physical properties of such serum, and also the demonstration of a changed condition or allergy in the body of the individual concerned. These possibilities will be dealt with in turn.

Several reactions are of accurate diagnostic value in testing for antibodies, particularly the agglutinin reaction, the reaction of alexin fixation, and, in a few cases, the percipitin reaction. The success in obtaining a positive reaction with a serum of a diseased individual for the presence of an antibody in such serum depends largely on the infecting microorganism and the type of disease it produces. Certain bacteria, as we have seen, produce agglutinins more readily than others, and the diagnostic value to the antibodies produced by a given microorganism is affected very markedly by the duration of the disease. In certain diseases the delayed appearance of antibodies may still be of diagnostic value, whereas other diseases are so acute that death or recovery takes place before such antibodies are present. The rapidity of appearance of antibodies again depends to some extent on the degree of involvement of the infectious process. The value of each of the tests we have mentioned in the diagnosis of infections may be summarily classified as follows:

The agglutination reaction is of great value in the following infections: typhoid fever, paratyphoid alpha fever, malta fever, and relapsing fever.

The agglutination reaction is of distinct though limited value in the following infections: paratyphoid beta fever, glanders, bubonic plague.

The agglutination reaction is of possible but doubtful value in the following infections: bacillary dysentery, epidemic meningitis, and lobar pneumonia.

The agglutination reaction is of no value in the following infections: syphilis, tuberculosis, gonorrhea, thrush (due to oidium albicans), and trichophyton infections. These latter instances include those diseases in which attempts have been made to utilize the reaction without definite success. There are other infections in which no serious estimate of the value of the agglutination reaction is available, and in some of these diseases at least it may eventually prove useful. 
The reaction of alexin fixation is of great value in the following infections: glanders and syphilis, in which latter case it is used in essential form although with modified principle in the Wassermann reaction.

The fixation reaction is of some value in the diagnosis of the following infections: typhoid fever, tuberculosis, bacillary dysentery, gonorrhea (chronic forms), whooping-cough, plague, thrush, relapsing fever, and echinococcus disease.

The precipitin reaction is said to be of value in echinococcus disease.

The passive transfer of anaphylaxis has been suggested as of value in a few diseases such as tuberculosis and cancer, but has not been generally utilized.

There are a number of other tests of suggestive or actual value in the diagnosis of infections that are dependent on some metabolic change in the individual that is reflected in certain of the physical properties of the blood serum. Such, for example, are the epiphanin reaction of Weichardt, which depends on the change in diffusion produced by a combination of an antiserum with its antigen. Of similar nature is the meiostagmin reaction of Ascoli dependent on a change in surface tension as measured by the size of drops of a mixture of antigen and antibody as compared with a control mixture and measured by a staglomometer. The reaction has been claimed to be useful in tuberculosis and typhoid fever. Other tests have depended on the increased globulin present particularly in the cerebrospinal fluid, as in the butyric acid test of Noguchi, and in Lange's colloidal gold test which is based on precipitates with the spinal fluid in different zones corresponding to various degrees of involvement of the central nervous system in syphilitic and other processes. The Abderhalden test for proteolytic ferments in the blood suggested as a means of diagnosis of pregnancy is of little value in the diagnosis of infections owing to its non-specific character as we have already mentioned.

Most important are the tests dependent on a changed reaction or allergy in the individual concerned, demonstrable by a local reaction on the application of a known antigen. The exact significance of these reactions apparently varies in particular as to whether we may consider them as instances of hypersusceptibility or of increased resistance. It is probable, indeed, that the two processes overlap. There are various methods that have been utilized in provoking these local reactions, notably the conjunctival application of the antigen, the cutaneous abrasion followed by local application (Von Pirquet), the percutaneous method produced by inunction, and finally the intradermal method produced by injecting a small amount of the antigen into the superficial 
layers of the skin. In general it may be said that the intradermal method is the most accurate and delicate. In a number of different infections localized susceptibility tests are of recognized diagnostic value. The use of tuberculin in tuberculosis and of mallein in glanders are of known significance, although more important when negative than when positive. The luetin reaction of Noguchi in syphilis is of value in tertiary cases provided care is taken to avoid those individuals to whom iodides have been administered, since it has been found that the administration of this drug in itself may give rise to a positive reaction. The abortin reaction has been used in cases of contagious abortion of cattle. An extract of the gonococcus has been employed in chronic localized infections of this nature. Plague, ringworm, favus, and trichophytosis all give local skin tests, under certain conditions. Streptothrix infections may be differentiated from tuberculosis by a preparation resembling tuberculin derived from one or the other of the varieties of this closely allied microorganism.

In addition to the local tests of definite diagnostic value certain tests should be mentioned in which they seem definitely indicative of protection, and which therefore may be regarded as of prognostic value. It was found, originally by Jenner and later made practical use of by Force and Beckwith $\left({ }^{47}\right)$, that individuals who are successfully vaccinated or who have recovered from smallpox give a characteristic intradermal reaction to vaccine virus. In a similar way, Gay and Force $\left({ }^{48}\right)$ have found that typhoid recoveries and individuals successfully vaccinated against typhoid react characteristically to injections of typhoidin. In a similar way it is questionable whether or not the tuberculin reaction may not indicate protection or successful resistance rather than advancing disease. In all these instances that have been mentioned a positive reaction is indicated by a reddish indurated nodule which appears in a few hours after intradermal injection. In the latter instances which we have mentioned as of prognostic value, the tests may be taken to indicate protection under normal conditions of infection. Of similar significance but of different appearance is the Schick test for diphtheria antitoxin. The intradermal method of injections of a small amount of diphtheria toxin may or may not give rise to a reddened area of inflammation. When this reddened area is absent the test is positive, and indicates the presence of a definite amount of antitoxin in the serum of the individual concerned and also his protection against diphtheria.

There are also numerous other tests of specific susceptibilities which may be regarded as definite indications of an increased susceptibility to the protein concerned. Such reactions are produced in the drug idiosyncrasies that may exist in certain individuals and be demonstrable 
by the local application of such substances as quinine, iodoform, atropine, and strychnia. We have already suggested that these conditions of local anaphylactic reaction may be due to sensitization and to a compound of the drug with the protein of the host.

Other instances of general hypersusceptibility may be indicated locally by application of the proper antigen concerned, as in hay fever, where one of several pollen proteins may alone produce a reaction, or in those cases of asthma associated with various susceptibilities to food and frequently produced by ingestion of these foods. Skin tests with the proteins of various meats, fish, or berries may give rise to local indications of this sort, and in some instances susceptibility to the inhalation of effluvia from animals may be determined by the application of an extract of hair from one of the animals. The test of local susceptibility again is applied in order to avoid anaphylactic shock which would occur on the injection of large amounts of serum.

\section{Prevention and Therapy of the Infectious Diseases by the Use of Antigens and Anti-sera}

The prevention or cure of the infectious diseases by so-called specific means depends, as in the case of their diagnosis, on the use of antigens or of antibodies, of vaccines, or of immune sera. In other words, we may discuss here the production of active or of passive immunity in its practical aspects. We have already considered briefly many of the factors that control the artificial production of these two advantageous conditions, such as the methods of producing active immunity, the types of virus or vaccines employed, the relative duration of active and of passive immunity, the method of transferring passive immunity and the properties of an antiserum employed in such transfer. Our interest here lies merely in summarizing the instances of successful and promising vaccine and serum prophylaxis and therapy.

Antigen prophylaxis. The first and still the most completely successful instance of antigen prophylaxis is in smallpox vaccination, where a modified living virus is used to induce a local form of the disease. In countries where vaccination is practiced twice in a lifetime, smallpox has practically ceased to exist; the protection afforded by this procedure is as nearly absolute as anything in biology can be. The next most successful instances of vaccine prophylaxis are in the case of the typhoid and paratyphoid fevers. Systemic immunization has enormously reduced the incidence of these diseases and their fatality both in army and in civilian life. The method, however, even when faithfully carried out, does not protect in one hundred per cent. of cases, 
as has often been assumed, for even a limited period of time, and it is probable that it protects very little under conditions of unusual infection. The duration of the protection, moreover, is limited to one or two years, which means that revaccination is necessary at such intervals. There is evidence that real, although by no means so striking, protection as in the case of typhoid vaccination can be secured in bubonic plague and in Asiatic cholera by vaccination. It seems possible also that vaccination against the pneumococcus is about to be realized. Good protection against certain infectious diseases of animals has been obtained by vaccination in chicken cholera, anthrax, blackleg, cattle plague, and symptomatic anthrax. There is a modified form of immunity against bovine tuberculosis that can be obtained by injecting a living attenuated human culture intravenously (Von Behring's bovo-vaccination). This method is being further perfected and is of great promise as a means of suppressing not only bovine but indirectly human tuberculosis.

The use of a dried attenuated virus in the prevention of rabies has. reduced the mortality in this disease from sixteen per cent. to a fraction of one per cent. It differs from the instances of prophylaxis already cited in that the treatment is begun after inoculation has taken place. An active immunity is produced, however, owing to the lengthy incubation period in the disease (forty days).

Another type of antigen prophylaxis, or better another type of antigen, is one modified in its toxicity by saturation with an immune serum. Sensitized vaccines have been used in cholera, plague, dysentery, and typhoid prophylaxis. They are less toxic and therefore may be given in comparatively larger doses, and there is reason to believe thcy produce a more durable immunity than untreated vaccine. Active immunization against diphtheria has also been attempted with apparent success by a balanced toxin-antitoxin mixture.

Antigen therapy. The interest in vaccine therapy was awakened by Sir Almoth Wright. He should be credited with starting us fairly on a profitable line of practical therapy of which the end is by no means yet attained; on the other hand his own work is biased and interpreted in the light of a somewhat unwarranted enthusiasm. Abashed by the repeated failures to affect infections of known etiology by passive immunization, Wright presented the possibilities of active immunization. In the case of chronic and subacute local infections there is little reason to question, on both theoretical and practical grounds, that the injection of the microorganism concerned, in the form of a vaccine, may "bring into play new areas of resistance" and prove of curative value. Wright's idea of following the progress of therapy of this kind by the opsonic index has proved impracticable, and it is also probable that his idea 


\section{0}

of the necessity of employing an autogenous vaccine is also exaggerated. It would even seem that the possibilities of treating acute and generalized infections by vaccine therapy, which at first seemed less reasonable on theoretical grounds, are being realized. The mechanism involved in such therapy is perhaps different from what Wright conceived it, as we shall see in a moment. The localized affections most benefited by antigen therapy are the staphylococcus infections, skin affections, and tuberculosis as treated by tuberculin.

Non-specific protein therapy. The type of therapy by antigens just discussed apparently depends for its success on the production of an active immunity against the infection concerned and is therefore highly specific. It is probable that at least some of the beneficial effects noted, however, are due to another non-specific factor that previously had been chanced upon in other lines of investigation. A number of instances of apparently successful therapy have been noted in the infectious diseases that have no specific relation to the etiological agent concerned, but are dependent on the fact that a foreign protein has been employed. .In spite of the interpretations that have been given for the effectiveness of "pyocanase" (Emmerich and Loew), " plasmine" (Buchner), and leucocytic extract (Hiss and Zinsser), it seems to us now self-evident that whatever good they did might have been obtained by any other foreign protein substance. Recent work has shown that certain general infections like typhoid fever and arthritis may be markedly benefited following the intravenous injection of any protein whether albumose or typhoid vaccine. These benefits are associated with, and we believe caused by, an increase or mobilization of the leucocytes; this mobilization may also be associated with or the cause of a mobilization of proteolytic ferments. We believe, however, that the advantages of a non-specific protein of this kind is enhanced at least in typhoid fever, by employing the antigen concerned in the disease (Gay) $\left({ }^{49}\right)$.

Antiserum prophylaxis. The short duration of passive serum immunity makes the field of usefulness of this type of protection very limited. It is only in those cases where infections may be assumed to have occurred or to be likely that it is widely practiced. Tetanus antitoxin is the best example of a really effective use of immune serum in prophylaxis. Lockjaw following fourth of July injuries and particularly after war wounds has practically ceased to exist since antitoxin has been universally employed. A similar usefulness seems promised in preventing at least certain forms of gas gangrene. Diphtheria antitoxin is also a valuable prophylactic serum. Small amounts confer an immunity lasting from two to four weeks; it is particularly advisable

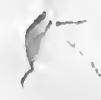


in asylums to prevent the outbreak of diphtheria and in families where cases have occurred.

Antiserum therapy. Antiserum therapy readily divides itself into two varieties, antitoxin serum therapy and antibacterial or anti-infectious serum therapy, based on the principal mode of infection of the particular microorganism in question. Diphtheria antitoxin has reduced the mortality from this disease from perhaps 40 to from 7 to $14 \%$ on the average. This by no means expresses the possible usefulness of the remedy, for it is found that when antitoxin is used on the first day of the disease the mortality is practically nil. From another standpoint it is found that the mortality rates from this disease in a city like Philadelphia have fallen from 125 to 34 per 100,000 since the introduction of antitoxin. In tetanus therapy the results are not so striking once symptoms are evident, but even here the mortality has been decreased perhaps 20\%. Great progress has been made in this disease by realizing the necessity for reaching the point of action of the toxin and therefore by injecting the serum intrathecally; many more cases have been saved since this method has become more common. The same is true to some extent of the intravenous injection of diphtheria antitoxin in desperate cases of diphtheria.

Antitoxins to the recently claimed soluble toxins of the cholera vibrios, and the Shiga dysentery bacillus have been used with apparently good results. A good antitoxin to $B$. botulinus has been produced but has not been used to any extent. The antitoxin to cobra snake venom has been used in India with good effect.

In view of the success that has been attained by at least one of the antibacterial sera (meningococcus), it seems surprising that a more widespread usefulness has not been obtained with antisera of this type. In cerebrospinal meningitis the mortality has been lowered from eighty to thirty per cent. or even less by the administration of the serum intrathecally. Another reason besides this adoption of the proper route of administration for the ultimate and increasing success of this form of therapy lies in the recognition of several varieties or types of the organism concerned. It is realized that corresponding antibodies for the particular type present must be included in the serum. This fact has been brought out even more clearly in the only other instance of distinctly successful human antibacterial therapy-lobar pneumonia. In this disease Type I pneumococcus infections are now treated with a definite result and Type II cases are apparently benefited, provided the corresponding type antiserum is employed. Anti-infectious serum therapy has been tried repeatedly and with at least some suggestion of success in infections due to $B$. influenza (meningitis), $B$. pestis, the 
gonococcus, the streptococcus, and the dysentery bacillus. Certain essential facts necessary to produce the good results which we feel certain will accrue in these and other infections still elude us. In addition to the choice of route and the recognition of types to be combated we have to consider the type of antigen to be employed. Should it be a strain grown on human media for instance, or sensitized? The technic of immunization is found to vary with each organism, and we have no certainty that the best method has been found in any case.

We have only to mention in conclusion the use of the serum of recovered cases as it has been tried in some diseases like typhoid, anterior poliomyelitis, and scarlet fever. The serum of the individual patient is also at times used (autoserum therapy). In some diseases the normal serum of animals has been used with apparent success, normal beef serum in anthrax, for example. All these latter instances suggest that at least part of the results hitherto attained may be due to a non-specific stimulation, as in the case of protein therapy discussed under antigen.

\section{BIBLIOGRAPHY}

(The articles listed include the more general and collective summaries and relatively few of the more specific articles quoted in the text.)

I. VIRCHOW, R.: Arch. für pathologische Anatomie, etc., I847, I, I.

-2. ALLBUTT, T. C.: Medicine. Encycl. Britannica, I9II, IIth ed., XVIII, 55.

3. GOOD, J. M.: A Physiological System of Nosology. Boston, I823.

4. KREHL, L., and MARCHAND, F.: Handbuch der allgemeinen Pathologie. Leipzig: Hirzel, 1908.

5. MacCALLUM, W. G.: A Text-book of Pathology. Philadelphia: Saunders, I9I6.

6. JOSLIN, E. P.: Treatment of Diabetes Mellitus. Philadelphia: Lea \& Febiger, I9I7.

7. GAY, F. P.: Jour. Med. Research, I9I2, XX, I75.

8. LEWIN, C: : Zeit. für Krebsforschung, I912, XI, 335.

9. TAYLOR, K.: Proceed. Soc. Exp. Biol. and Med., I9I5, XII, 216.

Io. CANNON, W. B.: Bodily Changes in Pain, Hunger, Fear, and Rage. New York: Appleton, 1915.

II. SEWALL, H.: Climate in Relation to Health and Disease. Oxford Medicine, vol. I.

12. HASSELBALCH, K. A., and LINDHARD, J.: Skandinavisches Arehiv für Physiol., IgII, XXV, 36r.

13. SCHNEIDER, E. C.: Jour. A. M. A., I918, LXXI, I384.

14. FUERST, E.: Beitr. z. path. Anat. u. z. allg. Path., I898, XXIV, 4I5.

15. MÖLLER, M.: Bibliotheca Med., I900, VIII, I.

16. VON HAUSMANN, W.: Ergebnisse der Physiologie, 1907, VI, 58.

17. WELLS, H. G.: Chemical Pathology. 3d ed. Philadelphia: Saunders, I9I8.

18. FAUST, E. S.: Arch. exp. Path. und Pharm., I900, XLIV, 217. 
19: RÜBESAMEN, W.: Arch. exp. Path. und Pharm., 1908, LIX, 227.

20. MEYER, H., and GOTTLIEB, R.: Experimentelle Pharmakologie. Zweite Auflage: Berlin, IgII.

21. DOERR, R.: Kolle und Wassermann Handbuch der pathogen. Mikroorganismen. 2d ed. I9I3, II, 947.

22. VOLTZ, W., and DEITRICH, W.: Biochem. Zeit. I9I4, LXVIII, I29.

23. CLOETTA, W.: Arch. exp. Path. und Pharm., 1906, LIV, I96.

24. ADAMI, J., and McCRAE, J.: Text-book of Pathology. 2d ed. Philadelphia: Lea \& Febiger, 1914, I5I.

25. VAUGHAN, V. C.: Protein Split Products in Relation to Immunity and Disease. Philadelphia: Lea \& Febiger, 19r3.

26. FORD,'W. W.: Cent. für Bakt., I9I3, LVIII, I29 and I93.

27. RABE, F.: Zeit. für exp. Path. und Therapie, I9II, IX, $35^{2}$.

28. VON ADELUNG, E.: Arch. Int. Med., I9I3, XI, I48.

29. GAY, F. P., and ROBERTSON, T. B.: Jour. Exp. Med., I912, XVI, 470.

30. OBERMAYER, F., and PICK, E. P.: Wien. klin. Wochenschr., 1903, XVI, 659.

31. GAY, F. P., and ROBERTSON, T. B.: Jour. Exp. Med., 1912, XVI, 479.

32. FORSSMAN, J.: Biochem. Zeit., I9II, XXXVII, 78.

33. METCHNIKOFF, E.: L'Immunité dans les maladies infectieuses. Paris: Masson \& Cie., 1901.

34. WRIGHT, A. E.: Studies on Immunization. New York: Wood \& Co., rgro.

35. EVANS, H. M.: Am. Jour. Phys., 1915, XXXVII, 243.

36. BORDET, J., and GAY, F. P.: Studies on Immunity. New York: Wiley \& Son, 1909 .

37. EHRLICH, E., and BOLDUAN, C. F.: Studies in Immunity. New York: Wiley \& Son, 1906.

38. NEUFELD, F.: Kolle und Wassermann's Handbuch. Ist ed. I903, II, 303.

39. RICHET, C.: Arch. di fisiol., 1903-04, I, I29.

40. LUCAS, W. P., and GAY, F. P.: Jour. Med. Research, I909, XX, 251.

4I. GAY, F. P., and SOUTHARD, E. E.: Jour. Med. Research, 1908, XVIII, 407.

42. BESREDKA, A.: Anaphylaxie et Antianaphylaxie. Paris: Masson \& Cie., 1917.

43. NOVY, F. G.: Jour. Infec. Dis., 1917, XX, 498-854.

44. VON PIRQUET and SCHICK: Die Serumkrankheit. Vienna, 1905.

45. ZINSSER, H.: Infection and Resistance. 2d ed. New York: Macmillan \& Co., 1918.

46. KOLMER, J.: Infection, Immunity, and Specific Therapy. 2d ed. Philadelphia: Saunders, I9I7.

47. FORCE, J. N., and BECKWITH, HELEN L.: Jour. A. M. A., 1915, LXV, 588.

48. GAY, F. P., and FORCE, J. N.: Arch. Int. Med., I9I4, XIII, 471.

49. GAY, F. P.: Typhoid Fever, chap. XIII. New York: Macmillan \& Co., 1918. 



\title{
CHAPTER X
}

\section{MEDICAL-SOCIAL SERVICE AS A FACTOR IN THE DIAGNOSIS AND TREATMENT OF DISEASE}

\author{
By Richard C. Cabot
}

I

Bотн in the causation and the relief of disease, bacteria and their products, together with certain physical and chemical agents, play the chief parts. But they are not the whole. Psychological, industrial, and educational factors, for instance, are also of some importance. Medicalsocial service, a branch of social work in general, deals with these factors and is therefore a useful tool in the medical kit.

In war medicine, these factors are at their minimum and medicalsocial service is relatively unimportant there. On the other hand, in the hospitals of great cities, especially in Out-Patient work, the social, economic, racial, domestic, and other influences dealt with by medicalsocial workers are at their maximum. Hence good medical practice is there impossible, or at least improbable, without the social worker's aid.

In private practice and especially in the general practice of country districts and small towns, the successful doctor usually does the social work himself. He deals as best he can with the mental, emotional, and industrial life of his patients in its bearings on their diseases. $\mathrm{He}$ is his own social worker as he is his own surgeon, laboratory man, and radiologist. 'This is possible because he knows each patient (and often his family) individually. He can see how each sufferer's maladies are the joint product of physical, chemical, and bacterial agencies and of the worries, deprivations, work conditions, and home conditions under which he lives.

But in the organized medical work of a great hospital or a metropolitan public school it is impossible for the physician to know all the important facts about his patient, unless those facts lie on the surface. The cut finger, the diphtheritic membrane, the gonorrheal discharge he can see, but the root causes of the stomach troubles, backaches, debilitated states which bring nearly half the patients to the hospital he cannot see at once and has neither the time nor the means to investigate thor- 
oughly. Yet without finding root causes, his treatment is bound to be a failure and his daily work a waste of time.

Whenever a person's sickness arises out of the way he lives (rather than out of some acute catastrophe like an explosion or a railway accident), the doctor must know how he lives. But in hospitals or public schools the doctor has no chance to grasp these essential factors. Hence the need of such help as a good social worker can give.

A child is pale, thin, listless in school work. Physical examination may show no clues for diagnosis. Questioning seldom helps. But a series of home visits by a woman who has the faculty of getting along pleasantly with school children and their families, who can investigate the details of diet, sleeping rooms and sleeping habits, the opportunities for contagion, the possible bearings of family income, family discord or paternal alcoholism on the children's health,to light the facts on which rational diagnosis and treatment can be based.

So far I have written of social work chiefly in diagnosis and in etiology as a part of diagnosis. But social work bears also on prognosis and on treatment. If malnutrition, dyspepsia, headaches, gonorrheal vulvovaginitis, scabies, or rheumatism are based on home conditions which we are practically powerless to change, then the prognosis is a blind alley, no thoroughfare, and we can turn our energies elsewhere.

If, on the other hand, the causative conditions can be changed by social-service work, then that is the treatment indicated. Sometimes really brilliant therapeutics can be thus achieved.

\section{II}

I have already tried to show, as under a low power of the microscope, the field of medical-social work and the tools likely to be useful there. Seen in more detail, its place and methods are as follows:

In the neurological and psychiatric clinics of a hospital Out-Patient Department and in the pediatric clinic, almost every case needs study and treatment by a social worker acting under direction of the Clinic Chief. To feed babies, to get older children properly nourished and fit to resist the common infections with success, is a matter of multitudinous detail. An exact knowledge of how the child lives, eats, sleeps, works, and plays is essential. The doctor cannot get this knowledge satisfactorily by questioning the child or its mother. Still less can he be sure that his directions and prescriptions are carried out exactly and persistently. $\mathrm{He}$ is at arm's length from his case. $\mathrm{He}$ cannot handle it. The social worker, acting as his agent both in the 
clinic and in the child's home, can see what is going on and can get things done-or at least ascertain that they are not done and that no good results can therefore be looked for.

In the neurological clinic the hemiplegics, arteriosclerotics, paretics, and epileptics must be gotten into institutions or their home companions must be shown the little that can be done to ease and cheer their lives. By teaching and occupation the social worker can save them much suffering, though medicine and surgery are practically helpless.

The "functional" cases, the tics, stammerers, psychoneurotics need re-education of a type which no clinic physician has time and few have ability to give. A properly trained social worker, by intensive effort, can do wonders for a few patients and accomplish substantial good for many more.

Still more important is the social worker as a magnet or focal point to which are drawn the functional neuroses usually hidden in the gynecological or general medical clinics where they are maltreated under diagnoses like gastritis, constipation, ptosis (gastric, intestinal, or uterine), endometritis, " anemia," and debility. In the orthopedic, in the general medical clinic, and in the departments of dermatology, syphilis, and tuberculosis, social-service work is important but somewhat less essential than in pediatrics and neurology.

In the surgical and throat departments there is still less need of anything beyond what doctors and nurses can give.

III

What the social worker does for disease can be grouped under four headings: (a) Discovery, (b) prevention, (c) education, $(d)$ disposition.

The discovery of concealed nests, foci, or cases of disease through visits to the patient's home, workshop, or school, can be carried out by health officers or public health nurses as well as by social workers, especially when the data sought for are obvious. Thus hidden nests of malaria, uncinariasis, pellagra, lead poisoning, and tuberculosis are now and then brought to light by public officials.

But in hospital work where the single case of phthisis, rickets, syphilis, or occupational disease is the natural starting-point and spur to the search for nests of cases like it, we need someone who can act as the doctor's and the hospital's agent, following a clue held there. Because the social worker is not a public official and comes from an institution which tries to assist rather than to discipline or check people. 
she * is in a good position psychologically to get the facts she is after. She is welcome. People are not afraid of her and are less likely to lie to her than to a public health official.

Besides the discovery of new cases of disease, the social worker, by her greater intimacy with the patient's family and by her chance to talk with him uninterrupted in his home and for a good while, may find new features in the cases already known and treated. Omissions in the history, new light on its interpretation,' further links in the chain of causation may be brought out thus. Why cannot the doctor do this better? First, because under present conditions of hospital organization he has not the time for home visits; i.e. he is more useful to more people by spending his time on such diagnoses and treatments as he can offer in conjunction with the other elements of the hospital teamconsultants, assistants, machines, and laboratories. This ties him down.

Moreover, he is not usually an expert in the give and take of intimate personal intercourse with people of the type who consult him at a free hospital. He cannot get at them as well, understand them as quickly or as far as a well-trained and sympathetic woman can.

Prevention through social worker's efforts springs from the discovery of incipient cases on home visits and through the detailed, hygienic explanations and therapeutic teaching presently to be referred to. She may thus prevent the relapses of mental disease, of peptic ulcer, of flat-foot, of industrial dermatitis, and to this extent prevent the existence of new cases of disease of "old" patients.

Education in the details of diet, sleeping arrangements, exercise, recreation, and the other departments of hygiene must be fitted to the individual like a suit of clothes if it is to be of use to him. General rules are of little value, especially if presented in printed circulars and in a hasty offhand way. The rules must be applied, reshaped, and modified to suit the individual's needs after these needs have been studied with care. Moreover, since these hygienic rules often call for the reform of tough old habit, one must use every effort to get a dynamic sufficient to make the patient put himself to so much trouble. The fear of disease and the doctor's authority can accomplish something towards making a man change his habits of diet, of work, or of thought. But usually we need also the persuasive force of someone who cares for the individual sufferer and is believed by him to understand his circumstances, his difficulties, and his point of view. The medical-social worker, acting for the doctor and transmitting his authority and his directions to a patient who believes that she under-

* Why a woman is preferable I will try to show later. 
stands him and feels a genuine interest in him, can accomplish more therapeutic education than anyone else now in sight.

By the disposition of patients I mean here the process of getting them into institutions, of getting financial or other aid for them through coöperation with other charitable agencies or with private individuals; all of these which are available in her district the social worker first lists and sizes up, then learns to use.

Hospitals, special and general, sanitoria, convalescent homes, homes for the aged, special funds for vacations, for recreation, for pure milk, for trade training (as in the case of mutilated persons), exemplify the tools which the social worker learns to use more or less effectively for hospital patients.

IV

The social-service department of a hospital should function as the $\mathrm{X}$-ray department does-not as an independent agent, but as part of a team under the direction of one guiding mind. The facts elicited by the social worker's studies, talks, and visits should (like X-ray data) be pooled with the data of physical examinations, the laboratory findings, etc. Then they are appreciated and of value; not otherwise.

So with her educational therapy. It will often go wide of the mark, unless it is supervised (like X-ray treatment or massage) by the doctor in charge of the case.

To turn a patient over to the social-service department once for all is a common but wholly mistaken practice.

Why has the work described here arisen only since 1905 ?

Because of the development of big Out-Patient Clinics where team work of many takes the place of one's doctor's attempt to do everything himself. The development of diagnostic and therapeutic teams and with this the stratification of medical jobs so that untrained people can do much of the job, leaving the doctor for his expert work, has helped to show us how many-sided is the task of helping a sick person towards recovery.

In the division of labor thus developed, place is found for one who deals in details, who knows the patient in his home, his work and his school, and who gradually becomes competent to trace out and record the mental elements present in all organic disease as well as in functional or neurotic maladies.

This is the most important point in the whole matter. Mental elements in the causation, in the symptomatology, in the prognosis and treatment of disease are recognized today more fully than ever before.

We know today more than we ever did before what worry, fear, 
grief, and other emotional strains can do in modifying and augmenting and prolonging disease. We also know something of what peace of mind, habits of concentration, recreative enjoyment, satisfaction in work, friendship or religion can do to banish or to alleviate disease.

No medical-social worker is an expert fit to succeed often in understanding or manipulating all these delicate and pervasive forces. But when she is born for her job and then trained on it, she can contribute perhaps as much to the hospital team work as any single person in it.

It is in organized medicine, then, as we have it in the best modern hospitals, sanitoria, schools, and factories that medical-social service has its chief function. Whenever medical organization takes a step forward, whenever group medicine in any form progresses, the sort of aid and technique here described will, I believe, find a part. 


\title{
CHAPTER XI
}

\section{ENVIRONMENT: ITS BEARING UPON THE MAINTENANCE OF HEALTH AND THE TREATMENT OF DISEASE}

\author{
By Walter B. James
}

IT is probable that in the last few decades less attention than formerly has been given to the study of environment in disease, because of the intensive, successful, and absorbing search for positive preventive and curative measures which has characterized the present and the recent preceding generations of physicians. Nevertheless, it seems wise to review this subject briefly in the light of recent advances in our knowledge of biology, of physiology, and of physics.

It is difficult to understand the relation of man to his environment as we see him today, without first remembering one or two fundamental biological principles. In the first place, it is necessary constantly to bear in mind Weissman's law, now practically universally admitted by biologists, namely, that acquired characters are not transmitted. It seems that we must also admit the truth of the logical corollary of this law, that neglected or abused characters are not lost, unless they are or become adverse in their relation to the race, and even then their disappearance is very gradual.

From this it arises that men are structurally not essentially different, and mentally too do not differ much from their remote progenitors, whose environment was much more nearly that of the lower animals. Celsus, a keen observer, writing in the first century, says, "All men of learning and almost all dwellers in large cities suffer with their stomachs." There has evidently been no marked change in this respect in these past almost two thousand years.

But environment, that is, the artificial features of it, is being constantly changed, and these changes tend to make the surroundings of man more and more different from those in which he was evolved and for which his body is really best suited. Moreover, they are rarely adopted from a study of the conditions, but rather in obedience to fashion or in the pursuit of comfort. A dog, if left to himself, will 
lie all day before the fire and only the need of food takes him through snow and cold on the long chase that is so necessary to his keeping fit.

Our clothing, homes, atmospheric temperature, food, and psychic environment we may and do change at will, but our bodies, our minds, our temperament can be changed only by the slow and tedious process of evolution through natural selection. The growing importance of mental hygiene, a comparatively new science, reminds us constantly that the mind and its operations also are closely related to its psychic environment, and that therefore the mental health of the individual is largely dependent upon this. The necessary limits to the length of this chapter make it impossible to do more than outline briefly a few of these relations with a view to stimulating further study of them.

It was formerly thought that the atmosphere affected the body only or chiefly through the absorption of its elements by the lungs, and that the symptoms that accompany a stay in an ill-ventilated room, drowsiness, headache, loss of appetite, nausea, etc., are the result of the absorption of poisons given off from men's bodies, the so-called anthropotoxins. It has been found that this is not the case, and that these symptoms are caused by the effect of the atmosphere upon the surface of the body, that is through the absence or lessening of the normal heat loss. In this connection the various respiratory membranes are to be thought of as internal body surfaces, which also are brought in direct physical contact with the atmosphere. Heat, humidity, and stillness are the essentials in a bad atmosphere, and coolness, dryness, and motion of the air constitute good ventilation. The carbon dioxide element may as a rule be neglected, for it is only under very extreme conditions that it ever reaches an amount that need be considered.

The writer once carried out a study of the humidity in various parts of a large hospital and under varying conditions. Under all conditions the humidity was much higher in the wards than out of doors, and it was also much higher with closed than with open windows. This helps to explain the sense of well-being and stimulation that comes from leaving the house and going out of doors or on opening windows wide. All of these agencies probably operate chiefly through the withdrawal of heat from the surface of the body, and through the effect of this upon the thermotactic centers, with the consequent stimulation of metabolism to replace the heat units lost.

There is another physiological principle which helps to explain a number of commonly observed facts. which have aroused of late a good deal of scientific interest. That is, the effect of changes of temperature upon human beings. In his "Civilization and Climate," Ellsworth Huntington describes how he proved, through a study of the productivity 
of factory workers, that their output increased with temperature change, and that no other elements, that go to make up the weather, seemed to have a real influence upon such productivity. He explains the generally admitted superiority of peoples who live in hard, rugged climates with much storminess and temperature variation, as compared with those who live in comfortable warm places with an even temperature, on the ground of the subjection of their bodies to frequent and extreme alternations of temperature.

A reasonable physiological explanation of this phenomenon seems to be that the maintenance of the balance of the circulation in the different parts of the body, the securing of a prompt supply of blood just where it is needed at any given time, and the avoidance of congestion depend upon the efficiency with which the vasotonic part of the nervous system does its work, as well as upon the state of training of the muscle coat of the arterioles. As the circulatory system does not contain enough blood to keep all of the body's organs in a state of activity at the same time, it follows that, just as in the case of the rolling stock of a railroad, efficiency depends upon the ability of the operating system to furnish the exact supply needed and just where it is needed, and to avoid congestion elsewhere. In both cases this can only be. secured, first by intelligence and second by.constant practice.

In this connection what is commonly called "catching cold" becomes of interest. While a cold may be the result of infection by some new strain of bacteria - new, that is, to this patient-in the great majority of cases this explanation is not satisfactory, and one is compelled to assume that it is directly the result of some exposure to cold or wet and therefore logically that it must be due to the fact that bacteria, which previously had been innocuous and friendly inhabitants of the mucous membrane in question, by reason of the exposure became suddenly pathogenic and unfriendly. In this latter case the natural assumption would be that it was the result of a congestion of the mucous membrane, which is the equivalent of an injury, and we know that bacteria which are unable to produce infection in a normal tissue will often do so in a tissue which has been damaged. It is probable that the congestion of the mucous membrane in the first place, following exposure of the surface of the body, occurred because nature's protective mechanism in that area, the vasotonic system, was not kept fit by training.

There are a number of familiar and interesting facts that have a bearing upon this. The lumberman, through habituation, has his boots full of water all day and often even sleeps in wet socks or falls into an icy river in the course of his day's work. and suffers no inconvenience 
therefrom, and colds and pneumonias are infrequent among workers of this kind. The city dweller, on the other hand, has found to his cost that he must wear rubbers in wet weather or have a cold in the head, and that if he falls into the river from a ferry boat he is almost sure to have a bad attack of bronchitis and probably pneumonia. Yet the city man if he changes his occupation and becomes a lumberman acquires the lumberman's immunity; while the lumberman, if he takes up city life, very soon acquires the city man's susceptibility to colds. The New England schoolboy goes bareheaded summer and winter, and there is a general agreement that this practice not only does not lead to colds and bronchitis but that it encourages robust health.

Again, the relation of women to men in this particular is interesting. The man, clad all day long in the same kind of woolen clothing from his ankles to his chin, finds that he can leave off his waistcoat or his coat only at the risk of taking cold. His wife wears high shoes or spats throughout the day when it is warm, and a cloth dress buttoned to the neck and with long sleeves, and when night comes, perhaps with a cold rain or snow and a considerable drop in the temperature, she puts on low shoes or slippers, openwork stockings, a much thinner dress, her arms and a large part of her body bare, leaves her head uncovered, and thus clad walks through the slush of the sidewalk, takes a motor to go to an entertainment, sits in a draughty dining-room, dances until she is very warm, and then sits with little if any additional wrap, and to the amazement of her husband is in the most robust health the following day in spite of his repeated predictions of pneumonia.

It is general medical experience that, at least among the well-to-do, it is the men who have most of the colds. The expectation of life in women past fifty is considerably greater than that of men, in spite of the fact that their husbands have been for years predicting early death from what they consider a most unwise exposure of their persons to the weather. The average man's clothing would easily fill a bushel basket. His slender and frail-looking daughter could almost put all of hers into a quart cup, and yet it is she who is entirely independent of the pocket handkerchief and when she does carry it uses it mainly as an ornament, while he is an abject slave to one containing almost a yard of cloth. Moreover, everyone knows that practically all of the disagreeable nose and throat noises that are heard in any household are made by the men and not by the women.

All of these facts are merely examples of the familiar hardening nrocess, and it is suggested that this consists essentially of the training a. $\mathrm{A}$ keeping in training of the whole vasomotor system, thereby securing speec and precision in the distribution and regulation of the body's 
blood supply and the prevention of local congestions: It should not be forgotten that the efficiency of the body and of the mind as machines is closely related to this mechanism.

It is probable that most of the minor and annoying disorders referred to above result from a failure to live in a proper and normal relation to our environment, that is, to an environment more nearly like, in this respect, that of our cave men progenitors, from whom after all we differ so little structurally, as was said at the beginning of this chapter. Women's relation to environment is far more normal and far more like that of the Cromagnon men and women.

Nowhere has a less rational and wise view been taken of the relation of dress to health than in the clothing of armies, especially in America. Every experienced family physician knows that when a young person is brought to him because of frequent catching of colds, with catarrh of the upper air passages, one of the first and most important things to examine into is the clothing and the manner of life. If the patient is found to be too much bundled up, to use too heavy underwear, to wear chest protectors, mufflers, and wristlets, and to take prolonged hot baths, the first thing to do is to put him into rational dress, to see that the surface of the skin is more and oftener exposed to the air, that cold baths are taken-in general, that the vasotonic mechanism is kept fit; and yet our army is clad in garments which almost hermetically seal the men's bodies from the soles of the feet to the chin. It seems at least possible that the enormous amount of respiratory disorders in our army cantonments is contributed to through taking out of civil life and civil loose, open dress a large number of young men and sealing them up in close-fitting, close-woven woolen and leather military uniforms.

It is probable that the benefits ascribed to the Kneipp cure, at one time in much vogue in Germany and in this country, which consisted in walking with bare feet in wet grass in the early morning and in putting on thin linen underwear after a bath without first drying the surface of the body, were due to its action as a hardening process and to the stimulation of certain parts of the body that are as a rule never exposed to changes of atmosphere. The writer has tried this process out of curiosity, and it is interesting to observe an almost instantaneous noticeable sensation, and a pleasurable one, upon the nasal mucous membrane. The value of hydrotherapeutics, especially of the Scotch douche, is perhaps best explained on this same ground, the training of the vasomotor system.

Similarly there is little doubt that part of the value of the sunshine cure for tuberculosis in young people as practiced by. Rollier at Leysin 
is due to a similar exposure of the skin of the entire body not only to the sun but to the atmosphere. Moreover, there is much reason for believing that this is one of the essential elements in all climatic cures, especially the climatic treatment of tuberculosis. Formerly it was assumed that the efficacy of the outdoor treatment of this disease depended upon the breathing of a certain kind of air. It now seems more likely that it depends upon the surface effect of the air and that the highness and the dryness of the air operate through a more rapid heat elimination and a consequent stimulus to metabolism.

In the Adirondacks and other similar places the patients regularly make more improvement during the winter than during the summer months. The practice that was begun some years ago of developing devices by which the patient's body could remain in a comfortable warm bedroom, while his head was outside the window so that he might breathe cold fresh air, has now been generally abandoned, probably for the reason that if a patient wants to benefit by a certain type of air, not only must he secure its entrance to his lungs but also have its effect upon his body generally, and such effect as is produced upon the lungs is probably through the action of these inner surfaces as body surfaces and not as organs of absorption.

It is generally found that cases of heart and kidney disease do best at or near the sea level and in climates that are warm and somewhat moist. It is possible that this is because in these diseases it is best to check rather than to stimulate metabolism. In the etiology of an important group of diseases, such as Bright's disease, arteriosclerosis, and related disorders, it is at least possible that maladjustment of the individual to his surroundings may play a part in causation, viewing these and including in them, climate, dress, labor, play, and all the other complex elements that go to make up man's environment. Bacon has said that "to be free-minded and cheerfully disposed at times of meiat and of sleep and of exercise, is one of the best precepts of long lasting."

In hospitals these principles are rarely thought of, and it is usual to see patients with heart and kidney disease, who need warmth and uniform temperatures, in the same ward with cases of pneumonia, tuberculosis, and other diseases which do best in a low and varying temperature or to see cases of acute meningitis with photophobia, that should be in darkness and quiet, in a room with light yellow walls, filled with the sunshine and the bustle that are good for the other diseases treated there.

In connection with environment the position of the body might well be considered a matter to which more attention should be given in practical medicine and especially in relation to the circulation. In health 
the circulation in all parts of the body is maintained independently of the effects of gravitation. In standing the feet do not swell unless the standing is excessively prolonged. In lying on the back the dependent portions of the individual free from cardiac or spinal disease do not become edematous until death ensues, when discoloration and edema of these parts occur at once. In a patient who dies in the upright position the same phenomenon is observed in the feet and legs. A dog or a rabbit suspended in a vertical position more than a certain not very long time dies with evidences of congestion of the abdominal viscera.

That is, man is built and his physiology regulated to spend twothirds of his time in a vertical position and about one-third in the horizontal. The dog and other quadrupeds are built to spend their whole lives horizontal. The dog or man dies with intense congestion of the head if suspended with the head down for even a very brief time. The opossum, on the other hand, can hang by its tail indefinitely, head downward, yet anatomically we can detect no difference. The circus performer by practice trains his vasotonic system so that he too, like the opossum, can hang head downward. All of which means that each is built for life in a certain plane and maintains his health and effectiveness best if his living conditions conform to the way he is built.

All of this indicates that there must be a mechanism present which automatically and entirely unconsciously adjusts the balance of the circulation to changes in the relation of the body's plane to the surface of the earth. The seat, at least of one important part of this mechanism, seems to be in the semicircular canals. This whole matter is of more than a purely academic interest and deserves more attention than has been paid to it.

The common experience that lying more than the customary number of hours in a horizontal position gives rise to dizziness on arising, probably means that this mechanism has become relaxed and that on arising the balance of the circulation is not immediately maintained and so cerebral anemia with corresponding congestion elsewhere ensues.

Such circulatory disturbance may very well have a somewhat deeper import than merely the discomfort of ringing in the ears or giddiness, and it is suggested that it may furnish the foundation for the growing tendency on the part of physicians and surgeons to aim at the shortening of convalescence and getting patients out of bed at the earliest possible moment, and especially patients in advanced life. The puerperal woman today sits up one or two weeks earlier than did her sister of twenty or thirty years ago, and the same is true of patients with appendix and other operations, and it is claimed that benefit to life and lessening of complications result. 
It is universally admitted by physicians of experience that it is wise to avoid keeping old people in bed as much as possible, and the striking coincidence between early rising and longevity was pointed out a good many years ago by Sir Herman Weber and Sir Lauder Brunton. These studies belong in the domain of medical physics, unfortunately a much neglected department of medical research.

The limits of the present chapter necessarily preclude the possibility of discussing seasickness, a most interesting malady from the point of . view of medical physics. The physical phenomena which accompany the oncoming of seasickness are identical with those of the oncoming of death and are easily recognizable by one familiar with the two conditions. The inability to concentrate the mind, for instance the inability to remember the cards and to fix the attention when playing whist at sea, then the gathering of cold dampness on the forehead, coldness of the tips of the fingers gradually creeping up into the hands, coldness of the tip of the nose, the shrunken look of the fingers and hands, the thin thready pulse, and with this the sickly greenish-gray color of the face with sinking of the eyes, and in the early stage a feeling of the absolute necessity for lying down-these phenomena have been frequently observed by many physicians in seasickness and in death, though the relationship has not been recognized.

The writer suggests that in both these conditions these physical phenomena are the result of the failure of venous vasotone resulting in an accumulation of blood in the large venous channels of the trunk and a corresponding lessening of the amount of blood in the arterial system. In other words, identical circulatory phenomena accompany seasickness and death, and this perhaps explains the fact that their accompanying sensations are so similar.

In the case of seasickness active nausea stimulates vasotone and the circulation balance is restored, while at the moment of death all nerve and muscle tone cease, vasotone disappears, elasticity of the arteries and arterioles however is maintained, and the entire blood supply soon finds its way into the venous system.

Very recently aviation medical research has opened up an interesting avenue for investigation into the relation of man to his environment, but the limits of this chapter preclude the possibility of entering this fascinating field.

Nowhere is environment more important than on the mental side of medicine. The stimulating effect upon the convalescent of being moved from one room to another, or in the case of long chronic disease of having the wallpaper of the sick-room changed, and even of changing the arrangement of the furniture, the striking impulse given to the 
convalescent by the first going out of the house, the stimulating effect of the physician's visit, all of these are matters of commonplace and everyday observation. Every hospital physician has seen many cases of disease with a protracted fever of low-grade or delayed resolution of a lesion corrected rapidly by having the individual up and dressed.

The successes achieved by Christian Science, mental healing, osteopathy, naturopathy, and a host of other irregular medical methods, all testify to the importance of this element in therapy, and testify also to the lack of imagination on the part of the medical profession shown in their frequent failure to recognize it. It seems at least possible that the study and practice of medicine of late years has tended to blunt the imaginative faculty. The more intensive and limited the line of thought, the deeper grow the brain tracks along which thought travels, until they become so deep that the mind is unable to look over the edge into the surrounding fields.

The great war has shown that, without any physical injury whatever, men may become speechless, deaf, blind, insensible to pain, or possessed of acute pain in any part of the body, and all this the result of a mental shock, operating through the mind, or speaking more broadly through the personality or consciousness, but profoundly influencing the function of any part of the body. Peace too has its shocks, and indeed life consists of a continuous series of impressions made upon our consciousness. Nature provides us with mental shock absorbers, and these constitute one of the most complex and obscure of all of our mechanisms. They must, however, be constantly in the minds of medical practitioners, and their subtle relation to the environment constitutes an important element in the causation of and in the battle with disease. Moreover, like other mechanisms, to be kept ready and in good order they require use and training, and so too careful shielding from mental shocks is but a poor preparation for the greater ones that are to come in the future.

The psychic influence of environment is strikingly illustrated in large hospitals for the insane, which in New York State approach six thousand in their census of patients, the institutions covering a very large area. Experienced medical superintendents have often noticed that a pronounced change for the better in the mental condition of patients is apt to follow a transfer from one institution to another, and the removal from one part of a large institution to another part. This improvement can be ascribed to nothing but the change in environment, and this principle should be made use of in institutions for the insane much more extensively and in a more carefully thought out way than has generally been the case. In the treatment of certain 
varieties of insanity it could probably be made a therapeutic asset of great importance. The newer plans for projected State hospitals in New York State are designed with special reference to the application of this therapeutic principle, and in a field in which there is sad need for this application of imagination and for the introduction of new plans of treatment.

We cannot think of life as an isolated fact, but only as a complex, the result of a large number of coördinated facts which should be in harmony with one another, but which unfortunately often are in disharmony. Health and disease too are not isolated facts, and it is to be desired that physicians, whose aim it is to promote health and to combat disease, should realize that man and his diseases should be viewed as parts of a complex in which the environment, both physical and mental, plays an important rôle, and one that should not be neglected by him, in practice, in teaching, and in research. 


\title{
CHAPTER XII \\ RADIATIONS AND THEIR EFFECTS
}

\author{
By W. T. Bovie
}

\section{STRUCTURE OF THE ATOM}

BECAUSE of the intimate relation of electricity, radiant energy, and chemical phenomena to atomic structure, no subject in physical science has produced a more profound influence on the theories of physics and chemistry during the last few years than that of the constitution of the atom. A complete exposition, therefore, of the nature of the biological action of various kinds of rays, as indeed a final physicochemical (mechanistic) exposition of any physiological process, must be made, not in the terms of the old indestructible, indivisible, and unchangeable atom of Dalton, but in the terms of the new atom which is, according to the "mechanists" of natural philosophy, intricate, complex, and changeable. While no one has wholly succeeded in describing a mechanical model of atomic structure, showing the nature and arrangement of the parts, we do know that an atom contains particles, at first called corpuscles, but now called electrons, which have a mass only ${ }_{1 \frac{1}{80} \sigma}$ part of that of a hydrogen atom. We know that the mass of all electrons, whatever their source, is the same; and all electrons carry a negative charge of electricity of the same magnitude. Because of the special properties possessed by electrons, their experimental investigation is less difficult than that of atoms or molecules, and it may truly be said that more is known of the electron than of the atom itself.

Although the structure of the atom can as yet only be inferred, we know that in some way it determines such fundamental phenomena as the conduction of electric currents, the absorption and emission of various kinds of radiation, the combination of the atoms into molecules, and hence must determine the fundamental phenomena of all physiological processes. Most natural philosophers believe that the atom has a central positively charged particle, called the nucleus, around which the electrons are grouped, either in orbital revolution about the nucleus as a center (the infinitesimal planetary atom of Bohr), or in fixed equilibrium positions, about which, under the influence of high temperature or electrical discharge, they can experience minute oscillations, 
but from which they cannot depart far without altering the structure of any molecule in which the atom is held (the static atom of Lewis).

The methods of investigating atomic structure are quite different from any of those familiar to the biologist, but the micro-anatomy of the atom may happily be described in familiar terms. There is a centrally located nucleus surrounded by a "cytoplasm" of revolving or vibrating electrons, the whole bounded without by a hypothetical "sphere of influence." The mechanism which forms the basis of individuality of the atom-i.e. that which keeps the atom capable of being identified as oxygen, gold, radium, etc.-is for the most part located in the nucleus. Many chemical and physical properties of the atom are, however, due to the number and the arrangement of the external electrons.

The diameter of an atom of hydrogen is in the neighborhood of ${ }_{101000}^{1}$ micron. The diameter of the nucleus has been estimated to be between $10 \frac{1}{0000}$ and $100^{2} \pi \overline{0}$ of this, or in the neighborhood of

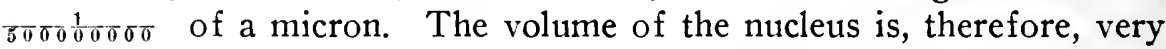
small as compared with the volume of the atom. In spite of its very small size, the nucleus contains very nearly the entire mass (weight) of the atom, for the number of electrons in an atom is at most only a very small multiple of its atomic weight. Nevertheless, the nuclei of atoms of high atomic weight appear to be very complicated structures.

Besides the captive electrons-i.e. those in orbital motion in the "planetary" atom, or those at equilibrium positions in the "static" atom-there are other electrons which are free and which may pass from one atom to another. It is believed that these free electrons, passing from atom to atom, from a point of higher electrical potential to one of lower potential, constitute the electric current in a metallic conductor. There are other electrons more firmly bound than these very free electrons, which are believed to be responsible for chemical valence and which by their position and number determine certain chemical and physical properties of the elements.

It may clear our mental picture of the atom to liken it to a miniature solar system, in which the sun is replaced by the positively charged nucleus and the planets by the negatively charged electrons, but the nucleus of the atom is much smaller when compared with the size of the atom than is the sun when compared with the dimensions of his planetary system. That is to say, if we were to magnify an atom until the diameter of its nucleus were equal to the diameter of the Sun, then the orbits of the planets would lie within the volume of the atom; the diameter of the orbit of Neptune being less than one-seventh the diameter of our magnified atom. 
From what has been said above, it will be seen that with nearly all of the mass of the atom contained in the nucleus, the diameter of which is only รотл that of the atom itself, an atom has, as a matter of fact, a very open structure. It is for the most part a portion of space bounded by a "sphere of influence," the ether of space continuing almost without any interruption right through the densest kind of atom.

The positions of the elements in the Periodic System of Mendeléeff appear to be determined by nuclear characteristics. It cannot be claimed, however, that the law or laws underlying this system are known or well understood, and a complete and satisfactory classification of the elements has not yet been made. The widely comprehensive term evolution, as. understood to-day, comprising as it does all of the vast complex of natural conditions which has resulted in the formation of the universe, includes the derivation of the elements by a gradual modification of some primordial substance. Some see in the periodic tabulation of the-elements the genealogical tree of a stupendous evolution of inorganic matter, beside which organic evolution is an affair of a day and to which it is a mere appendix. But the causes, directive forces, variations, and mutations of this gigantic evolution are entirely unknown. No one has said for certain which is the top and which the bottom of the genealogical series; whether the heavier atoms have been built up from a fundamental element, "protyle," as suggested by Prout over a hundred years ago, or whether all of the elements are the result of a disintegration of primary substance of high atomic weight. While an evolution involving a succession of changes from the simple to the complex is a more satisfactory hypothesis, no such changes have ever been observed. The disintegration process, on the other hand, is to be observed in all radioactive series. Here elements of lower atomic weight are formed from those of higher, and these are in turn transformed into other elements.

\section{RADIOACTIVITY}

Some of the laws governing this revolution of the elements have been stated more precisely perhaps than the laws in any other field of science. A law of the rate of transformation states that equal fractions of the element are transformed in equal times. The time required for onehalf of the element to be transformed is called the "half-life" period of the element. A table of half-life periods and some other radioactive constants compiled by $\mathrm{G}$. Wendt in 1916 is given as follows :- 


\begin{tabular}{|c|c|c|c|c|c|c|}
\hline Substances. & $\left(\right.$ Sec. $\left.^{\lambda}{ }^{-1}\right)$ & $P$ & 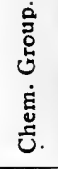 & 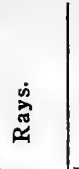 & 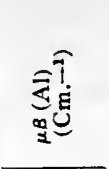 & ${ }^{\mu \gamma}\left(\mathrm{Cm} \cdot^{-1}\right)$ \\
\hline 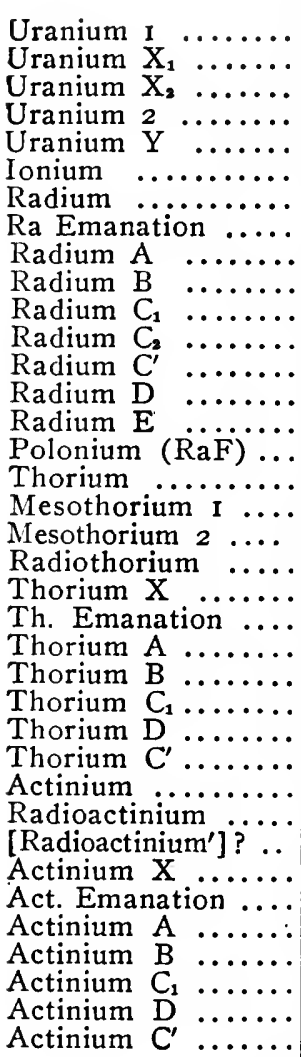 & $\begin{array}{l}4.3 \times 10^{-18} \\
3.3 \times 10^{-7} \\
0.01 \\
1.1 \times 10^{-14} \\
7.5 \times 10^{-8} \\
2.2 \times 10^{-13} \\
1.26 \times 10^{-11} \\
2.085 \times 10^{-6} \\
3.85 \times 10^{-8} \\
4.33 \times 10^{-4} \\
5.93 \times 10^{-4} \\
8.3 \times 10^{-3} \\
7 \times 10^{8} \\
1.39 \times 10^{-9} \\
1.66 \times 10^{-8} \\
5.90 \times 10^{-8} \\
1.2 \times 10^{-18} \\
4.0 \times 10^{-9} \\
3.1 \times 10^{-5} \\
1.09 \times 10^{-8} \\
2.20 \times 10^{-6} \\
0.0128 \\
5.0 \\
1.8 \times 10^{-6} \\
1.9 \times 10^{-4} \\
3.7 \times 10^{-3} \\
7 \times 10^{10} \\
1 \times 10^{-10} \\
4.25 \times 10^{-7} \\
3.2 \times 10^{-6} ? \\
7.6 \times 10^{-7} \\
0.18 \\
350 \\
3.2 \times 10^{-4} \\
5.37 \times 10^{-3} \\
2.26 \times 10^{-3} \\
700\end{array}$ & 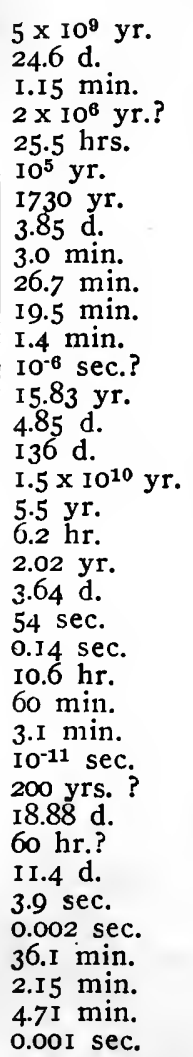 & $\begin{array}{l}6 \\
4 \\
5 \\
6 \\
4 \\
4 \\
2 \\
0 \\
6 \\
4 \\
5 \\
3 \\
6 \\
4 \\
5 \\
6 \\
4 \\
2 \\
3 \\
4 \\
2 \\
0 \\
6 \\
4 \\
5 \\
3 \\
6 \\
3 \\
4 \\
? \\
2 \\
0 \\
6 \\
4 \\
5 \\
3 \\
6\end{array}$ & $\begin{array}{l}a \\
\beta \\
\beta \\
a \\
\beta \\
a \\
a \beta \\
a \\
a \\
\beta \\
a \beta \\
\beta \\
c \\
\beta \\
\beta \\
a \beta ? \\
a \\
\beta \\
a \\
a \\
a \\
a \\
\beta \\
a \beta \\
\beta \\
a \\
a ? \\
a \beta \\
a ? \\
a \\
a \\
a \\
\beta \\
a \beta ? \\
\beta \\
a\end{array}$ & $\begin{array}{l}75 \\
\text { I3.5 } \\
\text { 130 } \\
43.3 \\
30 \\
30\end{array}$ & $\begin{array}{l}\{24 ; 0.70 ; 0.140 \\
354 ; 16 ; 0.27 \\
230 ; 40 ; 0.51 \\
0.115 \\
45 ; 0.99 \\
\text { like Ra D } \\
585 \\
26 ; 0.116\end{array}$ \\
\hline
\end{tabular}

$\lambda\left(\right.$ sec. $\left.^{-1}\right)=$ transformation constants, that is, the fraction of radioactive material transformed per second.

$P \quad=$ half-life period, i. e. the time required for the transformation of 50 per cent. of any amount of radioactive substance. To save space some of the values are represented as exponents of 10 ; for example, the half-life period of Thorium is $1.5 \times 10^{10}$ years, i. e. 15,000,000,000 years. The half-life period of Radium $C^{\prime}$ is $1 \times 10^{-6}$ sec., $i$. e. roforo $^{1}$ sec.

$\mu \beta\left(\mathrm{Al} . \mathrm{cm} .^{-1}\right)=$ the absorption index for $\beta$ rays in aluminum (in centimeters).

$\mu \gamma\left(\mathrm{Al} \cdot \mathrm{cm} \cdot^{-1}\right)=$ the absorption index for $\gamma$ rays in aluminum (in centimeters).

In order to obtain a clearer insight into the meaning of the rate of transformation law, let us consider roo atoms of the element Niton (radium emanation) which have been transformed from radium within an infinitesimal of time, so that they are all of the same age. The half-life period of Niton is 3.85 days. One-half or 50 of 
our atoms will be transformed then in 3.85 days, and according to the rate of transformation law, one-half of the remaining 50 , or 25 , will be transformed in the next 3.85 days. Now, if we possessed the perspicacity and dexterity of Maxwell's demons, we might arrange our IOo atoms of Niton in a row, in the order in which they are going to be transformed, so that as the transformation proceeds a wave of atomic disintegration would travel from one end of the row to the other. This wave of disintegration would, according to our transformation law, cover equal fractions of the remaining untransformed row in equal times. Stated in the shorthand of mathematics,

$$
i=I_{0} \varepsilon^{-\lambda t}
$$

where $i$ is the number of atoms remaining at the time $t . I_{0}$ the number of atoms at the beginning and $\varepsilon$ the base of the natural system of logarithms. $\lambda$ is called the transformation constant of the element. The value of this constant for radium emanation is $2.085 \times 10^{-8}$ $\left(\mathrm{sec}^{-1}\right)$.

The transformation constant is as characteristic of the radioactive element as is its atomic weight, or any other atomic property. It measures the average length of life of the element and is not altered by any known physical or chemical process. Even radioactive forces, such as the great atomic shocks which may be produced by the impact of alpha corpuscles, are powerless to induce radioactivity, or to influence in any measurable degree the rate of atomic disintegration. This introduction of a time factor as a fundamental property of matter has quite upset such philosophical ideas as the "indestructibility of matter" and even eternity itșelf, for it seems probable that all of the elements are members of radioactive series with transformation constants of such small orders of magnitude that our instruments do not detect the transformations.

Returning to oirr row of transforming atoms of Niton, we find that each atom inherited, during its infinitesimal time of birth, something, perhaps a mechanism within its nucleus, which fixed its span of life. When this mechanism has run its course, the atom disintegrates, radiates an unparalleled amount of energy, and from its remains a new element is born. And so we find it necessary to picture the nucleus of the atom, though inconceivably small, as being very complex, the seat of radioactive transformations, disordered perhaps, but constant in their mean state. It is held together by unknown powerful forces and is either a mosaic of the preformed and fully developed sub-atoms into which it will disintegrate, or it contains some kind of generative mechanism by which the atoms of the new elements are formed and differentiated. 
Doctrines of preformation and epigenesis are by no means new to the biologist, and he may follow the discussions of the natural philosophers with considerable fellow-feeling.

Sometimes the new-formed element has the same chemical characteristics as a previously known element, so that a distinction on the basis of a chemical analysis is impossible. Yet the new radioactive element is different, in that it is unstable and is sooner or later again transformed. Such chemically indistinguishable elements are called isotopes. And now we wonder whether any of the elements are really elementary in nature after all. What we call the element oxygen may be nothing more than a group of chemically indistinguishable isotopes. Thus does the stream of progress undermine and make unstable what we may have believed to be the very corner-stones of our temple of science.

Many of the elements of high atomic weight are radioactive. In every case it has been found that radioactivity is the result of a spontaneous and explosive disintegration of the atom accompanied by an evolution of energy far greater than that of any other known phenomenon. Three types of radiation are given off by radioactive substances. Two of these, the alpha and beta rays, are corpuscular in nature. The other, the gamma ray, is electromagnetic.

\section{ALPHA RAYS}

The alpha rays consist of positively charged corpuscles having a mass equal to that of an atom of helium. They are shot from the radioactive atom at speeds approaching 20,000 miles per second, a velocity which enables them to pass directly through other atoms, providing they do not collide with their nuclei. The alpha particle, when its positive. charges are neutralized by two electrons captured from surrounding matter, becomes the nucleus of an atom of the element helium.

\section{BETA RAYS}

The beta rays consist of negatively charged particles which are electrons. Beta particles are hurled from the disintegrating radioactive atom with velocities approaching that of light, velocities which are, in some cases, over 150,000 miles per second, and which are sufficient to carry the particle around the earth five times in a single second. Their high velocities have made it possible to determine quite definitely the nature of their mass, and it has been found to be entirely electromagnetic in origin. A beta particle or electron is in reality a disembodied electric charge, and a beta ray is a stream of these disembodied electric charges.

Both the alpha and beta rays are absorbed, i.e. their velocity is reduced by matter through which they pass. Absorption occurs when- 
ever the path of the particle lies very close to the nucleus of an atom of the absorbing medium. The amount of absorption is greater the greater the atomic density and the higher the atomic weight of the absorbing medium. The amount of absorption is independent of the chemical grouping of the atoms within the absorbing molecule.

\section{DELTA RAYS}

Knocking the electrons of the absorbing medium out of position, the flying alpha and beta particles leave a trail of disorder in their reckless flight. When valency electrons are dislocated a rearrangement of the atoms within the molecule of the absorbing medium may occur and the rays are said to have a chemical action. Some of the electrons of the absorbing atoms are hurled from their positions with such force that they form secondary streams of particles, the delta rays. The amount of chemical action is so greatly increased by these delta rays that it appears that most of the chemical effects of alpha and beta rays are to be ascribed to these secondary rays.

It is not surprising that alpha and beta particles traveling at such enormous velocities cause matter against which they strike to shine out in the dark, cause photographic plates to fog, make the air through which they pass a conductor of electricity, form clouds in moist air, and through their chemical action bring about peculiar physiological effects when they impinge on living matter.

\section{ELECTROMAGNETIC RAYS}

It is now generally believed that electromagnetic rays consist of wave-trains in the medium known as the ether, having their origin in rapidly oscillating or orbitally moving electrons whose periods are the same as the periods of the wave-motion. The wave impulse of all electromagnetic rays is propagated through space at the speed of light (about 186,000 miles per second), hence the wave-length, i.e. the distance between homologous parts of two adjacent waves, varies inversely as the frequency of the vibration. Electromagnetic waves are usually characterized by stating their wave-lengths. Were it not for the weight of precedent, it would be better to characterize them by the number of their vibrations per second, because most physical and chemical action is $a$ function of vibration frequency. Charts showing the relationship between the vibration frequency and the wave-length of various kinds of electromagnetic waves are shown in the figures on the next page. The names of the various parts of the spectrum will be found in the description of the figures. 


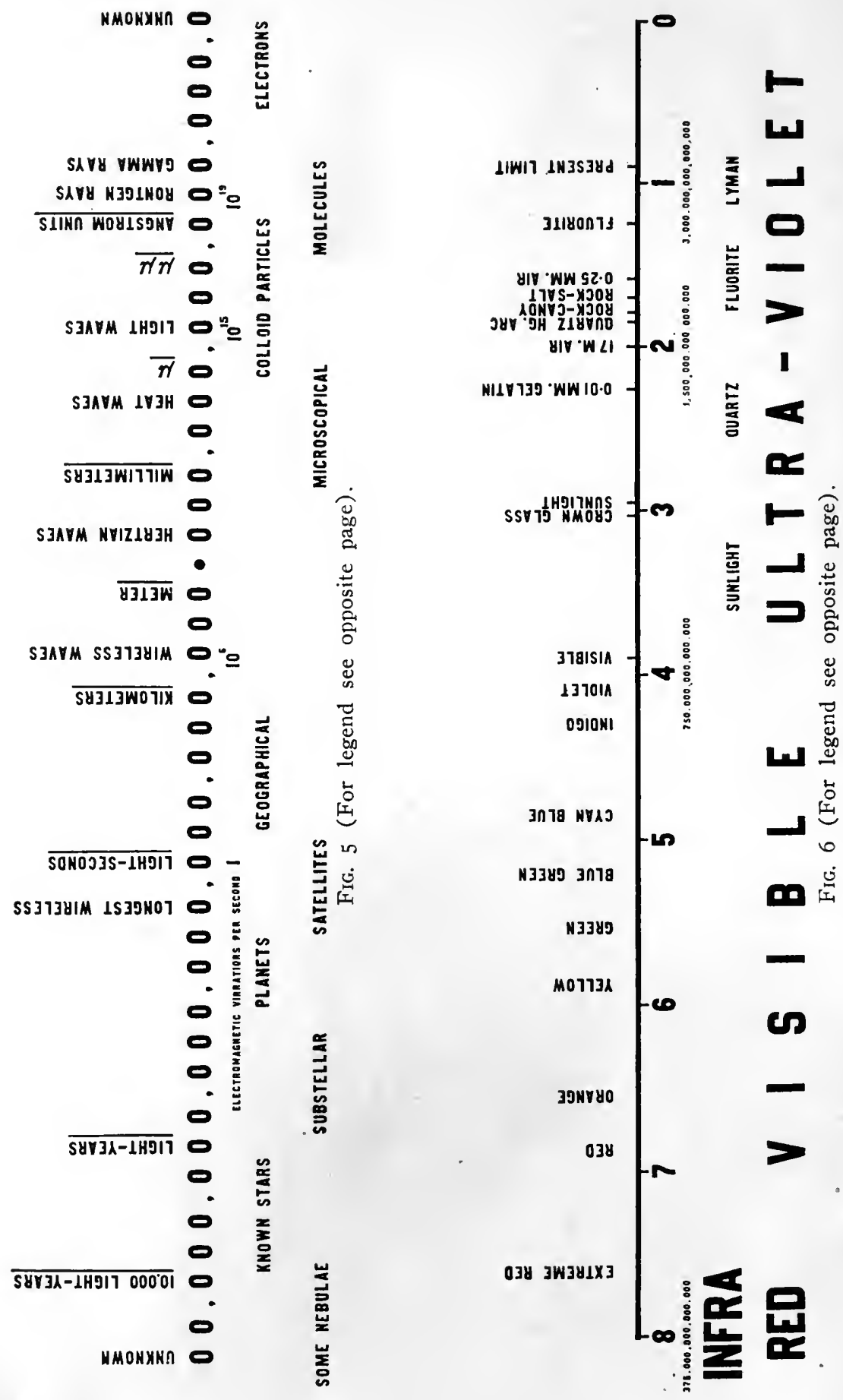


Fig. 5.- The Metric System of Length, and the Great Electromagnetic SPECTRUM. The ciphers represent the places in the Arabic system of arithmetical notation. The meter, the unit of measure, is in the unit's place followed by the decimal point. The ciphers at the right of the decimal point, therefore, represent the places for decimals of a meter; and those at the left represent multiples of a meter. Above the ciphers, the words underscored are the various units of length. The shortest unit of length, the Angstrom unit, is the one often used to indicate the length of light waves. Below the ciphers the words in large type suggest distances which are of the same order of magnitude as the lengths represented by the ciphers which the words subtend, e.g. known stars are from I to Io,000 light-years away (a light-year is the distance light is propagated in one year at the rate of 300,000 kilometers, or 186,000 miles, per second); microscopical distances are from millimeters to microns in length; colloid particles are from microns to millimicrons $(\mu \mu)$ in diameter; molecules are less than millimicrons in diameter; etc. The words, above the ciphers, not underscored, are the names of the various regions of the great electromagnetic spectrum. The names are placed with reference to the scale of measure of lengths so as to indicate the order of magnitude of their respective wave-lengths, e.g. wireless waves are some hundreds of meters long. The longest wireless waves ever produced are some number of light seconds long. These waves are probably the longest things man has ever produced. They are still traveling off in space, and are now several light-years away. Visible light-waves are between 0.4 and 0.8 microns long. Gamma waves, from radioactive substances, have very short wave-lengths. As shown in the figure, a train consisting of $1,000,000$ waves would be only one micron long.

When we are dealing with the biological effects of electromagnetic waves we are concerned with the vibration frequency, or the number of electromagnetic vibrations per second. Electromagnetic waves are all propagated through space at the same rate. Hence, the number of vibrations per second varies inversely as the wave-lengths. The number of vibrations per second is indicated by the small figures just beneath the ciphers. An electromagnetic wave one light second long obviously vibrates once per second. A wave 100 meters long vibrates $10^{\circ}$, or $1,000,000$ times per second. Light waves vibrate $10^{15}(1,000,000,000,000,000)$, or some quadrillion * times, while Roentgen rays vibrate $10^{13}$ (10,000,000,000,000,000,000), or some quintillion* times

per second. The gamma rays vibrate still faster. thousand billion and trillion respectively.

Fig. 6.-The Metric System of Length, Showing one Micron Dividen into Tenths, and the Light Region of the Great Electromagnetic Spectrum. (To save space only 8 of the tenths are shown.) Any of the ciphers in Fig. 5 may be replaced by the digits from one to nine. In Fig. 6 the cipher marked light waves, i.e. the cipher which occupies the place for one ten-millionth of a meter or one-tenth of a micron, has been thus expanded. As will be seen the spectrum of electromagnetic waves measured by tenths of a micron includes part of the infra-red, the visible, and the ultra-violet rays. The colors of the visible spectrum are written above their respective wave-lengths. The ultra-violet region has also been provisionally divided into regions which might be recognized as colors to eyes adapted for seeing ultraviolet rays. For convenience the names of the ultra-violet "colors" are printed below the line of wave-lengths. Sunlight ultra-violet includes the ultra-violet found in sunlight from the end of the visible to about wave-length 3,000 Angstrom units, $(0.3 \mu)$. Quartz ultra-violet includes the ultra-violet light emitted by the quartz mercury-vapor lamp but not found in sunlight, roughly from wave-length 3,000 to wave-length 2,000 Angstrom units. Fluorite ultra-violet includes the light which passes through fluorite but not through quartz. This region was explored by Schumann and is often referred to as the "Schumann region." The Lymann ultra-violet, the region recently explored by Theodore Lymann, includes the shortest ultra-violet light waves known.

Above the line in the ultra-violet region are a number of names so placed as to indicate the limits of transparency of the substances named. But few substances are transparent to the extreme ultra-violet rays. The substances named are selected from less than a dozen substances known to be transparent in this region of the ultraviolet.

The number of electromagnetic vibrations per second is indicated by the small figures below the line. It will be seen that a doubling of the vibration frequency occurs between wave-lengths 8,000 and 4,000 Angstrom units, giving us the whole visible spectrum from the extreme red to the violet. A second doubling of the vibration frequency occurs in the sunlight and quartz ultra-violet, while there is a third doubling in the fluorite ultra-violet. 
Sources of electromagnetic rays. Heat, visible, and ultra-violet waves are contained in sunlight. All substances having a temperature above absolute zero send forth electromagnetic rays at the cost of their own heat energy. This radiant energy was called "pure temperature radiation" by Helmholtz. In general it may be said that the intensity of the rays of all wave-lengths increases as the temperature of the radiator increases. For each temperature there is a maximum of radiation of a particular wave-length; and as the temperature increases this maximum is continually shifted in the direction of shorter wave-lengths, so that an incandescent body first glows red and then passes to a white heat. Light sources belonging to the class of "pure temperature" radiators include luminous flames, in which the radiation proceeds from glowing carbon particles, and various kinds of electric glow lamps, in which the filaments are heated to incandescence by the passage of an electric current. Radiation containing a high per cent. of ultraviolet is produced by the carbon arc, the cadmium and mercury vapor arcs, and the iron arc. Sources of lower intensity but very rich in ultra-violet are the electric sparks between various metallic electrodes. Ultra-violet of the shortest wave-lengths, such as fluorite light, is contained in the luminescence produced by a high-potential discharge through rarefied gases. The chemical luminescence, such as accompanies the oxidation of phosphorus or the oxidations which take place in the special light-producing organs of various animals, is not intense enough for therapeutic use.

Roentgen rays are produced when matter is bombarded by a stream of negative electrons. For the bombardment it is necessary that the electrons have a very high speed. In order to avoid the resistance of the air, the stream of electrons is produced in a vacuum tube, the X-ray bulb. The electrons, originating either from a high-potential discharge or from a hot metallic filament, are accelerated in an electric field. The wave-lengths of Roentgen rays are shorter the more suddenly the electrons are stopped. The wave-length is shorter (the X-rays are harder) the greater the potential gradient of the accelerating electric field and the greater the atomic density of the material (target) upon which the electrons impinge. Gamma rays have their origin in the sudden acceleration of electrons which accompanies the disintegration of radioactive atoms. In connection with the production of rays it must be borne in mind that a mutual transformation of one kind into another may take place when radiations are absorbed.

Resonance and phenomena depending on it. Electromagnetic rays are detected and investigated by the resonant vibrations which they set up in objects upon which they fall. It is necessary that the object 
used as a detector be capable of vibrating (electromagnetically) with the same or with some multiple of the same frequency as the rays which fall upon it. Any object capable of vibrating can always be set in motion by a train of vibrations which are of the same period, as when a note played on a musical instrument causes the same note on a neighboring stringed instrument to sound. The individual impulses, though very feeble, are added together, as when a child moves a heavy swing by pushing always at the moment when the push has the greatest effect.

Transparency. Electromagnetic rays have no effect upon objects which are incapable of vibrating in resonance with them. The ray passes through the object, and the object is said to be transparent to the particular ray in question; for example, rock-salt is transparent to heat, to visible, and to ultra-violet rays; ruby glass, to red light rays; paraffin wax, to Hertzian rays; white fluorite, the most transparent substance known, to heat, to visible, and to ultra-violet rays; thin deposits of metallic silver, to ultra-violet waves; thin aluminum plates to Roentgen rays and thin metals of all kinds to gamma rays. No substance is known which is transparent to the entire electromagnetic spectrum.

Reflection. If the object upon which the wave-train falls is capable of resonant vibration, and if the resonant vibration is not accompanied by friction, the ray is reflected. Usually only rays of certain vibration frequencies are reflected; for example, a red apple reflects red light rays. In the case of good conductors, like the metals, all of the light is reflected,- " metallic reflection," as by a silvered mirror.

Absorption. If the object upon which the wave-train falls is capable of resonant vibration, and if the resonant vibrations are accompanied by friction of some sort, the rays are absorbed, the temperature of the body may be raised, or the absorbed energy may bring about chemical changes.

Photoelectricity. When the resonance between the vibrating electron and electromagnetic impulses is close, the electron may, if the intensity of the radiation be great, vibrate beyond the sphere of influence of the atom of which it is a part. Such escaping electrons constitute secondary corpuscular rays which may bring about further chemical changes. When the electrons escape from atoms which are near the surface of the absorbing medium they may pass off into the surrounding air. Each escaping electron carries a negative charge of electricity, and escaping, leaves a positive charge behind on the absorbing body. This phenomenon is called the "photo-electric" effect of radiation. 


\section{ACTION OF RAYS}

It will be seen that the mechanism of the chemical action of all kinds of rays, both electromagnetic and corpuscular, is the same and consists in the dislocation of those electrons upon which the valency of the atom depends, and which function in holding the atoms together into the groups which we call molecules. Until we have information to the contrary, therefore, we will be more concerned with the laws which govern the localization and dosage of radiation, than with the specific effects of any particular kind of rays.

One of the oldest generalizations in photo-chemistry is due to Grothus and is usually known as Draper's "Photo-chemical Absorption Law." According to this law, only the rays absorbed are effective in producing chemical change. For a quantitative study of the dosage of rays we must, therefore, know (I) the laws governing the absorption of the rays within the tissues, (2) the composition of the rays used, and (3) the relative intensities of the various components. It must be remembered, however, that all the energy of the absorbed radiation is not necessarily productive of photo-chemical change.

Homogeneous medium and radiation. When both the absorbing medium and the radiation are homogeneous in character, i.e. when the absorbing medium has the same composition throughout and when the incident radiation is composed of wave-trains all of which have nearly the same wave-length, then, according to Lambert's law, each layer of absorbing medium of equal thickness absorbs an equal fraction of the radiation which traverses it. Hence, as the thickness of the absorbing medium increases in arithmetical progression, the radiation intensity decreases in geometrical progression. It is possible to express this relationship by assigning values to the absorption index $\mu$ in the equation

$$
i=I_{0} \varepsilon^{-\mu d}
$$

in which $i$ is the intensity of radiation passing through $d$ thickness of absorbing medium when the total intensity of the incident radiation is $I_{0}$ and $\varepsilon$ is the base of the natural system of logarithms.

For a given absorption medium (tissue) the value of $\mu$ will vary. with the wave-length of the incident radiation. If we write down the changing values of $\mu$ as we pass through a regular progressive series of wave-lengths, we form a mathematical picture of the amount of absorption of radiation of each wave-length traversing $d$ thickness of the absorbing medium, the values of $\mu$ rising and falling as we pass through the so-called absorption bands. Such mathematical descriptions (which are called "absorption spectra") are so characteristic of 


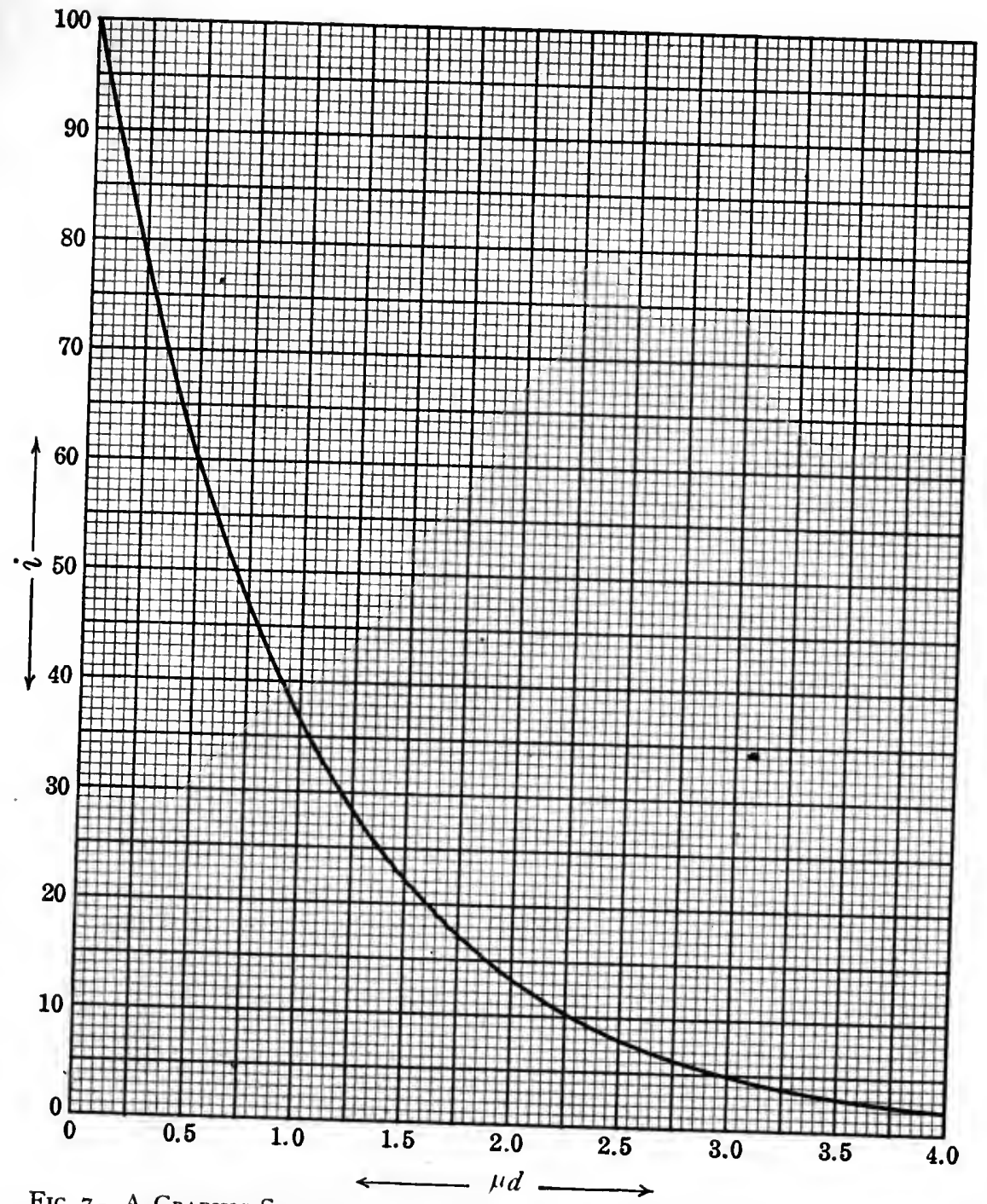

plotted as abscissae against the values of $i$ a $i$ ation $i=I_{0} \varepsilon-\mu_{d}$. The values of $\mu d$ are in per cent. of the incident radiation $I_{0}$. From the $i$ as ordinates. The values of $i$ are given tensity $i$ which will arrive at any point $I_{0}$. From the curve it is possible to read the inof the absorption coefficient $\mu$ of the $d$ in the absorbing medium, providing the value known. The values of $\mu$ and $d$ must, of course be in thedium under consideration is

many of the tissue components, such as hemoglobin, chlorophyll, hematoporphyrin, etc., that they may be used for their identification quite as precisely as we use their chemical formulæ, enabling us to make both 
qualitative and quantitative analysis for the presence of these substances under experimental conditions which would exclude ordinary chemical analysis. Unfortunately, more often than otherwise, absorption spectra are given qualitative rather than quantitative representation.

If we know the value of the absorption coefficient $\mu$ for any particular ray in any absorbing medium, we may calculate, by means of the formula given above, the intensity, in per cent. of the incident intensity, of the radiation which will pass through any point in the absorbing medium, providing we know its distance $d$ from the surface of incidence. The solution of this equation necessitates the use of a table of natural antilogarithms. Fig. 7 is a graphic solution of the equation, in which the values of $\mu d$ are plotted as abscissae against the values of $i$, as ordinates. The values of $i$ are given in per cent. of the incident radiation $I_{0}$. From the curve it is possible to read the intensity of radiation $i$, which will arrive at any point $d$ in the absorbing medium, providing the value of the absorption coefficient $\mu$ of the radiation in the medium under consideration is known. The values of $\mu$ and $d$ must, of course, be in the same units of length. The "volume" absorption for a given mass of absorbing medium included between the points $d_{1}$ and $d_{2}$ may easily be determined when we know both the amount of radiation entering and leaving the region.

For all ponderable matter there is a strong absorption band in that part of the spectrum which separates the ultra-violet region from the Roentgen region. It follows, therefore, that the absorption index of the components of protoplasm rises to very high values as we approach this band from either the ultra-violet .or Roentgen sides, and that the absorption index of protoplasm increases with decreasing wave-length in the ultra-violet region and decreases with decreasing wave-length in the Roentgen region of the spectrum.

This fact is given graphical expression in Fig. 8 in which the righthand curve represents the relation between absorption index and wavelength in the ultra-violet for egg-white (calculated for the most part from Henri's "Extinction Coefficients of Egg-white") and the left-hand curve represents the relation between absorption index and wave-length in the X-ray region for water (calculated from Colwell and Russ's values of the absorption index of aluminum).

It will be seen that both of these curves are convex toward the general absorption band, so that the values of $\mu$ rise faster and faster the closer the region of general absorption is approached. The absorption index values given for Roentgen rays are not as high as those given for ultra-violet rays because the Roentgen ray spectrum has not 


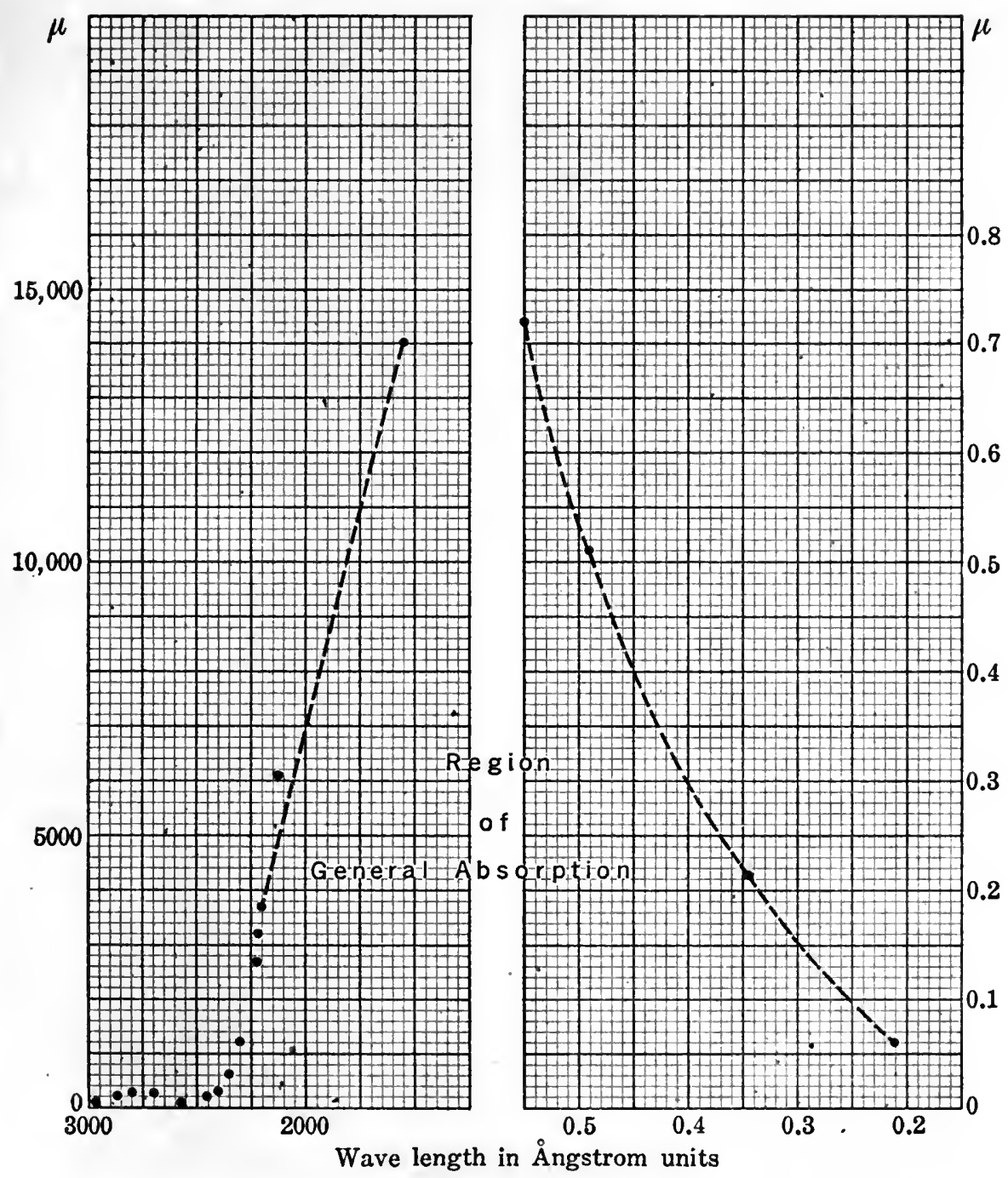

Fic. 8.-Curves Showing the Absorption Spectrum, Characteristic of Many of THE Tissue Components. The absorption indices, $\mu_{-}\left(\mathrm{cm}^{-1}\right)$ are plotted as ordinates against the wave-lengths in Angstrom units as abscissae. The ordinate values at the left are for the left-hand curve, those at the right are for the right-hand curve. The curve at the left in the ultra-violet region of the spectrum has been calculated from the extinction coefficients of egg-white. The curve at the right in the X-ray region of the spectrum has been calculated from the absorption index values of aluminum and represents values approximate for water.

been investigated as far into the region of general absorption as has the ultra-violet spectrum.

The absorption index values given above may be taken as approxi- 
mate representative values of the absorption index of bloodless tissues, since the absorption index curves for all of the more complex components of protoplasm so far investigated have the same general shape in the extreme ultra-violet. Moreover, the relation between chemical composition and absorption for the visible and ultra-violet regions of the spectrum is determined by the kind and arrangement of the atoms within the molecules, and increases, as a rule, with the increase in the molecular complexity.

In the Roentgen and gamma regions the absorption index is dependent only on, and increases with, the atomic density of the absorbing medium and is independent of the grouping of the atoms within the molecule. The atomic density of most tissues is very nearly that of the water which forms a large per cent. of their masses. The calcified tissues form the principal exception. The atomic density of an element is a function of, and increases with its atomic weight. With the exception of the blood none of the tissues are characterized by a predominance of elements of high. atomic weight. In the case of blood it has been suggested that the peculiar susceptibility of this tissue to the action of Roentgen and gamma rays is a result of its high iron content. The atoms of iron act as sources of secondary chemically active delta rays.

Now, if in the equation given above, we assign high values to the absorption index $\mu$ and solve for $i$ for various thicknesses $d$ of absorbing medium, we shall find, as shown in Fig. 9, in which the values of $i$ are plotted as ordinates against the values of $d$ as abscissae, that the values of $i$ fall off very rapidly at first, and then more slowly as the thickness of the absorbing medium increases. It will be observed that the amount of absorption is greatest near the side receiving the incident radiation, so that we have a localization of the place of action of the rays, and the amount of this localization increases with increasing values of $\mu$. These curves are, of course, fór homogenous radiation. Curves for heterogeneous radiation will have the same general form.

Heterogeneous medium. If the absorbing medium is heterogeneous in character, the intensity curves will depart from the simple exponential form, as is shown for example by the upper curve in Fig. 9, in which the absorption index of the region included between $d=5$ and $d=8$ microns is higher, and the amount of radiation absorbed per micron of thickness is greater in this region than in any other. Now in this case we would have a localization of the physiological action in the region of high absorption, provided that the physiological effects produced were proportional to the amount of radiation absorbed.

It is quite impossible to say $\grave{a}$ priori whether such an increase in 


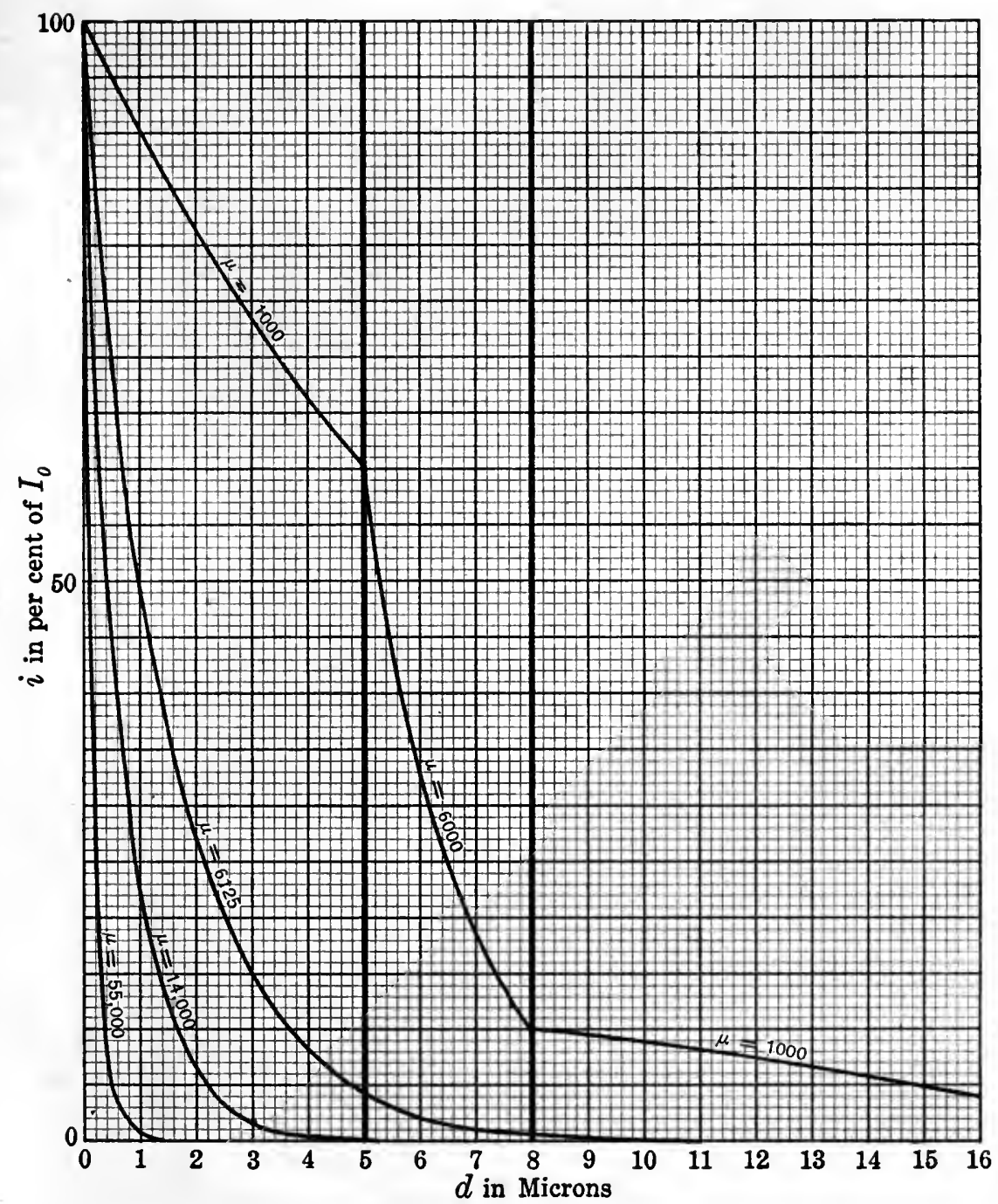

1

Fig. 9.-Some Graphical Solutions of the Equation $i=I_{\circ} \varepsilon^{-\mu} d$, in which the values of $d$ are plotted as abscissae and the values of $i$ in per cent. of $I_{0}$ as ordinates. The individual curves are for various values of $\mu$. The three lower curves are for a homogeneous medium, in which the value of $\mu$ is constant. The upper curve is for a heterogeneous medium in which the value of $\mu$ changes.

absorption will be accompanied by an increase in the physiological effect, because we do not know the mechanism by which the energy of the rays is transformed into physiological action. If the increased absorption were due merely to an increase in molecular concentration of the 
absorbing region, and further if the amount of physiological action were proportional to the degree rather than to the concentration of photochemical change, it is possible that no increase in the physiological effects would be observed. If, however, the increase in the absorption index were due to a difference in chemical composition of the absorbing medium, an increase in physiological action might reasonably be expected.

An application of these laws of absorption to biological problems is clearly shown in some experiments by the writer and Miss D. M. Hughes, on the action of ultra-violet rays on the unicellular organism, Paramecium, in which individual organisms were exposed to ultraviolet rays of different wave-lengths selected in accordance with the principles of absorption discussed above. In some of the experiments fluorite rays with a wave-length in the neighborhood of $\mathrm{I}, 600$ Ångstrom units were used. The absorption index of these rays in protoplasm (see Figs. 8 and 9) is in the neighborhood of 14,000 . Now the cytoplasm of Paramecium is about Io microns thick. Practically all of the radiation, therefore, was absorbed in the cytoplasm of the cell, and did not penetrate to the nucleus. In the other experiments quartz rays which are only slightly absorbed in the cytoplasm, but are strongly absorbed in the nucleus, were used. We were able, therefore, to localize the place of action of the rays within a single cell. When an organism was exposed to rays which are absorbed in the cytoplasm, it was first stimulated to more rapid movements, and then lost its powers of coördination so that it swam continuously in circles. For all exposures less than the amount necessary to produce cytolysis and death there was no interference with the rate of cell division. When, however, the organism was exposed to the quartz rays which are absorbed in the nucleus, the rate of cell division was altered, even though the exposure was so small that no effect upon the rate of motion of the organism could be observed. Cell division was always delayed by exposures to these rays, but the delay following short exposures was of very short duration and was followed by an acceleration so that the radiated organism experienced even more cell divisions than did the controls. Longer exposures resulted in a longer delay of cell division. Other metabolic processes appeared to be interfered with by exposures to rays which are absorbed in the nucleus, and the organism became transparent (starved) and finally died.

Heterogeneous radiation. It will be easily understood that alpha and beta particles traveling at a slow rate of speed will have less penetrating power, i.e. will be absorbed more easily, than those particles having a higher rate of speed. All sources of alpha and beta radiations 
emit rays covering a comparatively wide range of speeds. It has been found that such heterogeneous corpuscular radiations are absorbed according to an exponential law, which is quite similar to the one given above for the absorption of electromagnetic radiations.

When we know the kinds of rays which a given tissue will absorb and the laws of this absorption, we may proceed to select a suitable source of radiation. Sources giving large amounts of homogeneous radiation are not available. It is necessary, therefore, by a judicious use of ray-filters to screen out the undesirable rays. We may, for example, prevent excessive surface burning by passing the rays from radium bromide through a suitable metallic screen and thus filter out the soft beta and gamma rays, keeping in mind, of course, the fact that in this case some of the energy absorbed by the filter may be transformed and cause the filter to emit the very kind of rays we are endeavoring to eliminate. For a description of the numerous available sources of radiation and the proper methods of screening, the reader should consult special books on radiotherapy.

In order to emphasize the importance of the proper selection of the source of radiation, it may not be out of place to cite the experiences of Dr. Neils Finsen of the Finsen Institute in Copenhagen. Finsen used ultra-violet light to destroy the bacillus which causes lupus. In his first experiments. he used sunlight, but because of the large amount of violet and long waye-length of ultra-violet rays contained in sunlight the exposures were followed by excessive pigmentation, so that after several treatments the rays would not penetrate the skin sufficiently to destroy the bacterial colonies which lay some 3 or $4 \mathrm{~mm}$. beneath the surface. He then used the carbon-arc as a source of radiation and met with remarkable success. The radiation from the carbon-arc contains ultra-violet rays of shorter wave-length than those contained in sunlight. These rays, which have a higher absorption index in tissues than any of the rays contained in sunlight, are absorbed by the pigment forming cells of the skin which are thereby destroyed. The skin becomes bleached when exposed to the radiation of the carbon-arc and treatments can be continued as long as desired.

Because of the success which followed this change in the source of rays, Finsen at no small expense of time and energy had devised a quartz mercury-vapor arc, a source emitting ultra-violet rays of even shorter wave-length than those emitted by the carbon-arc. But then he obtained such an abundance of easily absorbed rays that there was excessive destruction of the epidermal tissues.

Distribution of radiant energy about its source. Not only must we know the composition of the radiation used, but we must also know 
the distribution of the radiant energy about the source. When the source is a point the distribution is symmetrical, and surfaces receiving equal intensity of illumination will be spheres described about the source as a center. The intensity of the radiation falling upon any such

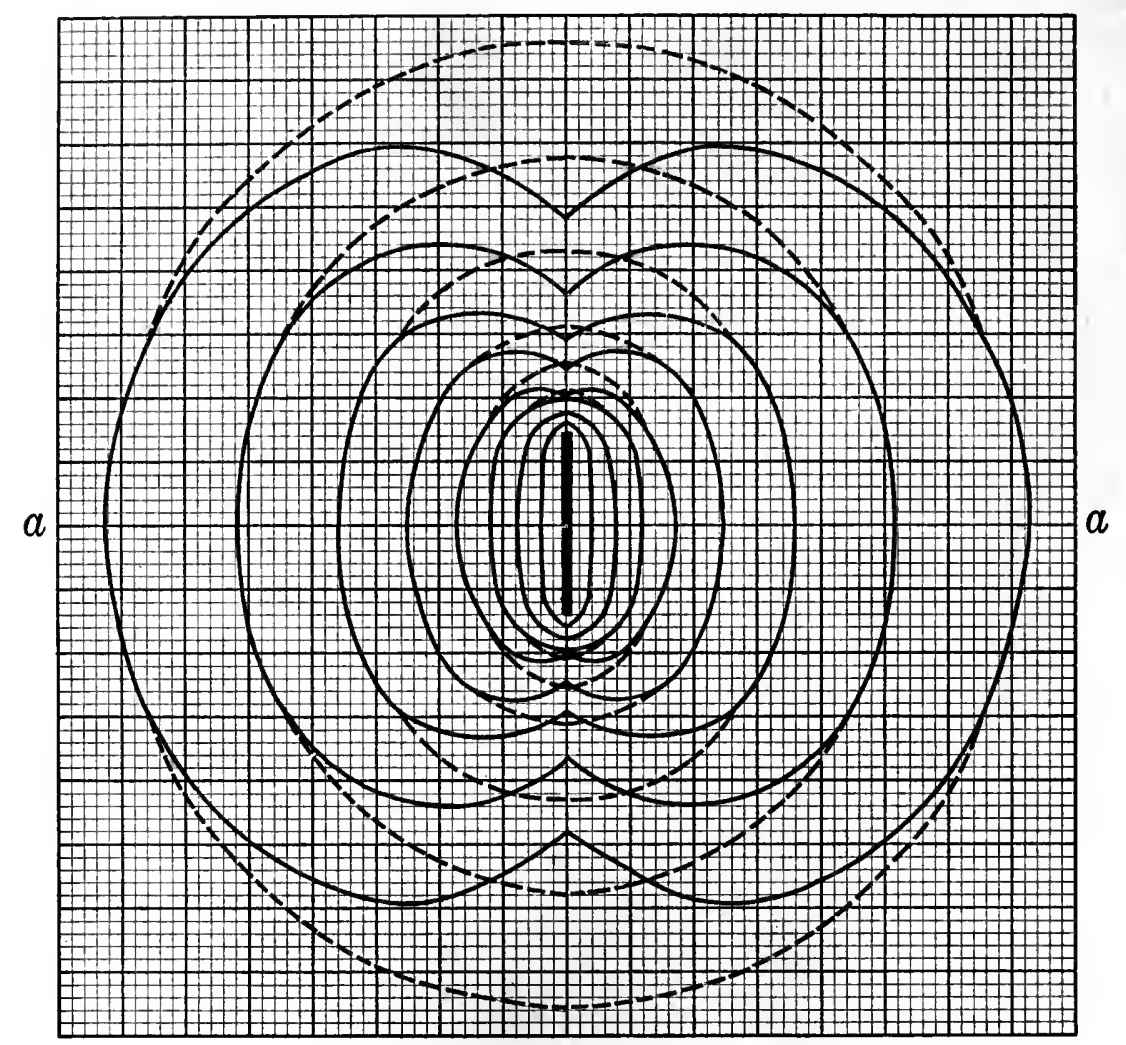

FIG. 10.

Figs. io and il.-Charts Showing the Sections of Surfaces of Equal Radiation Intensity About a Glass Tube of Radium Emanation. The tube is $15 \mathrm{~mm}$. long and $0.5 \mathrm{~mm}$. wide. The section passes through the major axis of the tube. The continuous lines are from experimental data. The dotted lines represent the graphical solution of a certain mathematical formula, and approach a concentric system of ellipses, having their foci near the ends of the tube. The entire surfaces of the figures as they would appear in three dimensions are surfaces of revolution which would be formed by revolving each section about the major axis of the radium tube. Fig. II is a section along the axis of $a a$ through the surfaces of revolution thus formed.

spherical surface is determined by the "inverse square law" of radiation, which states that the intensity varies inversely as the square of the distance between the source and the illuminated surface. If the shape of the source is other than a point, the surfaces of equal radiation 
intensity will depart from the spherical form, and mathematical representation, especially for distances very close to the source, is very difficult. In Fig. Io is shown sections of surfaces which I have made by measuring the chemical action of rays at various distances from

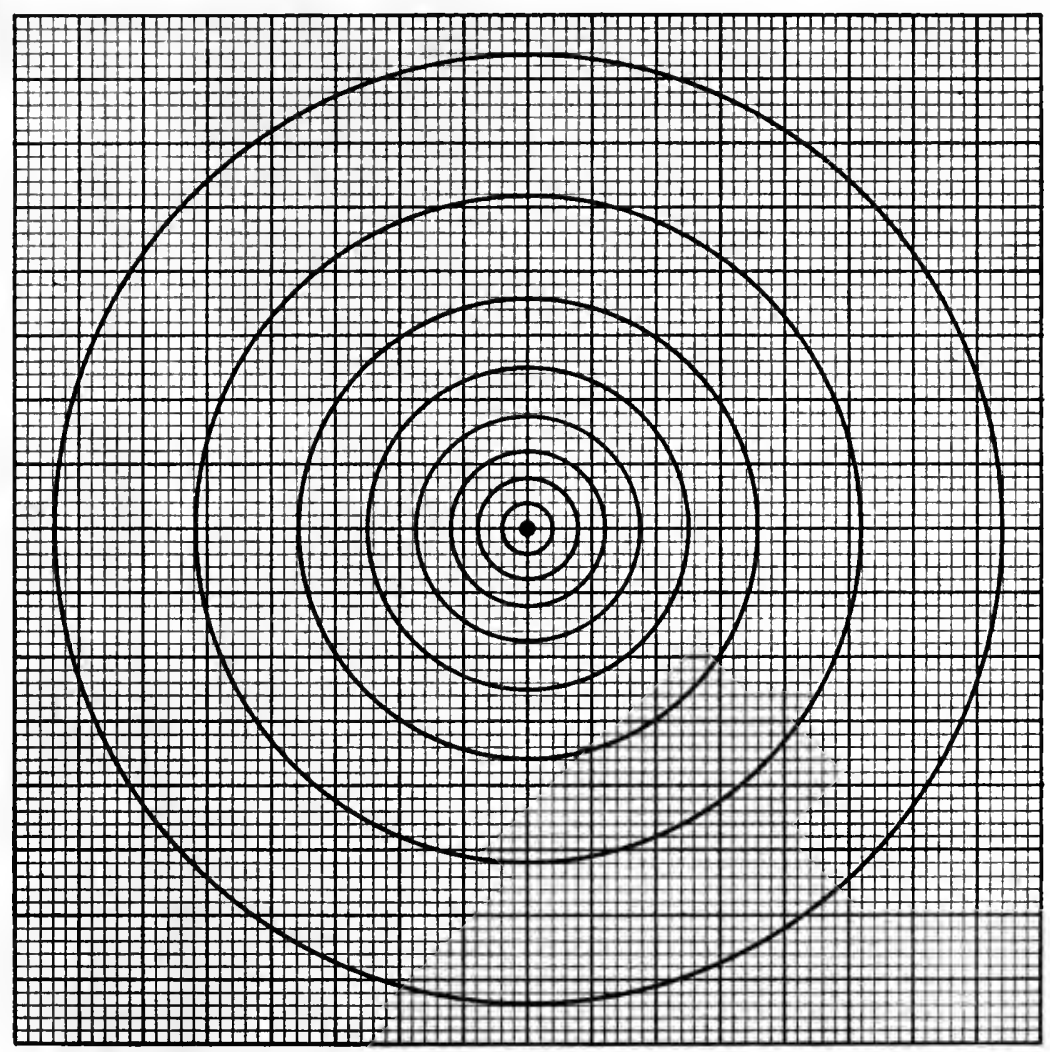

Fig. II.

the source. The source in these experiments was a glass tube, $0.5 \mathrm{~mm}$. in diameter and $15 \mathrm{~mm}$. long, containing radium emanation. The intensities were measured by the darkening of a photographic film. The distance between each surface and the source was such that the intensity of radiation falling on it would be one-half of that falling on the largest surface enclosed. The dotted lines in the figure are sections of surfaces formed according to a certain mathematical formula. The continuous lines are the sections of the experimental surfaces. The drawing-in of the experimental surfaces along the longitudinal axis of the source is undoubtedly caused by the increased absorption at the ends of the tube 
containing the emanation due to the thickening of the glass as the tube was sealed off. The section through these surfaces along the axis aa at right angles to the longitudinal axis of the emanation tube, is shown in Fig. I I. 'It will be seen that the sections are circles, and that the theoretical surfaces approach ellipsoids.

The measure of the exposure. The amount of photochemical change produced by radiation, and therefore the amount of biological action, is determined by the total quantity of energy received. We may look at radiation then as a flow of energy analogous to the flow of water or electricity, and inquire what relations exist between the rate of flow or intensity (the quantity of energy remaining constant) and the biological effect produced.

Talbot's law. A law of optics known as Talbot's law, which, as stated by Helmholtz, says "if any part of the retina is excited with intermittent light recurring periodically and regularly in the same way, and if the period is sufficiently short, a continuous impression will result which is the same as that which would result if the total light received during each period were uniformly distributed throughout the whole period." Because of the applicability of this law to the rotating sectored-disk-photometer, it has been investigated by many experimenters and appears to be most satisfactorily established, not only for white light, but for red, green, and blue light as well. A general applicability of Talbot's law to other physiological effects of radiation can be established only by experiment. It has been found applicable to the phototropic reactions of a number of animals.

The photochemical reciprocity law. "Not only does Talbot's law assume that the amount of photochemical change is a function of the total quantity of radiation received, but it also assumes another photochemical law which was formally stated some thirty years later by Bunsen and Roscoe. Bunsen and Roscoe's law states that within very wide limits, if in a series of exposures the products of light intensities times the exposure-times are equal, the amount of darkening of equally photo-sensitive silver chloride papers will be equal. This law is known as the Bunsen and Roscoe "Reciprocity Law." A great many experiments have been made both in photochemistry and photobiology to test the validity of this law, and it has been found to hold within certain limits for many photochemical and photobiological reactions. Deviations from the reciprocity law are to be found, however, for both strong and weak intensities. Deviations for the darkening of the photographic plate and for the growth of visual sensation in the human eye are shown in Figs. 12 and 13 . In the figures, the products of the intensity times the length of the exposure $(i \times t)$ required to produce a constant amount 
of photochemical change, are plotted as ordinates against the intensity (i) as abscissae. The human eye, by the way, after visual sensation has reached a maximum, is a notable exception to the reciprocity law, for, except during the growth of visual sensation, it does not possess the property of accumulating intensity. The stars do not appear brighter the longer we gaze at them nor can we double the apparent brightness of a candle by looking at it twice as long.

Influence of recovery. . The fact that organisms can recover from the effects of radiation gives sufficient reason to doubt, except within

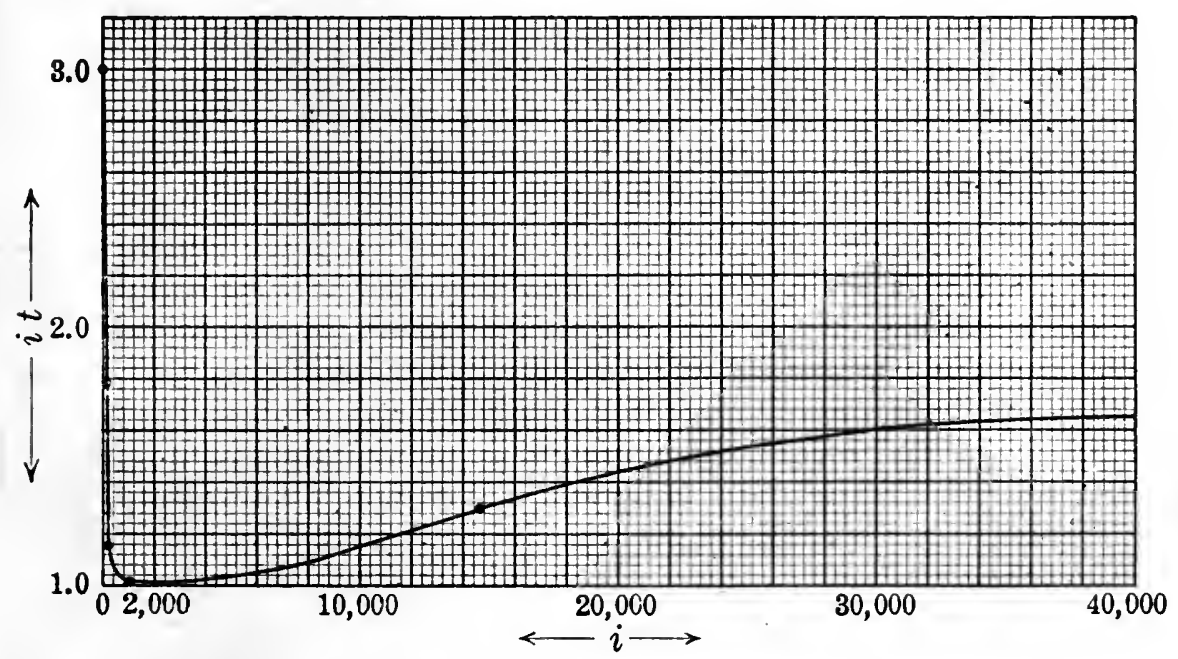

Fig. i2.-A Graphic Representation of the Relation Between Intensity and the Amount of Radiation Required to Produce a Constant Blackening of thr Protographic Plate. The amounts of radiation (products of intensity and time) producing a constant blackening are plotted as ordinates, and the corresponding intensities as abscissae. The curve shows that there is an optimum intensity with which the desired effect takes place with the least amount of exposure.

very narrow limits, the applicability of the reciprocity law to the physiological effects of rays, because if the intensity is low, processes of recovery act like reverse dark reactions and the physiological effects of the light do not accumulate.

The influence of recovery is shown in some experiments by the writer and Miss D: M. Hughes on the cytolytic action of fluorite rays on Paramecium caudatum. These organisms were exposed for 8 seconds to the rays from a fluorite discharge tube. The exposure was divided into two periods of 4 seconds each. In different experiments the interval of time between the two 4 -second exposure periods was 
varied. It was found that the percentage of organisms undergoing cytolysis decreased as the interval of time between the exposures increased from o to 60 minutes, showing that during the interval the organism recovered from the effects of the first exposure. The recovery was practically complete in 60 minutes. It was further found that the

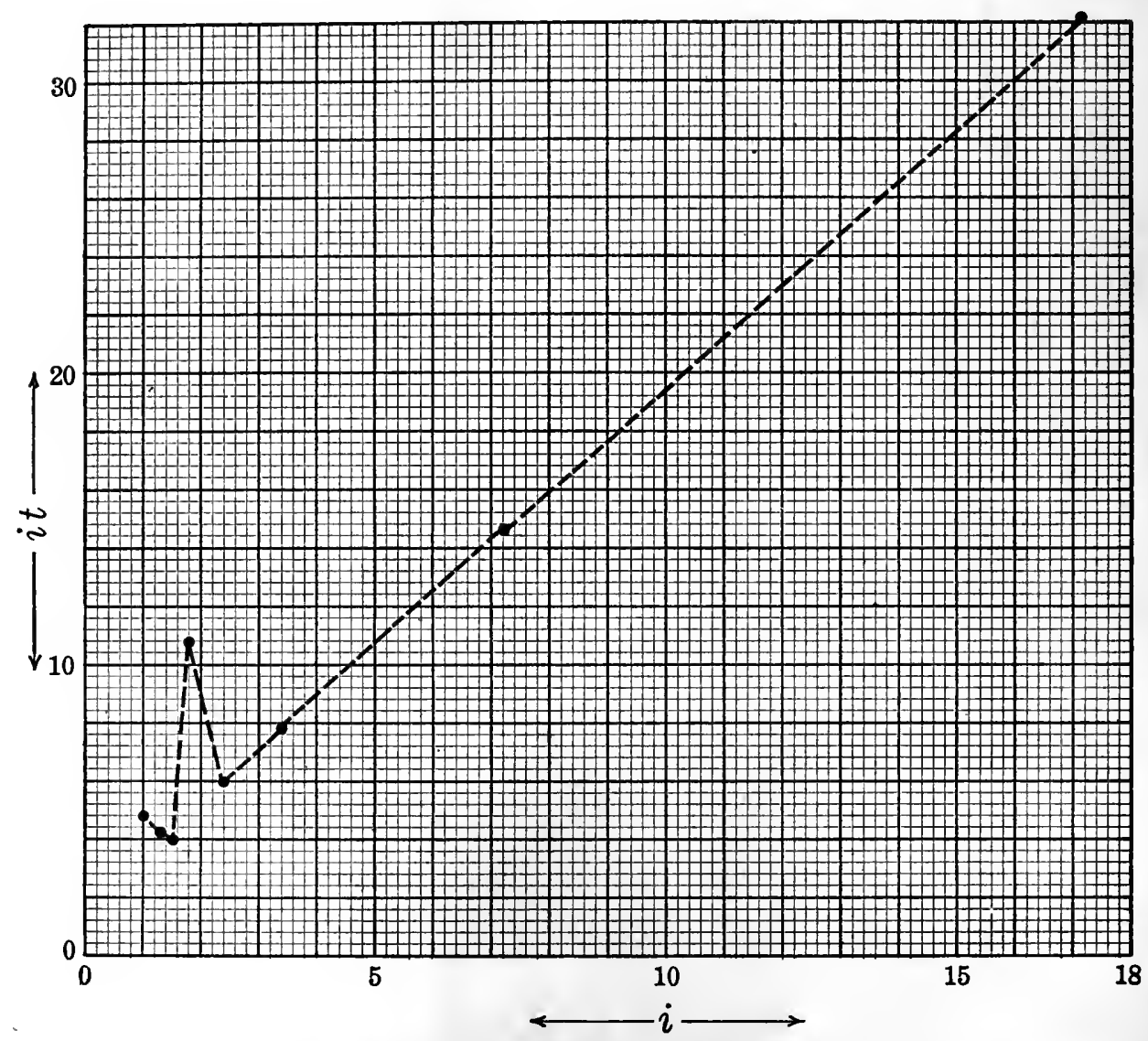

Fig. 13.-A Graphic Representation of the Relation Between Intensity and the A mount of Radiation Reguired to Produce the Threshold Sensation of Light IN THE HUMAN EYE. The amounts of radiation (products of intensity and time) are plotted as ordinates and the corresponding intensities as abscissae. The effect is shown to be accomplished with a smaller amount of energy when the intensity is low than when it is high.

rate of this recovery could be expressed very accurately by the monomolecular reaction formula

$$
X=X_{0} \varepsilon^{-K t}
$$

in which $X_{0}$ is the per cent. of cytolysis when the time interval is zero. 
$X$ the per cent. of cytolysis when the time interval is $t . \quad \varepsilon$ is the base of the natural system of logarithms. In these experiments the constant $K=0.0473$. That the recovery rate corresponds with an exponential function of time indicates, at least, an orderly occurrence of the proc-

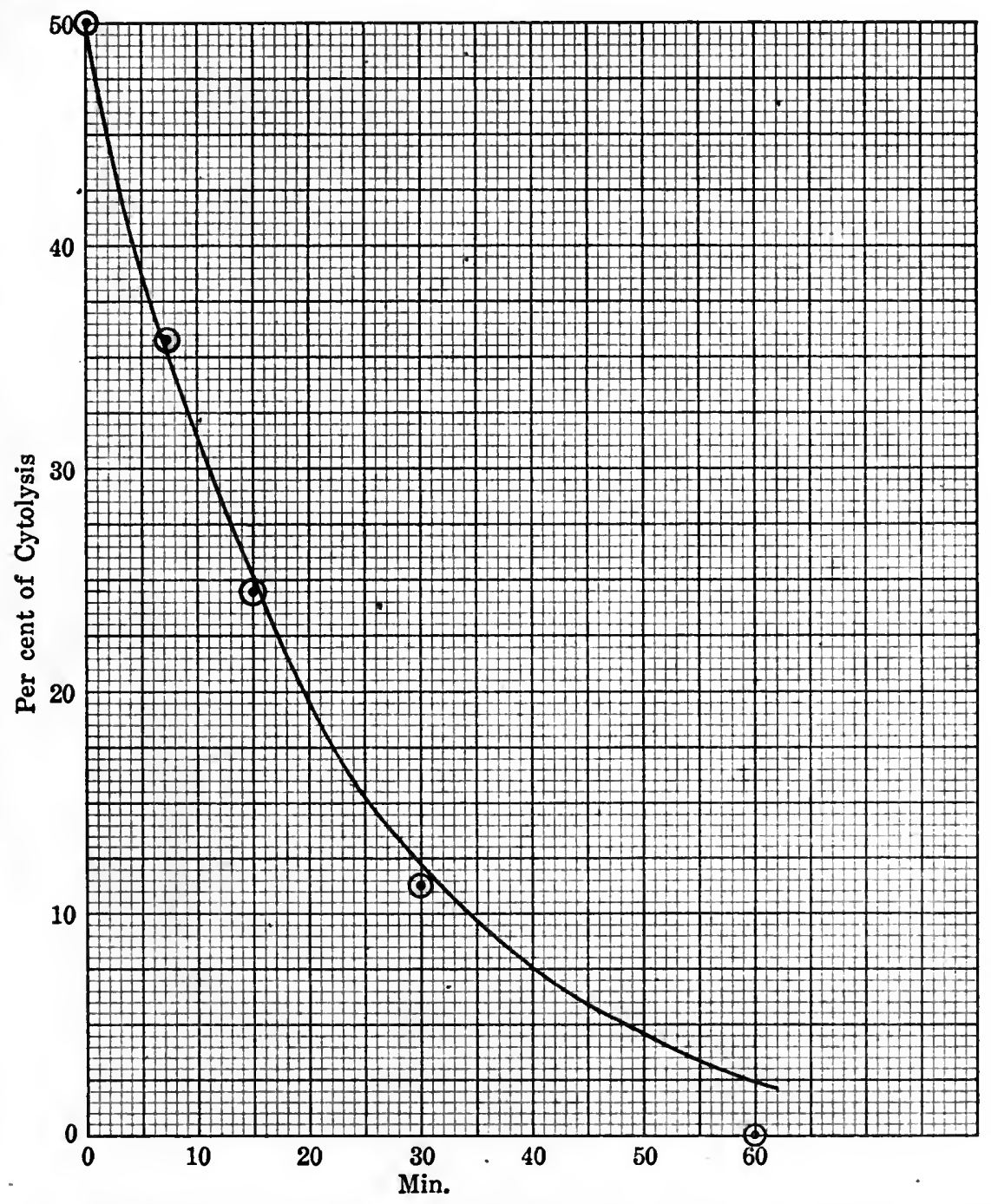

Fig. 14.-A Graphic Representation of the Recovery of Paramecium caudatum FROM FluORITE RAdiation as a Function OF Time. The per cents. of cytolysis are plotted as ordinates and the intervals of time between the two 4 -second exposures are plotted as abscissae. The curve is a graphic representation of the equation $X=X_{0} \varepsilon \cdot K t$, in which $K$ equals $0.0473, X_{0}$ equals the per cent. of cytolysis when the time interval is zero, and $X$ the per cent. of cytolysis after the intervals of time, $t$ $\varepsilon$ is the base of the natural system of logarithms. 
esses involved in recovery. But it is not clear whether the processes are physical or chemical in nature. A graphical solution of the equation given above, in which the values of $X$ are plotted as ordinates against various values of $t$ as abscissae, as given by the continuous line in Fig. I4. The experimental results are indicated by the points. It will be observed that the rate of recovery is very rapid during the first part of the interval of time between the two exposures. This is undoubtedly one reason why Talbot's law is applicable only if the period of the intermittent light be very short.

The relation between the amount of radiation and the effect produced. All photochemical investigations which have hitherto been

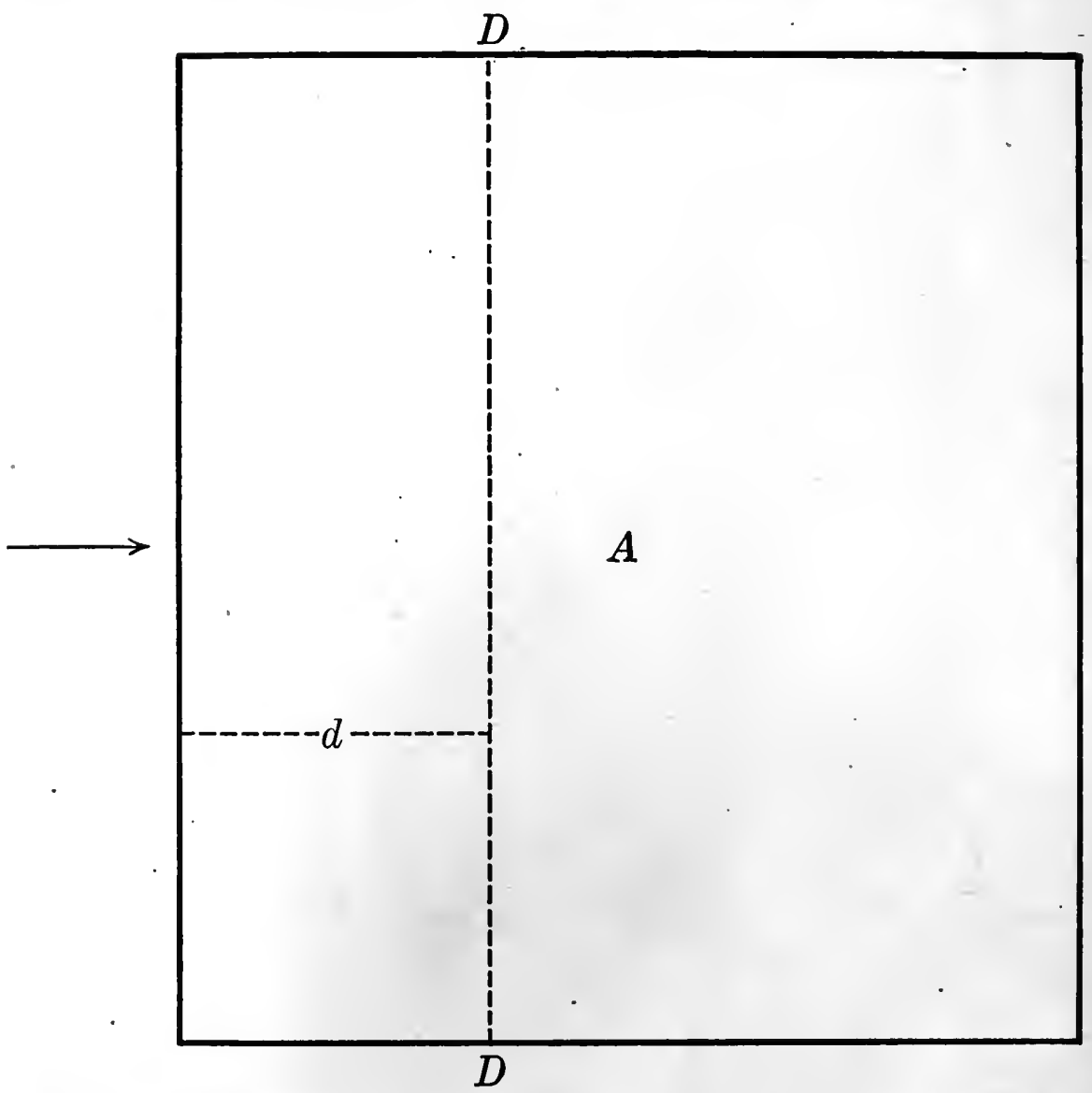

Fig. 15.-A Diagram to Illustrate the Influence of the Shape or Mass of the Radiated Tissue upon the EFfect Produced. The figure is a section through the mass lying in the plane of the direction of the rays which are supposed to be incident on the surface indicated by the arrow. Owing to the absorption of the rays the intensity of radiation arriving at the line $D-D$ will be a function of the distance $d$ of the line from the surface of incidence. 
made have proved that the amount of chemical action is proportional to the exposure, i.e. the product of the amount of radiation absorbed and of the time, and there is a priori no reason for supposing that the chemical action of radiation on protoplasm forms an exception to this general law. But owing to numerous disturbing conditions and the complicated kinetics of photochemical change, the law is probably never realized in radiobiology. The power of recovery possessed by practically all tissues acts like a reverse "dark" reaction and nullifies the effect of the rays. In the special photo-sensitive organs the mass of some active component is usually altered by the photochemical change, and thus becoming a limiting factor it controls the course of the reaction. In the photo-syntheses of carbohydrates in green plants it appears that an active principle is formed by the light, i.e. the light has an autocatalytic action.

Then there is always present the influence of the shape or mass of the tissues radiated. Consider a mass of homogeneous tissue $A$, Fig. I 5, illuminated by parallel rays of homogeneous radiation incident from the side marked with the arrow. Because of absorption the intensity of the radiation and therefore the amount of photochemical change along a line $D-D$ will be a function of the distance $d$ of the line from the surface of incidence. The relation between the rate of photochemical change and the distance $d$ can be easily determined by applying the laws of absorption discussed above. In practice, however, the rays are seldom either parallel or homogeneous, and a mathematical treatment of the problem is very difficult. The writer has found an experimental solution of the problem. A' glass tube $15 \mathrm{~mm}$. long and $0.5 \mathrm{~mm}$. in diameter containing radium emanation was embedded in a block of beeswax, and the wax cut away until a surface of equal radiation-intensity, as determined by the darkening of a photographic film, was formed. This process was repeated again and again, each time forming a surface having one-half the intensity of the surface just preceding, that is a surface requiring just twice the exposure to produce the same amount of photochemical change in the photographic film. The solid figures thus formed were ellipsoidal in shape with the radium tube lying along the principal axis. In order to compare the volumes of the space enclosed by the equal intensity surfaces the wax models were weighed. The weights are plotted as ordinates against the exposures as abscissae in Fig. I6. The continuous line drawn through these points shows the relation between the increase in weight and the length of exposure. It will be seen that the weight increases much more rapidly during the early part of the exposure. These figures are, of course, for the rays which affect the photographic film, but the same general relations will hold for the rays which destroy protoplasm. 


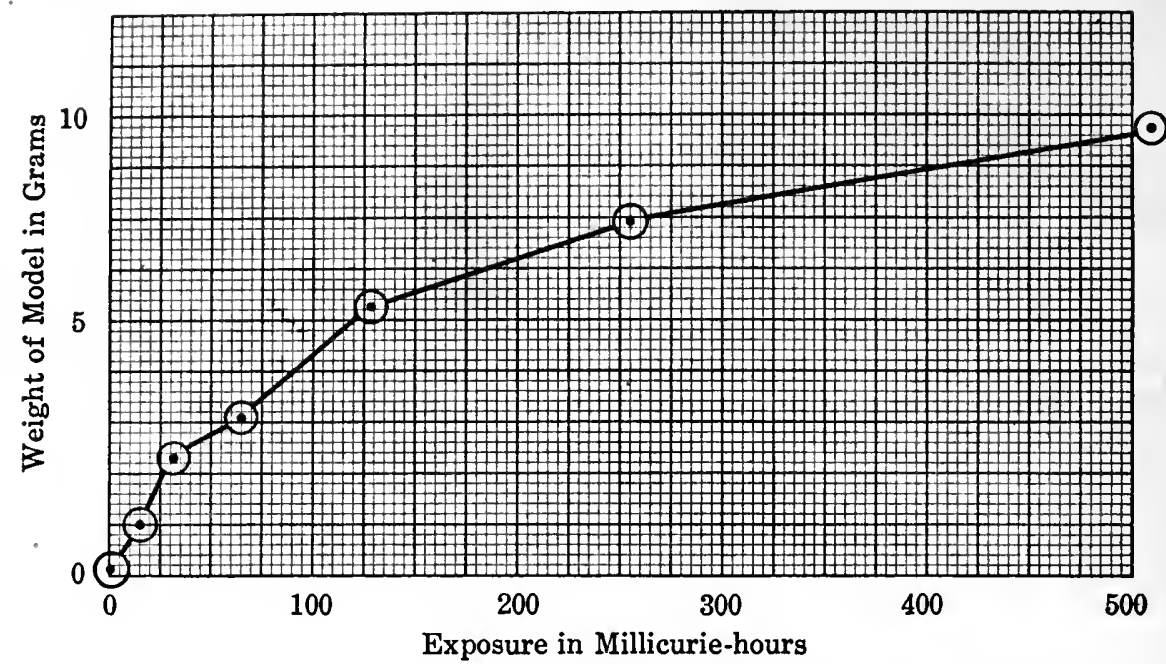

Fig. i6.-A Chart Showing the Results of Experiments Calculated to Determine the Influence of the Shape or Mass of the Tissue upon the Effects ProDUCED. The source of radiation was a glass tube $15 \mathrm{~mm}$. long, $0.5 \mathrm{~mm}$. in diameter containing radium emanation. The weights of wax models of surfaces of equal effect (darkening of the photographic film) are plotted as ordinates against exposures (in rnllicurie hours) as abscissae. The continuous line shows the relation between the weights of the models and the amount of exposure and may be interpreted as showing the relation between the mass of tissue affected by the rays and the amount of the exposure, it being supposed that the tube of emanation is buried deep within a mass of homogeneous tissue. It will be observed that the effect produced is relatively greater during the early parts of the exposure.

It is impossible, of course, in biological experiments, to measure the amount of initial photochemical change, for we observe only the physiological effects produced, and physiological effects are not good measures of the intensity or the amount of the stimulating agent. It would be an easy matter to find many examples illustrating this fact, but perhaps the two following are as good as any:-It is well known that a seedling illuminated from one side will bend towards the source of light. But by a closer study of phototropism, as this phenomenon is called, we learn that seedlings, receiving continuous unilateral illumination, bend first toward the light and then bend away, then again bend toward the light, and if the illumination be sufficiently strong or be continued for a sufficient length of time they again bend away. A continuous unilateral illumination results, therefore, in a series of alternate positive and negative phototropic responses, the duration of each response being longer than the one just preceding. Further, the amount of illumination which the seedling has received just previous to the unilateral stimulation is a determining factor in the outcome of the experiment. Now it is only the first positive phototropic response which follows the 
Bunsen and Roscoe reciprocity law; the others do not. It is obvious in this case that, if we were not aware of all of the possible phototropic responses of the seedling, we would be quite unable to find any constant relation between the physiological effect and either the amount or the intensity of the exposure.

The second example may be of more interest to the radiotherapist. I have found that when Paramecia are exposed to ultra-violet light (wave-length $=2800$ Angstrom units) cell division is inhibited and the duration of the inhibition increases, the intensity being constant, with the length of the exposure. But the short inhibitions which follow brief exposures are followed by an acceleration of cell division so that more individuals are produced by such radiated organisms than by nonradiated controls. The importance of the stimulating action of small doses of radiation (and a stimulation of functions other than cell division has been observed) lies in the fact that when large masses of tissue are exposed to radiation there are always regions at some distance from the point of application of the rays where the intensity is low and where a stimulating effect may result.

Scientific literature contains the records of many investigations which deal with the relation between the total amount of exposure (product of time and intensity) and the physiological effects produced. But because of inadequate physical data, such as intensity, amounts of reflection, absorption, etc., or the failure of the investigator to measure or record his experimental errors, such as those arising from unknown or uncontrollable variations in his technique, or those due to individual idiosyncrasies of the organism experimented upon, the results obtained have, for the most part, only qualitative significarice. Correlations of the findings of different investigators are, therefore, impossible. The subject is particularly difficult because the changes to be observed do not appear at the time of the exposure but only after a more or less prolonged interval, called the "latent period." During this time many physiological changes may occur in the organism experimented upon, and the effects of the exposure may be intensified or reduced and may even disappear altogether.

We may say that in general small doses of radiation, like small doses of many toxic drugs, have a stimulating action. Larger doses produce deferred physiological changes. With very large doses the latent period is greatly shortened and may entirely. disappear so that there is an immediate destruction of the exposed tissues.

It has been found that the phototropic responses of some plants and animals follow Weber's law, which states that for all intensities of 
excitation the increase of stimulus necessary to cause an increase of sensation is a constant fraction of the whole stimulus.

Fechner has attempted to reduce Weber's law to a mathematical expression in the psychophysical law that "the sensation is proportional to the natural logarithm of the stimulus." It has been shown that the lethal effect of X-rays on the flour-weevil Tribolium confusium (provided the resistance rather than the susceptibility of the organism to $\mathrm{X}$-rays is considered) bears a definite relation to the logarithm of the total X-ray dose. In the experiments referred to, the length of time between the exposure and death of the organism, that is, the duration of the latent period, was taken as a measure of the resistance of the organism to X-rays.

Effects of rays on protoplasm. Protoplasm has evolved in the presence of the heat and light contained in the solar spectrum, and no factor in its environment has had a more profound influence in directing its evolution. We find that these rays have called forth in both the animal and the vegetable kingdoms the nicest kind of adaptation. But we are as yet quite ignorant of the mechanism by which protoplasm perceives and is influenced by this imporiderable part of its environment.

Optimum intensity. There is for most living things an optimum intensity of illumination which is a necessary condition for the maintenance of a normal reaction between the organism and its environment. Naturally this optimum varies for the various species of animals and plants. For example, Ciona sp., a tunicate which normally lives under rocks in the sea, is killed upon exposure to the diffuse daylight of the laboratory. On the other hand, an exposure of fifteen seconds is sufficient to initiate regeneration of the hydroids of Eunedrium sp., a coelenterate, which is unable to regenerate without an exposure to light. A plant which has been growing in diffused daylight becomes incapable of being impressed by its surroundings when kept in a dark room for some time. This reaction is quite independent of the failure of photosynthesis. In animals the light produces a condition called " muscle tone" which in the insects, at.least, is responsible for their phototropic responses.

In the special photo-sensitive sense organs of animals it appears that either the light synthesizes a photo-product which decomposes in the dark or when illuminated by rays from another part of the spectrum, or that the light decomposes a precursor substance which is synthesized in the dark or when illuminated by rays from another part of the spectrum. We are at present quite ignorant of the nature of these photo-products or precursors as the case may be, or of the mechanism 
by which their formation or presence stimulates the optic nerve. We have been able in some cases to formulate the laws governing the rate at which these changes occur, and these laws are identical with other well-established laws of " dark" and " light" chemistry.

The above explanation of the action of photo-sensitive sense organs implies that some components of protoplasm are more sensitive to the light than are others. Indeed some kinds of protoplasm are by far the most sensitive of the known photo-sensitive substances. The photochemical substances involved in human vision are $\mathrm{I}, 000$ times more sensitive to light than are the silver salts used in the fastest photographic plate. It is only the eye's inability to accumulate intensity with exposure that makes the photographic plate superior for detecting stars of very low magnitude. The protoplasm at the tip of an ordinary oat seedling is even more sensitive to light than is the human eye.

Photodynamic effect. The extreme photo-sensitiveness of certain kinds of protoplasm is not due to the presence of photo-sensitive elements such as silver, many of the salts of which are altered under the influence of radiation, but in many cases appears to be the result of photodynamic sensitization, a process at present but very little understood but of very great importance in the economy of living things, and one which may in the future find a conspicuous place in radiotherapy. Photodynamic sensitization is brought about by the presence of fluorescent substances, that is, substances which have the property of giving off, when illuminated, light of a color differing from their own and from that of any component of the incident light. The light given off is usually of greater wave-length than the incident light, and the violet and ultra-violet rays are the best exciters of it.

In Photography:-The silver salts used in the photographic plate are normally more sensitive in the violet and ultra-violet part of the spectrum than they are in the red and orange part. If, however, a fluorescent stain, which absorbs, for example, red light, be added to the emulsion, the sensitiveness of the silver salt to red light will be increased so that it may equal or even exceed the sensitiveness to violet and ultra-violet light. It has been found that the stain must form a very close union (chemical?) with the silver salts, at least a union such that the stain is not removed by washing. Fluorescent stains are regularly employed in the manufacture of certain ortho-chromatic plates and for the production of the red-sensitive plates used for landscape photography during the recent war.

On Protoplasm:- When infusoria are placed in a $\mathrm{I}$ to $\mathrm{I}, 000,000$ solution of acridin (a fluorescent substance) and the culture placed in the dark, the organisms are not injured, but if the culture is placed in 
the diffused daylight of the laboratory the organisms are killed. Similar results have been obtained for many biological light reactions by many independent investigators and the following facts have been established:-

I. The photodynamic substance acts only in the wave-lengths which the fluorescent substance absorbs.

2. Photodynamic substances act on enzymes and all animal cells.

3. Only substances which fluoresce in watery solutions are active as sensitizers.

4. In some cases the photodynamic substance acts on the outside of the cell, in other cases on the inside.

5. The substance must fluoresce, but the amount of action does not vary with the amount of fluorescence.

6. The fluorescent waves are not effective, nor is there a photoelectric effect evident.

7. The fluorescent substance must come in contact with the cell. It seems, therefore, to be due to some action of the electrons.

8. The action of the fluorescent substance is not dependent on the presence of free oxygen.

Fluorescence is particularly common in the organic compounds formed by living protoplasm. The two best known fluorescent substances are the characteristic coloring materials of the plant and animal kingdoms, chlorophyll and hemoglobin. It appears that the ability which plants have of forming carbohydrates from carbon dioxid and water is due to the photodynamic action of the fluorescent chlorophyll. We can form carbohydrates from carbon dioxid and water in the laboratory by the use of ultra-violet light. In the plant in the presence of the fluorescent chlorophyll the union is made by red and orange light. In the animal kingdom the most attractive view of the function of the pigments associated with light sensitive organs, such as the visual purple of the vertebrate eye, is that they increase the delicacy of response or irritability of the organs to light.

In phototherapy two advantages may be gained by the skillful use of photodynamic substances. In the first place the sensitized tissues may be destroyed by rays which are unable to affect unsensitized tissues, and second, rays of a longer wave-length capable of penetrating farther into the tissues may be used. It has recently been found that these principles may be applied to the use of X-rays, thus providing us with a mechanism by which their bactericidal effects may be greatly enhanced.

It has often been pointed out that the distinction between the terms 
chemical and physical are arbitrary. Whether we term a particular change brought about by light as chemical or physical will depend upon the way in which we wish to analyze or utilize that change. All absorption of light alters the chemical potential of the absorbing medium, but whether a chemical change is observed will depend upon a variety of accessory factors not least among which will be our powers of perception.

Experience shows that light can bring about a variety of chemical changes:-polymerization and depolymerization, allotropic changes of atoms and isomeric changes of molecules, hydrolysis, oxidizations, reductions, syntheses, and dissociations. Examples of all kinds of light reactions are to be met with in a study of the biological action of rays. Therefore any theory of the physiological effects of radiation which ascribes the action to any particular kind of chemical reaction is of limited value.

The chemical nature of the action of radiation was recognized by Löbel in I815. Downs and Blunt ascribed the toxic action of light to the oxidizing power of the rays, and Hertel in 1905 concluded that the action is due to oxygen split off by the light. Schwarz in 1903 ascribed the toxic action to the splitting of lecithins, but Schaper ascribed the action not to the splitting of lecithins but to the toxic photo-products of the lecithins. Gager in I908, accepting the "biogen" theory of Verworn, suggested that radiation, either directly or indirectly, destroys the biogens of protoplasm. The Hertwigs thought that the rays act upon some part of the nuclear substances, perhaps the chromatin, which becomes a "living toxin" causing subsequent physiological effects. Heineke says, using the terminology of Ehrlich, that the "genoceptors" of the cell are more easily destroyed by radiation than the "nutroceptors." Neuberg concluded that radiation destroys the enzymes which are responsible for cell metabolism, and the writer has ascribed the toxic action of rays to photochemical changes occurring in the complex and unstable proteins of the cell.

A living organism is a complex of dynamic changes occurring in a colloidal substance called protoplasm. Because of the mutual interdependence between the course of these dynamic changes and the character of the colloidal protoplasm, it is essential to the continued existence of the organism that the dynamic changes take place in an orderly and controlled manner. Any alteration, either physical or chemical, which results from radiating protoplasm may be capable of producing both physiological and morphological disturbances. At present it matters little whether we say that the action of radiation is physical, chemical, or physico-chemical in nature. Changes of one kind will 
undoubtedly involve changes of another. Whether or not an alteration takes place will depend on, first, whether the rays are absorbed, and second, whether the absorbing substance or substances very intimately associated with it are photo-sensitive.

The initial change produced by the radiation may or may not be extensive. When special photo-sensitive sense organs are concerned, it is probably very slight. It will appear later as a structural or functional change in the organism only when the affected protoplasm becomes a critical limiting factor in the particular structure or function involved. Subsequent manifestations of the change will depend, therefore, largely upon the methods used in examining the radiated protoplasm. Pronounced changes may be observed in the tissues en masse. The microscope will reveal changes invisible to the unaided eye. Studies on permeability will disclose changes unrevealed by the microscope. None of the changes observed are the initial changes ("latent images;" as it were) produced by the radiation, but are after-effects developed by metabolic changes taking place in the cell, and they will vary in nature as the particular metabolic processes involved vary. Nevertheless certain processes, especially those involving the segregation of phases and components of the protoplasm (for example, the mitotic changes which occur during indirect cell division), may be more easily disturbed than others, not because substances of a more photo-sensitive nature are involved but because either the mechanism concerned is more intricate or a successful issue demands nicer coördinations and adjustments.

The separation of the initial change from the first visible effect was very clear in some experiments by the writer on the chemical action of ultra-violet light on egg albumen. Egg albumen exposed to ultraviolet light at $0^{\circ} \mathrm{C}$. showed no visible effects of the radiation, but when subsequently warmed to room temperature, it coagulated. Here was a photochemical change which I could "develop" to visibility by merely changing the temperature. I could change the length of the "latent period" at will. In some of the experiments I deferred the coagulation more than a month after the exposure.

In more recent experiments, I have found it possible by a slight increase in temperature to "develop" the initial change produced in the living cell by radiation. I have found that radiated protoplasm is sensitized to heat. For example, with a given intensity of radiation by fluorite rays, three per cent. of the radiated Paramecia cytolized when the temperature, during and after the radiation, was maintained at $16^{\circ} \mathrm{C} .-17^{\circ} \mathrm{C}$., while sixty-six per cent. cytolized if the temperature after radiation was raised only for sixty seconds to $24^{\circ} \mathrm{C} .-25^{\circ} \mathrm{C}$, , a temperature not far trom the optimum for normal, non-radiated 
Paramecia. Non-radiated Paramecia do not cytolize even when maintained at a temperature of $32^{\circ} \mathrm{C}$. for nearly two days. The similarity of these results to those of the coagulation of albumen is obvious. They are similar also to Nybergh's, on the influence of temperature on phototropism in seedlings, a fact which indicates that the action of light on the special sense organs may be similar in character to the abiotic action involved in these experiments.

We may, therefore, liken radiation to the injection of substances into cells by a method which does not necessitate their passing through the cell's limiting membranes, thus transcending the micro-pipette as used by Kite and others; the injected substances are, of course, the photo-products of the protoplasm. The greater the amount of photochange, or the more the products differ from the protoplasm from which they were formed (i.e. the more "foreign" they are), the greater will be the effect produced. Thus the biological action of radiation may increase with increasing exposure, although not necessarily by any constant ratio. In order to evaluate the effect produced, we may postulate that these substances react with the constituents of the cell and through the altered sequence of metabolic changes may affect the whole organism. It becomes at once evident that such an interaction depends not only upon the nature of the formed substance but quite as much upon the structure and nature of the protoplasm.

Our knowledge of the physiological effects of radiation is not sufficient to enable us to lay down fundamental principles concerning the hypersensitiveness of different kinds of protoplasm to the influence of rays. We know that some kinds of protoplasm, such, for example, as that composing the so-called "eye spot" of the single-celled organism, Euglena, are extremely sensitive (as compared with other parts of the cell) to the influence of light rays. In this case, the hypersensitive region is provided with a (photodynamic?) pigment which increases the absorption. Whether it is the pigmented protoplasm itself, or the protoplasm closely associated with it, which is hypersensitive, has not as yet been determined.

There is some evidence that certain parts of most cells-the nucleus, for example-are more sensitive to the influence of rays than other parts. Again, cells in a rapidly growing condition appear to be more sensitive to radiation than cells which are at rest. Evidence concerning these matters must be very carefully examined, however, before categorical statements of hypersensitiveness can be made, because our knowledge of the functions of the cell is limited, and what may appear to be specific hypersensitiveness may be nothing more than an expression of the-limitations of our experimental methods. We observe disturb- 
ances of functions only where we know how to look for them. Any apparent hypersensitiveness may be nothing more than a reflection of our experimental methods. The investigator himself is hypersensitive to those physiological disturbances which he has learned to observe.

In all of these effects it appears that the rays, through their chemical action, destroy some part of the protoplasm, and unless there is reparation of some kind, the cell is later unable to function and subsequent physiological processes are interfered with. The nature of the observed interference will later depend upon the particular activities which the cell has to undergo. When, for example, germ cells, egg or sperm are radiated, subsequent embryonic development is abnormal, monstrosities are produced, and if the radiation be long enough the organism dies.

With these notions in mind concerning the nature of the action of rays we will make a very brief survey of the therapeutic effects, remembering that the field of literature is governed rather by the limitations inherent in our experimental methods than by specificity in the biological action of rays.

\section{PRINCIPLES GOVERNING USE OF RADIATION IN THERAPEUTICS}

The use of radiation in therapeutics is based upon the following fundamental principles of the reaction of living tissues to the influence of rays: With small doses of radiation for every tissue there is a period of stimulated activity. With larger doses physiological processes are inhibited, and if the exposure be long enough death ensues. Certain kinds of cells, notably such as are immature or rapidly dividing, are more susceptible to the action of rays than others. After the physician has recognized the nature of the disease, he has, in so far as he understands these principles, a basis for selecting the proper technic of the application of rays.

For example, hair, which is a rapidly growing tissue, may be stimulated in growth, as in the treatment of alopecia by ultra-violet rays, or the exposed region may be entirely depilated, according as the dose is less or greater than a certain amount. X-ray operators, and those obliged to handle tubes of radioactive substances are early annoyed by irregularities in the growth of their nails. Growth and exfoliation of the epidermis are increased and it takes on an appearance of premature senility. Premature senility of rapidly growing tissues often follows exposure to rays. The spleen and other lymphoid tissues exhibit marked changes in function under the influence of X-rays in such small doses that no effect is noticed on the intervening tissues. Sex cells are 
destroyed with a readiness that necessitates extreme precaution on the part of technicians to prevent temporary or even permanent sterility.

Cells of pathological new growths, the regenerating tissues of wounds or injuries, cartilage in the process of ossification, perhaps the growing tissues resulting from bone grafts, and other instances of cells in a pathological or rapidly growing condition, are more susceptible than the normal tissues surrounding these regions. It is for this reason that rather deep-seated growths can be destroyed by radiations while the injury to the skin and other intervening tissues is very slight or nil. On the other hand, when the pathological state has existed for a long time, as in the forms of cancer where the cells are more vigorous, the growth is very difficult to destroy without the sacrifice of the normal tissues about it. $A^{\prime}$ number of persons hold to the theory that the destruction of pathological growths by radiation is due as much to the healthy stimulation of normal tissues and processes as it is to the killing of the diseased cells.

The increasing knowledge of the mechanism of the biological effects of rays has its influence on the methods of treatment. As a general rule, an effort is made to use a dose above that needed to destroy the diseased tissue, and yet not great enough to interfere with the functions of normal tissues (except in certain cases where this is a desirable effect). By using sources' of concentrated radiation it is often possible to expose the pathological tissues to inhibiting doses while the surrounding normal tissues receive only stimulating doses of rays.

The estimation of dose is rather difficult: only a person with long experience in the use of rays can realize the variations in resistance, not only with different types of disease, but with different persons having the same disease in an apparently similar form. For the radiation of deep-seated organs, filters of one kind or another are used to allow the passage of only the more penetrating rays. The filter absorbs the "soft" rays, i.e. those for which the tissues have a high absorption index. The transmitted rays, because of the higher absorption index, are not so rapidly absorbed near the surface of incidence. The amount of destruction of the superficial tissues is therefore lessened. In the more recent methods of treatment, combinations of two or more kinds of rays are often used with excellent results. For instance, in some cases of internal growths, the radium tube is inserted into the organ, while $\mathrm{X}$-rays are administered through the body-wall, thus exposing the tissues to a thorough cross-fire. Each kind of ray is useful in its own sphere, but must be chosen to suit the case.

Perhaps the most striking and widespread use of radiation is in the 
treatment of cancer and other tumors. Such growths exhibit varying degrees of susceptibility to this form of treatment, from the cellular teratomas and lymphosarcomas, which quickly melt away before the radiations, to the most resistant forms of epidermoid carcinoma. In spite of the remarkably gratifying effect produced at times in entirely curing the disease, radiotherapy has not reached such a sure stage in this connection that it is used in preference to operation where this is possible. Many appear to be of the opinion that radiations have their best use in preparing the tissues for operation, in destroying the remaining diseased cells after operation, or in rendering operable some inoperable cases. Where surgical methods are impossible, or inadvisable on account of the advanced stage of the disease, radiation may, at the very least, relieve pain, prevent foul discharge, and render the patient comfortable for a time. Caution must be practiced, however, in exposing to very strong radiations, for at times, after a temporary alleviation, the disease continues with increased vigor, and the body resistance is greatly weakened by the reduction of lymphocytes.

A desire is apparent among therapists to obtain some substance which may penetrate the diseased tissue and either of itself, or as a result of radiation, destroy the pathological cells. A number of preparations are being used to-day for injection into the growth or into the general circulation, or for external application. Colloidal preparations of some metals, salvarsan, thyroid extract, iron, arsenic, iodine, and bismuth paste have all been used with varying degrees of success. The value of these substances is largely due to the secondary radiations induced in them. Likewise, as suggested above, if the power of the blood, lymph, and other tissues to emit secondary radiaticns were thoroughly understood, perhaps much more exact application of this fact might be made than now is possible.

Local tuberculous affections are often an object of radio-treatment. Among these lupus is perhaps the one which has gained the most widespread attention. The actinic ray treatment as worked out by Finsen has been almost specific for this disease. The two theories abroad on the action of light in lupus are the bactericidal effect of the rays and the destruction of pathological tissues by the same. The Finsen treatment is considered by some to be inferior to radium treatment, on account of the more rapid effect of the latter, the ease of application, and the cosmetic results. On the other hand, much larger areas can be covered by ultra-violet rays; the radiant energy is to be had at much lower cost; and, because of the strong absorption of the rays, the treatments are not followed by systemic effects such as the red.ction of the number of lymphocytes, etc. 
During the recent war radiotherapy has found applications of almost sensational interest. Aside from its use in the types of disease met with in civil practice, the treatment of septic wounds and the use of rays in connection with plastic surgery have attracted considerable attention. In place of the disfiguring cheloid scars resulting from the healing of lacerated tissues or extensive surgery, a smooth, pliant, soft scar is made possible by judicious treatment with radium or other forms of radiation. The softening of the cicatricial tissue relieves pressure from nerve-endings, and diminishes or entirely removes pain. The closing of salivary fistulas and the depilation of skin flaps in facial reconstruction are a part of the work of the radiotherapist. The type of rays used throughout is selected with a view to the work to be accomplished.

Aside from these effects on special tissues, it has been observed that there often results from radiation a diminution in the size of glands in other regions of the body, and a noticeable effect on the general health. Patients may gain weight, improve in color, and have a general feeling of well-being. Nerve-pain is sometimes greatly relieved. The opinion is held that the effect of radiation on parts of the body other than those under immediate exposure may be due to the absorption of certain wave-lengths by the blood. Cases of malignant disease seem to respond best to radiations when the red cells and hemoglobin are high in value. For this reason, iron is sometimes given before treatment. It is thought that secondary radiations are produced in the blood, perhaps in the iron content. The increased hemoglobin-carrying power of the red corpuscles under the influence of light has been demonstrated by Graffenberger. Another effect of rays is on the leucocyte count. Leucocytes, in particular the lymphocytes, may be stimulated by small doses, or very readily reduced in number by large doses, probably as a result of the reaction of the spleen and lymphoid tissue. Radium is known, moreover, to increase the coagulating power of blood. On the whole, blood is one of the most readily affected of body tissues, and this fact should be taken into consideration by technicians. Either benefit may be derived by discreet radiation of relatively large areas of the body in order to produce a stimulation of general health, or harm may be done in too strong radiation of old diseased tissues by reducing the resistance of the body through the action on the blood.

The effect of rays on the general condition of health is applied in the chemical arc light bath, as worked out by Hasselbach. The rays produce a marked skin hyperemia, drawing the blood from the internal organs to the periphery, and making the breathing deeper and slower. 
In cases where such a skin hyperemia is desirable, the arc light bath is a worthy treatment.

Radiations are by no means a general therapeutic measure in every disease. There is a class of diseases, notably smallpox and measles, where experience has proved that light rays are injurious. In accordance with this fact, Finsen urged strongly the admittance of red light only, in such a manner that a photographic plate would receive no fogging, to a room containing a smallpox patient. The value of this method seems to lie in the cutting off of the harmful actinic rays. The facts appear to indicate that we are dealing here with photodynamic sensitization. The eruptions in xeroderma pigmentosa appear to have a photodynamic origin.

A's our knowledge of the etiology of physiological functions and diseases increases, and as we learn more concerning the relation between the organism and this imponderable part of its environment, radiations will find a place as therapeutic agents as secure as that now held by chemicals in rational scientific medicine. 


\section{ACIDOSIS AND THE PHYSICOCHEMICAL EQUILIBRIUM OF THE ORGANISM}

\section{By Lawrence J. Henderson}

Among the problems of physiology some seem to lead straight to a plain result whereby the question is definitively closed; but others, apparently not less simple in the beginning, grow larger and more complex as our knowledge increases, until they merge with all the other problems of the physical and chemical regulation of the body in the question of organization, which thus appears as a physicochemical phenomenon.

It is by slow degrees that this fundamental problem of general physiology has arrived at its modern phase. Throughout centuries of effort to solve the riddle of life, and of misguided search for a hidden vital principle or vital force, the scientific description of the organism and of its unique characteristics has slowly evolved. Beginning as a brilliant philosophical intuition born of Aristotle's medical and zoölogical experiences, then a mere tradition obscured by medieval scholasticism and almost forgotten in the struggle which marked the beginnings of modern science, our present understanding of organization was prepared by the labor of naturalists and physicians of the seventeenth and eighteenth centuries. Finally, in the nineteenth century, the rapid growth of experimental methods in the medical sciences completed a task which was beyond the powers of unaided observation and speculation, and to-day the uniqueness of organization among all the phenomena of nature is well established. For although there are certain interesting and, if somewhat trivial, true analogies to organization in the stability and regulation of inorganic systems and their activities, which greatly aid the understanding and show that organization need not necessarily be regarded as exclusively vital, the experience of all the sciences shows that such phenomena, wherever they occur, possess peculiarities which are lacking in other physical and chemical phenomena. This result, however, has not been won without arousing many of the most famous controversies in the history of biology and 
medicine, controversies in which nearly every historical figure of the biological and medical sciences has taken his part.

Yet, in spite of the great historical importance of the question, and the indispensable services of a true understanding of organization in explaining physiological and pathological processes, this subject, once in its many forms an unavoidable topic in all general medical discussions, is now almost forgotten, as if a conspiracy of silence excluded it from the lecture room and the hospital. The dissipation of general thought in a multitude of specialties, alike of laboratory and of clinic, has nearly obscured the question which absorbed so much of the thought of Aristotle and Descartes, of Cuvier and Claude Bernard, and of the clinicians of an earlier day. Nevertheless it is impossible to tunderstand the physicochemical equilibria of the organism without knowledge of the many aspects of organization, and nobody can hope to understand disease who has not studied the fundamental regulatory processes of the body, on the one hand as physicochemical processes, and on the other hand as illlustrations of the phenomenon of organization.

The earliest plain statement of the nature of organization is Aristotle's, which he developed at many points of his biological writings and perhaps best condensed in the following words:

"The animal organism must be conceived after the similitude of a well-governed commonwealth: When order is once established in it there is no more need of a separate monarch to preside over each separate task. The individuals each play their assigned part as it is ordered, and one thing follows another in its accustomed order. So in animals there is the same orderliness-nature taking the place of custom-and each part naturally doing his own work as nature has composed them. There is no need then of a soul in each part, but she resides in a kind of central governing place of the body, and the remaining parts live by continuity of natural structure, and play the parts Nature would have them play" $\left({ }^{1}\right)$.

This important idea has often engaged the attention of later philosophers, including both the Aristotelian schoolmen and the moderns, but in spite of occasional instances of philosophical contributions to biological thought, like the mechanistic views of Descartes; no important progress at this point was made until the nineteenth century. Meanwhile, those who pursued the subject were only too often misled into unfortunate vitalistic theories like those of the animistic school of Montpellier. But at length in the nineteenth century the modern idea of organization began to take form as a spontaneous result of the experiences of the laboratory. Bichat is the first important figure who clearly reveals this tendency. In spite of many unlucky speculations, which 
obscure the sound elements of his thought, he thereby exerted a powerful influence upon his successors.

A greater contemporary, Cuvier, was less philosophical, yet he too could not escape certain aspects of the question. In particular his mind seems to have been fascinated by that comparison of the living body to a vortex which again and again recurs in his works. This idea has been stated by Cuvier in the following language:

"La vie est donc un tourbillon plus ou moins rapide, plus ou moins compliqué, dont la direction est constante, et qui entraine toujours des molécules de mêmes sortes, mais où les molécules individuelles entrent et d'où elles sortent continuellement, de manière que la forme du corps vivant lui est plus essentielle que sa matière.

"Tant que ce mouvement subsiste, le corps oì il s'exerce est vivant; il vit. Lorsque le mouvement s'arrête sans retour, le corps meurt. Après la mort, les éléments qui le composent, livrés aux affinités chimiques ordinaires, ne tardent point à se séparer, d'où résulte plus ou moins promptement la dissolution du corps qui a été vivant. C'était donc par le mouvement vital que la dissolution était arrêtée, et que les éléments du corps étaient momentanément réunis." $\left({ }^{2}\right)$

"Il vient sans cesse des éléments du dehors en dedans; il s'en éch:ppe du dedans au dehors: toutes les parties sont dans un tourbillon continuel, qui est une condition essentielle du phénomène, et que nous ne pouvons suspendre long-temps sans l'arrêter pour jamais * * * Les branches les plus simples de l'histoire naturelle participent déjà à cette complication et à ce mouvement perpetuel, qui rendent si difficile l'application des sciences generales." $\left({ }^{3}\right)$

"Dans les corps vivans, chaque partie a sa composition propre et distincte; aucune de leurs molécules ne reste en place; toutes entrent et sortent successivement: la vie est un tourbillon continuel, dont la direction, toute compliquée qu'elle est, demeure constante, ainsi que l'espèce des molécules qui y sont entrainées, mais non les molécules individuelles elles-mêmes. . . Ainsi la forme de ces corps leur est plus essentielle que leur matière." $\left({ }^{4}\right)$

This conception of life, very different from that which might be expected from the master of comparative anatomy, is indispensable. In the first place it emphasizes the fact that the organism exists in a state of dynamic rather than static equilibrium, and secondly it directly suggests the peculiar character of the regulatory processes by which, both in health and in disease, life is preserved.

In Cuvier's day, however, the application of physics and chemistry 
was too rudimentary to permit the physiologists widely to apply this conception as a guide in research, and Cuvier's most highly developed ideas on organization are morphological. The study of metabolism, however, soon illustrated both the truth and the usefulness of Cuvier's fundamental idea. Here indeed the conception of equilibrium cannot be avoided. At an carly period in the history of the science it was discovered that a normal organism is in a state of nitrogen equilibrium. That is to say, the composition, so far as total nitrogen is concerned and presumably also in respect of compounds of nitrogen, is steadily preserved, through the regulation of a long chain of intricate chemical processes. Day by day the ingestion of nitrogen is approximately equal to the excretion. A modification of the diet may cause a temporary disturbance of the condition, but this is soon restored. The phenomena of growth and disease are found to involve more enduring changes. Hereupon, by the same process of reasoning which has long been employed in physical science, growth is declared to involve nothing more than other phenomena superimposed upon the underlying conditions, thereby modifying the observed facts in such manner that the fundamental state is partly obscured. And disease is after all, in its very essence, a disturbance of organization; in short, diseases of metabolism involve by definition disturbances of equilibria, which may or may not be compensated.

Further research reveals similar equilibria concerning carbon, sulphur, phosphorus, and the other elements. The results are extended to definite chemical compounds such as water, salt, sodium bicarbonate, glucose, and the like. It is perceived that the equilibria of temperature, of volume, of osmotic pressure, of alkalinity, which involve physicochemical states, are truly analogous phenomena.

Meanwhile it has always been clear that within certain limits the existence of these equilibria is essential to the preservation of life itself, and that they might have been taken for granted. The real question has been to define the normal and pathological fluctuations, their duration, their limits, and their relations to other phenomena. In short, so far as these problems are concerned, the study of metabolism has consisted in an attempt to describe as thoroughly as may be, and if possible to explain, the fluctuations of the approximately constant physical and chemical conditions of the body. In other words, the task of the investigator has been to make known the facts concerning the regulation of the ultimate physical and chemical constitution of the organism. In this undertaking he has always kept in mind the idea that the organism exists in a state of dynamic equilibrium, just as it was long ago conceived by Cuvier, and more vaguely by Hume and by Lucretius. 
The result of such developments in the study of metabolism has lately been discussed by von Wendt $\left(^{5}\right)$. This author clearly perceives that it is necessary to study metabolism as a whole in order to reveal its true nature as an example of organization. When protein metabolism, carbohydrate metabolism, or any other special aspect of the subject is studied by itself, many of the most important phenomena, and especially the regulation of physicochemical equilibria, are overlooked.

But when the process of metabolism as a whole is studied from the most general point of view, it becomes evident that the blood, in spite of its approximately constant volume and physicochemical properties, is continuously undergoing changes, chiefly as a result of three processes;- first, by absorption through the gastrointestinal tract and the lungs, secondly by losses to the cells and tissues, and thirdly by accessions, chiefly of catabolic products, from the cells and tissues. Such changes in the blood involve a great variety of regulatory processes, for certain substances may be stored up, while others must be excreted, either directly, or after chemical transformation. Further, there are certain substances which are harmful in themselves and which must, therefore, be so dealt with as to render them harmless. Finally, there are certain physicochemical changes in the blood which attend almost any modification of its composition and which appear. to call for very rapid readjustment. Such, in particular, are the changes of osmotic pressure and of reaction. These last changes are usually partly dealt with by exchanges between blood and cells, and then finally restored by the excretory process.

Thus it seems to be true that all such regulatory processes are so adjusted as to secure for the body the greatest possible capacity to undergo modifications of its physical and chemical composition with a minimum change in the fundamental physicochemical equilibria such as those of volume, temperature, osmotic pressure, alkalinity, and the like. In other words, the processes by which the composition of the blood is regulated are of such a nature that wide fluctuations of certain factors, which in the very nature of the case are bound to vary widely, such as the amount of water or salt absorbed from the intestine, or the amount of acid produced in the metabolism, shall produce as small a variation as possible in certain especially important characteristics of the blood, such as osmotic pressure and alkalinity. In the case of alkalinity this depends upon a peculiarity which is now generally known as buffer action. But it should be understood that buffer action in the widest sense is a characteristic of all such organic regulatory processes, and, of course, it may depend upon either physical or chemical mechanisms. The phenomena involved in these processes may be distinguished 
as regulatory metabolism in contrast to consumption or catabolism. There can be no doubt that this analysis of the problem, although in some respects a restatement of earlier views, is useful and substantially correct.

The idea of regulation, so familiar in the investigations of the temperature of the body, and in many other general problems of metabolism, is, moreover, the very conception to which all the other independent investigations of organization as a physiological problem also lead. Thus W. Roux has long since declared, and recently reasserted the belief, that the capacity of autonomous regulation is quite the most important of all the peculiarities of life. For example, he thinks that this is what makes possible the direct adaptation to the environment, or, in other words, the acquiring of characteristics. In like manner the action of hormones, the integrating function of the nervous system, and the phenomena of emotional excitement investigated by Cannon are all regulatory.

In the light of such scientific developments it is now possible to see that Herbert Spencer's half-forgotten conception of life as "the continuous adjustment of internal relations to external relations," thcugh doubtless far from satisfactory as a characterization of life itself, is really a true statement of the phenomena of organization. Vague though it may be, it is confirmed by the results of experimental morphology, of physiology, and of the science of metabolism, and I suspect that pathology affords some of the most striking justifications for such a view. Indeed pathology has its prerogatives, and of these not the least is to follow up the disturbances which, step by step, result from a single lesion or deranged activity until they close a vicious circle, to note the compensatory changes, regenerations, and repairs that oppose this process, and thus to perceive the organism as a whole acting so as to preserve that state of dynamic equilibrium which is essential to life itself.

And yet such a conception of organization is insufficient. With Cuvier, but far more clearly and in greater detail, we can now recognize the organism as a vortex in a state of dynamic equilibrium. Its stability through buffer action can sometimes be quantitatively described, and thereby the nature of the regulatory process made clear. But how much more complex than any other vortex is this living system, and how perfectly integrated are its activities and regulatory processes! Not only its size and its form, but its chemical composition and all its physicochemical processes are stable. And they are not only stable, they are also so integrated that the entire mechanism can function as a whole.

This is the aspect of the organism which has lately been emphasized 
by J. S. Haldane $\left({ }^{6}\right)$, whose point of view may be illustrated by the following considerations which will be later elaborated. A quantity of hydrochloric acid is introduced into the stomach, with the following effects. It is absorbed into the blood and reacts with basic substances; with sodium bicarbonate to form sodium chloride and free carbonic acid,. with alkali protein compounds so as to split off alkali and set the protein free. These processes take place both in the plasma and in the corpuscles. They produce changes in osmotic pressure which lead to clianges in the volume of the corpuscles. There is also a modification in the distribution of electrolytes between corpuscles and plasma. And, of course, there is an increase in the hydrogen ion concentration of the blood. This is followed by stimulation of the respiratory center, by an increase of the ventilation of the lungs, by an increase in the output of the heart, by a fall in the concentration of total and free carbonic acid in the blood, and by a diminution in the alveolar carbon dioxide tension. There is also an increase in the renal excretion of ammonia and of phosphates, an increase in the hydrogen ion concentration and titratable acidity of the urine and a change in the equilibrium between oxygen and hemoglobin. Meanwhile the colloidal systems of the organism have been modified by the interaction of the protein compounds and in other ways, so that, although it is not yet possible to pursue the question much farther, it seems clear that many structures, as for instance the muscles, suffer changes, but of course in such a manner as to produce a minimum of alteration in their functional activity. At length the excretory processes restore the original condition, but not until the acid-base equilibrium has interacted with the circulation, the nervous system, the respiration, the renal activity, and probably all the other functions of the body. Similar changes might have been produced by direct modification of the activity of the lung or of the kidney, and doubtless in many other ways. Indeed, such a cycle of changes may be initiated or modified by interference at any point. Of course, some kinds of interference involve very little experimental difficulty, while in other cases the practical difficulties may be insurmountable.

Thus it may be seen that there is no beginning and no end to the regulatory processes, and, indeed, that strictly speaking there do not exist. regulatory processes at all, but rather a single regulatory process, infinite in its ramifications and identical with the total activity of the organism. In such a system it is almost a hopeless task to distinguish cause and effect, for anything may be either cause or effect. So we come back to Aristotle's original conception from which, it is safe to say, science will never again escape. Moreover, there are many other lines of research in biology which also lead to this conclusion, such as 
the whole Darwinian movement, and the investigations of such men as K. E. von Baer and Claude Bernard.

More recently Pavlov's researches on the glands of digestion, the study of internal secretions and hormones, Sherrington's investigation of the integrative action of the nervous system, Cannon's study of the emotions, and many other independent lines of investigation have further cleared the ground; and at the present moment the physicochemical treatment of the problem of organization is widely, if somewhat vaguely, recognized as the ultimate goal of physiological research. An interesting statement of the present condition of physiology in this respect may be found in Haldane's little book, "Mechanism, Life, and Personality." Many physiologists doubt, however, if all the philosophical conclusions that Haldane draws can be regarded as well founded. In any case it is unprofitable to pursue this subject further, for a true understanding of this conception of the organism can arise only from familiarity with the facts.

Nevertheless one last consideration remains to be examined, the theory of the internal environment, or milieu interieur, as it was called by its author, Claude Bernard. It seems almost unaccountable that this idea should have exerted so little influence upon physiology, for Claude Bernard made every effort of which a man of genius was capable to establish it as the foundation of general physiology. And though his efforts to found this science were premature, because physical and chemical assistance were inadequate, there can be no doubt that his idea and his estimate of its importance were correct.

Year by year, in his lectures at the Collège de France and at the Sorbonne, Claude Bernard set forth this conception and grouped his new discoveries about it. For him it was plainly one of the guiding principles, if not the principal clue, in physiological research. In his opinion two environments must be distinguished, the external or cosmic environment, and the internal or organic environment. In lower organisms there is, so to speak, no distinct organic environment, and for that very reason organic activities are restricted. But, as we ascend in the scale, organization becomes more complex and histological structures more sensitive. Such structures can no longer persist in the external environment and, as a result, the internal environment is formed. Thus the blood is to be regarded as a physically and chemically stable fluid, in which the cells live "just as fishes live in water," and it must be clearly distinguished from the intracellular fluids which have a very different composition.

In the highest organisms this internal environment tends to become more and more stable and more and more independent of fluctuations in the external environment. For example, it acquires a constant tem- 
perature and peculiar nutritive constituents. In such organisms, therefore, the cells of the body are still more highly protected from extermal influences.

The internal environment is the product of the organism. Its composition is specially under the control of the organs of nutrition and excretion, which regulate its composition according to the needs of the cells of the body and keep it constant.

Such, in brief, is Claude Bernard's conception of the internal environment. Possibly somewhat defective through his unfamiliarity with the stability of the external environment of the simplest forms of life, it corresponds in all other respects with the science of to-day, which has brilliantly justified Bernard's prophetic vision. Obviously this view is not very different from that of von Wendt, developed a half-century later, with the help of all the new knowledge. Indeed, I think Claude Bernard's statement is the clearer and the more useful. Its great importance for the study of general physiology is, in his opinion, because this science is concerned with the definition of the fundamental conditions of the general phenomena of life, which are common to all organisms and to all living cells, and because in the blood and lymph of the highest organisms just those physicochemical conditions which are most important for the integrity of these fundamental phenomena are regulated. Indeed, as we now know, they may be preserved at the expense of other parts of the body, and regulated with an accuracy that could not possibly have been foreseen.

II

The recent developments of physiology, while undoubtedly enlarging our view of the facts, seem to establish Claude Bernard's doctrine of the necessity of constant physicochemical conditions in the blood. The volume of blood, the temperature, the viscosity, the osmotic pressure, and the alkalinity must all be kept constant, in order that physiological processes may run smoothly and not decline and cease. It is, however, only in the case of the alkalinity of the blood that we can, at present, not merely recognize this constancy but also clearly analyze the mechanism upon which, in large measure, it depends, and then, by pushing further our inquiries, at least provisionally describe the whole regulatory process in its manifold relations with many other physiological processes.

From its very beginning, Arrhenius's theory of ionization emphasized the peculiar importance of the ions of hydrogen and hydroxyl. As products of the electrolytic dissociation of water, these ions must be present in all aqueous liquids. As products of the dissociations of acids in the one case and of bases in the other, they must be essential factors, 
or at least the only constant factors, of acidity and alkalinity in aqueous solutions.

Methods for the estimation of the concentration of these ions were presently found, and before long successfully, if rather roughly, applied to physiological problems. Thus it was proved that the reaction of blood is nearly neutral and very constant.

Meanwhile the theory was extended, with the help of the mass law, until it became a quantitative theory of acidity, neutrality, and alkalinity. The principal results of this development of the subject, so far as they concern the biologists, are as follows:

Ionic equilibria of the types which are mainly involved in the acidbase equilibrium of the body have been found to obey the mass law with great accuracy. Accordingly, the two reactions of ionization,

$$
\begin{aligned}
\mathrm{H}_{2} \mathrm{O} & =\stackrel{+}{\mathrm{H}}+\overline{\mathrm{OH}} \\
\mathrm{H}_{2} \mathrm{CO}_{3} & =\stackrel{+}{\mathrm{H}}+\mathrm{H}_{\overline{\mathrm{CO}}}
\end{aligned}
$$

lead to conditions of equilibrium which are defined by the algebraic equations

$$
\begin{gathered}
\left.k_{1} \times\left[\mathrm{H}_{2} \mathrm{O}\right]=\stackrel{+}{\mathrm{H}}\right] \times[\overrightarrow{+} \mathrm{OH}] \\
k_{2} \times\left[\mathrm{H}_{2} \mathrm{CO}_{3}\right]=[\stackrel{+}{\mathrm{H}}] \times\left[\mathrm{HCO}_{3}\right] .
\end{gathered}
$$

Put into words, these equations merely declare that the concentration * of water molecules, $\left[\mathrm{H}_{2} \mathrm{O}\right]$, is always proportional to the product of the concentrations of hydrogen and hydroxyl ions and that there is always a similar proportionality between the concentration of carbonic acid molecules on the one hand and the product of the concentrations of hydrogen and $\mathrm{HCO}_{3}$ or bicarbonate ions on the other. These relationships hold simultaneously and under all circumstances, even when other acid or basic substances are present.

In the case of the dissociation of water, however, a simplification is possible, because the concentration of molecules of water is always very large and may be taken as constant. Accordingly, since the product of two constants is a third constant we may write the equation

$$
k_{\mathrm{H}_{2} \mathrm{O}}=[\stackrel{+}{\mathrm{H}}] \times[\overrightarrow{\mathrm{OH}}] \text {. }
$$

Therefore, the concentrations of the two ions vary inversely

$$
\stackrel{+}{\mathrm{H}}]=\frac{k_{\mathrm{H}_{2} \mathrm{O}}}{[\overline{\mathrm{OH}}]}
$$

* The chemical symbol of a substance enclosed in brackets stands for the concentration of the substance. 
This so-called water constant, $k_{\mathrm{H} 2 \mathrm{O}}$, is in fact only a true constant at constant temperature. It has, at a temperature slightly above $20^{\circ}$ Centigrade, the value

$$
10^{-14}=0.000,000,000,000,01 \text {. }
$$

Hence, when the concentrations of hydrogen and hydroxyl ions are equal, each has a concentration equal to the square root of the above number, i.e.

$$
10^{-7} \mathrm{~N}=0.000,000, \mathrm{I} \text { Normal. }
$$

In other words, the concentration of hydrogen ions in pure water is $0.000,000,1 \mathrm{~g}$ per liter, and of hydroxyl ions $0.000,001,7 \mathrm{~g}$ per liter. Upon this basis the definitions of neutrality, acidity, and alkalinity are as follows:

In every neutral solution

In every acid solution

$$
[\stackrel{+}{\mathrm{H}}]=10^{-7} \mathrm{~N}=[\overrightarrow{\mathrm{OH}}] \text {. }
$$

$$
[\stackrel{+}{\mathrm{H}}]>10^{-7} \mathrm{~N}>[\overline{\mathrm{OH}}] \text {. }
$$

In every alkaline solution

$$
[\stackrel{+}{\mathrm{H}}]<\mathrm{IO}^{-7} \mathrm{~N}<[\overrightarrow{\mathrm{OH}}] .
$$

It must be clearly understood that these relationships, like all those above mentioned, hold under all circumstances in aqueous solutions, and that they are, not sensibly modified by the presence of any known substances. It is also well to observe, in order to avoid misunderstandings, that there are several different ways of expressing concentrations. In the present paper the concentration of molecular or ionic species is designated by writing the symbol for the molecule or ion in brackets. Above, the magnitude of the concentration has been expressed in the usual chemical units, i.e. moles per liter. Thus, one gram of hydrogen, seventeen grams of hydroxyl, forty-four grams of carbon dioxide, or sixty-two grams of carbonic acid $\left[\mathrm{H}_{2} \mathrm{CO}_{3}\right]$ per liter constitute normal solutions, or in other words, have a concentration equal to unity.

When concentrations become very low, like the concentration of hydrogen and hydroxyl ions in the blood, it becomes somewhat inconvenient to express these concentrations as decimal fractions. To avoid this difficulty two methods are commonly adopted. The first consists in expressing the concentration as a multiple of a power of ten arbitrarily chosen so that the multiple shall be a small number. The second method, introduced by Sörensen, consists in expressing the concentration by means of the negative of the logarithm of that number (symbol $\mathrm{pH}$ ) which represents its concentration in ordinary terms, i.e. in moles per liter. 
The following table gives corresponding values of hydrogen and hydroxyl ion concentrations expressed in these three different manners.

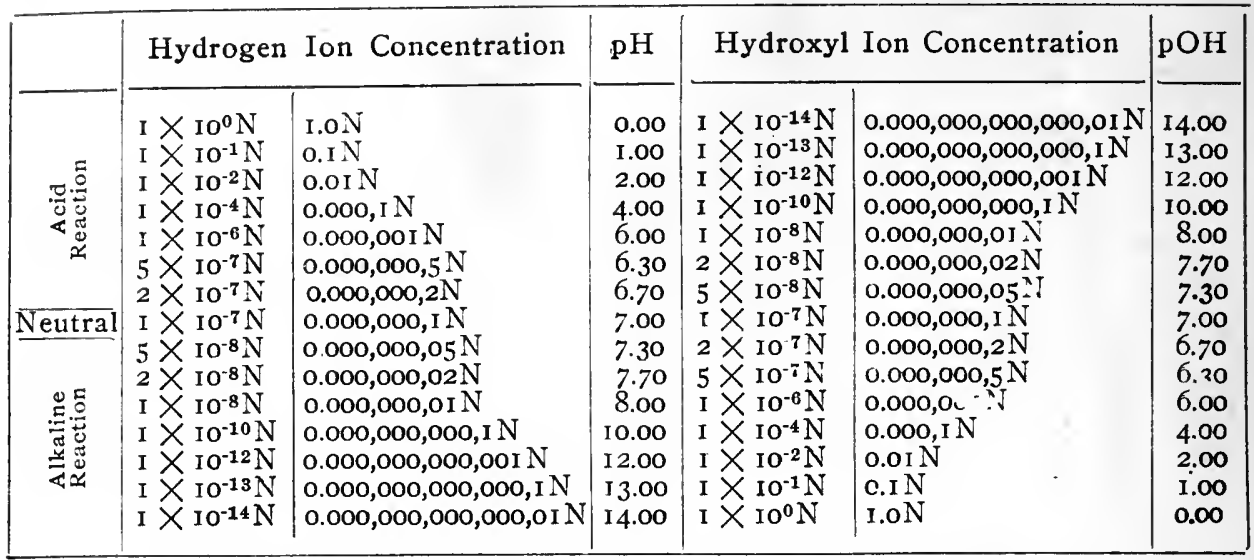

It should be noted that (for temperature slightly above $20^{\circ} \mathrm{C}$.) in every case

and

$$
[\stackrel{+}{\mathrm{H}}] \times[\overline{\mathrm{OH}}]=10^{-14}=0.000,000,000,000,0 \mathrm{I}
$$

$$
\mathrm{pH}+\mathrm{pOH}=\mathrm{I} 4.00 \text {. }
$$

In accordance with the preceding discussion it is easy to see that acids produce an acid reaction when dissolved in water because, through their own ionization, as in the case of carbonic acid, they give rise to hydrogen ions in excess of the small number present in pure water. Thereupon the hydroxyl ion concentration, which is determined by the equation

$$
[\stackrel{+}{\mathrm{H}}]=\frac{k_{\mathrm{H}_{2} \mathrm{O}}}{[\overrightarrow{\mathrm{OH}}]}
$$

suffers a proportional decrease. The case of the solution of a base is precisely analogous. Here the ionization of the base yields hydroxyl ions, and as the concentration of these varies, the concentration of the hydrogen ions varies inversely according to the requirements of the equation.

When, however, both an acid and a base are present in solution the condition is more complicated. This case is, however, also more important, since it is only in the artificially prepared solutions of the laboratory that we find acids, bases, or salts alone in solution. In both the inorganic and the organic solutions of nature acids and bases are present together, but not in chemically equivalent concentrations. 
Let us consider a solution containing both carbonic acid and sodium bicarbonate. Here we may at once write the following chemical reactions:

$$
\begin{gathered}
\mathrm{H}_{2} \mathrm{CO}_{3}=\stackrel{+}{\mathrm{H}}+\overline{\mathrm{HCO}}_{3} \\
\mathrm{NaHCO}_{3}=\stackrel{+}{\mathrm{Na}}+\overline{\mathrm{HCO}}_{3} \\
\mathrm{H}_{2} \mathrm{O}=\stackrel{+}{\mathrm{H}}+\overline{\mathrm{OH}} \\
\overline{\mathrm{CO}}_{3}=\stackrel{+}{\mathrm{H}}+\overline{\mathrm{CO}}_{3} \\
\overline{\mathrm{H} O}_{3}+\mathrm{H}_{2} \mathrm{O}=\mathrm{H}_{2} \mathrm{CO}_{3}+\overline{\mathrm{OH}} .
\end{gathered}
$$

Evidently the acidity or alkalinity of such a solution depends upon several factors, including the relative concentrations of acid and alkali and the strength of carbonic acid. But, since the application of the mass law to such reactions is valid, regardless of the presence of other substances, the problem may be readily solved $\left({ }^{7}\right)$.

The equation expressing the ionization equilibrium of carbonic acid, written above, is as follows:

or

$$
k \times\left[\mathrm{H}_{2} \mathrm{CO}_{8}\right]=[\stackrel{+}{\mathrm{H}}] \times\left[\mathrm{H}_{\overline{\mathrm{C}}}\right]
$$

$$
\stackrel{+}{\mathrm{H}}]=k \times \frac{\left[\mathrm{H}_{2} \mathrm{CO}_{3}\right]}{\left[\overline{\mathrm{H}}_{3}\right]} .
$$

In this case, since bicarbonate is present, there is very little dissociation of the acid and, accordingly, the concentration of its undissociated molecules may be taken as approximately equal to the total quantity of free acid. And, since salts are always largely dissociated, the concentration of the bicarbonate ions, $\left[\mathrm{HCO}_{3}\right]$, is not far from proportional to the total concentration of bicarbonate. Therefore if we represent this factor of proportionality by $\alpha$ we may write the approximate relationship $[\stackrel{+}{\mathrm{H}}]=\frac{k}{\alpha} \times \frac{\mathrm{H}_{2} \mathrm{CO}_{3}}{\mathrm{NaH}_{\mathrm{COO}}}$, where $\mathrm{H}_{2} \mathrm{CO}_{3}$ and $\mathrm{NaHCO}_{3}$ designate total free carbonic acid and total bicarbonate respectively.* Values of $\alpha$ will in all cases range between 1.0 and 0.5 , so that this factor is of liftle moment in view of the enormous fluctuations to which hydrogen and hydroxl ion concentrations are liable. And for many purposes the degree of dissociation of the salt may be neglected, thus leading to the simple relationship that the hydrogen ion concentration is proportional to the ratio of free acid to salt.

\footnotetext{
* Henceforth in this paper $\mathrm{NaHCO}$, will stand for the sum of all bicarbonates, including those of sodium, potassium, calcium, etc.
} 
Especially in many physiological problems has it been found both sufficiently accurate and convenient to regard the ratio of total free carbonic acid to total bicarbonate concentrations as proportional to, and the measure of, the hydrogen ion concentration. This relation, however, holds only when the ratio of acid to salt is neither very large nor very small.

It is therefore evident that in the solution of any weak acid, when the quantities of free and combined acid are equal; the value of $[\stackrel{+}{\mathrm{H}}]$ is $\frac{k}{c}$; if the ratio of acid to salt be $10: 1,[\stackrel{+}{\mathrm{H}}]$ is $\frac{10 k}{\alpha}$, if the ratio be I : IO, $[\stackrel{+}{\mathrm{H}}]$ is $\frac{0 . \mathrm{r} k}{\alpha}$. This is the total outcome of the theoretical analysis so far as it is necessary for a general understanding of the biological problem.

We may now turn again to the special case of carbonic acid. For this substance the value of $k$, expressed in our present units, is roughly $4.4 \times 10^{-\tau}$. Accordingly, in a dilute solution of carbonic acid and bicarbonate where $\alpha$ falls between 0.8 and 0.9 , and where $\frac{k}{\alpha}$ is approximately $6 \times 10^{-7}$, if the ratio of acid to salt be ro the concentration of hydrogen ions must be $60 \times 10^{-7} \mathrm{~N}$, if the ratio be 1.0 the concentration will be $6 \times 10^{-7} \mathrm{~N}$, and if the ratio be o.I the concentration will be $0.6 \times 10^{\top} \mathrm{N}$.

Thus we can see why carbonate solutions are almost always nearly neutral (e.g. IOO.10 ${ }^{-7} \mathrm{~N}>[\stackrel{+}{\mathrm{H}}]>0.01 \cdot 10^{-7} \mathrm{~N}$ ), and, taking account of the universal distribution of free and combined carbonic acid in the ocean, in lakes and streams, and in all organisms, we understand the primary cause of the approximate neutrality of nearly all natural solutions, both organic and inorganic, upon the earth. In blood the concentration of hydrogen ions is about $0.35 \times 10^{-7} \mathrm{~N}$., the degree of dissociation of bicarbonate about o.6. Substituting these values in the equation

we find the relationship

$$
[\stackrel{+}{\mathrm{H}}]=\frac{k}{\sigma} \times \frac{\mathrm{H}_{2} \mathrm{CO}_{3}}{\mathrm{NaHCO}_{3}}
$$

whence

$$
0.35 \times 10^{-7}=\frac{4.4 \times 10^{-7}}{0.6} \times \frac{\mathrm{H}_{2} \mathrm{CO}_{3}}{\mathrm{NaHCO}_{2}},
$$

$$
\frac{\mathrm{H}_{2} \mathrm{CO}_{3}}{\mathrm{NaHCO}_{3}}=\frac{\mathbf{I}}{2 \mathrm{I}}
$$


or in round numbers, since all these measurements are still subject to experimental revision,

$$
\frac{\mathrm{H}_{2} \mathrm{CO}_{8}}{\mathrm{NaHCO}_{8}}=\frac{\mathrm{I}}{20} \text {. }
$$

It is impossible to understand the efficiency with which neutrality is preserved in solutions containing carbonic acid without the discussion of an actual case. We may, therefore, consider a solution containing one kilogram of carbon dioxide dissolved in one hundred liters of water. Let sodium hydroxide be added to this solution. At the beginning of the experiment the concentration of hydrogen ions will be approximately $0.000, \mathrm{I} \mathrm{N}$., almost exactly one thousand times the concentration in neutral solution, and, of course, the concentration of hydroxyl ions must be $0.000,000,000$, I N., one one-thousandth the concentration in neutral solution. If the sodium hydrate be added in successive portions the change of reaction will be roughly according to the numbers in the following table:-

\begin{tabular}{|c|c|c|c|c|c|}
\hline $\begin{array}{l}\text { Added } \\
\text { NaOH } \\
\text { grams: }\end{array}$ & {$[\stackrel{+}{\mathrm{H}}]$} & {$[\overline{\mathrm{H}}]$} & $\begin{array}{l}\text { Added } \\
\text { NaOH } \\
\text { grams. }\end{array}$ & {$[\stackrel{+}{\mathrm{H}}]$} & {$[\mathrm{OH}]$} \\
\hline 0 & $1000 \times 10^{-7} \mathrm{~N}$ & $0.001 \times 10^{-7} \mathrm{~N}$ & 450 & $5.2 \times 10^{-7} \mathrm{~N}$ & $0.19 \times 10^{-7} \mathrm{~N}$ \\
\hline 50 & $91 \times 10^{-7} \mathrm{~N}$ & $0.011 \times 10^{-7} \mathrm{~N}$ & 500 & $4.4 \times 10^{-7} \mathrm{~N}$ & $0.23 \times 10^{-7} \mathrm{~N}$ \\
\hline 100 & $42 \times 10^{-7} \mathrm{~N}$ & $0.024 \times 10^{-7} \mathrm{~N}$ & 550 & $10^{-7} \mathrm{~N}$ & $0.29 \times 10^{-7} \mathrm{~N}$ \\
\hline 150 & $26 \times 10^{-7} \hat{N}$ & $0.038 \times 10^{-7} \mathrm{~N}$ & 600 & $2.6 \times 10^{-7} \mathrm{~N}$ & $0.39 \times 10^{-7} \mathrm{~N}$ \\
\hline 200 & $19 \times 10^{-7} \mathrm{~N}$ & $0.053 \times 10^{-7} \mathrm{~N}$ & $650^{\circ}$ & $2.1 \times 10^{-7} \mathrm{~N}$ & $0.48 \times 10^{-7} \mathrm{~N}$ \\
\hline 250 & $14 \times 10^{-7} \mathrm{~N}$ & $0.072 \times 10^{-7} \mathrm{~N}$ & 700 & $1.5 \times 10^{-7} \mathrm{~N}$ & $0.67 \times 10^{-7} \mathrm{~N}$ \\
\hline 300 & $10 \times 10^{-7} \mathrm{~N}$ & $0.10 \times 10^{-7} \mathrm{~N}$ & 750 & $1.0 \times 10^{-7} \mathrm{~N}$ & $1.0 \times 10^{-7} \mathrm{~N}$ \\
\hline 350 & $8.7 \times 10^{-7} \mathrm{~N}$ & $0.12 \times 10^{-7} \mathrm{~N}$ & 800 & $0.7 \times 10^{-7} \mathrm{~N}$ & $1.4 \times 10^{-7} \mathrm{~N}$ \\
\hline 400 & $7.0 \times 10^{-7} \mathrm{~N}$ & $0.14 \times 10^{-7} \mathrm{~N}$ & 850 & $0.35 \times 10^{-7} \mathrm{~N}$ & $2.9 \times 10^{-7} \mathrm{~N}$ \\
\hline
\end{tabular}

An examination of the table will show that the first 50 grams of alkali reduce the hydrogen ion concentration to less than one hundred times its value in neutral solution. From this point it falls more and more slowly until when 300 grams of alkali have been added the hydrogen ion concentration becomes only ten times and the hydroxyl ion concentration only one-tenth the values at neutrality. This degree of acidity may be obtained by dissolving 0.004 grams of hydrochloric acid in 100 liters of water, although the quantity of free carbonic acid remaining is one hundred thousand times as great as this. Thereafter neither acidity nor alkalinity rises even to this low intensity. Yet in pure water 0.005 grams of sodium hydroxide would produce a more alkaline reaction.

This consideration applies to the homogeneous equilibrium-the case of an isolated solution which is not in contact with the atmosphere- 
but when an atmosphere containing carbon dioxide is also present, the conditions become still more remarkable. Imagine, for instance, a solution containing one kilogram of sodium bicarbonate dissolved in one hundred liters of water and brought into a condition of equilibrium with an atmosphere containing one gram of carbon dioxide per liter. Let hydrochloric acid be added in successive small portions to this solution; let the solution be constantly stirred and shaken, and let the experiment be conducted slowly so that there shall always be equilibrium between the carbonic acid dissolved in the solution and that present as a gas in the atmosphere. Assuming the approximate values of 1.0 for the absorption coefficient of carbon dioxide and $\mathrm{I} \times \mathrm{IO}^{-14}$ for the water constant, the successive states of the solution will be approximately those recorded in the following table:-

Added
$\mathrm{HCl}$
grams
0
$\mathbf{1 0}$
50
100
150
200
250
300
310
318
320
330

$$
\begin{gathered}
\frac{\mathrm{H}_{2} \mathrm{CO}_{3}}{\mathrm{NaHCI}_{3}} \\
0.190 \\
0.197 \\
0.227 \\
0.277 \\
0.360 \\
0.516 \\
0.874 \\
3.34 \\
7.4 \\
8
\end{gathered}
$$

\section{[}

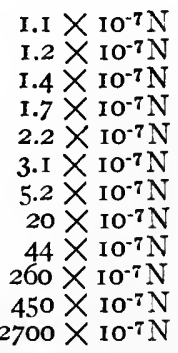

$[\overline{\mathrm{OH}}]$

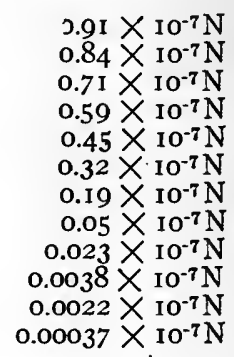

From the beginning of the experiment the acid reaction steadily increases, but at first with extraordinary slowness. Not until I 50 grams of hydrochloric acid have been added is the hydrogen ion concentration increased from one to two ten-millionths of a gram per liter. Thereafter the acidity rises somewhat more rapidly, but only after all the sodium bicarbonate has been exhausted does the acceleration become marked. Such a system manifests a stability of hydrogen ion concentration, or in other words a regulation of its reaction, which until a few years ago was scarcely known to the chemists at all. Such a close approach to neutrality can be attained with water only after elaborate and very difficult purification, yet when carbonic acid and sodium bicarbonate are both present it is automatically established. Of course the case here considered is an extreme one. In nature the concentrations of dissolved substances are less and the mixing sometimes less efficient. In the organism there is also a larger excess of bicarbonate, in accordance with the facts already considered. 
The cause of the greater constancy of the reaction in the case of the heterogeneous equilibrium is very simple. At the beginning of the experiment the free carbonic acid of the solution is exactly in equilibrium with that of the atmosphere. Therefore, when hydrochloric acid is poured in and reacts with sodium bicarbonate to form sodium chloride and to set free carbonic acid, according to the reaction

$$
\mathrm{NaHCO}_{3}+\mathrm{HCl}=\mathrm{NaCl}+\mathrm{H}_{2} \mathrm{O}+\mathrm{CO}_{2},
$$

every bit of the carbonic acid escapes into the atmosphere, and the total quantity of free acid left in the solution is just what it was before. Meanwhile, however, a certain quantity of sodium bicarbonate has been replaced by its chemical equivalent of sodium chloride. The latter substance, however, is perfectly neutral and has no effect which modifies the reaction of the solution. Thus the addition of the strong acid diminishes the quantity of weakly alkaline sodium bicarbonate, but does not increase the quantity of free acid present in the solution. Not until the bicarbonate is entirely decomposed, after 318 grams of hydrochloric acid have been added, does the hydrochloric acid begin to exert its own action as an acid, and then two grams cause an increase of acidity far greater than that produced by the first 300 grams.

From a consideration of the phenomena which have just been reviewed it may readily be seen that when the value of $k$ for an acid is nearly $\mathrm{I} \times 1 \mathrm{IO}^{-7}$ solutions containing that acid and its salts will be commonly neutral. But when the value of $k$ differs greatly from $\mathrm{I} \times 10^{-7}$ such solutions will be ordinarily acid or alkaline. This is due to the fact that when the ratio of acid to salt varies between ro: I and I : IO, the concentrations of hydrogen ions vary from $\mathrm{ro} k$ to o.I $k$.

Beside carbonic acid, there is but one biologically common acid substance, phosphoric acid after one hydrogen has been neutralized by base as in acid sodium phosphate, that possesses a value of $k\left(2 \times 10^{-7}\right)$ nearly equal to $i \times 10^{-7}$. Most weak acids have a value hundreds or thousands of times greater. Phosphate solutions are therefore commonly nearly neutral, and they share with carbonate solutions the function of preserving the constant alkalinity of the body.

It is easy roughly to demonstrate the general character of such acidbase equilibria with the help of the phosphates. Thus, for example, a solution of acid sodium phosphate has a faintly acid reaction, a solution of ordinary sodium phosphate an alkaline reaction, but almost any mixture of the two salts is neutral to ordinary indicators, and will take up strong acids or alkalies in large quantities without apparently changing its reaction. Of course every drop of acid or of alkali does change the reaction, but the change is so slight that it can not be detected by ordi- 
nary means. This depends upon the fact that strong acids and bases combine quantitatively with the alkaline or acid phosphate:

$$
\begin{gathered}
\mathrm{Na}_{2} \mathrm{HPO}_{4}+\mathrm{HCl}=\mathrm{NaCl}+\mathrm{NaH}_{2} \mathrm{PO}_{4} \\
\mathrm{NaH}_{2} \mathrm{PO}_{4}+\mathrm{NaOH}=\mathrm{Na}_{2} \mathrm{HPO}_{4}+\mathrm{H}_{2} \mathrm{O} .
\end{gathered}
$$

Accordingly there is only a change in the ratio between the concentrations of the two phosphate salts, and of hydrogen ion concentration in due proportion, according to the analysis already given.

If the solution is supposed to contain bicarbonates, as well as phosphates, the above experiment fully illustrates the general character of the process by which acids are immediately neutralized in the body. In blood plasma, however, the phosphates are of small importance. The proteins are also involved, but their share in the process is slight, though not physiologically insignificant. They owe their importance mainly to their character as amphoteric substances. In other words, they contain both basic and acid radicals which greatly weaken each other. Hence the ionization constants of the proteins are ordinarily very much less than $10^{-7}$. For this reason the proteins undergo little change in the quantities of acid and alkali combined with them in the neighborhood of the neutral point, that is to say, in the ranges of reaction which may occur in the body. It is only because of the very large quantities of protein present in the organism that they need to be considered at all. The case of hemoglobin is, however, an important exception.

In spite of the great difficulty of accurately determining the conditions in systems which contain proteins, there seems to be no doubt that the relation between proteins and acids or bases corresponds very accurately to the case of a simple acid-base equilibrium where a number of different acids and bases are simultaneously present. But the changes in such systems also involve colloidal changes, and although these are much less than has been assumed in some speculations which have failed to take account of the extreme constancy of the hydrogen ion concentration in blood, they nevertheless extend the sphere of action of acids in the physiological processes. Such phenomena, for instance, explain the effect of increased carbonic acid tension and also of acidosis to diminish the oxygen capacity of the blood, through a simple chemical reaction followed by a change in the distribution of electrolytes between corpuscles and plasma. In general it may be said that colloidal factors influence the progress of the change more than the condition of equilibrium.

III

The application of physical chemistry to physiology has encountered serious and highly characteristic difficulties, for the well-defined physico- 
chemical processes are those which take place in homogeneous systems containing small numbers of simple components. But we seek in vain for such simple systems isolated within the body. Instead we find in every case heterogeneous systems of indeterminate composition, which doubtless involve nearly all the complications that are possible in the presence of solids, liquids, and gases. In particular the difficulties which depend upon the presence of colloids and of more or less semipermeable membranes are always involved. Indeed many of the principles upon which the true description of such systems depends still remain unknown, or at least very uncertain.

In such colloidal systems the relationships between the different parts are often so complicated that we are unable to understand them. For example, the kidney is able to produce a concentrated solution of urea from a dilute solution, in a way that we cannot even imagine. An undiscovered machinery is here at work. As a result of such complications efforts to analyze the heterogeneous equilibrium and processes of the organism have led to very moderate achievements. When, however, a physiologist turns his attention to the true aqueous solutions of the body which are mingled with the colloidal substances the situation is very different. For these solutions, in spite of their complexity, in spite of the presence of colloids, and in spite of their contact with selectively semi-permeable membranes, must still obey the general laws of solutions. Thus the investigations of molecular concentration, of electrical conductivity, and of alkalinity within the organism have from the first led to important conclusions. These results, moreover, fully justify the study of the physicochemical characteristics of the liquids of the organism according to the methods that have been employed in the study of simpler systems.

The physicochemical point of view in the study of the alkalinity of the blood developed slowly. Many early investigations in the middle of the nineteenth century had clearly proved that the organism can survive the production or the introduction into the body of very large quantities of acid. A second step was made by Walter $\left({ }^{8}\right)$, who showed, with the help of earlier observations of Boussingault, that the carnivorous animals produce ammonia in large quantities and excrete it in union with acid when they suffer from acid intoxication.

Further indications of the resistance of the body to acid may be found in the neutralization of sulphuric acid and phosphoric acid produced in the metabolism, in the neutralization by the diabetic organism of large quantities of hydroxybutyric and acetoacetic acids, and in the facts concerning the physiology of lactic acid. A little later it was widely recognized that the blood possesses a great capacity to neutralize 
both acids and bases. Thus Friedenthal found that between forty and seventy times as much sodium hydroxide has to be added to blood sertim as to water in order to produce a pink color with phenolphthalein, and in order to obtain an acid reaction with methyl orange three hundred times as much hydrochloric acid must be added to blood serum as to water.

While the physiological importance of these facts was evident, their explanation was somewhat less so. Gradually, however, it came to be understood that some of these phenomena at least depended upon the properties of phosphates, carbonates, or proteins. In particular it seemed possible to explain the great diminution in the total quantity of carbonic acid in the blood after poisoning with acid as the result of a reaction between salts of carbonic acid and the ingested acid. Accordingly Walter (loc. cit.) introduced the practice of measuring the alkalinity of the blood by its carbonic acid content. This method has served a useful purpose $\left({ }^{9}\right)\left({ }^{10}\right)$ in the study of acid intoxication and, as Van Slyke has pointed out, fell into oblivion only on account of the difficulties of chemical manipulation which it involves.

Meanwhile steady advances in the knowledge of metabolism in diabetes, and of the rôle of ammonia in neutralizing hydroxybutyric acid and acetoacetic acid in this condition, turned the attention of physiologists as well as physicians toward the problems of metabolism and away from the physicochemical phenomena.

Still later, however, actual measurements of the hydrogen ion concentration of the blood and theoretical researches upon the acid-base equilibrium of the blood finally led to the interpretation of the underlying physicochemical phenomena.

The outstanding fact, as explained in the preceding pages, is the direct proportionality between hydrogen ion concentration and the ratio of total free carbonic acid to total bicarbonates (whether sodium, potassium, calcium, or other salts) as expressed by the equation

$$
\stackrel{+}{\mathrm{H}}]=\frac{k}{\alpha} \times \frac{\mathrm{H}_{2} \mathrm{CO}_{3}}{\mathrm{NaHCO}_{3}} .
$$

Such, briefly, are the principal stages in the development of our knowledge of the physical and chemical processes underlying the regulation of the reaction of the blood.*

Upon this physicochemical basis the physiological processes are erected. It is as a means of restoring bicarbonate and alkaline phos-

* I have elsewhere developed this subject more extensively. Cf. L. J. Henderson: Das Gleichgewicht zwischen Basen und Säuren im tierischen Organismus. Ergebnisse der Physiologie, VIII, 254. 
phate from the products of reaction of these substances with acids, or as a means to neutralize acid, and thus prevent its reaction with bicarbonates and phosphates, that ammonia is produced in the metabolism.

In like manner the acidity of the urine is the result of the reversal in the kidney of the reaction by which acids have been neutralized in the body. In the renal function phosphates almost alone are concerned. Therefore the process may be described as follows: In the blood, as the result of the production of acid, a certain amount of alkaline phosphate has been converted into acid phosphate, so that the ratio of acid phosphate to alkaline phosphate has been slightly increased. (Under normal circumstances this change is probably infinitesimal.) The kidney now removes relatively a still larger amount of acid than of alkaline phosphate, perhaps on account of changes in the blood bicarbonate rather than in the phosphate, and thus restores the ratio of base to acid in the blood. Here the essential factor is the ability of the kidney widely to vary the ratio of acid to alkaline phosphate without large variation of the hydrogen ion concentration of the urine. This very important fact once more depends upon the favorable value of $k$ for acid phosphate.

It is because, in the normal individual, both the production of ammonia and the ratio of acid to alkaline phosphate in the urine are variable within wide limits, and can be made to conform exactly to the varying ingestion and production of acid in the body, that the fundamental physicochemical apparatus can be kept intact and accurately adjusted.

A further factor in the process is the activity of the lung in excreting carbonic acid. This substance is the chief excretory product of the organism. As such it must be eliminated promptly and completely. Moreover, in that it leaves the body not in aqueous solution and as an acid, but almost exclusively in the form of gaseous carbon dioxide, there is no possibility of any variation of the permanent effect produced upon the reaction of the body by the elimination of a definite amount of it. In the final regulation by excretion it is not, therefore, concerned. And yet it has, in the process of excretion, a very important rôle in regulating the reaction of the body. This depends upon the fact that carbonic acid is not only a waste product, but also a normal constituent of the blood, and as such, a principal factor in the physicochemical regulation. Thus, if the ratio of carbonic acid to bicarbonates in a normal individual is $I: 20$, a large production of acid might cause a destruction of a fourth part of all the bicarbonates, producing in its place an equivalent amount of free carbonic acid. This, if nothing else occurred, would reduce the relative amount of bicarbonates from 20 to $I_{5}$, and simultaneously increase the free carbonic acid from $I$ to 6 . 
The ratio would now be $6: 15$, and since the hydrogen ion concentration is proportional to this ratio, this ion would suffer a nearly tenfold increase of concentration. But at this point, or more strictly speaking, continuously during the process, the excretory function intervenes. There is a tendency for the respiratory process to hold the tension of carbonic dioxide in the blood nearly constant. This is the reason why carbonic acid has sometimes been thought the respiratory hormone. Assuming that the exact quantity of carbonic acid set free by the reaction of neutralization were thus eliminated, the ratio would be reduced to $\mathrm{I}: \mathrm{I} 5$, and the hydrogen ion concentration would rise but one-fourth above its original value. More recent investigations, however, have shown that a tendency to acidity is accompanied by a lowering of the tension of carbon dioxide. Let us suppose that in this case the tension was lowered one-fourth. The free carbonic acid of the blood would then become 0.75 instead of 1.00 , and the ratio of acid to salt $0.75: 15$, which is exactly equal to $I: 20$, the original ratio. Accordingly, the hydrogen ion concentration would be restored exactly to its original value, and the regulation by excretion would be quite perfect. Now there is abundant evidence to show that something very much like this is always occurring in the body, and on the whole, I believe that the most delicate of all means to regulate the reaction of the body is to be found in this variation of the tension of carbonic acid during its excretion. Such considerations have strengthened the hypothesis that the hydrogen ion is the true respiratory hormone. Originally suggested as a guess, this theory has been supported by many investigations. But I think that it marks the opening rather than the closing of a chapter in physiology, for the subject is involved in many complexities.

The whole physiological equilibrium may now be concisely summed up. The hydrogen ion concentration of the body has been seen to depend upon the ratio

$$
\frac{\mathrm{H}_{2} \mathrm{CO}_{3}}{\mathrm{NaHCO}_{3}} \text {. }
$$

Acid reacting with this system causes a diminution of the denominator and an increase in the numerator of the fraction, the value of the fraction increases, and with it the hydrogen ion concentration. Hereupon the lung reduces the value of the numerator by diminishing the concentration of carbon dioxide in blood and alveolar air, the value of the fraction is restored more or less exactly to its original value and with it the concentration of the hydrogen ion. But the denominator is still below normal. To offset this, there occurs, on the one hand, a production of ammonia which takes the place in the urine of alkali existing as salt in the blood. This alkali recombines with carbonic acid, forming 
bicarbonate, and thus increasing the denominator. On the other hand, the kidney removes less alkali in combination with phosphates than exists in this state in the blood. This alkali, too, helps to regenerate sodium bicarbonate, and thus to increase the denominator. Both of these processes are so regulated that the denominator is restored to normal. The concentration of carbonic acid responds through the activity of the respiratory mechanism, and the organism returns to its normal state.

These processes, of course, go on simultaneously and not in succession. They are, moreover, far less simple than such an analysis admits, for on the one hand the interaction of phosphates and proteins has not been fully described, and on the other hand, many of these variations influence other conditions and processes in the organism.

Among these effects are the influence of carbonic acid concentration and of the hydrogen ion on the affinity of hemoglobin for oxygen and on the volume of the red corpuscles. More general is a necessary, but at present indeterminate, effect on the distribution of electrolytes in the body, on the osmotic pressure, on the state of colloids, and on the volume. I fully believe that such effects are real and that when acid is produced through long periods and in large quantities in particular organs or tissues, as during diabetes, they may well surpass the direct effects of the simple chemical reactions of acid in the pathological complex, and produce a condition very different indeed from that of experimental acidosis. For in such conditions the whole physicochemical composition of the cell, its concentrations and colloidal equilibria, might be sensibly altered.

The most important of these influences which depend upon the intervention of proteins is the action of hemoglobin in the acid-base equilibrium. Here the researches of recent years have finally led to a clear understanding of the more important elements of the phenomena.

The researches of Barcroft and his collaborators have defined the equilibrium between oxygen and hemoglobin in blood with great accuracy, and have proved that this equilibrium is disturbed in a perfectly definite manner by the variation of the tension of carbonic acid. These researches also indicate that this is due to an influence of carbonic acid upon the affinity of hemoglobin for oxygen.

The investigation of Christiansen, Douglas, and Haldane has proved, as was to be expected on theoretical grounds, that the oxygen tension of the blood modifies the capacity of the blood to bind carbonic acid. Further investigations upon these questions have lately been reported by Hasselbalch.

Disregarding the somewhat questionable colloidal elements of the phenomena, it seems at present safe to say that these results prove an 
interaction between two chemical equilibria, the oxygen-hemoglobin equilibrium and the acid-base equilibrium. This can only be brought about by the union of carbonic acid with hemoglobin, or by the exchange of alkali between hemoglobin and carbonic acid. The first possibility cannot be excluded merely on the ground that in blood plasma no more than a very small quantity of carbonic acid is in union with protein. For the protein globin which is united with hematin to form hemoglobin is a basic substance. There is, however, further evidence consisting of measurements of the equilibrium between carbonic acid and purified hemoglobin which were made some years ago by Bohr. At first sight Bohr's data suggest that a considerable reaction between carbon dioxide and hemoglobin must take place in the blood, and that this reaction may be responsible for the absorption of carbonic acid in the tissues and its escape in the lungs. When, however, the theory of the acid-base equilibrium is applied to these phenomena, it becomes clear that quite the opposite conclusion should be drawn. The reaction in Bohr's experiment must, in fact, have been decidedly acid, and the union of the weakly basic hemoglobin with carbonic acid at the same carbonic acid tension, but at a greatly reduced hydrogen ion concentration, must be very much less. This conclusion is also confirmed by the fact that the iso-electric point of hemoglobin is very close to absolute neutrality. This fact in itself is sufficient to show that both the magnitude and the variation of the quantity of base combined with hemoglobin in blood must be greater than the magnitude and the variation of the quantity of acid similarly combined.

The principal facts may, accordingly, be summed up by the following schematic representation of the chemical equilibria :-

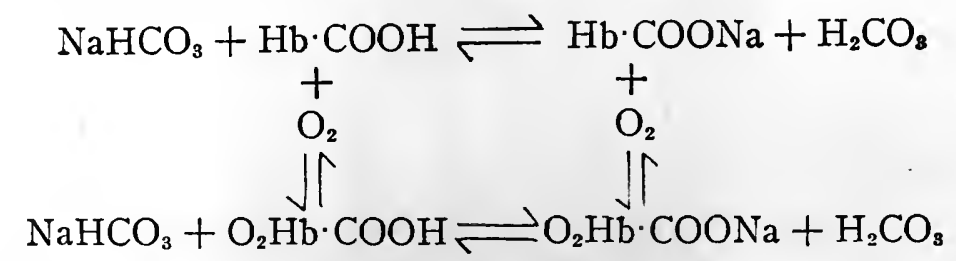

where the action of carbonic acid is to drive the reactions to the left and up, while the action of oxygen drives them down and to the right.

This is the explanation of the mechanism by which increase of carbonic acid tends to drive oxygen out of the blood and increase of oxygen similarly tends to drive out carbonic acid. These are in fact complementary aspects of the same phenomena. Further conclusions may also be drawn. First, it is now plain that the union of oxygen with hemoglobin must increase the acidity of hemoglobin, and that this is the reason why oxyhemoglobin can take away alkali from sodium 
bicarbonate and thus set free carbonic acid. In like manner the reverse reaction depends upon the weakening of the acidity of hemoglobin when oxygen escapes, and as a result the sodium compound of hemoglobin is decomposed by the action of carbonic acid and this leads to the formation of sodium bicarbonate.

Perhaps the strongest evidence for this interpretation of the facts concerning the equilibrium between oxygen and carbonic acid in blood is that it is now possible for the first time to explain how carbonic acid can escape from blood in such large quantities and with such a small change in the tension of carbonic acid.

So far as the acid-base equilibrium is concerned there is a further conclusion which must be pointed out. We have seen that it is when carbonic acid escapes from the blood that oxygen unites with hemoglobin and produces the more acid oxyhemoglobin, and it is when carbonic acid enters the blood that oxyhemoglobin dissociates and produces the less acid reduced hemoglobin. In other words, in the change from venous to arterial blood and in the change from arterial to venous blood, one acid is being substituted for another, and as a result there is a still further stabilization of the hydrogen ion concentration of the blood. 'In other words, when oxyhemoglobin dissociates, the blood is rendered less acid, and this decrease in acidity largely balances the increase in acidity which is due to the entrance of carbonic acid into the blood. Similarly, when oxyhemoglobin is regenerated, the blood is rendered more acid, and thereby the decrease in acidity, which depends upon the escape of car bonic acid, is partly offset.

This mechanism is to be regarded as the means for preventing large variation in hydrogen ion concentration between arterial and venous blcod, just as the control of respiration adjusts the hydrogen ion concentration of the blood as a whole.

IV

In the light of the above considerations, how is acidosis to be defined? Evidently it is a condition which may be brought about by the abnormal production of hydroxybutyric acid or acetoacetic acid. Yet there is no necessary connection between acidosis and this one abnormality of metabolism, for other acids may also produce the condition. Moreover, the difficulty may be due not to the excessive production of acid at all but to an insufficient excretion in nephritis.

Secondly, acidosis does not involve, except in the moribund, a sensible increase in the hydrogen ion concentration of the blood, still less a condition of acidity, or the presence of free acid in the blood. Acidosis is often characterized by high urinary ammonia, especially in the diabetic 
type of the condition, but sometimes in nephritic acidosis the urinary ammonia is low. The total amount of acid, in excess of that combined in the blood with base, excreted in the urine-the net acid excretiona quantity which added to the ammonia of the urine measures the total effect of the kidney in reducing acidity, is also variable. And the hydrogen ion concentration of the urine is a particularly uncertain factor.

More trustworthy is the measurement of the alveolar carbon dioxide. Under many circumstances, if not invariably, the concentration of carbonic acid in the alveolar air sinks below normal in acidosis. Nevertheless it seems to be proved that the proportionality between carbon dioxide tension in the alveolar air and the degree of acidosis is not in every pathological condition to be relied upon. Still less trustworthy, because not yet fully understood, is the diminished oxygen capacity of the blood.

Upon the whole I think that we come nearest to certainty if we say that acidosis must involve a diminution of the bicarbonate of the blood. Regardless of other changes, any increase of acidity or decrease of alkalinity of the body must decrease the total bicarbonate of the blood. This is the immediate chemical reaction, no less certain in the blood than in a test tube. Everything else is from the physicochemical standpoint secondary:- the stimulation of the respiration, the diminution of free carbonic acid in the blood and of the concentration of carbonic acid in the alveolar air, the heightened acidity and acid excretion of the urine, the increased ammonia, and those changes which modify the affinity of hemoglobin for oxygen. Such changes are, moreover, complex, and involve many other physiological activities.

Until the bicarbonate of the blood, the alkaline reserve, has been depleted, such a term as acidosis is meaningless; when this modification of the acid-base equilibrium has been established the whole chain of events involving breathing, oxidation, nitrogen metabolism, urinary secretion, and so on, has been set in motion. In spite of the fact that the blood has not an acid reaction and that there is in the blood no free acid, except the normal free carbonic acid, this is clearly a condition to which the term acidosis may reasonably be applied.

The causes of the condition may vary widely. It may be due to the production of acid, or the ingestion of acid, or to lack of alkali in the food; it may be due to failure to eliminate acid, e.g. acid phosphate, or to failure to produce and eliminate ammonia; but so far as can be seen it must always involve at least a diminution in the concentration of bicarbonate in the blood. As a practical maxim, we are therefore fully justified in saying that acidosis is a state of diminished bicarbonate in 
the blood. Accordingly it may also be said that the most complete proof of the existence of acidosis consists of evidence of diminution in the concentration of bicarbonate in the blood.

In the normal, healthy adult the amount of carbonic acid existing in chemical combination in blood plasma amounts to a concentration ranging from 50 to 65 volumes per cent. $\left(50-65 \mathrm{cc}\right.$. $\mathrm{CO}_{2}$ in $100 \mathrm{cc}$. of plasma). Nearly all of this combined carbonic acid is bicarbonate, and no important error is involved in assuming it to be exclusively so.

As Van Slyke has pointed out, the variation in this quantity is of the same relative magnitude as the variation of the pulse rate. Like the pulse rate or the body temperature the normal values may be overstepped even during health, as for instance during active muscular work. Accordingly a diminution below the normal in the quantity of sodium bicarbonate, like a rise in temperature or a rapid pulse, is not necessarily pathological, though it must be so regarded unless it is a very transitory phenomenon.

A diminution in the concentration of bicarbonate in the blood is, as we have seen, ordinarily accompanied by a proportional diminution in the concentration of free carbonic acid. This condition has been termed by Hasselbalch and Gammeltoft ${ }^{11}$ ) compensated acidosis. Later the activity of the respiratory apparatus may fail to keep the ratio $\frac{\mathrm{H}_{2} \mathrm{CO}_{3}}{\mathrm{NaHCO}_{3}}$ constant; as a result the hydrogen ion concentration rises and the stage of uncompensated acidosis sets in.

It is apparent that a mere estimation of the bicarbonate of the blood will not in itself permit a discrimination between a transitory and therefore pathologically insignificant diminution of the bicarbonate and a permanent and therefore significant one, and that it cannot lead to a discrimination between compensated and uncompensated acidosis with. out additional information.

Since it is the respiratory exchange in the lung which regulates the ratio $\frac{\mathrm{H}_{2} \mathrm{CO}_{3}}{\mathrm{NaHCO}_{3}}$ in the blood, it is only the arterial blood which can be regarded as giving a full indication of the results of the regulatory processes. Obviously venous blood must receive quantities of carbonic acid varying greatly with the activity of the body. As a result it must always be less alkaline than the arterial blood, but the difference is a variable one.

The further difference between the arterial and the venous blood depends upon the effect of carbon dioxide to change the distribution of electrolytes between corpuscles and plasma in such a way that with 
increased carbon dioxide tension there is an increase in the bicarbonate of the plasma. Especially for this reason it is necessary to know from what source blood is taken and what the conditions as regards carbonic acid tension were. It makes little difference, however, provided the individual is and has been for some time at rest, whether arterial or venous blood is employed. In case the more convenient venous blood is selected it is, however, desirable to avoid stasis.

A method, founded upon these considerations, has been worked out by Van Slyke and Cullen which readily permits the estimation of the combined carbonic acid of the blood plasma, and which is believed to provide a direct measure of the alkaline reserve of the blood and of the entire body. In the present state of knowledge there is strong reason to believe that this opinion is well founded. It should be remembered, however, that the relative rather than the absolute values of all measurements are the secure basis of conclusions. The method consists in withdrawing blood from a vein, treating with oxalate, and separating the plasma with the centrifuge. The plasma is then saturated with alveolar air from the lungs of the experimenter, or it may be mixed with air to which carbonic acid has been added so as to bring the concentration to 5.5 per cent. The total carbonic acid of the plasma is then determined with Van Slyke's apparatus designed for this purpose $\left({ }^{12}\right)$. This method permits measurements of sufficient exactness with very small quantities of blood. It is well adapted to clinical work and, certainly so far as relative values are concerned, is quite unexceptionable.

Other methods which do not directly measure the carbonic acid concentration of the blood may be employed as a means of calculating the diminution of the bicarbonate of the blood.: Thus, for instance, the mere titration of blood plasma may be taken as an approximate indication of variation of the bicarbonate, for, as the theory shows, this is the one constituent which is modified in acidosis and which also produces a considerable change in the titrable alkalinity. There can be no doubt that a theoretically sound titration method could readily be devised.

When, as a result of acidosis, the sodium bicarbonate concentration of the blood has been reduced and this change "compensated" by reduction in the carbonic acid tension so that the hydrogen ion concentration is unchanged or only slightly changed from the normal, it is a simple matter to demonstrate the acidosis with the concentration cell, according to a method introduced by Hasselbalch $\left({ }^{13}\right)$. This method consists in saturating the blood with carbon dioxide at a standard carbon dioxide tension. Under these circumstances, since the carbonic acid concentration is constant, the hydrogen ion concentration must vary 
inversely with the concentration of bicarbonates, and accordingly it is only necessary to measure the hydrogen ion concentration with the concentration cell in order to measure the degree of acidosis. The quantity thus determined is called by Hasselbalch the reduced hydrogen ion concentration.

Another means of estimating the degree of acidosis, which is equivalent to the measurement of the combined carbonic acid of the blood, consists in measuring the oxygen capacity of blood under standard carbon dioxide tension. This method; which is due to Barcroft and Peters, depends upon the fact that the oxygen capacity of hemoglobin is influenced by the hydrogen ion concentration in such a way that a certain constant in the equation for the equilibrium varies inversely with the hydrogen ion concentration. Since the latter quantity varies inversely with the blood bicarbonate, the method is equivalent to all those above mentioned. In all these cases, however, it must be remembered that we have no positive assurance that under peculiar circumstances the ordinary simple relationship may not be modified by a disturbance of other physiological processes.

A less direct method of detecting acidosis consists in the measurement of the concentration of carbon dioxide in alveolar air. This method depends upon the fact that the carbon dioxide of the alveolar air is normally in physical equilibrium with the free carbonic acid of the arterial blood. For this reason the alveolar carbon dioxide is proportional to the free carbonic acid and therefore, in compensated acidosis, to the bicarbonate of the blood. Accordingly Haldane's method for the determination of alveolar carbon dioxide may be used as a measurement of acidosis. Here, however, there is always the danger of independent interference with the respiration, and the results are, therefore, more open to criticism than those obtained from direct examination of the blood. There are also difficulties in the proper collection of alveolar air. These conditions are somewhat modified in the Plesch method which depends upon the rebreathing of air until the carbon dioxide tension has approached that of the venous blood. Accordingly the results, though different, are ordinarily parallel with those given by Haldane's method.

Once collected, the alveolar air may readily be analyzed for carbon dioxide by the simple and elegant method of Marriott, which depends upon saturating a standard solution of sodium bicarbonate with the air and measuring the hydrogen ion concentration of the resulting solution with an indicator. Under these circumstances the hydrogen ion concentration is evidently proportional to the concentration of carbonic acid, and therefore to the tension of carbonic acid in the air. In short, 
Marriott has adapted the physiological principle for the purpose of chemical analysis.

In severe diabetic acidosis the estimation of the condition with the help of the alveolar carbonic acid concentration is very useful. In the less severe stages it seems to be not so trustworthy, and decidedly inferior to the evidence which may be obtained from the analysis of the urine.

Because the kidney is the principal means of overcoming the tendency of the organism to become acid, through the production of acid substances, it is evident that the acid excretion in the urine plus the urinary ammonia must bear some relation to the degree of acidosis. According to Fitz and Van Slyke there is, in fact, a simple quantitative relation between the sum of these factors and the alkaline reserve of the body, both in normal men and in diabetics. These factors, however, are liable to variation in disease of the kidney, and like the determination of alveolar carbon dioxide, can hardly be regarded as direct measurements of the condition of acidosis.

A somewhat different test, which is also a method of treatment, has been introduced by Sellards as well as by Palmer and myself. This test consists in measuring the amount of sodium bicarbonate which the body will retain. It involves no experimental skill at all, and appears, at least under all ordinary circumstances, both in normal and in pathological individuals, to lead to trustworthy results. Palmer's extensive investigations upon this subject clearly show that when the bicarbonate of the blood, as a result of the administration of soda, has risen approximately to the highest normal levels, the urine becomes very alkaline. In fact it seems probable that the quantity of soda necessary to bring about the excretion of urine which is more alkaline than blood is a measure of the difference between the actual alkaline reserve of all the fluids of the body at the beginning of the experiment and the highest normal value of this quantity. But, whether or not it may be possible to establish a simple quantitative relationship between the alkali retention and the concentration of blood bicarbonate, it seems plain that this procedure measures the storing up of the alkali in an amount equal to the quantity which has been lost to the body. In the normal individual five or ten grams of sodium bicarbonate are sufficient to produce an alkaline reaction in the urine, and if more than ten grams are retained it seems safe to say that a condition of acidosis has existed.

In so far as it is possible to base treatment upon general scientific considerations, these facts also point to the rational treatment of acidosis. If sodium bicarbonate is administered at freq:ent intervals in quantities just sufficient to make the urine as alkaline as the blood, 
acidosis can not exist. The reaction of the urine can be followed closely enough even with litmus paper, a so-called amphoteric reaction indicating that sufficient alkali has been provided, and if the reaction does not become more alkaline than this there seems to be no danger of injuring the kidney.

Of course this method may be inadequate to cope with the more complex problems of diabetic acidosis, and it is very doubtful if the alkali can always penetrate in sufficient quantities to the seat of acid production. There is, moreover, no reason to suppose that it can influence the cause of the condition. Perhaps it is not too much to say that this is hardly a therapeutic measure at all. For next to water and sodium chloride the concentration of sodium bicarbonate is the greatest in blood, and it seems not unreasonable to care for a sufficient supply of this substance as one does for a supply of water.

There is the more reason for bearing these conclusions in mind because acidosis is one of the commonest of pathological states. Indeed I think that it is probably more common than fever. Therefore one may conclude that in serious illness the test for acidosis should always be made, especially because it is often a very simple matter to repair the defect. And there is some reason to suppose that such action may occasionally be of the greatest importance.

But the use of alkali must always be deliberate and founded upon the urinary reaction, for too much alkali may be very harmful indeed. Employed in large quantities in nephritis, it is a source of grave danger and, if possible, graver suffering to patients who can often expect from the physician little more than some relief from pain. Yet even in nephritis there is at present no reason to avoid the proper use of alkali. In fact, I have never known a kidney to be unable to excrete a small excess of it, and think that we may therefore always undertake the administration of soda according to the rule above laid down, with the conviction that when the quantity of sodium bicarbonate in the body is below normal, no harm is to be expected from the action of sodium bicarbonate. 


\section{BIBLIOGRAPHY}

I. ARISTOTLE: De motu animalium, II, 703a, 30-35, Oxford, 1912.

2. CUVIER: Le Règne animal, I4-I5, Paris, I836.

3. CUVIER: Rapport historique, I I4-1 I5, Paris, 1810.

4. CUVIER: Rapport historique, I51-152, Paris, 1810.

5. VON WENDT: Handbuch der Biochemie, ed. Oppenheimer, IV, Pt. I, 566-574, Jena, I9II.

6. HALDANE, J. S.: Organism and Environment, New Haven, 1917.

7. HENDERSON, L. J.: American Journal of Physiology, 1908, XXI, 427.

8. WALTER, F.: Archiv f. exp. Path. u. Pharm., I877, I, I48.

9. MEYER, H.: Archiv f. exp. Path. u. Pharm., I883, XVII, 304.

Io. MINKOWSKI, O.: Archiv f. exp. Path. u. Pharm., I885, XIX, 209.

II. HASSELBALCH and GAMMELTOFT: Biochemische Zeitschrift, I915, LXVIII, 205.

12. VAN SLYKE and CULLEN : Journal of Biological Chemistry, 1917, XXX, 289; VAN SLYKE, ibid., 1917, XXX, 347.

13. HASSELBALCH: Biochemische Zeitschrift, 1916, LXXIV, 56.

Works bearing upon the subjects discussed in the preceding chapter are so numerous that it is impossible to give more than a list of general discussions of the various topics. Reference to these will at once provide a more complete bibliography.

\section{Organization}

BERNARD, CLAUDE, Introduction à l'Etude de la médecine expérimentale. HALDANE, J. S.: Mechanism, Life, and Personality, London, I9i3.

Organism and Environment, New Haven, I9I7.

HENDERSON, L. J.: The Order of Nature, Cambridge, Mass., 1917.

\section{Acid-base Equilibrium}

HENDERSON, L. J.: American Journal of Physiology, 1908, XXI, 427. Ergebnisse der Physiologie, I909, VIII, 254.

\section{Hydrogen Ion Concentration}

SORENSEN : Ergebnisse der Physiologie, I912, XII, 393.

MICHAELIS: Die Wasserstoffionenkonzentration, Berlin, I9I4.

\section{Respiration}

DOUGLAS, C. GORDON : Ergebnisse der Physiologie, 19I4, XIV, 338. HALDANE, J. S.: Organism and Environment, New Haven, I9I7. HASSELBALCH: Biochemische Zeitschrift, I912, XLVI, 403.

\section{Hemoglobin}

BARCROFT: The Respiratory Function of the Blood, Cambridge, Eng., I9I4. 
Acid E.rcrction

AENDERSON, L. J.: Journal of Biological Chemistry, I9I I, IX, 403.

HENDERSON and PALMER: Journal of Biological Chemistry, 1913, XIII. 393; 1913, XIV, 81 ; 1914, XVII, 305; 1915, XXI, 37.

PALMER and HENDERSON: Archives of Internal Medicine, 1913, X1I, 153; I915, XVI, I09.

FITZ and VAN SLYKE: Journal of Biological Chemistry, 1917, XXX, 389.

\section{Acidosis}

HENDERSON, L. J.: Ergebnisse der Physiologie, I909, VIII, 254.

VAN SLYKE and CULLEN : Journal of Biological Chemistry, I917, XXX, 289. HASSELBALCH and GAMMELTOFT: Biochemische Zeitschrift, 1915. LXVIII, 205.

HASSELBALCH : Biochemische Zeitschrift, I916, LXXIV, 56; 1916, LXXVII, I 12.

HOWLAND and MARRIOTT: Bulletin Johns Hopkins Hospital, 1916, XXVII, 63.

SELLARDS, A. W.: The Principles of Acidosis, Cambridge, Mass., 1918.

WALTER, F.: Archiv für exper. Path. und Pharm., I877, VII, 148. 


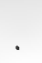




\section{THE}

\section{OXFORD MEDICINE ADVANCE PAGES}

EDITED BY

HENRY A. CHRISTIAN SIR JAMES MACKENZIE

CONTRIBUTORS Volume I

LEWELLYS F. BARKER

FRANK BILLINGS

WILLIAM T. BOVIE

RICHARD C. CABOT

HENRY A. CHRISTIAN

CHAS. BENEDICT DAVENPORT

EUGENE F. DU BOIS

FREDERICK P. GAY

LAWRENCE J. HENDERSON

A. WALTER HEWLETT

GUY HINSDALE

VOLUME I
WALTER B. JAMES

WILLIAM B. JOHNSTON

SIR JAMES MACKENZIE

JOHN J. MACKENZIE

ELMER V. MCCOLLUM

SIR WILLIAM OSLER

FRANCIS W. PEABODY

LEONARD G. ROWNTREE

HENRY SEWALL

DONALD D. VAN SLYKE

WILLIAM H. WELCH

PART 5

NEW YORK

OXFORD UNIVERSITY PRESS

AMERICAN BRANCH : 35 WEST 32 ND STREET 
COPYRIGHT, 1920

BY TAE

OXFORD UNIVERSITY PRESS

American Brance

PRINTED

IN

U. S. A. 
CONTENTS OF PART V, VOLUME I-OXFORD MEDICINE

\section{Chapter}

XIV. The Rationale of Clinical Diagnosis-Lewellys F.

Barker . . . . . . . . . . 505

\section{Chapter}

XV. Tests of Function-Henry A. Christian . . . . $57 \mathrm{I}$

\section{Chapter}

XVI. Respiration in Disease-Francis W. Peabody. . . $58 \mathrm{r}$

\section{Chapter}

XVII. Calorimetric Methods of Study of Disease-Eugene F.

Du Bois 


\title{
CHAPTER XIV
}

\section{THE RATIONALE OF CLINICAL DIAGNOSIS}

\section{(Medical Logic)}

\author{
By LEWELLYS F. BARKER
}

Table of Contents

Development of Clinical Diagnosis.

Relationships of the . Science of Clinical Diagnosis.

to Physics

to Chemistry

to Biology

to Psychology

to Sociology

to Preclinical Medical Sciences

T.ie Pure Science of Clinical Diagnosis.

The Applied Science of Clinical Diagnosis.

The Art of Clinical Diagnosis.

Recognition of the Problem.

Accumulation of Data.

Summarizing and Arranging the Data.

Elaboration by Reasoning of Diagnostic Inferences.

Testing of Inferences and Arriving at Diagnostic Conclusions.

IN making a diagnostic survey, we recognize the existence, or absence, of " disease" in a person through determining the presence, or absence, of certain symptoms or signs, drawing inferences from our findings, reasoning out the implications of these inferences, testing them for their validity, and finally arriving at the concluding belief that "disease " has been identified, or that the person is " healthy." It is, mainly, the logical basis and the technique of the process that will be discussed in the present article.

\section{Development of Clinical Diagnosis}

During the long period in which human beings have lived, enjoyed, and suffered, there has gradually grown up a body of opinions and beliefs regarding health and disease. At all times, animals and human beings have been subject to accidents and diseases that entail suffering 
or disability. A human being who suffers pain or who is conscious of disability desires, and seeks, relief.' Other human beings who see him suffer have their interest and sympathy aroused and desire to help him or to find someone who can help him. Even among the primitive healers, who called upon the gods to cure, or made use of magic arts and enchantments, there must have been a recognition of different kinds of suffering and of the need of a corresponding variety of remedial measures. Among these healers, too, there doubtless early arose, as incentive to discrimination, in addition to the desire to help and the desire for knowledge, the desire to be successful for the sake of livelihood, power, prestige, and other personal rewards. In the effort to satisfy these desires, partly private, partly social, the art of diagnosis had its crude, empirical beginnings. Listening to the complaints of those who suffered, watching their behavior, comparing the observations made on a given case with those made on others earlier in their experience, primitive healers gradually acquired experience and handed down their observations, opinions, beliefs, and customs to their successors (medical tradition).

The striking character of certain illnesses and accidents unquestionably helped to determine the order of development of medical observation and opinion. Wounds, hemorrhages, fractures, and dislocations, large tumors, phlegmons, convulsions, paralyses, chills, fevers, anasarca, deliria, violent pains, blindness, deafness, jaundice, persistent vomiting, madness, melancholy, and other gross manifestations doubtless earliest attracted attention; an acquaintance with these that permitted him to recognize them when he met them constituted the diagnostic knowledge of the ancient practitioner. It is easy, then, to understand why, in earlier times, external medicine should have developed before internal medicine, and why the observation of the natural course of disease should have yielded important facts that bore upon prognosis long before a therapy that could lay any claim to rationality could be applied.

The study of the natural course of disease and of prognosis was doubtless an inspiration to further diagnostic discrimination and resulted in the general growth of diagnostic knowledge. The course and the outcome were so different in different cases of hemorrhage, of fever, and of paralysis, for example, that curiosity must early have been stimulated to seek explanations by attempting an analysis of the different kinds of hemorrhage, of fever, and of paralysis. The primitive descriptions of disease were, perforce, vague and indefinite. The class names that were first used in diagnosis were names of what we now call symptoms and signs. The groupings that the earlier diagnosticians 
made use of were probably the best that could be selected for the purposes of their time; but we can be sure that then, as now, there were only a few who recognized that groupings are conceptual, and that they must be changed when the purposes for which they are wanted change. The rank and file are, at all times, prone to accept classes and classnames as given facts that need no further investigation. The value of a class-name is to assemble (for convenience in making general statements) individuals that have points of resemblance or "common attributes" despite the fact that they also differ from one another; the class-name stands for " unity in spite of difference." When, for example, diagnosticians assembled a group of cases under the class-name "paralysis," they sometimes forgot that the differences between one member of the class and other members of that class might, for certain purposes, be more important than the resemblances. Thus, a gumma pressing on the thoracic portion of the spinal cord causes paralysis; a metastatic carcinoma in the same region causes paralysis. For the purposes of description, the class-name "paralysis" has its value for both instances; for purposes of prognosis and therapy, other class-names (syphilis and cancer) are more important. In other words, in assigning an individual to a class, the value of the assignation depends upon the aim or purpose we have in view. We have gradually to find out the "degrees of generality" that exist, for one class possesses higher generality than another if it includes not only that other but also more. The older logicians recognized these scales of generality, and arranged tables of higher classes (general) and lower classes (special) in order-the so-called "tree" of division or classification (e.g. Porphyry's tree). And advance in diagnosis has resulted from discovering specific differences (differentiae) between members of general classes made for certain purposes, and discovering features that make assignation to other classes that we create for other purposes more important. By examining closely the members of a class for portions that for some clearly-seen purpose differ essentially from one another, diagnostic thought moves ever towards clearer definition. This movement from vagueness towards definiteness is on the one hand the effect, on the other the cause, of each advance in the growth of diagnostic knowledge.

Now, it is this movement of diagnostic thought, impelled by the desire for more effective treatment based upon more accurate and ever more definite knowledge, that accounts for the growth of the medical sciences as a whole and for the subdivision of diagnosis itself into three parts (pure science, applied science, and art). In order that diagnosis may become something more than merely regional and external, a knowledge of the interior of the body and its development, a knowledge of the 
mind and its development, and a knowledge of the environment of the body and mind, including a knowledge of the modes of interpenetration of associated minds, become important. Anatomy, physiology, pathology, and psychology are the sons and daughters of diagnosis, children that, in turn, contribute lavishly to the parental larder. Diagnosis gradually became internal as well as external; organal, histological, cytological, and chemical as well as regional; functional and dynamic as well as structural and static; psychic as well as somatic; social and situational as well as personal; etiological and pathogenetic as well as symptomatic and descriptive. Diagnosis has become partly a pure science based upon data derived from a large number of subsidiary sciences; partly an applied science in which the utilities of the truths of the pure science are perceived and the necessary adjustments for realizing them are made; and partly an art in the exercise of which practitioners employ more or less skillfully the inventions that the applied science affords.

\section{The Position and the Relationships of the Science of Clinical Diágnosis}

The meaning of diagnosis has, during the centuries, become gradually enlarged. The term "diagnosis" came to us through Latin from the Greek dia-, through, or thorough, and gignōskō, recognize. As applied to-day it is, of course, attached to a far more complex subject than could have been anticipated by those who first used the name. Through the development referred to above, a development that is going on in our time more rapidly than ever before, a pure science, an applied science, and an art of diagnosis may be said to have come into existence, for we now possess (I) laws and principles that hold in diagnosis ("pure," or "theoretical," diagnostic science), (2) perceptions of the possibilities of utility and inventions through which the principles are applied ("practical," or "applied," diagnostic science), and (3) skill and experience in employing these inventions in practice (diagnostic art).

The "pure" science of medical diagnosis treats of the phenomena and laws of disease, explains the processes by which pathological phenomena occur, tracing each phenomenon back through a series of antecedent conditions, and inquires into the anatomical, physiological, biological, chemical, physical, psychological, and social causes of diseasestates. This "pure" science of diagnosis, like every other "pure" science, is interested in facts and their regular occurrence. It reasons about the facts and discovers "truth," but it rests upon faith, namely, "faith that causation is universal," faith that "all effects have causes 
and all causes have effects," and faith that "beneficial results will follow the discovery of truth."

The progress of any science is irregular and more or less paroxysmal, but the general methods used for making progress are the same for all the sciences. Progress in the science of diagnosis results from the work of a huge army of clinical and laboratory investigators, each of whom has his individual peculiarities, has had his own special training, and lives and works in his own particular environment. It is not strange, therefore, that even men who are trying to solve the same problem should approach it in different ways, should use different methods, and should attain to somewhat different results. Still the general method employed by all serious scientific research workers is the same. A problem is set; a special technique suited to the particular purposes of the investigator is constructed; observations and experiments are made; the results are recorded. If these results prove to be important, they, and the methods by which they have been obtained, are published. Other workers, noticing the publication, try to verify or to disprove the results, using other similar materials but working by somewhat different methods in a different environment with a background of a different natural endowment and a different past experience. They criticize and are led through their criticisms to make further observations and experiments. All of the earlier results may be disproved or all of them may be verified; more often, some of the earlier results stand the crucial test and come to be admitted as truths by everybody, whereas others of the results fail to stand the test and are rejected. Gradually, the tested and accepted results in connection with special problems in a number of circumscribed fields make a considerable mass and attract the attention of some worker with a synthesizing mind who coördinates the new results in comprehensive papers. Later, the results thus coördinated get into textbooks and the knowledge is made widely accessible; the achievements of the relatively few workers in each circumscribed field can thus be appropriated by the many who are distributed over the whole area of medical research and medical practice. Thus, though advances in diagnosis may have been made by fits and starts, the forward strides have been due to the application of the method of science.

The science of medical diagnosis, or science of the thorough recognition of disease, is, like every other true science, a domain in which phenomena occur in regular order as the effects of natural or efficient causes, such that a knowledge of the causes renders it possible to predict the effects. In the last analysis, the causes are natural "forces"; they obey the Newtonian laws of motion. The word "law " in science implies " uniformity of movement." In the sciences of mechanics, astronomy, 
and physics, the phenomena can be, in large measure, reduced to exact measurement and in them the "theory of units" (mass, space, time) is easily applicable. In the more complex sciences, the phenomena cannot yet be reduced to exact measurement, except to a limited extent. We believe, however, that exact laws do prevail in medical science as well as in all natural domains, though our knowledge of these laws is as yet exceedingly imperfect. It is "faith in the order of the universe," belief that laws are uniform and invariable in the fields of life, mind, and society (as well as in other cosmic fields) that makes the sciences of biology, psychology, sociology, and medicine possible. Social, psychic, and biotic phenomena are exceedingly complex, but study of them by exact methods reveals the existence of uniformities among them. They occur in order. They are subject to law just as rigorously as are the phenomena of chemistry and physics. To discover the laws that healthphenomena and disease-phenomena obey, we must use the method of science, the method that has revealed to the physicist the laws to which heat, light, electricity, and magnetism conform. The diagnostician must know in order that he may accurately predict and successfully control.

A word may be said as to the place of diagnosis and of the other medical sciences in the classifications of all the general and special sciences that have been attempted. The fundamental sciences, as they have been arranged serially (and more or less genetically), are astronomy, physics, chemistry, biology, psychology, and sociology. As complexity increases in the series, the degree to which the phenomena can be exactly determined, what Comte called the "positivity," decreases. Comte made each of these coördinate fundamental sciences stand at the head of a hierarchy of sciences that can be arranged in a logical, or synoptical, order. It is obvious that, if we adopted such a classification, the natural place for the sciences of medicine would, like those of technology, be within the hierarchy of which sociology is the head.* Properly to understand the complex and less exact clinical science of diagnosis, a comprehensive grasp (but not necessarily a mastery of the details) of all the simpler and more exact sciences (physiological, biological, chemical, etc.) below it is necessary. Classifications of the sciences, imperfect as they are, do help us to understand the manifold relations of our own science and give useful pedagogic hints for the most suitable arrangement of curricula.

To understand just what diagnosis is, and what it is not, it is necessary to determine its boundaries, to differentiate it clearly from other

* No classification of the sciences as a whole can be regarded as entirely satisfactory. A very good attempt at a more elaborate classification than the simple one given by Comte is that of Karl Pearson. See his "Grammar of Science," 2d ed., I900. 
sciences, and especially, perhaps, from those to which it is most closely related. "The view or theory of the relations of the subject to other subjects and to the known world in general, as distinguished from the view or theory of it as isolated or in itself," has been given as a definition of the "philosophy" of a subject as distinguished from its "science." Though the distinction drawn between science and philosophy is now less sharp than that formerly drawn, there is something to be gained by considering separately the philosophy of a science in the sense mentioned above, namely, its relationships, and the definite delimitation of its field.

\section{Relations of Didgnosis and Physics}

Of all the natural sciences to which clinical diagnosis is related, none is more fundamental than the science of physics. Under physics are grouped the subjects (excluding chemistry and biology) that treat of the properties of matter and energy and of the action of the different forms of energy on matter. The conceptions of matter and energy, of mass and motion, and of space and time, dealt with by physics, lie at the basis of the scientific analysis of all natural phenomena, including those that we deal with in diagnosis.

The laws of dynamics, which deal with the action of force on bodies whether at rest or in motion, hold for the processes that go on within the human body, which the diagnostician studies. The prospective student of diagnosis, who in his early education gains some acquaintance with theoretical mechanics, not only acquires conceptions that aid him in the understanding of the problems of the pre-clinical and clinical medical sciences but also, through dealing with these ideal representations, secures a training in abstract thought that should be helpful to him in the whole of his subsequent career. The methods and principles of applied mechanics, the subject that deals with the theory of structures and with the theory of machines, are likely, as the medical sciences grow, to be ever more applicable to the solution of problems connected with the structures and mechanisms of the human machine.

That the study of the physics of heat, light, sound, electricity, and magnetism stand in intimate relation to the study of the functions of the human body in both normal and abnormal conditions, goes without saying. The diagnostician could not view intelligently the phenomena of fever and of metabolism if he were unacquainted with the effects produced by heat on material bodies, with the laws of transference of heat and with the laws that govern transformation of heat into other kinds of energy. Thermometry and calorimetry are simple and direct applications of physics to clinical diagnosis. And how unsatisfactory 
would be the work of the general as well as of the ophthalmic diagnostician who had not studied the physics of light, the laws of its rectilinear propagation, of its reflection and of its refraction, and the relations of light to the phenomena of vision and of color perception! In the construction of instruments of precision for diagnostic work, applied optics has made a very great contribution. To recall this fact vividly to mind, I need refer only to the microscope, the polariscope, the photographic camera, the ophthalmoscope, the speculum, the cystoscope, the bronchoscope, the sigmoidoscope, and the refractometer, and the uses to which they have been put in clinical diagnosis. The manifold applications of mirrors and lenses of different sorts in clinical diagnosis nowadays are contributions of optics that command the gratitude of every worker in the field of clinical diagnosis. Almost as important, too, are the applications of the physics of sound to the work of clinical inquiry. The art of auscultation in physical diagnosis can be practiced only inefficiently by one who is ignorant of the physical phenomena that correspond to the loudness, the pitch, and the quality of sounds. Instruments of precision like the stethoscope, the microphone, the phonocardiograph, the tuning fork, the continuous tone series, and the noise apparatus are direct contributions of applied phonetics to clinical diagnosis. It is astonishing, too, how various the applications of electrical science to diagnostic technic have been. In recent years, these have grown very rapidly. The testing of the functions of muscles and nerves by the faradic current and by the galvanic current 'were early' methods of employing electrical science in the service of medical diagnosis. The uses of electricity for the illumination, when instruments of inspection are employed on clinical examination, have been more recently recognized and these modes of applying electricity in medical practice have become ever more important and helpful to the diagnostician and therapeutist. Furthermore, the applications of electricity in roentgenological work are now manifold and by no means simple, as every expert X-ray worker sooner or later learns. A general knowledge of heat, light, sound, electricity, and magnetism is, therefore, obviously essential for good work in medical diagnosis.

One of the most important influences of the study of physics on the science of diagnosis is the understanding that the former subject gives of the different forms of energy, and of the law of conservation of energy during transformation, as they concern the human body. When we recall that stimuli are, in the last analysis, physical agencies, namely forms of energy that excite or depress the several functions of the living body, we realize how important a rôle applied physics must ultimately play in physiology and, accordingly, in diagnosis. The studies of direc- 
tive stimulation in lower forms of life (phototaxis, chemotaxis, thermotaxis, galvanotaxis, etc.) show us clearly certain of the directions that research must take if we are, later on, to understand more clearly than we do now the activities of the cellular constituents of the human body in health and in disease.

As diagnostic work grows gradually more precise, it makes ever greater use of certain standards of measurement that we owe to the physicists. Quantitative work in physics has been greatly facilitated by the selection for each measurable magnitude of a physical unit, or standard of reference; with the aid of these units, other similar quantities can, by comparison, be numerically defined. Fortunately, since physicists have become convinced that one form of physical energy is convertible into another and that the change takes place according to definite laws, it has been possible to coördinate these several physical units. The most fundamental units are those of length, mass, and timethe centimeter, the gram, and the second (hence the name CGS-system of units). All other physical units of measurement take account of these fundamental notions of length, mass, and time. In mechanics the unit of force is the dyne, the unit of work is the erg, the unit of power is the watt, the unit of energy is the joule. In the physics of heat the unit of heat is the calorie. In electricity the unit of resistance is the ohm, the unit of current is the ampère, the unit of electromotive force the volt, etc. These units are coming ever more into use in clinical diagnostic work. Their general adoption in scientific work is a forcible example of the fundamental relation in which physics stands to all the more complex sciences of nature.

The physical sciences, then, including, as they do, dynamics, mechanics, the physics of heat, light, sound, electricity, and magnetism. energetics, and the setting up of units to be used as standards of measurement, are seen to be essential as a part of the basis upon which a science of diagnosis can be built. Medical educators have been wise, therefore, in making knowledge of the theory and some skill in the use of the practical-technical methods of physics a prerequisite to the study of medicine and of diagnosis.

\section{Relations of Diagnosis and Chemistry}

The science of chemistry stands in almost as fundamental a relationship to the science of diagnosis as does the science of phyșics. Chemistry has to do with the study of the composition of substances and of the changes in composition that substances undergo, whereas physics, which we have just considered, studies rather the properties of substances. In physical chemistry, in process of development, we see a new 
science that attempts to correlate the physical properties of stibstances with their chemical composition. Our knowledge of the composition of the substances in the cells and in the fluids of the human body has already become very important for the student of the science of diagnosis. Indeed, it could scarcely be otherwise since the fact of the life of the human body is metabolism. The living substance is, on the one hand, ever undergoing decomposition (dissimilation or catabolism), and, on the other, is ever undergoing reconstruction (assimilation or anabolism). With the exact composition of the most complex types of chemical substances existing in the body-the hypothetical biogens-we are as yet unfamiliar, but of the importance in their composition of the long chains of amino-acids known as proteins we have now become convinced. Physiologists and physiological chemists have already gone far toward demonstrating to us how the foods taken into the body are changed in order that suitable building-stones may be available for the construction of the complex biogen-molecules of protoplasm, and they are also revealing to us the various stages in the degradation processes through which these biogens form secretions and excretions as endproducts of the body metabolism. The fascinating studies that deal with the morphological changes, with the chemical changes, and with the energy changes, that accompany the body metabolism were growing yearly more numerous before the outbreak of the great war; they will doubtless be resumed with even greater vigor now that the war is over and the nations can again settle down to the leisurely and undisturbed cultivation of medical science. The principles and the practical-technical methods of chemistry are now a part of the stock-in-trade of the wellequipped student of diagnosis.

If, then, the modern diagnostician needs to be tolerably familiar with the principles and the methods of chemical science, his preparatory studies should include inorganic chemistry, organic chemistry, analytical chemistry, and physical chemistry. The medical diagnosis of to-day makes extensive use of the principles, of the terminology, and of the machinery, of all these subdivisions of chemistry. The work of diagnosis in the clinical laboratory demands considerable practical acquaintance with the apparatus and the technique of chemical manipulations. In the clinical investigation of metabolism, especially, a knowledge of chemistry and an acquaintance with chemical methods is essential. I need refer only to the chemistry of the proteins and of their derivatives, and its applications to metabolism in the renal diseases and in the aminoacid diatheses; to the chemistry of the carbohydrates and its applications to metabolism in diabetes mellitus and allied disturbances; to the chemistry of the fats and its relations to obesity on the one hand, and 
to acidosis on the other; to the chemistry of the mineral substances in the body and its relations to the metabolism in rickets, in osteomalacia, and in tetany; to the chemistry of the nucleins, purins, and pyrimidins and its relations to the metabolism in gout; and, finally, to the as yet little known chemistry of the vitamins and its relations to the metabolism in beri-beri and in other diseases in which there is believed to be vitamin deficiency. If I were to-day a student in my teens, looking forward to the study of diagnosis and therapy, and could realize at that age as I do now the fundamental importance of physical and chemical science for the future of the biological and medical sciences, I should make a great effort to become firmly grounded in the different branches of physics and chemistry, securing also sufficient training in mathematics to permit of their higher study. Young students of to-day who will avail themselves of this hint before going on to the study of the biological and medical sciences will, I feel sure, be richly rewarded when they become the diagnosticians and therapeutists of twenty years from now. The time and energy expended in the acquisition of sound and thorough physical and chemical experience as a preliminary to medical study could scarcely be better employed.

\section{Relations of Diagnosis and Biology}

The relationship of the science of diagnosis to the science of biology is obvious and yet there should be no confusion or overlapping as regards their respective fields. As abstract sciences, biology deals with the laws of life, diagnosis with the laws of the recognition of health and of disease in living organisms. Each science is important for the other though each differs from the other. From biology we learn that the tendency of evolution is to "transfer the maximum amount of inorganic matter to the organized state " as a part of the general process of storing cosmical energy. The most complex chemical combination known is protoplasm, the "physical basis of life." In biotic organization, the unit is the cell, a very complex structure when compared with the relatively simple constitution of protoplasm. Biology treats of unicellular and multicellular organisms, of their structures and functions, of their origin, growth, and destiny. It reveals the advantages of living beings as organized mechanisms for the storage and expenditure of energy. It studies reactions between organisms and their environment and shows how .capacity for suitable adjustment makes for success and survival, and how inability adequately to adjust leads to failure and extinction. It explains the origin of anatomical structures and the relations of structures to functions. It discovers that at certain stages of biotic organization feeling becomes important for the preservation of life, that 
pleasure and pain are conditional to the existence of plastic organisms, and that, out of sentiency, mind develops. Knowing, feeling, and striving become ever more important factors in the living of higher organisms. Some kinds of structure, some varieties of function, and some modes of feeling, striving, and knowing are advantageous to organisms and make for their survival; others are disadvantageous and lead to disease or death of individuals and of species. Evolution, heredity, variation, adaptation, and selection, as studied by the biologist, are all important as building-stones in the foundation of a science of diagnosis.

The relation of diagnosis to one of the special biological sciences, anthropology, may be considered from two points of view. The student of diagnosis looks upon the science of anthropology as a part of the foundation for the science of diagnosis, whereas the anthropologist will look upon that part of diagnosis that deals with the recognition of health and of disease in man as belonging to the science of man. Undoubtedly, the two sciences overlap and the facts and phenomena of each are important for the other science. Anthropology as a descriptive science is really a branch of zoölogy. A knowledge of the peculiarly human characteristics, which anthropology supplies, is too often disregarded by students of the science of diagnosis who transfer, without criticism, conclusions drawn from observations upon experimental animals directly to the human sphere. Of the different departments of anthropology, it is somatology (dealing with man's physical constitution) and technology (dealing with man's products, material and institutional) that are most important for the student of diagnosis. Among the institutions man has produced are languages, customs, governments, religions, industries, art, and literature. How inadequate would be the work of the student of diagnosis who lacked familiarity with these achievements of man! Both the "natural history of man" and the " history of culture" supply data that are essential for the construction of a science of diagnosis.

The biological sciences deal, then, with living organisms, and patients who consult physicians are living organisms that conform to biological laws. All the special biological sciences, including ( I) Morphology, which deals with the statical aspects of the organic world, or with the structure of living organisms, (2) Physiology, which deals with the dynamical aspects of the same world, or with the properties, processes, and functions of living organisms, (3) Diștribution, which deals with the number of organisms of different kinds in different parts of the world, and (4) Evolution, or Etiology, which deals with the natural history of the cosmos, in as far as it concerns organic beings, can contribute to the science of diagnosis. That training in the principles 
and methods of the biological sciences should, like similar training in the sciences of physics and chemistry, be now regarded as an essential prerequisite to the study of medicine and diagnosis seens, therefore, a reasonable opinion for the medical educator to hold.

\section{Relàtions of Diagnosis and Psychology}

Turning to another subject, it is surprising how little attention has been paid by those who frame pre-medical curricula to the importance of the relations of the science of psychology to the science of diagnosis.

Psychology, as the science of mind, embraces not only the phenomena of "intellect" but also those of the "affections" and those of the "will." Students of human psychology study the knowing, the feeling, and the striving of man. Biology has shown us that feelings of pleasure induce lower animals to look for food and to eat it and to perform the acts that reproduce their kind, whereas feelings of pain lead them to make efforts to escape from enemies and from other dangers. Among higher animals, the knowing element became ever more helpful to the feeling and the striving elements of mind in attaining the purposes of the organism. Man is the most highly favored of all living creatures in this respect and by virtue of his intellect has not only obtained dominion over other forms of life, but has also become the conqueror of the physical forces of nature. Feeling is dynamic; intellect is directive; the will is an activity of purposive behavior that is determined by feeling and intellect.

In the animal series, the intellect seems to have developed, at first, as a means of increasing agreeable feeling, of overcoming obstacles to the satisfaction of desires. The "knowing" element gradually became a most important servant of the "feeling" and "striving" elements of the mind. The human pleasures include the realization of certain objective ends-the nutritive, the reproductive, the esthetic, the emotional, the moral, and the intellectual. The desire for self-realization and to obtain pleasure ("lower" or "higher") is the motive to effort. The intellect is to be looked upon as a directive agent to guide the organism in the achievement of its purposes, that is, in the satisfaction of its desires, and in the fulfilling of all its capacities. It manifests itself in foresight, cunning, shrewdness, sagacity, wisdom, tact, ingenuity, inventiveness, art, science, and philosophy.

The psychologist observes and studies his own consciousness and the behavior of men and of animals, and builds up his science upon the basis of the facts thus accumulated. He desires to understand and to explain his own behavior and that of other men and of animals. Knowledge of consciousness and of behavior will, he believes, yield the power 
to guide and control behavior. He defines behavior as the manner in which an organism possessing mind conducts itself in the active pursuit of its own welfare and in the effort to reach its own ends or to effect its own purposes. By studying himself and observing the phenomena of his own consciousness and his own behavior (as he, himself, thinks and feels and strives), he deepens his understanding of the behavior of all living things and draws conclusions regarding the consciousness he, believes they must possess when they exhibit behavior. By systematic studies of this sort the laws of mind are established.

In analyzing and describing the stream of his own consciousness, the psychologist meets with very great difficulties and the overcoming of these constitutes an important part of his problem. He designates the knowing aspect of mind as "cognitive," the feeling aspect as "affective," and the striving as "conative." He seeks to explain both what goes on in consciousness (and the accompanying behavior) on the basis of the "constitution" or the "structure" of the mind, as it develops during the life of the organism, a development that is determined partly by heredity and partly by environmental influences that favor or prevent the realization of the various hereditary possibilities. $\mathrm{He}$ conceives of the mind as constituted of a large number of "mental dispositions," which form organized systems. The totality of cognitive dispositions he speaks of as the "knowledge" possessed by the mind; the totality of affective and conative dispositions he refers to as the "character" of the individual. Acquaintance with the knowledge and character of a person gives the clue to his conduct in a given situation. Despite the difficulties of analysis and description, psychologists are gradually arriving at conceptions that are helpful both for theory and practice.

Now, the science of diagnosis is very largely dependent upon observation of the behavior of patients and necessitates inquiry into their mental states in their cognitive, affective, and conative aspects. Most physicians, whether or not they have had any academic training in psychology, acquire a certain power of estimating intellectual capacity and of recognizing types of character. For the higher reaches of diagnosis, however, a much fuller acquaintance with the laws of mind and the phenomena of human behavior than can be obtained without special training in psychology is requisite. Diagnostic science and the art of diagnosis are now being rapidly promoted by men who have been thoroughly trained in psychology as a whole or in one or more of its branches. A larger acquaintance with the psychology of the normal human adult, with the psychology of animals, with the psychology of children, with the individual psychology that deals with the peculiarities 
of individual minds, with abnormal psychology, and with the social psychology that studies the folk-mind, the crowd-mind, and the groupmind, and their influences upon individual minds through the processes of suggestion, sympathy, imitation, and interpenetration, will doubtless, . before long, be regarded as an essential part of the equipment of the earnest student of diagnosis.

\section{Relations of Diagnosis and Sociology}

We come next to the kinship of diagnosis and sociology. It is not easy sharply to separate sociology from psychology; social psychology is a link that joins these two sciences. In studying psychology, nowadays, the prospective student of diagnosis learns, as has just been said, of the importance of the crowd-mind or "mass-mind" exhibited by large masses, and of the group-mind that is manifested by smaller associations in every highly organized human society. He studies the principles of collective thinking, collective feeling, and collective acting, and he makes an effort to observe the influence of the social milieu upon the development of the individual mind. But the science of sociology itself is also closely related to the science of diagnosis, and a fairly comprehensive grasp of its methods and principles should be acquired by those who expect to study, and to practice, diagnosis. Diagnosis has to deal with the recognition of "disease" in individuals, but these individuals are members of social groups. To understand an individual thoroughly one must know much about the social groups to which he belongs and their origin by ascent or descent. A knowledge of sociology should therefore be helpful to the student and practitioner of diagnosis.

Sociology, the science of society, studies the structure, functions, and genesis of the social body, just as anatomy, physiology, embryology, and psychology study the individual organism. It discovers what it is in man's nature that induces him to associate himself with others; what the effects of association are upon his interests, his feelings, his emotions, his desires, and his acts; what purposes association and coöperation subserve and what means are adopted for favoring them; what relations become established among men as a result of different kinds of aggregation and coöperation; and what influence these relations exert upon the thought, the feelings, and the behavior of man. Sociologists like J. S. Mill, A. Comte, and Lester Ward have traced the broad outlines of the science and a host of workers are filling in the details. The data of sociology are drawn from a large number of special social sciences (ethnography, ethnology, technology, archeology, demography, history, economics, jurisprudence, politics, and ethics); these data form the basis of the reasoning and the generalizations of the more general 
science. The forces studied by the sociologist are psychic; they consist of human motives, the unsatisfied appetites and desires of men. These forces are preservative, reproductive, esthetic, moral, and intellectual-in other words, the "forces of individual preservation," the " forces of race continuance," and the "forces of race elevation." Feeling is the dynamic agent in society and intellect is the directing agent. Resulting from the collision of social forces, states of approximate equilibrium occur among them, and social structures (including the family, the clan, the tribe, the state, the church, and other voluntary associations) and social institutions (including marriage, customs, language, codes, religions, arts, literatures, and sciences) arise. These structures and institutions, while relatively stable, are constantly undergoing change; though there is at every time a social order, there is at all times some social progress, and this progress is described by the sociologist as partly the result of an unconscious evolution (social genesis), partly the result (and increasingly so now) of the conscious application of the intellect as a guide to human desires in avoiding obstacles to their satisfaction (individual and collective telesis). Knowledge of these social structures and functions and of their evolution, therefore, constitutes the science of sociology. None of the better diagnosticians of our time is likely to underestimate the importance for his own science of a knowledge of society, of social structures, of social institutions, or of social functions. For the physician is constantly called upon, nowadays, to recognize in his patients states in which there is maladjustment of the individual to his environment, states in which the reciprocal relations of the individual and of the social groups to which he belongs are unsatisfactory, states that cannot be properly understood or adequately modified by a therapeutic regimen when the individual is studied alone without concomitant consideration of the group, or groups, of socii to which he belongs. The prospective diagnostician should, therefore, receive sufficient training in the science of sociology and should familiarize himself with the laws of association and with the subtle psychic processes of interpenetration that characterize the activities of concrete groups.

\section{Relations of Diagnosis to the Preclinical Medical Sciences}

Though some acquaintance with the sciences of physics, chemistry, biology, anthropology, psychology, and sociology, including ethics and politics, is, as has been emphasized above, highly desirable as preparatory experience for the student of the science, and for the practitioner of the art, of diagnosis, a still more comprehensive training is necessary in certain distinctively medical sciences, namely, in a group of sciences intermediate between the fundamental sciences above referred to and 
the clinical sciences of diagnosis and therapy. This intermediate group of sciences, training in which is indispensable for the prospective clinician, is usually taught in the first two years of the course in the medical school; these sciences we may include under the general name of preclinical medical sciences. This group includes several subgroups: (I) an anatomical sub-group (gross human anatomy, microscopic anatomy, histology, cytology, and embryology); (2) a physiological sub-group (general and special physiology, physiological chemistry, and pharmacology); and (3) a pathological sub-group (general pathology, special pathological anatomy, and histology, bacteriology, parasitology, immunology, psychopathology, and social pathology). These preclinical sciences were largely developed in the first place by diagnosticians (as postclinical sciences), because their development was necessary for the growth of diagnosis and therapy, but as knowledge has grown and technique has become ever more complex, they have come to be cultivated as sciences for their own sake by men who devote their whole time and energies to the single provinces; and they are now taught, and should be taught (as far as developed); as prerequisite to work in diagnosis. The facts and principles of these preclinical sciences supply data that are necessary as a basis for the science of diagnosis. It is essential that the scientific diagnostician shall himself have had a general training in the methods and principles of each of these preclinical medical sciences and that he shall have acquired such a comprehensive grasp of them as will permit him, first, to keep pace with their progress during his lifetime, and, secondly, to make applications of them in any direction that will be helpful to his own science and art.

It must be emphasized, however; that the problems of the preclinical medical sciences, though closely related to the problems of the science of diagnosis, are by no means identical with them. There is much overlapping, but it is desirable that the purposes of each of the sciences should be kept clearly in view by those who represent it. The anatomist should work at the problems of his science for their own sake, for the sake of discovering facts and truths regarding the form and genesis of the structures in organisms, and especially in the human organism, without special reference to their applicability in the science of diagnosis. It is the business of the investigator in the science of diagnosis to make the application of the facts and truths of the anatomical sciences and their methods to the solution of diagnostic problems. The same is true as regards the physiological sub-group of the preclinical medical sciences. It holds even for the pathological sub-group, which many would look upon as an integral part of the science of diagnosis; but the aims, purposes, and methods of the representatives 
of the special sciences of pathological anatomy, pathological physiology, and bacteriology are, and should be, somewhat different from the aims, purposes, and methods of the representatives of clinical diagnosis. Closely related as the clinical and preclinical medical sciences are, they are still separate and distinct, and much is gained for both groups by the maintenance of this separation and distinction.

Each science will help its kindred sciences most by defining strictly the limits of its province, and cultivating industriously, intensively, and conscientiously its own fields within that province. The clinician must not expect the anatomist, the physiologist, and the pathologist to leave their own special tasks to solve his diagnostic problems for him, nor should the worker in clinical diagnosis be expected by his preclinical colleagues to neglect the crops on his own acreage by yielding to the temptation to till promising neighboring fields. When special needs are felt and lead to a division of work, dormant capacities are aroused and new powers are called into being. The differentiation of purposes and of labor is one of the most powerful influences for increasing the range of intellectual activities and for stimulating their development.

In diagnosis itself, the field is so large that no single person can expect to work equally well in all parts of it. A division of labor in diagnosis, partly methodological and partly regional and systematic, has proved profitable, as in science at large. As evidences of this division we find diagnostic workers distributing themselves more or less (I) according to the methods they employ (applied physics, applied chemistry, applied biology, applied psychology, applied sociology, applied physiology, applied pathology, etc.) and (2) according to the systems and regions they especially study (angiology, neurology, psychiatry, gastroenterology, dermatology, laryngology, ophthalmology, orthopedics, gynecology, etc.). Diagnosis by coöperative groups, with an integrator who, after collection of data and consultations with collaborators, synthesizes the total findings of the group and composes the clinical picture with balanced ordination of its parts, is the highest expression by the diagnostic art of to-day of the unity obtainable despite this differentiation, and of the profit derivable from specialization.

\section{The Pure Science of Clinical Diagnosis}

Though the pure science, the applied science, and the art of diagnosis are most often treated together, it is helpful, for purposes of analysis, to make the division and to understand how the three differ one from another.

By the pure science of diagnosis, we mean the part of the subject that deals with the general laws and principles of diagnosis, that is, 
with the laws and principles that govern the recognition of health and of disease. The laws of diagnosis are generalizations that epitomize in brief formulae uniformities of coexistence and of sequence among the phenomena of health and of disease. The causes of these uniformities are natural forces operating under like conditions. The forces concerned are physical, chemical, biotic, psychic, and social, and the pure science of diagnosis is gradually moving toward the recognition of the workings of these forces as manifested in the phenomena of health and of disease. In the construction of this pure science of diagnosis, the data are derived from all the sciences already referred to as fundamental for diagnosis, as well as from all the special diagnostic sciences. The storehouse of knowledge and of truths discovered in the science of diagnosis is already a very large one. Facts have been classified and are ever being more successfully reclassified as the laws underlying them and the causes of the uniformities are being ever better recognized. Subjective symptoms and physical signs are grouped together as symptom-complexes (or syndromes), and these are uniformities of coexistence and of sequence that betoken underlying causes acting under like conditions; and these causes and these conditions are slowly being determined by the host of investigators who are ever eagerly striving to discover them. Through observation, experimentation, and reflective thinking, knowledge concerning the symptoms of disease, disease-complexes, the sites of disease, the structural and functional alterations in disease and their genesis, the forces concerned, and the conditions under which they act, is being organized as a science of diagnosis with some well-established principles. The diagnosis of disease now includes (I) a recognition of disturbed function in disease (pathological-physiological diagnosis), (2) a recognition of the site and nature of the structural changes in disease (pathological-anatomical diagnosis), (3) a recognition of the causes of disease (etiological diagnosis), and (4) a recognition of the relation of causes to the sequence of conditions in the disease (pathogenetic diagnosis). The data accumulated by workers in all the medical sciences are gradually being summarized, arranged, and classified by workers in the science of diagnosis, so that the laws and principles underlying them are slowly becoming evident.

\section{The Applied Science of Diagnosis}

Pure science, applied science, and art, progress contemporaneously. Each plays into the hands of the other two; there are ever reciprocal contributions to healthy growth.

The applied science of diagnosis has the task of finding out how the laws and principles of the pure science of diagnosis can best be 


\section{4}

\section{THE RATIONALE OF CLINICAL DIAGNOSIS}

applied in the practical work of recognizing health or disease in persons who present themselves for examination. This applied science of diagnosis perceives the utilities of the truths of "pure" science and sets about devising the means of adjustment that are necessary to actualize them. It invents methods, tools, contrivances, and systems of procedure, in other words, the "machinery of diagnosis." It calls to the service of diagnosis, from the other sciences, any fact, truth, principle, or invention that it can make use of, directly or indirectly, as an aid. It becomes familiar with the methods and instruments of mathematics, chemistry, biology, psychology, physics, anatomy, physiology, pathology, bacteriology, and immunology, modifying them where necessary to meet its own needs. It extends the simpler methods of observation of patients by utilizing instruments of precision or special methods that enormously multiply and refine the possibilities of sense-impressions. Thus the sense of sight is extended by photography, and by the use of the microscope, the spectroscope, the ophthalmoscope, the bronchoscope, the cystoscope, the roentgenoscope, and a hundred other devices. The senses of smell and of taste are chemical senses that, unaided, carry us only a short way in collecting chemical data as compared with the fact-accumulation regarding chemical conditions in the blood, secretions, excretions, and effusions made possible by the clinical chemists' adaptations of methods devised by workers in physiological and pathological chemistry. The sense of hearing is extended by the stethoscope, the microphone, and the phono-cardiograph. The temperature-sense is supplemented by the clinical thermometer. The sense of touch and pressure is subtly refined, or replaced, by various ingenious devices such as the sphygmograph, the tonometer, the balance, the dynamometer, and the string galvanometer. The time-honored methods of inspection, palpation, percussion, auscultation, and mensuration have gradually become expanded into an observational and experimental technique that is subtle and complex, but which makes for ever greater objectivity and precision. Thus by devising practical technical methods that can be easily made use of in examining patients, the applied science of diagnosis is ever better able to turn to account the truths and principles that students of the pure science of diagnosis have established.

Workers in the applied science of diagnosis are also making many efforts better to organize the mode of conducting clinical examinations and more logically to arrange the several steps that necessarily must be taken to arrive at satisfactory diagnostic conclusions. That the procedure of collecting the data upon which a diagnosis is based and of making clinical records of the course. of disease-processes has been systematized in the interests of thoroughness, completeness, and accu- 
racy, can easily be seen by comparing the contents of the clinical histories kept to-day (anamnesis, status praesens, catamnesis, epicrisis) with those that have come down to us from the diagnosticians of earlier generations. Moreover, the methods of applying reflective thought to the consideration of the phenomena observed for the purpose of recognizing syndromes, lesions, causes, and prospects are being brought into accord with the general method of science and with the newer logic. The purpose of a diagnostic study decides what methods shall be applied and how. In every case there must first be a recognition of the existence of a diagnostic problem; observations and experiments are then made to locate, and more accurately to define, that problem; the phenomena observed are arranged and brooded over until suggestions of possible explanation, or recognition of meaning, occur to the mind; the implications of each interpretative suggestion are reasoned out; a comparison is made between each suggestion, with all its implications, and the facts, as already collected, or as extended by further observation and experiment; and finally, a decision is reached that there is sufficient reason for the acceptance of one or another of the diagnostic inferences through corroboration, and for the rejection of other suggestions that are proved invalid through failure of corroboration. If no suggestion that has been entertained can be found to be valid, no diagnosis is made; the mind is still kept open and judgment is kept suspended until the process has been gone through with again. An attempt to accumulate more facts has then to be made, the occurrence of further diagnostic suggestions is thus favored, and these in turn are reasoned out as to their bearings and tested for their validity. In this way the best diagnoses of which the examiner is capable, in the existing state of his knowledge, ability, and opportunities, are reached. The representatives of the applied science of diagnosis, through devising new and better methods of examination, through arranging for their more orderly employment, and through conforming to the usages of a sound logic, occupy therefore an important position mediating between that of the pure science of diagnosis and that of the diagnostic art.

\section{The Art of Clinical Diagnosis}

By the exercise of the art of diagnosis is meant the skillful carrying out of the plans and methods of the applied science of diagnosis (based upon the laws and principles of the pure science) in solving actual problems of recognizing health and disease in persons who present themselves for examination.

Expertness in performance and capacity to make and deliver a valuable product characterize the diagnostic artist, whether he is active 
in special domains only or whether he attempts to make a more general diagnostic survey. Many physicians have acquired an extensive knowledge of the classifications of disease, of pathological anatomy and physiology, and of etiology, have become familiar with descriptions of the practical-technical methods in use, and have observed skillful practitioners of the art of diagnosis at work, but have never arrived at expertness and facility, themselves, in the actual performance of diagnostic tasks. There are other physicians who, though they may have attained to real skill in the execution of certain diagnostic procedures, have never become good general diagnosticians owing to lack of a comprehensive grasp of the fundamental laws and principles of diagnosis or owing to insufficient acquaintance with the practical-technical methods of diagnostic work other than a few in which they have acquired accuracy and facility. Mere exactness in outlining an area of dulness by percussion, mere faultless objectivity in description of the sounds audible over the heart and lungs, or mere precision in the conduct of a roentgenoscopic examination of the cardiovascular stripe, in the recording of a sphygmogram, or in the quantitative estimation of blood sugar, valuable though any or all of these procedures may be in collecting data to be used in the reasoning process that precedes the arrival at a legitimate diagnostic conclusion, can be exhibited by men who dare lay no claim to mastery of the general art of diagnosis. As a matter of fact, a laboratory Diener may learn to carry out the technique of the Wassermann reaction, or of the differential count of the white corpuscles of the blood, just as accurately, and perhaps more speedily, than the physician who employs him and who has instructed him, but no one would think of regarding such a laboratory helper as proficient in the general art of medical diagnosis. Owing to the lower dignity of his employment, he must be regarded as an artisan rather than as an artist. An adept in an art of medical diagnosis that is not merely local or special in its aims must have acquired at least some skill in the collection of data in all the domains pertinent to general medical diagnosis and must possess that wide understanding of the truths and principles of diagnostic science and that ability in applying them that will permit him, on reflective thinking about the phenomena observed by himself or by those who are associated with him, to arrive at a diagnostic conclusion or belief that is warranted. Ability to do diagnostic work quickly, accurately, and effectively and capacity to produce diagnostic results that are adequate to the purpose in view are, then, the marks of an operator who is skilled in the art of diagnosis.

The attainment of real skill in the general diagnostic art is no easy matter. It presupposes in addition to good natural endowment, a thor- 
ough general, and special education for the developing artist. At the basis of our present-day conception of the training of medical students lies the recognition of $(I)$ the desirability of a collegiate education preliminary to the study of medicine, (2) the need of a thorough instruction in the preclinical sciences and in the organized body of knowledge that we call the pure science of medical diagnosis, and (3) of the importance of a closely-supervised systematic education in the practicaltechnical methods of accumulating facts pertinent to diagnosis and in the logical way of making use of these facts (by grouping them, by drawing inferences from them, by testing these inferences carefully for their validity and by finally reaching legitimate diagnostic conclusions). The requirements for admission to the better medical schools of our time are such that the students entering the schools have had ample opportunities for becoming habituated to the method of science and for acquiring a good general knowledge of nature and of man as an individual and as a member of social groups. The students have all had instruction in mathematics, physics, chemistry, and biology, and many of them have studied also psychology, logic, and sociology. In addition to a training in the use of their native language, they have acquired a reading knowledge of one or more modern foreign languages, have learned the technique of using libraries, and have discovered the value of consulting sources through bibliographies. The prospective medical students with such a preliminary training can scarcely have avoided becoming acquainted with the general methods and tools of scientific inquiry. They have learned how problems are set and solved. They have been taught the necessity of taking pains in collecting facts by the accurate and detailed observation of phenomena and have come to appreciate the special value of experimentation in which observations are made under rigidly controlled conditions. Under the guidance of good teachers they have begun to acquire the habit of reflective thinking in dealing with their perplexities. They have become unwilling to jump to conclusions and have learned to insist, when confronted with a difficulty, on temporarily suspending judgment and on collecting information that will more rigidly define and locate that difficulty; and when suggestions of possible solution of a problem have occurred to them on brooding over their facts, they have been taught to reason out the bearings of these suggestions, to compare them and their full implications with the actual facts before them, and thus to test the tentative ideas of solution for their validity; they have learned the importance, when necessary, of making more observations and experiments that will either corroborate or refute. In other words, they have had the opportunity to practice deliberative thinking before undertaking their 


\section{8}

medical studies proper. On entering the medical school they spend a couple of years in work in the simpler preclinical medical sciences before engaging in the much more complex work of the clinical sciences of diagnosis and therapy. In the laboratories of anatomy, of physiology, of physiological chemistry, of pharmacology, of pathology, and of bacteriology they continue their training in the applications of the method of science to the study of the phenomena dealt with by these special sciences, and they should come out of these laboratories with that background of knowledge and that familiarity with methods that is indispensable for any proper study of the science, and any skillful practice of the art, of medical diagnosis. In the clinical departments of the medical school the students then enter courses of instruction in the laws and principles of diagnosis and therapy, begin their education in the technical methods of these sciences and, under the closest supervision, make a start in the practice of the corresponding arts. Not only must the methods be learned (the applied sciences of diagnosis and therapy), but skill in carrying them out (the arts of diagnosis and therapy) must be acquired, in order that the students may acquire confidence in the reports that their sense-organs (thus refined) yield and in the warranty for the diagnostic conclusions that can be reached and the therapeutic regimens that can legitimately be outlined by the application of reflective thought to these reports. This training in the clinical departments includes instruction in history-taking, in general physical diagnosis, in clinical laboratory work, in X-ray work, and in the technique of a whole series of special and instrumental methods of examination. The students learn the clinical application of bacteriological and immunologicai methods to be used in the diagnosis of the infectious diseases; they are taught how to examine the respiratory apparatus, the circulatory apparatus, the blood, the digestive system, the urogenital system, the locomotor system, and the nervous system and its functions; and they also receive instruction in the methods of clinically investigating the processes of metabolism and the functions of the endocrine apparatus. After this more or less thorough drill in the use of the methods of collecting facts regarding each special domain, they begin, as clinical clerks working in hospital wards and dispensaries, to take up the complete diagnostic study of single unknown cases. In close association. with, and under the strict control of, experienced diagnosticians, they record anamneses, make physical and psychical examinations, resort to laboratory tests and X-ray tests, are present at and observe closely the examinations made by experts in special domains, summarize and rearrange the total findings, entertain tentative ideas of diagnosis based upon these, consider all the implications of such suggestions, and try to 
arrive finally at diagnostic ideas that can be corroborated. Though the students are encouraged to work independently as far as possible, they have also the great advantage that the facts they collect and their reasoning about the facts are subjected to frequent review and criticism by the resident hospital assistants and by the older and more experienced visiting physicians. Only after this long training in college, medical school, and hospital is the student fitted to undertake the perfecting of his skill in the art of diagnosis, and long experience in practice may still be required to make him truly expert.

\section{The Actual Process of Clinical Diagnosis}

If diagnostic results commensurate with the medical knowledge of the time are to be reached when an internist is asked by a patient to make a diagnostic study, the procedure that he must adopt will be somewhat prolonged and complex and may be divided into several different stages: ( $I$ ) the recognition of a problem to be solved, and the feeling of a diagnostic difficulty; (2) the accumulation of data that help to locate, and to define the diagnostic problem; (3) the consideration of the data (accumulated, summarized, and arranged) that suggestions of possible solution of the diagnostic problem may occur to the mind; (4) the elaboration by reasoning of the detailed bearings of the several suggestions of solution; and (5) the careful testing of the suggestions thus minutely worked out as to their bearings by comparison with the facts accumulated, supplemented when necessary by other facts obtained by further observations and experiments, this careful testing leading to disbelief in the unverifiable suggestions and finally to belief in the suggestions that are found to be valid; in other words, the arrival at diagnostic conclusions. Each of these five stages is a necessary part of any diagnostic study that aims at accuracy and completeness.

The course pursued by a worker in clinical diagnosis, then, is similar to that followed by everyone who engages in reflective or deliberative thinking in order to solve his problems. Thus the same five stages must be passed through by a business man of the higher type when he is confronted by a new and problematic industrial adventure. The same stopping-places occur in the path of an engineer who is given the task of constructing a bridge. And the same points are recognizable in the line along which any scientific investigator moves when he scents a problem that interests him, goes energetically to work to solve it, and finally meets. with success. There is only one satisfactory method for solving problems, no matter what the domain, and that method is the method of deliberative thinking, commonly known as the "method of science." 
Nowadays a modest internist makes no claim to powers of diagnosis von Gottes Gnaden; instead, he recognizes the necessity of subjecting himself gracefully to the laws of logic that must be obeyed not only by him but also by his fellow-workers in the higher branches of human endeavor. The clinician who sees and hears will often greatly wonder; he will then feed himself with questionings in order that reason may diminish his wonder. He will observe and experiment; he will brood over and speculate upon his findings; his thick-coming fancies will keep him from rest until he has tested them rigidly as to their validity in all their implications; he may even distrust his eyes and will wrangle with his reason until he has convinced himself that the evidence in favor of one set of conclusions, and of one only, is good and satisfying. He will observe so accurately, he will experiment so appropriately, he will imagine so vividly, and he will verify so conscientiously that his diagnostic conclusions will be readily defensible and will be concurred in by such other diagnosticians as are keen and honest observers, skill ful experimenters, and right reasoners. Feeling a difficulty, observing and experimenting to define and localize it, harboring hypotheses that may solve it, reasoning about the implications of these hypotheses, and finally verifying those that are valid, are the successive steps in the stairway of the process of diagnosis. Clinical diagnosis is, then, an arduous and composite process; its complexities and intricacies are unavoidable. But practice in the use of the scientific method gives strength, speed, and insight to him who employs it. Though the road followed by the reflective thinker may seem long, steep, and involved, it is the only safe way to as much of certainty in diagnosis as the knowledge and technique of a given time will permit.

\section{Stage I: The Recognition of a Problem to be Solved; Feeling a Diagnostic Difficulty}

It seems worth while to make the feeling of a diagnostic difficulty a definite stage in the actual process of clinical diagnosis. Formerly, more often than now, a common cause of incomplete diagnostic study was a lack of realization of the difficulties that lie in the way of accurate diagnosis. This was true especially in the times when dogmas prevailed among physicians. In those times a single symptom, say the complaint of the patient, often sufficed for the making of a diagnosis. Thus a headache, a cough, a palpitation, or a pain in the epigastrium, gave rise to no diagnostic perplexity, for the symptom itself was regarded as a diagnosis and the treatment could at once be undertaken, for the universal principle or dogma left no doubt as to the course of action to be pursued. If, nowadays, a patient complain of a headache, we at least 
make an effort to discover the cause of the headache in the hope that we may be able to apply a rational treatment; we are not content with any single prescription to be used in all cases of headache. Similarly, if a man complain of a backache, the scientific practitioner of to-day will not resort at once to manipulative or other therapy, but will first undertake a thorough investigation of the case; he will try to understand the pathogenesis of the condition before he decides upon the form of treatment to be applied. Thus, a realization of the difficulties of diagnosis protects one from the extreme. naivety in therapy that formerly prevailed.

Even those who have been educated in the best medical schools sometimes fail to apprehend clearly the extent of the diagnostic study that is necessary in certain cases to insure the patient that he shall receive the full benefit derivable from the diagnostic and therapeutic methods that are available. A practitioner may be tempted at times to make a "snap-shot" diagnosis and to be content with it, but if he yield to this temptation often and curtail his diagnostic studies correspondingly, he will have occasion sooner or later to rue some of his hasty conclusions. The larger the experience one has had in diagnosis the more often has he demonstrated that clinical conditions that at first seem exceedingly simple may turn out to be very complex. In many cases it is only after numerous data have been collected that the real nature of the physician's problem becomes apparent. In order, then, that the diagnostic study of a given patient shall be sufficiently comprehensive, the physician must have an adequate appreciation of the diagnostic difficulty that confronts him.

One of the principal causes of detrimental curtailment of diagnostic study probably lies in feeble curiosity. The instinct of curiosity is, of course, a part of our common endowment. When we see, or hear, something that we do not fully understand, this instinct should come into function. We should have a feeling of wonder and we should be driven by an impulse to approach and éxamine carefully the object that excites our wonder. Different persons are doubtless endowed in variable degree with this inborn impulse closely to examine objects that excite their wonder. The impulse grows stronger through exercise, weaker through neglect. It is probable that many persons endowed with an instinct of curiosity of normal strength fail to profit by it as they should owing to faulty education. A normal child exhibits regularly the workings of the instinct, and the medical student and the physician should, to a certain extent, try to remain childlike in this respect. In clinical diagnosis, especially, the mind should be kept ever on the alert, ever sensitive to anything out of the ordinary, ever eager for new experi- 
ence. The diagnostician should be always exploring, continually seeking new materials for thought. If he cultivate a healthy curiosity, if he foster the emotion of wonder, and if he keep strong the will to investigate in 'order that wonder may diminish, he will have provided the fundamental conditions that protect from one-sided and incomplete diagnostic studies and that insure the comprehensive survey, the accurate observation, the suitable experimentation, and the careful reasoning that lead to valid diagnostic conclusions.

\section{Stage II: The Accumulation of Data That Help to Localize and Define the Diagnostic Problem}

Once having realized that we are confronted by a diagnostic difficulty, that we face a problematic situation, we enter upon the second stage of the diagnostic procedure and begin to accumulate the data that will permit us more accurately to define and to localize the diagnostic problem. In other words, we avoid any immediate attempt at solution of the problem because we desire first to get a better idea of the nature of the difficulty before us. At this stage, therefore, restraint of inference and suspension of judgment are desirable. Even though suggestions of solution of the diagnostic problem arise in our minds as we proceed, it is best not to yield assent to them at this stage, even when they seem plausible, though it may be justifiable to pay as much attention to them as will help us to decide upon certain directions in which the investigation may be intensively undertaken, or to conclude that, in the particular instance, certain tests often made in clinical studies may safely be omitted. At this stage we must be sure that we drag our net over an area large enough to insure the enclosure of enough facts regarding the physical, psychical, and social status of our patient to make the diagnostic problem precise in localization and definition.

The accumulation of the data necessary for this purpose is greatly facilitated by the following of some systematic plan. Thus it is customary to train medical students to collect the more important facts regarding a patient in a certain regular way. The following of a routine method of procedure here has both advantages and disadvantages. Among the advantages are ( $\mathrm{I}$ ) speed in the performance of an habitual process, (2) comprehensiveness, and (3) convenience of arrangement after the facts have been collected. Among the disadvantages may be mentioned ( $\mathrm{I}$ ) the danger of stifling curiosity by too rigid adherence to a routine program and (2) the danger that routine may not be varied from time to time as knowledge grows, as methods become elaborated, and as changes of emphasis are seen to be important. However, the intelligent and experienced diagnostician should know not only when 
to deviate from a regular routine in a given case, but also how to modify his routine from month to month and from year to year in order that his practice may keep pace with the advances of his science and his art. The beginner in diagnosis does well, nevertheless, to adhere rather closely to a well-thought-out scheme for the collection of data regarding patients; after he has attained to accuracy and celerity in applying this routine scheme, he may begin to consider the occasions when he is justified in modifying it or in diverging from it. A systematic plan of collecting data is helpful both to the experienced and the inexperienced diagnostician.

We may, for convenience, deal with the systematic accumulation of data regarding a patient in five different parts:

I. The recording of the anamnesis.

2. The recording of the results of a general physical and psychical examination.

3. The recording of the results of the application of laboratory

4. The recording of the results of X-ray examinations.

5. The recording of the results of more intensive examinations of special domains.

ad 1.- The Recording of the Anamnesis. In collecting the data obtainable as answers to questions put to the patient or to his friends one must make sure that the questionnaire covers ( $I$ ) the main complaints of the patient, (2) his family history, (3) his personal history, and (4) the history of the illness for which he consults the practitioner, including the symptoms existing at the time. It does not matter, as a rule, in what order these several parts of the history are taken. Some physicians, after ascertaining the main complaint of the patient, prefer to begin with the family history, to follow this with the personal history, and to end up with a history of the present illness. Others prefer to take the history of the present illness first, and, later, to secure the family history and the personal history of the patient. The latter method has some advantages, for the patient is always more interested in talking about his present illness than in giving the details of the history of his family and of his earlier experiences. Thus sick people often exhibit a certain impatience if one begin with the family history rather than with the history of the illness itself, though after the latter has been given in detail, they. will willingly respond to inquiries regarding their family histories and their earlier personal histories. In the accompanying table a general outline is given of the principal points to be covered by the ordinary anamnesis: 
A. Main complaints of the patient and their duration.

B. Family history (parents; brothers, and sisters; consort; children; other relatives).

C. Personal history (habits of work, eating, drinking, smoking, exercising, resting, sleeping, relaxing, etc.) ; education; experience; diseases; operations; traumata; mental conflicts; social adaptations.

D. Present illness (onset; supposed causes; course; previous treatment; epitome of symptoms referable to different anatomical-physiological domains).

It is important when recording the anamnesis to ask questions that bear upon the presence or absence of certain prominent symptoms referable to definite domains of the body; such inquiries are best made also in systematic sequence. After one has formed the habit of such questioning, a catalogue of the more important indications can be easily held in the mind. But the beginner will do well, while recording the anamnesis, to have before him a list of these symptoms, to make sure that he overlook no inquiry that could be pertinent. In this connection, the following list of betokening symptoms is a serviceable one:

Prominent Symptoms.

Pain (topography; time relations; severity; quality; radiations; modifying influences; associated phenomena).

Headaches.

Dizziness.

Tinnitus.

Otorrhea.

Nasal catarrh.

Sore throat; hoarseness.

Cough; sputum, including hemoptysis.

Dyspnea.

Palpitation; irregular action of heart.

Retrosternal or precordial oppression or pain (relation to effort; radiation).

Swelling of ankles or face; varicose veins.

Ingesta (quality; quantity). Disturbances of appetite and of deglutition; trouble with teeth and gums.

Nausea; vomiting, including hematemesis.

Gaseous eructations; flatulence.

Constipation; diarrhea; blood or mucus in stools; hemorrhoids; fistulae.

Herniae.

Pollakiuria ; dysuria; polyuria ; nocturia ; hematuria ; pyuria.

Disturbance of sexual functions (male; female).

Symptoms referable to muscles, bones, or joints, including the spine.

Skin eruptions; pigmentations; pruritus; loss of hair or nails.

Disturbances of motility (paralysis; weakness; wasting ; rigidity; twitching; tremor; spasms; cramps; fits; ataxias; dysarthria; aphonia; aphasia; apraxia).

Disturbances of sensibility (anesthesia; hyperesthesia; paresthesia, especially tingling in the fingers and toes; defects of smell, taste, sight, and hearing).

Mental disturbances (nervousness; insomnia; amnesia ; "fainting spells" or other losses of consciousness; delusions [hypochondriacal, melanchotic, or paranoid] ; exaltation; depression; loss of interests; fears; indecision; inability to concentrate; feelings of unreality; social maladjustments).

Obesity; emaciation; changes in weight.

Signs of infection (fever; chills; sweats; petechiae; etc.).

The experience and common sense of the examiner must guide him in the application of his questionnaire in any given case. There may 
often be a temptation to try to make short cuts and to limit the questionnaire unduly. Such abbreviation should be permitted only most cautiously, for even an experienced physician may easily overlook important clues if he deviate too far from his definite systematic plan of inquiry, or if he reduce too much the number of inquiries he makes. A special warning to the beginner regarding interrogations concerning sexual, psychical, and social details may be in place. It is often difficult to judge how far one ought to go in his inquiry at the first interview when such details seem to be of importance. The most sagacious and adroit inquirer will here sometimes make mistakes. It is, therefore, important for a beginner to go slowly and cautiously when he approaches this part of the anamnesis. He should try to elicit the facts in an easy, conversational way, and he should especially avoid giving the impression that he is unnecessarily curious or offensively prying. It is only in certain cases that the details of the sexual life must be inquired into, and even then the mode and extent of the inquiry will necessarily be influenced by many circumstances, among which are the age, intelligence, character, and experience of the patient. In determining the mental status of the applicant, too, good judgment must be used in deciding upon the nature and extent of the questions to be asked. One never asks a patient, for example, whether he has delusions! But if there be reason to suspect the existence of pathological ideas in the patient's mind, his answers to the four questions (I) Are you sick? (2) Have you been sad, blue, gloomy, depressed? (3) Do you blame yourself at all, or anyone else, for your trouble? and (4) Has everyone treated you well? will usually reveal the presence or absence of hypochondriacal, melancholic, and paranoid ideas and will afford sufficient clues for the further prosecution, or for the suspension, of investigation in these directions. Psychoneurotic patients in whom it is often desirable to hunt carefully for so-called "psychogenic data" are often especially sensitive to inquiries regarding their personal lives and their adaptation to the social environment. If on cautious approach to this domain the patient be found unwilling to talk at the first interview, it may be wise to postpone this part of the inquiry for a time. A little later, after the confidence of the patient has been established by the thorough physical examination made and by the sympathetic attitude of the physician, it will be more easily possible to secure, should it be deemed important, the full avowal of the patient regarding his more intimate life. The reticence of patients regarding abnormal feelings and emotions, moods, ideas, and experiences is easily understandable, and even though questions relating to these necessarily form a part of the daily work of the medical practitioner, it can scarcely be expected that all the patients will willingly, 
and immediately, place their hearts upon their sleeves for his inspection. The larger the world-experience of the physician, the greater his acquaintance with abnormal, nervous, and mental states, the wider his sympathies, and the more winsome his personality, the easier it will be for him quickly to acquire the confidence of patients and an avowal of the sort referred to when it is desired for the purposes of diagnosis. When the account given by the patient suggests the existence of abnormalities of the intellect, of the emotions, or of the will, it may be helpful also to interview, privately, members of the patient's family or his business associates, in order to learn what impressions they may have formed of the patient's nervous and mental state and what alterations, if any, in his personality they have observed. By the prudent application of measures such as those described, the psychical, social, and, when necessary, the sexual status, of the patient can nearly always be satisfactorily estimated and recorded.

Besides the general features of the anamnesis above referred to there are certain special points that are worthy, perhaps, of particular mention. One of these is the significance that sometimes pertains to recording the precise time-relations of the appearance of different symptoms. Thus when a tumor of the acoustic nerve developing in the cerebello-pontine angle is present, the exact chronology of the appearance of the different symptoms may be very helpful for the diagnosis. And in other diseases (typhoid fever, malaria, syphilis) the temporal relations of the symptoms may be informative. A second special point in the anamnesis worthy of attention is the interpretation given by the patient himself of his illness as a whole, or of any single symptom. It is desirable to put such an interpretation down no matter how improbable or how erroneous it may seem to the examiner. Every practitioner must have been impressed by the remarkable interpretation-delusions that patients sometimes harbor. But when the patient's explanation of his condition is obviously delusional, some care must be taken to avoid too brusque a refusal of acceptance of his pathological interpretative ideas. Only after confidence has been gained through a thorough investigation and through the establishment of a sympathetic relationship dare the practitioner hope to change such firmly set opinions. Even then the bringing of conviction to the patient may not be possible except through a somewhat prolonged reeducative process. A third matter that may well be again emphasized in the recording of the anamnesis is the extension of the questionnaire so that it shall certainly cover the marks of disturbances of the several anatomical-physiological systems of the body. If the several prominent symptoms mentioned in the above table be inquired about and the answers recorded, it is not likely that many 
of the pathological phenomena self-observed by the patient will be omitted from the record and the examiner can be confident that he has at hand the data necessary for his guidance in the further progress of the diagnostic investigation; these particulars are helpful for the making of decisions regarding the necessity of more intensive explorations in certain domains. Attention to the exact chronology of the appearance of symptoms, the appropriate management of the patient's interpretative delusions when such exist, and a search for the subjective marks of systemic disturbances are, therefore, especially serviceable to the physician who is recording a patient's recollections.

The totality of facts that the anamnesis can yield when it is skillfully elicited and recorded, has an importance to the diagnostician in his appraisement of the physical, psychical, and social status of the patient under study that can scarcely be overestimated. Both the anamnesis and the general physical and psychical examination are, of course, essential for clinical diagnosis, and neither should be neglected. I have heard more than one good clinician, however, state that if they had to be guided by one or the other alone, they would prefer to follow the path shown by anamnestic records that they had elicited rather than by the results of other examinations. These were men, however, who through long experience had learned better how to assess the value of single subjective symptoms and groups of such symptoms than any beginner could hope to do. Fortunately, we do not have to be guided by the anamnesis alone or by the physical examination alone; we utilize both to supply us with the symptoms and signs that clarify for us the diagnostic problem by which we are confronted. But the point that I would emphasize here is that the facts obtained by recording the recollections of the patient form an indispensable part of the data we accumulate before we allow ourselves to consider the solution of any problem in clinical diagnosis.

ad 2.-Recording of the Results of a General Physical and Psychical Examination. On making the general physical and psychical examination it is desirable to dictate the findings to a stenographer, or to a stenotypist, familiar with medical terms, item by item as the examination proceeds, for in this way only can a full objective record be obtained. It is not safe to trust the results of such an examination even in so far as to attempt writing or dictating a report immediately after the examination has been made. The examination involves so many details that one who attempts to make his records subsequently will often forget points of importance. Moreover, the record made later is pretty sure to be colored by the examiner's total impression derived from the examination, and at this stage of the diagnostic study any such coloring is undesirable. The examiner should make an 
unprejudiced record of the findings in each region quite independent of any idea of what the ultimate diagnostic decisions are to be.

Before undertaking the general physical examination the patient should be completely undressed and placed between sheets with a towel across the breasts, and the lighting arrangements should be such as to permit of satisfactory inspection. How many errors in diagnosis would be avoided if practitioners always insisted upon the undressing of the patient before the examination is made! Many an aortic aneurysm, many a breast tumor, many a hernia, many a bubo, and many a gibbus go unrecognized because of disobedience to this fundamental rule. Where on account of prudery of the patient, or of great nervousness, or of other cause, a complete disrobing is not practicable, a note of this should be made in the record in order to call attention to the fact that the examination has been made under hindering conditions; later on, another examination can, perhaps, be made under more favorable conditions, if it be thought desirable. The patient should be under observation in good daylight, the source of the light preferably being on the side of the patient opposite to that of the examiner. For the valuation of pigmentations of the skin and of the conjunctiva, daylight is essential; for the rest of the examination, good artificial light is permissible if daylight be unavailable. Only when the patient's body is uncovered and adequately illuminated can one expect to make a satisfactory physical examination.

When recording the results of the general physical and psychical examination, it will be found convenient to subdivide the record into three parts: A. General points; B. Regional examinations; and C. General examination of the nervous system and sense organs. Thus the general points summarized in the accompanying table should first be recorded:

A. General Points.

r. Body temperature; pulse at both wrists; respiration.

2. Height; weight; calculated ideal weight; build or habitus; acra; nutrition; musculature.

3. Posture; gait; behavior.

4. Skin (color; thickness; moisture; eruptions; ulcers; pigmentation;

scars; striae; nodules; tumors; superficial blood vessels; edema).

5. Lymph glands (epitrochlear; superficial and deep cervical; occipital; posterior auricular; anterior auricular; submaxillary; axillary;

pectoral ; inguinal; subinguinal;
6. Blood pressure (systolic; diastolic).

Passing on to the exploration by regions, one examines, successively, the upper extremities, the head, the neck, the thorax, the abdomen and pelvis, and the lower extremities. There is a special reason for making the examination first mainly by regions rather than according to 
anatomical-physiological systems, for regional examinations better permit one to accumulate facts without too much regard, at the moment, to their bearings upon the conclusion toward which the whole examination is aimed; diagnostic inferences are to be avoided at this stage of the inquiry; suspension of judgment regarding the nature of the patient's ailment is at this time desirable. One can scarcely, with beginners in diagnosis, emphasize too strongly this restraint of inference and suspension of judgment while the facts are being accumulated. There is a great tendency among those who have never learned the importance and value of a general diagnostic survey to seize hold of some salient feature in the anamnesis or physical examination, to allow it to dominate all of the further investigations, and to permit it detrimentally to curtail the study of the patient as a whole. Points of importance to be noted in the regional examinations are summarized in the accompanying table:

B. Regional Examinations.

1. Head (skull; face; eyes; ears; nose; mouth; throat; glands).

2. Neck (form; thyroid; tracheal tug; esophagus; blood vessels; lymph glands ; cervical spine; cervical ribs; tumors; wryneck).

3. Thorax (form; bones; coverings; breasts; axillary hirci and glands; lungs; pleurae and mediastinum; heart and aorta).

4. Abdomen and pelvis (inspection, percussion, and auscultation of abdomen and abdominal viscera; examination of rectum and of urogenital apparatus).

5. Extremities (skin; bones; joints; muscles; nerves).

After having made a record of the general points and of the points noted under regional examinations, it is well even at this stage to make at least a general examination of the nervous system and sense organs, in order that the data referable to the nervous system accumulated during the regional examination may be supplemented sufficiently to prevent us from overlooking data that point to lesions, or to disturbances of function, of the nervous system. Points to be noted in this preliminary examination of the nervous system are summarized in the following table:

C. General Examination of the Nervous System.

I. Sensory functions (cutaneous, and deep sensibility; stereognosis; special senses, including vision, hearing, smell, and taste).

2. Motor functions (muscular power; finer movements, including speech and writing; coördination; tonus).

3. Reflexes (pupils; deep reflexes of extremities; superficial reflexes, plantar and abdominal; sphincters).

4. Autonomic functions (vasomotor; secretory; trophic).

5. Mental state * (orientation; memory; calculation; attention; sense deceptions; pathological ideas; mood; psychogenic data; etc.).

* If the exploration in this direction has been full enough and systematic enough in the recording of the anamnesis, it may be omitted here.

In making such a general physical and psychical examination we call upon our powers of clinical observation and of clinical experimenta- 
tion and these functions should be exercised in an orderly and balanced manner. In simple observation we note and record conditions that we do not alter. In an experiment we exert some influence upon the character of the event that we observe, that is, our observations are then made under altered conditions. Every clinical examination includes these two modes of experience long ago referred to by Herschel, the astronomer, as "passive and active observation." The technique of clinical observation and experimentation has to be learned slowly. In our better medical schools the students are drilled in one method after another until a certain amount of skill is acquired. But the practitioner goes on increasing his skill as his experience grows. The well-trained and experienced practitioner can make a general physical and psychical examination, such as that outlined above, very quickly and accurately. But even the well-trained man should examine himself from time to time for tendencies to error. One's methods of examination by observation and experiment are undoubtedly easily influenced by his special interests. The making of an objective record of facts without bias is not easy, especially if they come into conflict with one's own peculiar views. It is surprising how some men will always find tenderness at McBurney's point, or in the right hypochondrium, how others will always find a few crackles in one interscapular space, how others will nearly always find a vertebral spine out of alignment, how others will suspect the existence of a stricture of the ureter, and how others will always regard a patient's feelings and behavior as psychoneurotic in type. Men are very prone to find what they are looking for and it is easy to decide that very slight deviations from normal are worthy of being regarded as pathological findings if they be in the line of one's special clinical interests. Minute and accurate observations are of course desirable, but one must remember that the accurate recording of very minute deviations in one domain (the domain of one's special interest) if accompanied by failure of observation of grosser deviations from normal in other domains may result in an unbalanced study and in fallacious diagnostic inferences. When several special examiners have coöperated with an internist in the clinical study of a patient it is of interest, when going over all the findings, to see how often the special interests of the several collaborators have colored the record. The observations and experiments made upon a patient should always be conducted with proper regard to a sense of symmetry and proportion, for there should $b e$, in the clinical record, $a$ "due and harmonious admeasurement of the parts to each other and to the whole."

The report of the general physical and psychical examination, after it has been typewritten, is placed along with the record of the anamnesis, 
pending the arrival of the records of results of laboratory examinations, of X-ray examinations, and of examinations in special domains. It is best to accumulate all this material before attempting to summarize the data and to rearrange them according to the anatomical-physiological systems to which they may be especially related.

ad 3.- Recording of the Results of the Application of Laboratory Tests. The methods of the clinical laboratory, as developed in recent years, yield data of real importance for clinical diagnosis. When making a general diagnostic survey of a patient, suffering from some obscure malady, certain routine tests are now commonly made in hospitals and in the offices of consultants. These include examinations of the blood, of the sputum, of the stomach contents, of the feces, and of the urine. Just how much laboratory work shall be decided upon as a minimum routine requirement in every general diagnostic survey will vary with different clinicians. There is a general tendency at present to have made as a routine in every case that is at all obscure, unless for some reason one or more of them is contraindicated, the laboratory tests mentioned in the following table:

A. Routine Laboratory Tests.

I. Examination of blood.

Red blood corpuscles; count, with notes on size and form.

White blood corpuscles; count.

Differential count of white blood corpuscles in stained smears.

Platelets.

Search for parasites.

Wassermann reaction.

2. Examination of sputum (especially for (I) tubercle bacilli and other bacteria and parasites, (2) tissue fragments, (3) spirals, (4) elastic fibers, (5) cells, and (6) crystals).

3. Examination of stomach contents.

Free $\mathrm{HCl}$, combined $\mathrm{HCl}$, and total acidity.

Occult blood.

Lactic acid.

Oppler-Boas bacilli.

4. Examination of feces.

Macroscopic and microscopic appearances.

Undigested food (meat; fats; starch).

Occult blood.

Bile.

Parasites, or their eggs.

5. Examination of urine (night and day specimens).

Physical (color; reaction; specific gravity).

Chemical (albumin; sugar; bile; indican; diacetic acid).

Microscopical (red blood corpuscles; white blood corpuscles; casts).

Some clinicians will be satisfied with a less comprehensive routine requirement and there are others who will desire a more extensive series of laboratory tests in every case. But no matter what routine requirement one decides upon, it is often desirable, in special cases, to have certain other laboratory tests made. Thus when there are signs of 
infection with continuous fever of unknown origin a blood culture will be made, but it is quite unnecessary to make a blood culture as a routine examination in every patient who presents himself. A lumbar puncture with examination of the cerebrospinal fluid may seem desirable if a patient who has had lues years before presents nervous symptoms sug. gestive of involvement of the cerebrospinal nervous system, or if in any patient there be signs of meningeal irritation, or if one suspect the existence of an epidemic encephalitis or of a Heine-Medin infection; but it would be an unnecessary procedure to examine the cerebrospinal fluid as a routine measure in every patient who comes for examination. Again, if a peculiar arrhythmia present itself in the course of the regional examination, it may seem desirable to have polygraphic tracings of the radial and jugular pulse and of the movements of the heart's apex, or an electrographic study, though to apply the polygraph and the electrocardiograph to every patient in practice would be a waste of time and energy. Laboratory tests in great variety have been devised; but our clinical laboratories are gradually sifting out the less important ones, and we are slowly becoming familiar with the best methods for securing the different kinds of valuable information that the clinical laboratory can yield. Among the special laboratory tests occasionally required may be mentioned the following:

B. Special Laboratory Tests (to be made in certain cases).

I. Cerebrospinal fluid (lumbar puncture).

2. Tuberculin tests.

3. Excision of a gland, a piece of muscle, or a nodule, or making uterine scrapings, for histological examination.

4. Bacteriological smears and cultures (blood; sputum; urine; pus; prostatic milkings; cerebrospinal fluid, etc.).

5. Blood chemistry; and other special blood examinations (agglutinins; lysins; opsonins; coagulation-time; bleeding-time; content in coagulation-factors; etc.)

6. Renal function tests.

7. Metabolic studies.

8. Protein sensitization tests.

9. Pharmacodynamic tests (with epinephrin, pilocarpin, or atropin).

Io. Electrocardiography.

II. Sphygmography.

12. Exploratory punctures.

I3. Animal inoculations.

Some practitioners, especially young men recently trained in the medical schools, make all of the laboratory tests required themselves. Others make only their routine laboratory tests and depend upon special laboratory workers for the performance of the special tests. Still others have all their laboratory tests made for them by assistants, or by special clinical laboratory workers.

Since the results of laboratory tests have come to be so highly valued 
in diagnosis, clinical laboratories have been so greatly multiplied and the number of persons professing to do expert clinical laboratory work has so greatly grown, that it may be in place to sound a note of caution. Unfortunately the sudden demand for laboratory tests has occasioned a supply of laboratories and of laboratory workers that contribute results of variable value. Too often the work done is unsatisfactory. Much harm can result from inaccurate reports emanating from unreliable laboratory workers. Even the well-trained worker in the best clinical laboratory will make a mistake occasionally in the performance of some test. Especially is this true of the Wassermann reaction. Every effort should therefore be made to insure the avoidance of erroneous, or inaccurate, laboratory reports. The value of a general diagnostic survey is not infrequently vitiated by an unwarranted credence in a laboratory report.

It should further be emphasized that when practitioners call upon their co-workers in the clinical laboratories for the making of special tests, they should not expect the laboratory men to make their diagnoses for them. They should ask for, and expect, only reports upon the particular laboratory tests mentioned. The results of these tests should be valued in association with the results obtained by other methods of examination. It is only occasionally that a laboratory can report a result that is pathognomonic for diagnosis (positive Wassermann; positive streptococcus culture or typhoid culture from the blood; meningococcus, or tubercle bacilli, from the cerebrospinal fluid, etc.). One must remember, too, that even a pathognomonic finding by means of a laboratory test, though it reveal the existence of a certain disease in a patient, may not point to the pathological condition that is most important when the patient's whole state is considered. A man may have syphilis and a glioma of his brain at the same time. Another man may suffer from amebic dysentery and from leukemia at the same time. The report of a positive Wassermann reaction in the blood in the one instance, and the demonstration of the presence of amebae in the stools in the other, though not to be underestimated in value, would not point to the pathological conditions of paramount importance for the two patients mentioned. Our diagnostic study in any given case should be comprehensive enough to include, in the final summing-up, all the important deviations from the normal presented by the patient, arranged in the order of their relative importance. But no attempt at the ultimate diagnosis of the case should be permitted at the stage of examination now under description. The restraint of inference and the suspension of judgment that have been repeatedly emphasized should be continued until all of the data of our schema have been accumulated, including 
those already referred to and those obtainable by $\mathrm{X}$-ray examinations and by intensive examinations in special domains.

ad 4.-The Recording of the Results of $X$-ray Examinations. The X-ray laboratory, like the clinical laboratory, has, in recent times, made important contributions to the methods of clinical diagnosis and is, accordingly, now much appealed to for help in acçumulating data regarding patients undergoing diagnostic study. At first employed chiefly in surgical diagnosis, the X-ray laboratories to-day are utilized even more by internists than by surgeons. Many practitioners install a roentgenological department in their own office and do X-ray work themselves, or arrange for a roentgenological assistant. Others send their patients to X-ray laboratories conducted by physicians who limit their work to roentgenology. The great improvements that have been made in the manufacture of roentgenological apparatus have rendered the technique of $\mathrm{X}$-ray examinations much more simple than formerly, so that any intelligent person can be trained to make good roentgenograms of the bones, joints, teeth, lungs, heart and aorta, and alimentary canal. The accurate interpretation of the roentgenogram is, however, not such an easy matter. In the first place, no one but a medical man trained in anatomy, pathology, and the clinics can be expected adequately to interpret what can be seen in a roentgenogram, or what is visible on roentgenoscopic examination. Even among medical men who devote their whole time and energy to roentgenological work the interpretative powers vary greatly, depending partly upon native endowment and partly upon length and intensity of experience. There can be no doubt that roentgenoscopic examinations and roentgenograms carefully made and properly interpreted are valuable contributions to the data with which the modern diagnostician should be supplied when he is studying obscure conditions.

The importance of close coöperation between internists and roentgenologists is growing every day clearer. An internist who to-day is unable, himself, to interpret roentgenoscopic and roentgenographic findings is decidedly handicapped in his diagnostic work, for even though he receive objective reports from competent roentgenological experts it will be hard for him to value these reports in a proportionate way. Any clinician who has made an extensive study of $\mathrm{X}$-ray plates and who has familiarized himself with what can be seen on a fluoroscopic screen will testify to the great autodidactic advantage that results from combining personal roentgenological interpretation with the results obtainable by other clinical methods. Not that the hard-working internist can expect to become as proficient in the interpretation of plates and screen views as are professional roentgenologists who give their whole 
time and energy to roentgenological work. The close association of the expert internist with the expert roentgenologist is essential to the highest quality of work of each. The diagnostician who does not see the X-ray plates on his own patients misses a great deal and the roentgenologist who is never able to control the results of his X-ray examinations by the clinical history of the patient or by the physical examination made by the internist will fall into serious errors and will not grow as rapidly in X-ray interpretation as he should. Regular conferences should, therefore, be arranged between internists and associated roentgenologists.

Altogether too much reliance is placed at present by many practitioners upon the reports in the form of diagnoses rather than in the form of concrete objective descriptions of their actual findings that are made by some roentgenologists. The latter are perhaps not so much to blame for this as are the practitioners who pressingly solicit them to give specific diagnostic judgments based upon their X-ray plates. It may be very helpful, of course, to have the diagnostic impression of the experienced roentgenologist in addition to the objective description of his findings. But the diagnostician making the general survey of the patient should be on his guard against accepting too readily the diagnostic impression of the roentgenologist. The internist should pay much more attention to the objective description of the findings discovered by X-ray methods than to such diagnostic impressions, and should utilize these objective reports in connection with the data collected by all other methods in arriving at his diagnostic conclusion; otherwise he will, at times, be led astray by a positive diagnosis ventured by the roentgenologist.

General diagnosticians can, in turn, be very helpful to roentgenologists if they will report to the latter (I) the ultimate diagnostic conclusions to which they arrive after the study has been completed, and (2) a summary of the data upon which the complete diagnosis is based. We must gradually work out the methods by which roentgenology and internal medicine can be reciprocally most helpful. If the internist and the roentgenologist will each give his best and if arrangements can be made for frequent conferences and discussions regarding the findings in concrete cases, the accuracy of diagnostic studies requiring the coöperation of internists and roentgenologists. will be rapidly advanced.

When one is making a general diagnostic survey of an obscure case it is a real comfort to be supplied with the data that roentgenology can yield regarding the structures mentioned in the following table: 


\section{THE RATIONALE OF CLINICAL DIAGNOSIS}

Commoner Medical X-ray Examinations.

I. The paranasal sinuses.

2. Dead teeth and unerupted teeth.

3. The contents of the thorax (form; size; opacity or transparency).

4. The digestive tract as revealed in $X$-rays during and after ingestion of barium (deglutition: form, size, and motility of stomach and of different parts of intestine).

Roentgenograms of the paranasal sinuses and of suspicious teeth together with a roentgenoscopic report on the thorax and abdomen if made in the practitioner's own office can be done at very small expense, so small that many practitioners could include the charge for such reports, when made as a routine measure, in the general consultation fee. Only if the symptoms or physical signs point definitely to marked disturbance of the digestive functions, or if in the absence of such symptoms and signs the roentgenoscopic examination done for eliminative purposes reveal suspicious findings, need the more expensive serial roentgenograms of the gastrointestinal tract be made. The data obtainable by the simple and commoner X-ray examinations enumerated in the above table go far toward protecting the physician who is making a general diagnostic survey of a patient from making certain sins of omission and commission that are frequent.

In addition to such routine roentgenological examinations, certain special X-ray examinations may be indicated by the records of the anamnesis, by the results of the general physical examination, or by the preliminary roentgenological survey of the thorax and abdomen. A list of the roentgenological examinations most often used is included in the following table:

Special X-ray Examinations (to be made when indicated).

1. Stereoscopic roentgenogram of skull, of sella turcica, or of mastoid portion of temporal bone.

2. Stereoscopic roentgenograms of lungs and pleurae.

3. Teleroentgenogram of the heart.

4. Serial roentgenograms of the gastrointestinal tract.

5. Roentgenograms of the gall-bladder area.

6 . Roentgenograms of bones, joints, and spine.

7. Roentgenograms for renal, ureteral, and vesical calculi.

7. Pyelograms and ureterograms after thorium injection.

9. Ventriculograms after trephining and injecting air into the cerebral ventricles.

10. Bronchiograms after insufflation of a bronchus with bismuth subcarbonate through the bronchoscope.

One files the records of the results of any X-ray examinations made, along with the other reports, pending the collection of data derived from the intensive examinations of special domains that have become suspect from a consideration of the anamnestic and physical study.

ad 5.-The Recording of the Results of Intensive Examinations of Special Domains. In making a general diagnostic survey, an 
internist must ask himself what systems of the body of the patient require an especially intensive study and how the intensive study shall be conducted. While taking the anamnesis and dictating notes on the physical and the psychical status of his patient, the examiner will have had his attention arrested at intervals by the discovery of symptoms or signs that his experience has taught him are most frequently referable to disturbances of function in particular anatomical-physiological domains. Though in general restraining inference and suspending judgment regarding the final outcome of his study the positively abnormal findings that have thus arrested his attention will serve as clues to suggest certain special lines of inquiry; they guide him to the domains that, in the particular case, merit a more thorough study than that made in the course of a general routine examination. Thus the complaint of oppression in the chest on exertion or the observation of an increased blood pressure, of an arcus senilis or of a cardiac arrhythmia, may point to the desirability of an especially thorough study of the cardio-vascular system. In another case, a history of recurring epigastralgia, of gaseous eructations, or of persistent constipation will lead the examiner to undertake a special study of the digestive apparatus. In another, the history of frequent micturition during the night, of difficulty in starting the flow of urine, or of hematuria, may make an examination of the urogenital system by special methods imperative. Or again, the presence of a polyarthritis will suggest to the examiner the importance of studying intensively all those domains of the body in which focal infections that may give rise to metastatic infections of the joints occur. In such cases the question arises, How shall this intensive study of special domains to which certain symptoms or signs point be undertaken? How can the data pertaining to these particular domains be most accurately, most quickly, and most inexpensively collected?

During the past fifty years the technical methods of diagnosis and therapy have been greatly enriched through that process of division of labor among medical men that we know as the rise of specialism in medicine. Physicians and surgeons interested in special domains have devised a whole series of new methods of observation and of experiment, some of them involving the skillful use of instruments of a greater or less degree of complexity. The technique of ophthalmoscopy, of refraction, of otoscopy, of laryngoscopy, of esophagoscopy, of sigmoidoscopy, of cystoscopy, of ureteral catheterization, and the like, can be learned by any medical man of intelligence, but mastership in these practical-technical procedures is not easy and requires a practical experience extending over a considerable time for its acquisition. The result has been that many men have decided to "specialize" in order that they 
may acquire extraordinary skill in the diagnosis and treatment of disease in certain regions or systems of the body. Thus, to-day, besides the general practitioner, general internist, and surgeon, we see professional men who are known as specialists in diseases of children, in diseases of the eyes, in diseases of the ears, nose, and throat, in tuberculosis, in cardio-vascular diseases, in diseases of the blood, in dentistry, in diseases of the digestive tract, in gynecology, in urology, in orthopedics, in neurology, in psychiatry, in dermatology, in endocrinology, in roentgenology, and in clinical chemistry. No single person can therefore hope to be equally familiar with the facts and principles and equally skillful in applying the practicaltechnical methods of all these specialties; indeed, few men pretend to mastery of more than two or three of them. If the internist is to avail himself, then, of all the diagnostic methods that are helpful, he must, in certain cases at least, call specialists in particular domains to aid him by sharing in the labor of accumulating clinical data.

Among the pressing problems that medical educators of the present time have to solve are those concerned with the training of both general practitioners and specialists, and with the making of arrangements that will insure the mutual helpfulness of these two groups in the diagnosis of disease and the treatment of the sick. The ordinary curriculum of the medical school is now so crowded that the medical student in his undergraduate course, though he receives a thorough training in history taking and in the general methods of physical and psychical diagnosis, can scarcely be expected to do more in addition than to learn the main facts and principles of the several medical and surgical specialties and to acquire enough first-hand experience with special instruments like the ophthalmoscope, the nasopharyngoscope, the bronchoscope, the cystoscope, the ureteral catheter, the polygraph, and the electrocardiograph to permit him to understand their uses and to make clear to him the importance of their application as aids to diagnosis in certain special cases. There is not time in the undergraduate medical course for the student to obtain the experience in any special domain that justifies him in regarding himself as a medical or surgical specialist. To become an expert ophthalmologist, urologist, orthopedist, neurologist, or dermatologist, he must undertake special work extending over a considerable period after his graduation. The post-graduate schools are attempting to supply opportunities for quickly gaining the experience in specialistic work that will make men competent, but as yet only a beginning in this direction has been made. There is urgent need for the endowment of post-graduate schools with suitable hospitals attached in which men may be adequately trained in the work of the several 
specialties. At present a young physician who desires to specialize in some one branch does best to attach himself as an assistant to a real expert in the subject that interests him. Opportunities of this sort are, of necessity, limited in number. It is owing to the paucity of such opportunities for intensive post-graduate studies in the special branches, despite the growing demand for specialists in practice, that so much pseudo-specialism now exists. For the sake of the suffering public as well as for the advancement of scientific medicine this situation must be squarely faced by medical educators, by philanthropists, and by the state, its defects recognized and the remedy sought and applied.

The sick should reap the advantages that can be derived from the division of labor in medicine. Laymen have discovered that some expert specialists exist, but they are unable often to distinguish the true expert from the pseudo-expert. Having found that the general practitioner is not always wise enough to seek the aid of a true specialist when his help is needed, laymen have tended more and more to apply directly to medical or surgical specialists when they themselves believe that their malady pertains to a special domain. This tendency can only be harmful not only to the patients themselves but also to the general practitioners and, in the long run, to the specialists. In order that the best work shall be done in diagnosis and therapy, some means of coördinating the activities of general practitioners and specialists so that the best results will be obtained for all must be found. A general practitioner or an internist, who works alone and who does not call to his aid, at least in an obscure case, men who have had special training in particular domains will be sure to miss facts that are highly important for a complete understanding of his patient's condition. On the other hand, the specialist who works by himself, taking care of all patients who apply to him, whether or not they are referred to him by a general practitioner or an internist, is in danger of forgetting that he studies only one part of the body and that, though he may find abnormalities in his special domain, these may be less important for the patient's whole state than are other abnormalities that exist unknown to him in other domains. Some way or another must be found by which patients may profit by the division of medicine into specialties while at the same time they are protected from the dangers of a one-sided study.

The medical profession is now trying to solve the problem just stated by means of "group work" or " coöperative diagnosis." Diagnostic groups are being formed in which each member of the group possesses special skill in some particular kind of work and one member who acts as integrator tries to combine the single parts into a properly proportioned whole. In this connection I can perhaps not do better 
than to quote from an address given before the New York Academy of Medicine in I9I7, in which I briefly discussed this topic of coöperative diagnosis in obscure cases requiring the intensive exploration of several special domains :

"The integrator should preferably be a person who, though perhaps especially skilled in some one branch, is rather encyclopedic in training and comprehension, sympathetic and tolerably familiar with work in all the divisions of modern medicine and surgery, free from prejudices, disciplined by sufficient experience in hospital wards, in clinical laboratories, and in the autopsy room, and blessed with that common sense that is, in the last analysis, largely a sense of proportion.

"Specialism, thus resulting in the orderly coöperation of the members of a group, instead of acting as a disintegrating force, may be made to contribute to a higher unity, most helpful both to the public and to the profession. With organization in groups of the kind mentioned, it would matter but little to whom the patient applied for diagnosis; if the integrator be applied to first, he will secure the reports from other members of the group before undertaking the integration; if a specialist in some single anatomical domain be applied to first, he may make his own examination, refer the patient to the integrator for the conduct of the rest of the study, and receive from the latter the full and proportionate diagnostic report upon which a rational therapy can be planned. Obviously, mutual confidence and good-will must prevail among the members of such a group. Such groups already exist and the number of them is, I believe, destined rapidly to increase. The older competitive methods must give way to the newer coöperative methods in medicine as in all other walks of life. Nothing could be more unfortunate, however, than the formation of cliques when arranging for group work in diagnosis, and I would warn emphatically against this danger. It is obvious, I think, that such a system as I am referring to does not restrict any specialist or any integrator to activity in a single group; there is no reason why either should not participate in the activities of several different or overlapping coöperating groups, the important points being that the group at work on any single case shall be so constituted as to insure, first, expert study in each of the several bodily domains in which there is an indication of the need of special study, and, secondly, a combination of the parts of the study into a well-balanced whole, the systematic analysis being followed by an adequate synthesis.

"Now, in most cases, there is, of course, no necessity of examination by every member of a large group of specialists. In addition to the anamnesis, the general physical and psychical examination, the routine laboratory tests and X-ray tests already mentioned, there may be 
required special examinations in only one or two anatomical domains. In obscure cases, however, and especially in instances of chronic infections necessitating the search for hidden foci, we may feel the need of calling upon a number of experts for aid. How many cases of chronic infectious arthritis, for example, progress for months because the diagnostic studies have been limited to too few domains, when more complete studies might have located the primary foci that were responsible? No one can lay down hard and fast rules as to how extensive a study should be. The judgment and experience of the one who has the general conduct of the study in charge must decide after the anamnesis has been recorded and the general physical and psychical examination has been made. The main thing is that he who conducts the study shall be sensitive to the problems that confront him and know how to apply the best skill in attacking and solving them. The greater the talents and experience of the integrator, the better his insight and discernment, the more likely he will be to have a proper sense of the indicative importance of the various features of a puzzling case. The greater his familiarity with the making of general diagnostic surveys, the more he will avoid requesting examinations that are wholly superfluous, the less likely he will be to neglect a test that is essential in any single case. The taking of too much pains in one case may be foolish; the taking of too little in another may be disastrous."

Just how such coöperative diagnosis will ultimately be carried out is, as yet, somewhat doubtful. The general hospitals have been gradually working toward it, but there must be much reorganization of these hospitals if the best results of coöperative diagnosis are to be obtained and, especially, the men working in such hospitals must be brought to an understanding of the advantages of such group work and must be taught how to organize for it and how the organization must be managed in order that it may be efficient. Aside from the work of the general hospitals, coopperative diagnostic clinics have already arisen in different places in the United States. The Mayo Clinic at Rochester, Minnesota, and the "Pay Diagnostic Clinics" of Boston and of San Francisco are notable examples. In many places, group diagnosis is carried on in office buildings by coöperating physicians, surgeons, and specialists. In most places, however, the physician making a general diagnostic survey still has to send his patients to specialists in his own town or even to those in more distant places for reports of intensive studies in special domains. A general practitioner when isolated in the country has to do the best he can without such coöperative work, and it has been matter of surprise and pleasure to me to see how successful some men so situated are in the general diagnostic surveys that they 
make. Certainly recent medical graduates, who have had a thorough training in general medicine, surgery, and the more important medical specialties, as well as in laboratory work and in X-ray work, may, single-handed, do general diagnostic work of a very high order before they become too busy, though even these men could do still better work if they were members of groups in which a division of labor was arranged for. It seems to me possible that, in country districts, county hospitals, in which the work of coöperative diagnosis by a differentiated staff will be undertaken and be supported in part by public funds, may ultimately be organized. Great convenience for patients and for physicians results from arranging for the combination of the differentiated diagnostic activities under a single roof. Centers in which coöperative diagnostic groups can work effectively seem destined to grow in numbers and in public esteem.

The cost of making a complete diagnostic survey in an obscure case requiring the coöperative activities of a general internist and a group of specialists is an item that merits special comment. Unless ways can be devised for bringing the cost of such an examination comfortably within the means of all that require it, many who would benefit by it will be compelled to do without it. It must, of course, be borne in mind that the great bulk of medical practice as done at present is carried on without the making of a general diagnostic survey of the patients in the sense of this discussion. Indeed, for the host of minor ailments from which patients suffer, it would be superfluous to undertake the kind of general diagnostic survey here described. An elaborate investigation of every minor ailment would be a waste of the patient's time and money and of the physician's time and energy. Among his patients the physician of good judgment will have but little difficulty, however, in selecting a certain number that, for their own sake as well as for the reputation of the practitioner, should be advised to undergo a general diagnostic survey. Those selected would include the class of patients ordinarily referred to internists, surgeons, and medical and surgical specialists for consultation. For this group of cases it is desirable that methods for making quickly, efficiently, and inexpensively a general diagnostic survey shall be evolved. Thus far the well-to-do are becoming provided for in the private wards of general hospitals and in private group-clinics, and the poor are also being very well looked after in the public wards of those general hospitals in which the method of group diagnosis has been introduced. Provision has yet to be made, however, for satisfactory group diagnosis for those patients whose incomes preclude the use of free dispensaries or of the public wards of hospitals, but are not sufficiently large to permit them to pay the usual fees for the 
more expensive diagnostic survey carried on in private wards of hospitals or in private clinics by an expert integrator coöperating with a group of consulting specialists. Though groups of the latter sort study a certain number of patients of small or of moderate means, reducing the fees charged for the whole study to an amount that is no hardship or inconvenience to the patients, no matter what their incomes are, still the amount of such work that can be done by the groups thus far organized is relatively small in proportion to the public need. Moreover, many patients who would benefit by a general diagnostic survey hesitate to avail themselves of an organization in which the ordinary charge for a general study is beyond their means, even though the total charge be willingly reduced to a merely nominal sum. In Boston and in San Francisco an effort has been made to provide for this class of patients in the "Pay Clinics" that have there been organized, and at the Mayo Clinic the cost of an elaborate general diagnostic study has been kept low. There would seem to be room in all large cities for organizations of young men who are gradually making their reputations to be of service in this connection. This is work, too, for which community funds justifiably might be expended. Industrial establishments, towns, cities, counties, and states might do well to foster organizations for group diagnosis, making financial appropriations to aid them, and providing for regulation and supervision that would insure efficient and ethical conduct. The methods of the business organizer and business manager might well be adopted here, not for exploitation, but for the welfare and protection of the sick.

The filing of reports of the results of intensive examinations of special domains completes the preliminary collection of data necessary for the localization and definition of the diagnostic problem. The facts accumulated include the records of the anamnesis, of the general physical and psychical examination, of the laboratory tests made, of the $\mathrm{X}$-ray tests made, and of the intensive examinations made in special domains. These facts may now be summarized and arranged in groups, according to the anatomical-physiological systems to which they pertain. The time will then have arrived for brooding over the data gathered and for allowing the things that we have observed to bring into our minds things that we have not observed. Suggestions of solution of the diagnostic problem ought to begin to occur to us. We are ready, therefore, to enter upon the third stage of the diagnostic procedure. 
Stage III: Summarizing and Arranging the Data Accumulated, Pondering Them and Recording the Diagnostic Suggestions That Occur to the Mind

In order that suggestions of possible solution of our diagnostic problem may occur to our minds, we must weigh mentally the facts that we have accumulated in recording the anamnesis, in making the general physical and psychical examination, in the making of laboratory tests and of X-ray tests, and on intensive examination of special domains. We stop observing and experimenting for a time in order that there may occur to us ideas of what the things already observed may mean. We begin to draw inferences from the facts.

Consideration of the facts with this purpose in view is greatly facilitated, however, (I) by making a preliminary summary and (2) by rearranging the facts in a systematic way.

Thus, in order that one may take in at a glance the positive abnormal findings in the case, it will be found convenient, first, to summarize these findings under the general headings that correspond to their mode of accumulation.

Summary of Abnormal Findings.

I. Anamnesis.

2. General physical and psychical examination.

3. Laboratory tests.

4. Roentgenological examinations.

5. Intensive examinations in special domains.

From the large mass of data accumulated one selects for this preliminary summary only the points that represent definite deviations from normal conditions. This makes for brevity and for ease of survey, and the summary serves as a valuable control of the fact accumulation, for one can, from looking over it quickly, discover whether any important method of examination suggested by the results of the anamnesis and of the general physical and psychical examination has been omitted in the study as carried on up to this point. Furthermore, when the more important facts are thus closely crowded together in a summary, defects in the reports of suggestive symptoms or of physical findings or of special examinations may be easily recognized and remedied before one entertains ideas of'interpretation. One may find, for example, that the report of a dental consultation, or of an X-ray examination that has been requested, has not been sent in; or one may, on this quick review, become cognizant that he has neglected, on outlining the course of the study, to include the making of some observation, or of some special test, the necessity for which was clearly pointed to by one of the symptoms of which the patient complained or by one of the signs recorded at the first physical examination. 
The data accumulated may next be rearranged according to the anatomical-physiological domains to which they reasonably may be supposed to be related. This is another method of finding out whether our observations and experiments have been sufficiently inclusive. For this rearrangement of the facts in a systematic way I, myself, make use of a single sheet upon which the following form is printed, sufficient space being provided for the inclusion of the various symptoms and signs that are likely to be met with in any case in connection with any one of the anatomical-physiological systems:

Data Rearranged According to the Systems to Which They May Be Related.

Name:

Chief Complaints:

Age :

Body Temperature:

Time and Mode of Onset:

Habits:

Infections :

Operations; Traumata:

Respiratory System:

Circulatory System:

Blood and Hematopoietic System: R. B. C. Hb. $\quad$ W. B. C. WaR.

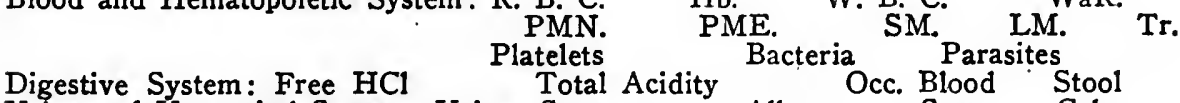
Urine and Urogenital System: Urine: Sp. gr. Alba Sugar Cyla.

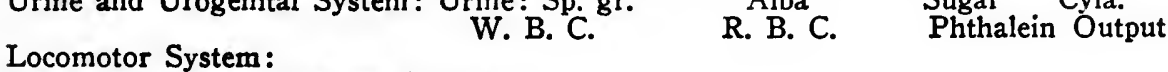

Nervous System and Sense Organs:

Metabolism and Endocrine System:

Remarks :

In this systematic rearrangement of the more important data, we include both positive and negative findings, jotting them down in as brief form as is compatible with quick apprehension, use being made of various symbols for purposes of abbreviation. Thus under the heading "Circulatory System" will be placed symptoms such as dyspnea, palpitation, precordial pain, and retrosternal oppression, should they be complained of, any physical signs referable to the heart and blood vessels (e.g. pulse rate; arrhythmias, systolic and diastolic blood pressure, position and character of apex beat, abnormal pulsations, heart murmurs, cyanosis, thickened vessels, arcus senilis, or edema), teleroentgenographic measurements and electrocardiagraphic results, if they have been recorded. Under the heading "Metabolism and Endocrine System" will be placed deviations from calculated ideal weight, notes from the anamnesis regarding gouty attacks or a gouty family history, diabetic symptoms, struma, tachycardia, fine tremor, eye signs common in the thyreopathies, abnormalities in the distribution of hair, pigmentations, condition of the acra, and the like. When placing a symptom like dyspnea, it may be well to include it not only under "Respiratory System" but also under "Circulatory System" and under "Metabolism" unless it has already become clear to what division the symptom 
predominantly belongs. Each integrator in rearranging the data will adopt or devise symbols and abbreviations that, though they are immediately intelligible, save space.

It will have been observed that, in making such a systematic rearrangement of the findings, the integrator has already begun to draw certain inferences and to make a series of particular judgments, for the assignment of given symptoms or signs to definite anatomicalphysiological domains is based upon knowledge, or prior experience, concerning the possible meanings of those symptoms or signs. The actual process of clinical diagnosis includes a search for clues, or marks, and the formation of judgments regarding the meaning of such clues, or marks as are discovered. The rearrangement of these clinical marks in groups according to the several anatomical-physiological systems to which they presumably pertain takes the facts out of the quarantine hitherto imposed upon them; isolation of the single facts gives way to association in groups as the integrator works at this stage of the diagnostic procedure. The materials thus dealt with prepare the way for the perception of further relations that may exist among the facts. Thus, the data pertaining to each anatomical-physiological domain may next be considered as a whole and judgments formed concerning their meaning and origin; later on, the relationship of the disturbances discovered in one anatomical-physiological domain to those found to exist in other domains may be sought for, with the idea of uniting two things in a third that is the foundation of the relationship (fundamentum relationis of the schoolmen). Such partial considerations as those just mentioned are necessary preliminaries to the localization of disease processes, and the assignment of place to the pathological phenomena is, in turn, necessarily antecedent to a proper understanding of the nature and cause of these phenomena; reflection upon the state of the patient as a whole, which we depend upon for supplying us with suggestions regarding the ultimate solution of our total diagnostic problem, can be advantageously entered upon only after we have made a long series of partial considerations and particular judgments and have already surmounted a number of local and minor diagnostic difficulties.

It may be worth while to advert for a moment to the kind of mental process we make use of when we have reached the stage of our diagnostic investigation in which we allow ourselves to entertain suggestions of explanation of the data that we have gathered, summarized, and systematically rearranged. We begin now to draw larger inferences, to form diagnostic hypotheses, to harbor interpretative ideas. Observation and experimentation, hitherto our main tasks, are now temporarily stopped. We begin to think and to make use of the creative imagination. 
With feet firmly fixed upon a basis of the facts observed, we try to pass by induction, or by deduction, to inference. We brood over the materials that we have selected and prepared in the hope that things that we have observed will lead us to ideas of things that cannot be observed. Contemplating the contents of our experience with the patient before us; we try to assimilate them with the contents of our own past experience (gained by studying patients and the medical sciences) and of the experience of other physicians as reported to us in medical literature, believing that, on such assimilation, suggestions will arise in our minds that we may tentatively entertain concerning things that our own present experience, by itself, does not hold. In other words, we now call upon our powers of reflection to make contributions beyond what our sense organs are able to yield to us. Were it not for this capacity of the mind to make leaps from facts to ideas, we should never go far in the process of clinical diagnosis. The mind of the diagnostician must bound forward by a leap, or by a succession of leaps, from the observed clinical facts to ideas of what these facts may mean. Thus, the integrating internist must be a mental gymnast; and he has to learn that expertness in the form of intellectual activity here described can scarcely be expected except after long experience, carefully directed. The regulation of the conditions under which the function of suggestion is allowed to take place is of the highest importance. Unless due care and attention have been exercised in the accumulation, selection, and arrangement of the facts from the consideration of which the diagnostic suggestions are to emerge, the conditions under which the creative imagination has to work will be faulty. Even when the conditions have been adequately regulated, a proper use of the function of suggestion implies the cultivation of both courage and caution as habits of mind. We should be bold enough to entertain several rival diagnostic conjectures that we test for validity, but we must be cautious enough to make sure that only hypotheses that are found, on testing them, to be valid are accepted as diagnostic conclusions. One sometimes hears medical men, well-meaning enough but innocent of any real acquaintance with the manner of working of the mind of the scientist, declare that "there is no place for imagination, or for hypothesis, in diagnostic work" and that "the real diagnostician should content himself with facts." But the truth is that everyone who does good work in clinical diagnosis is compelled, whether he is cognizant of it or not, to form hypotheses before he arrives at satisfactory diagnostic conclusions. A study of the conditions under which hypotheses should be permitted to arise and a knowledge of how to deal with these hypotheses once they have arisen in the mind would seem, then, to be indispensable for the higher walks of clinical diagnosis. 
Returning now to the actual occurrence of diagnostic suggestions to the mind when studying given cases, we may illustrate, by citing a few examples, how suggestions of meaning begin to arise on looking over the groups of symptoms and signs after their systematic tabulation in groups corresponding to single domains. Thus, if one finds recorded under the "Digestive System" morning diarrhea and the absence of free hydrochloric acid in the stomach juice, he will at once think of an achylia gastrica and of its possible relationship to a chronic gastritis, to an oral sepsis, or to a pernicious anemia. Or, if under the same system, one finds recorded a gastric hyperacidity, tenderness in the right lower quadrant, displacement of the stomach, markedly downward and to the right in the roentgenogram, and the history of recurring attacks of indigestion, he will think of the possible existence of some lesion in the right lower quadrant of the abdomen, say a chronic appendix. Or if there be recorded, in an obese person above the age of 40 , a gastric subacidity, a history of pain in the right upper quadrant of the abdomen (especially after riding horseback, after a night's ride in a sleeper, or after an automobile tour), of transitory attacks of jaundice, and of an earlier attack of typhoid fever, the idea of some gall-bladder trouble, probably gall-stones, will occur to the mind. Or, if one find recorded anorexia, an absence of free hydrochloric acid in the stomach juice, occult blood in the stool, and a definite filling defect in the roentgenogram of the stomach, the existence of carcinoma ventriculi will at once be suspected. Or, again, if under the "Circulatory System," one sees noted a retromanubrial dullness, a systolic blood pressure of I70, a diastolic pressure of 90, thickened radial arteries, an arcus senilis, a widened aorta or a transverse position of the heart in the X-ray, he will think at once of an arteriosclerotic process; or, if a definite thrill be palpable in the region of the apex of the heart and an asynchronism of the second sounds be audible in the pulmonic area, the first sound at the apex being abrupt, the existence of a mitral stenosis due to an earlier thrombo-endocarditis will no doubt suggest itself as a diagnostic idea. Or, if at the wrist a perpetually irregular pulse be felt and the record of the electrocardiogram shows a good many small waves arising in the atrium for every ventricular complex, the existence of atrial fibrillation will at once be thought of and a search for its etiology suggested. Or, if the pulse rate be 120 and there be no heart murmurs or marked enlargement of the heart, one will think at once of the possibility of a thyreopathy as an explanation and seek for corroborative data. Or, if, again, the pulse rate be 48 , one would leap to the idea of the existence of a conduction-disturbance in the atrioventricular bundle of the heart and would also find himself 
wondering whether this disturbance had its origin in some organic lesion within the heart itself, or had been due to a depression of the function of the bundle through vagus influences excited from a distance (intestinal irritation; increased intracranial pressure).

If, to take another example, under the "Urogenital System," in a woman of forty-two, one find recorded a prolonged metrorrhagia, say a flow of ten days each month, along with enlargement of the uterus, he will probably think of myomatosis with endometritis, and of carcinoma uteri, as rival explanatory hypotheses, each of which is rigorously to be tested for validity. If, under the "Nervous System," the data include nystagmus, loss of abdominal reflexes, and scanning speech, the integrator will think at once of lesions disseminated through the nervous system, the exact topography and nature of which he may try to determine. Or, if under the "Hemapoietic System," he finds jotted down a profound anemia with leukopenia, with a differential count of the white corpuscles showing a relative lymphocytosis of 94 per cent., along with enlargement of the spleen and with slight enlargement of the cervical lymph glands, the experienced internist may think of the possible existence of an aleukemic lymphadenosis, of a pernicious anemia, or of an anemia occurring in the course of a syphilis. As these examples illustrate, we deal, at this stage, separately with the symptoms and signs that pertain to each one of the several anatomical-physiological domains, cudgeling our brains for cues of possible meaning. When a group of signs and symptoms are present in a single domain, one should not be too easily satisfied with the occurrence to the mind of a single descriptive or explanatory hypothesis. Several possible hypotheses should be allowed to present themselves if they will and these should be pitted against one another as lusty rivals that are to be given opportunity to fight for supremacy. Hundreds of examples of syndromes might easily be given, were there need, but those mentioned will doubtless suffice to illustrate the mode of occurrence of diagnostic ideas to a mind that is pondering the symptoms and signs that have been referred to a given anatomical-physiological system.

As has been repeatedly emphasized in this article, on encouraging diagnostic suggestions to which a consideration of the facts as summarized and rearranged is to give rise, one tries to make scientific use of the imagination, a process that makes demands not only upon the intellect but also upon the affective-conative functions (the feelings and the will). From one's previous knowledge and experience he attempts to recognize in the group of facts before him either some wellknown uniformity of sequence or some easily identifiable uniformity of coexistence; only when no well-known one can be discovered by him, 
does he permit himself to think that he may be dealing with some new, or hitherto undescribed, syndrome. The aim of every scientific worker is to discover scientific laws to which the facts that he accumulates will conform. The scientific diagnostician desires also to summarize in a single statement, or in some brief formula, the disease process by which he is confronted and from which the whole group of facts that he has collected regarding the patient can be seen to flow. Out of a vast complexity of anamnestic data, of physical signs, of chemical reactions, and of biological tests, he strives to derive a unity, to detect the "one in the many"; by means of a disciplined imagination he attempts to formulate conceptions in which the whole range of facts may be resumed. He sets up groups of tentative or hypothetical conclusions that he is to scrutinize thoroughly and to examine adversely before admitting their validity. In order that a clinician may make a diagnosis as complete and as satisfactory as is possible in the state of medical knowledge that exists in his time, he must obviously, in addition to native ability, have a wide acquaintance with the main facts of all the medical sciences and he must have already become familiar with the classifications of groups of facts and with the descriptive formulae that have hitherto been made use of by other clinical workers. A certain esthetic element doubtless enters into the experience. The brief statement under which a large number of facts, or of perceptions and conceptions, is resumed must be felt to be adequate.

It was Karl Pearson, I believe, who emphasized that the continual gratification of the esthetic judgment is one of the chief delights of the pursuit of science. That this is true in the science of diagnosis, will be admitted by every advanced worker. The more comprehensive the diagnostic study made, and the more complete the understanding of the relationships of alterations of form and function to causes arrived at, the greater the esthetic appeal to the mind of the diagnostician of philosophic turn. This is why he, in making a clinical diagnosis, strives to arouse satisfactory suggestions of solution of his diagnostic problems by thinking systematically, first, of the possible pathological-physiological significance, secondly, of the possible pathological-anatomical basis, and thirdly, of the possible etiological and pathogenetic relationships of a given fact or group of facts. He thus secures his ideas of syndromes made up of functional disturbances, of the lesions present and their topographical relationships, of the nature of the disease processes that are going on in his patients and of their etiology. The several ideas that thus occur to him must, he knows, be subjected to such rigid criticism that the conclusions he finally arrives at will be equally valid for the minds of other clinicians who 
work in the same way. Intellect, emotion, and will,-all contribute, then, their share to the mental operations of this stage of the diagnostic inquiry.

One may ask the question, What reason have we to believe that different physicians, even when using the method of science, will, in studying a given case, arrive at similar, or identical, diagnostic conclusions? The reason why there can be, and often is, agreement in opinion among diagnosticians lies, one must believe, in a similarity of behavior of normally constituted minds. To the normal mind, the world outsidethe world of phenomena-presents itself in a certain way. The perceptive powers of normal minds must be very similar to one another. The same must be true of the reflective activities of the mind in normal persons. The mechanisms of association and of logical inference work similarly in different healthy people with the result that the mental contents of stored sense-impressions and of conceptions will be sufficiently similar to yield almost identical results in the same circumstances. The normal mind, when bombarded by a series of sense-impressions or perceptions, associates them with sense-impressions that have been stored in the memory; it combines these into conceptions or constructs; a train of thought is set up through association and the recognition of relationships; conceptions are formed and inferences begin to be drawn. Were it not that normal human beings perceive the same phenomena and reflect upon them in very similar manner, there could be no agreement regarding diagnostic conclusions, indeed there could be no such thing as science of any sort. As Pearson has well put it, "Human minds are, within limits, all receiving and sifting machines of one type." Minds that in their activities deviate too much from this normal type, we call disordered or insane. Within the range of normality, however, there is opportunity for considerable variation in activity. Minds that we call normal, though very similar in their activity, are by no means identical. We have abundant proof of this in the diagnostic suggestions that occur to different physicians who have had similar training and equality of opportunity for acquiring experience. To one mind, ideas of meaning may come easily and promptly, to another they come slowly and with difficulty. To one mind, a group of facts may quickly give rise to several ideas of possible meaning; to another mind, the stimulation by the same group of facts is barren of response. It is desirable of course that the number and range of ideas excited by the facts accumulated will suffice for the purpose of the study; there should not be too few of them and there should not be too many. Moreover, the quality of the ideas of solution that are aroused is even more significant than the promptness with which they come, or the abundance of the 
supply. One physician's mind may respond speedily with an abundance of suggestions and yet these suggestions may be inferior for the purpose in hand to those that arise in a mind whose response is slower but more profound. Rapidity of response is of course good in itself, but mere quickness will not compensate for either excessive prolificity or superficiality. A physician should, as far as possible, train his mind to make quick, balanced, and deep responses when he contemplates groups of clinical facts, in order that he may be supplied with enough worthy and substantial diagnostic ideas to test systematically for validity. Good native ability and prolonged training are essential for the best kind of diagnostic work. Though minds differ, the differences within normal limits are less important than the resemblances. Normally constituted minds are so nearly alike in their workings that diagnosticians of normal mental endowment who are well-educated in the contents and methods of the medical sciences, on studying similar pathological conditions, will, we may feel sure, arrive at similar conclusions.

In making a general diagnostic survey of a patient, the aim is to get as. complete an understanding as possible of the functioning of the whole man in his physical, psychical, and social aspects with the object of being of real help to him in improving his condition. As has been pointed out, the group of facts pertaining to each of the bodily domains (respiratory, circulatory, digestive, etc.) is first appealed to for suggestions of meaning and for calling forth in our mind ideas of similarity, of coexistence, or of sequence. We should not stop, however, with the recording of suggestions based upon the consideration of the data pertaining to these several systems, but should next turn to a survey of the whole series of suggestions that have thus arisen. For after testing systemic ideas for their validity, we want to know the relative importance of the several partial diagnoses that are found to be valid for an understanding of the condition of the patient as a whole. Until this general survey has been undertaken and completed, no final unified diagnostic conclusion with suitable ordination of all the factors in the case can be arrived at. By keeping the purpose of the diagnostic study vividly in mind, namely, the desire to find out what is wrong with the patient, in order to direct him how best to act, we shall find a suitable guide to the whole diagnostic procedure; this directing principle will enforce orderliness in the application of our methods, and it will give steadiness and continuity to our thinking as it moves toward its goal.

\section{Stage IV: The Elaboration by Reasoning of the Implications of Each Diagnostic Suggestion or Inference}

Before yielding assent to any suggestion that has issued from a consideration of the facts after they have been summarized and ar- 
ranged, no matter how plausible such a suggestion may seem, it should have been traced to its full consequences and its validity carefully tested. The acceptance of an idea as valid before it has been elaborated so that its full bearings may be clearly seen and compared with the facts that exist is the mark of an uncritical thinker. There is no room in clinical diagnosis for light-hearted and over-ready belief. Any tendency to infer wildly, rashly, or fallaciously must be vigorously combated. One should familiarize himself with the canons of legitimate inference and make sure that he is governed by them. When resorting to this reasoning process in which all the implications of each suggestion deemed worthy of testing are developed and are compared with the facts that have been accumulated regarding the patient, it will frequently occur that the diagnostician will discover the need of supplementing his first store of facts by further observation or by further experiment. It may even be necessary to apply methods other than those that have been used in a search for new materials to support, or to render untenable, an idea of interpretation that has occurred to the mind. It is only after we have entirely unfolded a diagnostic idea in detail that we can compare the several particulars that compose it with the facts as we have observed them and decide whether or not sameness can be recognized and identity established. When the facts observed are found to be in accord with the implications of a diagnostic suggestion as fully reasoned out, we accept the suggestion as valid and have a feeling of belief in it.

This process of developing the implications of diagnostic suggestions by reasoning may be illustrated by considering, as examples, the diagnostic suggestions that occur to us as solutions of the diagnostic problem presented by a patient who exhibits an acute febrile process with leukopenia. The patient, let us say, has complained of headache, of pain in his back, of loss of appetite, and of disinclination for exertion. The temperature of his body has been found. to be $102.5^{\circ}$ Fahrenheit, his pulse rate is 84 and the pulse is slightly dicrotic. A few rhonchi are audible over the lungs, the spleen is palpable, and the white-cell count of the blood is 4,800 . When confronted by this group of facts, the diagnostician will at once think of infectious processes associated with splenomegaly and leukopenia, and he will recall that two of the commoner infections of this sort are typhoid fever and malaria. His next step will be to develop the implications of each of these two diagnostic suggestions by reasoning. He will say to himself, "If the suggestion of typhoid fever be correct, we should find in studying the history of this patient's illness, an insidious onset of the symptoms, a characteristic temperature curve, a relative bradycardia, an initial bronchitis, headache, anorexia, palpable spleen, perhaps rose spots, a leukopenia, an 
early bacillaemia, an absence of coryza and herpes, an epidemiological record that gives the clue to the source of a bacillus typhosus in the case, the presence of the typhoid bacillus in plate-cultures made from the feces on the Drigalski-Conradi medium, or on Endo's fuchsin agar, the presence of specific agglutinins in the blood after the disease has lasted for a certain length of time, etc." $\mathrm{He}$ will also elaborate the suggestion of malarial fever and will say to himself, "If this patient has malaria, his temperature-chart should be that of either an intermittent fever (if it be a tertian or a quartan case), or a continuous or remittent fever (if it be an estivo-autumnal case); the patient will have had chills, sweats, headaches, anorexia, palpable spleen, herpes labialis, leukopenia, anemia, a history of exposure to the bite of an Anopheles mosquito, and perhaps neuralgic pains; in his blood, the presence of malarial parasites should be demonstrable, and pigment containing leukocytes may also be discernible; marked amelioration of the symptoms will follow the administration of quinine, etc." If the diagnostician be a careful and experienced worker, he will have thought not only of the commoner infections associated with leukopenia, such as typhoid fever and malaria, but also of the somewhat less common conditions so associated, such as paratyphoid fever, measles, mumps, glanders, and dengue. It will have occurred to him still further that leukopenia is sometimes met with in certain very severe forms of infection, like pneumonia and septicemia, that in ordinary circumstances are associated with leukocytosis. He will then develop the full implications of these diagnostic ideas also. These several diagnostic suggestions, thus fully developed as to their implications, will be looked upon by him as so many intellectual keys with which he will successively try to fit the lock.

If none of the keys he has forged is found to fit, he must try some modification of one of them or make still other keys to try. It may be that some complicating process of a secondary nature is changing the clinical picture so that it deviates from type. When, in a case, a survey of the data as a whole suggests the existence of a certain disease-process, one should give this process careful consideration, even though some of the data recorded seem to be inconsistent with it. Thus, if the symptoms and signs on the whole suggest the existence of typhoid fever, one should not throw this diagnostic suggestion into the discard simply because a leukocytosis is present, for, although leukopenia is the rule in typhoid fever, we do sometimes find a leukocytosis in that disease, owing to a complicating pyogenic process (phlebitis, pneumonia, cholecystitis, etc.). Or, to take another example, if the knee-jerks and anklejerks are absent in a patient, and anesthesias and paresthesias of his 
lower extremities have been recorded, the diagnostician will not rule out the idea of tabes dorsalis, at once, simply because an Argyll-Robertson pupil is not present, but will still keep this diagnostic suggestion in mind along with other conjectures of possible solutions of the diagnostic problem (funicular myelitis; polyneuritis; etc.). He will then reason each of the suggestions out fully as to its implications, and, if necessary, will make further observations or experiments that will decide whether identity exists. He may require to extend the blood examination, to undertake the examination of the cerebrospinal fluid, or to map out the exact topography of the sensory disturbances. It may even be necessary considerably to enlarge the anamnestic record in the case. If there be no anemia, if the cerebrospinal fluid yield a positive Wassermann reaction and contain many lymphocytes and more globulin than normal, if the topography of the sensory disturbance be segmental in type, and if the revised anamnesis reveal the history of luetic infection, of periods when lancinating pains occurred and show the absence of any abuse of alcohol and of any poisoning by lead, arsenic, or other substances that cause neuritis, the idea of tabes dorsalis as a satisfactory diagnosis may be accepted as valid even though no Argyll-Robertson pupil be demonstrable. Thus a diagnostic suggestion that, on elaboration, seems to be inconsistent with some of the data present, may, on modification, be found to be adequate as a solution of a diagnostic problem.

The original diagnostic suggestions that come up in our minds are always inchoate; they require to be developed. From principles that have already been established in medicine and with which we have become familiar through our earlier clinical experience and through our study of medical books and journals, we deduce the fullness and completeness of their meaning. The data accumulated by the analytical processes of the anamnesis, and by means of the general physical and psychical examination, the laboratory tests, the X-ray tests, and the special tests, suggest to us, when we brood over them, wholes into which they may be synthesized. Such suggested wholes are then again disintegrated by a reasoning process of deduction into their known constituent parts. Further observation and experimentation may then be required before identity can be established between one of these suggested wholes with its constituent elements and the actual whole to which the symptoms and signs in our patient really belong. Indeed, one of the great advantages of the consideration of all the possible bearings of the general diagnostic notions that we tentatively harbor is that it often leads us to expand substantially our collection of particular data. The full development, by reasoning, of all the implications of the diagnostic suggestions that occur to us is, then, an essential part of the diagnostic procedure. 
Stage $V:$ The Testing of Diagnostic Suggestions (Elaborated by Reasoning) for Their Validity and Arriving at Diagnostic Conclusions or Beliefs

It has been repeatedly emphasized that before we accept a diagnostic suggestion, inference, or hypothesis, after developing its bearings and implications, as a true explanation or description of the facts in a case, we must test it carefully for its validity. Having found out by a process of deductive reasoning precisely what it implies, we must demonstrate that there is identity between its implications and the actual data that we have accumulated, or can accumulate, regarding the patient. If the diagnostic suggestion as developed by ratiocination be found to be out of accord with the facts collectable we dare not give credence to it. Accordance in composition with what has been, or can be, observed is the sole real test for the validity of a diagnostic suggestion.

If, on looking over our amassed data, we find some single fact that seems to be in absolute conflict with some implication of our reasonedout suggestion, though the other facts are in entire conformity, we shall do well to question the accuracy of our observation on the one hand and the flawlessness of our reasoning on the other. If the discordant fact be confirmed by a second observation and if it can be shown that there has been no fallacy in reasoning out the implications of a diagnostic suggestion, the latter, unless it can be so modified as to do away with the discrepancy, must be regarded as untenable. Any absolute conflict between clinical facts and diagnostic suggestion is fatal to the suggestion as a whole, for in the phrase of the logicians, "falsus in uno, falsus in omni." It must surely be quite clear that what is true of one thing must be true also of its equivalent.

In this last, or fifth stage, of the diagnostic procedure we have to deal, then, with the verification, or corroboration, of our conjectural ideas. It will be recalled that in the third stage of our inquiry we allowed the particular facts that we had accumulated regarding the patient to call forth in our minds suggestions of a general nature that might explain these facts, or that might at least classify them under a common head; we there tried, by an inductive process, to pass from certain results or consequences (our collected data) to some general conceptions from which they might be presumed to flow. It will also be remembered that in the fourth stage of our study these suppositional general conceptions were, by a process of deduction, reasoned out fully as to their bearings and implications; through ratiocination we determined what particular clinical facts or consequences ought to be present in the patient if the general ideas were valid. Now we come to the last stage of the diagnostic inquiry, in which our task consists in com- 
paring the whole meaning embodied in the diagnostic suggestion, that is, the whole of the consequences that flow from it, with the actual clinical facts that we have gathered; or that we can gather by further observation and experimentation. We have, at this stage, to trace out fully the degree of similarity that obtains between the facts that exist and the facts that should exist if the ideas that have occurred to us are true. We must ascertain whether there is a sufficient degree of likeness or sameness to justify the acceptance of the idea that we have tentatively entertained and rationally elaborated; and if we have provisionally considered other diagnostic ideas as rivals to it, we must demonstrate that the distinguishing criteria of these rivals are absent. Unless a diagnostic idea as fully reasoned out can be verified we dare not believe it to be true.

The secondary observation and experimentation stimulated by our attempt to establish identity between observable particulars and the implications of a tentative idea may strengthen or weaken the diagnostic conjecture and result in corroborating it, or in refuting it. Thus in the case of infection with fever, leukopenia, and palpable spleen to which we have referred, it may be found possible on reexamination of the patient to discover that we had previously overlooked a roseola; or we may find on the patient's lip a slight herpes that had not been noticed at the first examination or that had been passed over as insignificant; or in a blood culture made in bile-bouillon we may be able to grow a motile bacillus which, on being tested, turns out to be the bacillus paratyphosus; or again, on making another careful search of a stained smear of the blood, we may find a single crescent-shaped malarial parasite, or some small intracorpuscular forms that earlier had escaped observation; or, a week or two after the first examination, during which time the diagnosis has remained in doubt, we may become able to demonstrate specific agglutinins for bacillus typhosus in the blood, though the Widal reaction had been negative at the first examination. Thus, where neither corroboration nor entire rejection may be justifiable on comparison of the facts originally collected with the implications of the conjectural idea of diagnosis entertained, additions that will bring a decision may sometimes be made to the clinical data.

As long as the data are insufficient for the determination of identity between the facts of experience and the reasoned-out implications of a diagnostic idea, the scientific diagnostician will reserve his judgment. And though doubt as to diagnosis will seem intolerable to him as long as a chance of a justifiable decision remains open, he will nevertheless often be compelled to suspend a conclusion when a more ignorant, or a less cautious, mind, unwilling to bear a painful feeling of incapacity, 
will indulge in a positive decision and advance in a wrong direction. The only safe way to arrive at accurate diagnostic conclusions or beliefs is to follow the slow process that has been indicated, namely, fact accumulation by observation and experiment, lying with the facts that tentative ideas of solution may be engendered, reasoning these out fully as to their implications, comparing these implications with the facts to see whether or not identity can be established, if necessary making further observations or experiments to extend the facts, and testing one suggestion after another until at least some one of them can be corroborated and accepted as valid; then, and not until then, should a diagnostician permit himself to feel that his problem has been solved.

The best diagnostic brain, fortified by a large experience, will sometimes make mistakes in diagnosis, even when all the precautions that have been referred to have been observed. Indeed, it has been among the highest type of clinicians, from the earliest times on, that can be found most often the evidences of willingness to acknowledge that "experience is fallacious and judgment difficult." No medical man is so expert or so careful that he never arrives at diagnostic conclusions that, later on, have to be revised. Exploratory operations on the living and complete autopsies upon fatal cases, are most salutary correctives of diagnostic jauntiness. The diagnostician who follows his patients to the operating room, or their bodies, should they die, to the morgue, learns lessons in modesty and takes the best course for the avoidance of presumption on the one hand and undue diffidence on the other. The physician who conscientiously applies the method of science to clinical diagnosis, who recognizes how difficult diagnosis in a given case may be, who tries to make accurate observations himself, who is willing sometimes to enlist the aid of expert observers in special domains in the collection of data, who deduces fully the consequences that flow from any diagnostic suggestions that occur to him, and who insists upon accordance between these reasoned consequences and the clinical facts before he permits himself to arrive at a diagnostic conclusion, can feel sure that he is working in the right way and can know that, as he grows in knowledge and experience, he will become an ever more expert diagnostician.

The extent to which a diagnostic study is carried will depend partly upon the purpose for which the study is made and partly upon the natural endowment and the experience of the man making it. The purpose of the general practitioner varies somewhat from that of the consulting physician, and the purpose of the latter is different to a certain extent from that of the man who devotes his life to original investigation. The particular aim that the diagnostician has in view (welfare of the single 
patient; or additions to knowledge that may contribute to the welfare of future generations) will, in some degree, determine the methods of clinical investigation employed and the scope of the diagnostic inquiry.

The natural capacity, the experience, and the ideals of the diagnostician will have their influence upon the work that he does. They will reveal themselves in problem recognition, in the practical technique of fact accumulation, in creative imagination, in reasoning power, in verification, in philosophic grasp, and in esthetic appreciation. It is well, now and then, perhaps to take stock of the personal qualities that make for success in diagnosis. What Faraday said of the philosopher is very applicable to the diagnostician of the higher type. He " should be a man willing to listen to every suggestion, but determined to judge for himself. He should not be biased by appearances; have no favorite hypothesis; be of no school; and in doctrine have no master. He should not be a respecter of persons, but of things. Truth should be his primary object. If to these qualities be added industry, he may indeed hope to walk within the veil of the temple of nature."

\section{BIBLIOGRAPHY}

ANGELL, J. R.: Psychology: An Introductory Study of the Structure and Function of Human Consciousness. 4th ed. New York: Henry Holt \& Co., 1908.

BALDWIN, J. M.: Thought and Things. 3 vols. New York: The Macmillan Company.

BARKER, L. F.: The General Diagnostic Study by the Internist, etc. N. Y. Med. J., 1918.

BOSANQUET, B.: The Essentials of Logic. New York: The Macmillan Company, 1895 .

BRADLEY, F. H.: The Principles of Logic (Anastatic Reprint). New York: G. E. Stechert \& Co., 1912.

CABOT, R. C.: Differential Diagnosis. Philadelphia: W. B. Saunders Company, I9II.

DAVIS, M. M.: Group Medicine, Am. Jour. Public Health, Boston, I9I9.

DEWEY, J.: How We Think. Boston: D. C. Heath \& Co., 1916.

FOLLETT, M. P.: The New State: Group Organization the Solution of Popular Government. London: Longmans, Green \& Co., 1918.

FRENCH, H.: Index of Differential Diagnosis. New York: Wm. Wood \& Co., 1917.

HORNE, H. H.: Psychological Principles of Education: A Study in the Science of Education. New York: The Macmillan Company, 1906.

JAMES, W.: The Principles of Psychology. 2 vols. New York: Henry Holt $\&$ Co., 1890 .

JEVONS, W. S.: The Principles of Science: A Treatise on Logic and Scientific Method. New York: The Macmillan Company, 1900.

JONES, BENCE: The Life and Letters of Faraday. 2 vols. London: Longmans, Green \& Co., 1870. 
LASÉGUE, C.: De la logique scientifique et de ses applications médicales. Paris: Arch. gén. de med., I868, 6. s., XI, 7I5-732.

McDOUGALL, W.: An Introduction to Social Psychology. 9th ed. London: Methuen \& Co., 1915.

MACKENZIE, J.: Symptoms and Their Interpretation. New York: P. B. Hoeber, 1914.

MILL, J. S.: System of Logic, Ratiocinative and Inductive. 8th ed. New York: Harper \& Brothers, I900.

MILLER, J. E.: The Psychology of Thinking. New York: The Macmillan Company, 1917.

MINTON, W.: Logic: Inductive and Deductive. New York: Charles Scribner's Sons, 1905 .

MÜLLER, F.: Der Ausbau der klinischen Untersuchungsmethoden. Ztschr. $f$. ärzt. Fortbild., Jena, I906, III, 433.

OESTERLEN, F.: Medical Logic. Transl. and edited by G. Whitley, M.D. London: Sydenham Society, 1855.

O'SHEA, M. V.: Social Development and Education. Boston: Houghton Mifflin \& Co, 1909.

OSLER, SIR W.: Internal Medicine as a Vocation. In: Equanimitas and Other Essays. Philadelphia, 1904.

PEARSON, K.: The Grammar of Science. 2nd ed. London: A. \& C. Black, 1900.

PRATT, J. H.: The Method of Science in Clinical Training. Boston Med. and Surg. Jour., I912, CXLVI, 835.

RICHMOND, MARY E.: Social Diagnosis. New York: Russell Sage Foundation, I9I7.

SIDGWICK, A.: The Application of Logic. London: The Macmillan Company, I9I0.

SOUTHARD, E. E.: Diagnosis per exclusionem in ordine: general and psychiatric' remarks. Trans. Ass. Am. Physicians. Philadelphia, I918, XXXIII, 267.

THAYER, W. S.: On the Importance of Fundamental Methods of Physical Examination in the Practice of Medicine. Mobile: South. M. J., 1914, VII, 933-942.

THOMPSON, J. A.: Introduction to Science. New York: Henry Holt \& Co., IgII. 


\title{
CHAPTER XV
}

\section{TESTS OF FUNCTION}

\author{
By Henry A. Christian
}

\author{
TABLE OF Contents \\ Introduction. \\ Tests of Renal Function. \\ Tests of Digestive Function. \\ Tests of Circulatory Function. \\ Basal Metabolism as a Measure of Function.
}

IN diagnosis at the present time much use is made of methods of testing the function of various organs. Diagnosis is concerned not alone with determining what parts of the body are diseased and the causes thereof, but also with estimating the extent of the disease by measuring the degree of change from the normal in the function of the part or parts damaged. The development of these methods has been a large factor in giving to our present-day medicine its distinctive functional aspect. By these tests of function we seek to learn what an organ can do in regard to its total function or in respect to some specialized part of its activity. Knowing this we have a measure of the damage wrought as a basis for diagnosis and prognosis, and by repeating tests from time to time we are in a position to judge of the progress in either direction of the disease process and of the effects of treatment on it.

Tests of function are not very recent developments, but in the last few years they have been used much more generally and many new methods of testing function have been described. In essence they consist of means of measuring the activity of an organ. To accomplish this very many different principles are applied varying with the type of function we seek to measure. To illustrate this method of study several different types will be described and their application discussed in order that the reader may have a better idea of the ways in which much of our present knowledge of functional medicine has been acquired.

\section{Tests of Renal Function}

The kidney in a functional sense is almost a purely excretory organ. Its work is to rid the body of certain products of metabolism; to the body it furnishes practically nothing. Being an excretory organ it lends 
itself well to tests of renal function; it is relatively easy to measure the results of its activity by determining the rate at which it excretes different substances under varying conditions. By these means we can measure renal activity. To correlate such results with structural changes, however, presents great difficulties. The kidney is very complex in structure and undoubtedly different parts of its structure possess different excretory activities; there is much evidence for a selective excretory activity of different parts of the epithelial structure. Unfortunately in the normal kidney there is uncertainty as to the locus of excretion of the various components of the urine; just how much reabsorption takes place in the tubules of substances excreted through the glomeruli and what substances are so affected is not definitely known. Then with injury to one part compensatory over-activity of other parts is very probable. All of these factors enter to make it exceedingly difficult to determine disturbances or lesions in any particular portion of the renal structure. Numerous tests have been devised to measure disturbances in the selective excretory activity of the kidney tubule or glomerulus, such as the rate of excretion of potassium iodide and of lactose, but they have proven of little help, chiefly because it has not been certain that the given substance, for example, is excreted by the glomerulus and not elsewhere, or if it is excreted by the glomerulus under normal conditions it is not known whether a decreased excretion indicates a decreased glomerular excretory activity or a possibly increased tubular absorption. For these reasons we have been unable to say whether in a given case the pathological lesion involved mainly the tubule or some other renal structure; on the other hand, we can by tests determine whether the kidney in certain respects is doing its work well or poorly, i.e. determine its total function.

One of the best tests of renal function is the rate of excretion of phenolsulphonephthalein, a test introduced by Rowntree and Geraghty $\left.{ }^{1}\right)$. This is a non-toxic dyestuff which with the addition of alkali turns a bright red. I c.c. of a solution containing $6 \mathrm{mgm}$. of the dyestuff is injected intramuscularly. There is no local reaction; it is promptly absorbed and circulated to the kidney. The kidney excretes it in the urine; there is no irritant action on the kidney. If after a definite period of time the urine is collected, diluted to one liter, rendered alkaline, and compared in some form of colorimeter with a test solution containing $6 \mathrm{mgm}$. of the dyestuff in one liter of water similarly rendered alkaline, the amount excreted can be expressed as a percentage of the amount injected. The normal kidney excretes from 60 to 80 per cent. of the amount injected in two hours after excretion begins. A lessened per cent. of phenolsulphonephthalein, if absorption of the in- 
jected amount is normal, indicates a decreased excretory activity of the kidney which may depend on a lesion of the kidney or on disturbed circulation through the kidney. Kendall $\left({ }^{2}\right)$ has shown that the dyestuff under certain conditions may be changed to a colorless compound, so that the decrease in the amount found in the urine may not be due entirely to a slowed excretion.

This test is very useful both as a measure of total renal excretion and as an index of differences of renal function in the two kidneys when separate specimens are collected by ureteral catheterization; here it helps in the diagnosis of surgical conditions of the kidney. The great simplicity and safety of this test make it of especial value. In its simplest form it can be carried out readily by any physician with apparatus of slight cost. A number of other tests utilizing other coloring matters have been described, but the phenolsulphonephthalein is, in many ways, the best. It is a test of total renal function; beyond indicating a decrease in renal function and serving as a measure of its degree, it has no particular diagnostic value; it is not a test of the presence of a nephritis, for example. Nephritis may exist and yet renal function measured by phenolsulphonephthalein may lie within normal limits. On the other hand, its excretion may be decreased without there being a nephritis, as in chronic passive congestion of the kidney.

Another measure of renal efficiency lies in the ability of the kidney to excrete water and solids in relation to the intake of liquid and solid food. The normal kidney excretes these promptly in relation to their intake. If a considerable amount of fluid is given the amount of urine quickly increases and the amount of total solids as measured by the specific gravity decreases. If, on the other hand, solid food with relatively little fluid content is taken, the amount of urine decreases and its specific gravity increases. Under normal conditions in the kidney, on the average diet, the bulk of the urine is excreted in the day; usually in the night period 400 c.c. or less is excreted. These facts serve as the basis of a very useful test of renal function, usually spoken of as the two-hour renal test.

The patient, who has been on a fairly uniform diet for several days, is given a diet consisting of breakfast of the usual type, a noon meal containing relatively high protein, extractive and sodium chloride content, and a quite simple supper. Several different menus have been used (v. Monakow $\left({ }^{8}\right)$, Mosenthal $\left({ }^{4}\right)$, and others). The one we use at the Peter Bent Brigham Hospital is as follows:

Morning meal.-Orange 50 grams, oatmeal 150 grams and milk 25 c.c., I egg 50 grams, toast 20 grams and butter 5 grams, sugar ro grams and cream 20 c.c. to be used with 150 c.c. of tea or coffee. 
Midday meal.-Milk (in soup) I 50 c.c., steak 75 grams, potato 100 grams and butter Io grams, peas roo grams and butter Io grams, bread 20 grams and butter 5 grams, ice cream composed of milk too c.c., cream 20 c.c., sugar Io grams, and I egg 50 grams, sugar Io grams, $\mathrm{NaCl} 3.5$ grams (to be used by patient on above food) and water 500 c.c. with meal.

Evening meal.-Tomatoes 75 grams and butter Io grams, lettuce 20 grams with celery 50 grams and olive oil 20 c.c., bread 20 grams and butter 5 grams, baked apple Ioo grams with sugar 20 grams and cream 20 c.c., water 500 c.c. with meal. 250 c.c. water during evening.

With the patient having these meals the urine is collected in twohour portions throughout the day from 7. a.m. to 9 p.m., and in a single night portion from 9 p.m. to 7 a.m. Each portion is measured and its specific gravity taken; the sodium chloride and nitrogen content of each portion may be determined in addition.

The normal kidney under these circumstances will show a very considerable variation in the amount and specific gravity of the urine from period to period; with renal damage these differences decrease, tending toward fixation in both amount and specific gravity, indicative of a delayed or a decreased excretion. The delay in promptness of excretion causes the night amount of urine to increase beyond the average normal amount of 400 c.c. In just the same way a decrease in amount and a tendency to fixation in percentage concentration of sodium chloride and nitrogen occurs with renal damage. These various constituents in the urine, water, total solids, sodium chloride, and nitrogen often do not change in the same ratio, probably because of varying lesions differently affecting the selective excretory power of the kidney. Anyhow, such differences may be taken as indicating that the kidney can handle certain constituents better than others, and suggest in each case more or less restriction of water, salt, or protein in the diet, as the case may be.

This so-called two-hour test is particularly serviceable as indicating the lesser degrees of renal disturbance, for we often get changes by this test when other tests indicate almost no departure from the normal. In more marked renal damage the two-hour test shows the decreased excretion and fixation in amount, but after these have reached a certain degree relatively little more change occurs with increasing renal lesion, so that here the two-hour test is not of service in measuring increasing degrees of renal damage, though it indicates that the damage exists and is extensive.

As the kidney is an excretory organ and the substances which it excretes are present, in part at least, in the blood in the form they have 
in the urine, when their excretion is interfered with, it would be expected that they would be found in the blood in increased amount, and these increases would serve as an index of renal insufficiency. Such is the case. Estimates of these substances long have been possible, but as the methods required a considerable amount of blood or were in themselves difficult to carry out, they were not of much practical use. More recently, however, much simpler methods have been devised and of a nature requiring only small amounts of blood. With the advent of these methods, practical application to our patients has been possible. Folin $\left(^{5}\right)$ has done much to develop these methods and has described colorimetric methods for quantitating total non-protein nitrogen, urea, uric acid, creatin, and creatinin, all substances which are increased in the blood when different degrees of renal lesion exists. Marshall's ( ${ }^{\circ}$ ) urease method of determining blood urea has been particularly helpful in clinical work and is the one most generally used in the study of nephritis. McLean and Van Slyke $\left({ }^{7}\right)$ have developed a good method of estimating blood chlorides.

Without burdening the reader with the details of the technique of these various methods, it is sufficient to point out that the underlying principle of their use as a test of renal function lies in the assumption that the increase of these substances in the blood depends on decreased renal excretion, and that the amount in the blood is proportionate to the degree of renal damage. In general this is true, but not wholly so. Their amount in the blood is to a considerable extent dependent on food intake. With the renal lesion unchanged the blood urea nitrogen, for example, will increase or decrease with variations in protein intake. This must not be lost sight of in interpreting results. Then destruction of body protein is another factor in increases, though obviously such a source is an indication of serious disease. Finally there are some conditions in which blood urea and total non-protein nitrogen increase where the relation to renal function is not so clear. Such a condition is intestinal obstruction. This has been particularly well shown by Whipple $\left({ }^{8}\right)$ and his associates in experimental studies of the effects of duodenal secretion and of duodenal obstruction. Uric acid, according to Myers and Fine $\left({ }^{\circ}\right)$, increases in the blood in nephritis earlier than the other protein substances. An increase in uric acid also occurs in gout even when there is no demonstrable nephritis. With these various factors in mind the estimation in the blood of these several substances becomes of much aid in the diagnosis and prognosis of renal lesions.

The ratio between non-protein nitrogen in blood and urine has been expressed in various formulae (Ambard's coefficient and McLean's index) in the effort to give a numerical value for renal function. The 
relationship between blood and urine nitrogen obviously is important, but the formulae so far introduced seem to be too artificial to express adequately the relationship.

\section{Tests of Digestive Function}

The gastrointestinal tract very early was investigated by means of tests of function. Gastric analysis following various types of test meals is in essence a function test. The meal is given and after a certain time the content of the stomach is sampled with a view to determining the character and relative quantity of the various active substances in the gastric juice. The function, in part, of the stomach is to secrete gastric juice of a certain kind under the stimulus of the presence of certain foods. Has the normal amount of free and combined hydrochloric acid appeared? Has pepsin been secreted? These are questions of function answered by the chemical analysis of the gastric juice. This knowledge is of value in both the diagnosis and treatment of stomach disease.

The stomach tube, by the fractional method of gastric analysis as especially developed by Rehfuss $\left({ }^{10}\right)$, gives valuable information as to the rate and prolongation or delay of secretion of components of the gastric juice. This method really enables us to sample and quantitate the gastric juice at successive periods of digestion and obtain still more complete knowledge of gastric secretion.

The same methods of gastric analysis give information in regard to gastric motility, emptying time, pyloric obstruction, etc. This information is furnished more completely by the X-ray examination of the stomach after a meal mixed with a substance opaque to the rays has been given. Here we are able to study with minuteness the peristaltic waves as they pass along the stomach and note their influence in mixing gastric contents with gastric juices and eventually emptying the gastric contents into the duodenum-a study of gastric motor function.

By the same X-ray methods intestinal motor function is studied. Rates of progress of intestinal contents through different portions of the intestine can be observed. Stasis and its dilating effects can be noted. In all parts of the gastrointestinal tract the effect on function of local lesions, such as ulcer or tumor, can be observed with the X-ray in so far as they influence motility.

With the duodenal tube $\left({ }^{11}\right)$ samples of bile and pancreatic secretion can be obtained for study and some knowledge obtained as to the function of the liver and pancreas, two very important accessory glands of the gastrointestinal tract. Methods of studying the secretory activity of the liver, such as that introduced by Rowntree $\left({ }^{12}\right)$ in the use of tetra- 
chlorphthalein, which is a dyestuff excreted solely through the biliary tract, have been introduced, and though sound in principle, so far these methods have been of relatively little practical value.

Test intestinal meals have been used. The studies of Schmidt and Strasburger $\left({ }^{18}\right)$ are good examples of these methods. They prepare a standard meal containing an oatmeal porridge rich in butter fat, potato soup, and finely cut up beef cooked rare, as the essential parts. Following this meal certain amounts of undigested meat fibers and starch granules, fat droplets, fatty acid crystals, and soaps should be found in a bit of stool examined under the microscope. Striking increases in these point to very rapid progression of material through the intestine or to insufficient activity of pancreatic and other enzymes or to bile deficiencies. With other data obtained from the study of the patient, judgment as to intestinal function can be obtained by such a method.

A number of methods $\left({ }^{14}\right)$ for measuring the activity in the stool of various enzymes contributed by the intestinal tract to its contents have been used, but on the whole these have not been of very great practical help. They are, however, distinct methods of studying digestive function, and further improvements in them may make them of greater use in the study of patients.

\section{Tests of Circulatory Function}

Measures of cardiac function, in particular measures of cardiac reserve, are needed in every case we meet showing disturbed circulation. To a very large extent we have depended for this on subjective symptoms, on respiratory rate, on cardiac rate, etc., as influenced by exertion. These are of very great value in forming a judgment as to how well the circulation is maintained under the ordinary conditions of the daily demands of life. These are, however, all complex functions involving cardiac activity, vasomotor changes, respiration, etc., and in ratio as they are complex, they are difficult of evaluation. Such changes are not well suited as values to compare from period to period in the individual, and are particularly unsuited for comparison between different individuals, especially because of that very unmeasurable factor, individual susceptibility to change, which depends in so large a measure on the nervous mechanism and its balanced or unbalanced activities. What is needed is some measured value which expresses cardio-circulatory efficiency under rest and measured exercise as a basis for comparison between different periods in the same individual and between different individuals, and various attempts have been made to provide such tests.

A number of ways have been used to express circulatory function. The increase in heart rate following exercise, and especially the length of 
time required for a return to the normal rate during rest following exercise, has been used as a measure of function, and this gives reasonably satisfactory evidence of a disturbed circulatory mechanism. Methods of this type have been utilized in the cardiovascular examinations for army service, and when such exercises as hopping 50 to roo times on one foot produced an increased heart rate slowly recovered from or undue breathlessness, these individuals were regarded as unsuited for military service. Such tests, however, obviously are unsuited to patients with any degree of cardiac decompensation and only poorly express circulatory efficiency.

By measuring reasonably accurately the amount of work done in exercises by an ergometer and determining the systolic blood pressure and pulse rate at frequent intervals thereafter, Barringer $\left({ }^{15}\right)$ claims that he has a satisfactory measure of the heart's functional capacity. With a normal individual a rise in blood pressure and in pulse rate follows work to return promptly to the level preceding the work. The greater the work the higher are the subsequent systolic blood pressure and pulse rate until a certain amount of work is done. Beyond this the curve changes so that now the blood pressure rise is delayed and does not appear until the pulse rate is decreasing. According to Barringer the amount of work necessary to produce this change does not vary much from day to day in the normal individual. With varying grades of cardiac insufficiency much smaller amounts of work are needed to produce the delayed rise, and these facts serve as a basis for a measure of functional capacity of the circulation. This method of Barringer has been criticized adversely by Rapport $\left({ }^{16}\right)$, who has used an improved technique which permits of more rapid and frequent determinations of pressure and pulse rate. Certainly the simple determination of blood pressure in itself is an extremely inadequate index of cardiac function, for we know that even during evident and marked decompensation blood pressure remains at a normal or elevated level, and this level is maintained almost to the end unless there is a marked peripheral vascular dilatation.

Blood pressure and pulse rate changes under other conditions have been utilized as indications of circulatory function. In studying soldiers with cardiovascular asthenia, the changes following the subcutaneous injection of 0.5 c.c. of a $I$ to 1000 adrenalin solution have been taken to indicate function $\left({ }^{17}\right)$. If pressure and rate increase, accompanied by symptoms such as flushing, sweating, increased vascular pulsation, increased tremor, restlessness, and more or less marked general nervousness, the test is positive and indicates a neurovascular instability likely to unfit for vigorous exertion. Though this is not an effect from cardio- 
circulatory disease, it is in a sense an indication of poor function involving cardio-circulatory apparatus as well as other body functions.

Rather different in principle are tests that are intended to measure circulatory efficiency by estimating respiratory activity-an indirect sort of test of cardiac function. Such a method is that for measuring pulmonary vital capacity by means of a spirometer $\left({ }^{18}\right)\left({ }^{19}\right)$. This in essence consists in determining the maximum amount that the individual can expire and comparing it with the average for normals of the same sex and approximate height. A decrease from the average normal indicates circulatory insufficiency when local pulmonary disease is excluded, and changes in vital capacity indicate a change in cardiac efficiency.

Another principle that has been used to test cardiac function consists in observing the change in heart size, mainly changes in the right border, produced by holding one's breath or following exercise and noting the time required for the heart to return to its previous size. This can be done most accurately when the heart is viewed under the fluoroscope.

The string galvanometer in the form of the electrocardiogram has thrown some light on cardiac function, but this information is of relatively little help as a test of cardiac function, though certain changes, as widening and notching of the $Q \mathrm{R} \mathrm{S}$ complex $\left({ }^{20}\right)$ and inversion of $\mathrm{T}$ waves $\left({ }^{21}\right)$ in certain leads indicate a seriously damaged heart muscle.

None of these tests, however, are very satisfactory as measures of circulatory function, and so far as the heart and peripheral vessels are concerned a careful history of symptoms in relation to exercise remains the most valuable basis for estimating cardiac efficiency.

\section{Basal Metabolism as a Measure of Funtion}

Basal metabolism determinations serve as another example of methods of measuring function. These, for example, are particularly serviceable along with other data, as indicating thyroid activity. These methods are described and discussed by Du Bois in Chapter XVII and by Peabody in Chapter XVI of this volume.

The several methods discussed above indicate the underlying principles and the scope of functional tests; no completely inclusive cataloguing of these methods has been undertaken; a number of tests of function will be found referred to in various chapters of the System in connection with the descriptions of disease conditions.

\section{BIBLIOGRAPHY}

I. ROWNTREE, L. G., and GERAGHTY, J. T.: Jour. Pharm. and Exper. Therap., 1909-10, I, 579.

2. KENDALL, E. C.: Jour. Am. Med. Assoc., 1917, LXVIII, 343. 
3. v. MONAKOW, PAUL: Deutsch. Arch. f. klin. Med., IgI I, CII, 248.

4. MOSENTHAL, H. O.: Arch. of Int. Med., 1915, XVI, 733.

5. FOLIN, O., and DENIS, W.: Jour. Biol. Chem., 1912, XI, 527; 1913, XIII, $469 ; 1916, \mathrm{XXVI}, 491$.

DENIS, W.: Jour. Biol. Chem., I918, XXXV, 513.

FOLIN, O., and WU, H.: Jour. Biol. Chem., I9I9, XXXVIII, 8I.

6. MARSHAL, E. K.: Jour. Biol. Chem., I9r3, XV, 487.

7. McLEAN, F. C., and VAN SLYKE, D. D., Jour. Biol. Chem., 1915, XXI, $36 \mathrm{I}$.

VAN SLYKE, D. D., and DONLEAVY, J. J.: Jour. Biol. Chem., I9I9, XXXVII, 55I.

8. COOKE, J. V., RODENBAUGH, F. H., and WHIPPLE, G. H.: Jour. Exp. Med., I9I6, XXIII, 7I7.

WHIPPLE, G. H., and VAN SLYKE, D. D.: Jour. Exp. Med., I918, XXVIII, 213.

COOKE, J. V., and WHIPPLE, G. H.: Jour. Exp. Med., I9I8, XXVIII, 243.

9. MYERS, V. C., and FINE, M. S.: Arch. of Int. Med., I9I6, XVII, 570.

ro. REHFUSS, M. E., BERGEIM, O., and HAWK, P. B.: Jour. Am. Med. Assoc., I9I4, LXIII, 909.

REHFUSS, M. E.: Am. Jour. Med. Sc., I914, CXLVII, 848.

II. EINHORN, MAX: Am. Jour. Med. Sc., I9I6, CLI, 202.

12. ABEL, JOHN J., and ROWNTREE, L. G.: Jour. Pharm. and Exp. Therap., I909, I, 23I.

ROWNTREE, L. G., HURWITZ, S. H., and BLOOMFIELD, L. A.: Bull. Johns Hopkins Hosp., I9I3, XXIV, 327.

13. SCHMIDT, A., and STRASBURGER, J.: Die Fäzes des Menschen, Berlin, IgI5.

I4. BROWN, T. R.: Boston Med. and Surg. Jour., IgI6, CLXXV, 775.

I5. BARRINGER, T. B.: Arch. of Int. Med., 19I6, XVII, 363 and 670; 19I7, $\mathrm{XX}, 829$.

16. RAPPORT, D. L.: Arch. of Int. Med., I9r7, XIX, 98I.

17. PEABODY, F. W., CLOUGH, H. D., STURGIS, C. C., WEARN, J. T., and TOMPKINS, E. H.: Jour. Am. Med. Assoc., I9I8, LXXI, I9I2.

I8. PEABODY, F. W., and WENTWORTH, J. A.: Arch. of Int. Med., I9I7, $\mathrm{XX}, 443$.

19. McCLURE, C. W., and PEABODY, F. W.: Jour. Am. Med. Assoc., I9I7, LXIX, I954.

20. OPPENHEIMER, B. S., and ROTHSCHILD, M. A.: Proceed. Soc. for Exp. Biol. and Med., 1916, XIV, 57, and Jour. Am. Med. Assoc., 1917, LXIX, 429.

CARTER, E. P.: Arch. of Int. Med., I9I8, XXII, 33I.

NEUHOF, S.: Arch. of Int. Med., 1918, XXII, 45.

21. LEWIS, T.: Mechanism of the Heart Beat, Shaw \& Sons, London, I9II, p. 74 .

SMITH, F. M.: Arch. of Int. Med., I9I8, XXII, 8. 


\title{
CHAPTER XVI
}

\section{RESPIRATION IN DISEASE}

\author{
By FRANCIS W. PEABODY
}

\section{TABLE OF Contents}

Introduction.

Minute-Volume of Pulmonary Ventilation.

Vital Capacity of the Lungs.

Lung Volumes.

Irregularity of Respiration.

BEFORE taking up the subject of the respiration in pathological conditions, it may be well to consider briefly a few facts concerning normal breathing and the methods by which the respiration responds to any unusual demands that may be made upon it. It is in general customary to separate sharply the "internal respiration," between the tissues and the blood, from the "external" or "pulmonary respiration," between the blood stream and the air outside the body. Such a distinction is, however, justifiable in only a limited sense, and, although serving a purpose, it tends to obscure the broader point of view from which the subject should properly be approached. In attempting to analyze too minutely the different phases of the respiration, the student of physiology or of physiological pathology is liable to lose sight of the fact that he is in reality investigating, not a single independent function, but a group of interacting functions, each influencing and being in turn influenced by the other. This intimate relationship and interdependence exists not only between the various functions of the respiration, but also between the respiration as a whole and the circulation of the blood.

It is, indeed, perhaps unfortunate that the respiration and the circulation are so generally regarded and studied as separate entities, for a better understanding of each would result if attention was more frequently focused on what might be termed the "circulatory-respiratory" mechanism. $U_{p}$ to a certain point the circulation and the respiration must be studied separately, and the various details of each require intensive investigation along more and more sharply defined lines if the limits of our knowledge are to be expanded. But when, as clinicians, we approach the problem of disease, it is essential to look at the organism 
as a whole and to appreciate how closely the various physiological functions are interrelatęd. In the normal living organism respiration and circulation go hand in hand. Thus for instance the blood carries the hormone of the respiration to the medulla, and the stimulation of the respiratory center causes an increase in the pulmonary ventilation. This increase in ventilation helps to supply the coronary vessels of the heart with a normal blood and it also exerts a direct action on the circulation by facilitating, during inspiration, the blood-flow from the great veins to the right side of the heart. But behind the circulation and the respiration, dependent on them and at the same time the most important factor in their regulation, lie all the complex chemical processes which go on in the tissues and which are customarily summed up under the general caption of the metabolism. The continuation of life itself is determined largely by the carrying on, within certain rather definite normal limits, of the metabolic processes of the body cells, and the normal activity of these metabolic processes is in great degree dependent on the adequate provision of oxygen and on the adequate removal of carbon dioxid. Under normal conditions the body metabolism is probably at its lowest ebb during deep sleep, but for purposes of general comparison, the usually adopted standard is the so-called basal metabolism, - the metabolism when the body is at complete rest, and when the digestive processes are at a minimum. Under these conditions, the contraction of the heart and the tonus of the blood vessels are so regulated as to provide sufficient oxygen to meet the needs of the cells and to remove the carbon dioxid that results from metabolic activity. At the same time, the movements of the lungs are regulated so that the rate and depth of respiration is such as to provide, in an economical manner, the entrance into them of sufficient atmospheric air to insure the proper saturation of the blood with oxygen and the discharge from it of excess carbon dioxid. But during the course of normal life there are many factors,- such as the taking of food, muscular movement, or nervous activity,--which increase the metabolism and thus create an increased demand for oxygen on the part of the cells involved. To this increased demand the organism responds by a complex series of adjustments on the part of the respiration and circulation which are aimed at allowing the body to carry on its activities at the new, higher level. The changes in the circulation are such as to increase the blood-flow. In general they consist in an increase in the rate of the heart, an increase in the output of the heart per beat, a rise of blood pressure, and vascular contraction or dilatation in the various parts of the body. As a result of this, the blood-flow through the lungs is augmented. More blood passes through the pulmonary 
vessels, and the time during which the blood is in the pulmonary capillaries is decreased. In order, therefore, that the blood shall be adequately aërated, the composition of the air in the alveoli of the lungs must be kept such that its oxygen tension is high enough to saturate the increased amount of hemoglobin which passes the lungs in a decreased time, and the carbon dioxid tension must be kept so low that the increased amount of carbon dioxid in the venous blood will diffuse out readily into the air of the alveolar spaces. To accomplish this the air in the lungs, and especially that in the alveoli, must be more completely diluted by atmospheric air. This is brought about by increasing the rate and the depth of respiration,-both of which actions increase the volume of air entering the lungs in a given time, or the so-called minute-volume of pulmontary respiration.

These adjustments may be brought about with astonishing rapidity, as is seen at the onset of severe physical work. The response of the circulation is probably initiated, and perhaps regulated, by nervous reflexes. That of the respiration is directly regulated by the metabolic processes themselves, for the stimulus to the respiratory center is the increase of the $\mathrm{H}$-ion concentration of the blood. It would appear that these adjustments to an increase of metabolism are adapted to the needs of the organism in an astonishingly accurate and economical manner. Blood-flow and pulmonary ventilation are raised to fulfill the demands of the tissues, but not much beyond these demands, and there is a harmonious interrelation between the increase of blood-flow and that of pulmonary ventilation. No considerable increase of body metabolism could be met by increasing the blood-flow or the pulmonary ventilation alone. When, however, the circulation and the respiration are functioning normally the metabolism may be raised four or five times, and in trained persons nearly ten times, above the basal value without the organism suffering from an insufficient supply of oxygen or from an accumulation of carbon dioxid. It will thus be seen that the reserve on the part of the "respiratory-circulatory mechanism" is comparatively great.

Conditions analogous to those which have just been mentioned are also met with in the various pathological states which are associated with an increased metabolism. Febrile diseases and hyperthyroidism are the conditions which are perhaps most definitely characterized by increased heat production, but the same thing, to a lesser extent or less constantly, may be found in diabetes, anemia, advanced nephritis, and various other diseases. Here again the body meets the increase of metabolism in the normal way by increasing the rate of blood-flow and of pulmonary ventilation. Inasmuch as the increase of metabo- 
lism due to disease is rarely more than roo per cent. above the normal basal value, it is comparatively easy for the body to meet the demands of the tissues as long as the circulation and the respiration are normal. Various other abnormal conditions such as acidosis, oxygen lack, and nervous influences behave in a manner similar to an increase in metabolism and stimulate the respiratory center. The result is again an increase in minute-volume of pulmonary respiration. At the same time there are other pathological conditions which prevent the organism from responding to these stimuli and which limit the degree to which the minute-volume can be raised. An increase in minute-volume is brought about largely by increasing the volume of each respiration, and so it is that those factors which prevent deep breathing are important in the pathology of the respiration. These influences manifest themselves in various ways, but chiefly by causing a diminution in the vital capacity of the lungs. What may be termed the "reserve" of the pulmonary respiration,-its power of responding to demands over and above what is required at rest,-may be expressed by the difference between the ventilation of the lungs at rest and the highest degree of pulmonary ventilation which the subject is capable of maintaining.

Under normal conditions, with the body at complete rest, the movements of the thorax and diaphragm are such that about four or five liters of air enter and leave the lungs per minute, and this is sufficient for the gaseous requirements of the body. When the metabolism is increased so that the body needs greater amounts of oxygen and larger quantities of carbon dioxid must be removed, the respiratory center is stimulated, with the result that the rate, and more especially the depth, of breathing is increased. By this means the volume of air breathed per minute (the minute-volume) can be enormously increased. When walking quietly the pulmonary ventilation is two or three times what it is when lying down, and in some observations made on healthy young men, it was found that when riding a bicycle at a high rate of speed, the minutevolume of air breathed averaged nearly ten times what it was while they were sitting on the bicycle at rest. This gives a rough index of the normal "reserve" of the pulmonary respiratory mechanism. Many of the pathological conditions affecting the respiration act essentially by reducing this reserve, and it is evident that they may do so either by increasing the minute-volume of air breathed while at rest, or by decreasing the maximum minute-volume which the patient is capable of breathing. These two influences will be considered under the headings of "The Minute-Volume of Pulmonary Ventilation" and "The Vital Capacity of the Lungs." In addition to these certain other conditions bring about interesting and important "Irregularities of the Respira- 
tion." When one considers these various abnormalities, it is important to remember that disturbances of the respiration, the circulation, and of the blood itself often go hand in hand, and it is only by a somewhat arbitrary distinction that the present discussion is limited to the external respiration.

\section{The Minute-Volume of Pulmonary Ventilation}

The most common form of abnormal respiration met with in disease is hyperpnea, or an increase in the amount of air breathed per unit of time above the normal value for the subject when at rest. Hyperpnea may be due to an increase in the depth of respiration, or in the rate of respiration, or it may be due to a combination of the two. If the abnormality is essentially one of rate, one may speak of a tachypnea or polypnea. It is important, however, that hyperpnea should not be confused with dyspnea. Dyspnea, as the derivation of the word indicates, is a difficult or labored breathing, and there is implied in it an element of subjective discomfort. This subjective distress apparently arises from the fact that either the circulation or the respiration or both are not able to keep pace with the gaseous requirements of the body. The reserve power of the circulation or respiration has been used up. In so far as dyspnea is dependent on the respiration, it is caused by an inadequate aëration of the blood in the lungs. This may be due to several causes, one of which is an inability of the subject to increase his pulmonary ventilation sufficiently to produce an adequate degree of hyperpnea. Dyspnea is usually associated with hyperpnea, but whether in a given instance hyperpnea is associated with dyspnea will depend on the degree to which the pulmonary ventilation is increased, and on the ability of the subject to raise his minute-volume to that degree easily.

There are various factors which may cause an increase in the minutevolume of pulmonary respiration. Thus it may be brought about by an increase of metabolism, whether this be due to a physiological process, as exercise, or to a pathological process, as in Graves disease. Hill and Flack $\left({ }^{1}\right)$ have shown the effect of temperature in raising the ventilation by immersing subjects in a hot bath at a temperature of $105^{\circ}$ to $I 10^{\circ} \mathrm{F}$. The minute-volume rose to 20 or 30 liters, and even, in one instance, to 50 liters. Another type of hyperpnea is that caused by stimulation of the respiratory center in pathological conditions associated with acidosis. Hyperpnea is also seen frequently in various nervous disturbances, such as hysteria.

Closely related to the subject of pulmonary ventilation is the question of alveolar ventilation or the volume of air which actually reaches the alveolar spaces per minute. From the point of view of the respiratory 
exchange, the important thing is, not how much air enters the mouth and nose in a given time, but how much air enters the alveoli of the lungs and is able to take part in the gaseous exchange with the blood. With each breath a part of the air inspired remains in the buccal and nasal cavities, in the larynx, trachea, and bronchi. This portion of the inspired air does not take part in the respiratory exchange of gases, and the area which it occupies is known as the dead space. Haldane $\left({ }^{2}\right)$ and Henderson, Chillingworth, and Whitney $\left({ }^{3}\right)$ have shown that the dead space for oxygen is larger than that for carbon dioxid, and the latter explain this on the basis that considerable amounts of carbon dioxid may diffuse out from the blood in the walls of the respiratory passages. The dead space is to be regarded as a physiological, and not as an anatomical conception. As Haldane says (loc. cit.): "The magnitude of this space depends on the physiological efficiency of the respiratory surface in relation to the supply of venous blood and fresh air." Much work has been done on the determination of the size of the dead space, both with normal respiration at rest and under various conditions such as hyperpnea, but the results obtained by different investigators are not in harmony. The generally accepted value for the dead space in normal persons at rest is not far from 120 to 140 c.c. The authors just cited agree in finding that the dead space is much larger when the respirations are deep, either at rest or with exercise, but Pearce $\left({ }^{4}\right)$ states that he found only a small variation in the dead space between the conditions of rest and exercise consisting of walking $3^{1 / 2}$ miles an hour, and Krogh and Lindhard $\left({ }^{5}\right)$ say that with the maximum inflation of the lungs the increase in the size of the dead space may amount to roo c.c.

Until the vexed question as to the size of the dead space in normal individuals is definitely settled, it is perhaps best to be somewhat skeptical with regard to the observations which have been made on it in pathological conditions. Such determinations as have been made, however, indicate that there may be a considerable change in the dead space in different diseases. Thus there is good evidence to show that the dead space is increased in emphysema. An increase in the size of the dead space is a factor which may cause hyperpnea, because with a large dead space there will be less of each individual respiration that will reach the alveoli than with a small dead space. If, for instance, a subject breathes Io times a minute, and each respiration is of 400 c.c., his total minutevolume is 4,000 c.c. With a dead space of 120 c.c. only 280 c.c. of each respiration will take part in the gaseous exchange, and the alveolar ventilation will, therefore, equal $280 \times$ Io or 2,800 c.c. per minute. This is presumably the amount of alveolar ventilation required by the metabolic activities of the body. If, then, the dead space were 
increased to 200 c.c., only 200 c.c. of each respiration of 400 c.c. would reach the alveoli, and the minute-volume of alveolar ventilation would be $200 \times$ IO or 2,000 c.c. But this is below the needs of the body, and the ventilation must be raised so as to compensate for the increase in dead space. This may be done by increasing the rate or depth of breathing. If the volume per respiration is kept constant at 400 c.c., the requisite alveolar ventilation will be obtained if the rate is increased to 14 per minute $\left(400-200 \times I_{4}=2,800\right)$, but the total minute-volume will then be 5,600 c.c. On the other hand, if the rate is kept constant, the necessary alveolar ventilation will be reached if the volume of each respiration is increased to 480 c.c. $(480-200 \times 10=2,800)$. In this case, the total pulmonary ventilation will be 4,800 c.c. It will be seen that an increase in the size of dead space necessarily brings about a hyperpnea in order that the alveolar ventilation may be maintained, but the hyperpnea will be less in degree if compensation is brought about by increasing the depth of respiration than if it is done by increasing the rate of respiration. The dead space may also be an element in the production of hyperpnea in cases where the respiratory surface is cut down, as occurs when a large pleural effusion compresses one lung. In such an instance the area of the dead space is not affected, but the proportion of each inspiration that remains in the dead space is larger than normal. The result will again be that the necessary alveolar ventilation must be obtained by the development of an increased pulmonary ventilation.

Strictly speaking, it would of course be of greater clinical significance to determine the alveolar ventilation than it is to determine the total pulmonary ventilation, for it is the former that takes an active part in the exchange of gases with the blood. Hitherto, however, this has not been very practicable. The calculation of the alveolar ventilation involves a knowledge of the size of the dead space, and the methods for obtaining this are too complicated and difficult to apply extensively to patients. There is also, as has been indicated, some doubt as to their reliability. The alveolar ventilation may, of course, be calculated by assuming a normal or standard figure for the dead space, but, especially in pathological conditions, such an assumption may be far from accurate and may lead to large errors. Until, therefore, more information is gathered concerning the dead space in normal persons and in disease, it may be best to devote attention chiefly to the total ventilation,- a value that can be readily determined.

The method of determining the total pulmonary ventilation is on the whole very simple. The subject should lie at rest in bed for at least I5 minutes before the observation is begun. It is only for the most 
highly accurate and strictly comparable results that it is necessary that he should be in the fasting state required in basal metabolism observations, but it is on the whole better not to make determinations within one or two hours after a hearty meal. The subject is then made to breathe through valves separating the inspired from the expired air, and the latter is measured for a known period of time. The best way to collect the expired air is in a large (IOo liter) spirometer, but as accurate results can be obtained if the expired air is allowed to pass through a carefully calibrated gas meter. When patients cannot be moved to the laboratory it is often convenient to collect the air in a Douglas bag $\left({ }^{6}\right)$ and then measure it by passing it through a gas meter. In order to avoid any error due to the breathing being uneven from minute to minute, the expired air should be measured over a period of at least 5 minutes. Results that are comparable from one patient to another are best obtained if the temperature of the expired air and the existing barometric pressure are taken, and the volume of air reduced to standard temperature and pressure conditions, dry $\left(0^{\circ} \mathrm{C}\right.$., and $760 \mathrm{~mm}$.). The chief difficulty consists in the fact that it is not always easy to collect the expired air without interfering to some degree with the normal respiration of the subject. The easiest way is to have the valves attached to a rubber mouthpiece, with a tube to breathe through about $1.5 \mathrm{~cm}$. in diameter, and a wide flange to fit between the teeth and cheeks to prevent leakage. The nose is then closed with a clip. This method has the disadvantage that most people do not breathe naturally through their mouths, and when the nose is closed they have a tendency to overventilate. It is therefore better to use some form of mask which covers both mouth and nose, and allows the subject to breathe through either. Even with this arrangement some trouble is often found with nervous patients, and especially with patients who are dyspneic and who fear that the apparatus may cut off some of the air. It is in all cases essential that the subjects should breathe through the apparatus for some minutes before the measuring of the air is begun, so that they may get used to it, and may get over any abnormalities of respiration which may arise when the apparatus is first applied.

The rate of respiration is most accurately determined by means of a pneumograph attached to the patient's chest, and registering by a simple tambour on a revolving drum. The time intervals should be marked on the drum by some form of time-marker. Having thus obtained the volume of air expired over a known period of time, and the rate of respiration, the minute-volume of ventilation and the volume per single respiration can be calculated.

The normal figures for the minute-volume of pulmonary ventilation 
show a considerable amount of variation, depending largely on the size of the individual. Even with the same alveolar ventilation, the pulmonary ventilation in the same individual will vary somewhat according to whether he is breathing slowly and deeply or rapidly and superficially. There is thus no such close relationship between pulmonary ventilation and body surface-area as there is between the latter and basal metabolism. Sufficient accurate data are not at hand to enable us to know whether there is a close relation between alveolar ventilation and body surface-area. The average minute-volume of pulmonary respiration for adults, at rest, is between 4 and 6 liters, measured at standard temperature and pressure, dry. Debenham and Poulton $\left({ }^{7}\right)$ have made careful observations on Io normal men and give as their results figures from 4.87 to 7.55 liters. The average is 6.06 liters. These volumes are, however, measured at $37 .^{\circ} \mathrm{C}$. and prevailing atmospheric pressure saturated with moisture. Reduced to $0^{\circ} \mathrm{C}$., dry, the average minutevolume would be about 5.0 liters. The subjects of these observations were studied while resting on a bed and in the "fasting" state. Schlesinger and Pembrey $\left({ }^{8}\right)$ found the average minute-volume to be 7. 105 liters, uncorrected for temperature, in a group of medical students who were examined during the course of their usual day's work.

Debenham and Poulton give as the mean tidal air (volume per respiration) 4ro c.c., with a maximum of 524 c.c. and a minimum of 3 IO c.c. Corrected to standard temperature and pressure, the average would be about 335 c.c. The same authors also determined the dead space in their subjects and calculated the average alveolar ventilation as 3.94 liters per minute.

Hyperpnea may occur in pathological conditions as the only or the chief abnormality of the respiration, or it may be one factor in a clinical picture which is complicated by other processes interfering with the pulmonary respiration. As an uncomplicated feature it is most often seen in association with an increase of metabolism, an acidosis, or in certain conditions which are probably of a nervous origin.

Investigations of the gaseous exchange have shown that an increase of metabolism may be present in many pathological conditions. No attempt will be made to consider all of these, but some of the more important may be referred to. Thus many observations have shown a rise of the basal metabolism in fever. Typhoid fever has perhaps been as thoroughly studied as any febrile disease, and Coleman and $\mathrm{Du}$ Bois $\left({ }^{\circ}\right)$ state that the average increase is approximately 40 per cent., although " figures over 50 per cent. are frequently encountered." A rise in metabolism may also take place in anemia. Meyer and Du Bois $\left({ }^{10}\right)$ found an increase of 7 to 33 per cent. in the oxygen consumption in two severe cases of anemia with a hemoglobin percentage of less than 
25 , but in two milder cases, with a hemoglobin percentage between 40 and 44 , there was no definitely pathological increase in metabolism. In leukemia the metabolism may be much above normal, and the increase appears to be rather closely parallel to the rise in the leucocyte count. The increase in metabolism is not to be accounted for by the degree of anemia. Thus in Case 5 (Table III) the metabolism was 20 per cent. above normal at a time when the hemoglobin was 75 per cent. and the leucocyte count about 90,000, and in Case 6 the metabolism was 65 per cent. above normal when the hemoglobin was 50 per cent. and the leucocytes numbered approximately 950,000. The basal metabolism has also been studied in many cases of exophthalmic goiter, and in general it has been found that there is a close relationship between the rise in metabolism and the severity of the disease. In marked cases the metabolism rises to a higher degree in this condition than in any other disease that as yet has been investigated carefully. In Table III are given the results obtained in the study of the basal metabolism of cases of exophthalmic goiter and myelogenous leukemia at the Peter Bent Brigham Hospital. The expired air was collected in a large Tissot spirometer, and the metabolism calculated in the usual way on the basis of oxygen consumption and respiratory quotient. These cases enable one to see definitely the relation of increased metabolism to hyperpnea, for it was possible to make observations showing great variations in the metabolism in the same individual. In the patients with exophthalmic goiter the fall in basal metabolism followed in three instances an operation for thyroidectomy, and in the patients with leukemia it followed radium treatment which brought their leucocyte counts down to normal figures. It will be noticed that the pulmonary ventilation increases with the rise in metabolism, but there is no very definite relationship between the two. The extent to which the increase in minute-volume of air breathed depended on an increase of rate or of depth varied in the different cases. It is especially noteworthy that even with the highest figures for basal metabolism, the minute-volume was not as much as twice what it was when the metabolism was normal. The "reserve" of the pulmonary mechanism is so great that the minutevolume can be doubled easily if the lungs are normal. It is unlikely, therefore, that a rise in metabolism, uncomplicated by other features, is in itself an important factor in causing dyspnea while a patient is at rest. Its chief significance is when it is associated with other conditions which interfere with the respiration, and also on account of the fact that the increased minute-volume at rest serves to cut down the "reserve" which will be called on when any further demands, such as occur in exercise, are made on the respiratory system. 


\section{TABLE III}

\section{Relation of Pulmonary Ventilation to Metabolism}

(Metabolism is expressed in percentage of normal according to the standards of age and surface area of Du Bois)

\begin{tabular}{|c|c|c|c|c|c|c|c|c|c|}
\hline $\begin{array}{l}\text { Case } \\
\text { No. }\end{array}$ & Diagnosis & Age & Sex & $\begin{array}{l}\text { Metabo- } \\
\text { lism in } \\
\text { per cent. } \\
\text { of Normal }\end{array}$ & $\begin{array}{c}\text { Minute- } \\
\text { Volume, } \\
\text { Liters. } \\
\text { S. T. P. D. }\end{array}$ & $\begin{array}{l}\text { Rate } \\
\text { of } \\
\text { Resp. }\end{array}$ & $\begin{array}{c}\text { Volume } \\
\text { per } \\
\text { Resp. } \\
\text { c.c. }\end{array}$ & $\begin{array}{l}\text { Pulse } \\
\text { Rate }\end{array}$ & Remarks \\
\hline I & $\begin{array}{l}\text { Exophthalmic } \\
\text { Goiter }\end{array}$ & 21 & M. & $\begin{array}{l}+83 \\
+65 \\
+22\end{array}$ & $\begin{array}{l}9.32 \\
7.67 \\
6.16\end{array}$ & $\begin{array}{l}24.1 \\
20.9 \\
16.3\end{array}$ & $\begin{array}{l}388 \\
367 \\
379\end{array}$ & $\begin{array}{r}127 \\
129 \\
88\end{array}$ & $\begin{array}{l}\text { After thy- } \\
\text { roidectomy }\end{array}$ \\
\hline 2 & $\begin{array}{l}\text { Exophthalmic } \\
\text { Goiter }\end{array}$ & 19 & F. & 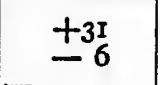 & $\begin{array}{l}6.46 \\
4.54\end{array}$ & $\begin{array}{l}22.7 \\
18.4\end{array}$ & $\begin{array}{l}285 \\
247\end{array}$ & $\begin{array}{l}8 \mathrm{r} \\
69\end{array}$ & \\
\hline 3 & $\begin{array}{c}\text { Exophthalmic } \\
\text { Goiter }\end{array}$ & $\begin{array}{l}25 \\
28\end{array}$ & M. & $\begin{array}{l}+73 \\
+46 \\
-4\end{array}$ & $\begin{array}{r}\text { II.IO } \\
8.51 \\
6.16\end{array}$ & 15.9 & 353 & $\begin{array}{r}135 \\
118 \\
88\end{array}$ & $\begin{array}{l}\text { After thy- } \\
\text { roidectomy }\end{array}$ \\
\hline 4 & $\begin{array}{c}\text { Exophthalmic } \\
\text { Goiter }\end{array}$ & 38 & M. & $\begin{array}{l}+69 \\
+3\end{array}$ & $\begin{array}{l}8.84 \\
5.32\end{array}$ & $\begin{array}{l}21.3 \\
17.8\end{array}$ & $\begin{array}{l}415 \\
298\end{array}$ & $\begin{array}{r}128 \\
78\end{array}$ & $\begin{array}{l}\text { After thy- } \\
\text { roidectomy }\end{array}$ \\
\hline 5 & $\begin{array}{c}\text { Myelogenous } \\
\text { leukemia }\end{array}$ & 29 & M. & $\begin{array}{l}+20 \\
-9\end{array}$ & $\begin{array}{l}6.52 \\
4.87\end{array}$ & $\begin{array}{l}14.3 \\
13.8\end{array}$ & $\begin{array}{l}456 \\
355\end{array}$ & $\begin{array}{l}75 \\
60\end{array}$ & $\begin{array}{l}\text { After ra- } \\
\text { dium treat- } \\
\text { ment }\end{array}$ \\
\hline 6 & $\begin{array}{c}\text { Myelogenous } \\
\text { leukemia }\end{array}$ & 26 & M. & $\begin{array}{l}+65 \\
+36 \\
+12\end{array}$ & $\begin{array}{l}9.75 \\
7.56 \\
6.16\end{array}$ & $\begin{array}{l}12.3 \\
15.4 \\
12.8\end{array}$ & $\begin{array}{l}796 \\
492 \\
484\end{array}$ & $\begin{array}{l}80 \\
68 \\
63\end{array}$ & $\begin{array}{c}\text { After ra- } \\
\text { dium treat- } \\
\text { ment }\end{array}$ \\
\hline
\end{tabular}

In connection with the hyperpnea associated with the increase of metabolism in fever, mention may be made here of an unusual degree of hyperpnea which is occasionally seen in the late stages of severe acute infections. This is illustrated by two patients observed at the Peter Bent Brigham Hospital. The first was a middle-aged woman, with a marked unilateral exophthalmos, which with other signs led to the diagnosis of thrombosis of the cavernous sinus. On the day after admission to the hospital she was comatose and the breathing showed an extraordinary hyperpnea. Respiration was regular. Each individual breath was much deeper than normal and the rate of respiration was 50 per minute. The minute-volume could not be measured, but it is scarcely possible that it was less than 20 to 25 liters per minute. The temperature was $105^{\circ} \mathrm{F}$; the pulse rate was 160 per minute; the hemoglobin was 90 per cent.; 
and the leucocyte count was 24,000 per c.mm. The deep, regular respiration suggested that seen in severe diabetic acidosis, except that it was more rapid than is usually the case in that condition. The carbon dioxid tension in the blood by the Van Slyke method was, however, $26.6 \mathrm{~mm}$. This figure is somewhat below normal, but does not indicate a degree of acidosis which could at all account for the hyperpnea. It seems more probable that the low value was the result of the hyperpnea, and the washing out of carbon dioxid from the blood. The breath sounds were heard all over both sides of the chest, but there were harsh breathing and a few râles at the left base posteriorly. This was taken to indicate a beginning pneumonia, but the involvement of the lungs was slight and not such as to explain the hyperpnea. There was no cyanosis. The spinal fluid was cloudy and contained $r, 800$ leucocytes per c.mm. The blood culture was positive for staphylococcus albus. On the following day the patient died. An exactly similar picture occurred at the end of a long protracted case of cerebrospinal meningitis, at a time when there were signs suggestive of an early pneumonia at the right base behind. The rate of respiration was 46 per minute, the volume per respiration was 479 c.c., and the minute-volume was 22.3 liters. When completely saturated with oxygen the blood contained 19.3 volumes per cent., and the oxygen content of the venous blood was 7.22 volumes per cent. The hemoglobin was 90 per cent. The carbon dioxid tension of the blood was $23.1 \mathrm{~mm}$., indicating only a moderate degree of acidosis,-wholly insufficient to account for the hyperpnea. The temperature was $102.5^{\circ} \mathrm{F}$. The blood culture was negative. Conner and Stillman ( $\left.{ }^{11}\right)$ mention the occurrence of this type of dyspnea in their studies on the respiration in meningitis. Very rapid regular breathing was a constant terminal phenomenon in fatal cases. The cause of this extreme hyperpnea seen occasionally in cases of severe acute infection is not clear.

The respiratory center is extremely sensitive to any increase in $\mathrm{H}$-ion concentration of the blood, and one of the most characteristic evidences of the development of an acidosis in the organism is the production of hyperpnea. The effect of acidosis on the respiration has been most carefully studied in reference to diabetes, in which there may be a great production of $\beta$-oxybutyric and of aceto-acetic acid. These acid bodies are neutralized by combination with the fixed bases of the blood and tissues, and on account of the large amount of so-called "buffer salts" in the blood, comparatively great quantities of acid may be produced without the actual reaction of the blood being shifted. When, however, a certain point is reached, and the accumulation of acids in the blood is so great that the reaction of the blood begins to change, the increase of acidity stimulates the respiratory center. As a result of this, the 
minute-volume of air breathed is increased, more carbon dioxid is washed out of the blood, and the increase of non-volatile acids in the blood is compensated for by a decrease in carbon dioxid, so that the final reaction of the blood remains unaltered. It is probably only in the terminal stages of fatal cases that the actual reaction of the blood changes. At other times the acidosis is recognized by the decrease in carbon dioxid tension of the blood. Minor degrees of acidosis, such as cause a slight fall in the tension of the carbon dioxid of the blood, bring about a slight rise in the minute-volume of air breathed, while the higher degrees of acidosis in which the carbon dioxid tension falls from the normal of approximately $40 \mathrm{~mm}$. to I5 or Io $\mathrm{mm}$. cause an intense hyperpnea. It is with these low carbon dioxid tensions that one sees the very deep respiration which is typical of "air hunger" or the so-called "Kussmaul breathing." In such cases the minute-volume is often from 20 to 30 liters. In diabetes the acidosis is due primarily to an excessive formation of acids, but a similar result occurs in nephritis, in which an acidosis may be brought about owing to an inability to excrete acids normally. Mild degrees of nephritis are usually not associated with an acidosis sufficient to cause decrease in the tension of the blood carbon dioxid, but in advanced cases, with an output of phenolsulphonephthalein less than Io per cent. in two hours, a low carbon dioxid tension may be found. In cases of uremia, the carbon dioxid tension may fall to below Io $\mathrm{mm}$. and in such cases a marked hyperpnea develops, with deep respirations, exactly similar to the "air hunger" seen in diabetic coma $\left({ }^{12}\right)$. "Air hunger" dependent on acidosis, accompanied by very low carbon dioxid tension, has been described by Howland and Marriott $\left({ }^{13}\right)$ in severe intestinal conditions with diarrhea in children, and by Sellards $\left({ }^{14}\right)$ in the marked acidosis of cholera. Slight degrees of acidosis may occur in many conditions, such as pneumonia $\left({ }^{15}\right)$ and other febrile diseases, as well as in starvation, and in persons on a diet low in carbohydrates $\left({ }^{16}\right)$.

The degree to which hyperpnea may develop as a result of acidosis is indicated by observations on a patient with nephritis studied at the Peter Bent Brigham Hospital. The case was unusual in that a marked disturbance of respiration amounting to true dyspnea arose rather suddenly in a man with an acute nephritis and a phenolsulphonephthalein output of I9 per cent. in two hours. The blood urea nitrogen was $44 \mathrm{mg}$. per Ioo c.c. blood. The patient was perfectly conscious, with deep, slightly rapid respiration. The rate of respiration was 26 per minute, and the minute-volume of air breathed was 24.2 liters. The average volume per respiration was thus about $I, 200$ c.c. The hyperpnea in this case was, however, not as marked as is frequently seen in diabetic coma. 
The effect of lesser degrees of acidosis on the minute-volume of respiration is illustrated in the following table compiled from cases studied by Higgins, Peabody, and Fitz (loc. cit.) in a study of the acidosis developing in men on a diet low in carbohydrates. The extent of the acidosis is indicated by the fall in the tension of carbon dioxid in the alveolar air.

TABLE IV

\begin{tabular}{c|c|c}
\hline Case No. & $\begin{array}{c}\text { Alveolar Co2 } \\
\text { Tension } \\
\text { m.m. }\end{array}$ & $\begin{array}{c}\text { Minute-Volume } \\
\text { Liters. S. T. P. D. . }\end{array}$ \\
\hline I & 38.6 & 4.99 \\
& 29.1 & 6.98 \\
\hline 2 & 38.7 & 4.55 \\
& 30.1 & 5.73 \\
\hline 3 & 38.1 & 4.91 \\
& 35.7 & 6.00 \\
\hline
\end{tabular}

The hyperpnea due to acidosis may be decreased by the administration of alkali, and if sufficient alkali is given to raise the carbon dioxid of the blood to a normal value, the hyperpnea will usually disappear. The effect of alkali on the hyperpnea of acidosis is shown in Table V. The patient was a man with advanced chronic nephritis and beginning uremia. The urea nitrogen was greatly increased, there being $106 \mathrm{mg}$. per roo c.c. blood. After the first observation was made, 80 grams of sodium bicarbonate were given. This was enough to change the reaction of the urine from acid to alkaline. At the same time, the blood carbon dioxid tension rose from 23 to $52 \mathrm{~mm}$. and the minute-volume fell from 6.36 to 4.06 liters. It is doubtful whether the slight rise in basal metabolism is of any significance, but it is noteworthy that this rise was associated with a fall in minute-volume.

TABLE V

\begin{tabular}{c|c|c|c|c|c|c}
\hline Date & Metabolism* & $\begin{array}{c}\text { Minute- } \\
\text { Volume } \\
\text { Liters. } \\
\text { S. T. P. D. }\end{array}$ & $\begin{array}{c}\text { Rate } \\
\text { of } \\
\text { Resp. }\end{array}$ & $\begin{array}{c}\text { Volume } \\
\text { per Resp. } \\
\text { c.c. }\end{array}$ & $\begin{array}{c}\text { Pulse } \\
\text { Rate }\end{array}$ & $\begin{array}{c}\text { Carbon Dioxid } \\
\text { Tension of } \\
\text { Blood } \\
\text { mm. }\end{array}$ \\
\hline Apr. II & -9 & 6.36 & 13.7 & 464 & 69 & 23 \\
\hline Apr. 15 & normal & 4.06 & 15.2 & 268 & 82 & 52 \\
\hline
\end{tabular}

* Metabolism calculated according to body-surface and referred to normal standards of Du Bois. 
Much more striking results are, of course, achieved when alkali is given to patients with higher grades of acidosis. Table VI illustrates the effect of the administration of 120 grams of sodium bicarbonate to a man in early uremia with a carbon dioxid combining capacity of $\mathbf{I 7 . 6}$ volumes per cent. In this patient there was practically no excretion of phenolsulphonephthalein in two hours. The total non-protein nitrogen of the blood was $138 \mathrm{mg}$. per 100 c.c.; the hemoglobin was 54 per cent., and the vital capacity was $I, 900$ c.c.

TABLE VI

\begin{tabular}{c|c|c|c|c}
\hline $\begin{array}{c}\text { CO, Combining } \\
\text { Capacity } \\
\text { vols. per cent. }\end{array}$ & $\begin{array}{c}\text { Minute- } \\
\text { Volume } \\
\text { Liters }\end{array}$ & $\begin{array}{c}\text { Respiration } \\
\text { Rate }\end{array}$ & $\begin{array}{c}\text { Volume } \\
\text { per Resp. } \\
\text { c.c. }\end{array}$ & $\begin{array}{c}\text { Pulse } \\
\text { Rate }\end{array}$ \\
\hline 17.6 & 15 & 22 & 730 & 98 \\
\hline 69.7 & 5 & 25 & 200 & \\
\hline
\end{tabular}

In the conditions that have just been described, the acidosis was due to the increased production or to the retention of non-volatile acids. That a similar hyperpnea may be brought about by an acidosis due to carbon dioxid has been shown experimentally by Scott $\left({ }^{17}\right)$ in observations made on decerebrate cats breathing air containing increasing percentages of carbon dioxid. His results indicate that a true carbon dioxid acidosis may exist. That a similar condition may occur clinically has been indicated by Peters $\left({ }^{18}\right)$ and Peabody $\left({ }^{19}\right)$. Both of these observers have found that in certain cases of acutely decompensated heart disease, with dyspnea, and usually with cyanosis, the carbon dioxid tension in the venous blood may be considerably higher than it is in the alveolar air. This is probably due to an interference with the passage of carbon dioxid from the blood into the alveolar air. On the other hand, observations by Hürter $\left({ }^{20}\right)$ on the arterial blood gases failed to show any abnormal increase in the carbon dioxid in several patients with decompensated heart disease, chronic bronchitis, pulmonary tuberculosis, or pleural exudate. If a retention of carbon dioxid did occur it would cause an increase in the $\mathrm{H}$-ion concentration of the blood which would stimulate the respiratory center, and would be a factor in the production of dyspnea.

With regard to methods for the determination of acidosis, little need be said in this place, as they are now generally well known to clinicians. The subject has recently been admirably reviewed by Sellards $\left({ }^{2 x}\right)$, who also describes the details of various methods. Direct determinations of the $\mathrm{H}$-ion concentration of the blood are most accurately performed by 
electrochemical methods, but the technique is extremely difficult and the variations in blood reaction are so slight that no actual change is usually found except in severe cases just before death. The simplified dialysis method of Levy, Rowntree, and Marriott $\left({ }^{22}\right)$ is comparatively easy to carry out, but it is less satisfactory than the indirect methods depending on the determination of the carbon dioxid tension of the blood. The actual reaction of the blood remains remarkably constant during life, and as the amount of non-volatile acid in the blood rises, there is a compensatory fall in the carbon dioxid. The determination of the degree of the decrease in carbon dioxid serves, therefore, as an index of the increase of other acids. The carbon dioxid tension may be determined directly by examination of the blood, or indirectly by analysis of the alveolar air, for in most instances (see above) the tension in the blood and in the alveolar air is approximately the same. The simplest and most satisfactory method for the determination of the carbon dioxid tension or the carbon dioxid combining power of the blood is that of Van Slyke $\left({ }^{23}\right)$. The technique is easy and accurate results may be obtained very quickly on a few cubic centimeters of blood. Perhaps one of the chief advantages of making the determination on the blood itself is that any effort on the part of the patient is obviated, and it is not always easy to obtain satisfactory samples of alveolar air from patients who are sick or who cannot coöperate. There are various methods for collecting specimens of alveolar air. The oldest, and one of the most satisfactory, is that of Haldane and Priestley $\left({ }^{24}\right)$, in which the subject expires deeply into a long tube and the last part of the expiration is taken for analysis. There are certain technical difficulties which cause trouble with untrained subjects, and the fact that many sick patients cannot give a deep enough respiration limits its clinical use. It is easier to obtain samples by means of the Plesch-Higgins method $\left({ }^{25}\right)$, in which the patient rebreathes rather deeply from a rubber bag, and the results are quite satisfactory for clinical purposes in spite of the fact that the values are closer to those of the venous than of the arterial carbon dioxid. The method devised by Lindhard $\left({ }^{26}\right)$, by which multiple small samples are taken at the end of respirations of comparatively normal depth, has been used by Boothby and Peabody, and with especial success by Debenham and Poulton $\left({ }^{7}\right)$. Probably the most accurate method is that recently brought out by Pearce $\left({ }^{4}\right)$, but it is not easily applicable to clinical work. In all of these methods the samples of alveolar air must be analyzed in some type of special apparatus. The apparatus most 'commonly used is that of Haldane $\left({ }^{27}\right)$, and this is undoubtedly the most accurate and satisfactory method, but Henderson $\left({ }^{28}\right)$ has devised a much simpler and cheaper form that is 
quite accurate enough for general clinical purposes. Many of the technical difficulties of gas analysis are obviated in the use of the apparatus of Fridericia $\left({ }^{29}\right)$. This consists of a special form of glass tube, into which the patient expires, and the carbon dioxid in the last portion of the expiration is absorbed by adding sodium hydrate directly. The volume of carbon dioxid in the expired sample of alveolar air is read on a graduated scale on the tube. The method is simple and rapid and the apparatus is well adapted to bedside work, but in the obtaining of proper samples of alveolar air one has the same difficulties as in the Haldane method, of which it is really a modification.

Of less importance than an increase of the $\mathrm{H}$-ion concentration of the blood in the production of hyperpnea is a decrease in oxygen tension of the arterial blood. This, however, occurs frequently, and while it usually depends on a low hemoglobin content, it may also be due to an incomplete oxygenation of the blood during its passage through the lungs, to a diminished oxygen tension in the inspired air, or to the formation of abnormal hemoglobin compounds. The following instances taken from the study of Tompkins, Drinker, and Brittingham $\left({ }^{30}\right)$ on the effect of transfusion on the metabolism in anemia, illustrate the degree to which the minute-volume rises as the hemoglobin falls.

TABLE VII

\begin{tabular}{c|c|c|c}
\hline Case No. & $\begin{array}{c}\text { Hemoglobin } \\
\text { per cent. }\end{array}$ & $\begin{array}{c}\text { Metabolism } \\
\text { per cent. of Normal }\end{array}$ & $\begin{array}{c}\text { Minute-Volume } \\
\text { Liters }\end{array}$ \\
\hline 3 & 30 & - II \\
-I & 4.56 \\
\hline 12 & 90 & +9 & 4.48 \\
\hline 14 & 40 & -2 & 4.50 \\
& 60 & + II & 3.99 \\
\hline 15 & 30 & -10 & 5.11 \\
& 74 & +15 & 4.19 \\
\hline
\end{tabular}

Another severe case of secondary anemia in which the hemoglobin was 2 I per cent. (Palmer.method) and the oxygen combining capacity was 5.7 volumes per cent. (normal about I 8.5 volumes per cent.) had a minute-volume of 9.34 liters. The rate of respiration was 13 per minute and the volume per respiration was 720 c.c. Observations made when testing aviators in the United States Army as to their ability to stand low oxygen tensions in the inspired air show that there is generally some increase in the minute-volume when the oxygen has dropped from 
the normal of about 2 I per cent. to percentages of from I6 to 15 per cent. Below this there is a slight gradual increase in the respiration down to concentrations of from $\mathrm{I} 2.5$ to 9 per cent. of oxygen. From this point to about 8.5 or 6 per cent. oxygen the minute-volume increases much more rapidly, and at percentages of between 8 and 6 the majority of men examined showed an increase of 5.5 liters over the volume breathed when atmospheric air was being inspired. At tensions corresponding to an altitude of 25,000 feet the average minute-volume was approximately I4 liters $\left({ }^{31}\right)$. As to the effect of incomplete saturation of the blood in patients breathing atmospheric air little is known, because comparatively few determinations have been made of the oxygen content of the arterial blood of man in disease. It would seem to be quite possible, however, that in certain conditions, such as severe generalized bronchitis, there might well be an interference with the proper oxygenation of the blood. The presence of marked cyanosis may be an indication of this. Pearce $\left({ }^{32}\right)$ suggested that the cyanosis in pneumonia is due to the fact that part of the blood flows through a portion of the involved lung and is thus not exposed to the alveolar air. Stadie $\left({ }^{88}\right)$ has found this to be the case in patients with the influenzal type of broncho-pneumonia. In normal controls the arterial blood was from 85 to 98 per cent. saturated with oxygen, and in patients with pneumonia it remained at this level until twelve to twenty-four hours before death, when it fell rapidly to as low as 32 per cent. This was not associated with any striking diminution in the oxygen combining capacity of the blood. The low arterial oxygen seemed to parallel closely the degree of cyanosis. Hürter $\left({ }^{20}\right)$, who made the first systematic observations on the arterial blood gases in man, also found a slight oxygen deficiency in two cases of pneumonia. In four cases of heart disease in whom the circulation was compensated, the oxygen content of the arterial blood was, according to Hürter, within the normal limits, but in two out of three decompensated cases there was a slight decrease in the oxygen content. The cyanosis of congenital heart disease is generally supposed to be due to the arterial blood containing a mixture of oxyhemoglobin and reduced hemoglobin, and in this condition the minute-volume has been found to be above normal even at rest. The increased minute-volume in cases of acutely decompensated heart disease with great cyanosis may also be in part due to stimulation of the respiratory center by low oxygen content of the arterial blood. In a case of chronic bronchitis and in one of pulmonary tuberculosis, Hürter found the oxygen content of the arterial blood to be normal, but in another patient with pulmonary tuberculosis, and in a patient with a pleural effusion and slight cyanosis, the oxygen content was a little below normal. Conditions analogous to these are 
seen when a part of the hemoglobin is converted into a compound which is not easily dissociable and does not combine with oxygen. This occurs in poisoning by illuminating gas, when carbon monoxidehemoglobin is formed, and under the various circumstances in which methemoglobin is found. Peabody $\left({ }^{84}\right)$ has described the formation of methemoglobin in the terminal stages of pneumococcus pneumonia, and Harrop $\left({ }^{85}\right)$ has found a decrease in the oxygen combining capacity of the blood at the period of collapse in two fatal cases of pneumonia following influenza.

Another type of clinical condition in which marked hyperpnea may arise is that in which there is increased cerebral pressure from any cause. In such cases the disturbance of respiration is probably due to pressure on the respiratory center. An extraordinary increase in the minutevolume may also be seen in hysteria. Paroxyms of intense dyspnea may arise and may persist for remarkably long periods. Sometimes the increase in volume of the individual respiration is the striking feature, but more often there is a great increase in rate, and the breathing is very shallow. Observations have been reported on such a case by Peabody, Wearn, and Tompkins $\left({ }^{86}\right)$. The patient was an extremely neurotic soldier, who developed a marked hyperpnea when the mask was put on his face to study his basal metabolism. Before the mask was put on his respiratory rate was 36 per minute; after the mask was put on the rate reached 102. The minute-volume in the first period was 7.86 liters, and in the second period it was I 1.69 liters. His metabolism was normal, and the oxygen consumption was practically the same in the two periods ( 243 c.c. in the first period, and 233 c.c. in the second period). It is characteristic of hysterical hyperpnea that it decreases during sleep and when the patient does not know he is being observed.

Various drugs produce a hyperpnea. Thus Higgins and Means $\left({ }^{87}\right)$ found a slight increase of the minute-volume of air breathed after the administration of atropin and caffein. With atropin the hyperpnea seemed to depend on an increase in metabolism, and with caffein on a stimulation of the respiratory center. Adrenalin also causes a rise in minute-volume which depends on an increase of metabolism. Tompkins and Wearn $\left({ }^{83}\right)$ found that this increase was much greater in persons who are hypersensitive to the drug, and that it depends on a rise in metabolism.

\section{The Vital Capacity of the Lungs}

The vital capacity of the lungs is the volume of the greatest possible expiration after the deepest possible inspiration. Its measurement is thus an expression of the depth to which the respiration can be increased. In normal breathing, at rest, the volume of each respiration is only a 
small proportion, about ro per cent., of the vital capacity, but with the very deep breathing which is brought about by severe muscular exercise or in advanced diabetic acidosis, each respiration may amount to as much as 30,40 , or even 50 per cent. of the total vital capacity. It is readily seen that anything which decreases the vital capacity of the lungs may interfere with the breathing in that it reduces the reserve of the respiration. A normal man, for instance, breathes 400 c.c. per respiration, at the rate of 15 , or a minute-volume of 6.0 liters at rest. If his vital capacity is 5,000 c.c., and if he is able, during exercise, to breathe 40 , per cent. of this (or 2,000 c.c.) at each respiration, and at a rate of 30 per minute, it will be possible for him to raise his minute-volume to 6o.o liters. On the other hand, if his vital capacity is reduced to 3,000 c.c. and if he breathes the same percentage of it at the same rate, it will only be possible for him to raise his minute-volume to 36.0 liters. The vital capacity is thus an important factor in determining the degree to which the minute-volume can be raised in the development of hyperpnea.

Since 1846 , when Hutchinson $\left({ }^{39}\right.$ ) first introduced the use of the spirometer, the question of the vital capacity of the lungs in disease has been frequently investigated in medical clinics. Much information has been obtained, but the value of the determinations has been limited by the difficulties in establishing normal standards for comparison. In I 855 Arnold $\left({ }^{40}\right)$ published an extensive and carefully compiled monograph in which he showed that age, sex, height, size, and expansion of chest, and habit of life may all have a considerable effect on the vital capacity of the lungs. Attempts have been made to find a simple relationship between vital capacity and some readily obtainable body measurement, or a formula which would give a constant for the relation between vital capacity and body size, but none that is entirely satisfactory has been found. In an attempt to establish normal standards for the vital capacity, Peabody and Wentworth $\left({ }^{41}\right)$ studied I 40 healthy persons, including physicians, nurses, medical students, and a number of ward patients, who would be classed as normal from the point of view of the respiration. The subjects ranged between 20 and 50 years of age, but the majority were between 20 and 30 years. They were, on the whole, a representative group of normal people in every-day life. Since the vital capacity decreases with advancing years, it would have been better if more elderly normal persons had been studied, but in spite of this lack the normal standards established appear to be of practical value. It was felt to be important to have as simple a method of standardizing results as possible and, after attempting various ways, it was found that a classification based on sex and height was practical and sufficiently 
accurate. In the determination of the normal standards of vital capacity, therefore, the observations on 140 normal persons were grouped according to sex, and for each sex three subgroups were made on the basis of height. The average normal vital capacity was determined for each subgroup. Having thus established the normal standards, subsequent determinations were referred to these and expressed in percentage of the appropriate normal. Ninety-six normal males were studied and divided into three groups according to their height. Group I included those who were $182.5 \mathrm{~cm}$. ( 6 feet) tall or over, and the normal standard computed was 5, I00 c.c. Group II consisted of men between $173.5 \mathrm{~cm}$. ( 5 feet, $8 \mathrm{r} / 2$ inches) and $182.5 \mathrm{~cm}$. (6 feet) tall, and the average vital capacity, which was 4,800 c.c., was taken as the normal. Group III was comprised of persons whose height was between $173.5 \mathrm{~cm}$. (5 feet, $8 \mathrm{r} / 2$ inches) and $159.5 \mathrm{~cm}$. ( 5 feet, 3 inches). The normal standard of this group was 4,000 c.c. With one exception the vital capacity in all the males examined varied between 86 and I2 I per cent. of the normal figure, while 84 per cent. were within Io per cent. of the normal. The largest vital capacity was 7,I80 c.c., or I4I per cent. This was found in a powerful man, who had recently been a member of the varsity crew, track and football teams in a large university. Four other members of this group had a vital capacity of 6,000 c.c. or over, and all had undergone severe athletic training. Table VIII gives the details of the determinations of vital capacity in the normal males and shows the actual and percentage variations from the standard adopted in each group.

\section{TABLE VIII}

The Vital Capacity of the lungs of Normal Males

\begin{tabular}{|c|c|c|c|c|c|c|c|c|c|}
\hline Group & $\begin{array}{l}\text { Number } \\
\text { Studied }\end{array}$ & $\begin{array}{l}\text { Height in } \\
\text { Feet and } \\
\text { Inches }\end{array}$ & $\begin{array}{c}\text { Normal } \\
\text { Vital } \\
\text { Capacity } \\
\text { c.c. }\end{array}$ & $\begin{array}{l}\text { Number } \\
\text { Within } \\
\text { Io per } \\
\text { cent. of } \\
\text { Normal }\end{array}$ & $\begin{array}{l}\text { Highest } \\
\text { Vital } \\
\text { Capacity } \\
\text { c.c. }\end{array}$ & $\begin{array}{c}\text { Lowest } \\
\text { Vital } \\
\text { Capacity } \\
\text { c.c. }\end{array}$ & $\begin{array}{c}\text { Highest } \\
\text { per } \\
\text { cent. }\end{array}$ & $\begin{array}{c}\text { Lowest } \\
\text { per } \\
\text { cent. }\end{array}$ & $\begin{array}{l}\text { Number } \\
\text { Below } \\
90 \text { per } \\
\text { cent. of } \\
\text { Normal }\end{array}$ \\
\hline I & 14 & $6^{\prime}+$ & 5,100 & 9 & 7,180 & 5,030 & I4I & 99 & 0 \\
\hline II & 44 & $\begin{array}{c}\text { Over } 5^{\prime} 81 / 2^{\prime \prime} \\
\text { to } 6^{\prime}\end{array}$ & 4,800 & $4 I$ & 5,800 & 4,300 & I2I & 90 & 0 \\
\hline III & 38 & $\begin{array}{l}5^{\prime}, 3^{\prime \prime} \text { to } \\
5^{\prime} 81 / 2^{\prime \prime}\end{array}$ & 4,000 & $3 I$ & 5,080 & 3,450 & 127 & 86 & I \\
\hline
\end{tabular}

Special attention is called to the last column in Table VIII. It will be seen from this that only one of the ninety-six normal subjects had a vital capacity which was more than ro per cent. below the appropriate 
normal standard. For practical purposes, then, the significant fact is the demonstration that healthy males almost invariably have a vital capacity of 90 per cent. or more of the normal standard. A decrease in the vital capacity below 90 per cent. will therefore suggest some pathological condition.

The women were also subdivided into three groups according to their height. Group I was composed of those who measured over $167 \mathrm{~cm}$. (5 feet, 6 inches) tall, and the vital capacity was 3,275 c.c. Group II consisted of those who were from $162 \mathrm{~cm}$. (5 feet, 4 inches) up to and including $167 \mathrm{~cm}$. ( 5 feet, 6 inches). The normal standard for this group was found to be 3,050 c.c. Group III was made up of persons from $154.5 \mathrm{~cm}$. ( 5 feet, I inch) up to and including those who were $162 \mathrm{~cm}$. ( 5 feet, 4 inches) tall. The standard vital capacity for this group was 2,825 c.c. Table IX gives an analysis of variations from the normal standards in the different groups.

TABLE IX

The Vital Capacity of the Lungs of Normal Females

\begin{tabular}{|c|c|c|c|c|c|c|c|c|c|}
\hline Group & $\begin{array}{l}\text { Number } \\
\text { Studied }\end{array}$ & $\begin{array}{l}\text { Height in } \\
\text { Feet and } \\
\text { Inches }\end{array}$ & $\begin{array}{l}\text { Normal } \\
\text { Vital } \\
\text { Capacity } \\
\text { c.c. }\end{array}$ & $\begin{array}{l}\text { Number } \\
\text { Within } \\
\text { Io per } \\
\text { cent. of } \\
\text { Normal }\end{array}$ & $\begin{array}{l}\text { Highest } \\
\text { Vital } \\
\text { Capacity } \\
\text { c.c. }\end{array}$ & $\begin{array}{l}\text { Lowest } \\
\text { Vital } \\
\text { Capacity } \\
\text { c.c. }\end{array}$ & $\begin{array}{c}\text { Highest } \\
\text { per } \\
\text { cent. }\end{array}$ & $\begin{array}{c}\text { Lowest } \\
\text { per } \\
\text { cent. }\end{array}$ & $\begin{array}{l}\text { Number } \\
\text { Below } \\
90 \text { per } \\
\text { cent. of } \\
\text { Normal }\end{array}$ \\
\hline I & I0 & Over $5^{\prime} 6^{\prime \prime}$ & 3,275 & 5 & 4,075 & 2,800 & 124 & 86 & 2 \\
\hline II & 13 & $\begin{array}{l}\text { Over } 5^{\prime} 4^{\prime \prime} \\
\text { to } 5^{\prime} 6^{\prime \prime}\end{array}$ & 3,050 & 9 & 3,425 & 2,660 & II 2 & 88 & 2 \\
\hline III & 2I & $5^{\prime} 4^{\prime \prime}$ or less & 2,825 & 16 & 3,820 & 2,500 & 135 & 89 & I \\
\hline
\end{tabular}

As far as these figures go, then, one may state fairly definitely that in normal persons the vital capacity is at least 85 per cent., and almost always 90 per cent., or more of the normal standards adopted for each group. In elderly persons a slight decrease from these standards may be expected.

Lundsgaard and Van Slyke $\left({ }^{42}\right)$ have attempted to find a more accurate basis for the comparison of measurements of vital capacity in different individuals, and they have investigated the relationship between chest volume and vital capacity. The chest is considered to be a geometrical figure, and the product of the three dimensions, length, depth, and breadth, is assumed to represent a volume proportional to the chest volume. "The height of the chest is taken as the length of the sternum from incisio intraclavicularis to a point just below articu- 
latio sterno-xiphoidea. The depth is then taken as the horizontal distance from the middle of the sternum at the insertion of the third rib to the spinal column, and the breadth is the distance between the sixth ribs in the midaxillary line. The points between which the measures are taken are almost without any muscular covering." The ratio between the vital capacity and chest volume is calculated as Io0 vital capacity $\frac{\text { IoO vital capacity }}{\text { chest volume at rest }}$, and the average ratio is given as 45 . A study of a larger series of cases seems to show that this figure is too low. In I6 normal cases of Lundsgaard and Van Slyke which could be compared by the two methods, I was more than Io per cent. below, and 3 more than Io per cent. above the normal standard when the relationship to chest volume was used, while 2 were more than Io per cent. below and 5 more than ro per cent. above by the standards of Peabody and Wentworth. By either method the variations from the normal should probably be considered as at least Io per cent. either way. In pathological conditions it is a decrease below the normal that is of especial significance. Physical training and deep breathing exercises are important factors in increasing the vital capacity. On account of these and other conditions which have already been mentioned as influencing the vital capacity, it is not likely that any absolutely accurate means of determining normal standards in a simple way will be found, and the variations under different circumstances in a given individual will be of more significance than the variations from any arbitrarily chosen standard.

The simplest method of determining the vital capacity is by means of a small, well-balanced spirometer of about 8 liters capacity, such as is illustrated in Fig. 17. The subject takes the deepest possible inspiration and then expires completely into the spirometer. The volume of the expiration can be read with an accuracy of 50 c.c., at least, from the calibrations on the wheel. Difficulty is sometimes experienced in that many persons tend to continue inspiration after having inserted the tube into the mouth. It is therefore well to start with the spirometer partly filled with air. By means of a short pin which is placed on the wheel at the zero point, and a pointer that is easily movable and catches on the pin, it is easy to arrange so that even after an inspiration the pointer will give the actual zero point at the beginning of expiration. In order to obtain accurate results, proper coöperation on the part of the subject is, of course, necessary, and this is sometimes difficult to obtain in sick or very weak persons.

It has long been known that the vital capacity of the lungs may be decreased in heart disease, and a systematic study of the occurrence 
and significance of this decrease was made by Peabody and Wentworth (loc. cit.). As a result of 224 observations on patients with various types of cardiac disease, they conclude that there is a close relation between decrease in vital capacity and the tendency to dyspnea. Com-

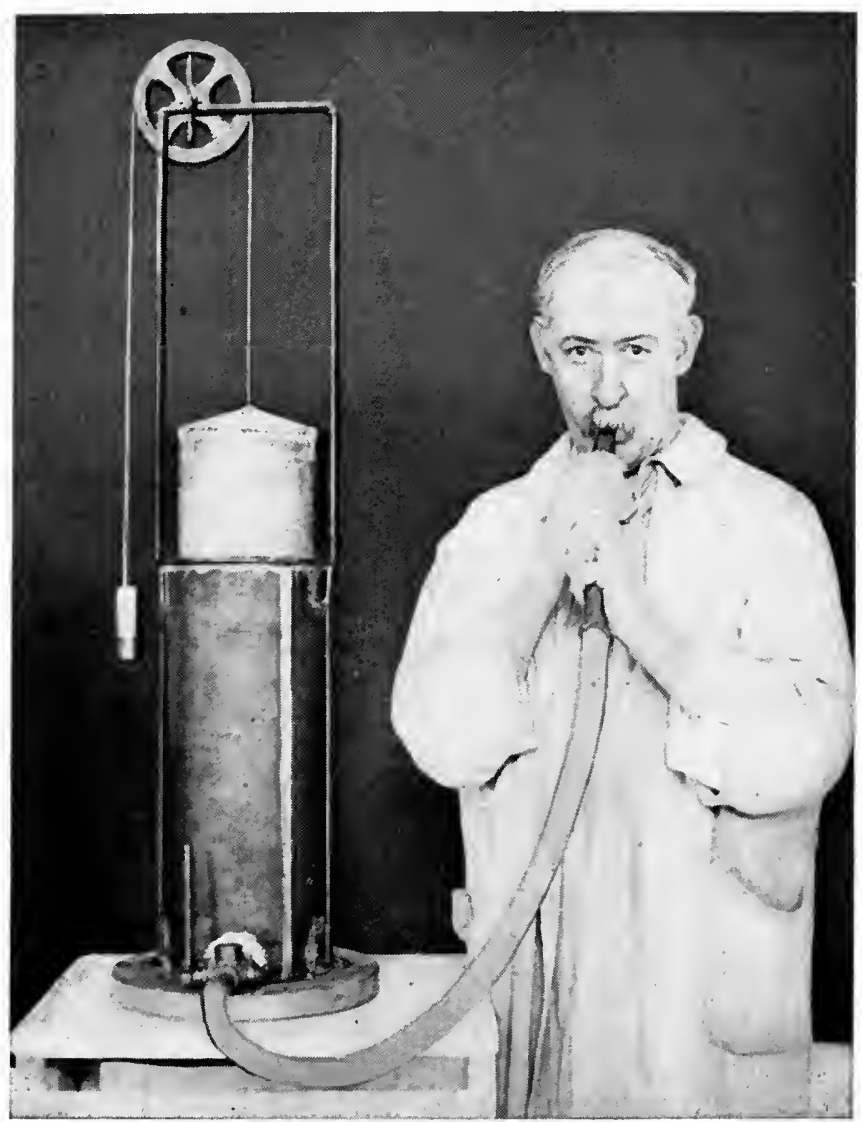

Fig. 17.-Spirometer for Determination of Vital Capacity of the Lungs.

pensated patients, who do not complain of dyspnea on exertion, have a normal vital capacity. Patients with more serious disease, in whom dyspnea is a prominent symptom, have a low vital capacity, and the decrease in vital capacity runs parallel to the clinical condition. Changes in the clinical condition are usually associated with changes in the vital capacity. As a patient improves, his vital capacity tends to rise, and as he becomes worse, it tends to fall. They find that determinations of 
the vital capacity in cases of cardiac disease are often of practical value, as they give quantitative information as to the tendency to dyspnea, and thus, indirectly, as to the clinical condition and the reserve power of the patient. If the vital capacity of patients with heart disease is expressed in terms of the normal standards for sex and height, as given above, four distinct groups may be recognized, in which there is a close relationship between clinical condition and percentage decrease of vital capacity from the normal figure. Among the patients in whom the vital capacity is 90 per cent. or more of the normal standard, very few complained of symptoms referable to their hearts. Dyspnea was not more prominent in their histories than it would be found to be in a similar group of normal persons. Many of them entered the hospital for other diseases and the cardiac condition was found only in the course of the routine examination. Twenty-three out of twenty-five were able to work, and the majority without much restriction. They were almost invariably in a good state of compensation, and if, as rarely happened, they were prevented from leading a normal life, this was usually on account of cardiac pain or some other disturbance. Patients with a vital capacity of 70 to 90 per cent. differed from those having a higher vital capacity in that almost all gave a history of dyspnea on moderate exertion, and had a distinctly limited cardiac reserve. About half of them were able to do a moderate amount of work. A number of them had had periods of more or less severe cardiac decompensation in which there had been a further drop in the vital capacity. These are, therefore, to be regarded as border line cases in whom the activities must be somewhat limited, but in whom under favorable circumstances there is little evidence of cardiac insufficiency. The third group of cases, in whom the vital capacity was from 40 to 70 per cent. of the normal, was characterized by the fact that all its members were in a much less favorable clinical condition than were those who had a higher vital capacity. Dyspnea on even moderate exertion was always noted in the history, and was, indeed, usually the most prominent symptom complained of. Even within the group there was a fairly definite relation between the vital capacity and the clinical condition. Thus all patients with a vital capacity of from 40 to 45 per cent. of the normal were in bed. Some of them were slightly dyspneic even when completely at rest, while the others became dyspneic on the least exertion. With a vital capacity of from 45 to 60 per cent. of the normal, patients were rarely dyspneic while in bed, and most of them could walk slowly around the ward without becoming short of breath. A few were living at home and could come to the outdoor department, but they all had to walk slowly and avoid stairs or hills. When the vital capacity was between 
60 to 70 per cent., the patients have usually been able to walk fairly comfortably, many could come to the hospital on foot and could even go upstairs without any special distress. Only 7 per cent. of the persons in this group were at work when they were examined, and in all cases the work done was very light. Attacks of severe cardiac decompensation occurred with considerable frequency in this group of patients, and the mortality among those who at some time have been members of the group was rather high. The fourth group consisted of cardiac patients with a vital capacity which was 40 per cent. of the normal or less. They were usually bedridden and severely decompensated. Many of them had dyspnea even when completely at rest. Patients whose vital capacity falls as low as this during their first period of cardiac insufficiency may improve so much that they are able to return to a fairly normal life, but the occurrence of such a low vital capacity in later attacks of decompensation makes for a distinctly unfavorable prognosis. Few patients who at any time fell into this group showed great clinical improvement, and more than one-half had died.

Table $X$ summarizes these observations on the relation between the vital capacity of the lungs and the clinical condition of patients with heart disease.

TABLE X.

The Relation of the Vital Capacity of the Lungs to the Clinical Condition in Patients with Heart Disease*

\begin{tabular}{r|c|c|c|c|c}
\hline Group & $\begin{array}{c}\text { Vital Capacity } \\
\text { per cent. }\end{array}$ & $\begin{array}{c}\text { Number of } \\
\text { Cases }\end{array}$ & $\begin{array}{c}\text { Mortality } \\
\text { per cent. }\end{array}$ & $\begin{array}{c}\text { Symptoms } \\
\text { of Decom- } \\
\text { pensation } \\
\text { per cent. }\end{array}$ & $\begin{array}{c}\text { Working } \\
\text { per } \\
\text { cent. }\end{array}$ \\
\hline I & $90+$ & 25 & 0 & 0 & 92 \\
II & 70 to 90 & 41 & 5 & 2 & 54 \\
III & 40 to 70 & 67 & 17 & 39 & 7 \\
IV & Under 40 & 23 & 6 6I & 100 & 0 \\
\hline
\end{tabular}

The cause of the decrease of the vital capacity of the lungs in heart disease is somewhat complex, as there may be several factors involved, each of which can act to limit the movement of the lungs. Some of these

* Certain cases were tested several times, and owing to changes in vital capacity they appear in more than one group. In the "mortality" column they are included only in the lowest group into which they fell. "Symptoms of decompensation" indicates dyspnea while at rest in bed or on very slight exertion. Under "working" are included only those actually at work and able to continue. Many other patients in Group II were able to work, but they are not included as they were still in the hospital. 
affect the movement of the chest wall and prevent a normal expansion of the thoracic cavity. Weakness of the intercostal muscles, rigidity of the bony framework or ankylosis of the costal joints may act in this way. Alterations in the lung tissue itself may cause a diminution in its elasticity, as is seen in emphysema. Accumulations of fluid in the pleural cavities prevent the normal expansion of the lungs, and great cardiac hypertrophy, pericardial effusion, or mediastinal tumors may produce a similar result. The normal inspiratory depression of the diaphragm is interfered with by intrathoracic conditions, which make it assume a flattened position even during quiet respiration, and by intraabdominal conditions which push it upward. Hepatic enlargement, tympanites, and ascites are examples of such conditions. Fluid within the bronchi and smaller air passages may prevent the entrance of air into considerable portions of the lung. That is, of course, frequently found in the dependent parts of the lungs in severely decompensated patients. The effect produced by a generalized bronchitis is not wholly clear from the results which have as yet been accumulated. The decrease in vital capacity associated with this condition is frequently much less than one would expect, and observations suggest that the intense dyspnea sometimes occurring in cardiac patients who acquire an acute bronchitis is largely dependent on some factor other than a change in the vital capacity.

In many cases, and particularly in acutely decompensated patients, conditions such as those just mentioned appear to account for the decrease in the vital capacity of the lungs in a wholly satisfactory manner. In other instances, especially where dyspnea is only experienced on moderate exertion, all of these factors may be absent, and yet the vital capacity may be low enough to explain fully the history of shortness of breath. The cause of the low vital capacity in such cases is not absolutely certain, but the work of Siebeck $\left({ }^{48}\right)$ on the determination of the lung volumes in heart disease bears on the question and suggests that the decrease in vital capacity may be due to an overfilling or engorgement of the pulmonary vessels, and a consequent diminution of the elasticity of the lungs. If this is proved to be true it would signify that the determinations of vital capacity may give direct evidence as to the state of the pulmonary circulation. Such information would be of great value in the study of cardiac insufficiency. Other methods of examination bear chiefly on the greater circulation, and disturbances in the pulmonary circuit are only recognizable when of sufficient grade to cause the passage of fluid out of the vessels into the air spaces and the production of râles.

Similar detailed studies have been made by Garvin, Lundsgaard, and 
Van Slyke $\left({ }^{44}\right)$ on 31 men and 20 women with pulmonary tuberculosis. The results agreed with former observations in showing that the vital capacity is usually decreased in this disease, and further, that the decrease in vital capacity bears a close. relation to the extent of the pathological process. Thus in 9 men with incipient pulmonary tuberculosis, the average vital capacity was 87 per cent. of the normal according to chest volume, and 89 per cent. according to the standards for height of Peabody and Wentworth. In I 3 cases with moderately advanced lesions the vital capacity was 68 per cent. of the normal referred to chest volume and 74 per cent. if referred to height. In 9 patients with severe pulmonary tuberculosis, the average vital capacity was 62 per cent. according to the normal standard based on chest volume, and 60 per cent. according to the standards for height.

There are, of course, many other pathological conditions in which the movement of the lungs is restricted, and the vital capacity is reduced. This is the case, for instance, in many patients with emphysema, with pneumonia, or with pleural effusions.

A secondary effect on the respiration, caused by a reduction in the vital capacity of the lungs, is the production of an hyperpnea. If the vital capacity is considerably decreased, the breathing tends to become more rapid and more shallow, and the minute-volume becomes greater. This is due to the fact that the "dead space" is not decreased in proportion to the respiratory surface. A larger part of each respiration thus remains in the dead space and does not come in contact with the respiratory epithelium. As a result of this the oxygenation of the blood is maintained by raising the minute-volume of air breathed, and this is done chiefly at the expense of the rate of respiration. The effect of reduction in the vital capacity on the minute-volume is shown by the following observations made on a patient with one side of the chest almost completely filled by pleural effusion, before and after tapping.

TABLE XI

\begin{tabular}{l|c|c|c|c|c|c}
\hline & $\begin{array}{c}\text { Vital } \\
\text { Capacity } \\
\text { c.c. }\end{array}$ & $\begin{array}{c}\text { Vital } \\
\text { Capacity } \\
\text { per cent. }\end{array}$ & $\begin{array}{c}\text { Minute- } \\
\text { Volume } \\
\text { Liters }\end{array}$ & $\begin{array}{c}\text { Volume } \\
\text { per } \\
\text { Respiration } \\
\text { c.c. }\end{array}$ & $\begin{array}{c}\text { Rate } \\
\text { of } \\
\text { Respiration }\end{array}$ & $\begin{array}{c}\text { Metabolism } \\
\text { per cent. of } \\
\text { Normal }\end{array}$ \\
\hline Before Thoracentesis & 1,950 & 49 & 8.06 & 360 & 22.5 & 25 \\
After Thoracentesis. & 2,700 & 68 & 6.96 & 503 & 14.0 & 18 \\
\hline
\end{tabular}

This effect on the minute-volume is, however, apparently only of definite consequence if the decrease in vital capacity is of a considerable degree. Peabody, Wentworth, and Barker $\left({ }^{45}\right)$, in a study of the basal metabolism 
and minute-volume in patients with heart disease, found that if the cases were divided into two groups, depending on whether the vital capacity was above or below 60 per cent. of the normal, minute-volume averaged about 30 per cent. higher in the group with the low vital capacity, while the basal metabolism only averaged Io per cent. higher. The rate averaged I 3 per minute, and the volume per respiration 47 I c.c. for the cases with the higher vital capacity, while the rate was 22 and the volume per respiration 408 c.c. in the cases with low vital capacity.

\section{Lung Volumes}

In addition to studies on the vital capacity many observations have been made on other measurements of lung volumes. Those which have received particular attention are: the total capacity at full inspiration; the residual air, which is left in the lungs on full expiration; the middle capacity, or the amount of air in the lungs at a point halfway between normal inspiration and normal expiration; the complementary air, which is the difference between the middle capacity and the total capacity; and the reserve air, or the difference between middle capacity and residual air.

The usual method used for determining these volumes has been by means of the inspiration of a given amount of air containing a known percentage of oxygen or hydrogen, and the analysis of it after it has been mixed with the air in the lungs. From the dilution which the gas undergoes, the volume of the air in the lungs can be calculated. Lundsgaard and Van Slyke, however, in the article quoted above, have found from observations on I I normal men and 7 normal women that there is a numerical relationship between external chest measurements and total capacity, middle capacity, and residual air. By the use of the ratios thus established the lung capacities normal for a chest of given measurements can be estimated.

In emphysema Siebeck $\left({ }^{48}\right)$, Bohr $\left({ }^{48}\right)$, and Bittorf and Forschbach $\left({ }^{47}\right)$ all found an increase in the residual air, an increase in the middle capacity, and a decrease in the reserve air. The complementary air may be normal, increased, or decreased according to Bittorf and Forschbach, but the vital capacity is characteristically decreased. In pulmonary tuberculosis Garvin, Lundsgaard, and Van Slyke (loc. cit.) state that the total capacity and middle capacity are normal in incipient cases, but that the residual air may be increased. In moderate and in advanced cases they agree with Siebeck in that the total capacity is decreased, the middle capacity is decreased or may be normal, while the residual air is normal or sometimes increased. Siebeck says the residual air is increased in proportion to the total capacity. In pleurisy 
with effusion, Siebeck and Bittorf and Forschbach state that the reserve air is decreased and the complementary air may also be decreased. This results in a low vital capacity and a low total capacity. The middle capacity is decreased, but is about normal in its percentage relation to the total capacity, and the residual air is usually decreased.

Investigations by Rubow $\left({ }^{48}\right)$ and by Bittorf and Forschbach show that in heart disease the residual air is approximately normal in volume. The low vital capacity is due essentially to a diminution in the reserve air, but the complementary air may also be decreased. Most observations indicate that the middle capacity in heart disease is normal or somewhat decreased, but that it is increased in its percentage relationship to the total capacity.

Clinical interest in the determination of the lung volumes in disease was largely stimulated by the work of Bohr (loc. cit.), who laid great stress on the importance of the middle capacity. According to the teaching of this eminent Danish physiologist, an increase of the middle capacity in emphysema is a useful compensatory change by means of which the circulation through the lungs is facilitated, and the same explanation was applied to the increase in the middle capacity in exercise. Rubow believed that the relative increase of the middle capacity in heart disease was similarly a mechanism by which the pulmonary circulation is aided and the ventilation of the lungs increased. He even went so far as to ascribe the dyspnea in certain cases to the muscular exertion necessary to raise the middle capacity. At the present time, however, there seems to be a general doubt as to the physiological and pathological significance of variations in the middle capacity. Changes in the residual air are undoubtedly of importance. A decrease probably indicates a diminution of the respiratory surface. An increase means that each inspiration is less effective in altering the composition of the alveolar air. This condition is seen in emphysema in which, as has been shown by Hoover $\left({ }^{49}\right)$, the subject is put to a further disadvantage by the fact that the dead space is also increased.

\section{IRREGULARITY OF RESPIRATION}

Apart from the slight irregularity of rate and depth of breathing that occurs with more or less constancy in normal persons even when at rest, the most important type of respiratory arrhythmia is the periodic form known as Cheyne-Stokes respiration. This type of breathing consists essentially of alternating periods of complete apnea and of grouped respirations. The apneic phase is commonly shorter than the respiratory phase, and the latter is usually characterized by a waxing and waning 
in the size of the individual breaths, the onset being marked by shallow respiration, the middle by respirations of greater volume, and the termination again by shallow breathing. This is, however, not a constant feature, and the beginning of the period of breathing may be marked by deep respirations. Cheyne-Stokes respiration is not in itself a pathological phenomenon, as it is frequently seen in normal persons during sleep,-particularly in infants and in old persons and in hibernating animals. It may also occur normally in people at high altitudes. Under pathological circumstances it is not at all infrequent, and it arises in many different conditions. Among the more common conditions with which it is associated are: cerebral lesions accompanied by increased intracranial pressure, arteriosclerosis, uremia, myocardial insufficiency, lesions of the aortic valve, morphine poisoning, and profound stupor arising from various other causes. Cheyne-Stokes respiration is perhaps most often seen in patients with diseases of the circulatory system and in them it forms a very characteristic clinical picture. Sometimes it persists both by day and by night, but usually it disappears in the daytime, recurring in the evening, and it is very frequently the cause of what is generally referred to as " nocturnal dyspnea." The usual story is that just as the patient is dozing off to sleep he is wakened by a severe paroxysm of dyspnea, which makes him sit up straight in bed gasping for breath. After a short period of distress, he falls asleep again, only to be awakened by another attack of dyspnea, following which he once more goes to sleep until, after a short interval, he is aroused again by a period of labored breathing. If pneumographic tracings are taken it will be found that Cheyne-Stokes respiration is present and that the patient is dozing off in the periods of apnea and awakening in the periods of respiration. This condition is sometimes called "cardiac asthma," but the term is singularly inappropriate, as the characteristic feature is the apneic pause, which is, of course, not a part of the true asthmatic attack. In the milder cases, anything which rouses the patient, such as the entering into conversation, will occasionally make the periodicity of respiration become less marked or even disappear.

Cheyne-Stokes breathing is accompanied by phasic variations in the blood pressure, and many investigations have been made on the relation of the changes in blood pressure to the changes in respiration. Barbour $\left({ }^{50}\right)$ says: "All theories of periodic respiration which do not take into account periodic circulatory interference have yet to explain why the breathing does not remain rhythmical." Cushing $\left({ }^{51}\right)$ first showed in experiments on dogs that the blood pressure tends to keep higher than the intracranial tension, and that an increase in the latter brings about a rise in the former. The changes of blood pressure associated with 
increased intracranial tension cause alternating periods of blood-flow and anemia in the brain, and simultaneously with these there may occur phasic variations of the breathing, in such a way that the periods of high blood pressure and blood-flow in the brain are accompanied by respiration, while the periods of low blood pressure and anemia are accompanied by apnea. Eyster $\left({ }^{52}\right)$ continued this work experimentally and amplified the results by the study of clinical cases with increased intracranial pressure in whom he found the same relationship between the changes of blood pressure and the alternating periods of respiration and apnea. The intervals of apnea are accounted for by the fact that the excitability of the respiratory center is reduced by the anemia, and the increase in the stimulus to respiration which develops during apnea is not as great proportionally as the decrease in the excitability. The vasomotor center is stimulated to greater activity during the periods of anemia. It thus raises the blood pressure, increases the flow of blood to the medulla, and by this means raises the excitability of the respiratory center, so that finally the latter responds to the abnormally high stimulus and respiration begins. The increased supply of well-aërated blood then causes the vasomotor center to relax. A fall of blood pressure results, and with this an anemia which decreases the excitability of the respiratory center below the threshold of the stimulus, so that respiration ceases and the period of apnea begins. In a second group of clinical patients, in whom Cheyne-Stokes respiration was associated with cardiovascular or renal disease, Eyster found an almost exactly reversed relationship between the blood pressure changes and the phases of respiration. In these cases the blood pressure began to fall at about the middle of apnea and continued to fall until the third or fourth respiration, when it began to rise again, increasing through the rest of the respiratory period and reaching its maximum at approximately the middle of the succeeding interval of apnea. In both types of cases the increase of blood pressure was accompanied by a rise of pulse rate and the fall of blood pressure by a slowing of the pulse. The same relationship between blood pressure and respiration was found by Pollock ${ }^{(53}$ ) in a similar series of cases, and by Clark and Hamill $\left({ }^{54}\right)$ in four cases, including one of severe opium poisoning. In the latter there was a prolongation of the a-v interval in addition to the slowing of the pulse and fall of blood pressure during apnea. Fulton ${ }^{(55}$ ) has reported a case in which Cheyne-Stokes respiration was associated with auricular flutter. There was a rise of blood pressure during apnea and an increase of pulse from approximately 45 during the period of dyspnea to 80 during apnea. Fulton cites cases from the literature that do not fall definitely into either of these two groups, and concludes with regard 
to this classification that " not all of the cases of Cheyne-Stokes breathing will fit readily into the two groups, but that these two groups as originally described by Eyster probably embrace most of the cases and that the exceptions are likely to be isolated instances." In experiments on cats under the influence of morphin Barbour was able to demonstrate that both of these types of Cheyne-Stokes respiration may occur. In both groups, in those in which respiration is accompanied by rise of blood pressure as well as in those in which apnea is accompanied by rise of blood pressure, he believes that the variations in blood pressure are the essential cause of the periodic breathing. The apparent paradox that rises of blood pressure may produce exactly opposite effects is explained by the fact that they may supply the medulla with blood of different constitution. In the "cardiac type," he considers that the respiratory center is greatly depressed by some cause such as morphin, edema of the brain, uremia, or circulatory insufficiency. Because of this the center does not respond normally, and a period of apnea results which may persist so long that asphyxia of the heart with a fall of blood pressure and a slowing of the pulse rate results. This leads to an anemia of the respiratory center, but in spite of its depression, the latter "responds to the double stimulus of anemia and $\mathrm{CO}_{2}$ by a series of respirations. These relieve the cardiac asphyxia and thus improve the medullary circulation and the $\mathrm{CO}_{2}$ tension is reduced. Both respiratory stimuli being thus eliminated, the condition of apnea recurs." In the type of Cheyne-Stokes respiration described by Eyster as occurring in association with increased intracranial pressure, and called by Barbour the "vasomotor type," the latter holds that the "heart is less affected and the waves in the blood pressure arise from periodic stimulation of the vasomotor center by asphyxial products. The respiratory center whose excitability to these products has been reduced to about the level at which the vasomotor center responds to them shows parallel periodicity." Observations have been made by Pembrey and Allen $\left({ }^{88}\right)$ on the composition of the alveolar air during the changes in the respiration in Cheyne-Stokes breathing. At the onset of respiration, following apnea, the carbon dioxid is high and the oxygen low. At the end of the period of respiration the percentage of carbon dioxid in the alveolar air falls and the oxygen rises. They believe that the essential feature in producing the periodicity of the respiration is a decrease of the excitability of the respiratory center. Only when the combined stimulus of increasing carbon dioxid and decreasing oxygen has reached a certain level does the center respond, and then it reacts so extensively that it reduces the stimulus below the threshold and apnea follows. - Similar observations were made by Douglas and Haldane $\left({ }^{57}\right)$ in artificially 
produced Cheyne-Stokes respiration in man, but they lay especial emphasis on the effect of oxygen-want. It was found that the respiratory center responded when the carbon dioxid tension was below normal, and this is explained by the suggestion that the low oxygen tension leads to the formation of lactic acid in the respiratory center, and that this augments the carbon dioxid in its effect as the stimulus to the respiration. When the respiratory phase begins the lactic acid is oxidized and the carbon dioxid removed so that the respiratory stimulus becomes greatly reduced and a period of apnea follows. White and his associates $\left({ }^{58}\right)$ found in a clinical case of prolonged Cheyne-Stokes breathing that the $\mathrm{H}$-ion concentration of the blood was abnormally high at the onset of dyspnea, but about normal at the beginning of apnea. They argue from this that the excitability of the respiratory center was depressed, since it did not respond to a stimulus of normal intensity. It is, of course, very difficult to disprove the theory of Douglas and Haldane of a local formation of abnormal acid in the respiratory center, but observations reported by Peabody $\left({ }^{19}\right)$ on the carbon dioxid tension of the blood show that there was no general acidosis of severe grade, and that in the individual case there was no significant fall in the carbon dioxid tension with the onset of periodic dyspnea. That want of oxygen may be a factor of importance in the production of Cheyne-Stokes respiration is also indicated by the reports of Pembrey and Beddard and French $\left({ }^{59}\right)$, of Pembrey $\left({ }^{80}\right)$, and of White and his co-workers $\left({ }^{58}\right)$, who showed that the periods of apnea may be made to disappear if oxygen in high concentration is given to the patient to breathe. The same result may be brought about if the stimulus to respiration is increased by raising the concentration of carbon dioxid in the inspired air. It is, however, of interest in this connection, to note a fact that is very striking when one is attempting to study the respiration of patients with Cheyne-Stokes breathing. This is that even the application of a mask and valves; through which the subject can breathe a wholly adequate amount of atmospheric air, may suffice to cause the cessation of the periodicity of respiration in the less marked cases. The tendency of patients who have mild degrees of Cheyne-Stokes breathing to assume a regular normal rhythm as the result of being roused. by almost any nervous stimulus is wholly in accord with what is generally accepted as the underlying feature of periodic respiration. Whatever disagreement there may be as to the details of the physiology of Cheyne-Stokes breathing, most authors are in harmony in believing that it is fundamentally dependent on a decreased excitability of the respiratory center.

Somewhat similar to Cheyne-Stokes breathing is the respiratory arrhythmia known as Biot's breathing. This is characterized by constant 
variation in the rate and depth of breathing, the irregular occurrence of periods of apnea of varying duration, and by frequent deep sighing respirations. It is particularly associated with meningitis. Conner and Stillman ( ${ }^{11}$ ) found it to be present in 27 per cent. of 43 cases of meningitis, its incidence among the adults and non-tuberculous cases being greater than among the children and tuberculous cases. In the same group Cheyne-Stokes respiration was observed in 53 per cent. of the cases. The latter occurs in so many clinical conditions that it is, of course, of very little diagnostic significance, but as the result of the study of a large series of pneumographic records on adults and children. Conner and Stillman believe that Biot's breathing is almost pathognomonic of meningitis.

\section{BIBLIOGRAPHY}

I. HILL, L., and FLACK, M.: Jour. Physiol., 1909, XXXVIII; Proc., p. lvii.

2. HALDANE, J. S.: Am. Jour. Physiol., 19I5, XXXVIII, 20.

3. HENDERSON, Y., CHILLINGWORTH, F. P., and WHITNEY, J. L.: Am. Jour. Physiol., 1915, XXXVIII, I.

4. PEARCE, R. G.: Am. Jour. Physiol., I9I7, XLIII, 73.

5. KROGH, A., and LINDHARD, J.: Jour. Physiol., 1917, LI, 59.

6. DOUGLAS, C. G.: Jour. Physiol., IgII, XLII ; Proc., p. xvii.

7. DEBENHAM, L. S., and POULTON, E. P.: Quart. Jour. Med., I9I9, XII, 38.

8. SCHLESINGER, E. G., and PEMBREY, M. S.: Jour. Physiol., I908, XXXVII; Proc., p. lxix.

9. COLEMAN, W., and DU BOIS, E. F.: Arch. Int. Med., I915, XV, 887.

10. MEYER, A. L., and DU BOIS, E. F.: Arch. Int. Med., 1916, XVII, 965.

II. CONNER, L. A., and STILLMAN, R. G.: Arch. Int. Med., 1912, IX, 203.

12. PEABODY, F. W.: Arch. Int. Med., 1915, XVI, 955.

13. HOWLAND, J., and MARRIOTT, W. MCK.: Bull. Johns Hopkins Hosp., 1916, XXVII, 63.

14. SELLARDS, A. W.: Philippine Jour. Sc., Igrr, VI, Sect. B, 53.

15. PEABODY, F. W.: Jour. Exper. Med., 1912, XVI, 701.

I6. HIGGINS, H. L., PEABODY, F. W., and FITZ, R.: Jour. Med. Research, I916, XXXIV, 263.

17. SCOTT, R. W.: Am. Jour. Physiol., 1917, XLIV, 196.

18. PETERS, J. P., Jr.: Am. Jour. Physiol., I9I7, XLIII, II3.

19. PEABODY, F. W.: Am. Jour. Med. Sc., 1918, CLV, 100.

20. HÜRTER: Deutsch. Arch. f. klin. Med., 1912, CVIII, I.

21. SELLARDS, A. W.: The Principles of Acidosis and Clinical Methods for its Study. Cambridge: Harvard University Press, I917.

22. LEVY, R. L., ROWNTREE, L. G., and MARRIOTT, W. McK.: Arch. Int. Med., I9I5, XVI, 388.

23. VAN SLYKE, D. D.: Jour. Biol. Chem., I9I7, XXX, 347.

24. HALDANE, J. S., and PRIESTLEY, J. G.: Jour. Physiol., 1905, XXXII, 225.

25. BOOTHBY, W. M., and PEABODY, F. W.: Arch. Int. Med., 19I4, XIII, 497. 
26. LINDHARD, J.: Jour. Physiol., I9I I, XLII, 337.

27. HALDANE, J. S.: Methods of Air Analysis, London, 1912.

28. HENDERSON, Y.: Jour. Biol. Chem., I9I7, XXXI, 217.

29. FRIDERICIA, L. S.: Berliner. klin. Wochenschr., I9I4, LI, I268.

30. TOMPKINS, E. H., BRITTINGHAM, H. H., and DRINKER, C. K.: Arch. Int. Med., I9I9, XXIII, 44I.

31. Air Service Medical, Government Printing Office, Washington, I9I9, p. I7I.

32. PEARCE, R. G.: Trans. Assoc. Am. Phys., I9I7, XXXII, 7I.

33. STADIE, W. C.: Proc. Soc. Exper. Biol. and Med., IgI9, XVI, 88.

34. PEABODY, F. W.: Jour. Exper. Med., I913, XVIII, 7.

35. HARROP, G. A., Jr.: Bull. Johns Hopkins Hosp., I9I9, XXX, io.

36. PEABODY, F. W., WEARN, J. T., and TOMPKINS, E. H.: Med. Clin. N. A., I908, II, $50 \%$.

37. HIGGINS, H. L., and MEANS, J. H.: Jour. Pharmacol. and Exper. Therap., I9I5, VII, I.

38. TOMPKINS, E. H., and WEARN, J. T.: (not yet published).

39. HUTCHINSON, JONATHAN : Med. Chir. Tr., London, 1846, 29.

40. ARNOLD, F.: Ueber die Athmungsgrösse des Menschen, Heidelberg, 1855.

4I. PEABODY, F. W., and WENTWORTH, J. A.: Arch. Int. Med., 1917, XX, 443.

42. LUNDSGAARD, C., and VAN SLYKE, D. D.: Jour. Exper. Med., I9I8, XXVII, 65.

43. SIEBECK, R.: Deutsch. Arch. f. klin. Med., I9io, C, 204.

44. GARVIN, A., LUNDSGAARD, C., and VAN SLYKE, D. D.: Jour. Exper. Med., I9I8, XXVII, 87 and I29.

45. PEABODY, F. W., WENTWORTH, J. A., and BARKER, B. I.: Arch. Int. Med., I9I7, XX, 468.

46. BOHR, C.: Deutsch. Arch. f. klin. Med., I907, LXXXVIII, 385.

47. BITTORF, A., and FORSCHBACH, J.: Zeitschr. f. klin. Med., I910, LXX, 474.

48. RUBOW, V.: Deutsch. Arch. f. klin. Med.,'1908, XCII, 255.

49. HOOVER, C. F.: Arch. Int. Med., I9I3, XI, 52.

50. BARBOUR, H. G.: Jour. Pharmacol. and Exper. Therap., 1914, V, 393.

51. CUSHING, H.: Johns Hopkins Hosp. Bull., I90I, XII, 290.

Am. Jour. Med. Sc., I902, CXXIV, 375.

52. EYSTER, J. A. E.: Johns Hopkins Hosp. Bull., I906, XVII, 296.

Jour. Exper. Med., I906, VIII, 565.

53. POLLOCK, L. J.: Arch. Int. Med., I912, IX, 406.

54. CLARK, A. J., and HAMILL, P.: Jour. Pharmacol. and Exper. Therap., I9I4, V, 357.

55. FULTON, F. T.: Heart, I9I5, VI, 77.

56. PEMBREY, M. S., and ALLEN, R. W.: Jour. Physiol., 1905, XXXII; Proc., p. xviii.

57. DOUGLAS, C. G., and HALDANE, J. S.: Jour. Physiol., 1909, XXXVIII, 401.

58. WHITE, W. H., RYFFEL, J. H., POULTON, E. P., JOHNSON, W., and CHISOLM, R. A.: Quart. Jour. Med., I9I4, VIr, 389.

59. PEMBREY, M. S., BEDDARD, A. P., and FRENCH, H.: Jour. Physiol., I906, XXXIV; Proc., p. vi.

6o. PEMBREY, M. S.: Jour. Path. and Bacter., Ig08, XII, 258. 


\title{
CHAPTER XVII
}

\section{CALORIMETRIC METHODS OF STUDY OF DISEASE}

\author{
By EUGENE F. DU BOIS
}

Introduction.

\section{TABLE of Contents}

Some Clinical Applications of Calorimetric Observations.

Questions Answered by the Respiratory Calorimeter.

The Respiratory Calorimeter.

Indirect Calorimetry.

Direct Calorimetry.

THE respiration calorimeter is a complicated apparatus which brings to notice simple facts. Originally it served as a connecting link between physiology and the more exact science of physics. It has joined to these quite recently the science of clinical medicine and has helped to simplify the subject by demonstrating that the diseased human organism obeys the fundamental laws which govern inanimate objects. The soul of a man cannot yet be studied by such a machine, but his body is a very definite mixture of physical substances and lends itself to careful measurements.

Speaking rather brutally, a man is merely a combination of water, salts, protein, fat, carbohydrate, and certain other organic compounds. These are contained within a moist skin which loses heat and water vapor to the surrounding atmosphere. As the tissues oxidize foodstuffs they produce heat from materials brought in the blood; the blood obtains part of the reacting substances from the alimentary tract and part from the lungs. This same blood serves to distribute heat throughout the body in very much the manner in which a hot-water system distributes the warmth of a furnace.

The whole process can be studied when a man is enclosed in a respiration calorimeter. Thermometers measure the heat radiated from the skin or stored in the body. Chemical methods determine the water of vaporization, the oxygen consumed, and the carbon dioxid produced in the combustion of food. If also the nitrogen of the urine be determined we can calculate the grams of protein, fat, and carbohydrate consumed and the amount and distribution of the heat thus produced. It is possible to study these vital phenomena in disease and determine the manner in which they are affected by different factors. 


\section{I8 CALORIMETRIC METHODS OF STUDY OF DISEASE}

To be more specific, we might select a few questions which can be answered by such an apparatus. For instance: How much carbohydrate can this patient with severe diabetes oxidize in his tissues? Which is the best carbohydrate? How many calories does he require per day? What is the effect of fasting? What is the basal metabolism of this woman with hyperthyroidism? What happens when this malarial patient has a chill? Where does he get the heat which raises his temperature suddenly? How does he get rid of it when the temperature falls? How much water does a patient lose from his skin and lungs? Where does the heat go when a man's legs get cold? Why is it impossible to make some children fat when they eat large amounts of food?

The history of clinical calorimetry is one of slow development and gradual adaptation. Standard types of apparatus used in physical measurements were applied first to the study of heat production in animals, next to the study of normal men, and finally were used with patients. There is a striking similarity between the calorimeter which measures the oxidation of food in a sick man and the one which determines the heating value of a sample of food or coal. The industrial chemist weighs out a portion of the material to be tested, places it in a strong, steel cylinder with a large excess of oxygen, and ignites it by means of a glowing platinum wire. The heat given off is measured by noting the rise in temperature of the water which surrounds the cylinder.

Some of the early calorimeters in which animals and men were studied consisted of chambers surrounded by water jackets. Knowing the weight of the water and the number of degrees' rise in temperature, it was possible to measure the heat given off from the body. To this was added the heat lost in the vaporization of water from the skin and lungs. Arrangements were made to ventilate the chamber and either prevent or correct for loss of heat in the air. A simple apparatus of this type was used by Isaac Ott ( $\left.{ }^{1}\right)$ of Philadelphia in 1892 in the study of a man with malarial fever. Ten years later Likatscheff and Avroroff $\left({ }^{2}\right)$ of St. Petersburg made similar studies, using the famous calorimeter of Paschutin. This latter apparatus consisted of a large chamber with a water jacket but was also provided with the means of analyzing the carbon dioxid given off during each period of two hours. The instrument was capable of accurate work and the experiments were carefully planned. We must give the Russians the credit for being the first to make elaborate studies in the field of clinical calorimetry. Unfortunately, the volume of their work was small and it was buried in journals which could not be read by the large mass of investigators. Their results are also impaired by the fact that they did not realize the importance of keeping their experimental subjects absolutely quiet. In 
fact, it was not until about the year 1908 that anyone realized that the uncontrolled activity. of subjects in respiration chambers caused such a large error that the results which had been obtained could not be compared with each other or with figures published after 1908.

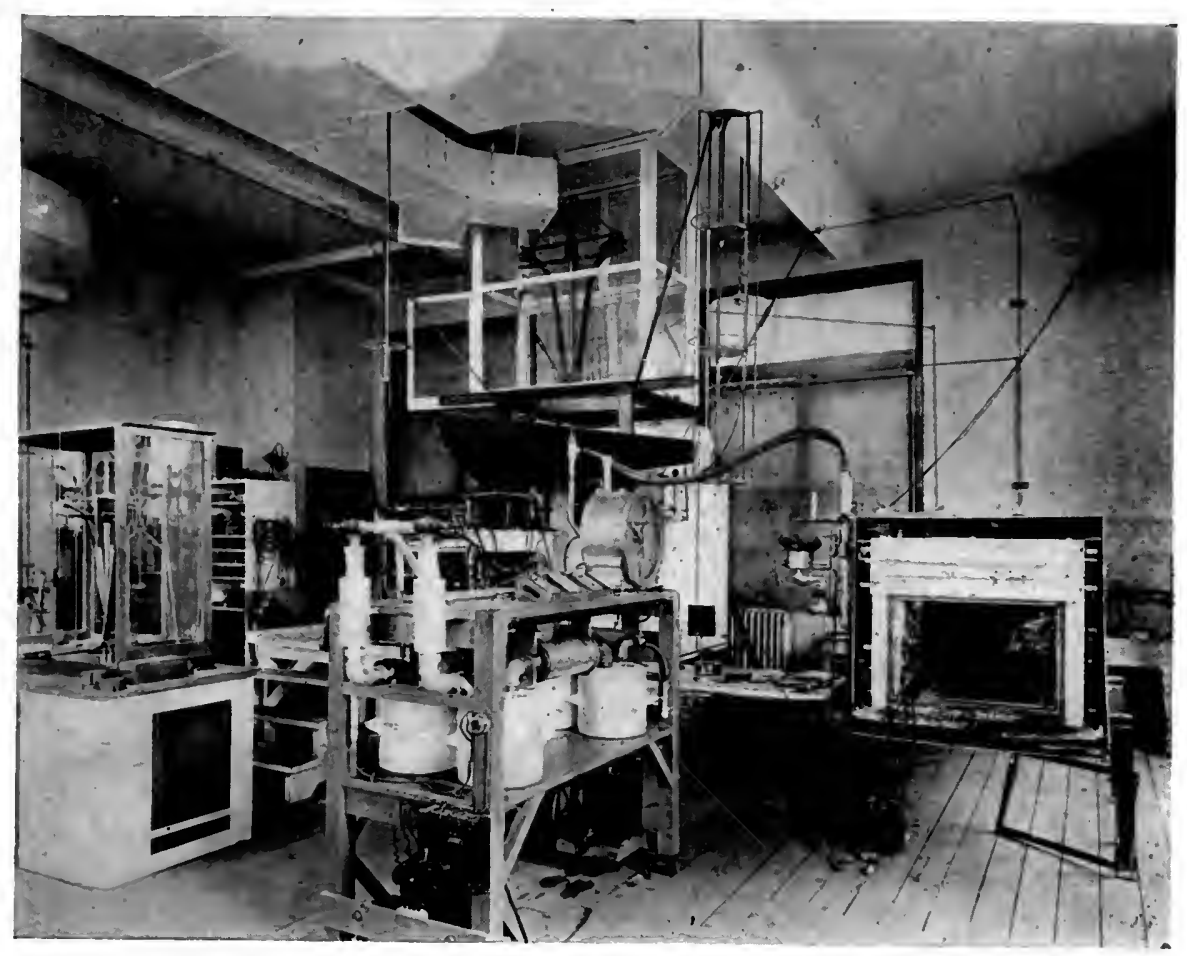

FIg. 18.-Bed calorimeter of the Nutrition Laboratory of Boston.

The foundations of the modern science of nutrition were built by the experiments on animals and normal men made in the respiration chamber of Pettenkofer $\left({ }^{3}\right)$ and Voit, the respiration calorimeter of Atwater and Benedict $\left({ }^{4}\right)\left({ }^{5}\right)$ (Fig. I8), and the dog calorimeters of Rubner $\left({ }^{6}\right)$ and Lusk $\left({ }^{7}\right)$ (Fig. 19). Much help was given by the simpler types of respiration apparatus used by Zuntz, Durig, Magnus-Levy, Benedict, and others. These are fully described by Carpenter $\left({ }^{8}\right)$, who compares the various designs. Some consist of spirometers or gas meters into which the patient breathes through a mouthpiece or mask for periods of ten to twenty minutes. Others resemble the ventilating system of a calorimeter and the patient either lies in a closed chamber or is connected with the apparatus by means of a nosepiece or mouthpiece. The heat production is calculated from the oxygen consumption by the method of 


\section{CALORIMETRIC METHODS OF STUDY OF DISEASE}

indirect calorimetry. Up to the last few years these devices were the only ones which had made really important additions to our knowledge of the respiratory exchanges in disease. No large mass of clinical work was attempted with calorimeters until 1908, when Benedict and Jos-

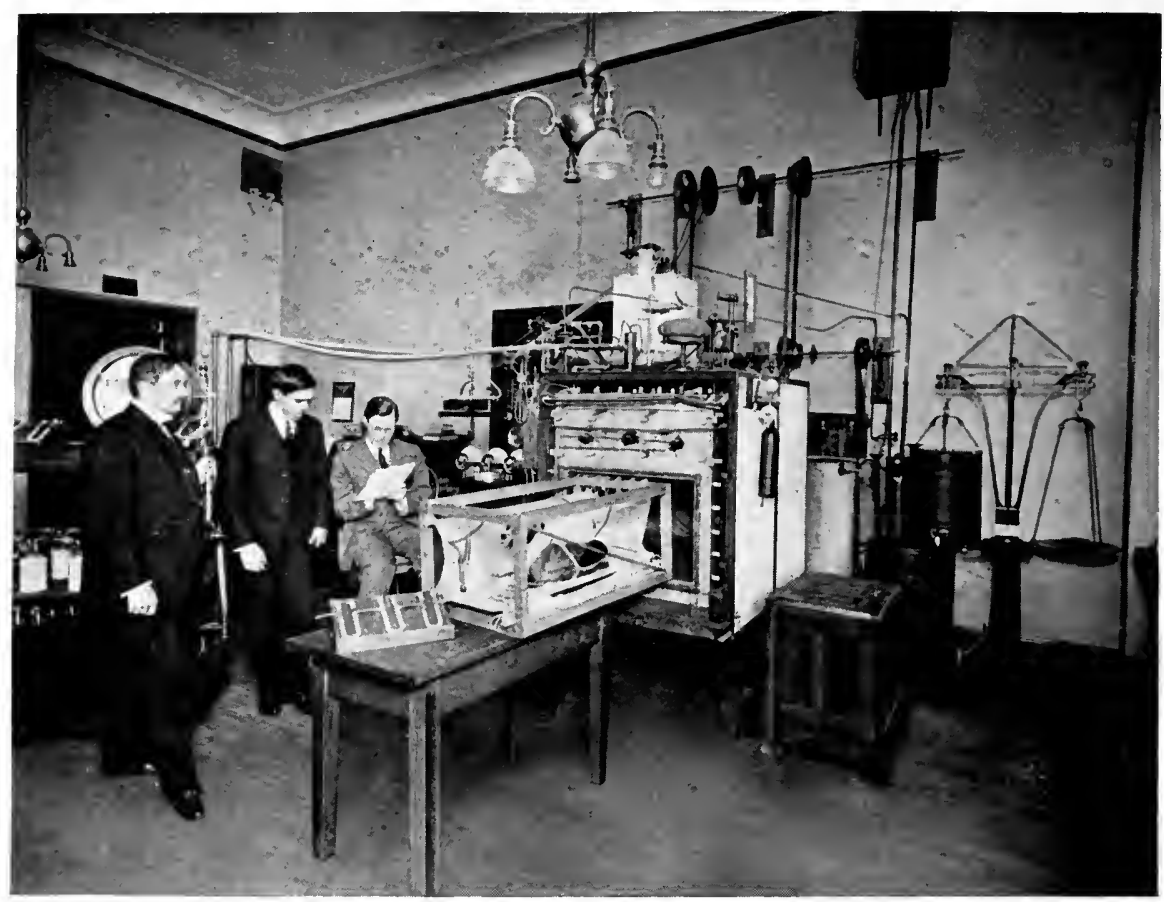

FIG. 19.-This figure shows a small respiration calorimeter built by H. B. Williams for the Physiological Laboratory, Cornell Medical College, New York City. A dog, wearing a bandage which holds a rectal thermometer in place, is shown lying on a cot suspended from a frame which may at any time be slid into the open chamber of the calorimeter. This accomplished, the front is then sealed. The animal respires within the chamber, the water and carbonic acid which he eliminates are removed by circulating the air through absorbing chemicals, and fresh oxygen is admitted automatically to replace the oxygen absorbed by the animal. The heat produced by the $\mathrm{dog}$ is removed by a current of water flowing through a system of pipes within the calorimeter.

lin $\left({ }^{9}\right)\left({ }^{10}\right)$ in Boston began a very extensive series of experiments with diabetics, using the small Benedict apparatus and the Atwater-RosaBenedict respiration calorimeter. This work was done in the Nutrition Laboratory of the Carnegie Institution of Washington, a splendidly equipped plant where extensive studies had already been made in the metabolism of normal subjects. In this same laboratory Carpenter and Murlin ( ${ }^{11}$ ) studied the metabolism of pregnant women and for the 


\section{CALORIMETRIC METHODS OF STUDY OF DISEASE 62I}

first time compared in clinical cases the methods of direct and indirect calorimetry.

Working in Lusk's laboratory and using the small calorimeter which was built for dogs, Howland $\left({ }^{12}\right)$ in I9I I established the fact that the methods of direct and indirect calorimetry agree closely in the case of infants. Under Lusk's direction two years later work with a larger

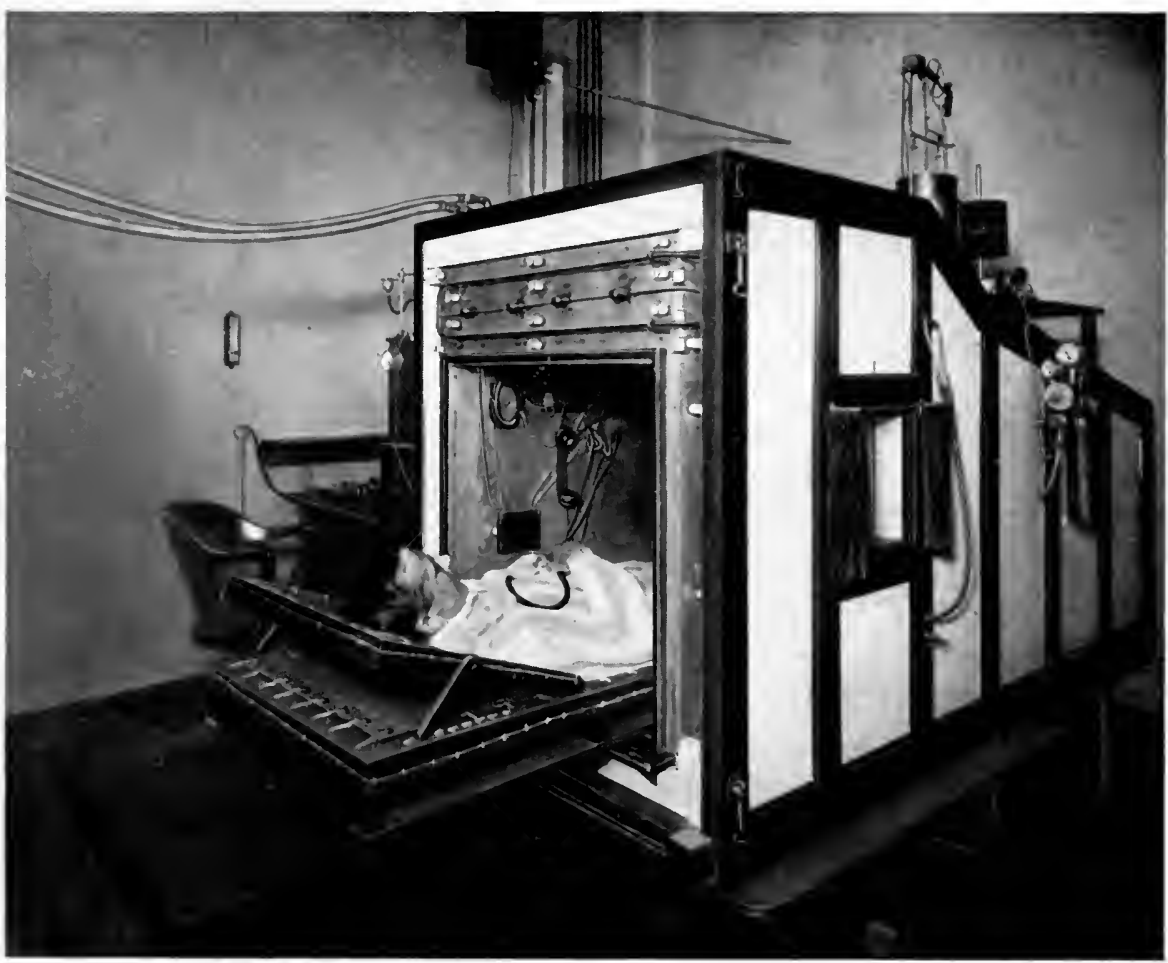

FIG. 20.-Calorimeter of the Russell Sage Institute of Pathology in Bellevue Hospital.

respiration calorimeter was begun by the Russell Sage Institute of Pathology $\left({ }^{13}\right)$ in a small room adjoining the medical wards of the Second Medical (Cornell) Division of Bellevue Hospital. (Figs. 20 and 2I.) This apparatus was constructed solely for the purpose of clinical calorimetry. The patients studied were kept in a specially organized metabolism ward where all food was weighed and all excreta saved for analysis by a corps of graduate nurses. A number of diseases were thus studied in detail under ideal conditions which have never been duplicated.

Since practically all clinical calorimetry has been performed in 


\section{CALORIMETRIC METHODS OF STUDY OF DISEASE}

Atwater-Rosa-Benedict respiration calorimeters, it may be well to describe this instrument briefly. The reader who is interested in the details of the technic and calculation will find an unusually clear exposition in Lusk's "Science of Nutrition" $\left({ }^{7}\right)$.

The calorimeter is a closed circuit absolutely shut off from the surrounding atmosphere. Everything eliminated by the subject is caught

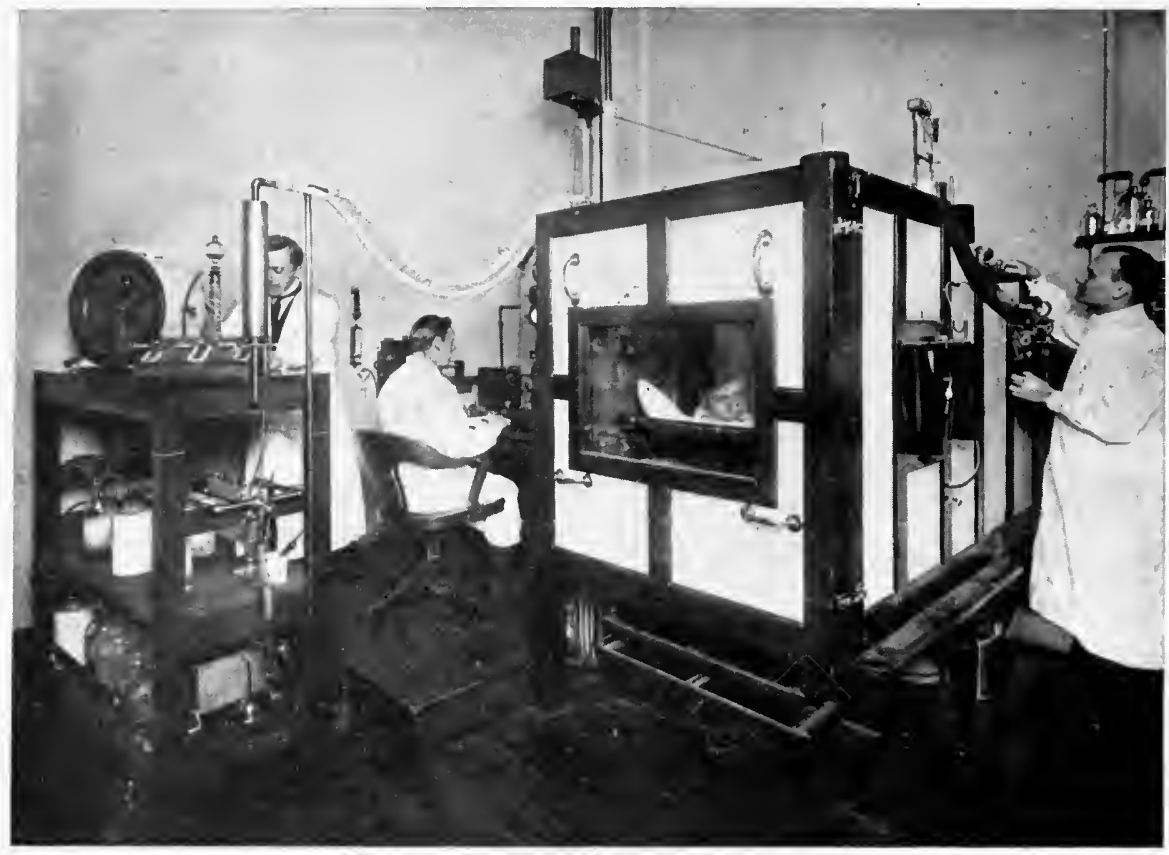

FIG. 2r.-Sage calorimeter. The patient has disobeyed the order to remain quiet and has turned to look at the camera. The observer at the left is taking a sample of the air in the box. The man seated at the table is reading the electrical thermometers. The man at the right is adjusting the device for admitting oxygen.

and measured. This is shown schematically in Fig. 22. The protecting wall is lined with cork. Next to this comes the outer copper wall, separated by an air space from the inner copper wall. By means of electrical thermometers, thermocouples, heating wires and cooling coils, the outer copper wall is maintained at exactly the same temperature as the inner wall. Therefore there is no tendency for heat to travel from one wall to the other. In a similar fashion, the air which enters the calorimeter is brought to exactly the same temperature as the air which leaves the box. All the heat of radiation and conduction is removed by means of a stream of water flowing at constant rate through pipes at the top of the chamber. The temperature of the water is measured 


\section{CALORIMETRIC METHODS OF STUDY OF DISEASE 623}

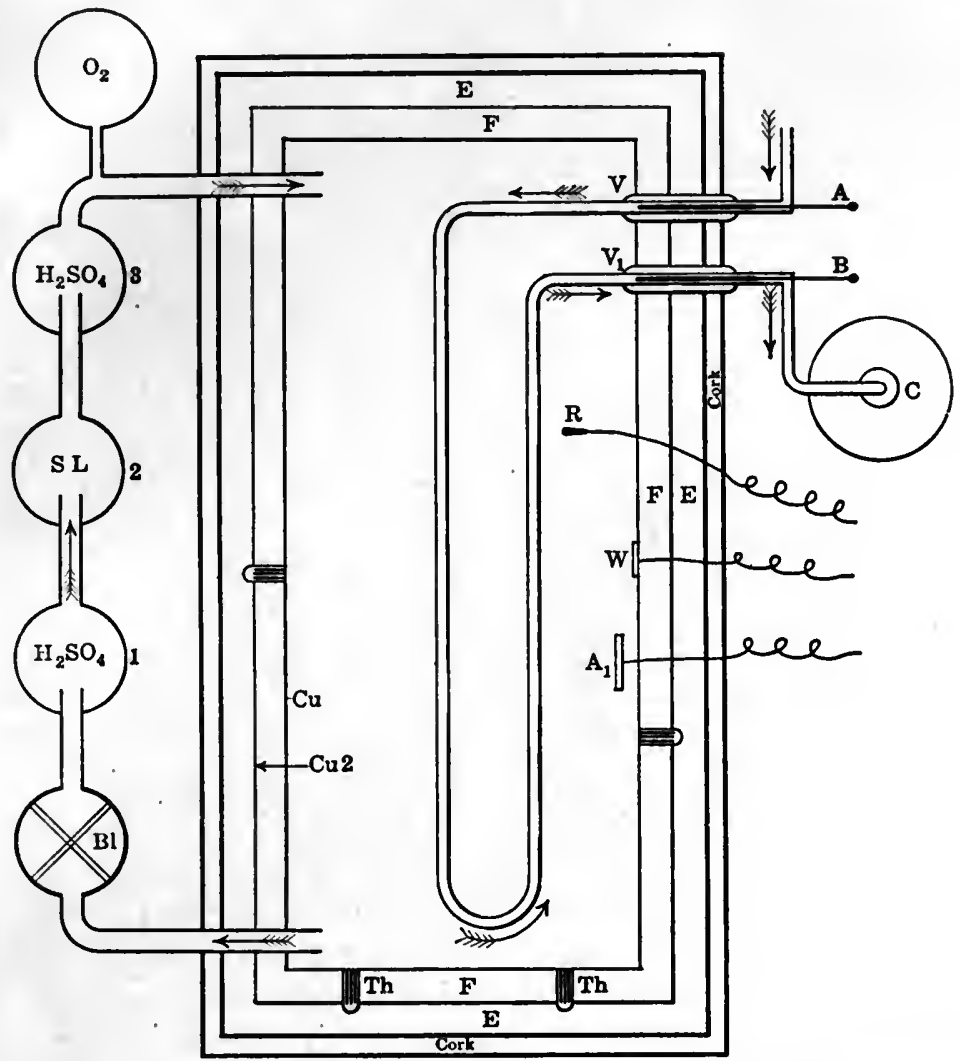

FIG, 22.-Schematic diagram of Atwater-Rosa-Benedict respiration calorimeter.

Ventilating System:

$\mathrm{O}_{2}$, Oxygen introduced as consumed by subject.

3, $\mathrm{H}_{2} \mathrm{SO}_{4}$, to catch moisture given off by soda-lime.

2 , Soda-lime to remove $\mathrm{CO}_{2}$.

$I, \mathrm{H}_{2} \mathrm{SO}_{4}$, to remove moisture given off by patient.

$B l$, Blower to keep air in circulation.

Indirect Calorimetry:

Increase in weight of $\mathrm{H}_{2} \mathrm{SO}_{4}$ :

(I) $=$ water elimination of subject.

Increase in weight of soda-lime:

(2) + increase in weight of $\mathrm{H}_{2} \mathrm{SO}_{4}$.

(3) $=\mathrm{CO}_{2}$ elimination.

Decrease in weight of oxygen tank = oxygen consumption of subject.

Heat-Absorbing System :

$A$, Thermometer to record temperature of ingoing water.

$B$. Thermometer to record temperature of outgoing water.
$V$, Vacuum Jacket:

$C$, Tank for weighing water which has passed through calorimeter each hour. $W$, Thermometer for measuring temperature of wall.

$A_{1}$, Thermometer for measuring temperature of the air.

$R$, Rectal thermometer for measuring temperature of subject.

Direct Calorimetry :

Average different of $A$ and $B \times$ liters of water $+(\mathrm{gm}$. water eliminated $\times 0.586$ ) \pm (change in temperature of wall $\bar{x}$ hydrothermal equivalent of box) \pm (change of temperature of body $X$ hydrothermal equivalent of body) $=$ total calories produced.

$T h$, thermocouple; $C u$, inner copper

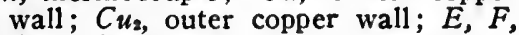
dead air spaces. 


\section{CALORIMETRIC METHODS OF STUDY OF DISEASE}

as it enters and as it leaves the calorimeter and the total water for each experimental period. is caught and weighed. - If we multiply the weight of water in kilograms by the average rise in temperature we obtain the calories of radiation and conduction. The calories lost in the vaporization of water are determined by multiplying the grams of water given off by the patient by the figure 0.584 , which represents the heat rendered latent by the vaporization of one gram of water at a temperature of $23^{\circ} \mathrm{C}$. The rectal temperature of the patient is measured by an electrical thermometer and the amount of heat stored in or lost from the body is calculated from the body weight and specific heat.

The air in the chamber is used over and over again but is kept pure by circulation through a system of absorber bottles. A powerful blower draws the air from the foot of the box, drives it through the absorbers, and then returns a mixture of dry oxygen and nitrogen to the head of the box. The first absorber contains concentrated sulphuric acid and removes all water vapor; the next contains soda-lime which removes all carbon dioxid; the third contains sulphuric acid to catch the moisture from the damp soda-lime. These are all weighed before and after each period, the gain in weight of the first absorber giving the water of evaporization, the combined gain in weight of the soda-lime and the last sulphuric acid bottle giving the carbon dioxid.

Meanwhile, the subject has been absorbing oxygen from the air, causing a shrinkage of volume of the contents of the calorimeter. This is compensated by an automatic device which admits oxygen from a weighed steel flask exactly as it is consumed. By weighing this flask again at the end of the period it is possible to determine with nicety the oxygen consumption of the subject. The principles of the technic are fairly simple, but in practice it is necessary to make a number of corrections for changes in temperature, barometer, residual air in the calorimeter, heat lost or gained from objects in the calorimeter, etc. The average experiment requires two or three observers and involves about 40 weighings, 500 temperature readings, and the writing of more than 4,000 figures. The accuracy of the apparatus is checked at frequent intervals by burning known amounts of alcohol and determining the error of the individual measurements. For instance, in the season of 1915-16 the average of such errors of the Sage calorimeter $\left({ }^{14}\right)$ was as follows:

$\begin{array}{lcr}\text { Heat } & \text { (Direct Calorimetry) } & +0.63 \\ \text { Oxygen } & \text { (and Indirect Calorimetry) } & +0.25 \\ \text { Carbon dioxid } & & -0.42 \\ \text { Water } & & +2.5 \\ \text { Respiratory Quotient } 0.665 \text { (Theoretical for alcohol } 0.6666 \text { ) }\end{array}$




\section{CALORIMETRIC METHODS OF`STUDY OF DISEASE 625}

In determinations upon men water shows the largest error on account of the moisture held in the subject's clothing. All the other figures are surprisingly accurate.

The management of patients to be studied in the calorimeter requires special attention. They must be selected with some care as to intelligence and disposition and must not be put into the apparatus until they have confidence in the nurses and physician in charge. As a rule they respond quickly to the good food and extra attentions received in the metabolism ward. Then they are given a short dummy experiment, a sort of dress rehearsal which removes all apprehension. Early on the morning of the observation the patient receives a cup of black coffee and perhaps a slice of bread and is put in the calorimeter about ten o'clock. For the next three hours he lies quietly in bed turning over on his side once or twice an hour, looking out of the window, perhaps reading, but most of the time vegetating mentally. A normal man under these conditions is intensely bored after a couple of hours and resents being confined to bed. Patients make much better subjects, since the calorimeter is for them a diversion. They are in no way uncomfortable and, if care be used, patients who are seriously ill can be studied without harm. As a matter of fact, during the period of experimentation they are under closer observation than at any other time in their lives. The pulse is counted frequently, the temperature read every four minutes, and each movement of the body recorded on a smoked drum.

The respiration calorimeter makes two different sets of determinations, one using chemical and the other physical methods. We shall discuss first the chemical method of indirect calorimetry and show how it is possible to calculate a man's heat production if we know during a given experimental period the amount of oxygen absorbed, the carbon dioxid given off by the lungs, and the nitrogen of the urine.

When we take a chemical substance, such as starch, and oxidize it, the process may be expressed as follows:

$$
\mathrm{C}_{6} \mathrm{H}_{12} \mathrm{O}_{6}+6 \mathrm{O}_{2}=6 \mathrm{CO}_{2}+6 \mathrm{H}_{2} \mathrm{O}
$$

Carbohydrates, as their name indicates, contain just enough oxygen to combine with the hydrogen in the molecule and form water. This means that the volume of oxygen absorbed in the above reaction will equal the volume of carbon dioxid produced. In other words, the respiratory quotient $\frac{\mathrm{CO}_{2}}{\mathrm{O}_{2}}=1.00$. Fat and protein do not contain enough oxygen to combine with the hydrogen in their molecules, so they must absorb some of this gas to form water in addition to the oxygen required to produce carbon dioxid. Since fat and protein have fairly definite compositions, it is possible to calculate the oxygen absorbed and carbon 


\section{CALORIMETRIC METHODS OF STUDY OF DISEASE}

dioxid produced when a gram of each substance is oxidized, thus obtaining the respiratory quotients. We can also burn these substances in a bomb calorimeter and obtain the heat of combustion. By simple mathematics we can then find out how much heat must be liberated when a liter of oxygen is used in the oxidation of fat or protein or starch (Table XII).

TABLE XII

Oxidation of FoodstufFs

\begin{tabular}{|c|c|c|c|c|c|c|}
\hline \multirow{2}{*}{$\begin{array}{l}\text { I Gram } \\
\text { Substance }\end{array}$} & \multirow{2}{*}{$\begin{array}{c}\mathrm{O}_{2} \\
\text { Absorbed } \\
\text { c.c. }\end{array}$} & \multirow{2}{*}{$\begin{array}{l}\mathrm{CO}_{2} \\
\text { Formed } \\
\text { c.c. }\end{array}$} & \multirow{2}{*}{ R. Q. } & \multirow{2}{*}{ Calories } & \multicolumn{2}{|c|}{ Calories Computed from } \\
\hline & & & & & I Liter $\mathrm{O}_{2}$ & I Liter $\mathrm{CO}_{2}$ \\
\hline Protein ... & 966.3 & 773.9 & $0.8 \mathrm{or}$ & 4.316 & 4.485 & 5.579 \\
\hline Fat $\ldots . . .$. & $2,019.3$ & $1,427.3$ & 0.707 & $9.46 \mathrm{I}$ & 4.686 & 6.629 \\
\hline Starch .... & 828.8 & 828.8 & 1.000 & 4.182 & 5.047 & 5.047 \\
\hline
\end{tabular}

We also know that, for each roo grams of meat protein oxidized, I6.28 grams of nitrogen will appear in the urine. This enables us to calculate how much protein has been metabolized in an experimental period and to determine the grams of oxygen (grams $\mathrm{N} \times 8.45$ ) absorbed and the carbon dioxid (grams $\mathrm{N} \mathrm{x} 9.35$ ) produced in the process. Subtracting these figures from the totals, we have left the carbon dioxid and oxygen of carbohydrate and fat. If the respiratory quotient $\frac{\mathrm{CO}_{2}}{\mathrm{O}_{2}}$ of this residuum be 1.00 , we know that carbohydrate is being oxidized; if the respiratory quotient be 0.707 , fat is indicated. Except in rare instances the figure for the non-protein quotient is between these points and it is possible to calculate exactly the ratio between the two. We have already found the protein calories and the total non-protein calories. Knowing the ratio between the fat and carbohydrate it is possible to calculate the actual grams of fat and carbohydrate metabolized during the experiment.

The importance of this method of study is obvious to the clinician. One can give a diabetic an experimental meal and determine just how much carbohydrate he oxidizes each hour. One can study patients with various diseases and find out exactly what the tissues are metabolizing under various conditions. The respiration apparatus is a sort of metabolic X-ray capable of taking serial pictures of function rather than of structure.

Meanwhile, the calorimeter has been making a set of physical measurements and determining the heat production by the method of direct 
calorimetry. The human body gives off to the surrounding air about three-quarters of the total heat eliminated by means of radiation and conduction, one-quarter is lost in the vaporization of water from skin and lungs. Some is lost in warming cold food and drink and eliminating their end products in urine, feces, and expired air. If the average body temperature changes considerably, the heat stored in or lost from the tissues may be large in proportion to the total produced. For instance, we can take the following examples:

Subject A. Period of I Hour. Weight, 70 kg. Temperature Rising

Radiation and conduction

Vaporization

Food and urine

Total heat eliminated

Plus heat stored in body

Total heat produced
$43.020 \mathrm{~kg}$. water flowing through calorimeter raised an average of $2.15^{\circ} \mathrm{C}$.

14.0 $23.9 \mathrm{gm}$. water from skin and lungs multiplied by 0.584 (latent heat of vaporization)

3.4 Drank $200 \mathrm{gm}$. water at $20^{\circ} \mathrm{C}$. Voided $200 \mathrm{gm}$. urine at $37^{\circ} \mathrm{C}$.

Temperature rose $0.2^{\circ} \mathrm{C} .70 \mathrm{~kg}$. body equivalent to $58 \mathrm{~kg}$. water

Subject A. Temperature Faling

Radiation and conduction

Vaporization

Total heat eliminated

Minus heat lost from body

Total heat produced

$\begin{array}{ll}63.2 & 20 \mathrm{~kg} . \text { water raised } 3.15^{\circ} \mathrm{C} . \\ \frac{20.4}{83.6} & 34.8 \mathrm{gm} . \text { water } \times 0.586 \\ \frac{1 \mathrm{I} .6}{72.0} & \text { Temperature fell } 0.2^{\circ} \mathrm{C} .\end{array}$

These calculations are based on the assumption that the specific heat of the body is 0.83 , a figure that has been used for many decades without satisfactory experimental support. Water, which is the unit, has the specific heat of 1.00 . Lean flesh gives a figure of 0.83 ; fat, 0.45 ; compact bone, 0.30 . It is obvious that the specific heat of an edematous man would be much greater than that of a fat man of the same weight, and that it would therefore require more calories to raise his body temperature an equal number of degrees. At the present time, however, we are obliged to use the standard figure of 0.83 for all cases, since we cannot very well analyze our subjects before each experiment.

The calorimeter has introduced a new method of determining the average changes in body temperature $\left({ }^{15}\right)$. We know that with a carefully tested instrument the methods of direct and indirect calorimetry agree closely. We can therefore determine a man's heat production by the indirect method and his heat elimination by the direct method. The difference between these represents the amount of heat stored in or lost from the body. Knowing the body weight and approximate specific heat, we can calculate the rise or fall of the average body temperature. Experiments in malarial and other fevers have shown that the rectal 


\section{8}

temperature does not always represent the average changes throughout the body. Every clinician has at times noticed that, with a rising rectal temperature, there may be a distinct cooling of the feet and hands. We must not forget that a man who weighs 70 kilograms has a surface area

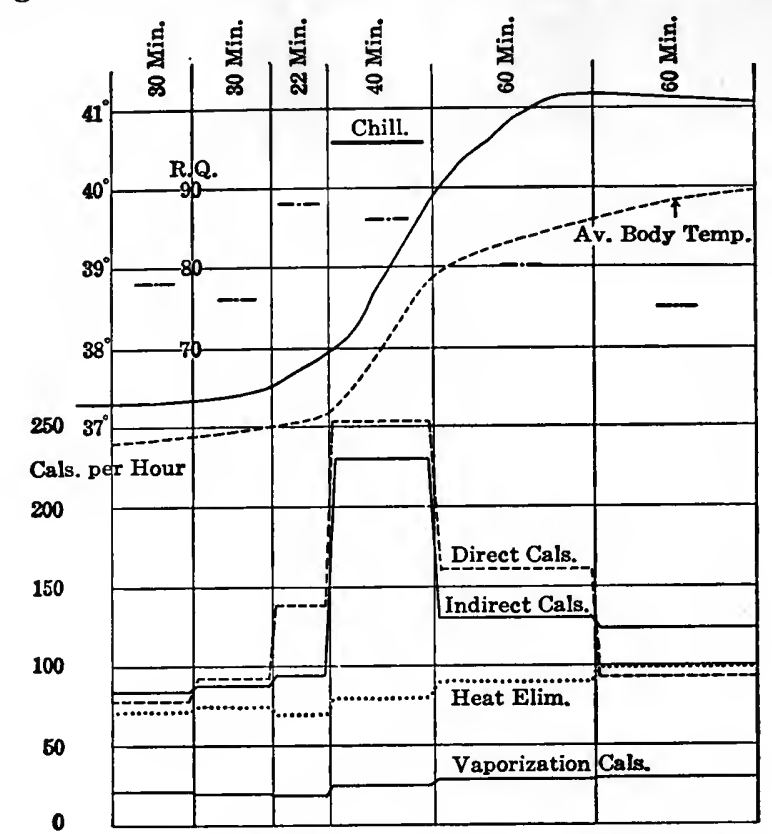

Fig. 23.-Changes in Metabolism Caused by a Malarial Chill.

The curves show the relationship of heat production and heat elimination before, during, and after a malarial chill. The uppermost line shows the changes in rectal temperature. The accompanying dash line shows the changes in the average body temperature. The middle dash line represents the heat production in calories as measured by the direct method; the solid line, the heat production by the indirect method; the dotted line, the heat eliminated, and the lowest solid line, the calories lost in the vaporization of water. The respiratory quotients are represented above the rectal temperature by dashes for each period.

of about I.8 square meters, and that I5 kilograms of tissue lies within $\mathrm{I} \mathrm{cm}$. of the surface. The temperature of this surface layer is intermediate between that of the deeper structures and the surrounding atmosphere. Also, the conduction of heat through this layer is variable, depending on the blood-flow. Curiously enough, the heat elimination may be exactly the same with a warm skin as with a cold skin. In the former case the blood from the interior is cooled at the surface of the body; in the latter case it is cooled by the structures a few centimeters beneath the surface. All of these points are illustrated by the above diagram (Fig. 23) showing the results of an observation on a patient with a malarial chill $\left({ }^{15}\right)$. 


\section{CALORIMETRIC METHODS OF STUDY OF DISEASE 629}

Two thirty-minute periods were studied before there was any significant rise in temperature. In the third period a distinct rise gave warning of the approaching paroxysm. In the fourth period the patient shivered violently and his heat production, which had been at the level of 86 calories an hour, rose to 230 calories. In forty minutes he stored about IOO calories within his body. After the chill his temperature continued to rise, but the rectal temperature rose more rapidly than the average body temperature. During the period of high but level rectal temperature the average for the body was still rising. Although the heat production had shown a sharp rise during the chill and a drop immediately afterwards, there was practically no change in the rate of heat elimination. This, in spite of the fact that the skin must have passed through all the changes that characterize a malarial paroxysm. Other experiments have shown the same phenomenon and have proved that the rise of temperature in malaria is due almost entirely to an increased heat production. The fall in temperature is due chiefly to a marked increase in heat elimination and especially vaporization. The heat production returns almost to normal soon after the chill.

The following case $\left({ }^{16}\right)$ illustrates the manner in which a patient with nephritis may eliminate more water through skin and lungs than through his kidneys (Table XIII) :

TABLE XIII

\begin{tabular}{|c|c|c|c|c|c|}
\hline & Lee $H$. & $\begin{array}{l}\text { Weight } \\
\text { kg. }\end{array}$ & $\begin{array}{l}\text { Urine Vol. } \\
\text { c.c. }\end{array}$ & $\begin{array}{c}\text { Fluid Intake } \\
\text { c.c. }\end{array}$ & $\begin{array}{l}\text { Water Vaporized } \\
\text { from Skin } \\
\text { and Lungs } \\
\text { c.c. per day }\end{array}$ \\
\hline Jan. & $5 \ldots \ldots \ldots$ & $\ldots$ & 260 & 1,278 & 514 \\
\hline$"$ & $6 \ldots \ldots \ldots$ & 61.5 & 240 & 980 & \\
\hline “ & $7 \ldots \ldots \ldots$ & $\cdots$ & 600 & 1,160 & \\
\hline “ & $8 \ldots \ldots \ldots$ & 60.7 & 940 & $\mathrm{I}, \mathrm{1} 40$ & " \\
\hline
\end{tabular}

In the four days the fluid intake was 4,558 c.c.; urine output, 2,040 c.c.; output of water through skin and lungs, 2,054 c.c.; according to the two-hour experiment on January 5. The positive balance of 464 c.c. to the body was more than counteracted by a slight diarrhea, and the patient was actually losing weight.

By means of calorimeter experiments it has been possible to compare the water elimination of a large number of normal men and patients dressed in pajamas, covered with a blanket, and exposed to a temperature of 22 to $25^{\circ} \mathrm{C}$. and a relative humidity of between 30 and 50 per cent. $\left({ }^{14}\right)$. Under such conditions normal men on an average excrete 


\section{CALORIMETRIC METHODS OF STUDY OF DISEASE}

through skin and lungs about 700 grams of water a day, losing in this manner 24 per cent. of the heat produced. Patients with high metabolism vaporize more water, but the increase is usually proportional to the total heat elimination. Of course, patients with temperatures falling sharply lose a much larger proportion through vaporization from the skin. The rather surprising uniformity found in a number of widely different conditions is shown in Table XIV.

\section{TABLE XIV}

Water Vaporization of Different Groups of Subjects Studied Under Standard Conditions of Clothing, Temperature, and Ventilation. SUmmary Table

\begin{tabular}{|c|c|c|}
\hline Group of Subjects & $\begin{array}{l}\text { Number of } \\
\text { Experiments } \\
\text { in Group }\end{array}$ & $\begin{array}{l}\text { Percentage of } \\
\text { Calories Lost in } \\
\text { Vaporization }\end{array}$ \\
\hline 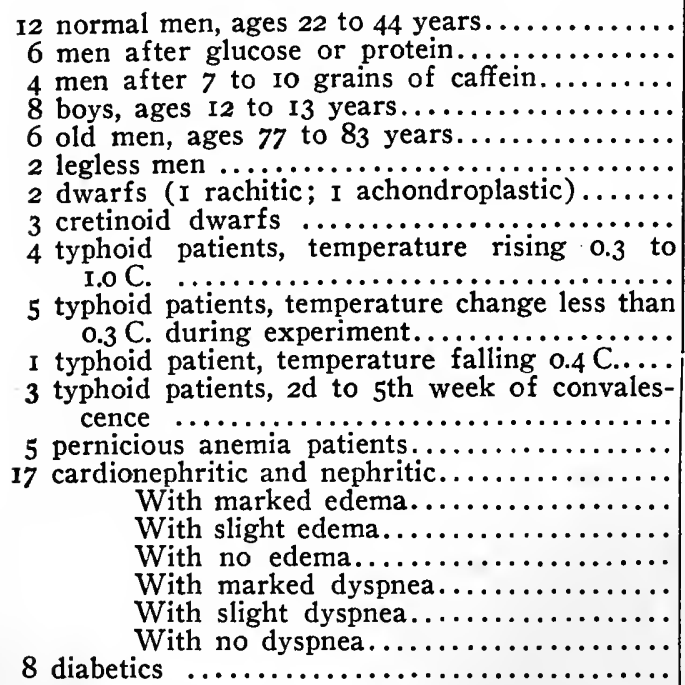 & $\begin{array}{r}29 \\
9 \\
5 \\
8 \\
6 \\
2 \\
2 \\
4 \\
9 \\
6 \\
6 \\
1 \\
9 \\
9 \\
24 \\
8 \\
5 \\
8 \\
6 \\
10 \\
8 \\
30\end{array}$ & $\begin{array}{l}24 \\
23 \\
26 \\
27 \\
27 \\
25 \\
23 \\
22 \\
22\end{array}$ \\
\hline
\end{tabular}

Perhaps the most useful function of the calorimeter in clinical medicine has been to demonstrate that the methods of direct and indirect calorimetry, on the whole, agree closely in pathological cases. There has been a tendency for investigators to make rather wild speculations when the results of respiration experiments with patients do not seem to follow the laws established with animals and normal men. They assume abnormal reactions or storages of substances or even hint that the law of conservation of energy may be distorted in disease. The calorimeter has shown that the ordinary laws of nutrition will explain the processes in disease with the possible exception of certain phases of severe diabetes. The law of conservation of energy applies just as 


\section{CALORIMETRIC METHODS OF STUDY OF DISEASE 63I}

strongly in the clinic as elsewhere. Every new theory of metabolism must have its pluses and minuses balanced as carefully as the books of a banking house.

Owing to technical difficulties, the calorimeter does not always give close agreements between the methods of direct and indirect calorimetry in short periods or small groups of experiments. A slight error is introduced when a patient is taken from a cool ward and placed in a warm respiration chamber, and there is a tendency towards making the direct measurement a trifle too low. This will be seen in Table XV.

TABLE XV

Groups of Subjects Studied in the Sage Calorimeter Comparison of Direct and Indirect Methods

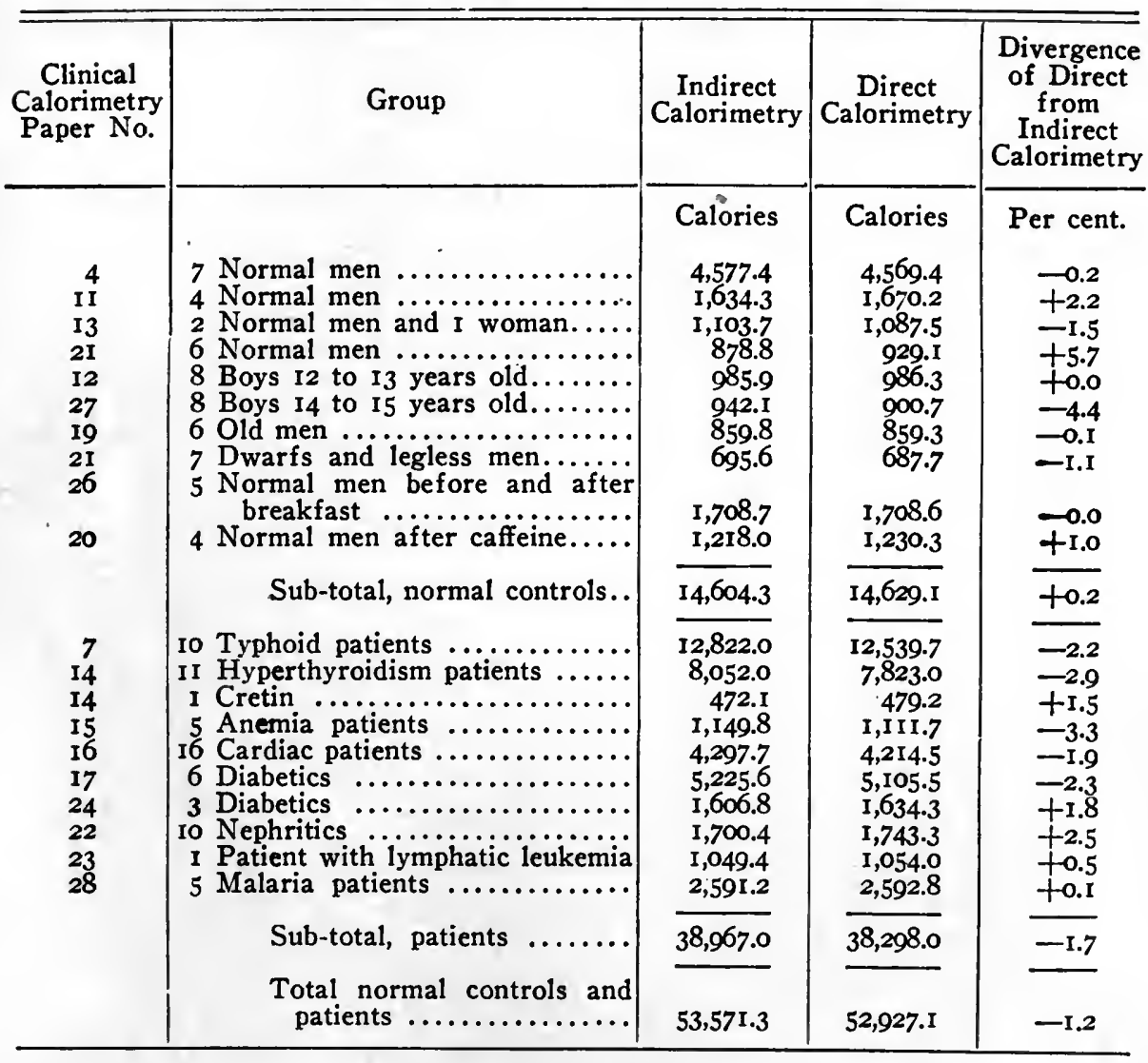

A classical demonstration of the possibilities of the calorimeter was made by Benedict $\left({ }^{17}\right)$ in the study of a man who fasted 3 I days, taking nothing but distilled water. In this case the data obtained before the 
fast were used as the basis of comparison. The method of indirect calorimetry demonstrated a marked reduction in total metabolism. A diminution in the water of vaporization was also shown. The respiratory quotients proved that practically all carbohydrate was exhausted by the fifth day and that subsequently protein furnished I4 to 20 per cent. of the calories, and fat the remainder. Table XVI gives a few of the many analyses made in this investigation, which has thrown much light on normal and pathological conditions.

TABLE XVI

Benedict's Fasting Man

Subject L. Height, $170.7 \mathrm{~cm}$. Only distilled water was taken during this fast.

\begin{tabular}{|c|c|c|c|c|}
\hline & \multicolumn{4}{|c|}{ Day of Fasting } \\
\hline & Ist & IIth & 2rst & 3rst \\
\hline 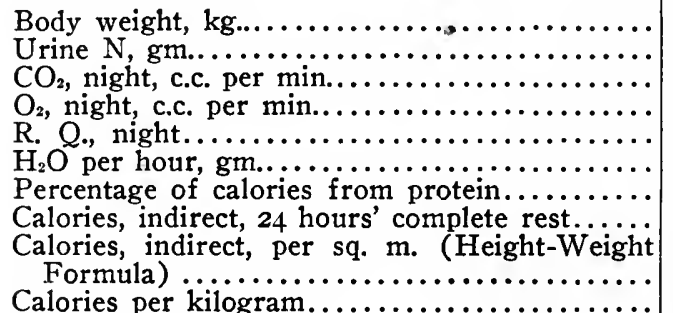 & $\begin{array}{l}59.60 \\
7.10 \\
165 . \\
212 . \\
0.78 \\
22.8 \\
10.6 \\
1,441 . \\
843 . \\
24.2\end{array}$ & $\begin{array}{l}53.88 \\
10.25 \\
\text { I28. } \\
\text { I76. } \\
0.72 \\
18.3 \\
19.6 \\
\text { I,I93. } \\
732 . \\
\text { 22. }\end{array}$ & $\begin{array}{l}50.49 \\
7.93 \\
\text { r12. } \\
\text { r54. } \\
0.73 \\
14.6 \\
16.5 \\
\text { I,032. } \\
653 . \\
20.4\end{array}$ & $\begin{array}{c}47.39 \\
6.94 \\
\text { Ir5. } \\
\text { 160. } \\
0.72 \\
17.9 \\
14.4 \\
1,072 . \\
701 . \\
22.6\end{array}$ \\
\hline
\end{tabular}

* Previous day 1,025 calories.

$\dagger$ Previous day 66r calories.

Unfortunately, in most clinical studies it is impossible to obtain data on the patient after complete recovery from his illness. This brings us to the question of how we shall handle the figures obtained in calorimeter experiments in order to demonstrate the changes caused by the pathological factor under investigation. In this, as in all other research, the normal control is the most important consideration. Quite recently a large number of respiration experiments have been made on normal men, giving us information as to the factors of age, sex, height, and weight. Magnus-Levy, Benedict, and many others have made comparisons of the oxygen consumption per minute of the patient and a group of normal controls of the same height and weight. This method cannot be criticized if the group of normals be large enough to rule out the variation of plus or minus ten per cent. found quite often in normals. Another method devised for use in the clinic has been to compare the results in terms of calories per square meter of body surface. Rubner has shown that with animals of varying sizes the heat production is roughly pro- 
portional to the surface area. The Sage investigators have devised a rapid and fairly accurate method for determining surface area from height and weight $\left({ }^{18}\right)$ and have constructed a curve showing the calories per square meter per hour for people of different ages $\left({ }^{10}\right)\left({ }^{20}\right)$, finding that 86 per cent. of normals are within ro per cent. of this line. The results in pathological cases are referred to this curve and expressed in percentages above or below the normal, much as one would speak of hemoglobin or McLean index.

Very recently Harris and Benedict $\left({ }^{21}\right.$ ) have published another set of standards in which the normal metabolism of an individual can be calculated from his height, weight, and age, according to certain factors determined by statistical methods.

The importance of the normal standard and the difficulty of calculating the results obtained in a group of pathological cases is best illustrated by a concrete example. In I9I2 Benedict and Joslin $\left({ }^{10}\right)$ studied a number of patients with severe diabetes in a respiration calorimeter and used the group method of comparing the results with the normal. They found that the oxygen consumption of the diabetics averaged 2I per cent. higher than the normals. In r9I6 Allen and $\mathrm{Du}$ Bois $\left({ }^{22}\right)$ recalculated these same results according to the surface area standards and obtained figures indicating that diabetics averaged only 3 per cent. higher than the normals. In I9I7 Lusk ( $\left.{ }^{7}\right)$, using a similar method, estimated the increased metabolism of this group of diabetics at II per cent. In I919 Harris and Benedict $\left({ }^{21}\right)$, using their new biometric standard, found that the patients with severe diabetes gave an average metabolism Ii.55 per cent. higher than the normal.

In a severe case of diabetes mellitus the calorimeter will furnish a wealth of detail regarding the processes of metabolism, when aided by careful urinary analyses. This is shown in the following table made from results obtained with a patient, Cyril K., nineteen years old $\left({ }^{23}\right)$. He was brought to Bellevue Hospital with the most severe type of complete diabetes, his $D: N$ ratio showing that he was excreting in the urine all the glucose which could be derived from the protein molecule. It was also possible to show that he was excreting in the urine all the beta-oxybutyric acid which could be formed as an end product of the fat metabolized on December I5 and I6. After treatment by alkalies, low diet, and fasting he recovered from his dangerous acidosis and regained his tolerance for carbohydrates so that in the course of two months he was able to derive 62 per cent. of his calories from carbohydrate. It is interesting to note that the metabolism, which was slightly higher than the normal when he was first observed, dropped far below the average during the period of undernutrition. 
TABLE XVII

Metabolism of Patient with Severe Diabetes (Cyril $\mathrm{K}$.)

\begin{tabular}{|c|c|c|c|c|c|c|c|c|}
\hline \multirow{2}{*}{ Period } & \multirow{2}{*}{ Date } & \multirow{2}{*}{$D: N$} & \multirow{2}{*}{ R.Q } & \multicolumn{3}{|c|}{$\begin{array}{l}\text { Percentage of } \\
\text { Calories from }\end{array}$} & \multirow{2}{*}{$\begin{array}{c}\text { Total } \\
\text { Metabolism } \\
\text { Variation } \\
\text { from } \\
\text { Average } \\
\text { Normal }\end{array}$} & \multirow{2}{*}{$\begin{array}{c}\text { Urine } \\
\text { Beta- } \\
\text { oxybut. } \\
\text { Acid }\end{array}$} \\
\hline & & & & Protein & Fat & $\begin{array}{l}\text { Carbo- } \\
\text { hydrate }\end{array}$ & & \\
\hline & 1915 & & & & & & & gm. \\
\hline I. First fasting..... & Dec. 8-10 & 2.7 & .... & .. & .. & .. & ... & 39.6 \\
\hline 2. Mixed diet $\ldots \ldots \ldots$ & $“$ II-I4 & I.9 & $\dddot{\cdots}$ & $\because$ & 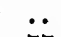 & .. & $\cdots$ & 56.5 \\
\hline 3. High protein-fat ... & “ $15-17$ & 3.9 & 0.700 & 23 & 77 & 0 & $-\mathrm{II}$ & 77.8 \\
\hline 4. Second fasting $\cdots$ & “ I8-2I & 2.7 & 0.714 & I8 & 8I & I & -2 & 45.7 \\
\hline (Interval 2 months) & " 22 & I.I & 0.734 & 20 & 74 & 6 & -10 & 10.9 \\
\hline 6. Temporary recovery & Feb. Io & 0.0 & 0.915 & $3 \mathbf{I}$ & 7 & 62 & -36 & 0.0 \\
\hline
\end{tabular}

The respiration calorimeter will never be used in many clinics on account of the cost and the difficulty of technic. For practical purposes almost all the information needed can be obtained by means of simpler respiration apparatus, using the method of indirect calorimetry, since it has been shown that this gives results identical with the direct method. It must be recognized, however, that the indirect method does not tell the whole story. The man who wishes to obtain the proper conception of any disease must consider the wealth of information furnished by the respiration calorimeter.

\section{BIBLIOGRAPHY}

I. OTT, ISAAC: Fever, Its Thermotaxis and Metabolism. New York, I9I4.

2. LIKATSCHEFF, A. A., and AVROROFF, P. P.: Investigations of Gaseous and Heat Exchange in Fevers. Reports of the Imperial Military Academy, St. Petersburg, I902, V, Pts. 3 and 4. (Abstracted in OTT: Fever.)

3. PETTENKOFER, M. J.: Ann. d. Chem. u. Pharmakol., I862, Supplement 2.

4. ATWATER, W. O., and BENEDICT, F. G.: A Respiration Calorimeter with Appliances for the Direct Determination of Oxygen, Pub. 42, Carnegie Institution of Washington, 1905.

5. BENEDICT, F. G., and CARPENTER, T. M.: Respiration Calorimeter for Studying the Respiratory Exchange and Energy Transformations of Man, Pub. 123, Carnegie Institution of Washington, Igro.

6. RUBNER, M.: Kalorimetrie, Tigerstedt's Handbuch der physiologischen Methodik, Leipzig, IgI I, I, Pt. 3, p. 150.

7. LUSK, G.: Elements of the Science of Nutrition, 3rd ed., Philadelphia, I9I7. 
8. CARPENTER, T. M.: Pub. 216, Carnegie Institution of Washington, 1915.

9. BENEDICT, F. G., and JOSLIN, E. P.: Metabolism in Diabetes Mellitus, Pub. 136, Carnegie Institution of Washington, 1910.

10. BENEDICT, F. G., and JOSLIN, E. P.: A Study in Metabolism in Severe Diabetes, Pub. 176, Carnegie Institution of Washington, I912.

I1. CARPENTER, T. M., and MURLIN, J. R.: Arch. Int. Med., I9I I, VII, i84.

12. HOWLAND, JOHN : Hoppe-Seyler's Ztschr. f. physiol. Chem., Igri, LXXIV, I.

13. LUSK, G.: Arch. Int. Med., I915, XV, 793. (See also RICHE, J. A., and SODERSTROM: Ibid., I9I5, XV, 805, and GEPHART, F. C., and DU BOIS, E. F.: Ibid., I915, XV, 829.)

14. SODERSTROM, G. F., and DU BOIS, E. F.: Arch. Int. Med., 1917, XIX, 931.

15. BARR, D. P., and DU BOIS, E. F.: Arch. Int. Med., 1918, XXI, 627.

16. AUB, J. C., and DU BOIS, E. F.: Arch. Int. Med., I9I7, XIX, 865.

17. BENEDICT, F. G.: A Study of Prolonged Fasting, Pub. 203, Carnegie Institution of Washington, I9I5.

18. DU BOIS, D., and DU BOIS, E. F.: Arch. Int. Med., I916, XVII, 863.

19. DU BOIS, E. F.: Arch. Int. Med., I916, XVII, 887.

20. AUB, J. C., and DU BOIS, E. F.: Arch. Int. Med., I917, XIX, 823.

21. HARRIS, J. A., and BENEDICT, F. G.: Scientific Monthly, 1919, VIII, 385.

22. ALLEN, F. M., and DU BOIS, E. F.: Arch. Int. Med., IgI6, XVII, IoIo.

23. GEPHART, F. C., AUB, J. C., DU BOIS, E. F., and LUSK, G.: Arch. Int. Med., I9I7, XIX, 9I5. 



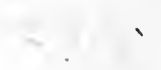





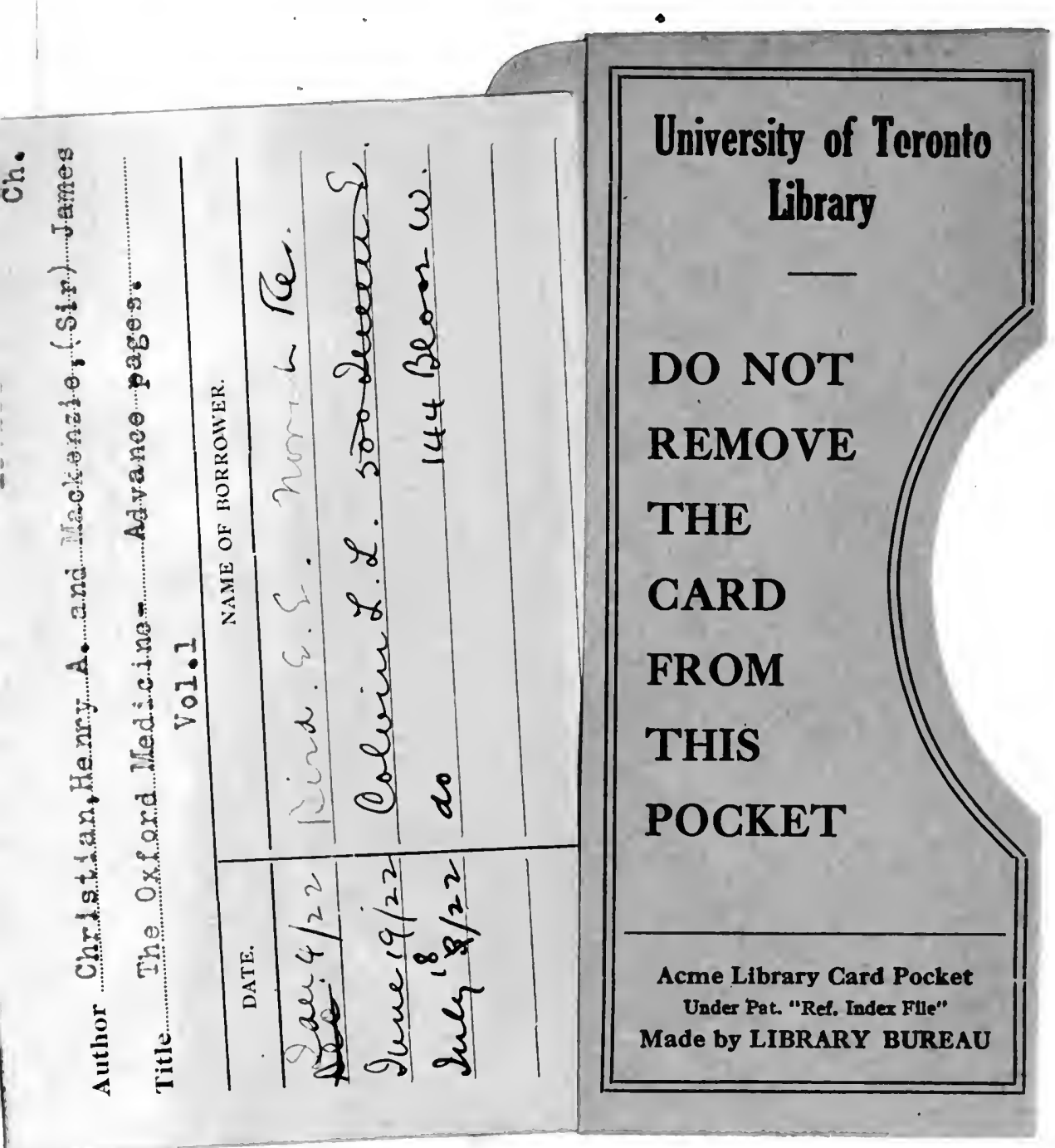


